\title{
Stereodivergent Synthesis of Enantioenriched 2,3-Disubstituted Dihydrobenzofurans via One-Pot C-H Functionalization/Oxa-Michael Addition Cascade
}

\author{
Dong-Xing Zhu, Jian-Guo Liu, and Ming-Hua $\mathrm{Xu}^{*}$ \\ Shenzhen Grubbs Institute and Department of Chemistry, Guangdong Provincial Key Laboratory of \\ Catalysis, Southern University of Science and Technology, 1088 Xueyuan Boulevard, Shenzhen \\ 518055, China and State Key Laboratory of Drug Research, Shanghai Institute of Materia Medica, \\ Chinese Academy of Sciences, 555 Zuchongzhi Road, Shanghai 201203, China
}

\section{Table of Contents}

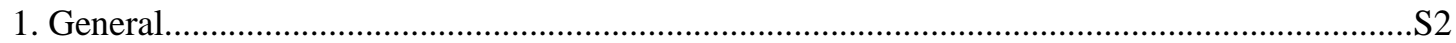

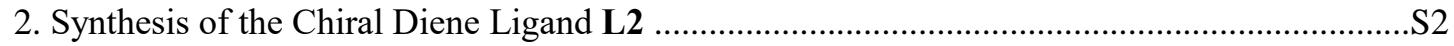

3. General Procedure for C-H Functionalization/Oxa-Michael Addition Cascade...........................S4

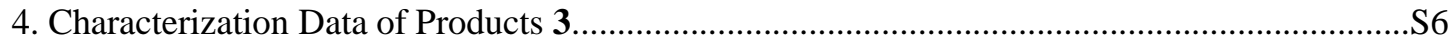

5. Determination of the Diastereoselectivity Ratio and Enantiomeric Excess Values...................S18

6. Optimization of Reaction Conditions for Stereodivergent Synthesis of 3,4-Disubstituted

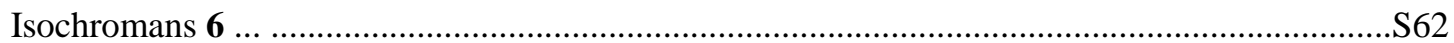

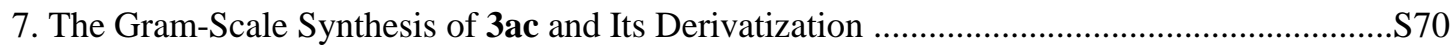

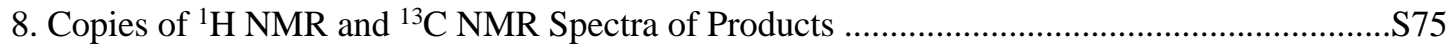




\section{General}

All anaerobic and moisture-sensitive manipulations were carried out with standard Schlenk techniques under predried argon. Solvents were dried and distilled by standard procedures. NMR spectra were recorded on a Varian spectrometer $\left(400 \mathrm{MHz}\right.$ for ${ }^{1} \mathrm{H}, 100 \mathrm{MHz}$ or $125 \mathrm{MHz}$ for ${ }^{13} \mathrm{C}$ ). Chemical shifts are reported in $\delta \mathrm{ppm}$ referenced to an internal $\mathrm{SiMe}_{4}$ standard for ${ }^{1} \mathrm{H} \mathrm{NMR}$ and chloroform- $d(\delta 77.36)$ for ${ }^{13} \mathrm{C}$ NMR. Optical rotations were measured on a Perkin-Elmer $241 \mathrm{MC}$ polarimeter. HPLC was performed on a JASCO 2000 instrument by using Daicel columns with 2-propanol/hexane as the eluent.

\section{Synthesis of the Chiral Diene Ligand L2}
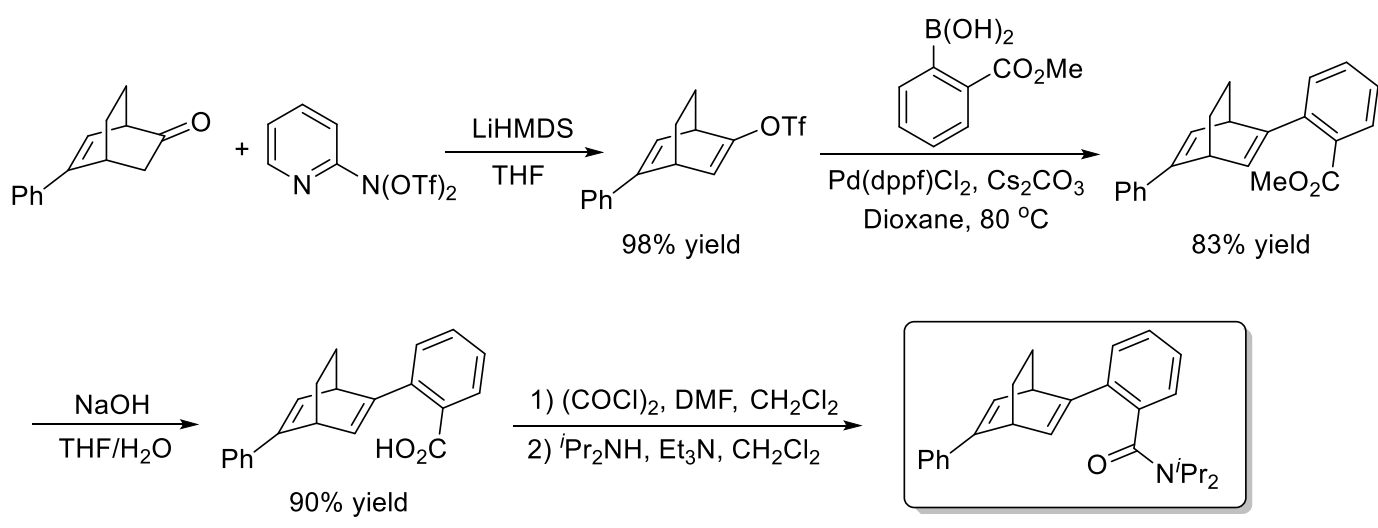

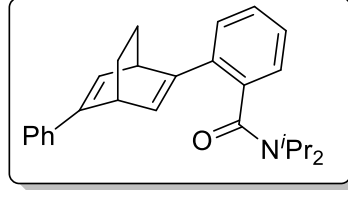

L2, 92\% yield

To a solution of $(1 R, 4 R)$-5-phenylbicyclo[2.2.2]oct-5-en-2-one (792 mg, 4 mmol, 1 equiv) and 2-[N,N-bis(trifluoromethyl(sulfonyl)amino]pyridine (2.86 g, $8 \mathrm{mmol}, 2$ equiv) in THF in -20 ${ }^{\circ} \mathrm{C}$ was added Lithium bis(trimethylsilyl)amide $(1 \mathrm{M} / \mathrm{L}, 12 \mathrm{~mL}, 12 \mathrm{mmol}, 3$ equiv) dropwise and allowed to stir at $0{ }^{\circ} \mathrm{C}$ for $3 \mathrm{~h}$. Then the reaction was quenched with saturated ammonium chloride aqueous solution and extracted with EA for three times, the combined organic layer was washed with $10 \%$ aqueous $\mathrm{NaOH}$ solution and brine, then dried over $\mathrm{Na}_{2} \mathrm{SO}_{4}$, concentrated in vacuo. The crude product was purified by silica gel column chromatography using petroleum ether/ethyl acetate to afford the corresponding product $(1.302 \mathrm{~g}, 98 \%$ yield $)$.

\section{$(1 R, 4 R)-5$-phenylbicyclo[2.2.2]octa-2,5-dien-2-yl trifluoromethanesulfonate ${ }^{[1]}$}

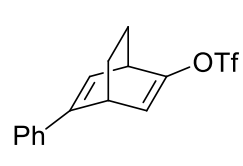

${ }^{1} \mathrm{H}$ NMR data of this product is consistent with the reported value. ${ }^{[1]}$

${ }^{1} \mathrm{H}$ NMR (300 MHz, $\left.\mathrm{CDCl}_{3}\right) \delta$ 7.41-7.23 (m, 5H), $6.55(\mathrm{dd}, J=6.0,2.1 \mathrm{~Hz}, 1 \mathrm{H})$,

$6.17(\mathrm{dd}, J=7.2,2.7 \mathrm{~Hz}, 1 \mathrm{H}), 4.20-4.17(\mathrm{~m}, 1 \mathrm{H}), 3.75-3.72(\mathrm{~m}, 1 \mathrm{H}), 1.86-1.81$

(m, 1H), 1.63-1.51 (m, 3H). 
To a $50 \mathrm{~mL}$ schlenk tube under an $\mathrm{N}_{2}$-atmosphere was charged with $(1 R, 4 R)$-5-phenylbicyclo[2.2.2] octa-2,5-dien-2-yl trifluoromethanesulfonate $(660 \mathrm{mg}, 2 \mathrm{mmol}, 1$ equiv), 2-(methoxycarbonyl)phenylboronic acid $\left(720 \mathrm{mg}, 4 \mathrm{mmol}, 2\right.$ equiv), $\mathrm{Pd}(\mathrm{dppf}) \mathrm{Cl}_{2}(41 \mathrm{mg}$, $0.05 \mathrm{mmol}, 2.5 \mathrm{~mol} \%$ ), $\mathrm{Cs}_{2} \mathrm{CO}_{3}(975 \mathrm{mg}, 3 \mathrm{mmol}, 1.5$ equiv) in dioxane, the resulting mixture was stirred at $110{ }^{\circ} \mathrm{C}$ for $8 \mathrm{~h}$, then the reaction was diluted with saturated ammonium chloride aqueous solution and extracted with EA for three times, the combined organic layer was washed with brine, dried over $\mathrm{Na}_{2} \mathrm{SO}_{4}$, concentrated in vacuo. The crude product was purified by silica gel column chromatography using petroleum ether/ethyl acetate to afford the corresponding product (525 mg, 83\% yield).

\section{Methyl 2-((1R,4R)-5-phenylbicyclo[2.2.2]octa-2,5-dien-2-yl)benzoate}

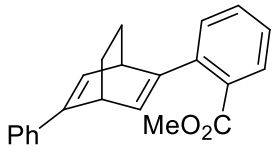

${ }^{1} \mathrm{H}$ NMR $\left(300 \mathrm{MHz}, \mathrm{CDCl}_{3}\right) \delta 7.73(\mathrm{~d}, J=7.8 \mathrm{~Hz}, 1 \mathrm{H}), 7.48-7.23(\mathrm{~m}, 8 \mathrm{H})$, $6.68(\mathrm{dd}, J=6.3,1.8 \mathrm{~Hz}, 1 \mathrm{H}), 6.32(\mathrm{dd}, J=6.3,1.8 \mathrm{~Hz}, 1 \mathrm{H}), 4.20(\mathrm{~d}, J=3.9$ $\mathrm{Hz}, 1 \mathrm{H}), 3.81-3.77(\mathrm{~m}, 4 \mathrm{H}), 1.58-1.52(\mathrm{~m}, 4 \mathrm{H})$.

To a solution of the above ester ( $525 \mathrm{mg}, 1.66 \mathrm{mmol}, 1$ equiv) in $20 \mathrm{~mL}$ of THF/ $\mathrm{H}_{2} \mathrm{O}$ was added $1 \mathrm{~N} \mathrm{NaOH}\left(8.3 \mathrm{~mL}, 8.3 \mathrm{mmol}, 5\right.$ equiv), The reaction mixture was stirred at $60{ }^{\circ} \mathrm{C}$ for $6 \mathrm{~h}$, then the reaction mixture was acidified by $2 \mathrm{M} \mathrm{HCl}$ to $\mathrm{PH}=2$, extracted with EA for three times, the combined organic layer was washed with brine, dried over $\mathrm{Na}_{2} \mathrm{SO}_{4}$, concentrated in vacuo. The crude product was purified by silica gel column chromatography using petroleum ether/ethyl acetate to afford the corresponding acid product ( $451 \mathrm{mg}, 90 \%$ yield).

\section{2-((1R,4R)-5-phenylbicyclo[2.2.2]octa-2,5-dien-2-yl)benzoic acid}

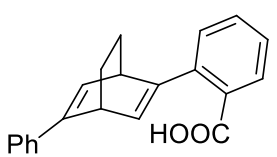

${ }^{1} \mathrm{H}$ NMR $\left(300 \mathrm{MHz}, \mathrm{CDCl}_{3}\right) \delta 7.96(\mathrm{dd}, J=7.8,1.2 \mathrm{~Hz}, 1 \mathrm{H}), 7.53-7.20(\mathrm{~m}$, $8 \mathrm{H}), 6.71(\mathrm{dd}, J=6.3,1.8 \mathrm{~Hz}, 1 \mathrm{H}), 6.38(\mathrm{dd}, J=6.3,1.8 \mathrm{~Hz}, 1 \mathrm{H}), 4.20(\mathrm{dd}, J$ $=6.3,2.1 \mathrm{~Hz}, 1 \mathrm{H}), 3.79(\mathrm{dd}, J=6.3,2.1 \mathrm{~Hz}, 1 \mathrm{H}), 1.59-1.45(\mathrm{~m}, 4 \mathrm{H})$.

A vessel was charged with the above obtained acid (302 mg, $1 \mathrm{mmol})$, dry DCM (20 mL) and catalytic amount of DMF. The reaction mixture was cooled to $0{ }^{\circ} \mathrm{C}$ and stirred for 5 minutes. Then $(\mathrm{COCl})_{2} \quad$ was added dropwise and stirred at room temperature for $4 \mathrm{~h}$. The resulting mixture was concentrated under reduced pressure to afford acid chloride quantitatively which was used directly without further purification for the next step. The crude acid chloride was dissolved in $\operatorname{dry}^{\mathrm{CH}_{2} \mathrm{Cl}_{2}}$ and $\mathrm{Et}_{3} \mathrm{~N}$ and ${ }^{i} \mathrm{PrNH}_{2}$ was added, after stirred at room temperature for $4 \mathrm{~h}$, solvent was evaporated to dryness under reduced pressure and the crude compound was purified by column chromatography over silica gel to furnish the ligand ( $354 \mathrm{mg}, 92 \%$ yield). Tow rotamers were observed in the ${ }^{1} \mathrm{H}$ NMR, the ratio is $0.67 / 0.33$. 


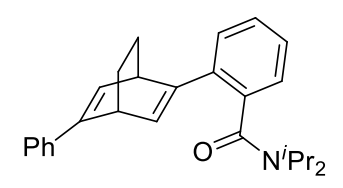

$[\alpha]_{\mathrm{D}}^{20}=-25.6\left(c 1.11, \mathrm{CHCl}_{3}\right) ;{ }^{1} \mathrm{H} \mathrm{NMR}\left(300 \mathrm{MHz}, \mathrm{CDCl}_{3}\right) \delta 7.42-7-20$ $(\mathrm{m}, 8 \mathrm{H}), 7.14(\mathrm{~d}, J=1.2 \mathrm{~Hz}, 0.67 \mathrm{H}), 7.11(\mathrm{~d}, J=1.2 \mathrm{~Hz}, 0.33 \mathrm{H}), 6.75(\mathrm{dd}$, $J=6.4,2.0 \mathrm{~Hz}, 0.67 \mathrm{H}), 6.65(\mathrm{dd}, J=6.4,2.0 \mathrm{~Hz}, 0.33 \mathrm{H}), 6.58(\mathrm{dd}, J=$ 6.4, $2.0 \mathrm{~Hz}, 0.67 \mathrm{H}), 6.53(\mathrm{dd}, J=6.4,2.0 \mathrm{~Hz}, 0.33 \mathrm{H}), 4.20-4.14(\mathrm{~m}, 1.36$ $\mathrm{H}), 4.07-4.04(\mathrm{~m}, 0.67 \mathrm{H}), 3.55-3.38(\mathrm{~m}, 1.36 \mathrm{H}), 3.33-3.26(\mathrm{~m}, 0.67 \mathrm{H}), 1.69-1.63(\mathrm{~m}, 1 \mathrm{H})$, 1.56-1.44 (m, 7H), $1.31(\mathrm{~d}, J=6.8 \mathrm{~Hz}, 2 \mathrm{H}), 0.98-0.94(\mathrm{~m}, 4 \mathrm{H}), 0.87(\mathrm{~d}, J=6.8 \mathrm{~Hz}, 2 \mathrm{H}) ;{ }^{13} \mathrm{C} \mathrm{NMR}$ $\left(100 \mathrm{MHz}, \mathrm{CDCl}_{3}\right) \delta 170.1,170.0,147.2,146.5,146.1,144.1,138.7,138.5,137.4,136.8,136.1$, $133.5,132.4,130.2,129.7,128.9,128.7,128.4,128.3,127.3,127.1,126.9,126.7,126.3,126.2$, 125.1, 125.0, 50.9, 46.0, 45.7, 42.8, 42.3, 40.5, 40.2, 26.4, 25.9, 25.8, 25.6, 21.1, 21.0, 20.8, 20.4, 20.0. HRMS (ESI) for $\mathrm{C}_{27} \mathrm{H}_{32} \mathrm{NO}[\mathrm{M}+\mathrm{H}]^{+}$: calcd 386.2478, found 386.2483 .

[1] Abele, S.; Inauen,R.; Spielvogel, D.; Moessner, C. Scalable Synthesis of Enantiomerically Pure Bicyclo[2.2.2] octadiene Ligands. J. Org. Chem. 2012, 77, 4765-4773.

\section{General Procedure for C-H Functionalization/Oxa-Michael Addition Cascade}

\subsection{Synthesis of 2,3-disubstituted dihydrobenzofurans 3}

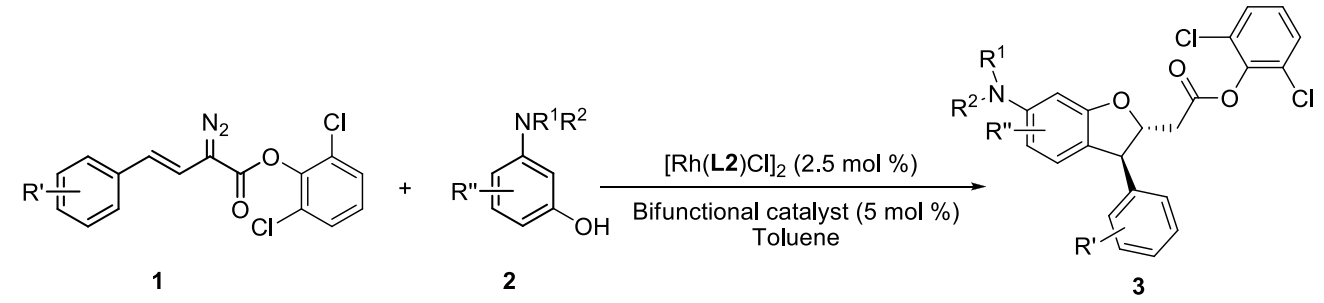

Under Ar atmosphere, one tube with $[\mathrm{Rh}(\mathbf{L} 2) \mathrm{Cl}]_{2}(2.6 \mathrm{mg}, 0.0025 \mathrm{mmol}, 2.5 \mathrm{~mol} \%)$ in 2.0 $\mathrm{mL}$ of toluene was stirred at room temperature. A mixture of arylvinyldiazoacetates $(0.1 \mathrm{mmol})$, 3-aminophenol derivatives $(0.2 \mathrm{mmol})$ and cinchona alkaloid catalyst $(3.0 \mathrm{mg}, 0.005 \mathrm{mmol}, 5$ mol \%) in $2.0 \mathrm{~mL}$ of toluene was added in one portion and then the resulting mixture was stirred at $\mathrm{rt}$ for $24 \mathrm{~h}$. The solvent was removed under reduced pressure and the residue was purified by silica gel column chromatography using petroleum ether/ethyl acetate to afford the corresponding product 3 . 


\subsection{Synthesis of 3,4-disubstituted isochromans 6}

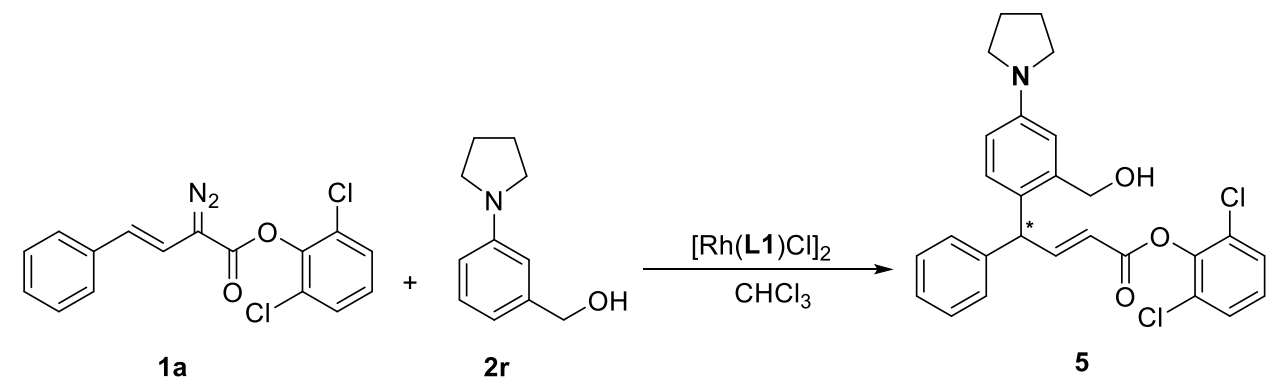

Under Ar atmosphere, one tube with $[\mathrm{Rh}(\mathbf{L 1}) \mathrm{Cl}]_{2}(3.2 \mathrm{mg}, 0.003 \mathrm{mmol}, 1.5 \mathrm{~mol} \%)$ in 2.0 $\mathrm{mL}$ of $\mathrm{CHCl}_{3}$ was stirred at room temperature, a mixture of (E)-2,6-dichlorophenyl 2-diazo-4-phenylbut-3-enoate 1a (66.6 mg, $0.2 \mathrm{mmol})$ and (3-(pyrrolidin-1-yl)phenyl)methanol $\mathbf{2 r}$ $(0.4 \mathrm{mmol})$ in $2.0 \mathrm{~mL}$ of $\mathrm{CHCl}_{3}$ was added in one portion and then the resulting mixture was stirred at $\mathrm{rt}$ for $6 \mathrm{~h}$. The solvent was removed under reduced pressure and the residue was purified by silica gel column chromatography using petroleum ether/ethyl acetate to afford the corresponding product 5 (65 mg, 68\% yield, $86 \%$ ee).

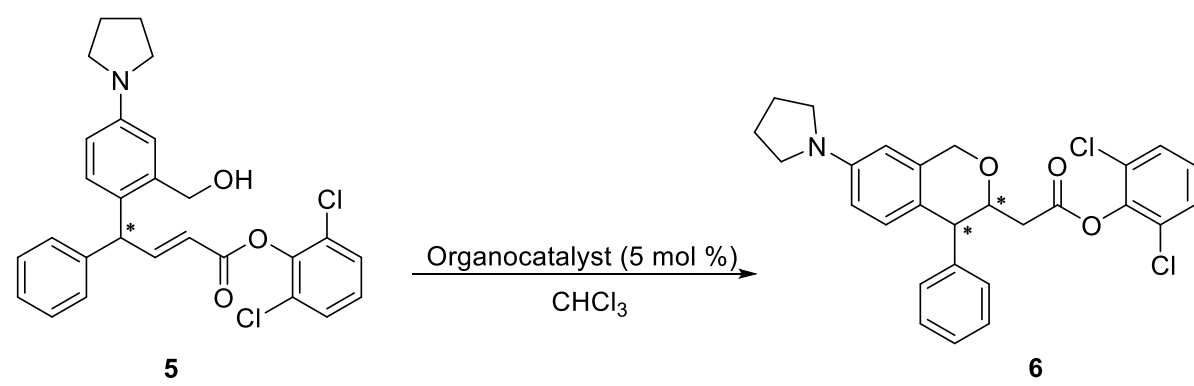

To a vessel charged with the C-H functionalization product 5 ( $86 \%$ ee, $48.2 \mathrm{mg}, 0.1 \mathrm{mmol})$ in anhydrous $\mathrm{CHCl}_{3}$, organocatalyst (5 mol \%) was added. Then, the mixture was stirred at room temperature for $24 \mathrm{~h}$. The solvent was removed under reduced pressure and the residue was purified by silica gel column chromatography using petroleum ether/ethyl acetate to afford the corresponding product 6 . 


\section{Characterization Data of Products 3}

\section{2,6-dichlorophenyl 2-((2R,3S)-3-phenyl-6-(pyrrolidin-1-yl)-2,3-dihydrobenzofuran-2-yl)}

acetate $(R, S-3 a)$

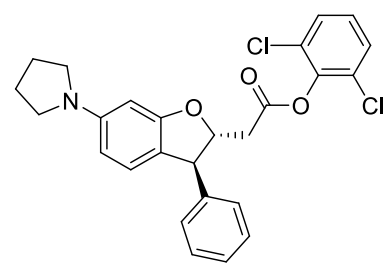

White solid, $41.9 \mathrm{mg}, 89 \%$ yield, $99: 1 \mathrm{dr}, 99 \%$ ee; m.p.201.5-203.5 ${ }^{\circ} \mathrm{C}$, $[\alpha]_{\mathrm{D}}{ }^{20}=40.8\left(c 1.08, \mathrm{CHCl}_{3}\right) ;{ }^{1} \mathrm{H} \mathrm{NMR}\left(400 \mathrm{MHz}, \mathrm{CDCl}_{3}\right) \delta 7.36(\mathrm{~d}$, $J=8.4 \mathrm{~Hz}, 2 \mathrm{H}), 7.33-7.30(\mathrm{~m}, 2 \mathrm{H}), 7.26-7.23(\mathrm{~m}, 3 \mathrm{H}), 7.15(\mathrm{t}, J=8.0$ $\mathrm{Hz}, 1 \mathrm{H}), 6.87(\mathrm{~d}, J=8.4 \mathrm{~Hz}, 1 \mathrm{H}), 6.18(\mathrm{~d}, J=0.8 \mathrm{~Hz}, 1 \mathrm{H}), 6.13(\mathrm{~d}, J$ $=8.0 \mathrm{~Hz}, 1 \mathrm{H}), 5.12-5.07(\mathrm{~m}, 1 \mathrm{H}), 4.37(\mathrm{~d}, J=5.6 \mathrm{~Hz}, 1 \mathrm{H}), 3.29(\mathrm{t}, J=$ $6.0 \mathrm{~Hz}, 4 \mathrm{H}), 3.25-3.12(\mathrm{~m}, 2 \mathrm{H}), 2.02-1.99(\mathrm{~m}, 4 \mathrm{H}) ;{ }^{13} \mathrm{C}$ NMR $(100$ $\left.\mathrm{MHz}, \mathrm{CDCl}_{3}\right) \delta 167.3,160.9,149.6,144.2,143.4,129.3,129.1,129.0,128.2,127.6,127.4,125.9$, 116.6, 105.3, 93.7, 87.3, 54.1, 48.2, 39.6, 25.8; HRMS (ESI) for $\mathrm{C}_{26} \mathrm{H}_{24} \mathrm{Cl}_{2} \mathrm{NO}_{3}[\mathrm{M}+\mathrm{H}]^{+}$: calcd 468.1128 , found 468.1133 .

\section{2,6-dichlorophenyl 2-((2S,3S)-3-phenyl-6-(pyrrolidin-1-yl)-2,3-dihydrobenzofuran-2-yl)} acetate $(S, S-3 a)$

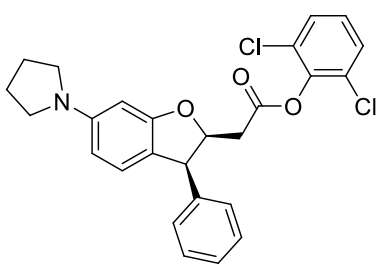

Pale yellow solid, $44.7 \mathrm{mg}, 96 \%$ yield, $93: 7 \mathrm{dr}, 98 \%$ ee; m.p.141.7-143.6 ${ }^{\circ} \mathrm{C},[\alpha]_{\mathrm{D}}{ }^{20}=-3.1$ (c 1.08, $\left.\mathrm{CHCl}_{3}\right) ;{ }^{1} \mathrm{H}$ NMR (400 $\left.\mathrm{MHz} \mathrm{CDCl}_{3}\right) \delta$ 7.37-7.23 (m, 5H), $7.13(\mathrm{t}, J=8.0 \mathrm{~Hz}, 1 \mathrm{H}), 7.07(\mathrm{~d}, J$ $=6.8 \mathrm{~Hz}, 2 \mathrm{H}), 6.94(\mathrm{~d}, J=8.0 \mathrm{~Hz}, 1 \mathrm{H}), 6.21(\mathrm{~s}, 1 \mathrm{H}), 6.16(\mathrm{~d}, J=8.0$ $\mathrm{Hz}, 1 \mathrm{H}), 5.48-5.42$ (m, 1H), 4.68 (d, $J=8.4 \mathrm{~Hz}, 1 \mathrm{H}), 3.37-3.30$ (m, $4 \mathrm{H}), 2.90-2.84(\mathrm{~m}, 1 \mathrm{H}), 2.60-2.54(\mathrm{~m}, 1 \mathrm{H}), 2.03-2.00(\mathrm{~m}, 4 \mathrm{H}) ;{ }^{13} \mathrm{C}$ NMR $\left(100 \mathrm{MHz}, \mathrm{CDCl}_{3}\right) \delta 167.8,161.1,149.5,144.1,140.2,129.4,129.3,128.9,127.5,126.1$, 117.5, 105.4, 94.7, 82.9, 50.9, 48.2, 36.5, 25.8; HRMS (ESI) for $\mathrm{C}_{26} \mathrm{H}_{24} \mathrm{Cl}_{2} \mathrm{NO}_{3}[\mathrm{M}+\mathrm{H}]^{+}$: calcd 468.1128 , found 468.1133 .

\section{2,6-dichlorophenyl 2-((2S,3R)-3-phenyl-6-(pyrrolidin-1-yl)-2,3-dihydrobenzofuran-2-yl)} acetate $(S, R-3 a)$

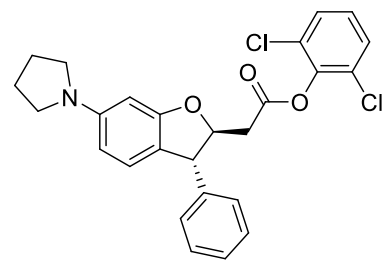

Pale yellow solid, $46.5 \mathrm{mg}, 99 \%$ yield, 99:1 dr, $99 \%$ ee; m.p.194.6-196.3 $\left.{ }^{\circ} \mathrm{C},[\alpha]_{\mathrm{D}}{ }^{20}=-38.9(c) 1.08, \mathrm{CHCl}_{3}\right) ;{ }^{1} \mathrm{H}$ NMR $(400$ $\left.\mathrm{MHz}, \mathrm{CDCl}_{3}\right) \delta 7.35(\mathrm{~d}, J=8.4 \mathrm{~Hz}, 2 \mathrm{H}), 7.32-7.29(\mathrm{~m}, 2 \mathrm{H}), 7.25-7.22$ $(\mathrm{m}, 3 \mathrm{H}), 7.14(\mathrm{t}, J=8.0 \mathrm{~Hz}, 1 \mathrm{H}), 6.86(\mathrm{~d}, J=8.4 \mathrm{~Hz}, 1 \mathrm{H}), 6.17(\mathrm{~d}, J=$ $0.8 \mathrm{~Hz}, 1 \mathrm{H}), 6.12(\mathrm{~d}, J=8.0 \mathrm{~Hz}, 1 \mathrm{H}), 5.11-5.06(\mathrm{~m}, 1 \mathrm{H}), 4.36(\mathrm{~d}, J=$ $5.6 \mathrm{~Hz}, 1 \mathrm{H}), 3.28(\mathrm{t}, J=6.0 \mathrm{~Hz}, 4 \mathrm{H}), 3.25-3.11(\mathrm{~m}, 2 \mathrm{H}), 2.02-1.99(\mathrm{~m}$, $4 \mathrm{H}) ;{ }^{13} \mathrm{C}$ NMR $\left(100 \mathrm{MHz}, \mathrm{CDCl}_{3}\right) \delta 167.3,160.9,149.6,144.2,143.4,129.3,129.1,129.0,128.2$, 127.6, 127.4, 125.9, 116.6, 105.3, 93.7, 87.3, 54.1, 48.2, 39.6, 25.8; HRMS (ESI) for $\mathrm{C}_{26} \mathrm{H}_{24} \mathrm{Cl}_{2} \mathrm{NO}_{3}[\mathrm{M}+\mathrm{H}]^{+}$: calcd 468.1128, found 468.1133 .

\section{2,6-dichlorophenyl 2-((2R,3R)-3-phenyl-6-(pyrrolidin-1-yl)-2,3-dihydrobenzofuran-2-yl)} acetate $(\boldsymbol{R}, \boldsymbol{R}-3 \mathbf{a})$

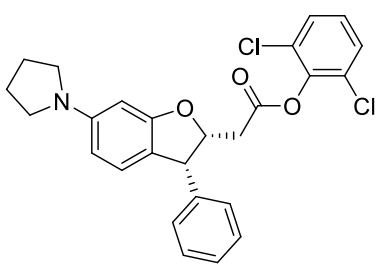

Pale yellow solid, $46.8 \mathrm{mg}, 99 \%$ yield, $98: 2 \mathrm{dr}, 99 \%$ ee; m.p.142.8-144.6 ${ }^{\circ} \mathrm{C},[\alpha]_{\mathrm{D}}{ }^{20}=10.5\left(c 1.0, \mathrm{CHCl}_{3}\right) ;{ }^{1} \mathrm{H}$ NMR $(400 \mathrm{MHz}$, $\left.\mathrm{CDCl}_{3}\right) \delta 7.37-7.23(\mathrm{~m}, 5 \mathrm{H}), 7.13(\mathrm{t}, J=8.0 \mathrm{~Hz}, 1 \mathrm{H}), 7.07(\mathrm{~d}, J=6.8$ $\mathrm{Hz}, 2 \mathrm{H}), 6.94(\mathrm{~d}, J=8.0 \mathrm{~Hz}, 1 \mathrm{H}), 6.21(\mathrm{~s}, 1 \mathrm{H}), 6.16(\mathrm{~d}, J=8.0 \mathrm{~Hz}$, $1 \mathrm{H}), 5.48-5.42(\mathrm{~m}, 1 \mathrm{H}), 4.68(\mathrm{~d}, J=8.4 \mathrm{~Hz}, 1 \mathrm{H}), 3.34-3.30(\mathrm{~m}, 4 \mathrm{H})$, 2.90-2.84 (m, 1H), 2.60-2.54 (m, 1H), 2.03-2.00 (m, 4H); ${ }^{13} \mathrm{C}$ NMR 
$\left(100 \mathrm{MHz}, \mathrm{CDCl}_{3}\right) \delta 167.8,161.1,149.5,144.1,140.2,129.4,129.3,128.9,127.5,126.1,117.5$, 105.4, 94.7, 82.9, 50.9, 48.2, 36.5, 25.8; HRMS (ESI) for $\mathrm{C}_{26} \mathrm{H}_{24} \mathrm{Cl}_{2} \mathrm{NO}_{3}[\mathrm{M}+\mathrm{H}]+$ : calcd 468.1128, found 468.1133 .

\section{2,6-dichlorophenyl 2-((2R,3R)-3-(2-fluorophenyl)-6-(pyrrolidin-1-yl)-2,3-dihydrobenzofuran}

-2-yl)acetate (3b)

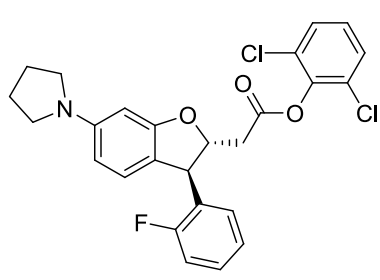

Pale yellow solid, $46.9 \mathrm{mg}$, 96\% yield, 98:2 dr, 99\% ee; m.p.165.9-169.7 $\left.{ }^{\circ} \mathrm{C},[\alpha]_{\mathrm{D}}{ }^{20}=50.5(c) 1.13, \mathrm{CHCl}_{3}\right) ;{ }^{1} \mathrm{H}$ NMR $(400$ $\left.\mathrm{MHz}, \mathrm{CDCl}_{3}\right) \delta 7.34(\mathrm{~d}, J=8.0 \mathrm{~Hz}, 2 \mathrm{H}), 7.22-7.18(\mathrm{~m}, 1 \mathrm{H}), 7.15-7.04$ (m, 4H), $6.90(\mathrm{~d}, J=8.0 \mathrm{~Hz}, 1 \mathrm{H}), 6.17-6.13(\mathrm{~m}, 2 \mathrm{H}), 5.15-5.10(\mathrm{~m}$, $1 \mathrm{H}), 4.71(\mathrm{~d}, J=5.6 \mathrm{~Hz}, 1 \mathrm{H}), 3.30-3.16(\mathrm{~m}, 6 \mathrm{H}), 2.01-1.98(\mathrm{~m}, 4 \mathrm{H})$; ${ }^{13} \mathrm{C}$ NMR $\left(100 \mathrm{MHz}, \mathrm{CDCl}_{3}\right) \delta 167.2,161.1,161.0(\mathrm{~d}, J=243.9 \mathrm{~Hz})$, 149.7, 144.3, 130.2 (d, $J=13.8 \mathrm{~Hz}), 129.6$ (d, $J=4.0 \mathrm{~Hz}), 129.3,128.9,128.8,128.7,127.5$, 125.9, 124.9 (d, $J=3.5 \mathrm{~Hz}), 115.7$ (d, $J=12.0 \mathrm{~Hz}), 105.3,94.0,86.4,48.2,46.3(\mathrm{~d}, J=2.9 \mathrm{~Hz})$, 40.0, 25.8; HRMS (ESI) for $\mathrm{C}_{26} \mathrm{H}_{23} \mathrm{Cl}_{2} \mathrm{FNO}_{3}[\mathrm{M}+\mathrm{H}]+$ : calcd 486.1029, found 486.1034 .

\section{2,6-dichlorophenyl 2-((2R,3S)-3-(3-methoxyphenyl)-6-(pyrrolidin-1-yl)-2,3-dihydrobenzo} furan-2-yl)acetate (3c)

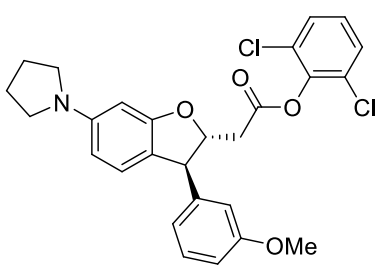

Pale yellow solid, $42.5 \mathrm{mg}, 85 \%$ yield, 99:1 dr, 99\% ee; m.p.165.3-167.4 ${ }^{\circ} \mathrm{C},[\alpha]_{\mathrm{D}}{ }^{20}=42.4\left(c 1.0, \mathrm{CHCl}_{3}\right) ;{ }^{1} \mathrm{H}$ NMR $(400 \mathrm{MHz}$, $\left.\mathrm{CDCl}_{3}\right) \delta 7.35(\mathrm{~d}, J=8.0 \mathrm{~Hz}, 2 \mathrm{H}), 7.23(\mathrm{t}, J=8.4 \mathrm{~Hz}, 1 \mathrm{H}), 7.14(\mathrm{t}, J$ $=8.0 \mathrm{~Hz}, 1 \mathrm{H}), 6.89-6.78(\mathrm{~m}, 4 \mathrm{H}), 6.17(\mathrm{~d}, J=1.6 \mathrm{~Hz}, 1 \mathrm{H}), 6.12(\mathrm{~d}, J$ $=8.0 \mathrm{~Hz}, 1 \mathrm{H}), 5.10(\mathrm{q}, J=6.0 \mathrm{~Hz}, 1 \mathrm{H}), 4.34(\mathrm{~d}, J=6.0 \mathrm{~Hz}, 1 \mathrm{H}), 3.77$ (s, 3H), $3.28(\mathrm{t}, J=6.0 \mathrm{~Hz}, 4 \mathrm{H}), 3.25-3.15(\mathrm{~m}, 2 \mathrm{H}), 2.00(\mathrm{t}, J=6.0$ $\mathrm{Hz}, 4 \mathrm{H}) ;{ }^{13} \mathrm{C}$ NMR $\left(100 \mathrm{MHz}, \mathrm{CDCl}_{3}\right) \delta 167.3,160.9,160.2,149.6,144.9,144.2,130.0,129.3$, 128.9, 127.6, 125.9, 120.6, 116.3, 113.9, 112.7, 105.3, 93.9, 87.2, 55.5, 54.1, 48.2, 39.6, 25.8; HRMS (ESI) for $\mathrm{C}_{27} \mathrm{H}_{26} \mathrm{Cl}_{2} \mathrm{NO}_{4}[\mathrm{M}+\mathrm{H}]+$ : calcd 498.1233, found 498.1226.

\section{2,6-dichlorophenyl 2-((2R,3S)-6-(pyrrolidin-1-yl)-3-m-tolyl-2,3-dihydrobenzofuran-2-yl)} acetate (3d)

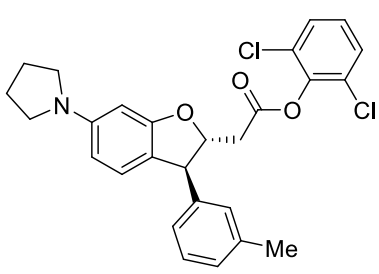

White solid, $44.0 \mathrm{mg}$, $91 \%$ yield, $99: 1 \mathrm{dr}$, $99 \%$ ee; m.p.154.3-157.6 ${ }^{\circ} \mathrm{C}$, $[\alpha]_{\mathrm{D}}^{20}=15.9\left(c 1.15, \mathrm{CHCl}_{3}\right) ;{ }^{1} \mathrm{H}$ NMR $\left(400 \mathrm{MHz}, \mathrm{CDCl}_{3}\right) \delta 7.35(\mathrm{~d}$, $J=8.4 \mathrm{~Hz}, 2 \mathrm{H}), 7.20(\mathrm{t}, J=8.0 \mathrm{~Hz}, 1 \mathrm{H}), 7.14(\mathrm{t}, J=8.4 \mathrm{~Hz}, 1 \mathrm{H})$, 7.07-7.03 (m, 3H), $6.86(\mathrm{~d}, J=8.0 \mathrm{~Hz}, 1 \mathrm{H}), 6.18(\mathrm{~d}, J=1.6 \mathrm{~Hz}, 1 \mathrm{H})$, $6.13(\mathrm{dd}, J=8.0,1.6 \mathrm{~Hz}, 1 \mathrm{H}), 5.09(\mathrm{q}, J=6.0 \mathrm{~Hz}, 1 \mathrm{H}), 4.33(\mathrm{~d}, J=$ $6.0 \mathrm{~Hz}, 1 \mathrm{H}), 3.29$ (t, $J=6.0 \mathrm{~Hz}, 4 \mathrm{H}), 3.25-3.11(\mathrm{~m}, 2 \mathrm{H}), 2.32(\mathrm{~s}, 3 \mathrm{H})$, $2.00(\mathrm{t}, J=6.4 \mathrm{~Hz}, 4 \mathrm{H}) ;{ }^{13} \mathrm{C} \mathrm{NMR}\left(100 \mathrm{MHz}, \mathrm{CDCl}_{3}\right) \delta 167.3,160.9,149.5,144.2,143.2,138.7$, 129.2, 128.9, 128.8, 128.1, 127.6, 125.9, 125.4, 116.7, 105.2, 93.8, 87.4, 54.0, 48.2, 39.6, 25.8, 21.8; HRMS (ESI) for $\mathrm{C}_{27} \mathrm{H}_{26} \mathrm{Cl}_{2} \mathrm{NO}_{3}[\mathrm{M}+\mathrm{H}]+$ : calcd 482.1284, found 482.1293 .

\section{2,6-dichlorophenyl 2-((2R,3S)-3-(3-bromophenyl)-6-(pyrrolidin-1-yl)-2,3-dihydrobenzofuran} -2 -yl)acetate (3e)

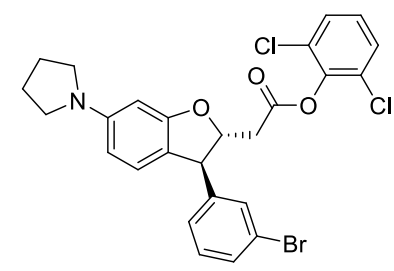

Pale yellow solid, $48.8 \mathrm{mg}, \quad 89 \%$ yield, $99: 1 \mathrm{dr}, 99 \%$ ee; m.p.163.3-164.8 ${ }^{\circ} \mathrm{C},[\alpha]_{\mathrm{D}}{ }^{20}=41.2\left(c 1.0, \mathrm{CHCl}_{3}\right) ;{ }^{1} \mathrm{H}$ NMR $(400 \mathrm{MHz}$, $\left.\mathrm{CDCl}_{3}\right) \delta$ 7.39-7.35 (m, 4H), 7.18-7.13 (m, 3H), $6.87(\mathrm{~d}, J=8.0 \mathrm{~Hz}$, $1 \mathrm{H}), 6.17(\mathrm{~d}, J=2.0 \mathrm{~Hz}, 1 \mathrm{H}), 6.14(\mathrm{dd}, J=8.4,2.0 \mathrm{~Hz}, 1 \mathrm{H}), 5.07$ (q, 
$J=6.0 \mathrm{~Hz}, 1 \mathrm{H}), 4.33(\mathrm{~d}, J=5.6 \mathrm{~Hz}, 1 \mathrm{H}), 3.29(\mathrm{t}, J=6.0 \mathrm{~Hz}, 4 \mathrm{H}), 3.25-3.10(\mathrm{~m}, 2 \mathrm{H}), 2.01(\mathrm{t}, J=$ $6.0 \mathrm{~Hz}, 4 \mathrm{H}) ;{ }^{13} \mathrm{C} \mathrm{NMR}\left(100 \mathrm{MHz}, \mathrm{CDCl}_{3}\right) \delta 167.2,160.8,149.7,145.9,144.1,131.1,130.6,130.5$, 129.2, 128.9, 127.7, 126.9, 125.9, 123.2, 115.7, 105.5, 93.9, 87.0, 53.7, 48.2, 39.5, 25.8; HRMS (ESI) for $\mathrm{C}_{26} \mathrm{H}_{23}{ }^{79} \mathrm{BrCl}_{2} \mathrm{NO}_{3}[\mathrm{M}+\mathrm{H}]^{+}$: calcd 546.0233, found: 546.0232; $\mathrm{C}_{26} \mathrm{H}_{23}{ }^{81} \mathrm{Br}^{35} \mathrm{Cl}_{2} \mathrm{NO}_{3}$ $[\mathrm{M}+\mathrm{H}]^{+}$: calcd 548.0212, found 548.0216.

\section{2,6-dichlorophenyl 2-((2R,3S)-3-(4-methoxyphenyl)-6-(pyrrolidin-1-yl)-2,3-dihydrobenzo} furan-2-yl)acetate (3f)

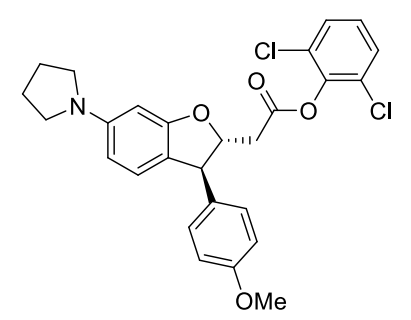

Pale yellow solid, $41.6 \mathrm{mg}, \quad 84 \%$ yield, $99: 1 \mathrm{dr}, 99 \%$ ee; m.p.123.5-126.7 ${ }^{\circ} \mathrm{C},[\alpha]_{\mathrm{D}}{ }^{20}=34.9\left(c 1.0, \mathrm{CHCl}_{3}\right) ;{ }^{1} \mathrm{H} \mathrm{NMR}(400 \mathrm{MHz}$, $\left.\mathrm{CDCl}_{3}\right) \delta 7.36(\mathrm{~d}, J=8.4 \mathrm{~Hz}, 2 \mathrm{H}), 7.17-7.12(\mathrm{~m}, 3 \mathrm{H}), 6.87-6.84(\mathrm{~m}$, $3 \mathrm{H}), 6.17(\mathrm{~d}, J=2.0 \mathrm{~Hz}, 1 \mathrm{H}), 6.13(\mathrm{dd}, J=8.0,2.0 \mathrm{~Hz}, 1 \mathrm{H}), 5.04(\mathrm{q}$, $J=6.0 \mathrm{~Hz}, 1 \mathrm{H}), 4.33(\mathrm{~d}, J=6.0 \mathrm{~Hz}, 1 \mathrm{H}), 3.79(\mathrm{~s}, 3 \mathrm{H}), 3.28(\mathrm{t}, J=6.4$ $\mathrm{Hz}, 4 \mathrm{H}), 3.24-3.11(\mathrm{~m}, 2 \mathrm{H}), 2.00(\mathrm{t}, J=6.4 \mathrm{~Hz}, 4 \mathrm{H}) ;{ }^{13} \mathrm{C}$ NMR $(100$ $\left.\mathrm{MHz}, \mathrm{CDCl}_{3}\right) \delta 167.3,160.8,159.0,149.5,144.2,135.4,129.3,128.9$, $127.6,125.9,116.8,114.4,105.2,93.9,87.5,55.6,53.3,48.2,39.5,25.8$; HRMS (ESI) for $\mathrm{C}_{27} \mathrm{H}_{26} \mathrm{Cl}_{2} \mathrm{NO}_{4}[\mathrm{M}+\mathrm{H}]+$ : calcd 498.1233 , found 498.1223 .

\section{2,6-dichlorophenyl 2-((2R,3S)-6-(pyrrolidin-1-yl)-3-p-tolyl-2,3-dihydrobenzofuran-2-yl)} acetate (3g)

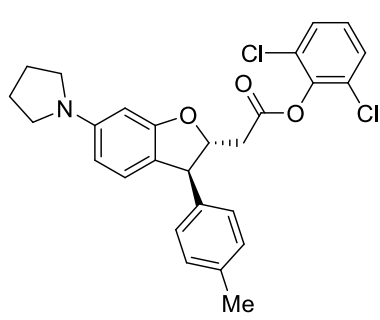

Pale yellow solid, $39.9 \mathrm{mg}, \quad 83 \%$ yield, $99: 1 \mathrm{dr}$, $99 \%$ ee; m.p.159.1-162.8 ${ }^{\circ} \mathrm{C},[\alpha]_{\mathrm{D}}^{20}=33.1\left(c 1.0, \mathrm{CHCl}_{3}\right),{ }^{1} \mathrm{H}$ NMR $(400 \mathrm{MHz}$, $\left.\mathrm{CDCl}_{3}\right) \delta 7.35(\mathrm{~d}, J=8.0 \mathrm{~Hz}, 2 \mathrm{H}), 7.16-7.12(\mathrm{~m}, 5 \mathrm{H}), 6.85(\mathrm{~d}, J=8.0$ $\mathrm{Hz}, 1 \mathrm{H}), 6.17(\mathrm{~s}, 1 \mathrm{H}), 6.12(\mathrm{~d}, J=8.0 \mathrm{~Hz}, 1 \mathrm{H}), 5.06(\mathrm{q}, J=6.0 \mathrm{~Hz}$, $1 \mathrm{H}), 4.33(\mathrm{~d}, J=6.4 \mathrm{~Hz}, 1 \mathrm{H}), 3.29-3.11(\mathrm{~m}, 6 \mathrm{H}), 2.33(\mathrm{~s}, 3 \mathrm{H}), 2.00(\mathrm{t}$, $J=6.0 \mathrm{~Hz}, 4 \mathrm{H}) ;{ }^{13} \mathrm{C} \mathrm{NMR}\left(100 \mathrm{MHz}, \mathrm{CDCl}_{3}\right) \delta 167.3,160.9,149.5$, $144.2,140.3,137.0,129.7,129.3,128.9,128.2,127.6,125.9,116.8$, 105.2, 93.9, 87.5, 53.7, 48.2, 39.5, 25.8, 21.4; HRMS (ESI) for $\mathrm{C}_{27} \mathrm{H}_{26} \mathrm{Cl}_{2} \mathrm{NO}_{3}[\mathrm{M}+\mathrm{H}]+$ : calcd 482.1284, found 482.1272 .

2,6-dichlorophenyl 2-((2R,3S)-3-(4-chlorophenyl)-6-(pyrrolidin-1-yl)-2,3-dihydrobenzofuran2-yl)acetate (3h)

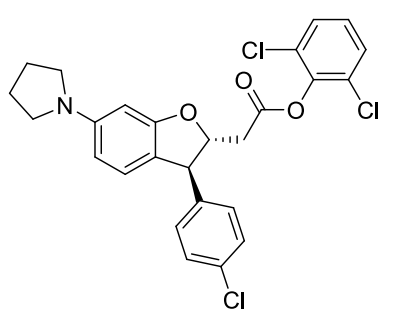

Pale yellow solid, $45.5 \mathrm{mg}$, 91\% yield, 99:1 dr, 99\% ee; m.p.143.9-145.2 ${ }^{\circ} \mathrm{C},[\alpha]_{\mathrm{D}}{ }^{20}=48.5\left(c 1.0, \mathrm{CHCl}_{3}\right) ;{ }^{1} \mathrm{H}$ NMR $(400 \mathrm{MHz}$, $\left.\mathrm{CDCl}_{3}\right) \delta 7.36(\mathrm{~d}, J=8.0 \mathrm{~Hz}, 2 \mathrm{H}), 7.27(\mathrm{~d}, J=8.0 \mathrm{~Hz}, 2 \mathrm{H})$, 7.17-7.13 (m, 3H), $6.85(\mathrm{~d}, J=8.0 \mathrm{~Hz}, 1 \mathrm{H}), 6.17(\mathrm{~d}, J=1.6 \mathrm{~Hz}, 1 \mathrm{H})$, $6.13(\mathrm{dd}, J=8.0,2.0 \mathrm{~Hz}, 1 \mathrm{H}), 5.04(\mathrm{q}, J=6.0 \mathrm{~Hz}, 1 \mathrm{H}), 4.35(\mathrm{~d}, J=$ $5.2 \mathrm{~Hz}, 1 \mathrm{H}), 3.29$ (t, $J=6.0 \mathrm{~Hz}, 4 \mathrm{H}), 3.26-3.10(\mathrm{~m}, 2 \mathrm{H}), 2.01(\mathrm{t}, J=$ $6.0 \mathrm{~Hz}, 4 \mathrm{H}) ;{ }^{13} \mathrm{C} \mathrm{NMR}\left(100 \mathrm{MHz}, \mathrm{CDCl}_{3}\right) \delta 167.2,160.8,149.7,144.1,142.1,133.1,129.5,129.2$, 129.1, 129.0, 127.7, 125.9, 115.9, 105.4, 93.8, 87.1, 53.4, 48.2, 39.5, 25.8; HRMS (ESI) for $\mathrm{C}_{26} \mathrm{H}_{23}{ }^{35} \mathrm{Cl}_{3} \mathrm{NO}_{3}[\mathrm{M}+\mathrm{H}]^{+}$: calcd 502.0738, found 502.0734; $\mathrm{C}_{26} \mathrm{H}_{23}{ }^{35} \mathrm{Cl}_{2}{ }^{37} \mathrm{ClNO}_{3}[\mathrm{M}+\mathrm{H}]^{+}$: calcd 504.0709, found: 504.0707. 
2,6-dichlorophenyl 2-((2R,3S)-6-(pyrrolidin-1-yl)-3-(4-(trifluoromethyl)phenyl)-2,3dihydrobenzofuran-2-yl)acetate (3i)

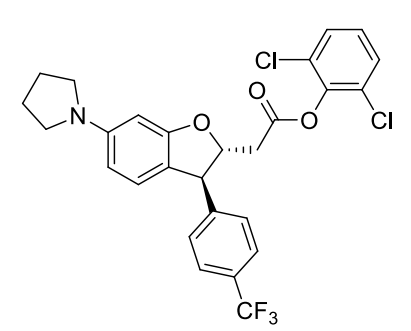

Pale yellow solid, $41.6 \mathrm{mg}$, 78\% yield, 99:1 dr, 99\% ee; m.p.147.3-149.5 ${ }^{\circ} \mathrm{C},[\alpha]_{\mathrm{D}}{ }^{20}=53.0\left(c 1.0, \mathrm{CHCl}_{3}\right) ;{ }^{1} \mathrm{H} \mathrm{NMR}(400 \mathrm{MHz}$, $\left.\mathrm{CDCl}_{3}\right) \delta 7.55(\mathrm{~d}, J=8.0 \mathrm{~Hz}, 2 \mathrm{H}), 7.38-7.33(\mathrm{~m}, 4 \mathrm{H}), 7.15(\mathrm{t}, J=8.0$ $\mathrm{Hz}, 1 \mathrm{H}), 6.85(\mathrm{~d}, J=8.0 \mathrm{~Hz}, 1 \mathrm{H}), 6.18(\mathrm{~d}, J=1.6 \mathrm{~Hz}, 1 \mathrm{H}), 6.14(\mathrm{dd}$, $J=8.0,2.0 \mathrm{~Hz}, 1 \mathrm{H}), 5.11-5.07(\mathrm{~m}, 1 \mathrm{H}), 4.44(\mathrm{~d}, J=5.2 \mathrm{~Hz}, 1 \mathrm{H})$, 3.31-3.12 (m, 6H), $2.01(\mathrm{t}, J=6.4 \mathrm{~Hz}, 4 \mathrm{H}) ;{ }^{13} \mathrm{C} \mathrm{NMR}(100 \mathrm{MHz}$, $\left.\mathrm{CDCl}_{3}\right) \delta 167.2,160.8,149.8,147.7,144.1,129.6(\mathrm{q}, J=33.2 \mathrm{~Hz})$, $129.2,129.0,128.5,127.7,126.0(\mathrm{q}, J=3.8 \mathrm{~Hz}), 125.9,124.5(\mathrm{q}, J=270.4 \mathrm{~Hz}), 115.6,105.5$, 93.9, 86.9, 53.8, 48.2, 39.6, 25.8; HRMS (ESI) for $\mathrm{C}_{27} \mathrm{H}_{23} \mathrm{Cl}_{2} \mathrm{~F}_{3} \mathrm{NO}_{3}[\mathrm{M}+\mathrm{H}]+$ : calcd 536.101, found 536.1002 .

\section{2,6-dichlorophenyl 2-((2R, 3S)-3-(3,4-dimethoxyphenyl)-6-(pyrrolidin-1-yl)-2,3-} dihydrobenzofuran-2-yl)acetate (3j)

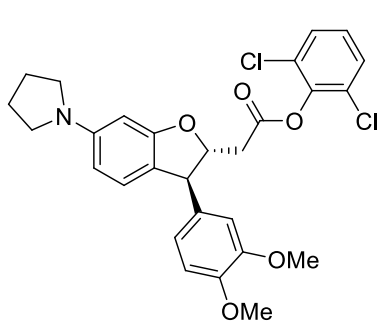

Pale yellow solid, $38.2 \mathrm{mg}, 72 \%$ yield, $99: 1 \mathrm{dr}$, $99 \%$ ee; m.p.150.8-153.4 ${ }^{\circ} \mathrm{C},[\alpha]_{\mathrm{D}}{ }^{20}=20.4\left(c 1.0, \mathrm{CHCl}_{3}\right) ;{ }^{1} \mathrm{H}$ NMR $(400 \mathrm{MHz}$, $\left.\mathrm{CDCl}_{3}\right) \delta 7.35(\mathrm{~d}, J=8.0 \mathrm{~Hz}, 2 \mathrm{H}), 7.14(\mathrm{t}, J=8.0 \mathrm{~Hz}, 1 \mathrm{H}), 6.87(\mathrm{~d}, J$ $=8.0 \mathrm{~Hz}, 1 \mathrm{H}), 6.82-6.76(\mathrm{~m}, 3 \mathrm{H}), 6.17(\mathrm{~s}, 1 \mathrm{H}), 6.13(\mathrm{~d}, J=8.0 \mathrm{~Hz}$, $1 \mathrm{H}), 5.07-5.02(\mathrm{~m}, 1 \mathrm{H}), 4.33(\mathrm{~d}, J=6.4 \mathrm{~Hz}, 1 \mathrm{H}), 3.86(\mathrm{~s}, 3 \mathrm{H}), 3.81$ (s, $3 \mathrm{H}), 3.28(\mathrm{t}, J=6.0 \mathrm{~Hz}, 4 \mathrm{H}), 3.24-3.11(\mathrm{~m}, 2 \mathrm{H}), 2.00(\mathrm{t}, J=6.4 \mathrm{~Hz}$, $4 \mathrm{H}) ;{ }^{13} \mathrm{C}$ NMR $\left(100 \mathrm{MHz}, \mathrm{CDCl}_{3}\right) \delta 167.3,160.8,149.52,149.50$, $148.4,144.2,135.7,129.2,128.9,127.6,125.8,120.4,116.6,111.5,111.2,105.3,93.8,87.4,56.2$, 56.1, 53.7, 48.2, 39.4, 25.8; HRMS (ESI) for $\mathrm{C}_{28} \mathrm{H}_{28} \mathrm{Cl}_{2} \mathrm{NO}_{5}[\mathrm{M}+\mathrm{H}]+$ : calcd 528.1339, found 528.135 .

2,6-dichlorophenyl 2-((2R,3S)-3-(3,5-dimethoxyphenyl)-6-(pyrrolidin-1-yl)-2,3dihydrobenzofuran-2-yl)acetate (3k)

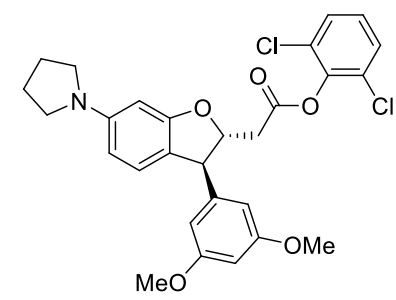

Pale yellow solid, $52.6 \mathrm{mg}$, 99\% yield, 99:1 dr, 99\% ee; m.p.129.5-131.4 ${ }^{\circ} \mathrm{C},[\alpha]_{\mathrm{D}}{ }^{20}=34.9\left(c 1.0, \mathrm{CHCl}_{3}\right) ;{ }^{1} \mathrm{H} \mathrm{NMR}(400 \mathrm{MHz}$, $\left.\mathrm{CDCl}_{3}\right) \delta 7.35(\mathrm{~d}, J=8.0 \mathrm{~Hz}, 2 \mathrm{H}), 7.15(\mathrm{t}, J=7.6 \mathrm{~Hz}, 1 \mathrm{H}), 6.88(\mathrm{~d}, J$ $=8.0 \mathrm{~Hz}, 1 \mathrm{H}), 6.40(\mathrm{~d}, J=1.2 \mathrm{~Hz}, 2 \mathrm{H}), 6.35(\mathrm{~d}, J=2.0 \mathrm{~Hz}, 1 \mathrm{H}), 6.16$ $(\mathrm{s}, 1 \mathrm{H}), 6.12(\mathrm{~d}, J=8.0 \mathrm{~Hz}, 1 \mathrm{H}), 5.09(\mathrm{q}, J=6.0 \mathrm{~Hz}, 1 \mathrm{H}), 4.30(\mathrm{~d}, J=$ $6.0 \mathrm{~Hz}, 1 \mathrm{H}), 3.75$ (s, $6 \mathrm{H}), 3.28$ (t, $J=6.0 \mathrm{~Hz}, 4 \mathrm{H}), 3.23-3.10(\mathrm{~m}, 2 \mathrm{H})$, $2.00(\mathrm{t}, J=6.0 \mathrm{~Hz}, 4 \mathrm{H}) ;{ }^{13} \mathrm{C} \mathrm{NMR}\left(100 \mathrm{MHz}, \mathrm{CDCl}_{3}\right) \delta 167.2,161.4,160.9,149.6,145.7,144.2$, 129.3, 128.9, 127.6, 125.9, 116.1, 106.3, 105.4, 99.3, 93.9, 87.1, 55.7, 54.3, 48.2, 39.6, 25.8; HRMS (ESI) for $\mathrm{C}_{28} \mathrm{H}_{28} \mathrm{Cl}_{2} \mathrm{NO}_{5}[\mathrm{M}+\mathrm{H}]+$ : calcd 528.1339, found 528.1344 .

2,6-dichlorophenyl 2-((2R,3S)-6-(pyrrolidin-1-yl)-3-(3,4,5-trimethoxyphenyl)-2,3dihydrobenzofuran-2-yl)acetate (3l)

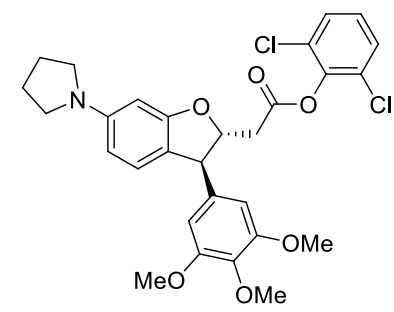

Pale yellow solid, $48.0 \mathrm{mg}, 86 \%$ yield, 99:1 dr, 99\% ee; m.p.117.2-119.4 ${ }^{\circ} \mathrm{C},[\alpha]_{\mathrm{D}}{ }^{20}=28.8\left(c\right.$ 1.56, $\left.\mathrm{CHCl}_{3}\right) ;{ }^{1} \mathrm{H}$ NMR $(400$ $\left.\mathrm{MHz}, \mathrm{CDCl}_{3}\right) \delta 7.35(\mathrm{~d}, J=8.0 \mathrm{~Hz}, 2 \mathrm{H}), 7.14(\mathrm{t}, J=8.0 \mathrm{~Hz}, 1 \mathrm{H})$, $6.89(\mathrm{~d}, J=8.0 \mathrm{~Hz}, 1 \mathrm{H}), 6.45(\mathrm{~s}, 2 \mathrm{H}), 6.17-6.12(\mathrm{~m}, 2 \mathrm{H}), 5.08$ (q, $J=$ $6.4 \mathrm{~Hz}, 1 \mathrm{H}), 4.31$ (d, $J=6.0 \mathrm{~Hz}, 1 \mathrm{H}), 3.83(\mathrm{~s}, 3 \mathrm{H}), 3.80(\mathrm{~s}, 6 \mathrm{H}), 3.28$ 
$(\mathrm{t}, J=6.0 \mathrm{~Hz}, 4 \mathrm{H}), 3.24-3.11(\mathrm{~m}, 2 \mathrm{H}), 2.00(\mathrm{t}, J=6.0 \mathrm{~Hz}, 4 \mathrm{H}) ;{ }^{13} \mathrm{C}$ NMR $\left(100 \mathrm{MHz}, \mathrm{CDCl}_{3}\right) \delta$ $167.3,160.8,153.7,149.6,144.2,138.9,137.2,129.2$, 128.9, 127.6, 125.9, 116.2, 105.4, 105.1, 93.9, 87.2, 61.1, 56.5, 54.4, 48.2, 39.6, 25.8; HRMS (ESI) for $\mathrm{C}_{29} \mathrm{H}_{30} \mathrm{Cl}_{2} \mathrm{NO}_{6}[\mathrm{M}+\mathrm{H}]+$ : calcd 558.1445 , found 558.1439 .

\section{2,6-dichlorophenyl 2-((2R,3S)-3-(furan-2-yl)-6-(pyrrolidin-1-yl)-2,3-dihydrobenzofuran}

-2 -yl)acetate $(3 \mathrm{~m})$

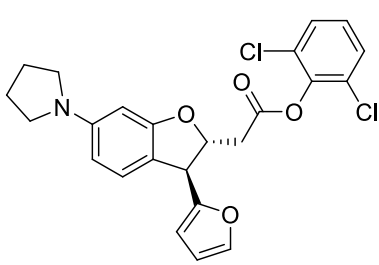

Pale yellow solid, $42.2 \mathrm{mg}$, 92\% yield, 99:1 dr, 99\% ee; m.p.122.5-123.8 ${ }^{\circ} \mathrm{C},[\alpha]_{\mathrm{D}}{ }^{20}=18.7$ (c 1.26, $\left.\mathrm{CHCl}_{3}\right) ;{ }^{1} \mathrm{H}$ NMR $(400$ $\left.\mathrm{MHz}, \mathrm{CDCl}_{3}\right) \delta$ 7.37-7.35 (m, 3H), $7.14(\mathrm{t}, J=8.0 \mathrm{~Hz}, 1 \mathrm{H}), 7.03(\mathrm{~d}, J$ $=8.4 \mathrm{~Hz}, 1 \mathrm{H}), 6.32-6.30(\mathrm{~m}, 1 \mathrm{H}), 6.16-6.11(\mathrm{~m}, 3 \mathrm{H}), 5.24(\mathrm{q}, J=6.0$ $\mathrm{Hz}, 1 \mathrm{H}), 4.51(\mathrm{~d}, J=6.4 \mathrm{~Hz}, 1 \mathrm{H}), 3.29-3.16(\mathrm{~m}, 6 \mathrm{H}), 2.00$ (t, $J=6.4$ $\mathrm{Hz}, 4 \mathrm{H}) ;{ }^{13} \mathrm{C}$ NMR $\left(100 \mathrm{MHz}, \mathrm{CDCl}_{3}\right) \delta 167.1,160.7,155.4,149.8$, 144.2, 142.5, 129.3, 128.9, 127.6, 125.7, 113.2, 110.6, 106.4, 105.2, 94.1, 84.2, 48.2, 47.1, 39.7, 25.8; HRMS (ESI) for $\mathrm{C}_{24} \mathrm{H}_{22} \mathrm{Cl}_{2} \mathrm{NO}_{4}[\mathrm{M}+\mathrm{H}]+$ : calcd 458.0920, found 458.0935 .

\section{2,6-dichlorophenyl 2-((2R,3S)-6-(pyrrolidin-1-yl)-3-(thiophen-2-yl)-2,3-dihydrobenzofuran} -2-yl)acetate (3n)

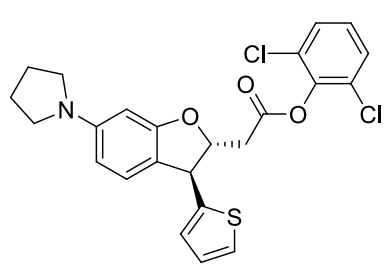

Pale yellow solid, $45.7 \mathrm{mg}, 96 \%$ yield, 99:1 dr, 99\% ee; m.p.162.1-163.8 ${ }^{\circ} \mathrm{C},[\alpha]_{\mathrm{D}}{ }^{20}=3.3\left(c 1.21, \mathrm{CHCl}_{3}\right) ;{ }^{1} \mathrm{H}$ NMR $(400 \mathrm{MHz}$, $\left.\mathrm{CDCl}_{3}\right) \delta 7.36(\mathrm{~d}, J=8.0 \mathrm{~Hz}, 2 \mathrm{H}), 7.20(\mathrm{dd}, J=8.8,1.2 \mathrm{~Hz}, 1 \mathrm{H}), 7.14$ $(\mathrm{t}, J=8.0 \mathrm{~Hz}, 1 \mathrm{H}), 7.01(\mathrm{~d}, J=8.4 \mathrm{~Hz}, 1 \mathrm{H}), 6.97-6.95(\mathrm{~m}, 2 \mathrm{H})$, 6.16-6.14 (m, 2H), $5.11(\mathrm{q}, J=6.4 \mathrm{~Hz}, 1 \mathrm{H}), 4.69(\mathrm{~d}, J=6.4 \mathrm{~Hz}, 1 \mathrm{H})$, 3.30-3.27 (m, 4H), 3.25-3.16 (m, 2H), 2.01-1.98 (m, 4H); ${ }^{13} \mathrm{C} \mathrm{NMR}$ $\left(100 \mathrm{MHz}, \mathrm{CDCl}_{3}\right) \delta 167.1,160.6,149.8,146.8,144.2,129.2,128.9,127.6,127.3,125.9,125.1$, 124.8, 115.8, 105.3, 93.9, 87.3, 48.9, 48.2, 39.1, 25.8; HRMS (ESI) for $\mathrm{C}_{24} \mathrm{H}_{22} \mathrm{Cl}_{2} \mathrm{NO}_{3} \mathrm{~S}[\mathrm{M}+\mathrm{H}]^{+}$: calcd 474.0692, found 474.0705 .

\section{2,6-dichlorophenyl 2-((2R,3R)-6-(pyrrolidin-1-yl)-3-(thiophen-3-yl)-2,3-dihydrobenzofuran} -2-yl)acetate (3o)

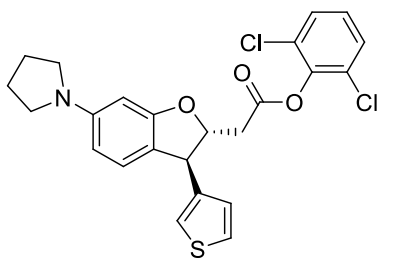

Pale yellow solid, $44.5 \mathrm{mg}$, 94\% yield, 99:1 dr, 99\% ee; m.p.173.5-175.2 ${ }^{\circ} \mathrm{C},[\alpha]_{\mathrm{D}}{ }^{20}=28.8\left(\right.$ c $\left.1.16, \mathrm{CHCl}_{3}\right) ;{ }^{1} \mathrm{H}$ NMR $(400$ $\left.\mathrm{MHz}, \mathrm{CDCl}_{3}\right) \delta 7.36(\mathrm{~d}, J=8.0 \mathrm{~Hz}, 2 \mathrm{H}), 7.29-7.26(\mathrm{~m}, 1 \mathrm{H}), 7.15(\mathrm{t}$, $J=8.0 \mathrm{~Hz}, 1 \mathrm{H}), 7.04(\mathrm{~d}, J=2.0 \mathrm{~Hz}, 1 \mathrm{H}), 7.00-6.93(\mathrm{~m}, 2 \mathrm{H})$, 6.16-6.12 (m, 2H), 5.09 (q, $J=6.0 \mathrm{~Hz}, 1 \mathrm{H}), 4.49(\mathrm{~d}, J=5.6 \mathrm{~Hz}, 1 \mathrm{H})$, 3.29-3.11 (m, 6H), $2.00(\mathrm{t}, J=6.0 \mathrm{~Hz}, 4 \mathrm{H}) ;{ }^{13} \mathrm{C}$ NMR $(100 \mathrm{MHz}$, $\left.\mathrm{CDCl}_{3}\right) \delta 167.3,160.6,149.6,144.2,143.8,129.2,128.9,127.6,127.4,126.6,125.8,121.8,115.9$, 105.2, 93.9, 86.3, 49.0, 48.2, 39.4, 25.8; HRMS (ESI) for $\mathrm{C}_{24} \mathrm{H}_{22} \mathrm{Cl}_{2} \mathrm{NO}_{3} \mathrm{~S}[\mathrm{M}+\mathrm{H}]+$ : calcd 474.0692, found 474.0693 .

2,6-dichlorophenyl 2-((2R,3S)-3-(naphthalen-2-yl)-6-(pyrrolidin-1-yl)-2,3-dihydrobenzofuran -2 -yl)acetate (3p)

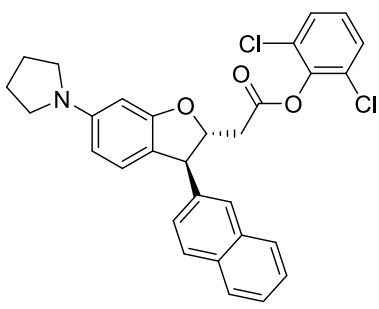

Pale yellow solid, $50.4 \mathrm{mg}$, 97\% yield, 99:1 dr, 99\% ee; m.p.154.3-157.1 ${ }^{\circ} \mathrm{C},[\alpha]_{\mathrm{D}}{ }^{20}=73.0\left(c 0.90, \mathrm{CHCl}_{3}\right) ;{ }^{1} \mathrm{H}$ NMR $(400$ $\left.\mathrm{MHz}, \mathrm{CDCl}_{3}\right) \delta$ 7.83-7.79 (m, 3H), $7.71(\mathrm{~s}, 1 \mathrm{H}), 7.49-7.44(\mathrm{~m}, 2 \mathrm{H})$, 7.40-7.34 (m, 3H), $7.13(\mathrm{t}, J=8.0 \mathrm{~Hz}, 1 \mathrm{H}), 6.89(\mathrm{~d}, J=8.0 \mathrm{~Hz}, 1 \mathrm{H})$, 
$6.23(\mathrm{~d}, J=1.2 \mathrm{~Hz}, 1 \mathrm{H}), 6.15(\mathrm{dd}, J=8.0,1.6 \mathrm{~Hz}, 1 \mathrm{H}), 5.24-5.19(\mathrm{~m}, 1 \mathrm{H}), 4.56(\mathrm{~d}, J=6.0 \mathrm{~Hz}$, 1H), 3.32-3.18 (m, 6H), $2.02(\mathrm{t}, J=6.4 \mathrm{~Hz}, 4 \mathrm{H}) ;{ }^{13} \mathrm{C} \mathrm{NMR}\left(100 \mathrm{MHz}, \mathrm{CDCl}_{3}\right) \delta 167.3,160.9$, 149.6, 144.2, 140.6, 133.8, 132.9, 129.2, 129.0, 128.9, 128.1, 127.9, 127.6, 126.9, 126.5, 126.4, 126.0, 125.9, 116.6, 105.3, 93.9, 87.2, 54.3, 48.2, 39.6, 25.8; HRMS (ESI) for $\mathrm{C}_{30} \mathrm{H}_{26} \mathrm{Cl}_{2} \mathrm{NO}_{3}$ $[\mathrm{M}+\mathrm{H}]+$ : calcd 518.1284 , found 518.1297 .

\section{2,6-dichlorophenyl 2-((2R,3R)-3-(4-methoxyphenyl)-6-(pyrrolidin-1-yl)-2,3-dihydro} benzofuran-2-yl)acetate (3q)

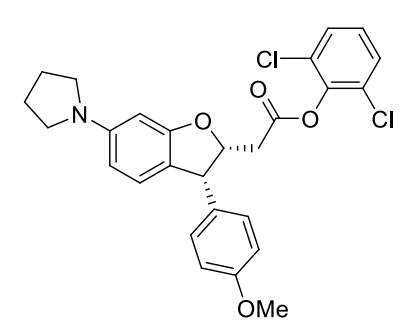

Yellow oil, $35.0 \mathrm{mg}, 70 \%$ yield, $97: 3 \mathrm{dr}, 99 \%$ ee; $[\alpha]_{\mathrm{D}}^{20}=8.8(c$ 1.0, $\left.\mathrm{CHCl}_{3}\right) ;{ }^{1} \mathrm{H} \mathrm{NMR}\left(400 \mathrm{MHz}, \mathrm{CDCl}_{3}\right) \delta 7.34(\mathrm{~d}, J=8.4 \mathrm{~Hz}, 2 \mathrm{H}), 7.13$ (t, $J=8.0 \mathrm{~Hz}, 1 \mathrm{H}), 6.97(\mathrm{~d}, J=8.4 \mathrm{~Hz}, 2 \mathrm{H}), 6.92(\mathrm{~d}, J=8.0 \mathrm{~Hz}, 1 \mathrm{H})$, $6.83(\mathrm{~d}, J=8.8 \mathrm{~Hz}, 2 \mathrm{H}), 6.18(\mathrm{~d}, J=1.6 \mathrm{~Hz}, 1 \mathrm{H}), 6.14(\mathrm{dd}, J=8.4$, $2.0 \mathrm{~Hz}, 1 \mathrm{H}), 5.42-5.37(\mathrm{~m}, 1 \mathrm{H}), 4.64(\mathrm{~d}, J=8.0 \mathrm{~Hz}, 1 \mathrm{H}), 3.79(\mathrm{~s}, 3 \mathrm{H})$, $3.29(\mathrm{t}, J=6.4 \mathrm{~Hz}, 4 \mathrm{H}), 2.88-2.82(\mathrm{~m}, 1 \mathrm{H}), 2.59-2.53(\mathrm{~m}, 1 \mathrm{H})$, 2.05-1.99 (m, 4H); ${ }^{13} \mathrm{C}$ NMR (100 MHz, $\left.\mathrm{CDCl}_{3}\right) \delta 167.9,161.0$, 159.0, 149.4, 144.2, 132.2, 130.4, 129.3, 128.9, 127.5, 126.1, 117.7, 114.3, 105.4, 93.7, 83.0, 55.6, 50.1, 48.2, 36.5, 25.8; HRMS (ESI) for $\mathrm{C}_{27} \mathrm{H}_{26} \mathrm{Cl}_{2} \mathrm{NO}_{4}[\mathrm{M}+\mathrm{H}]+$ : calcd 498.1233, found 498.1231.

2,6-dichlorophenyl 2-((2R,3R)-3-(4-chlorophenyl)-6-(pyrrolidin-1-yl)-2,3-dihydrobenzofuran $-2-y l)$ acetate (3r)

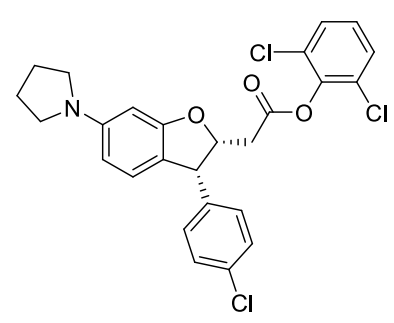

Yellow oil, $35.1 \mathrm{mg}, 70 \%$ yield, $97: 3 \mathrm{dr}, 99 \%$ ee; $[\alpha]_{\mathrm{D}^{20}}=-22.6(c 1.4$, $\left.\mathrm{CHCl}_{3}\right) ;{ }^{1} \mathrm{H} \mathrm{NMR}\left(400 \mathrm{MHz}, \mathrm{CDCl}_{3}\right) \delta 7.34(\mathrm{~d}, J=8.0 \mathrm{~Hz}, 2 \mathrm{H}), 7.26$ $(\mathrm{d}, J=8.4 \mathrm{~Hz}, 2 \mathrm{H}), 7.14(\mathrm{t}, J=8.0 \mathrm{~Hz}, 1 \mathrm{H}), 6.98(\mathrm{~d}, J=8.4 \mathrm{~Hz}, 2 \mathrm{H})$, $6.89(\mathrm{~d}, J=8.0 \mathrm{~Hz}, 1 \mathrm{H}), 6.18(\mathrm{~s}, 1 \mathrm{H}), 6.14(\mathrm{~d}, J=8.0 \mathrm{~Hz}, 1 \mathrm{H})$, $5.42-5.37(\mathrm{~m}, 1 \mathrm{H}), 4.63(\mathrm{~d}, J=8.0 \mathrm{~Hz}, 1 \mathrm{H}), 3.29(\mathrm{t}, J=6.4 \mathrm{~Hz}, 4 \mathrm{H})$, 2.93-2.87 (m, 1H), 2.60-2.54 (m, 1H), 2.03-1.99 (m, 4H); ${ }^{13} \mathrm{C}$ NMR $\left(100 \mathrm{MHz}, \mathrm{CDCl}_{3}\right) \delta 167.6,161.0,149.6,144.1,138.8,133.3,130.8$, 129.2, 129.1, 128.9, 127.6, 126.0, 117.2, 105.6, 93.7, 82.6, 50.3, 48.2, 36.3, 25.8; HRMS (ESI) for $\mathrm{C}_{26} \mathrm{H}_{23} \mathrm{Cl}_{3} \mathrm{NO}_{3}[\mathrm{M}+\mathrm{H}]^{+}$: calcd 502.0738, found 502.0736; $\mathrm{C}_{26} \mathrm{H}_{23}{ }^{35} \mathrm{Cl}_{2}{ }^{37} \mathrm{ClNO}_{3}[\mathrm{M}+\mathrm{H}]^{+}$: calcd 504.0709, found: 504.0707.

\section{2,6-dichlorophenyl 2-((2R,3R)-3-(naphthalen-2-yl)-6-(pyrrolidin-1-yl)-2,3-dihydro} benzofuran-2-yl)acetate (3s)

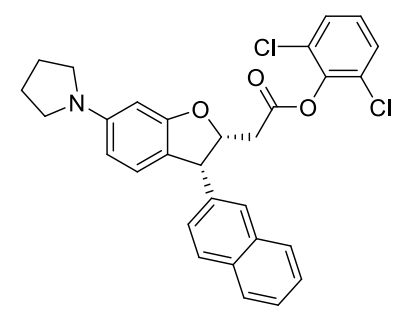

Yellow oil, $49.9 \mathrm{mg}, 96 \%$ yield, $98: 2 \mathrm{dr}, 99 \%$ ee; $[\alpha]_{\mathrm{D}}^{20}=-44.9(c$ 1.0, $\left.\mathrm{CHCl}_{3}\right) ;{ }^{1} \mathrm{H}$ NMR $\left(400 \mathrm{MHz}, \mathrm{CDCl}_{3}\right) \delta$ 7.84-7.78 (m, 3H), $7.61(\mathrm{~s}$, $1 \mathrm{H}), 7.51-7.46(\mathrm{~m}, 2 \mathrm{H}), 7.32(\mathrm{~d}, J=8.0 \mathrm{~Hz}, 2 \mathrm{H}), 7.17(\mathrm{~d}, J=8.4 \mathrm{~Hz}$, 1H), 7.11 (t, $J=8.0 \mathrm{~Hz}, 1 \mathrm{H}), 6.97$ (d, $J=8.0 \mathrm{~Hz}, 1 \mathrm{H}), 6.27$ (s, 1H), $6.17(\mathrm{~d}, J=8.0 \mathrm{~Hz}, 1 \mathrm{H}), 5.56-5.51(\mathrm{~m}, 1 \mathrm{H}), 4.86(\mathrm{~d}, J=8.4 \mathrm{~Hz}, 1 \mathrm{H})$, $3.33(\mathrm{t}, J=6.4 \mathrm{~Hz}, 4 \mathrm{H}), 2.97-2.90(\mathrm{~m}, 1 \mathrm{H}), 2.65-2.60(\mathrm{~m}, 1 \mathrm{H})$, 2.05-2.01 (m, 4H); ${ }^{13} \mathrm{C}$ NMR (100 MHz, $\left.\mathrm{CDCl}_{3}\right) \delta 167.8,161.1$, $149.5,144.1,137.7,133.7,133.0,129.2,128.8,128.7,128.2,128.0,127.9,127.5,127.4,126.4$, 126.2, 126.1, 117.6, 105.5, 93.8, 82.9, 51.1, 48.2, 36.5, 25.8; HRMS (ESI) for $\mathrm{C}_{30} \mathrm{H}_{26} \mathrm{Cl}_{2} \mathrm{NO}_{3}$ $[\mathrm{M}+\mathrm{H}]+$ : calcd 518.1284 , found 518.1284 . 


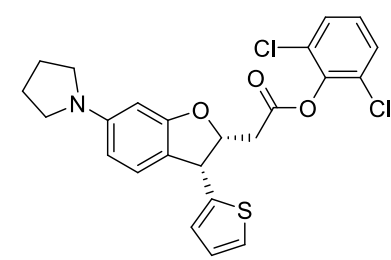

Yellow oil, $47.2 \mathrm{mg}, 99 \%$ yield, $98: 2 \mathrm{dr}, 99 \%$ ee; $[\alpha]_{\mathrm{D}}^{20}=46.0(c 1.0$, $\left.\mathrm{CHCl}_{3}\right) ;{ }^{1} \mathrm{H} \mathrm{NMR}\left(400 \mathrm{MHz}, \mathrm{CDCl}_{3}\right) \delta 7.36(\mathrm{~d}, J=8.0 \mathrm{~Hz}, 2 \mathrm{H}), 7.22$ $(\mathrm{d}, J=4.8 \mathrm{~Hz}, 1 \mathrm{H}), 7.14(\mathrm{t}, J=8.0 \mathrm{~Hz}, 1 \mathrm{H}), 7.05(\mathrm{~d}, J=8.4 \mathrm{~Hz}, 1 \mathrm{H})$, 7.00-6.98 (m, 1H), $6.86(\mathrm{~d}, J=2.0 \mathrm{~Hz}, 1 \mathrm{H}), 6.18-6.16(\mathrm{~m}, 2 \mathrm{H})$, $5.41-5.35(\mathrm{~m}, 1 \mathrm{H}), 4.99(\mathrm{~d}, J=8.0 \mathrm{~Hz}, 1 \mathrm{H}), 3.32(\mathrm{t}, J=6.0 \mathrm{~Hz}, 4 \mathrm{H})$, 3.01-2.95 (m, 1H), 2.76-2.70 (m, 1H), 2.05-2.01 (m, 4H); ${ }^{13} \mathrm{C}$ NMR $\left(100 \mathrm{MHz}, \mathrm{CDCl}_{3}\right) \delta 167.9,160.6,149.7,144.1,144.0,129.2,128.9,127.6,127.4,126.5,126.2$, 125.3, 117.0, 105.4, 93.7, 82.7, 48.1, 45.9, 36.0, 25.8; HRMS (ESI) for $\mathrm{C}_{24} \mathrm{H}_{22} \mathrm{Cl}_{2} \mathrm{NO}_{3} \mathrm{~S}[\mathrm{M}+\mathrm{H}]+$ calcd 474.0692, found 474.0691 .

2,6-dichlorophenyl 2-((2R, 3S)-6-(dimethylamino)-3-phenyl-2,3-dihydrobenzofuran-2-yl) acetate (3aa)

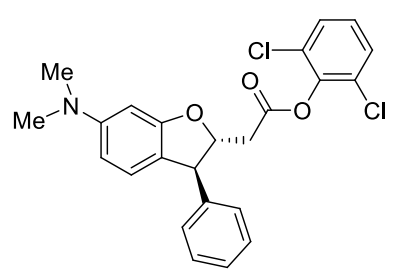

White solid, $39.6 \mathrm{mg}$, 89\% yield, 98:2 dr, 99\% ee; m.p.201.2-203.5 ${ }^{\circ} \mathrm{C},[\alpha]_{\mathrm{D}^{20}}=52.1\left(c 0.6, \mathrm{CHCl}_{3}\right) ;{ }^{1} \mathrm{H}$ NMR $\left(400 \mathrm{MHz}, \mathrm{CDCl}_{3}\right) \delta$ 7.37-7.23 (m, 7H), $7.14(\mathrm{t}, J=8.4 \mathrm{~Hz}, 1 \mathrm{H}), 6.88(\mathrm{~d}, J=8.4 \mathrm{~Hz}, 1 \mathrm{H})$, $6.34(\mathrm{~d}, J=2.0 \mathrm{~Hz}, 1 \mathrm{H}), 6.29(\mathrm{dd}, J=8.4,2.0 \mathrm{~Hz}, 1 \mathrm{H}), 5.13-5.08(\mathrm{~m}$, $1 \mathrm{H}), 4.38(\mathrm{~d}, J=6.4 \mathrm{~Hz}, 1 \mathrm{H}), 3.26-3.12(\mathrm{~m}, 2 \mathrm{H}), 2.95(\mathrm{~s}, 6 \mathrm{H}) ;{ }^{13} \mathrm{C}$ $\mathrm{NMR}\left(100 \mathrm{MHz}, \mathrm{CDCl}_{3}\right) \delta 167.3,160.9,152.4,144.2,143.1,129.2$, 129.1, 128.9, 128.3, 127.6, 127.4, 125.8, 117.8, 106.1, 94.9, 87.4, 54.0, 41.2, 39.5; HRMS (ESI) for $\mathrm{C}_{24} \mathrm{H}_{22} \mathrm{Cl}_{2} \mathrm{NO}_{3}[\mathrm{M}+\mathrm{H}]+$ : calcd 442.0971, found 442.0966 .

\section{2,6-dichlorophenyl 2-((2R,3S)-6-(diethylamino)-3-phenyl-2,3-dihydrobenzofuran-2-yl)} acetate (3ab)

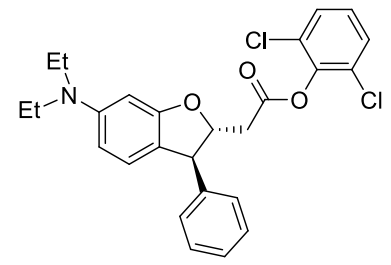

Pale yellow solid, $45.4 \mathrm{mg}$, 97\% yield, 99:1 dr, 99\% ee; m.p.140.3-142. $8^{\circ} \mathrm{C},[\alpha]_{\mathrm{D}}{ }^{20}=45.1\left(c 1.0, \mathrm{CHCl}_{3}\right) ;{ }^{1} \mathrm{H}$ NMR $(400 \mathrm{MHz}$, $\left.\mathrm{CDCl}_{3}\right) \delta 7.35-7.24(\mathrm{~m}, 7 \mathrm{H}), 7.13(\mathrm{t}, J=8.0 \mathrm{~Hz}, 1 \mathrm{H}), 6.84(\mathrm{~d}, J=8.4$ $\mathrm{Hz}, 1 \mathrm{H}), 6.28(\mathrm{~s}, 1 \mathrm{H}), 6.23(\mathrm{~d}, J=8.4 \mathrm{~Hz}, 1 \mathrm{H}), 5.11-5.06(\mathrm{~m}, 1 \mathrm{H})$, $4.36(\mathrm{~d}, J=5.6 \mathrm{~Hz}, 1 \mathrm{H}), 3.34(\mathrm{q}, J=6.8 \mathrm{~Hz}, 4 \mathrm{H}), 3.25-3.12(\mathrm{~m}, 2 \mathrm{H})$, $1.17(\mathrm{t}, J=6.8 \mathrm{~Hz}, 6 \mathrm{H}) ;{ }^{13} \mathrm{C} \mathrm{NMR}\left(100 \mathrm{MHz}, \mathrm{CDCl}_{3}\right) \delta 167.3,161.2$, 149.6, 144.2, 143.2, 129.2, 129.1, 128.9, 128.3, 127.6, 127.4, 125.9, 116.5, 105.3, 93.9, 87.3, 54.1, 44.9, 39.5, 12.9; HRMS (ESI) for $\mathrm{C}_{26} \mathrm{H}_{26} \mathrm{Cl}_{2} \mathrm{NO}_{3}[\mathrm{M}+\mathrm{H}]+$ : calcd 470.1284, found 470.1273.

2,6-dichlorophenyl 2-((2R, 3S)-6-(dibenzylamino)-3-phenyl-2,3-dihydrobenzofuran-2-yl) acetate (3ac)

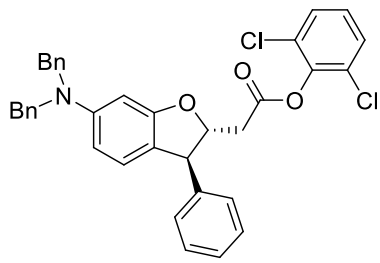

Pale yellow solid, $47.0 \mathrm{mg}, 79 \%$ yield, $99: 1 \mathrm{dr}$, 98\% ee; m.p. $146.5-147.7{ }^{\circ} \mathrm{C},[\alpha]_{\mathrm{D}}{ }^{20}=31.8\left(c \quad 0.85, \mathrm{CHCl}_{3}\right) ;{ }^{1} \mathrm{H}$ NMR $(400$ $\left.\mathrm{MHz}, \mathrm{CDCl}_{3}\right) \delta 7.36-7.24(\mathrm{~m}, 17 \mathrm{H}), 7.13(\mathrm{t}, J=8.0 \mathrm{~Hz}, 1 \mathrm{H}), 6.79(\mathrm{~d}$, $J=8.0 \mathrm{~Hz}, 1 \mathrm{H}), 6.35(\mathrm{~d}, J=2.4 \mathrm{~Hz}, 1 \mathrm{H}), 6.29(\mathrm{dd}, J=8.0,2.0 \mathrm{~Hz}$, $1 \mathrm{H}), 5.09(\mathrm{q}, J=6.0 \mathrm{~Hz}, 1 \mathrm{H}), 4.64-4.60(\mathrm{~m}, 4 \mathrm{H}), 4.36(\mathrm{~d}, J=6.4 \mathrm{~Hz}$, 1H), 3.24-3.11 (m, 2H); ${ }^{13} \mathrm{C}$ NMR (100 MHz, $\left.\mathrm{CDCl}_{3}\right) \delta 167.2,161.0,151.1,144.1,142.9,138.8$, 129.2, 129.1, 129.0, 128.9, 128.3, 127.6, 127.4, 127.3, 127.0, 125.8, 118.1, 106.0, 94.9, 87.4, 54.8, 54.0, 39.4; HRMS (ESI) for $\mathrm{C}_{36} \mathrm{H}_{30} \mathrm{Cl}_{2} \mathrm{NO}_{3}[\mathrm{M}+\mathrm{H}]+$ : calcd 594.1597, found 594.1601. 


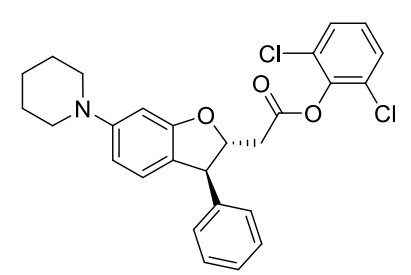

White solid, $43.8 \mathrm{mg}$, 91\% yield, 99:1 dr, 99\% ee; m.p.143.7-145.4 ${ }^{\circ} \mathrm{C},[\alpha]_{\mathrm{D}}{ }^{20}=43.9\left(c 1.27, \mathrm{CHCl}_{3}\right) ;{ }^{1} \mathrm{H}$ NMR $\left(400 \mathrm{MHz}, \mathrm{CDCl}_{3}\right) \delta$ 7.37-7.22 (m, 7H), $7.14(\mathrm{t}, J=8.0 \mathrm{~Hz}, 1 \mathrm{H}), 6.88(\mathrm{~d}, J=8.4 \mathrm{~Hz}, 1 \mathrm{H})$, $6.53(\mathrm{~d}, J=1.2 \mathrm{~Hz}, 1 \mathrm{H}), 6.50(\mathrm{~d}, J=8.0 \mathrm{~Hz}, 1 \mathrm{H}), 5.13-5.09(\mathrm{~m}, 1 \mathrm{H})$, $4.39(\mathrm{~d}, J=6.4 \mathrm{~Hz}, 1 \mathrm{H}), 3.26-3.12(\mathrm{~m}, 6 \mathrm{H}), 1.74-1.69(\mathrm{~m}, 4 \mathrm{H})$, 1.61-1.57 (m, 2H); $\left.{ }^{13} \mathrm{C} \mathrm{NMR} \mathrm{(100} \mathrm{MHz,} \mathrm{CDCl}_{3}\right) \delta 167.2,160.7$, 154.0, 144.2, 142.8, 129.2, 129.1, 128.9, 128.3, 127.6, 127.5, 125.7, 120.2, 110.0, 98.7, 87.4, 54.1, 51.1, 39.5, 26.2, 24.6; HRMS (ESI) for $\mathrm{C}_{27} \mathrm{H}_{26} \mathrm{Cl}_{2} \mathrm{NO}_{3}[\mathrm{M}+\mathrm{H}]+$ : calcd 482.1284, found 482.1282.

2,6-dichlorophenyl 2-((2R,3S)-6-(azepan-1-yl)-3-phenyl-2,3-dihydrobenzofuran-2-yl)acetate (3ae)

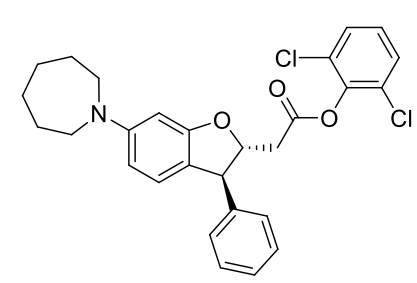

Pale yellow solid, $47.2 \mathrm{mg}$, 95\% yield, 99:1 dr, 99\% ee; m.p.156.7-158.6 ${ }^{\circ} \mathrm{C},[\alpha]_{\mathrm{D}}{ }^{20}=39.2\left(c 1.65, \mathrm{CHCl}_{3}\right) ;{ }^{1} \mathrm{H}$ NMR $(400$ $\left.\mathrm{MHz}, \mathrm{CDCl}_{3}\right) \delta$ 7.37-7.26 (m, 7H), $7.14(\mathrm{t}, J=8.0 \mathrm{~Hz}, 1 \mathrm{H}), 6.84(\mathrm{~d}$, $J=8.4 \mathrm{~Hz}, 1 \mathrm{H}), 6.31(\mathrm{~s}, 1 \mathrm{H}), 6.25(\mathrm{~d}, J=8.4 \mathrm{~Hz}, 1 \mathrm{H}), 5.12-5.09(\mathrm{~m}$, $1 \mathrm{H}), 4.38(\mathrm{~d}, J=5.6 \mathrm{~Hz}, 1 \mathrm{H}), 3.46-3.43(\mathrm{~m}, 4 \mathrm{H}), 3.27-3.14(\mathrm{~m}, 2 \mathrm{H})$, 1.83-1.76 (m, 4H), 1.59-1.55 (m, 4H); $\left.{ }^{13} \mathrm{C} \mathrm{NMR} \mathrm{(100} \mathrm{MHz,} \mathrm{CDCl}_{3}\right)$ $\delta 167.3,161.2,150.7,144.2,143.2,129.2,129.0,128.9,128.3,127.6,127.3,125.9,116.3,104.8$, 93.4, 87.3, 54.1, 49.8, 39.5, 28.1, 27.5; HRMS (ESI) for $\mathrm{C}_{28} \mathrm{H}_{28} \mathrm{Cl}_{2} \mathrm{NO}_{3}[\mathrm{M}+\mathrm{H}]+$ : calcd 496.1441, found 496.1457 .

2,6-dichlorophenyl 2-((2R,3S)-6-morpholino-3-phenyl-2,3-dihydrobenzofuran-2-yl)acetate (3af)

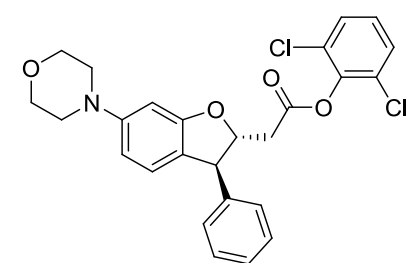

White solid, $17.1 \mathrm{mg}, 35 \%$ yield, $98: 2 \mathrm{dr}$, 99\% ee; m.p.120.7-122.3 ${ }^{\circ} \mathrm{C},[\alpha]_{\mathrm{D}}{ }^{20}=39.1\left(c 1.00, \mathrm{CHCl}_{3}\right) ;{ }^{1} \mathrm{H}$ NMR $\left(400 \mathrm{MHz}, \mathrm{CDCl}_{3}\right) \delta$ 7.37-7.22 (m, 7H), 7.14 (t, $J=8.4 \mathrm{~Hz}, 1 \mathrm{H}), 6.91(\mathrm{~d}, J=8.0 \mathrm{~Hz}, 1 \mathrm{H})$, $6.51(\mathrm{~d}, J=2.0 \mathrm{~Hz}, 1 \mathrm{H}), 6.47(\mathrm{dd}, J=8.0,2.4 \mathrm{~Hz}, 1 \mathrm{H}), 5.15-5.10$ (m, $1 \mathrm{H}), 4.40(\mathrm{~d}, J=6.0 \mathrm{~Hz}, 1 \mathrm{H}), 3.87-3.85(\mathrm{~m}, 4 \mathrm{H}), 3.26-3.13(\mathrm{~m}$, $6 \mathrm{H}) ;{ }^{13} \mathrm{C}$ NMR (100 MHz, $\left.\mathrm{CDCl}_{3}\right) \delta 167.1,160.8,153.1,144.1,142.6,129.2,129.1,128.9,128.3$, 127.6, 127.5, 125.9, 121.4, 109.1, 98.1, 87.5, 67.2, 53.9, 49.8, 39.4; HRMS (ESI) for $\mathrm{C}_{26} \mathrm{H}_{24} \mathrm{Cl}_{2} \mathrm{NO}_{4}[\mathrm{M}+\mathrm{H}]+$ : calcd 484.1077, found 484.1081.

tert-butyl 4-((2R,3S)-2-(2-(2,6-dichlorophenoxy)-2-oxoethyl)-3-phenyl-2,3-Dihydrobenzo furan-6-yl)piperazine-1-carboxylate (3ag)

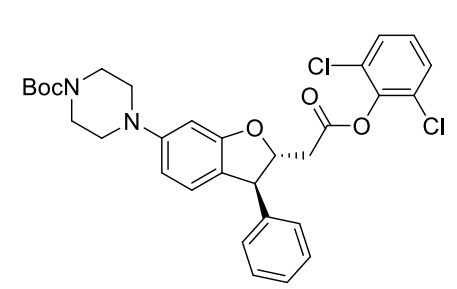

Pale yellow solid, $40.5 \mathrm{mg}$, 69\% yield, $98: 2 \mathrm{dr}$, 99\% ee; m.p.123.1-125.8 ${ }^{\circ} \mathrm{C},[\alpha]_{\mathrm{D}}{ }^{20}=30.6\left(c 1.14, \mathrm{CHCl}_{3}\right) ;{ }^{1} \mathrm{H}$ NMR $(400$ $\left.\mathrm{MHz}, \mathrm{CDCl}_{3}\right) \delta 7.36-7.21(\mathrm{~m}, 7 \mathrm{H}), 7.14(\mathrm{t}, J=8.0 \mathrm{~Hz}, 1 \mathrm{H}), 6.90$ $(\mathrm{d}, J=8.0 \mathrm{~Hz}, 1 \mathrm{H}), 6.51(\mathrm{~d}, J=1.2 \mathrm{~Hz}, 1 \mathrm{H}), 6.46(\mathrm{~d}, J=8.0 \mathrm{~Hz}$, 1H), 5.14-5.09 (m, 1H), 4.39 (d, $J=6.0 \mathrm{~Hz}, 1 \mathrm{H}), 3.59-3.56(\mathrm{~m}$, $4 \mathrm{H}), 3.25-3.13(\mathrm{~m}, 6 \mathrm{H}), 1.49(\mathrm{~s}, 9 \mathrm{H}) ;{ }^{13} \mathrm{C} \mathrm{NMR}\left(100 \mathrm{MHz}, \mathrm{CDCl}_{3}\right)$ $\delta$ 167.1, 160.7, 155.1, 153.0, 144.1, 142.6, 129.2, 129.1, 129.0, 128.3, 127.6, 127.5, 125.9, 121.6, 110.0, 98.9, 87.5, 80.2, 54.0, 49.9, 39.4, 28.8; HRMS (ESI) for $\mathrm{C}_{31} \mathrm{H}_{33} \mathrm{Cl}_{2} \mathrm{NO}_{5}[\mathrm{M}+\mathrm{H}]+$ : calcd 
583.1761, found 583.1763.

2,6-dichlorophenyl 2-((2R,3S)-6-(4-benzylpiperazin-1-yl)-3-phenyl-2,3-dihydrobenzofuran -2-yl)acetate (3ah)

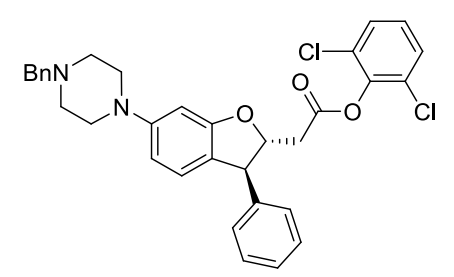

Pale yellow solid, $31.3 \mathrm{mg}, 54 \%$ yield, $97: 3 \mathrm{dr}$, $99 \%$ ee; m.p.145.3-146.2 ${ }^{\circ} \mathrm{C},[\alpha]_{\mathrm{D}}{ }^{20}=32.8\left(\right.$ c $\left.1.0, \mathrm{CHCl}_{3}\right) ;{ }^{1} \mathrm{H}$ NMR $(400$ $\left.\mathrm{MHz}, \mathrm{CDCl}_{3}\right) \delta$ 7.39-7.23 (m, 12H), $7.14(\mathrm{t}, J=8.4 \mathrm{~Hz}, 1 \mathrm{H}), 6.90$ $(\mathrm{d}, J=8.4 \mathrm{~Hz}, 1 \mathrm{H}), 6.52(\mathrm{~d}, J=1.6 \mathrm{~Hz}, 1 \mathrm{H}), 6.48(\mathrm{dd}, J=8.0,1.6$ $\mathrm{Hz}, 1 \mathrm{H}), 5.12(\mathrm{q}, J=6.0 \mathrm{~Hz}, 1 \mathrm{H}), 4.40(\mathrm{~d}, J=6.0 \mathrm{~Hz}, 1 \mathrm{H}), 3.59$ (s, 2H), 3.26-3.12 (m, 6H), $2.62(\mathrm{t}, J=4.8 \mathrm{~Hz}, 4 \mathrm{H}) ;{ }^{13} \mathrm{C}$ NMR $(100$ $\left.\mathrm{MHz}, \mathrm{CDCl}_{3}\right) \delta 167.2,160.7,153.1,144.1,142.7,138.2,129.6,129.2,129.1,128.9,128.6,128.3$, 127.6, 127.5, 127.4, 125.7, 120.8, 109.5, 98.3, 87.4, 63.4, 54.0, 53.4, 49.6, 39.4; HRMS (ESI) for $\mathrm{C}_{33} \mathrm{H}_{31} \mathrm{Cl}_{2} \mathrm{~N}_{2} \mathrm{O}_{3}[\mathrm{M}+\mathrm{H}]+$ : calcd 573.1706, found 573.1701.

2,6-dichlorophenyl 2-((2R,3S)-4-fluoro-3-phenyl-6-(pyrrolidin-1-yl)-2,3-dihydrobenzofuran -2-yl)acetate (3ai)

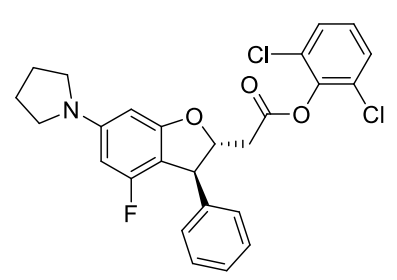

White solid, $46.2 \mathrm{mg}$, 95\% yield, 97:3 dr, 99\% ee; m.p.156.3-158.2 ${ }^{\circ} \mathrm{C},[\alpha]_{\mathrm{D}}^{20}=58.2\left(c 0.91, \mathrm{CHCl}_{3}\right) ;{ }^{1} \mathrm{H}$ NMR $\left(400 \mathrm{MHz}, \mathrm{CDCl}_{3}\right) \delta$ 7.37-7.22 (m, 7H), $7.14(\mathrm{t}, J=8.0 \mathrm{~Hz}, 1 \mathrm{H}), 5.96(\mathrm{~d}, J=2.0 \mathrm{~Hz}, 1 \mathrm{H})$, $5.82(\mathrm{~d}, J=12.0,2.0 \mathrm{~Hz}, 1 \mathrm{H}), 5.15-5.11(\mathrm{~m}, 1 \mathrm{H}), 4.48(\mathrm{~d}, J=4.4 \mathrm{~Hz}$, $1 \mathrm{H}), 3.28-3.11(\mathrm{~m}, 6 \mathrm{H}), 2.01(\mathrm{t}, J=6.0 \mathrm{~Hz}, 4 \mathrm{H}) ;{ }^{13} \mathrm{C}$ NMR $(100$ $\left.\mathrm{MHz}, \mathrm{CDCl}_{3}\right) \delta 167.1,162.3(\mathrm{~d}, J=11.6 \mathrm{~Hz}), 160.7(\mathrm{~d}, J=242.9$ $\mathrm{Hz}), 150.8(\mathrm{~d}, J=12.0 \mathrm{~Hz}), 144.1,142.3,129.2,129.1,128.9,127.7,127.6,127.5,102.7$ (d, $J=$ $21.0 \mathrm{~Hz}$ ), $92.3(\mathrm{~d}, J=24.7 \mathrm{~Hz}), 89.9,87.8,51.6,48.3,39.6,25.8$; HRMS (ESI) for $\mathrm{C}_{26} \mathrm{H}_{23} \mathrm{Cl}_{2} \mathrm{FNO}_{3}[\mathrm{M}+\mathrm{H}] *$ : calcd 486.1034 , found 486.1048 .

2,6-dichlorophenyl 2-((2R,3S)-5-fluoro-3-phenyl-6-(pyrrolidin-1-yl)-2,3-dihydrobenzofuran -2-yl)acetate (3aj)

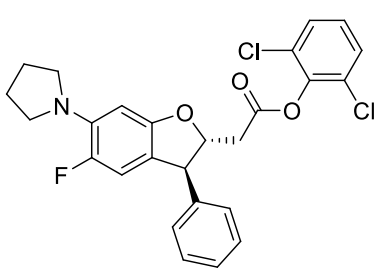

White solid, $37.0 \mathrm{mg}, 76 \%$ yield, 98:2 dr, 99\% ee; m.p.169.3-172.4 ${ }^{\circ} \mathrm{C},[\alpha]_{\mathrm{D}}{ }^{20}=52.7\left(c 1.0, \mathrm{CHCl}_{3}\right) ;{ }^{1} \mathrm{H} \mathrm{NMR}\left(400 \mathrm{MHz}, \mathrm{CDCl}_{3}\right) \delta$ 7.36-7.21 (m, 7H), $7.14(\mathrm{t}, J=8.0 \mathrm{~Hz}, 1 \mathrm{H}), 6.65(\mathrm{~d}, J=12.4 \mathrm{~Hz}, 1 \mathrm{H})$, $6.27(\mathrm{~d}, J=7.2 \mathrm{~Hz}, 1 \mathrm{H}), 5.11-5.06(\mathrm{~m}, 1 \mathrm{H}), 4.38(\mathrm{~d}, J=6.0 \mathrm{~Hz}, 1 \mathrm{H})$, 3.39-3.36 (m, 4H), 3.24-3.11 (m, 2H), $1.95(\mathrm{t}, J=6.4 \mathrm{~Hz}, 4 \mathrm{H}) ;{ }^{13} \mathrm{C}$ NMR $\left(100 \mathrm{MHz}, \mathrm{CDCl}_{3}\right) \delta 167.2,156.0,148.0(\mathrm{~d}, J=232.4 \mathrm{~Hz})$, $144.2,142.5,138.4(\mathrm{~d}, J=11.4 \mathrm{~Hz}), 129.2,129.1,128.9,128.2,127.6,116.9$ (d, $J=8.0 \mathrm{~Hz})$, $112.8(\mathrm{~d}, J=24.3 \mathrm{~Hz}), 97.1$ (d, $J=4.8 \mathrm{~Hz}), 87.6,54.4,50.3(\mathrm{~d}, J=5.3 \mathrm{~Hz}), 39.4,25.6(\mathrm{~d}, J=1.6$ $\mathrm{Hz}$ ); HRMS (ESI) for $\mathrm{C}_{26} \mathrm{H}_{23} \mathrm{Cl}_{2} \mathrm{FNO}_{3}[\mathrm{M}+\mathrm{H}]+$ : calcd 486.1034, found 486.1036 .

2,6-dichlorophenyl 2-((2R,3S)-5-methyl-3-phenyl-6-(pyrrolidin-1-yl)-2,3-dihydrobenzofuran -2-yl)acetate (3ak)

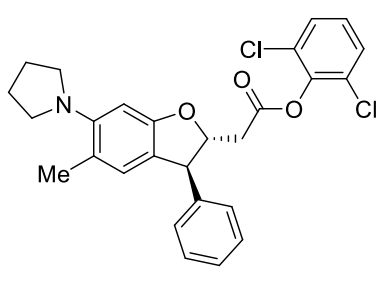

Pale yellow oil, $31.5 \mathrm{mg}, 65 \%$ yield, $98: 2 \mathrm{dr}$, $98 \%$ ee; $[\alpha]_{\mathrm{D}}{ }^{20}=34.5(c$ $\left.1.0, \mathrm{CHCl}_{3}\right) ;{ }^{1} \mathrm{H} \mathrm{NMR}\left(400 \mathrm{MHz}, \mathrm{CDCl}_{3}\right) \delta$ 7.36-7.23 (m, 7H), $7.14(\mathrm{t}$, $J=8.0 \mathrm{~Hz}, 1 \mathrm{H}), 6.74(\mathrm{~s}, 1 \mathrm{H}), 6.52(\mathrm{~s}, 1 \mathrm{H}), 5.11-5.06(\mathrm{~m}, 1 \mathrm{H}), 4.37(\mathrm{~d}$, $J=6.0 \mathrm{~Hz}, 1 \mathrm{H}), 3.24-3.10(\mathrm{~m}, 6 \mathrm{H}), 2.20$ (s, 3H), 1.94-1-91 (m, 4H); ${ }^{13} \mathrm{C}$ NMR $\left(100 \mathrm{MHz}, \mathrm{CDCl}_{3}\right) \delta 167.3,158.4,150.7,144.2,142.9$, 
$129.2,129.1,128.9,128.3,128.0,127.6,127.4,121.8,120.9,98.4,87.2,54.4,51.5,39.5,25.3$, 20.6; HRMS (ESI) for $\mathrm{C}_{27} \mathrm{H}_{26} \mathrm{Cl}_{2} \mathrm{NO}_{3}[\mathrm{M}+\mathrm{H}]+$ : calcd 482.1284, found 482.1275 .

\section{2,6-dichlorophenyl 2-((2R,3S)-7-methyl-3-phenyl-6-(pyrrolidin-1-yl)-2,3-dihydrobenzofuran} -2-yl)acetate (3al)

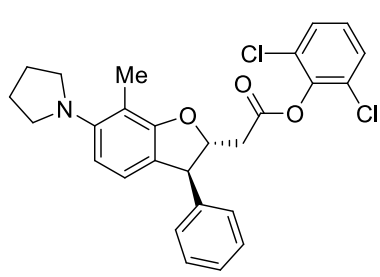

Pale yellow solid, $46.4 \mathrm{mg}$, 96\% yield, 99:1 dr, 99\% ee; m.p.76.1-78.3 ${ }^{\circ} \mathrm{C},[\alpha]_{\mathrm{D}}{ }^{20}=41.1\left(c 1.07, \mathrm{CHCl}_{3}\right) ;{ }^{1} \mathrm{H}$ NMR $(400 \mathrm{MHz}$, $\left.\mathrm{CDCl}_{3}\right) \delta$ 7.37-7.23 (m, 7H), 7.16-7.11 (m, $\left.1 \mathrm{H}\right), 6.74(\mathrm{~d}, J=8.4 \mathrm{~Hz}$, $1 \mathrm{H}), 6.45(\mathrm{~d}, J=8.4 \mathrm{~Hz}, 1 \mathrm{H}), 5.15-5.10(\mathrm{~m}, 1 \mathrm{H}), 4.40(\mathrm{~d}, J=6.4 \mathrm{~Hz}$, $1 \mathrm{H}), 3.25-3.10(\mathrm{~m}, 6 \mathrm{H}), 2.24(\mathrm{~s}, 3 \mathrm{H}), 1.93(\mathrm{t}, J=6.0 \mathrm{~Hz}, 4 \mathrm{H}) ;{ }^{13} \mathrm{C}$ NMR $\left(100 \mathrm{MHz}, \mathrm{CDCl}_{3}\right) \delta 167.3,159.5,150.8,144.2,142.9,129.3$, 129.1, 128.9, 128.3, 127.6, 127.4, 122.3, 121.3, 111.3, 109.1, 86.9, 54.7, 51.7, 39.8, 25.3, 13.0; HRMS (ESI) for $\mathrm{C}_{27} \mathrm{H}_{26} \mathrm{Cl}_{2} \mathrm{NO}_{3}[\mathrm{M}+\mathrm{H}]+$ : calcd 482.1284, found 482.1299 .

\section{2,6-dichlorophenyl 2-((2R,3S)-7-benzyl-3-phenyl-3,5,6,7-tetrahydro- $2 \mathrm{H}$-furo[3,2-f]indol} -2-yl)acetate (3am)

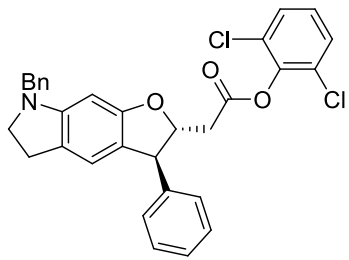

Colorless oil, $51.5 \mathrm{mg}, 97 \%$ yield, $99: 1 \mathrm{dr}, 99 \%$ ee; $[\alpha]_{\mathrm{D}}{ }^{20}=33.3(c$ 1.35, $\left.\mathrm{CHCl}_{3}\right) ;{ }^{1} \mathrm{H}$ NMR (400 MHz, $\left.\mathrm{CDCl}_{3}\right) \delta$ 7.40-7.26 (m, 12H), 7.14 (t, $J=8.0 \mathrm{~Hz}, 1 \mathrm{H}), 6.72(\mathrm{~s}, 1 \mathrm{H}), 6.16(\mathrm{~s}, 1 \mathrm{H}), 5.14-5.09(\mathrm{~m}, 1 \mathrm{H}), 4.35$ (d, $J=5.6 \mathrm{~Hz}, 1 \mathrm{H}), 4.25(\mathrm{~s}, 2 \mathrm{H}), 3.37-3.32(\mathrm{~m}, 2 \mathrm{H}), 3.26-3.12(\mathrm{~m}, 2 \mathrm{H})$, $2.86(\mathrm{t}, J=8.0 \mathrm{~Hz}, 2 \mathrm{H}) ;{ }^{13} \mathrm{C} \mathrm{NMR}\left(100 \mathrm{MHz}, \mathrm{CDCl}_{3}\right) \delta 167.3,159.6$, $154.1,144.2,143.3,138.6,129.2,129.1,128.9,128.8,128.3,127.6$, $127.5,127.4,123.1,121.2,118.0,90.9,87.4,54.6,54.3,54.0,39.5,28.2$; HRMS (ESI) for $\mathrm{C}_{31} \mathrm{H}_{26} \mathrm{Cl}_{2} \mathrm{NO}_{3}[\mathrm{M}+\mathrm{H}]+$ : calcd 530.1284, found 530.1282.

\section{2,6-dichlorophenyl 2-((2R,3S)-6-benzyl-3-phenyl-3,6,7,8-tetrahydro- $2 \mathrm{H}$-furo[2,3-e]indol} -2-yl)acetate (3an)

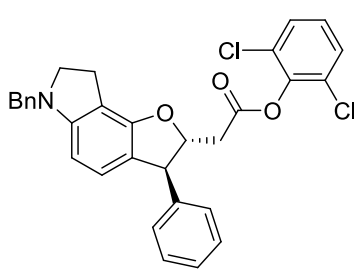

Colorless oil, $48.4 \mathrm{mg}, 91 \%$ yield, $97: 3 \mathrm{dr}, 99 \%$ ee; $[\alpha]_{\mathrm{D}}^{20}=44.0(c 1.0$, $\left.\mathrm{CHCl}_{3}\right) ;{ }^{1} \mathrm{H} \mathrm{NMR}\left(400 \mathrm{MHz}, \mathrm{CDCl}_{3}\right) \delta$ 7.39-7.25 (m, 12H), $7.14(\mathrm{t}, J=$ $8.0 \mathrm{~Hz}, 1 \mathrm{H}), 6.72(\mathrm{~d}, J=8.0 \mathrm{~Hz}, 1 \mathrm{H}), 6.11(\mathrm{~d}, J=8.0 \mathrm{~Hz}, 1 \mathrm{H})$, 5.16-5.11 (m, 1H), $4.36(\mathrm{~d}, J=6.4 \mathrm{~Hz}, 1 \mathrm{H}), 4.26(\mathrm{~s}, 2 \mathrm{H}), 3.42-3.37(\mathrm{~m}$, $2 \mathrm{H}), 3.26-3.13$ (m, 2H), 2.99 (t, $J=8.0 \mathrm{~Hz}, 2 \mathrm{H}) ;{ }^{13} \mathrm{C}$ NMR $(100 \mathrm{MHz}$, $\left.\mathrm{CDCl}_{3}\right) \delta 167.2,155.9,155.1,144.2,143.1,138.8,129.2,129.1,128.9$, $128.8,128.3,128.2,127.6,127.41,127.40,124.4,120.6,110.4,100.6,87.7,54.4,54.1,54.0,39.5$, 25.4; HRMS (ESI) for $\mathrm{C}_{31} \mathrm{H}_{26} \mathrm{Cl}_{2} \mathrm{NO}_{3}[\mathrm{M}+\mathrm{H}]+$ : calcd 530.1284, found 530.1294.

\section{2,6-dichlorophenyl 2-((2R,3S)-6-benzyl-3-phenyl-2,3,6,7,8,9-hexahydrofuro[2,3-f]} quinolin-2-yl)acetate (3ao)

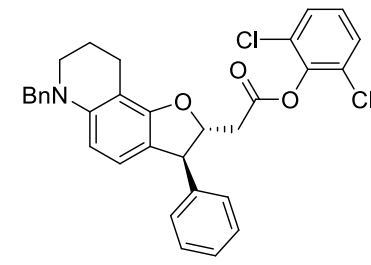

Colorless oil, $49.0 \mathrm{mg}$, 90\% yield, $97: 3 \mathrm{dr}, 99 \%$ ee; $[\alpha]_{\mathrm{D}}^{20}=22.8(c$ 0.535, $\left.\mathrm{CHCl}_{3}\right) ;{ }^{1} \mathrm{H}$ NMR $\left(400 \mathrm{MHz}, \mathrm{CDCl}_{3}\right) \delta$ 7.37-7.22 (m, $\left.12 \mathrm{H}\right)$, $7.14(\mathrm{t}, J=8.0 \mathrm{~Hz}, 1 \mathrm{H}), 6.62(\mathrm{~d}, J=8.0 \mathrm{~Hz}, 1 \mathrm{H}), 6.12(\mathrm{~d}, J=8.0 \mathrm{~Hz}$, $1 \mathrm{H}), 5.14-5.09(\mathrm{~m}, 1 \mathrm{H}), 4.53-4.41(\mathrm{~m}, 2 \mathrm{H}), 4.34(\mathrm{~d}, J=6.4 \mathrm{~Hz}, 1 \mathrm{H})$, 3.37-3.35 (m, 2H), 3.24-3.11 (m, 2H), $2.78(\mathrm{t}, J=6.0 \mathrm{~Hz}, 2 \mathrm{H})$, 2.05-1.99 (m, 2H); ${ }^{13} \mathrm{C}$ NMR $\left(100 \mathrm{MHz}, \mathrm{CDCl}_{3}\right) \delta 167.3,157.9,146.9$, 144.2, 143.1, 139.4, 129.2, 129.0, 128.9, 128.8, 128.3, 127.6, 127.3, 127.1, 126.9, 123.1, 117.0, 
105.1, 104.6, 87.3, 56.3, 54.5, 50.1, 39.9, 22.1, 21.6; HRMS (ESI) for $\mathrm{C}_{32} \mathrm{H}_{28} \mathrm{Cl}_{2} \mathrm{NO}_{3}[\mathrm{M}+\mathrm{H}]+$ : calcd 544.1441, found 544.1458.

2,6-dichlorophenyl 2-((2R,3S)-8-benzyl-3-phenyl-2,3,5,6,7,8-hexahydrofuro[3,2-g]quinolin -2-yl)acetate (3ap)

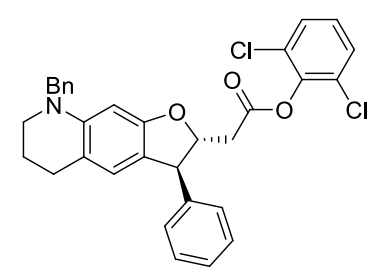

Colorless oil, $52.9 \mathrm{mg}, 97 \%$ yield, $99: 1 \mathrm{dr}, 99 \%$ ee; $[\alpha]_{\mathrm{D}}{ }^{20}=25.3(c$ 1.39, $\left.\mathrm{CHCl}_{3}\right) ;{ }^{1} \mathrm{H} \mathrm{NMR}\left(400 \mathrm{MHz}, \mathrm{CDCl}_{3}\right) \delta$ 7.36-7.24 (m, 12H), 7.12 $(\mathrm{t}, J=8.0 \mathrm{~Hz}, 1 \mathrm{H}), 6.63(\mathrm{~s}, 1 \mathrm{H}), 6.15(\mathrm{~s}, 1 \mathrm{H}), 5.06-5.01(\mathrm{~m}, 1 \mathrm{H})$, 4.48-4.42 (m, 2H), $4.33(\mathrm{~d}, J=6.0 \mathrm{~Hz}, 1 \mathrm{H}), 3.35(\mathrm{t}, J=5.2 \mathrm{~Hz}, 2 \mathrm{H})$, 3.21-3.08 (m, 2H), $2.70(\mathrm{t}, J=6.0 \mathrm{~Hz}, 2 \mathrm{H}), 2.01-1.95(\mathrm{~m}, 2 \mathrm{H}) ;{ }^{13} \mathrm{C}$ NMR $\left(100 \mathrm{MHz}, \mathrm{CDCl}_{3}\right) \delta 167.3,159.2,146.8,143.2,139.0,129.2$, 129.0, 128.94, 128.90, 128.3, 127.5, 127.4, 127.1, 126.9, 125.5, 116.7, 115.6, 93.4, 87.0, 55.9, 54.3, 53.8, 50.2, 39.4, 28.3, 22.9; HRMS (ESI) for $\mathrm{C}_{32} \mathrm{H}_{28} \mathrm{Cl}_{2} \mathrm{NO}_{3}[\mathrm{M}+\mathrm{H}]+$ : calcd 544.1441, found 544.1454 .

2,6-dichlorophenyl 2-((2R,3R)-6-(diethylamino)-3-phenyl-2,3-dihydrobenzofuran-2-yl) acetate (3aq)

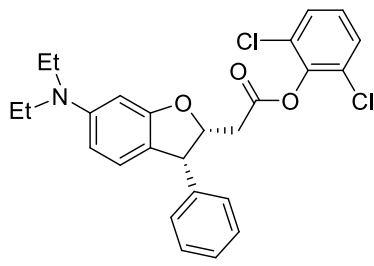

Colorless oil, $41.9 \mathrm{mg}, 89 \%$ yield, $96: 4 \mathrm{dr}, 99 \%$ ee; $[\alpha]_{\mathrm{D}}{ }^{20}=8.3(c 1.0$, $\left.\mathrm{CHCl}_{3}\right) ;{ }^{1} \mathrm{H} \mathrm{NMR}\left(400 \mathrm{MHz}, \mathrm{CDCl}_{3}\right) \delta$ 7.35-7.25 (m, 5H), 7.15-7.07 $(\mathrm{m}, 3 \mathrm{H}), 6.92(\mathrm{~d}, J=8.4 \mathrm{~Hz}, 1 \mathrm{H}), 6.30(\mathrm{~d}, J=2.0 \mathrm{~Hz}, 1 \mathrm{H}), 6.25(\mathrm{dd}, J$ $=8.0,2.0 \mathrm{~Hz}, 1 \mathrm{H}), 5.47-5.42(\mathrm{~m}, 1 \mathrm{H}), 4.67(\mathrm{~d}, J=8.4 \mathrm{~Hz}, 1 \mathrm{H}), 3.36$ (q, $J=6.8 \mathrm{~Hz}, 4 \mathrm{H}), 2.88-2.82(\mathrm{~m}, 1 \mathrm{H}), 2.58-2.53(\mathrm{~m}, 1 \mathrm{H}), 1.19(\mathrm{t}, J=$ $6.8 \mathrm{~Hz}, 6 \mathrm{H}) ;{ }^{13} \mathrm{C}$ NMR $\left(100 \mathrm{MHz}, \mathrm{CDCl}_{3}\right) \delta 167.8,161.4,149.5,144.1,140.1,129.4,129.3$, $128.93,128.90,127.5,126.1,117.4,105.4,93.9,82.8,50.9,45.0,36.5,12.9$; HRMS (ESI) for $\mathrm{C}_{26} \mathrm{H}_{26} \mathrm{Cl}_{2} \mathrm{NO}_{3}[\mathrm{M}+\mathrm{H}]+$ : calcd 470.1284 , found 470.1289 .

\section{2,6-dichlorophenyl 2-((2R,3R)-3-phenyl-6-(piperidin-1-yl)-2,3-dihydrobenzofuran-2-yl)} acetate (3ar)

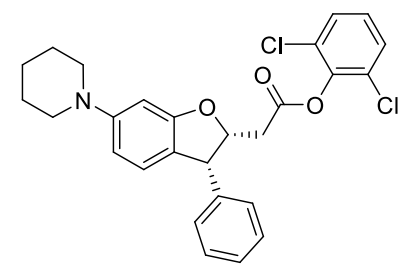

Pale yellow oil, $43.5 \mathrm{mg}$, 90\% yield, $97: 3 \mathrm{dr}, 99 \%$ ee; $[\alpha]_{\mathrm{D}}^{20}=13.6(c$ 1.0, $\left.\mathrm{CHCl}_{3}\right) ;{ }^{1} \mathrm{H}$ NMR $\left(400 \mathrm{MHz}, \mathrm{CDCl}_{3}\right) \delta$ 7.35-7.26 (m, 5H), 7.13 $(\mathrm{t}, J=8.0 \mathrm{~Hz}, 1 \mathrm{H}), 7.05(\mathrm{~d}, J=8.0 \mathrm{~Hz}, 2 \mathrm{H}), 6.95(\mathrm{~d}, J=8.0 \mathrm{~Hz}, 1 \mathrm{H})$, $6.55(\mathrm{~d}, J=1.6 \mathrm{~Hz}, 1 \mathrm{H}), 6.52(\mathrm{dd}, J=8.0,1.6 \mathrm{~Hz}, 1 \mathrm{H}), 5.48-5.42(\mathrm{~m}$, $1 \mathrm{H}), 4.68(\mathrm{~d}, J=8.0 \mathrm{~Hz}, 1 \mathrm{H}), 3.19-3.16(\mathrm{~m}, 4 \mathrm{H}), 2.89-2.83(\mathrm{~m}, 1 \mathrm{H})$, 2.59-2.53 (m, 1H), 1.75-1.69 (m, 4H), 1.61-1.58 (m, 2H); ${ }^{13} \mathrm{C} \mathrm{NMR}$ $\left(100 \mathrm{MHz}, \mathrm{CDCl}_{3}\right) \delta 167.7,160.9,153.9,144.1,139.7,129.4,129.2,129.0,128.9,127.6,127.5$, 125.9, 121.1, 110.1, 96.5, 82.9, 51.1, 50.9, 36.4, 26.2, 24.6; HRMS (ESI) for $\mathrm{C}_{27} \mathrm{H}_{26} \mathrm{Cl}_{2} \mathrm{NO}_{3}$ $[\mathrm{M}+\mathrm{H}]+:$ calcd 482.1284 , found 482.1286 .

\section{2,6-dichlorophenyl 2-((2R,3R)-4-fluoro-3-phenyl-6-(pyrrolidin-1-yl)-2,3-dihydrobenzofuran} -2-yl)acetate (3as)

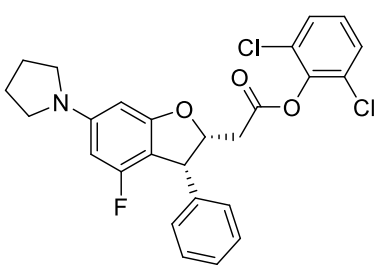

Pale yellow oil, $47.8 \mathrm{mg}, 98 \%$ yield, $96: 4 \mathrm{dr}, 99 \%$ ee; $[\alpha]_{\mathrm{D}}{ }^{20}=8.9(c$ 1.53, $\left.\mathrm{CHCl}_{3}\right) ;{ }^{1} \mathrm{H}$ NMR $\left(400 \mathrm{MHz}, \mathrm{CDCl}_{3}\right) \delta 7.37-7.25(\mathrm{~m}, 5 \mathrm{H}), 7.13$ $(\mathrm{t}, J=8.0 \mathrm{~Hz}, 1 \mathrm{H}), 7.06(\mathrm{~d}, J=7.6 \mathrm{~Hz}, 2 \mathrm{H}), 5.98(\mathrm{~s}, 1 \mathrm{H}), 5.83(\mathrm{~d}, J=$ $12.0 \mathrm{~Hz}, 1 \mathrm{H}), 5.46-5.40(\mathrm{~m}, 1 \mathrm{H}), 4.75(\mathrm{~d}, J=8.0 \mathrm{~Hz}, 1 \mathrm{H}), 3.27$ (t, $J=$ $6.0 \mathrm{~Hz}, 4 \mathrm{H}), 2.89-2.82(\mathrm{~m}, 1 \mathrm{H}), 2.64-2.58$ (m, 1H), 2.03-1.99 (m, 4H); 
${ }^{13} \mathrm{C}$ NMR $\left(100 \mathrm{MHz}, \mathrm{CDCl}_{3}\right) \delta 167.6,162.6(\mathrm{~d}, J=11.6 \mathrm{~Hz}), 160.3(\mathrm{~d}, J=243.1 \mathrm{~Hz}), 150.6(\mathrm{~d}, J$ $=12.0 \mathrm{~Hz}$ ), 144.1, 138.7, 129.2, 129.0, 128.93, 128.90, 127.7, 127.6, 104.3 (d, $J=21.6 \mathrm{~Hz}), 92.3$ (d, $J=24.7 \mathrm{~Hz}$ ), 89.7, 83.4, 48.6, 48.3, 36.2, 25.8; HRMS (ESI) for $\mathrm{C}_{26} \mathrm{H}_{23} \mathrm{FCl}_{2} \mathrm{NO}_{3}[\mathrm{M}+\mathrm{H}]+$ calcd 486.1034 , found 486.1035 .

2,6-dichlorophenyl 2-((2R,3R)-6-benzyl-3-phenyl-3,6,7,8-tetrahydro-2H-furo[2,3-e]indol-2-yl )acetate (3at)

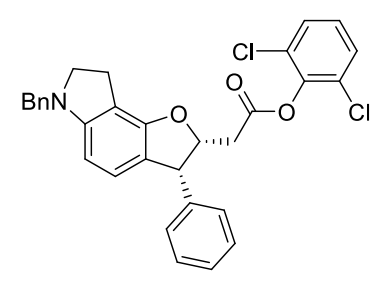

Pale yellow oil, $50.6 \mathrm{mg}, 96 \%$ yield, $99: 1 \mathrm{dr}, 99 \%$ ee; $[\alpha]_{\mathrm{D}}^{20}=31.9(c$ $\left.1.0, \mathrm{CHCl}_{3}\right) ;{ }^{1} \mathrm{H}$ NMR $\left(400 \mathrm{MHz}, \mathrm{CDCl}_{3}\right) \delta 7.41-7.28(\mathrm{~m}, 10 \mathrm{H})$, 7.16-7.10 (m, 3H), $6.82(\mathrm{~d}, J=8.0 \mathrm{~Hz}, 1 \mathrm{H}), 6.16(\mathrm{~d}, J=8.0 \mathrm{~Hz}, 1 \mathrm{H})$, 5.51-5.45 (m, 1H), $4.71(\mathrm{~d}, J=8.4 \mathrm{~Hz}, 1 \mathrm{H}), 4.33-4.24(\mathrm{~m}, 2 \mathrm{H})$, 3.48-3.36 (m, 2H), 3.04-3.00 (m, 2H), 2.94-2.87 (m, 1H), 2.59-2.54 (m, $1 \mathrm{H}) ;{ }^{13} \mathrm{C} \mathrm{NMR}\left(100 \mathrm{MHz}, \mathrm{CDCl}_{3}\right) \delta 167.8,156.1,155.0,144.1,139.9$, $138.8,129.4,129.2,128.93,129.91,128.8,128.2,127.6,127.4,124.6,121.3,110.4,100.8,83.3$, 54.5, 54.2, 50.8, 36.5, 25.4; HRMS (ESI) for $\mathrm{C}_{31} \mathrm{H}_{26} \mathrm{Cl}_{2} \mathrm{NO}_{3}[\mathrm{M}+\mathrm{H}]+$ : calcd 530.1284, found 530.1284 . 


\section{Determination of the Diastereoselectivity Ratio and Enantiomeric Excess}

Values

Due to the relatively low solubility of the obtained 2,3-disubstituted dihydrobenzofuran products 3 in hexane/i-propanol, their optical purities were determined by measuring the ee values of the corresponding alcohol derivatives after LAH reduction.

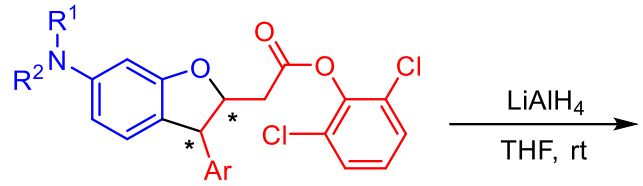

3<smiles>[R]N([R1])c1ccc2c([14Cl])c(CCO)oc2c1</smiles>

4

To a solution of the above ester $(0.1 \mathrm{mmol})$ in $10 \mathrm{~mL}$ of anhydrous THF was added $\mathrm{LiAlH}_{4}(15$ $\mathrm{mg}, 0.4 \mathrm{mmol}$ ), after stirring at room temperature for $10 \mathrm{~min}$, the reaction was quenched with ice water and saturated aqueous sodium potassium tartrate was added, and the solution was stirred at room temperature for another $30 \mathrm{~min}$. Then the aqueous layer was extracted with EA for three times, the combined organic layers were dried over $\mathrm{Na}_{2} \mathrm{SO}_{4}$ and concentrated under reduced pressure. The crude product was purified by silica gel column chromatography using petroleum ether/ethyl acetate to afford the corresponding alcohol 4. 
<smiles>OCCC1Oc2cc(N3CCCC3)ccc2C1c1ccccc1</smiles>

White solid, $29.2 \mathrm{mg}$, 94\% yield, 99:1 dr, 99\% ee; m.p.101.4-103.5 ${ }^{\circ} \mathrm{C}$, $[\alpha]_{\mathrm{D}}^{20}=16.1\left(c 1.00, \mathrm{CHCl}_{3}\right) ;{ }^{1} \mathrm{H}$ NMR $\left(400 \mathrm{MHz}, \mathrm{CDCl}_{3}\right) \delta$ 7.33-7.21 (m, 5H), $6.79(\mathrm{~d}, J=8.0 \mathrm{~Hz}, 1 \mathrm{H}), 6.13-6.09(\mathrm{~m}, 2 \mathrm{H}), 4.71-4.66(\mathrm{~m}$, $1 \mathrm{H}), 4.23(\mathrm{~d}, J=7.8 \mathrm{~Hz}, 1 \mathrm{H}), 3.88(\mathrm{t}, J=6.0 \mathrm{~Hz}, 2 \mathrm{H}), 3.28$ (t, $J=6.4$ $\mathrm{Hz}, 4 \mathrm{H}), 2.20-1.98(\mathrm{~m}, 7 \mathrm{H}) ;{ }^{13} \mathrm{C}$ NMR $\left(100 \mathrm{MHz}, \mathrm{CDCl}_{3}\right) \delta 161.0$, $149.5,143.3,129.0,128.4,127.3,125.7,117.3,105.0,93.6,91.4,60.9,54.8,48.2,37.6,25.7$; HRMS (ESI) for $\mathrm{C}_{20} \mathrm{H}_{24} \mathrm{NO}_{2}[\mathrm{M}+\mathrm{H}]^{+}$: calcd 310.1802, found 310.1798 .

HPLC: Chiralpak AD-H column $(250 \mathrm{~mm})$; detected at $220 \mathrm{~nm}$; hexane $/ i$-propanol $=90 / 10$; flow $=0.7 \mathrm{~mL} / \mathrm{min}$; Retention time: $10.3 \mathrm{~min}, 20.9 \mathrm{~min}$ (major)

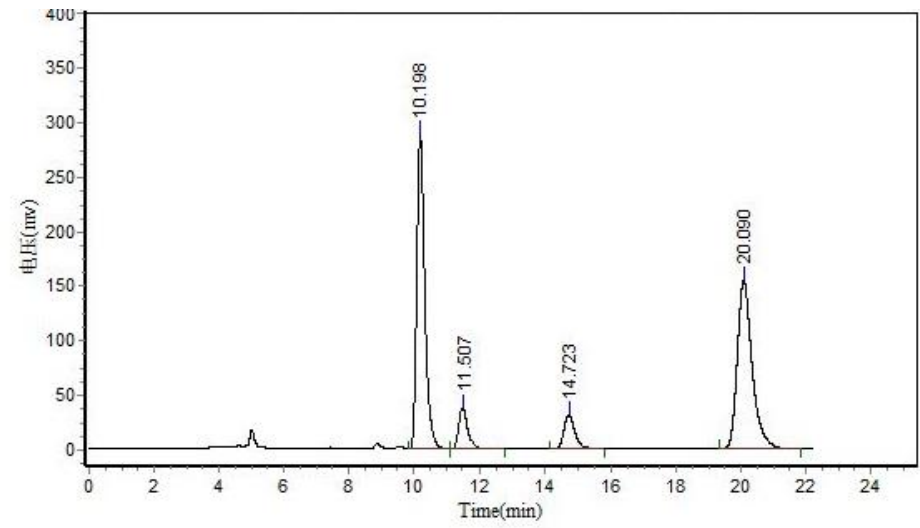

\begin{tabular}{|c|c|c|c|c|c|}
\hline \multicolumn{6}{|c|}{ Results } \\
\hline Peak No. & Peak ID & Ret Time & Height & Area & Conc. \\
\hline 1 & & 10.198 & 287936.438 & 4922798.500 & 43.5722 \\
\hline 2 & & 11.507 & 39072.328 & 733967.750 & 6.4964 \\
\hline 3 & & 14.723 & 31480.623 & 738886.625 & 6.5400 \\
\hline 4 & & 20.090 & 153834.000 & 4902380.000 & 43.3914 \\
\hline Total & & & 512323.389 & 11298032.875 & 100.0000 \\
\hline
\end{tabular}

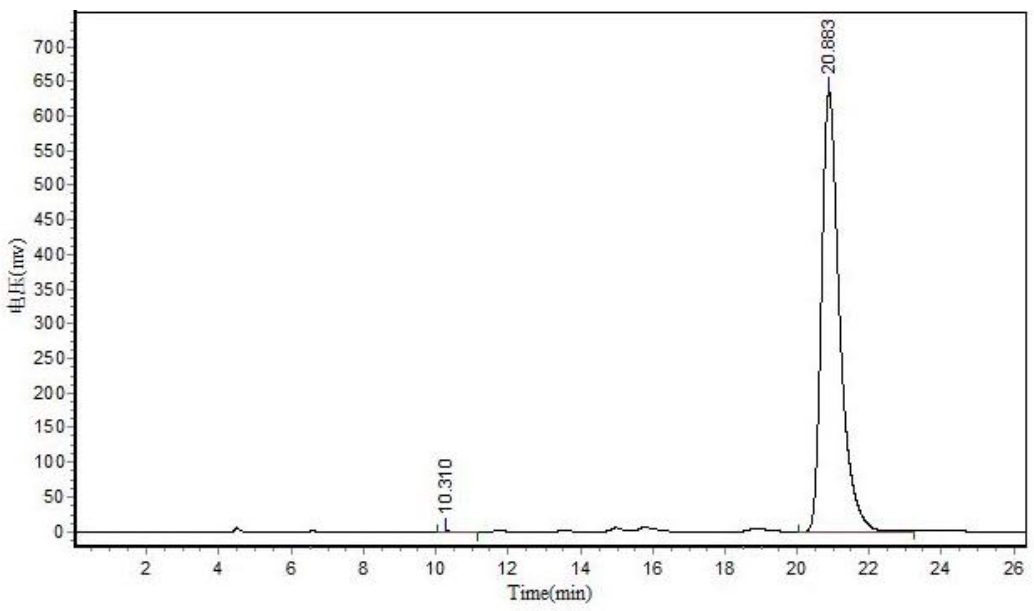

Results

\begin{tabular}{|c|c|c|c|c|c|}
\hline \multicolumn{6}{|c|}{ Results } \\
\hline Peak No. & Peak ID & Ret Time & Height & Area & Conc. \\
\hline 1 & & 10.310 & 1114.709 & 20093.498 & 0.0901 \\
\hline 2 & & 20.883 & 636874.063 & 22273342.000 & 99.9099 \\
\hline Total & & & 637988.772 & 22293435.498 & 100.0000 \\
\hline
\end{tabular}


2-((2S,3S)-3-phenyl-6-(pyrrolidin-1-yl)-2,3-dihydrobenzofuran-2-yl)ethanol ((2S, 3S)-4a)<smiles>OCCC1Oc2cc(N3CCCC3)ccc2C1c1ccccc1</smiles>

White solid, $27.6 \mathrm{mg}$, $90 \%$ yield, $93: 7 \mathrm{dr}$, $98 \%$ ee; m.p.126.8-130.4 ${ }^{\circ} \mathrm{C}$, $[\alpha]_{\mathrm{D}}{ }^{20}=2.1\left(c \quad 1.1, \mathrm{CHCl}_{3}\right) ;{ }^{1} \mathrm{H} \mathrm{NMR}\left(400 \mathrm{MHz}, \mathrm{CDCl}_{3}\right) \delta$ 7.28-7.19 (m, 3H), $7.00(\mathrm{~d}, J=7.2 \mathrm{~Hz}, 2 \mathrm{H}), 6.91(\mathrm{~d}, J=8.0 \mathrm{~Hz}, 1 \mathrm{H}), 6.15-6.12$ $(\mathrm{m}, 2 \mathrm{H}), 5.09-5.03(\mathrm{~m}, 1 \mathrm{H}), 4.50(\mathrm{~d}, J=8.0 \mathrm{~Hz}, 1 \mathrm{H}), 3.80-3.76(\mathrm{~m}$, $2 \mathrm{H}), 3.29(\mathrm{t}, J=6.0 \mathrm{~Hz}, 4 \mathrm{H}), 2.02-1.99(\mathrm{~m}, 5 \mathrm{H}), 1.54-1.41(\mathrm{~m}, 2 \mathrm{H})$; ${ }^{13} \mathrm{C}$ NMR $\left(100 \mathrm{MHz}, \mathrm{CDCl}_{3}\right) \delta 161.1,149.4,141.0,129.3,128.6,127.1,126.1,117.8,105.2,93.6$, 86.3, 61.2, 51.5, 48.2, 34.9, 25.8; HRMS (ESI) for $\mathrm{C}_{20} \mathrm{H}_{24} \mathrm{NO}_{2}[\mathrm{M}+\mathrm{H}]^{+}$: calcd 310.1802, found 310.1800 .

HPLC: Chiralpak AD-H column $(250 \mathrm{~mm})$; detected at $220 \mathrm{~nm}$; hexane/ $i$-propanol $=90 / 10$; flow $=0.7 \mathrm{~mL} / \mathrm{min}$; Retention time: $11.7 \mathrm{~min}$ (major), $15.7 \mathrm{~min}$.

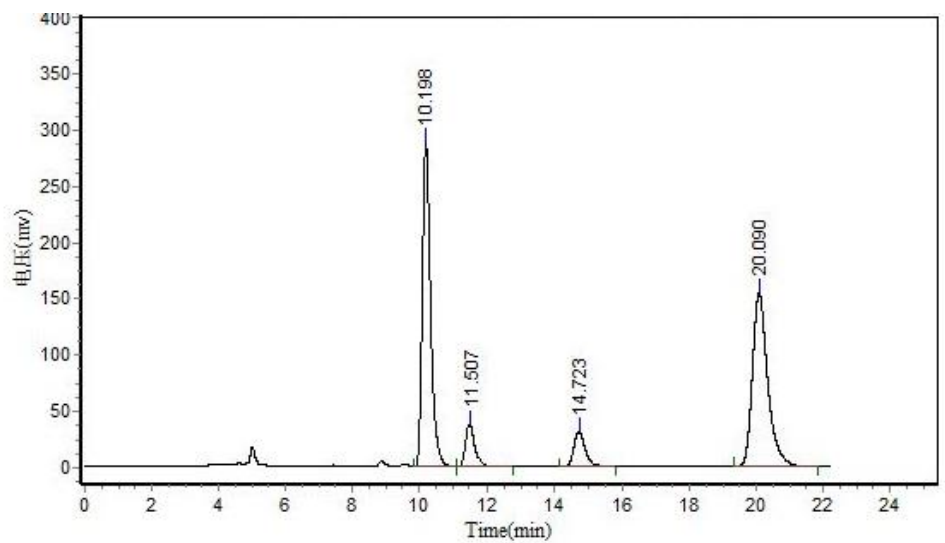

\begin{tabular}{|c|c|c|c|c|c|}
\hline \multicolumn{6}{|c|}{ Results } \\
\hline Peak No. & Peak ID & Ret Time & Height & Area & Conc. \\
\hline 1 & & 10.198 & 287936.438 & 4922798.500 & 43.5722 \\
\hline 2 & & 11.507 & 39072.328 & 733967.750 & 6.4964 \\
\hline 3 & & 14.723 & 31480.623 & 738886.625 & 6.5400 \\
\hline 4 & & 20.090 & 153834.000 & 4902380.000 & 43.3914 \\
\hline Total & & & 512323.389 & 11298032.875 & 100.0000 \\
\hline
\end{tabular}
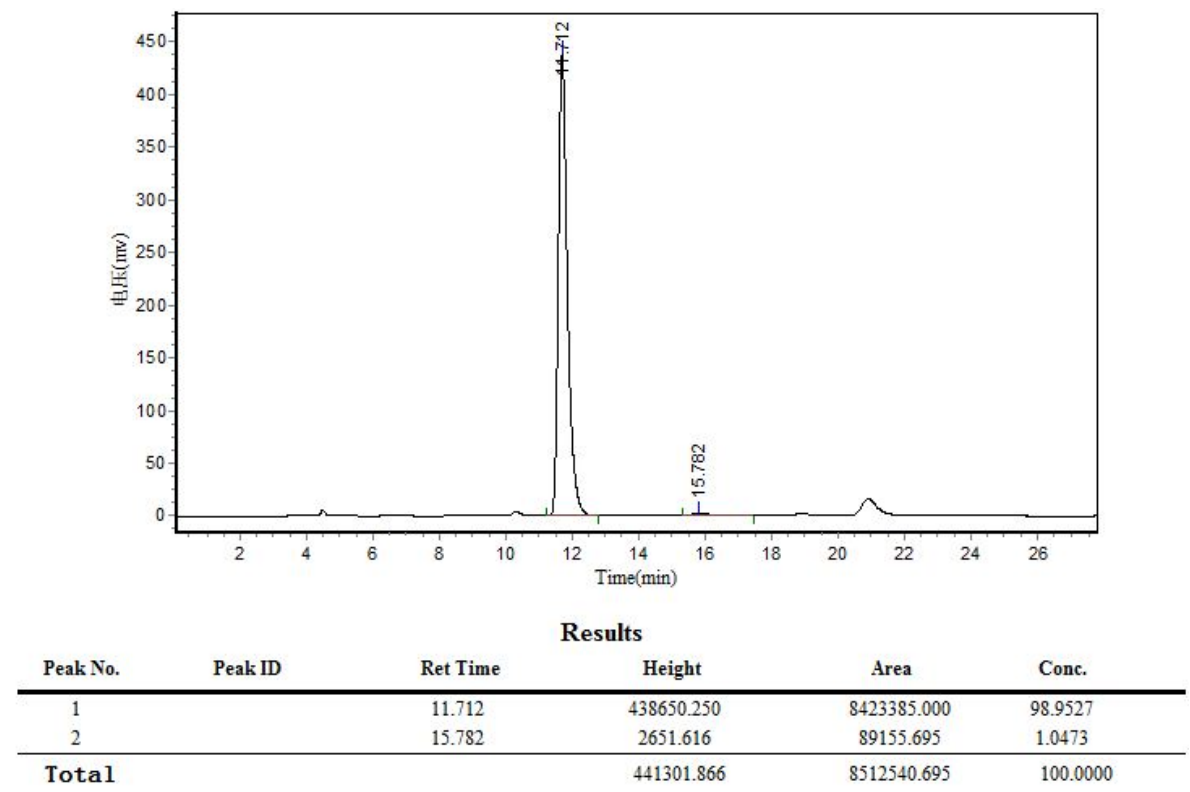
2-((2S,3R)-3-phenyl-6-(pyrrolidin-1-yl)-2,3-dihydrobenzofuran-2-yl)ethanol ((2S,3R)-4a)<smiles>OCCC1Oc2cc(N3CCCC3)ccc2[C@H]1c1ccccc1</smiles>

White solid, $27.4 \mathrm{mg}, 89 \%$ yield, $99: 1 \mathrm{dr}$, $99 \%$ ee; m.p.102.6-104. ${ }^{\circ} \mathrm{C}$, $[\alpha]_{D^{20}}=-14.3\left(c \quad 1.00, \mathrm{CHCl}_{3}\right) ;{ }^{1} \mathrm{H}$ NMR $\left(400 \mathrm{MHz}, \mathrm{CDCl}_{3}\right) \delta$ 7.34-7.22 (m, 5H), $6.79(\mathrm{~d}, J=8.0 \mathrm{~Hz}, 1 \mathrm{H}), 6.13-6.09(\mathrm{~m}, 2 \mathrm{H})$, 4.71-4.66 (m, 1H), $4.23(\mathrm{~d}, J=7.8 \mathrm{~Hz}, 1 \mathrm{H}), 3.88(\mathrm{t}, J=6.0 \mathrm{~Hz}, 2 \mathrm{H})$, $3.27(\mathrm{t}, J=6.4 \mathrm{~Hz}, 4 \mathrm{H}), 2.18-1.98(\mathrm{~m}, 7 \mathrm{H}) ;{ }^{13} \mathrm{C}$ NMR $(100 \mathrm{MHz}$, $\left.\mathrm{CDCl}_{3}\right) \delta 161.0,149.5,143.3,129.0,128.4,127.3,125.7,117.3,105.0,93.6,91.4,60.9,54.8,48.2$, 37.6, 25.7; HRMS (ESI) for $\mathrm{C}_{20} \mathrm{H}_{24} \mathrm{NO}_{2}[\mathrm{M}+\mathrm{H}]^{+}$: calcd 310.1802, found 310.1798.

HPLC: Chiralpak AD-H column $(250 \mathrm{~mm})$; detected at $220 \mathrm{~nm}$; hexane $/ i$-propanol $=90 / 10$; flow $=0.7 \mathrm{~mL} / \mathrm{min}$; Retention time: $10.3 \mathrm{~min}$ (major), $21.0 \mathrm{~min}$.

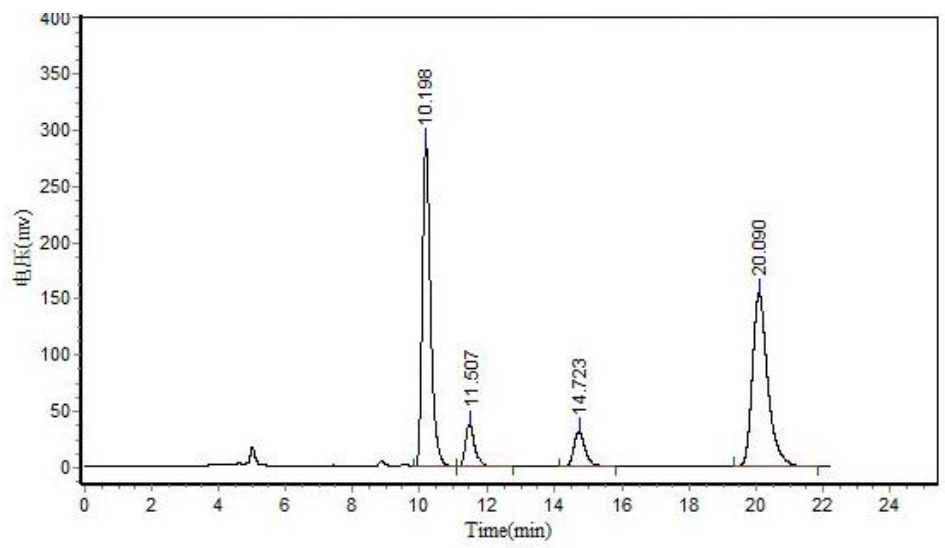

\begin{tabular}{|c|c|c|c|c|c|}
\hline \multicolumn{6}{|c|}{ Results } \\
\hline Peak No. & Peak ID & Ret Time & Height & Area & Conc. \\
\hline 1 & & 10.198 & 287936.438 & 4922798.500 & 43.5722 \\
\hline 2 & & 11.507 & 39072.328 & 733967.750 & 6.4964 \\
\hline 3 & & 14.723 & 31480.623 & 738886.625 & 6.5400 \\
\hline 4 & & 20.090 & 153834.000 & 4902380.000 & 43.3914 \\
\hline Total & & & 512323.389 & 11298032.875 & 100.0000 \\
\hline
\end{tabular}

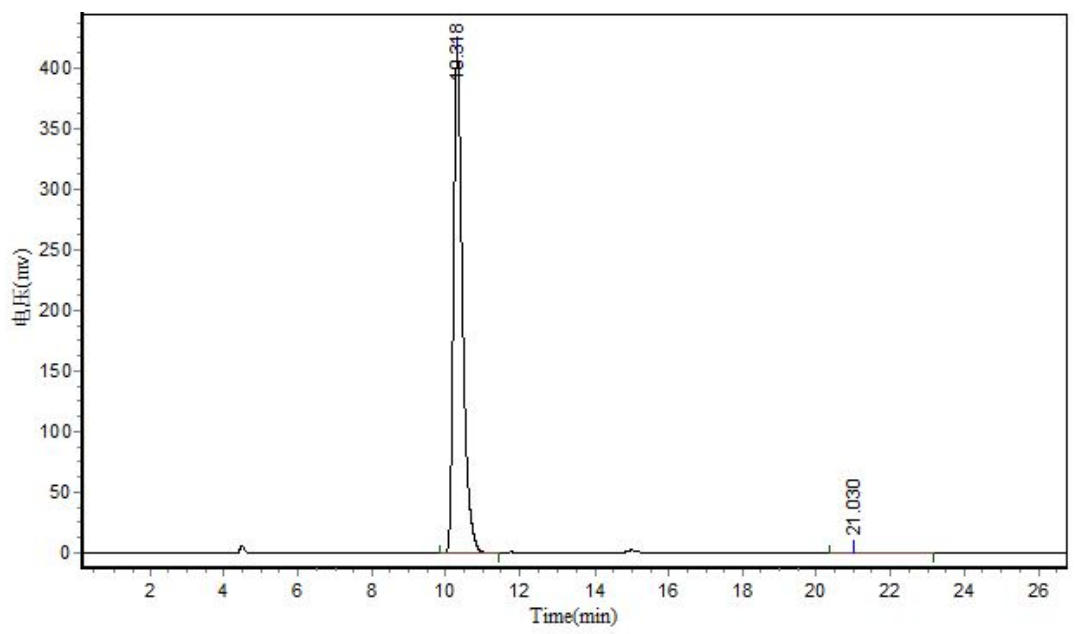

Results

\begin{tabular}{|c|c|c|c|c|c|}
\hline Peak No. & Peak ID & Ret Time & Height & Area & Conc. \\
\hline 1 & & 10.318 & 414579.000 & 7129880.500 & 99.5363 \\
\hline 2 & & 21.030 & 655.651 & 33214.500 & 0.4637 \\
\hline Total & & & 415234.651 & 7163095.000 & 100.0000 \\
\hline
\end{tabular}


<smiles>OCCC1Oc2cc(N3CCCC3)ccc2[C@H]1c1ccccc1</smiles>

White solid, $29.0 \mathrm{mg}, 94 \%$ yield, 98:2, 99\% ee; m.p.128.4-131. ${ }^{\circ} \mathrm{C}$, $[\alpha]_{\mathrm{D}}{ }^{20}=-2.2\left(c 1.0, \mathrm{CHCl}_{3}\right) ;{ }^{1} \mathrm{H}$ NMR $\left(400 \mathrm{MHz}, \mathrm{CDCl}_{3}\right) \delta$ 7.28-7.19 (m, 3H), $7.01(\mathrm{~d}, J=7.2 \mathrm{~Hz}, 2 \mathrm{H}), 6.91(\mathrm{~d}, J=8.0 \mathrm{~Hz}, 1 \mathrm{H}), 6.16-6.12$ $(\mathrm{m}, 2 \mathrm{H}), 5.08-5.03(\mathrm{~m}, 1 \mathrm{H}), 4.50(\mathrm{~d}, J=8.0 \mathrm{~Hz}, 1 \mathrm{H}), 3.79-3.75(\mathrm{~m}$, 2H), 3.29 (t, $J=6.0 \mathrm{~Hz}, 4 \mathrm{H}), 2.02-1.99(\mathrm{~m}, 5 \mathrm{H}), 1.58-1.39(\mathrm{~m}, 2 \mathrm{H})$; ${ }^{13} \mathrm{C}$ NMR $\left(100 \mathrm{MHz}, \mathrm{CDCl}_{3}\right) \delta 161.1,149.4,141.0,129.3,128.6,127.1,126.1,117.8,105.2,93.6$, 86.3, 61.2, 51.5, 48.2, 34.9, 25.8; HRMS (ESI) for $\mathrm{C}_{20} \mathrm{H}_{24} \mathrm{NO}_{2}[\mathrm{M}+\mathrm{H}]^{+}$: calcd 310.1802, found 310.1800 .

HPLC: Chiralpak AD-H column $(250 \mathrm{~mm})$; detected at $220 \mathrm{~nm}$; hexane $/ i$-propanol $=90 / 10$; flow $=0.7 \mathrm{~mL} / \mathrm{min}$; Retention time: $11.7 \mathrm{~min}, 14.9 \mathrm{~min}$ (major).

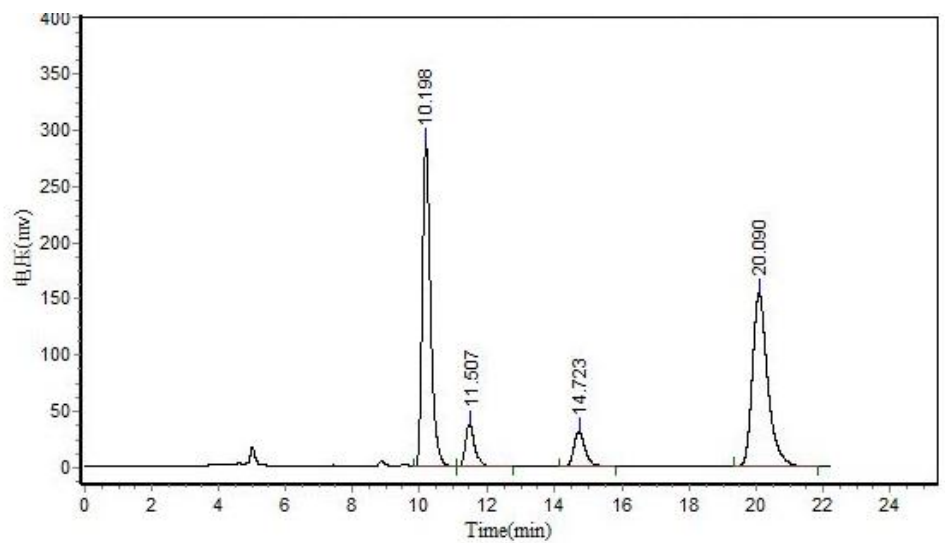

\begin{tabular}{|c|c|c|c|c|c|}
\hline \multicolumn{6}{|c|}{ Results } \\
\hline Peak No. & Peak ID & Ret Time & Height & Area & Conc. \\
\hline 1 & & 10.198 & 287936.438 & 4922798.500 & 43.5722 \\
\hline 2 & & 11.507 & 39072.328 & 733967.750 & 6.4964 \\
\hline 3 & & 14.723 & 31480.623 & 738886.625 & 6.5400 \\
\hline 4 & & 20.090 & 153834.000 & 4902380.000 & 43.3914 \\
\hline Total & & & 512323.389 & 11298032.875 & 100.0000 \\
\hline
\end{tabular}

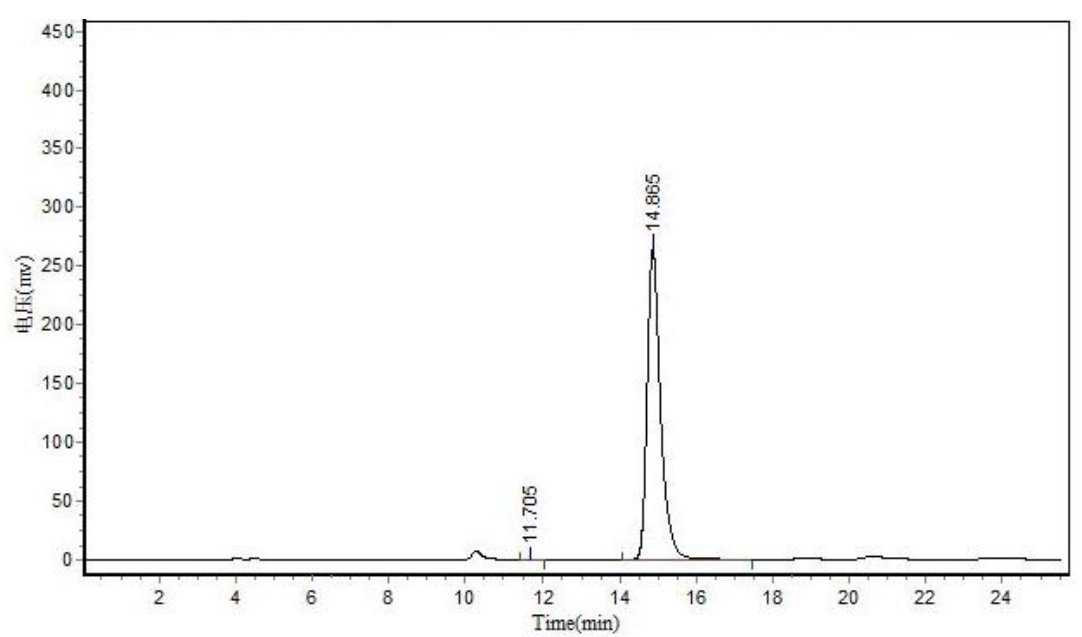

Results

\begin{tabular}{|c|c|c|c|c|c|}
\hline \multicolumn{6}{|c|}{ Necos } \\
\hline Peak No. & Peak ID & Ret Time & Height & Area & Conc. \\
\hline 1 & & 11.705 & 86.669 & 1479.301 & 0.0230 \\
\hline 2 & & 14.865 & 264434.750 & 6440377.500 & 99.9770 \\
\hline Total & & & 264521.419 & 6441856.801 & 100.0000 \\
\hline
\end{tabular}




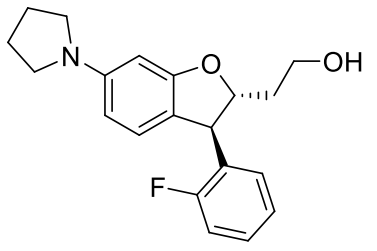

Pale yellow oil, $29.1 \mathrm{mg}, 89 \%$ yield, $98: 2 \mathrm{dr}, 99 \%$ ee; $[\alpha]_{\mathrm{D}}^{20}=17.6(c$ $\left.1.0, \mathrm{CHCl}_{3}\right) ;{ }^{1} \mathrm{H}$ NMR $\left(400 \mathrm{MHz}, \mathrm{CDCl}_{3}\right) \delta$ 7.23-7.18 (m, $\left.1 \mathrm{H}\right)$, 7.13-7.03 (m, 3H), $6.83(\mathrm{~d}, J=8.8 \mathrm{~Hz}, 1 \mathrm{H}), 6.12-6.10(\mathrm{~m}, 2 \mathrm{H})$, 4.73-4.68 (m, 1H), $4.60(\mathrm{~d}, J=7.2 \mathrm{~Hz}, 1 \mathrm{H}), 3.88(\mathrm{t}, J=6.0 \mathrm{~Hz}, 2 \mathrm{H})$, $3.27(\mathrm{t}, J=6.4 \mathrm{~Hz}, 4 \mathrm{H}), 2.17-1.97(\mathrm{~m}, 7 \mathrm{H}) ;{ }^{13} \mathrm{C} \mathrm{NMR}(100 \mathrm{MHz}$, $\left.\mathrm{CDCl}_{3}\right) \delta 161.2(\mathrm{~d}, J=239.2 \mathrm{~Hz}), 161.1,149.5,130.4(\mathrm{~d}, J=13.7 \mathrm{~Hz}), 129.7(\mathrm{~d}, J=4.2 \mathrm{~Hz})$, $128.6(\mathrm{~d}, J=8.2 \mathrm{~Hz}), 125.8,124.8(\mathrm{~d}, J=3.5 \mathrm{~Hz}), 115.6(\mathrm{~d}, J=22.0 \mathrm{~Hz}), 115.4,105.1,93.7,90.4$, 60.8, 48.2, 46.7 (d, $J=2.8 \mathrm{~Hz}), 37.9,25.8$; HRMS (ESI) for $\mathrm{C}_{20} \mathrm{H}_{23} \mathrm{FNO}_{2}[\mathrm{M}+\mathrm{H}]^{+}$: calcd 328.1707, found 328.1703 .

HPLC: Chiralpak IG-3 column $(250 \mathrm{~mm})$; detected at $254 \mathrm{~nm}$; hexane $/ i$-propanol $=80 / 20$; flow $=$ $0.5 \mathrm{~mL} / \mathrm{min}$; Retention time: $13.9 \mathrm{~min}, 15.1 \mathrm{~min}$ (major).
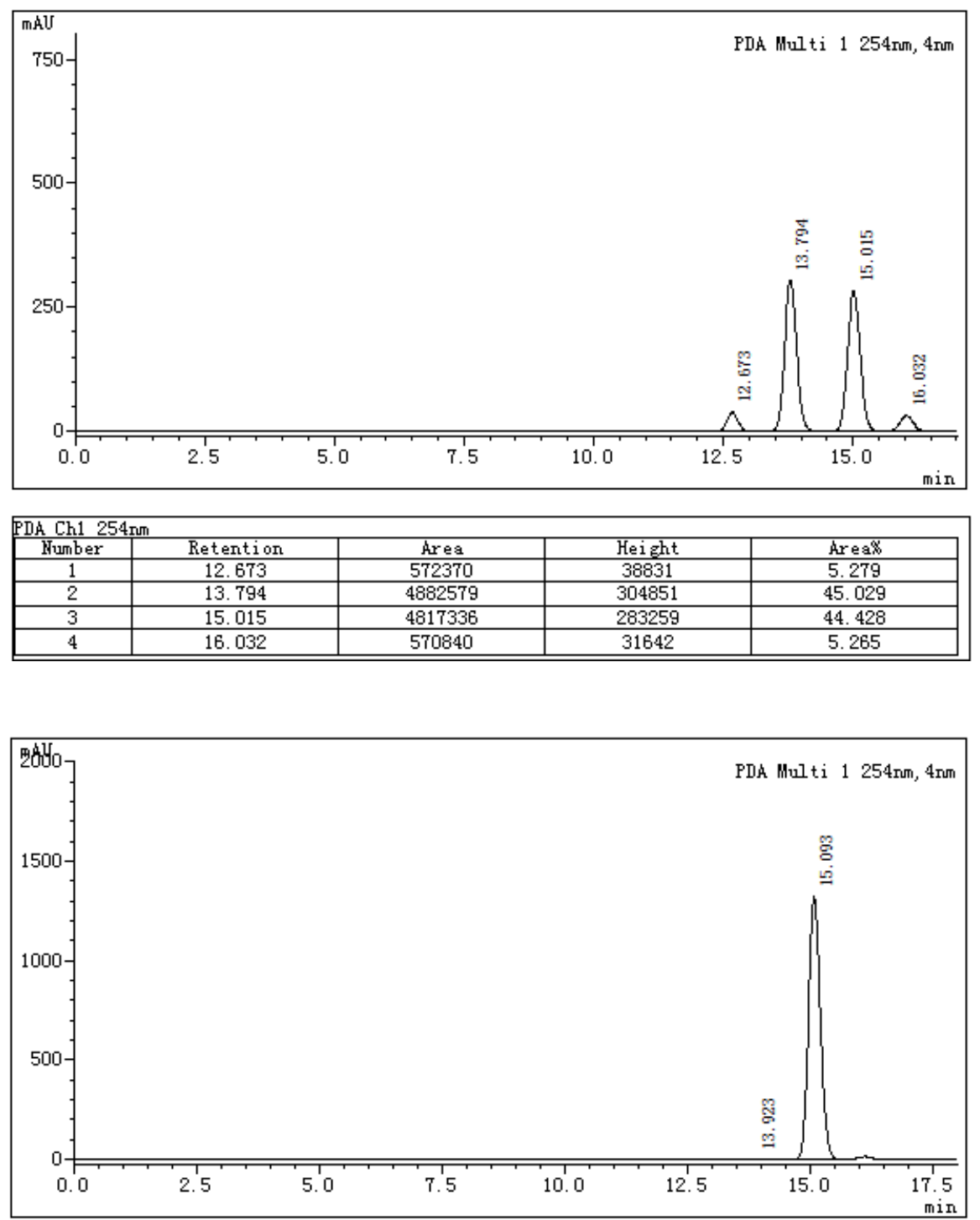

\begin{tabular}{|c|c|c|c|c|}
\hline PDA Ch1 254rnm & \multicolumn{4}{|c|}{} \\
\hline Humber & Retention & Area & Hei ght & Area \\
\hline 1 & 13.923 & 69023 & 3474 & 0.310 \\
\hline 2 & 15.093 & 22199758 & 1324162 & 99.690 \\
\hline \hline
\end{tabular}




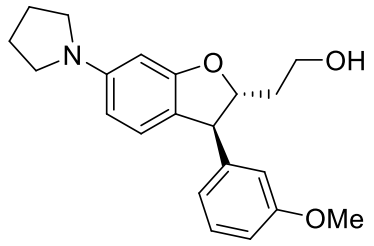

Colorless oil, $30.2 \mathrm{mg}, 89 \%$ yield, $99: 1 \mathrm{dr}, 99 \%$ ee; $[\alpha]_{\mathrm{D}}^{20}=17.1(c$ 1.0, $\left.\mathrm{CHCl}_{3}\right) ;{ }^{1} \mathrm{H}$ NMR $\left(400 \mathrm{MHz}, \mathrm{CDCl}_{3}\right) \delta 7.24(\mathrm{t}, J=8.0 \mathrm{~Hz}, 1 \mathrm{H})$, 6.83-6.79 (m, 4H), 6.12-6.10 (m, 2H), 4.72-4.67 (m, 1H), $4.21(\mathrm{~d}, J=$ $8.0 \mathrm{~Hz}, 1 \mathrm{H}), 3.88(\mathrm{t}, J=6.0 \mathrm{~Hz}, 2 \mathrm{H}), 3.78(\mathrm{~s}, 3 \mathrm{H}), 3.28(\mathrm{t}, J=6.0 \mathrm{~Hz}$, $4 \mathrm{H}), 2.16-1.99(\mathrm{~m}, 7 \mathrm{H}) ;{ }^{13} \mathrm{C}$ NMR $\left(100 \mathrm{MHz}, \mathrm{CDCl}_{3}\right) \delta 161.0,160.2$, 149.4, 144.9, 130.0, 125.7, 120.8, 117.0, 114.1, 112.5, 105.0, 93.5, 91.2, 60.9, 55.5, 54.9, 48.2, 37.7, 25.8; HRMS (ESI) for $\mathrm{C}_{21} \mathrm{H}_{26} \mathrm{NO}_{3}[\mathrm{M}+\mathrm{H}]^{+}$: calcd 340.1907, found 340.1905.

HPLC: Chiralpak OD-H column $(250 \mathrm{~mm})$; detected at $254 \mathrm{~nm}$; hexane $/ i$-propanol $=90 / 10$; flow $=0.7 \mathrm{~mL} / \mathrm{min}$; Retention time: $17.1 \mathrm{~min}$ (major), $28.4 \mathrm{~min}$.

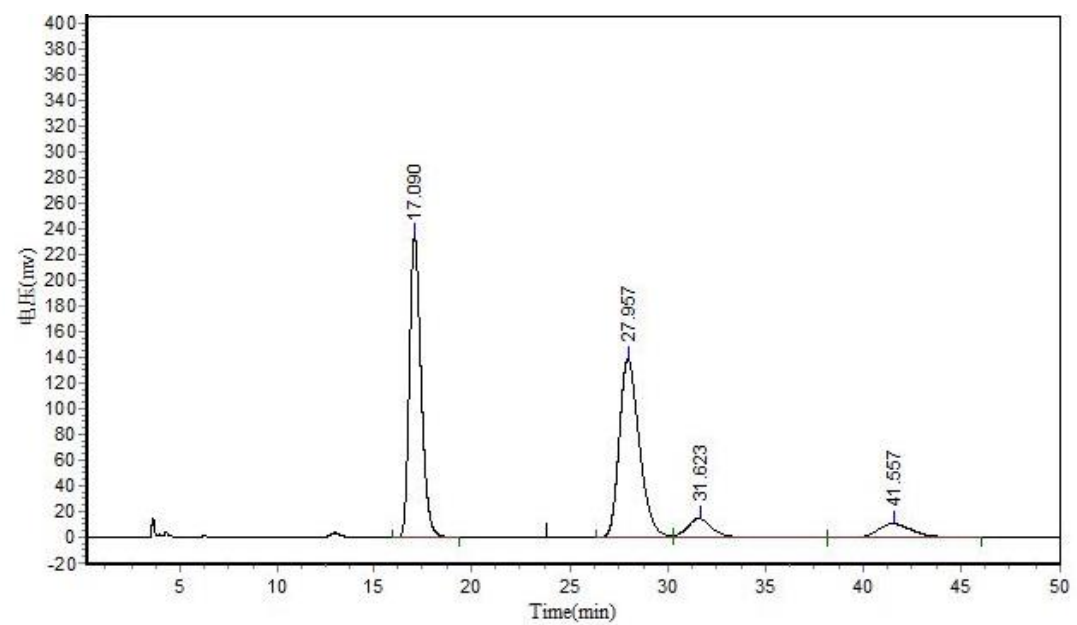

\begin{tabular}{|c|c|c|c|c|c|}
\hline \multicolumn{6}{|c|}{ Results } \\
\hline Peak No. & Peak ID & Ret Time & Height & Area & Conc. \\
\hline 1 & & 17.090 & 236537.141 & 9834083.000 & 43.4549 \\
\hline 2 & & 27.957 & 139290.688 & 10069142.000 & 44.4936 \\
\hline 3 & & 31.623 & 14846.816 & 1387157.875 & 6.1296 \\
\hline 4 & & 41.557 & $10 \$ 26.671$ & 1340159.125 & 5.9219 \\
\hline Total & & & 401501.315 & 22630542.000 & 100.0000 \\
\hline
\end{tabular}

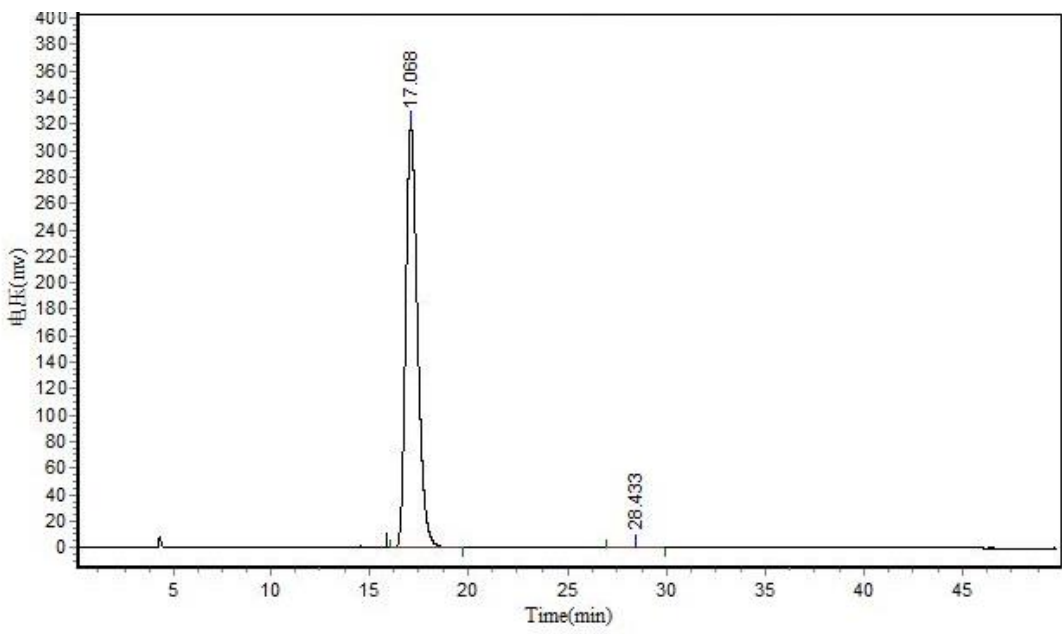

\begin{tabular}{|c|c|c|c|c|c|}
\hline \multicolumn{6}{|c|}{ Results } \\
\hline Peak No. & Peak ID & Ret Time & Height & Area & Conc. \\
\hline 1 & & 17.068 & 319811.813 & 13280096.000 & 99.8616 \\
\hline 2 & & 28.433 & 234.608 & 18410.627 & 0.1384 \\
\hline Total & & & 320046.420 & 13298506.627 & 100.0000 \\
\hline
\end{tabular}


<smiles>Cc1cccc(C2c3ccc(N4CCCC4)cc3OC2CCO)c1</smiles>

White solid, $28.5 \mathrm{mg}, 88 \%$ yield, $99: 1 \mathrm{dr}$, 99\% ee; m.p.78.3-79.8 ${ }^{\circ} \mathrm{C}$, $[\alpha]_{\mathrm{D}}^{20}=10.5\left(c 1.0, \mathrm{CHCl}_{3}\right) ;{ }^{1} \mathrm{H}$ NMR $\left(400 \mathrm{MHz}, \mathrm{CDCl}_{3}\right) \delta 7.21(\mathrm{t}, J$ $=7.2 \mathrm{~Hz}, 1 \mathrm{H}), 7.08-7.02(\mathrm{~m}, 3 \mathrm{H}), 6.80(\mathrm{~d}, J=8.0 \mathrm{~Hz}, 1 \mathrm{H})$, 6.13-6.10 (m, 2H), 4.71-4.66 (m, 1H), $4.20(\mathrm{~d}, J=8.0 \mathrm{~Hz}, 1 \mathrm{H}), 3.88$ $(\mathrm{t}, J=6.0 \mathrm{~Hz}, 2 \mathrm{H}), 3.28(\mathrm{t}, J=6.4 \mathrm{~Hz}, 4 \mathrm{H}), 2.33(\mathrm{~s}, 3 \mathrm{H}), 2.17-1.99$ $(\mathrm{m}, 7 \mathrm{H}) ;{ }^{13} \mathrm{C}$ NMR $\left(100 \mathrm{MHz}, \mathrm{CDCl}_{3}\right) \delta 160.9,149.3,143.2,138.6,129.0,128.9,128.0,125.7$, 125.6, 117.3, 104.9, 93.5, 91.4, 60.8, 54.8, 48.2, 37.6, 25.8, 21.7; HRMS (ESI) for $\mathrm{C}_{21} \mathrm{H}_{26} \mathrm{NO}_{2}$ $[\mathrm{M}+\mathrm{H}]^{+}$: calcd 324.1958, found 324.1962.

HPLC: Chiralpak AD-H column $(250 \mathrm{~mm})$; detected at $254 \mathrm{~nm}$; hexane $/ i$-propanol $=90 / 10$; flow $=0.7 \mathrm{~mL} / \mathrm{min}$; Retention time: $8.9 \mathrm{~min}, 15.3 \mathrm{~min}$ (major).
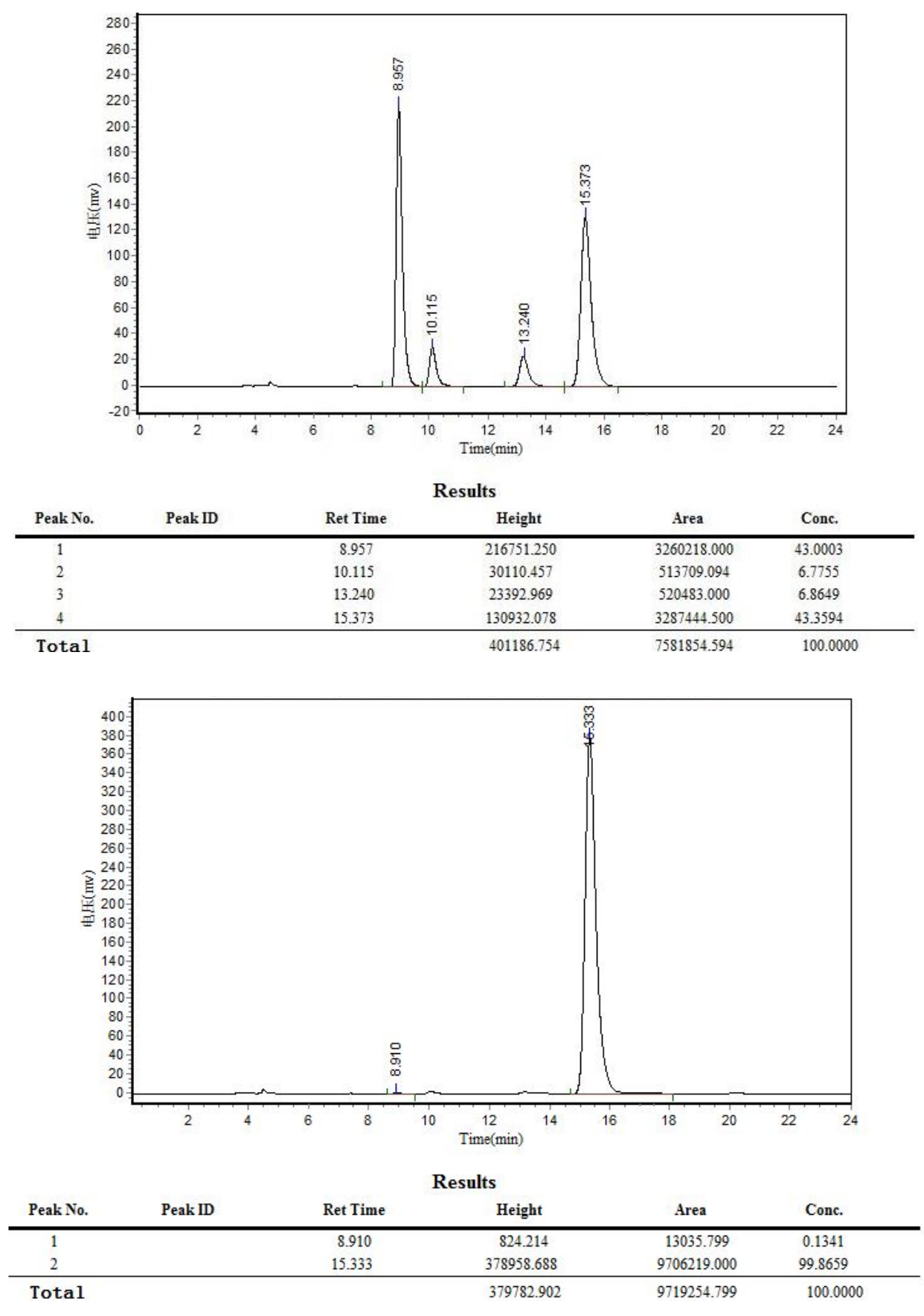


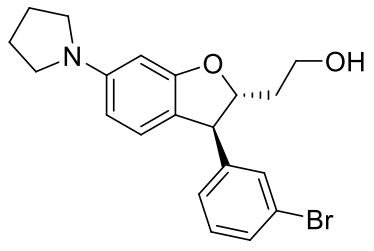

White solid, $35.0 \mathrm{mg}$, 90\% yield, $99: 1 \mathrm{dr}$, 99\% ee; m.p.87.8-90.5 ${ }^{\circ} \mathrm{C}$, $[\alpha]_{\mathrm{D}}{ }^{20}=23.1\left(c 1.0, \mathrm{CHCl}_{3}\right) ;{ }^{1} \mathrm{H} \mathrm{NMR}\left(400 \mathrm{MHz}, \mathrm{CDCl}_{3}\right) \delta$ 7.38-7.36 (m, 2H), 7.20-7.13 (m, 2H), $6.78(\mathrm{~d}, J=7.6 \mathrm{~Hz}, 1 \mathrm{H}), 6.11-6.09(\mathrm{~m}$, $2 \mathrm{H}), 4.68-4.63(\mathrm{~m}, 1 \mathrm{H}), 4.19(\mathrm{~d}, J=7.6 \mathrm{~Hz}, 1 \mathrm{H}), 3.87(\mathrm{t}, J=6.0 \mathrm{~Hz}$, $2 \mathrm{H}), 3.27(\mathrm{t}, J=6.4 \mathrm{~Hz}, 4 \mathrm{H}), 2.13-1.98(\mathrm{~m}, 7 \mathrm{H}) ;{ }^{13} \mathrm{C} \mathrm{NMR}(100 \mathrm{MHz}$, $\left.\mathrm{CDCl}_{3}\right) \delta 160.9,149.6,145.9,131.3,130.6,130.4,127.1,125.7,123.1,116.4,105.2,93.6,91.0$, 60.6, 54.5, 48.2, 37.6, 25.8; HRMS (ESI) for $\mathrm{C}_{20} \mathrm{H}_{23}{ }^{79} \mathrm{BrNO}_{2}[\mathrm{M}+\mathrm{H}]^{+}$: calcd 388.0907, found 388.0915; $\mathrm{C}_{20} \mathrm{H}_{23}{ }^{81} \mathrm{BrNO}_{2}[\mathrm{M}+\mathrm{H}]^{+}$: calcd 390.0886, found: 390.0898 .

HPLC: Chiralpak AD-H column $(250 \mathrm{~mm})$; detected at $254 \mathrm{~nm}$; hexane $/$ - propanol $=90 / 10$; flow $=0.7 \mathrm{~mL} / \mathrm{min}$; Retention time: $10.0 \mathrm{~min}, 18.1 \mathrm{~min}$ (major).

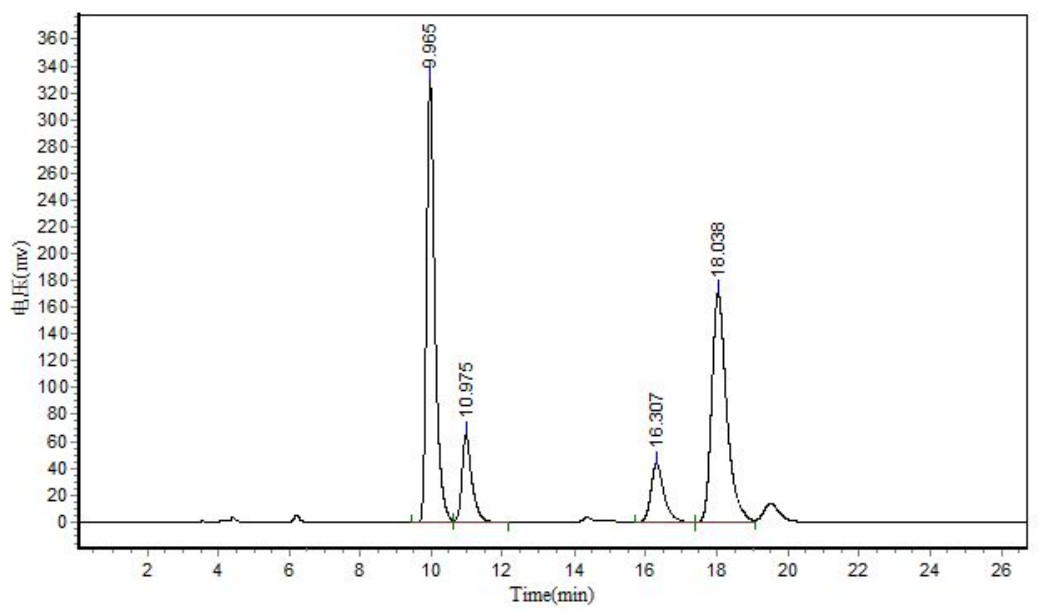

\begin{tabular}{|c|c|c|c|c|c|}
\hline \multicolumn{6}{|c|}{ Results } \\
\hline Peak No. & Peak ID & Ret Time & Height & Area & Conc. \\
\hline 1 & & 9.965 & 329774.031 & 5448621.500 & 42.1459 \\
\hline 2 & & 10.975 & 65712.375 & 1283910.375 & 9.9312 \\
\hline 3 & & 16.307 & 43891.102 & 1152544.000 & 8.9151 \\
\hline 4 & & 18.038 & 171016.297 & 5042924.500 & 39.0078 \\
\hline Total & & & 610393.805 & 12928000.375 & 100.0000 \\
\hline
\end{tabular}

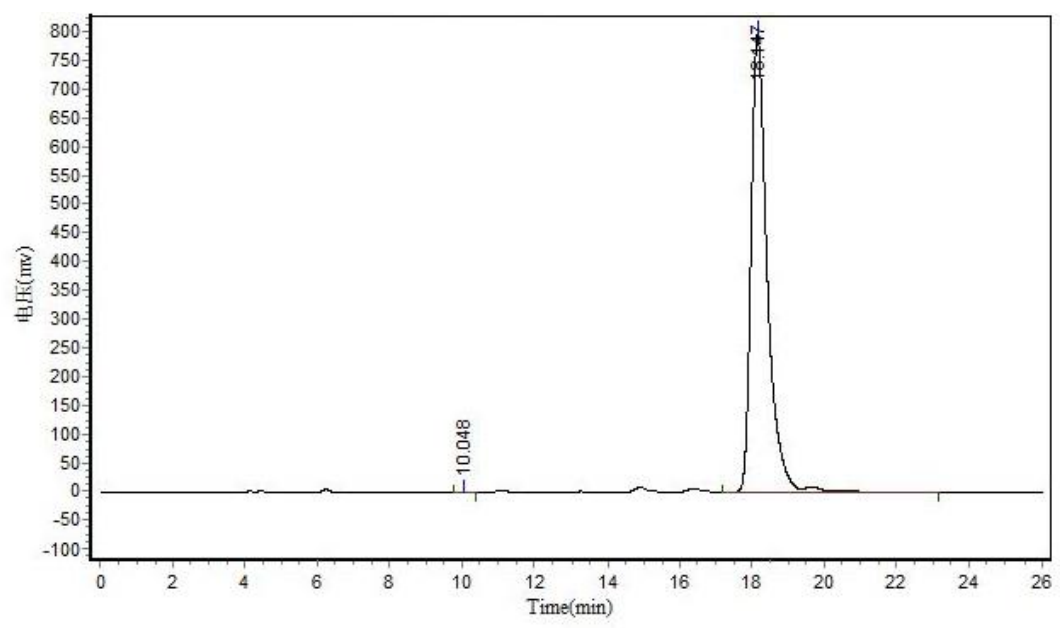

\begin{tabular}{|c|c|c|c|c|c|}
\hline \multicolumn{6}{|c|}{ Results } \\
\hline Peak No. & Peak ID & Ret Time & Height & Area & Conc. \\
\hline 1 & & 10.048 & 738.312 & 11114.645 & 0.0446 \\
\hline 2 & & 18.147 & 794794.063 & 24882382.000 & 99.9554 \\
\hline Total & & & 795532.375 & 24893496.645 & 100.0000 \\
\hline
\end{tabular}


<smiles>COc1ccc(C2c3ccc(N4CCCC4)cc3OC2CCO)cc1</smiles>

White solid, $30.9 \mathrm{mg}, 91 \%$ yield, $99: 1 \mathrm{dr}, 99 \%$ ee; m.p.105.6-107. ${ }^{\circ} \mathrm{C}$, $[\alpha]_{\mathrm{D}}{ }^{20}=6.3\left(c 1.0, \mathrm{CHCl}_{3}\right) ;{ }^{1} \mathrm{H}$ NMR $\left(400 \mathrm{MHz}, \mathrm{CDCl}_{3}\right) \delta 7.14(\mathrm{~d}, J=$ $8.4 \mathrm{~Hz}, 2 \mathrm{H}), 6.85(\mathrm{~d}, J=8.4 \mathrm{~Hz}, 2 \mathrm{H}), 6.78(\mathrm{~d}, J=8.0 \mathrm{~Hz}, 1 \mathrm{H})$, 6.12-6.09 (m, 2H), 4.65-4.60 (m, 1H), $4.19(\mathrm{~d}, J=8.0 \mathrm{~Hz}, 1 \mathrm{H}), 3.87(\mathrm{t}$, $J=6.0 \mathrm{~Hz}, 2 \mathrm{H}), 3.80(\mathrm{~s}, 3 \mathrm{H}), 3.27(\mathrm{t}, J=6.4 \mathrm{~Hz}, 4 \mathrm{H}), 2.14-1.98(\mathrm{~m}$, $7 \mathrm{H}) ;{ }^{13} \mathrm{C} \mathrm{NMR}\left(100 \mathrm{MHz}, \mathrm{CDCl}_{3}\right) \delta 160.9,158.9,149.4,135.2,129.4$, 125.6, 117.5, 114.4, 105.0, 93.5, 91.6, 60.9, 55.6, 54.0, 48.2, 37.5, 25.8; HRMS (ESI) for $\mathrm{C}_{21} \mathrm{H}_{26} \mathrm{NO}_{3}[\mathrm{M}+\mathrm{H}]^{+}$: calcd 340.1907, found 340.1903.

HPLC: Chiralpak AD-H column $(250 \mathrm{~mm})$; detected at $254 \mathrm{~nm}$; hexane $/ i$-propanol $=90 / 10$; flow $=1.1 \mathrm{~mL} / \mathrm{min}$; Retention time: $13.4 \mathrm{~min}, 42.7 \mathrm{~min}$ (major).

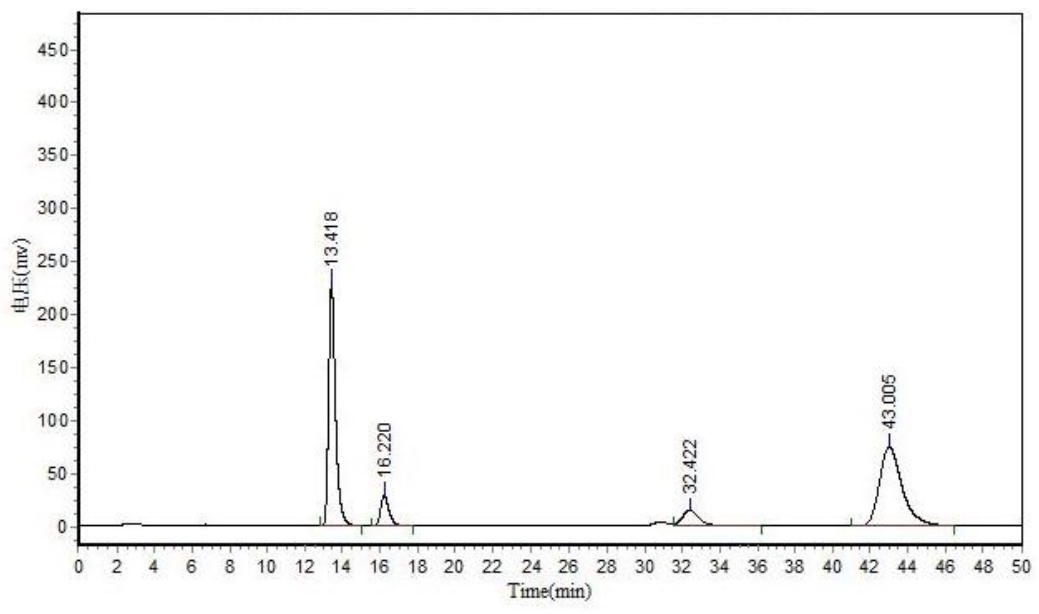

\begin{tabular}{|c|c|c|c|c|c|}
\hline \multicolumn{6}{|c|}{ Results } \\
\hline Peak No. & Peak ID & Ret Time & Height & Area & Conc. \\
\hline 1 & & 13.418 & 228945.734 & 5806399.500 & 41.6869 \\
\hline 2 & & 16.220 & 29782.564 & 902722.375 & 6.4811 \\
\hline 3 & & 32.422 & 15236.500 & 953972.188 & 6.8490 \\
\hline 4 & & 43.005 & 75007.992 & 6265502.000 & 44.9830 \\
\hline Total & & & 348972.791 & 13928596.063 & 100.0000 \\
\hline
\end{tabular}

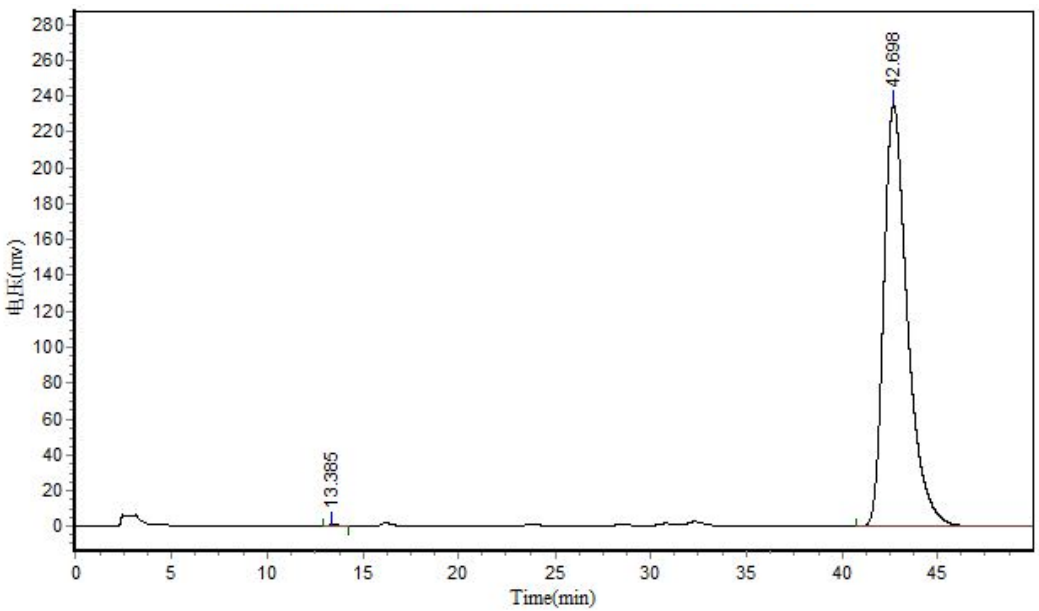

\begin{tabular}{|c|c|c|c|c|c|}
\hline \multicolumn{6}{|c|}{ Results } \\
\hline Peak No. & Peak ID & Ret Time & Height & Area & Conc. \\
\hline 1 & & 13.385 & 829.935 & 22281.000 & 0.1097 \\
\hline 2 & & 42.698 & 234859.141 & 20280192.000 & 99.8903 \\
\hline Total & & & 235689.076 & 20302473.000 & 100.0000 \\
\hline
\end{tabular}


<smiles>O=[N+]([O-])c1ccc(C2c3ccc(N4CCCC4)cc3OC2CCO)cc1</smiles>

White solid, $27.9 \mathrm{mg}, 86 \%$ yield, $99: 1 \mathrm{dr}$, 99\% ee; m.p.95.3-97. $8^{\circ} \mathrm{C}$, $[\alpha]_{\mathrm{D}}^{20}=9.2\left(c \quad 0.64, \mathrm{CHCl}_{3}\right) ;{ }^{1} \mathrm{H} \mathrm{NMR}\left(400 \mathrm{MHz}, \mathrm{CDCl}_{3}\right) \delta$ 7.14-7.10 $(\mathrm{m}, 4 \mathrm{H}), 6.78(\mathrm{~d}, J=8.0 \mathrm{~Hz}, 1 \mathrm{H}), 6.11-6.08(\mathrm{~m}, 2 \mathrm{H}), 4.67-4.62(\mathrm{~m}$, $1 \mathrm{H}), 4.20(\mathrm{~d}, J=7.6 \mathrm{~Hz}, 1 \mathrm{H}), 3.87(\mathrm{t}, J=5.6 \mathrm{~Hz}, 2 \mathrm{H}), 3.27(\mathrm{t}, J=6.0$ $\mathrm{Hz}, 4 \mathrm{H}), 2.33$ (s, 3H), 2.15-1.98 (m, 7H); $\left.{ }^{13} \mathrm{C} \mathrm{NMR} \mathrm{(100} \mathrm{MHz,} \mathrm{CDCl}_{3}\right)$ $\delta 161.0,149.4,140.2,136.9,129.7,128.4,125.7,117.4,105.0,93.5$, 91.7, 61.0, 54.5, 48.2, 37.5, 25.8, 21.4; HRMS (ESI) for $\mathrm{C}_{21} \mathrm{H}_{26} \mathrm{NO}_{2}[\mathrm{M}+\mathrm{H}]^{+}$: calcd 324.1958, found 324.1960 .

HPLC: Chiralpak AD-H column $(250 \mathrm{~mm})$; detected at $254 \mathrm{~nm}$; hexane $/ i$-propanol $=90 / 10$; flow $=0.7 \mathrm{~mL} / \mathrm{min}$; Retention time: $9.8 \mathrm{~min}, 28.9 \mathrm{~min}$ (major).

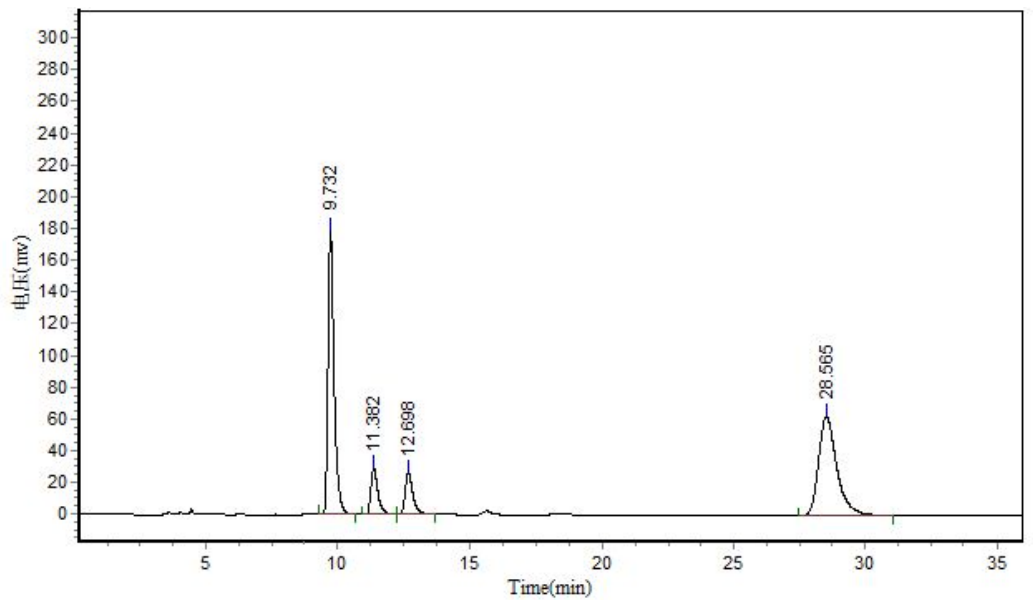

Results

\begin{tabular}{|c|c|c|c|c|c|}
\hline \\
\hline Peak No. & Peak ID & Ret Time & Height & Area & Conc. \\
\hline 1 & & 9.732 & 178388.609 & 2865946.000 & 41.2319 \\
\hline 2 & & 11.382 & 29657.859 & 546702.875 & 7.8653 \\
\hline 3 & & 12.698 & 26724.158 & 550041.625 & 7.9134 \\
\hline 4 & & 28.565 & 62516.133 & 2988104.500 & 42.9894 \\
\hline Total & & & 297286.760 & 6950795.000 & 100.0000 \\
\hline
\end{tabular}

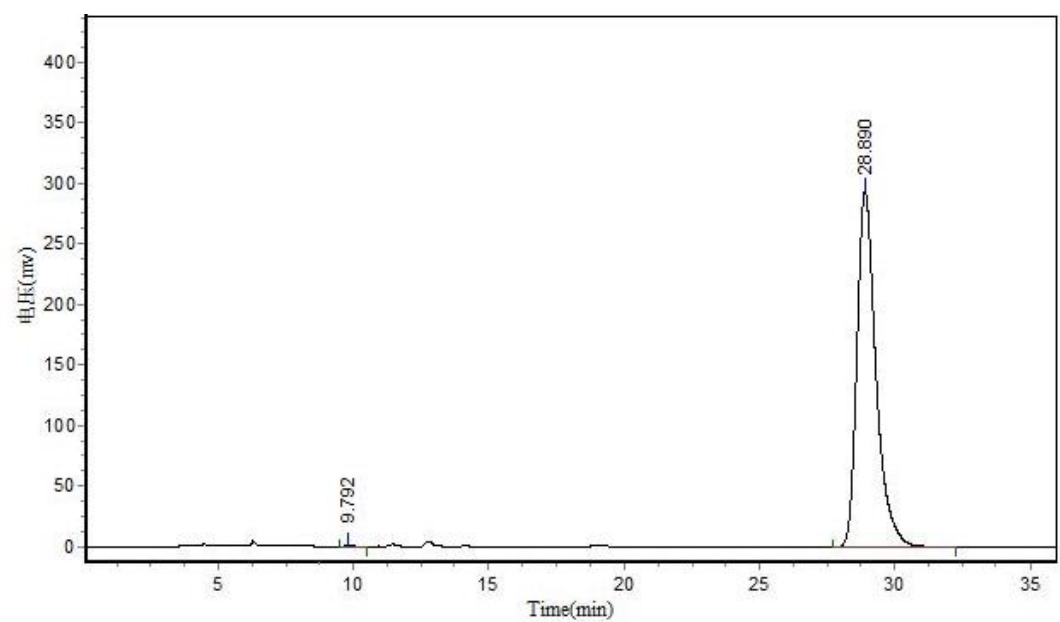

\begin{tabular}{|c|c|c|c|c|c|}
\hline \multicolumn{6}{|c|}{ Results } \\
\hline Peak No. & Peak ID & Ret Time & Height & Area & Conc. \\
\hline 1 & & 9.792 & 832.215 & 13677.800 & 0.0952 \\
\hline 2 & & 28.890 & 293113.875 & 14356851.000 & 99.9048 \\
\hline Total & & & 293946.090 & 14370528.800 & 100.0000 \\
\hline
\end{tabular}


<smiles>OCCC1Oc2cc(N3CCCC3)ccc2C1c1ccc(Cl)cc1</smiles>

White solid, $31.3 \mathrm{mg}, 91 \%$ yield, $99: 1 \mathrm{dr}, 99 \%$ ee; m.p.114.8-117.4 ${ }^{\circ} \mathrm{C}$, $[\alpha]_{\mathrm{D}}^{20}=29.0\left(c 1.0, \mathrm{CHCl}_{3}\right) ;{ }^{1} \mathrm{H}$ NMR $\left(400 \mathrm{MHz}, \mathrm{CDCl}_{3}\right) \delta 7.28(\mathrm{~d}, J$ $=8.4 \mathrm{~Hz}, 2 \mathrm{H}), 7.15(\mathrm{~d}, J=8.4 \mathrm{~Hz}, 2 \mathrm{H}), 6.76(\mathrm{~d}, J=8.0 \mathrm{~Hz}, 1 \mathrm{H})$, 6.12-6.09 (m, 2H), 4.66-4.61 (m, 1H), $4.20(\mathrm{~d}, J=7.6 \mathrm{~Hz}, 1 \mathrm{H}), 3.87$ (t, $J=6.0 \mathrm{~Hz}, 2 \mathrm{H}), 3.27(\mathrm{t}, J=6.4 \mathrm{~Hz}, 4 \mathrm{H}), 2.15-1.98(\mathrm{~m}, 7 \mathrm{H}) ;{ }^{13} \mathrm{C} \mathrm{NMR}$ $\left(100 \mathrm{MHz}, \mathrm{CDCl}_{3}\right) \delta 160.9,149.5,141.9,133.0,129.7,129.1,125.6$, 116.7, 105.1, 93.5, 91.0, 60.6, 54.2, 48.2, 37.6, 25.8;HRMS (ESI) for $\mathrm{C}_{20} \mathrm{H}_{23}{ }^{35} \mathrm{CINO}_{2}[\mathrm{M}+\mathrm{H}]^{+}$: calcd 344.1412, found 344.1415; $\mathrm{C}_{20} \mathrm{H}_{23}{ }^{37} \mathrm{ClNO}_{2}[\mathrm{M}+\mathrm{H}]^{+}$: calcd 346.1382, found: 346.1401 .

HPLC: Chiralpak AD-H column $(250 \mathrm{~mm})$; detected at $254 \mathrm{~nm}$; hexane $/ i$-propanol $=90 / 10$; flow $=0.7 \mathrm{~mL} / \mathrm{min}$; Retention time: $10.0 \mathrm{~min}, 21.6 \mathrm{~min}$ (major).

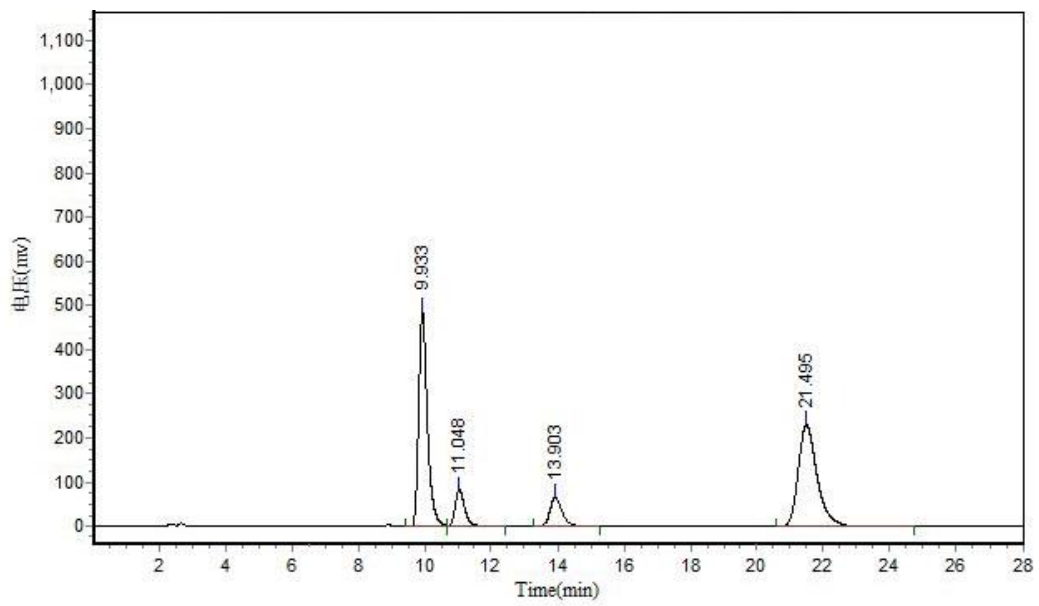

Results

\begin{tabular}{|c|c|c|c|c|c|}
\hline \\
\hline Peak No. & Peak ID & Ret Time & Height & Area & Conc. \\
\hline 1 & & 9.933 & 484673.750 & 8868383.000 & 41.2614 \\
\hline 2 & & 11.048 & $\$ 4050.891$ & 1705837.000 & 7.9366 \\
\hline 3 & & 13.903 & 67000.547 & 1742103.250 & 8.1054 \\
\hline 4 & & 21.495 & 230225.141 & 9176871.000 & 42.6966 \\
\hline Total & & & 865950.328 & 21493194.250 & 100.0000 \\
\hline
\end{tabular}

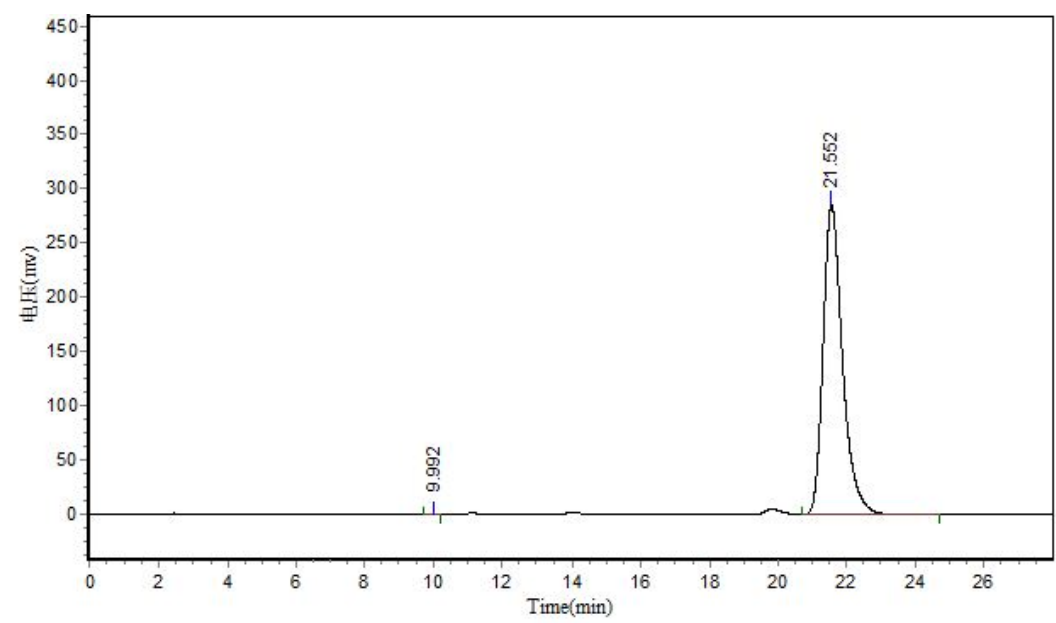

Results

\begin{tabular}{|c|c|c|c|c|c|}
\hline Peak No. & Peak ID & Ret Time & Height & Area & Conc. \\
\hline 1 & & 9.992 & 825.271 & 16005.300 & 0.1421 \\
\hline 2 & & 21.552 & 285865.406 & 11245877.000 & 99.8579 \\
\hline Total & & & 286690.678 & 11261882.300 & 100.0000 \\
\hline
\end{tabular}


2-((2R,3S)-6-(pyrrolidin-1-yl)-3-(4-(trifluoromethyl)phenyl)-2,3-dihydrobenzofuran-2-yl) ethanol (4i)

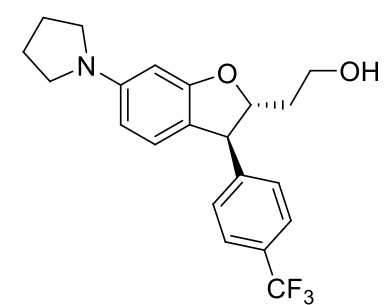

Pale yellow solid, $34.4 \mathrm{mg}, 91 \%$ yield, 99:1 dr, 99\% ee; m.p.55.7-57.2 ${ }^{\circ} \mathrm{C},[\alpha]_{\mathrm{D}}^{20}=32.8\left(c 0.97, \mathrm{CHCl}_{3}\right) ;{ }^{1} \mathrm{H}$ NMR $\left(400 \mathrm{MHz}, \mathrm{CDCl}_{3}\right) \delta 7.56$ (d, $J=8.0 \mathrm{~Hz}, 2 \mathrm{H}), 7.33(\mathrm{~d}, J=8.0 \mathrm{~Hz}, 2 \mathrm{H}), 6.76(\mathrm{~d}, J=8.0 \mathrm{~Hz}, 1 \mathrm{H})$, $6.12(\mathrm{~s}, 1 \mathrm{H}), 6.10(\mathrm{~d}, J=8.0 \mathrm{~Hz}, 1 \mathrm{H}), 4.71-4.66(\mathrm{~m}, 1 \mathrm{H}), 4.29(\mathrm{~d}, J=$ $7.6 \mathrm{~Hz}, 1 \mathrm{H}), 3.88(\mathrm{t}, J=6.0 \mathrm{~Hz}, 2 \mathrm{H}), 3.27$ (t, $J=6.4 \mathrm{~Hz}, 4 \mathrm{H})$, 2.17-1.98 (m, 7H); ${ }^{13} \mathrm{C}$ NMR $\left(100 \mathrm{MHz}, \mathrm{CDCl}_{3}\right) \delta 161.0,149.6,147.7$, $129.6(\mathrm{q}, J=32.1 \mathrm{~Hz}), 128.7,126.0(\mathrm{q}, J=3.8 \mathrm{~Hz}), 125.7,124.5$ (q, $J$ $=270.3 \mathrm{~Hz}$ ), 116.4, 105.2, 93.6, 90.9, 60.6, 54.6, 48.2, 37.7, 25.8; HRMS (ESI) for $\mathrm{C}_{21} \mathrm{H}_{23} \mathrm{~F}_{3} \mathrm{NO}_{2}$ $[\mathrm{M}+\mathrm{H}]^{+}$: calcd 378.1675, found 378.1680.

HPLC: Chiralpak OD-H column $(250 \mathrm{~mm})$; detected at $254 \mathrm{~nm}$; hexane $/ i$-propanol $=90 / 10$; flow $=0.7 \mathrm{~mL} / \mathrm{min}$; Retention time: $10.7 \mathrm{~min}$ (major), $12.1 \mathrm{~min}$.

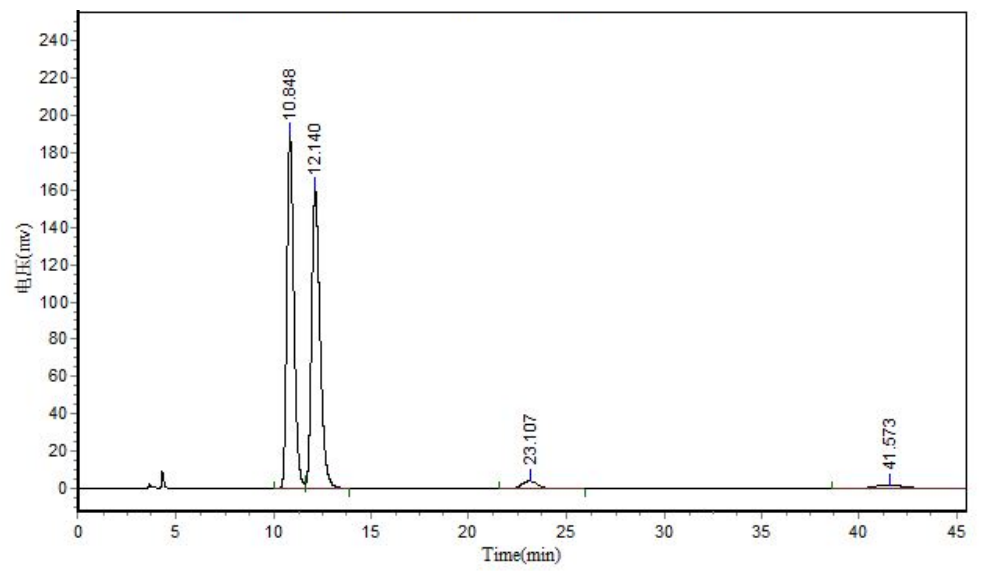

\begin{tabular}{|c|c|c|c|c|c|}
\hline \multicolumn{6}{|c|}{ Results } \\
\hline Peak No. & Peak ID & Ret Time & Height & Area & Conc. \\
\hline 1 & & 10.848 & 189512.547 & 4772510.500 & 47.0392 \\
\hline 2 & & 12.140 & 159854.938 & 4845671.000 & 47.7603 \\
\hline 3 & & 23.107 & 4229.153 & 261041.000 & 2.5729 \\
\hline 4 & & 41.573 & 2065.487 & 266585.625 & 2.6275 \\
\hline Total & & & 355662.124 & 10145808.125 & 100.0000 \\
\hline
\end{tabular}

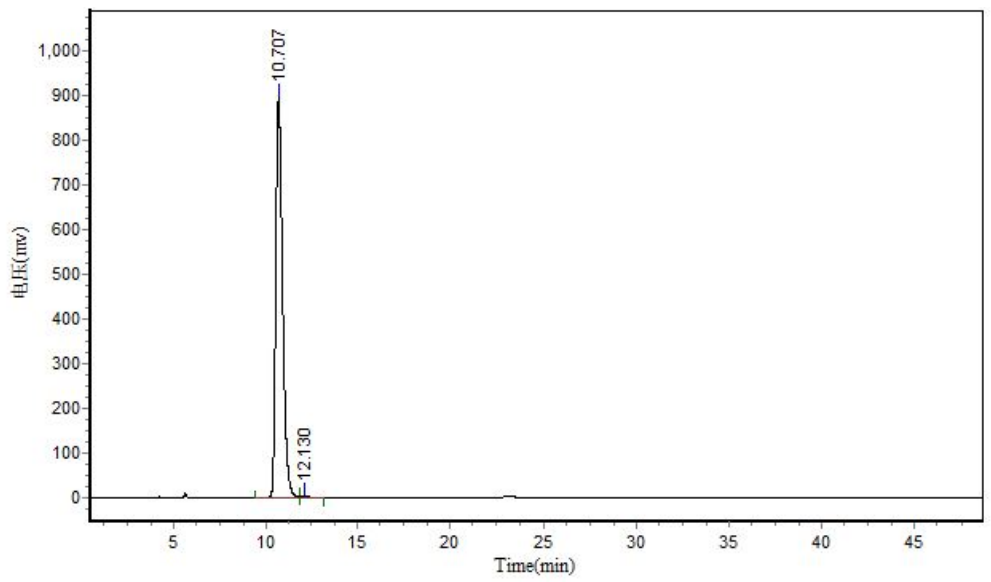

Results

\begin{tabular}{|c|c|c|c|c|c|}
\hline \multicolumn{6}{|c|}{ nes } \\
\hline Peak No. & Peak ID & Ret Time & Height & Area & Conc. \\
\hline 1 & & 10.707 & 895868.688 & 22934700.000 & 99.4951 \\
\hline 2 & & 12.130 & 2905.786 & 116376.047 & 0.5049 \\
\hline Total & & & 898774.474 & 23051076.047 & 100.000 \\
\hline
\end{tabular}


2-((2R,3S)-3-(3,4-dimethoxyphenyl)-6-(pyrrolidin-1-yl)-2,3-dihydrobenzofuran-2-yl)ethanol $(\mathbf{4 j})$<smiles>COc1ccc(C2c3ccc(N4CCCC4)cc3OC2CCO)cc1OC</smiles>

Pale yellow oil, $31.3 \mathrm{mg}, 84 \%$ yield, $99: 1 \mathrm{dr}, 99 \%$ ee; $[\alpha]_{\mathrm{D}}{ }^{20}=-0.88(c$ $\left.1.0, \mathrm{CHCl}_{3}\right) ;{ }^{1} \mathrm{H}$ NMR $\left(400 \mathrm{MHz}, \mathrm{CDCl}_{3}\right) \delta 6.82-6.73(\mathrm{~m}, 4 \mathrm{H})$, 6.12-6.09 (m, 2H), 4.67-4.61 (m, 1H), $4.18(\mathrm{~d}, J=8.0 \mathrm{~Hz}, 1 \mathrm{H})$, 3.89-3.86 (m, 5H), $3.81(\mathrm{~s}, 3 \mathrm{H}), 3.27(\mathrm{t}, J=6.0 \mathrm{~Hz}, 4 \mathrm{H}), 2.14-1.98(\mathrm{~m}$, $7 \mathrm{H}) ;{ }^{13} \mathrm{C} \mathrm{NMR}\left(100 \mathrm{MHz}, \mathrm{CDCl}_{3}\right) \delta 160.9,149.5,149.4,148.4,135.6$, $125.7,120.7,117.3,111.5,111.3,105.1,93.5,91.5,60.9,56.2,54.6$, 48.2, 37.6, 25.8; HRMS (ESI) for $\mathrm{C}_{22} \mathrm{H}_{28} \mathrm{NO}_{4}[\mathrm{M}+\mathrm{H}]^{+}$: calcd 370.2015, found 370.2013.

HPLC: Chiralpak AD-H column $(250 \mathrm{~mm})$; detected at $254 \mathrm{~nm}$; hexane $/ i$-propanol $=90 / 10$; flow $=0.7 \mathrm{~mL} / \mathrm{min}$; Retention time: $22.8 \mathrm{~min}, 51.4 \mathrm{~min}$ (major).

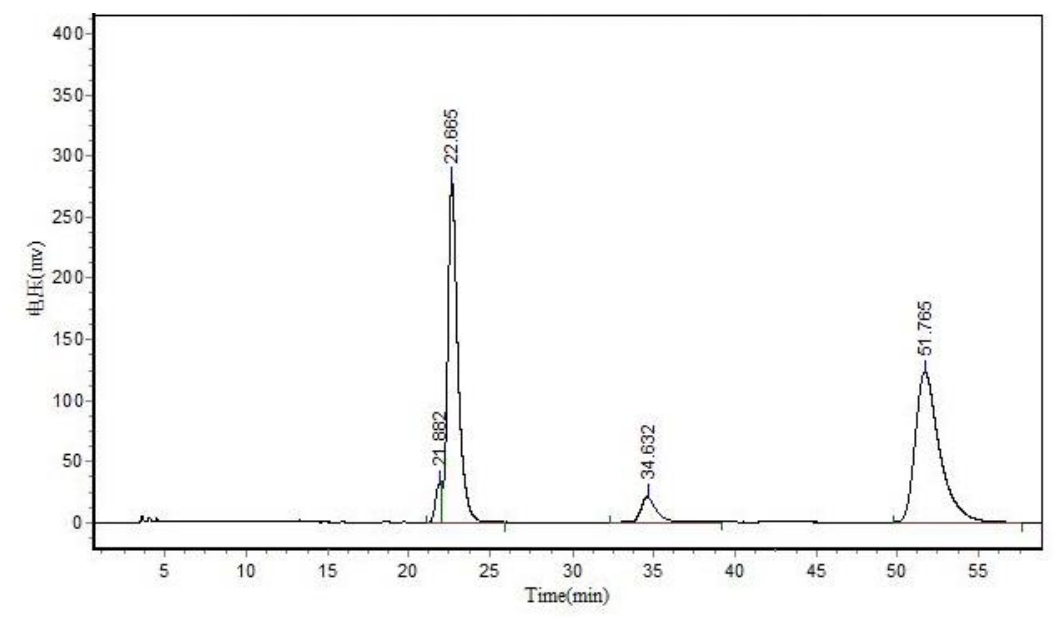

\begin{tabular}{|c|c|c|c|c|c|}
\hline \multicolumn{6}{|c|}{ Results } \\
\hline Peak No. & Peak ID & Ret Time & Height & Area & Conc. \\
\hline 1 & & 21.882 & 32037.594 & 866025.125 & 3.1770 \\
\hline 2 & & 22.665 & 279244.406 & 12097351.000 & 44.3791 \\
\hline 3 & & 34.632 & 21026.762 & 1460467.875 & 5.3577 \\
\hline 4 & & 51.765 & 123274.453 & 12835286.000 & 47.0862 \\
\hline Total & & & 455583.215 & 27259130.000 & 100.0000 \\
\hline
\end{tabular}

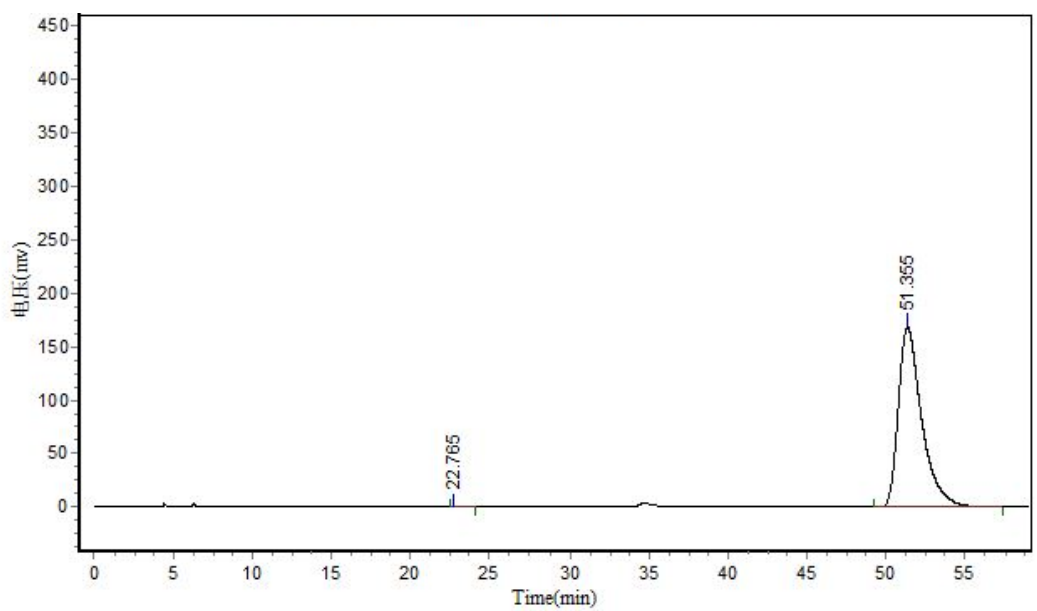

\begin{tabular}{|c|c|c|c|c|c|}
\hline \multicolumn{6}{|c|}{ Results } \\
\hline Peak No. & Peak ID & Ret Time & Height & Area & Conc. \\
\hline 1 & & 22.765 & 643.534 & 26262.404 & 0.1511 \\
\hline 2 & & 51.355 & 168073.203 & 17359692.000 & 99.8489 \\
\hline Total & & & 168716.737 & 17385954.404 & 100.000 \\
\hline
\end{tabular}


2-((2R,3S)-3-(3,5-dimethoxyphenyl)-6-(pyrrolidin-1-yl)-2,3-dihydrobenzofuran-2-yl)ethanol $(4 \mathbf{k})$<smiles>COc1cc(OC)cc(C2c3ccc(N4CCCC4)cc3OC2CCO)c1</smiles>

Pale yellow oil, $32.6 \mathrm{mg}, 89 \%$ yield, $99: 1 \mathrm{dr}, 99 \%$ ee; $[\alpha]_{\mathrm{D}}{ }^{20}=14.6(c$ $\left.1.0, \mathrm{CHCl}_{3}\right) ;{ }_{1}^{1} \mathrm{H}$ NMR $\left(400 \mathrm{MHz}, \mathrm{CDCl}_{3}\right) \delta 6.81(\mathrm{~d}, J=8.4 \mathrm{~Hz}, 1 \mathrm{H})$, 6.37-6.35 (m, 3H), 6.10-6.08 (m, 2H), 4.70-4.65 (m, 1H), 4.15 (d, $J=$ $8.0 \mathrm{~Hz}, 1 \mathrm{H}), 3.87(\mathrm{t}, J=6.0 \mathrm{~Hz}, 2 \mathrm{H}), 3.75(\mathrm{~s}, 6 \mathrm{H}), 3.26(\mathrm{t}, J=6.0 \mathrm{~Hz}$, 4H), 2.14-1.99 (m, 7H); ${ }^{13} \mathrm{C}$ NMR $\left(100 \mathrm{MHz}, \mathrm{CDCl}_{3}\right) \delta 161.3,160.9$, $149.5,145.7,125.8,116.8,106.5,105.1,99.1,93.5,91.2,60.9,55.7$, 55.1, 48.2, 37.7, 25.8; HRMS (ESI) for $\mathrm{C}_{22} \mathrm{H}_{28} \mathrm{NO}_{4}[\mathrm{M}+\mathrm{H}]^{+}$: calcd 370.2015, found 370.2016.

HPLC: Chiralpak AD-H column $(250 \mathrm{~mm})$; detected at $254 \mathrm{~nm}$; hexane/ $i$-propanol $=90 / 10$; flow $=0.7 \mathrm{~mL} / \mathrm{min}$; Retention time: $16.6 \mathrm{~min}, 30.1 \mathrm{~min}$ (major).

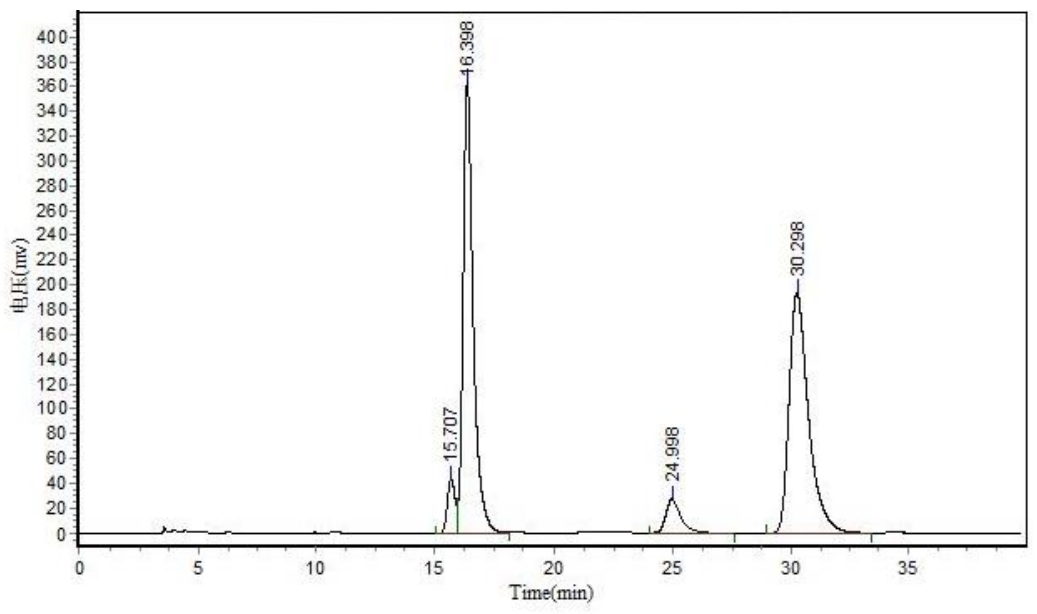

\begin{tabular}{|c|c|c|c|c|c|}
\hline \multicolumn{6}{|c|}{ Results } \\
\hline Peak No. & Peak ID & Ret Time & Height & Area & Conc. \\
\hline 1 & & 15.707 & 43664.574 & 995242.625 & 4.1310 \\
\hline 2 & & 16.398 & 363641.375 & 10825480.000 & 44.9335 \\
\hline 3 & & 24.998 & 27232.107 & 1225346.500 & 5.0861 \\
\hline 4 & & 30.298 & 193583.875 & 11046175.000 & 45.8495 \\
\hline Total & & & 628121.932 & 24092244.125 & 100.000 \\
\hline
\end{tabular}

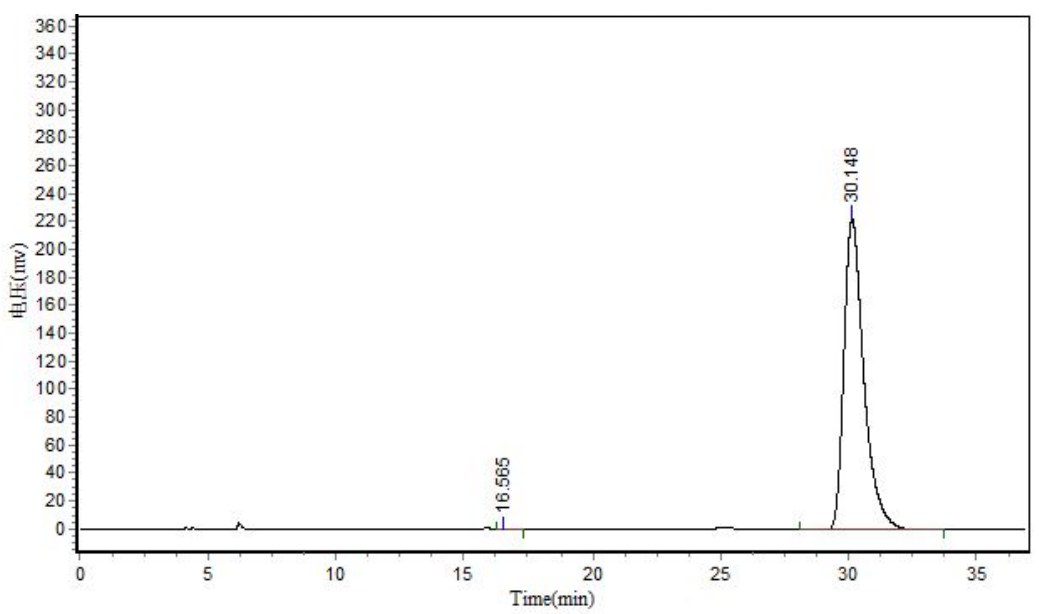

\begin{tabular}{|c|c|c|c|c|c|}
\hline \multicolumn{6}{|c|}{ Results } \\
\hline Peak No. & Peak ID & Ret Time & Height & Area & Conc. \\
\hline 1 & & 16.565 & 303.704 & 8634.528 & 0.0712 \\
\hline 2 & & 30.148 & 222937.656 & 12126343.000 & 99.9288 \\
\hline Total & & & 223241.361 & 12134977.528 & 100.000 \\
\hline
\end{tabular}


2-((2R,3S)-6-(pyrrolidin-1-yl)-3-(3,4,5-trimethoxyphenyl)-2,3-dihydrobenzofuran-2-yl) ethane (4I)<smiles>COc1cc(C2c3ccc(N4CCCC4)cc3OC2CCO)cc(OC)c1OC</smiles>

White solid, $34.0 \mathrm{mg}$, $85 \%$ yield, $99: 1 \mathrm{dr}$, $99 \%$ ee; m.p.107.1-108. ${ }^{\circ} \mathrm{C}$, $[\alpha]_{\mathrm{D}}^{20}=6.7\left(c 1.0, \mathrm{CHCl}_{3}\right) ;{ }^{1} \mathrm{H}$ NMR $\left(400 \mathrm{MHz}, \mathrm{CDCl}_{3}\right) \delta 6.81(\mathrm{~d}, J=$ $8.4 \mathrm{~Hz}, 1 \mathrm{H}), 6.41$ (s, 2H), 6.10-6.08 (m, 2H), 4.67-4.62 (m, 1H), 4.14 $(\mathrm{d}, J=8.0 \mathrm{~Hz}, 1 \mathrm{H}), 3.87(\mathrm{t}, J=6.0 \mathrm{~Hz}, 2 \mathrm{H}), 3.82(\mathrm{~s}, 3 \mathrm{H}), 3.79(\mathrm{~s}, 6 \mathrm{H})$, $3.25(\mathrm{t}, J=6.4 \mathrm{~Hz}, 4 \mathrm{H}), 2.11-1.96(\mathrm{~m}, 7 \mathrm{H}) ;{ }^{13} \mathrm{C} \mathrm{NMR}(100 \mathrm{MHz}$, $\left.\mathrm{CDCl}_{3}\right) \delta 160.9,153.7,149.4,138.9,137.1,125.7,116.9,105.2,105.1$ 93.5, 91.1, 61.1, 60.8, 56.4, 55.3, 48.2, 37.6, 25.8; HRMS (ESI) for $\mathrm{C}_{23} \mathrm{H}_{30} \mathrm{NO}_{5}[\mathrm{M}+\mathrm{H}]^{+}$: calcd 400.2118, found 400.2114 .

HPLC: Chiralpak IF-3 column $(250 \mathrm{~mm})$; detected at $254 \mathrm{~nm}$; hexane $/ i$-propanol $=65 / 35$; flow $=$ $0.5 \mathrm{~mL} / \mathrm{min}$; Retention time: $18.6 \mathrm{~min}$ (major), $23.2 \mathrm{~min}$.

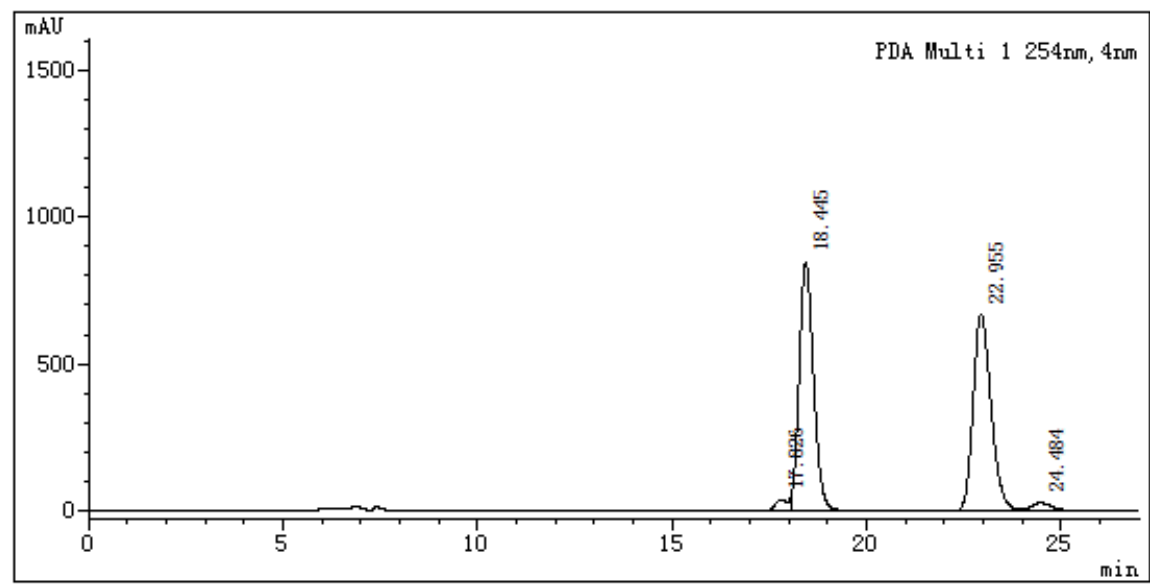

\begin{tabular}{|c|c|c|c|c|}
\hline \multicolumn{5}{|c|}{ Dh Ch1 254nm } \\
\hline Kumber & Retention & Area & Hei ght & Area \\
\hline 1 & 17.826 & 1079464 & 39141 & 2.384 \\
\hline 2 & 18.445 & 21572732 & 845376 & 47.649 \\
\hline 3 & 22.955 & 21549490 & 669753 & 47.598 \\
\hline 4 & 24.484 & 1072705 & 27505 & 2.369 \\
\hline
\end{tabular}

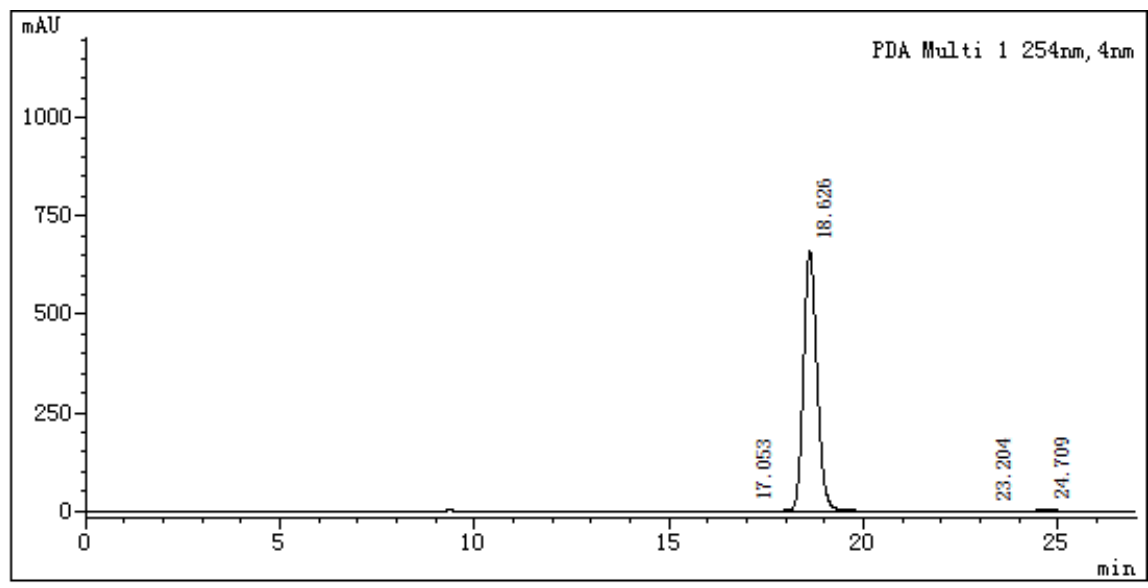

\begin{tabular}{|c|c|c|c|c|}
\hline \multicolumn{5}{|c|}{ PDA Ch1 254nm } \\
\hline Humber & Retention & Area & Height & Area \\
\hline 1 & 17.053 & 25462 & 758 & 0.157 \\
\hline 2 & 18.626 & 16011505 & 660751 & 98.866 \\
\hline 3 & 23.204 & 28415 & 946 & 0.175 \\
\hline 4 & 24.709 & 129724 & 3604 & 0.801 \\
\hline
\end{tabular}




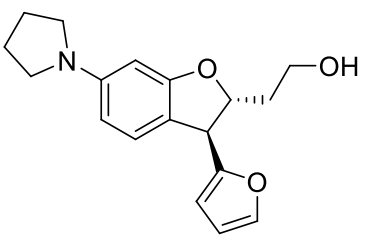

White solid, $25.2 \mathrm{mg}, 84 \%$ yield, $99: 1 \mathrm{dr}$, $99 \%$ ee; m.p.87.6-89.2 ${ }^{\circ} \mathrm{C}$, $[\alpha]_{\mathrm{D}}^{20}=-13.2\left(c 1.0, \mathrm{CHCl}_{3}\right) ;{ }^{1} \mathrm{H}$ NMR $\left(400 \mathrm{MHz}, \mathrm{CDCl}_{3}\right) \delta 7.36(\mathrm{~d}, J$ $=0.8 \mathrm{~Hz}, 1 \mathrm{H}), 6.98(\mathrm{~d}, J=8.0 \mathrm{~Hz}, 1 \mathrm{H}), 6.32-6.31(\mathrm{~m}, 1 \mathrm{H}), 6.14-6.09$ (m, 3H), 4.86-4.81 (m, 1H), 4.37 (d, $J=7.6 \mathrm{~Hz}, 1 \mathrm{H}), 3.91(\mathrm{t}, J=6.0$ $\mathrm{Hz}, 2 \mathrm{H}), 3.26(\mathrm{t}, J=6.4 \mathrm{~Hz}, 4 \mathrm{H}), 2.18-2.08(\mathrm{~m}, 3 \mathrm{H}), 2.00-1.97(\mathrm{~m}$, $4 \mathrm{H}) ;{ }^{13} \mathrm{C}$ NMR $\left(100 \mathrm{MHz}, \mathrm{CDCl}_{3}\right) \delta 160.8,155.8,149.7,142.3,125.6,113.9,110.6,106.1,104.9$, 93.7, 87.9, 60.6, 48.2, 47.6, 38.0, 25.8; HRMS (ESI) for $\mathrm{C}_{18} \mathrm{H}_{22} \mathrm{NO}_{3}[\mathrm{M}+\mathrm{H}]^{+}$: calcd 300.1594, found 300.1589 .

HPLC: Chiralpak OD-H column $(250 \mathrm{~mm})$; detected at $254 \mathrm{~nm}$; hexane $/ i$-propanol $=90 / 10$; flow $=0.7 \mathrm{~mL} / \mathrm{min}$; Retention time: $15.4 \mathrm{~min}, 18.1 \mathrm{~min}$ (major).
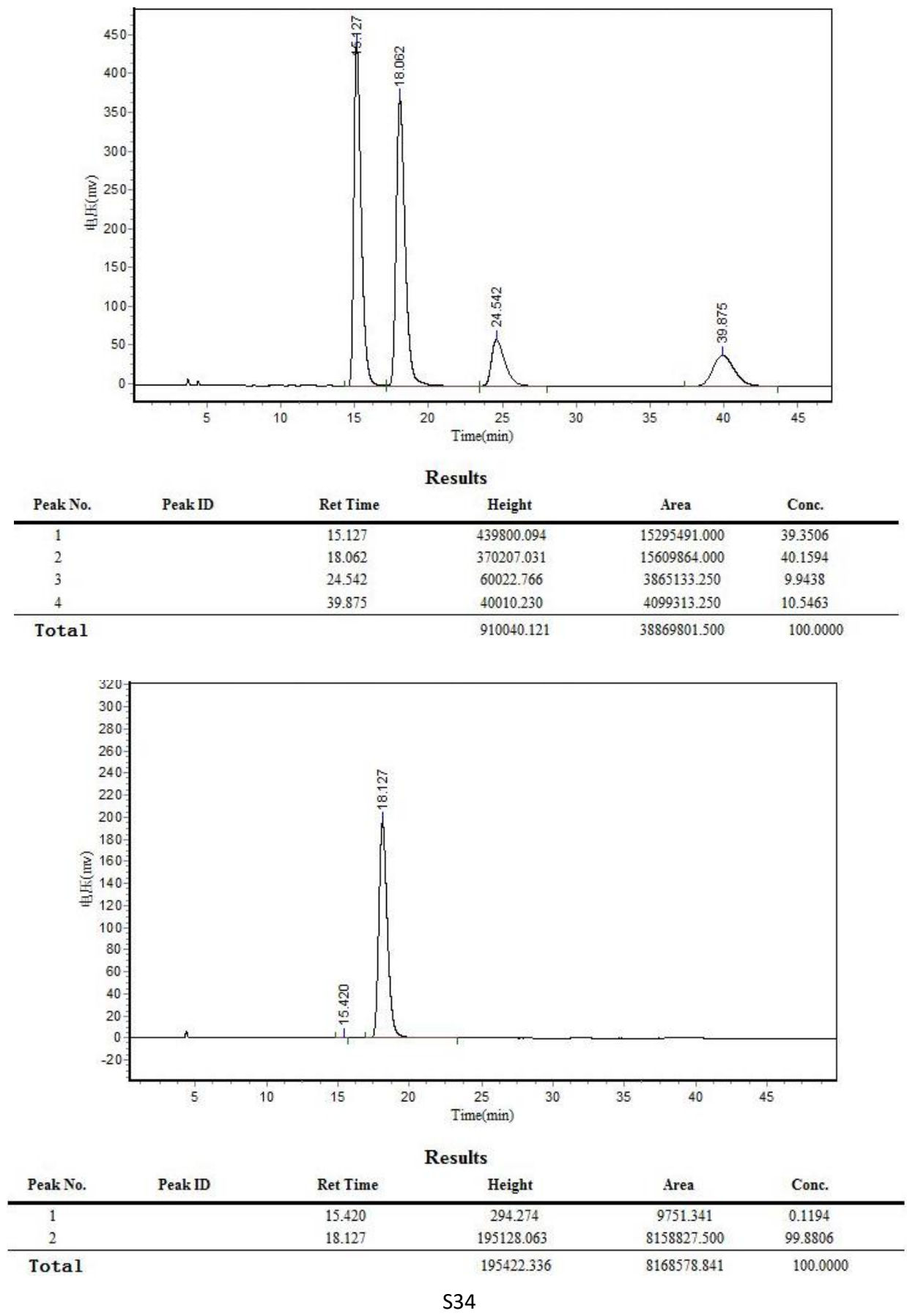
2-((2R,3S)-6-(pyrrolidin-1-yl)-3-(thiophen-2-yl)-2,3-dihydrobenzofuran-2-yl)ethanol (4n)

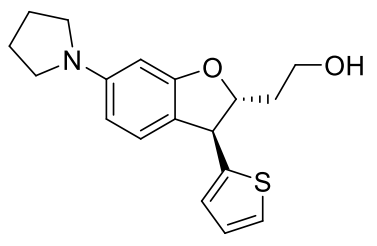

White solid, $28.8 \mathrm{mg}, 91 \%$ yield, $99: 1 \mathrm{dr}, 99 \%$ ee; m.p. $78.7-80.2^{\circ} \mathrm{C}$, $[\alpha]_{\mathrm{D}^{20}}=-20.5\left(c 1.0, \mathrm{CHCl}_{3}\right) ;{ }^{1} \mathrm{H} \mathrm{NMR}\left(400 \mathrm{MHz}, \mathrm{CDCl}_{3}\right) \delta 7.20(\mathrm{~d}, J$ $=5.2 \mathrm{~Hz}, 1 \mathrm{H}), 6.98-6.93(\mathrm{~m}, 3 \mathrm{H}), 6.13-6.10(\mathrm{~m}, 2 \mathrm{H}), 4.73-4.67(\mathrm{~m}$, $1 \mathrm{H}), 4.54(\mathrm{~d}, J=8.4 \mathrm{~Hz}, 1 \mathrm{H}), 3.89$ (t, $J=6.0 \mathrm{~Hz}, 2 \mathrm{H}), 3.27$ (t, $J=6.4$

$\mathrm{Hz}, 4 \mathrm{H}), 2.14-1.98(\mathrm{~m}, 7 \mathrm{H}) ;{ }^{13} \mathrm{C}$ NMR $\left(100 \mathrm{MHz}, \mathrm{CDCl}_{3}\right) \delta 160.7$, 149.7, 146.7, 127.3, 125.7, 125.0, 124.7, 116.6, 105.0, 93.6, 91.2, 60.8, 49.6, 48.2, 37.3, 25.8; HRMS (ESI) for $\mathrm{C}_{18} \mathrm{H}_{22} \mathrm{NO}_{2} \mathrm{~S}[\mathrm{M}+\mathrm{H}]^{+}$: calcd 316.1366, found 316.1368 .

HPLC: Chiralpak OD-H column $(250 \mathrm{~mm})$; detected at $254 \mathrm{~nm}$; hexane $/ i$-propanol $=90 / 10$; flow $=0.7 \mathrm{~mL} / \mathrm{min}$; Retention time: $18.2 \mathrm{~min}, 22.0 \mathrm{~min}$ (major).

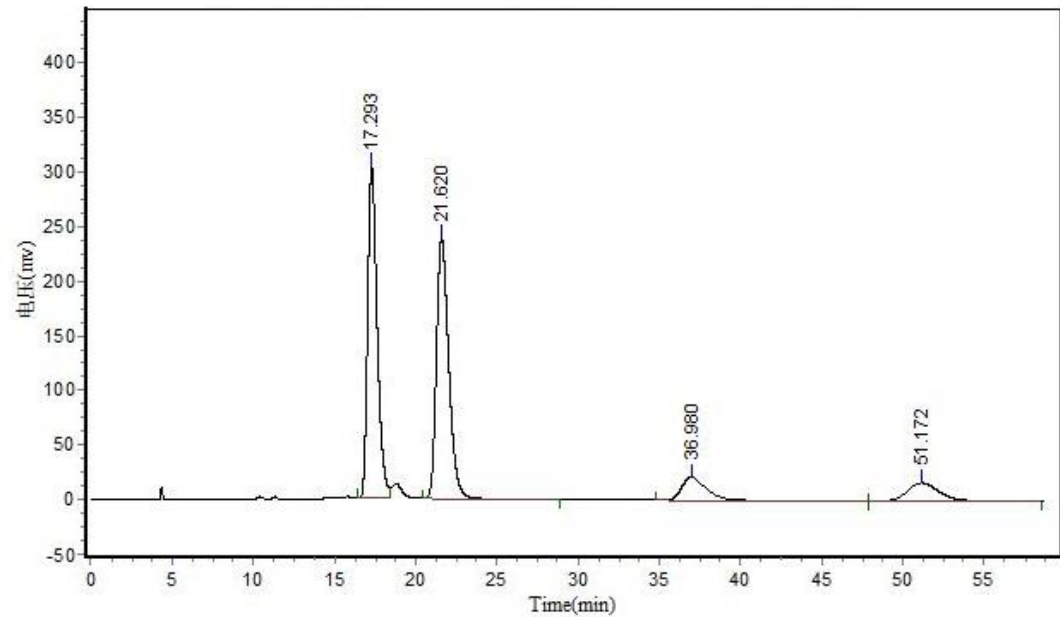

\begin{tabular}{|c|c|c|c|c|c|}
\hline \multicolumn{6}{|c|}{ Results } \\
\hline Peak No. & Peak ID & Ret Time & Height & Area & Conc. \\
\hline 1 & & 17.293 & 302468.938 & 12361014.000 & 41.9446 \\
\hline 2 & & 21.620 & 237974.281 & 12533319.000 & 42.5293 \\
\hline 3 & & 36.980 & 21184.703 & 2283733.250 & 7.7494 \\
\hline 4 & & 51.172 & 16464.463 & 2291766.500 & 7.7767 \\
\hline Total & & & 578092.385 & 29469832.750 & 100.000 \\
\hline
\end{tabular}

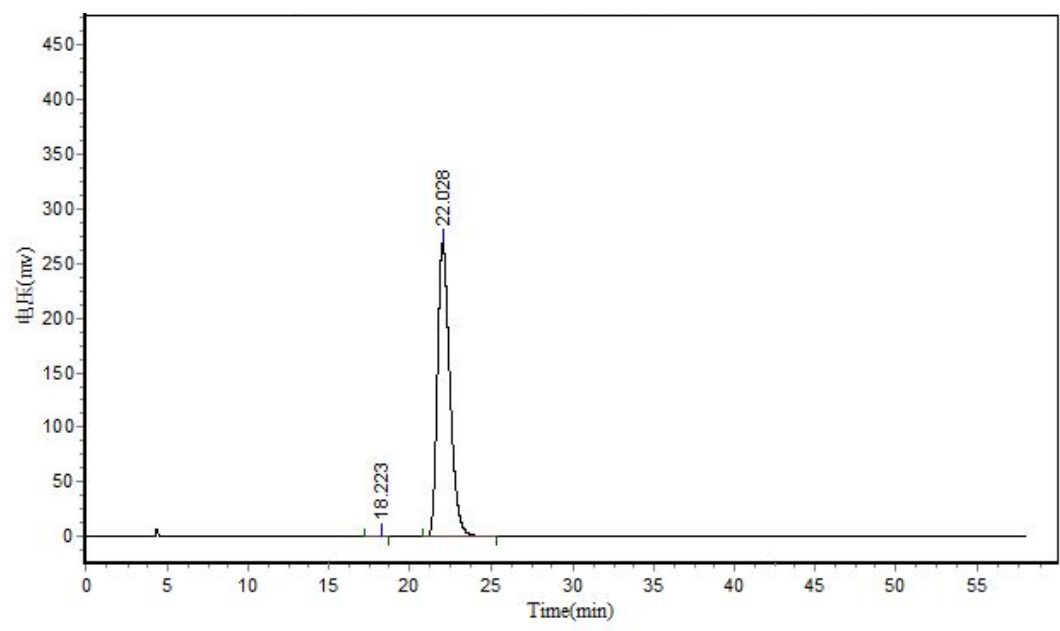

\begin{tabular}{|c|c|c|c|c|c|}
\hline \multicolumn{6}{|c|}{ Results } \\
\hline Peak No. & Peak ID & Ret Time & Height & Area & Conc. \\
\hline 1 & & 18.223 & 200.059 & 10018.349 & 0.0697 \\
\hline 2 & & 22.028 & 268638.219 & 14367638.000 & 99.9303 \\
\hline Total & & & 268838.278 & 14377656.349 & 100.0000 \\
\hline
\end{tabular}


<smiles>OCCC1Oc2cc(N3CCCC3)ccc2C1c1ccsc1</smiles>

Colorless oil, $29.6 \mathrm{mg}, 94 \%$ yield, 99:1 dr, 99\% ee; $[\alpha]_{\mathrm{D}}^{20}=18.3(c 1.0$ $\left.\mathrm{CHCl}_{3}\right) ;{ }^{1} \mathrm{H}$ NMR $\left(400 \mathrm{MHz}, \mathrm{CDCl}_{3}\right) \delta$ 7.30-7.28 (m, 1H), 7.07 (d, $J=$ $1.6 \mathrm{~Hz}, 1 \mathrm{H}), 6.95(\mathrm{~d}, J=4.8 \mathrm{~Hz}, 1 \mathrm{H}), 6.86(\mathrm{~d}, J=8.4 \mathrm{~Hz}, 1 \mathrm{H})$, 6.11-6.09 (m, 2H), 4.70-4.65 (m, 1H), 4.35 (d, $J=8.0 \mathrm{~Hz}, 1 \mathrm{H}), 3.88(\mathrm{t}$, $J=5.2 \mathrm{~Hz}, 2 \mathrm{H}), 3.26(\mathrm{t}, J=6.4 \mathrm{~Hz}, 4 \mathrm{H}), 2.15-1.97(\mathrm{~m}, 7 \mathrm{H}) ;{ }^{13} \mathrm{C} \mathrm{NMR}$ $\left(100 \mathrm{MHz}, \mathrm{CDCl}_{3}\right) \delta 160.7,149.5,143.6,127.4,126.6,125.5,121.7,116.8,104.9,93.7,90.3$, 60.8, 49.7, 48.2, 37.6, 25.8; HRMS (ESI) for $\mathrm{C}_{18} \mathrm{H}_{22} \mathrm{NO}_{2} \mathrm{~S}[\mathrm{M}+\mathrm{H}]^{+}$: calcd 316.1366, found 316.1370 .

HPLC: Chiralpak AD-H column $(250 \mathrm{~mm})$; detected at $254 \mathrm{~nm}$; hexane $/ i$-propanol $=95 / 5$; flow $=$ $1.0 \mathrm{~mL} / \mathrm{min}$; Retention time: $16.3 \mathrm{~min}, 39.6 \mathrm{~min}$ (major).

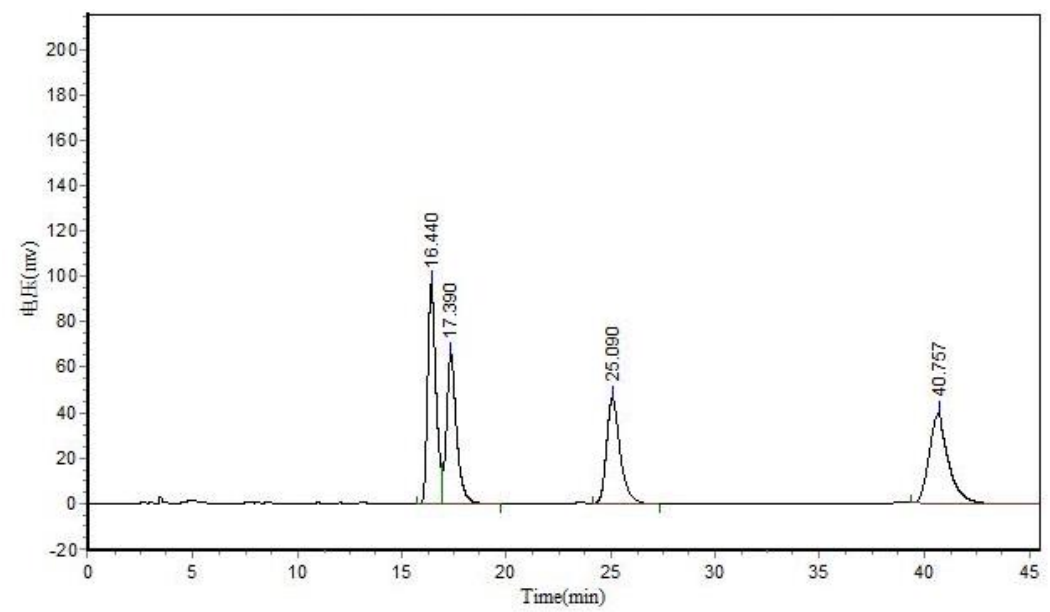

Results

\begin{tabular}{|c|c|c|c|c|c|}
\hline Peak No. & Peak ID & Ret Time & Height & Area & Conc. \\
\hline 1 & & 16.440 & 96519.797 & 2708225.500 & 27.8697 \\
\hline 2 & & 17.390 & 65933.477 & 2215755.500 & 22.8018 \\
\hline 3 & & 25.090 & 46727.898 & 2163180.750 & 22.2608 \\
\hline 4 & & 40.757 & 40385.703 & 2630298.000 & 27.0678 \\
\hline Total & & & 249566.875 & 9717459.750 & 100.0000 \\
\hline
\end{tabular}

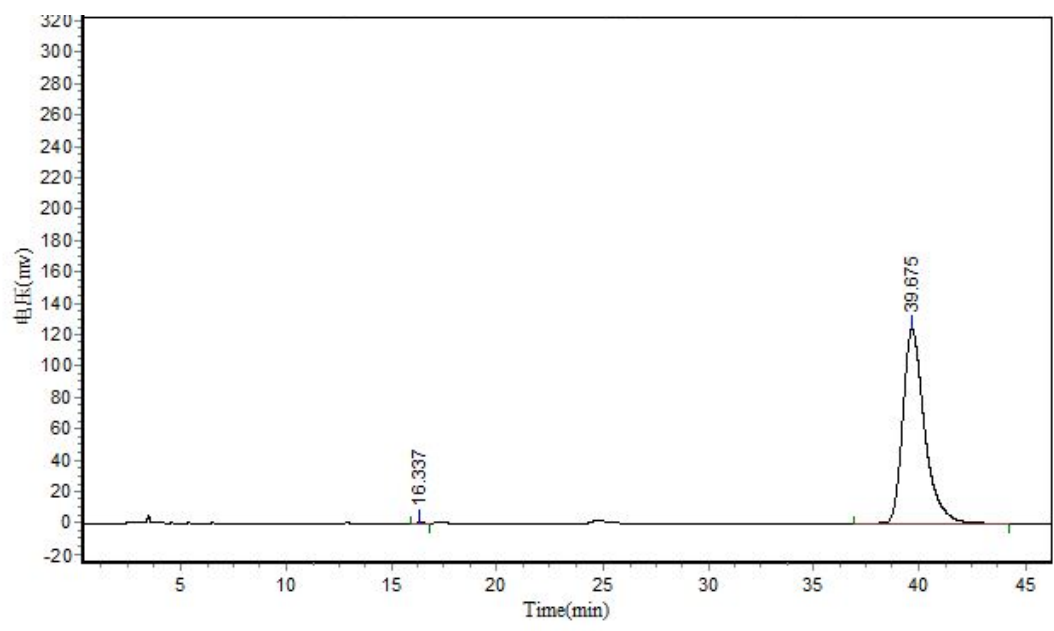

Results

\begin{tabular}{|c|c|c|c|c|c|}
\hline \multicolumn{6}{|c|}{$\mathbf{N}$} \\
\hline Peak No. & Peak ID & Ret Time & Height & Area & Conc. \\
\hline 1 & & 16.337 & 195.510 & 4591.500 & 0.0514 \\
\hline 2 & & 39.675 & 123905.492 & 8929646.000 & 99.9486 \\
\hline Total & & & 124101.002 & 8934237.500 & 100.0000 \\
\hline
\end{tabular}




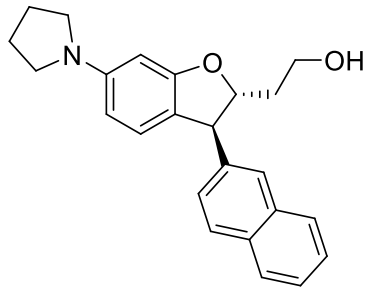

White solid, $33.1 \mathrm{mg}, 92 \%$ yield, $99: 1 \mathrm{dr}, 99 \%$ ee; m.p. $74.2-76.3^{\circ} \mathrm{C}$, $[\alpha]_{\mathrm{D}}^{20}=86.4\left(c\right.$ 1.44, $\left.\mathrm{CHCl}_{3}\right) ;{ }^{1} \mathrm{H}$ NMR $\left(400 \mathrm{MHz}, \mathrm{CDCl}_{3}\right) \delta$ 7.83-7.79 (m, 3H), $7.71(\mathrm{~s}, 1 \mathrm{H}), 7.50-7.44(\mathrm{~m}, 2 \mathrm{H}), 7.35(\mathrm{~d}, J=8.4 \mathrm{~Hz}, 1 \mathrm{H}), 6.81$ $(\mathrm{d}, J=8.0 \mathrm{~Hz}, 1 \mathrm{H}), 6.18(\mathrm{~s}, 1 \mathrm{H}), 6.12(\mathrm{~d}, J=8.0 \mathrm{~Hz}, 1 \mathrm{H}), 4.82-4.77$ $(\mathrm{m}, 1 \mathrm{H}), 4.41(\mathrm{~d}, J=8.0 \mathrm{~Hz}, 1 \mathrm{H}), 3.88(\mathrm{t}, J=6.0 \mathrm{~Hz}, 2 \mathrm{H}), 3.29(\mathrm{t}, J=$ $6.0 \mathrm{~Hz}, 4 \mathrm{H}), 2.21-1.99(\mathrm{~m}, 7 \mathrm{H}) ;{ }^{13} \mathrm{C} \mathrm{NMR}\left(100 \mathrm{MHz}, \mathrm{CDCl}_{3}\right) \delta 161.1$, $149.5,140.6,133.8,132.9,128.9,128.0,127.1,126.5,126.4,126.0,125.8,117.3,105.1,93.6$, 91.2, 60.8, 55.0, 48.2, 37.7, 25.8; HRMS (ESI) for $\mathrm{C}_{24} \mathrm{H}_{26} \mathrm{NO}_{2}[\mathrm{M}+\mathrm{H}]^{+}$: calcd 360.1958, found 360.1956 .

HPLC: Chiralpak AD-H column $(250 \mathrm{~mm})$; detected at $254 \mathrm{~nm}$; hexane $/ \mathrm{i}$-propanol $=90 / 10$; flow $=0.7 \mathrm{~mL} / \mathrm{min}$; Retention time: $12.6 \mathrm{~min}, 37.7 \mathrm{~min}$ (major).

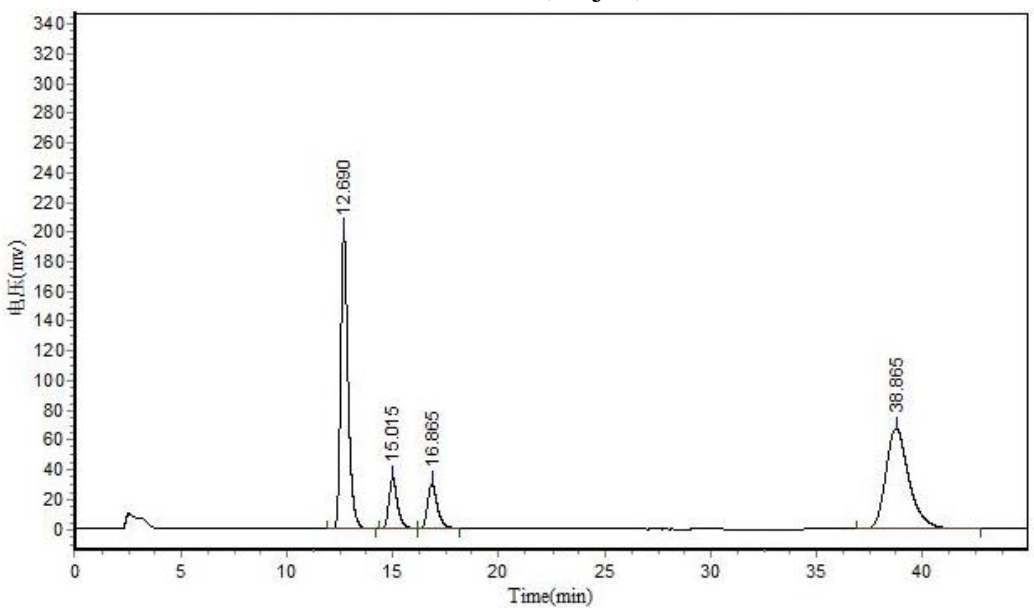

\begin{tabular}{|c|c|c|c|c|c|}
\hline \multicolumn{6}{|c|}{ Results } \\
\hline Peak No. & Peak ID & Ret Time & Height & Area & Conc. \\
\hline 1 & & 12.690 & 200296.625 & 4885418.000 & 40.1760 \\
\hline 2 & & 15.015 & 34417.031 & 1001617.375 & 8.2370 \\
\hline 3 & & 16.865 & 30922.205 & 1045197.625 & 8.5953 \\
\hline 4 & & 38.865 & 68060.688 & 5227818.000 & 42.9917 \\
\hline Total & & & 333696.549 & 12160051.000 & 100.0000 \\
\hline
\end{tabular}

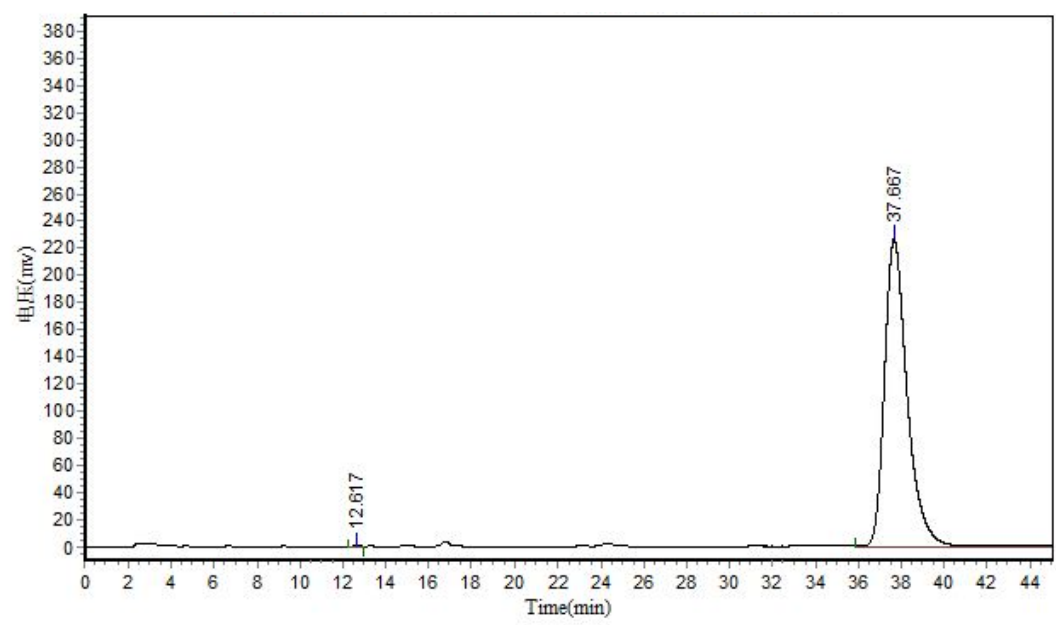

\begin{tabular}{|c|c|c|c|c|c|}
\hline \multicolumn{6}{|c|}{ Results } \\
\hline Peak No. & Peak ID & Ret Time & Height & Area & Conc. \\
\hline 1 & & 12.617 & 326.928 & 6048.500 & 0.0365 \\
\hline 2 & & 37.667 & 226076.094 & 16583476.000 & 99.9635 \\
\hline Total & & & 226403.022 & 16589524.500 & 100.0000 \\
\hline
\end{tabular}


<smiles>COc1ccc(C2c3ccc(N4CCCC4)cc3OC2CCO)cc1</smiles>

Colorless oil, $30.8 \mathrm{mg}, 91 \%$ yield, $97: 3 \mathrm{dr}, 99 \%$ ee; $[\alpha]_{\mathrm{D}}^{20}=3.7(c 1.0$, $\left.\mathrm{CHCl}_{3}\right) ;{ }^{1} \mathrm{H}$ NMR $\left(400 \mathrm{MHz}, \mathrm{CDCl}_{3}\right) \delta$ 6.92-6.89 (m, 3H), $6.79(\mathrm{~d}, J$ $=8.4 \mathrm{~Hz}, 2 \mathrm{H}), 6.13-6.11(\mathrm{~m}, 2 \mathrm{H}), 5.04-4.98(\mathrm{~m}, 1 \mathrm{H}), 4.45(\mathrm{~d}, J=8.4$ $\mathrm{Hz}, 1 \mathrm{H}), 3.79-3.75(\mathrm{~m}, 5 \mathrm{H}), 3.28$ (t, $J=6.4 \mathrm{~Hz}, 4 \mathrm{H}), 2.05-1.95$ (m, $5 \mathrm{H}), 1.56-1.39(\mathrm{~m}, 2 \mathrm{H}) ;{ }^{13} \mathrm{C} \mathrm{NMR}\left(100 \mathrm{MHz}, \mathrm{CDCl}_{3}\right) \delta 161.0,158.8$, 149.3, 133.1, 130.2, 126.1, 118.0, 113.9, 105.2, 93.5, 86.4, 61.3, 55.5, 50.7, 48.2, 34.9, 25.8; HRMS (ESI) for $\mathrm{C}_{21} \mathrm{H}_{26} \mathrm{NO}_{3}[\mathrm{M}+\mathrm{H}]^{+}$: calcd 340.1907, found 340.1905.

HPLC: Chiralpak AD-H column $(250 \mathrm{~mm})$; detected at $220 \mathrm{~nm}$; hexane/ $i$-propanol $=90 / 10$; flow $=1.1 \mathrm{~mL} / \mathrm{min}$; Retention time: $16.5 \mathrm{~min}, 32.5 \mathrm{~min}$ (major).

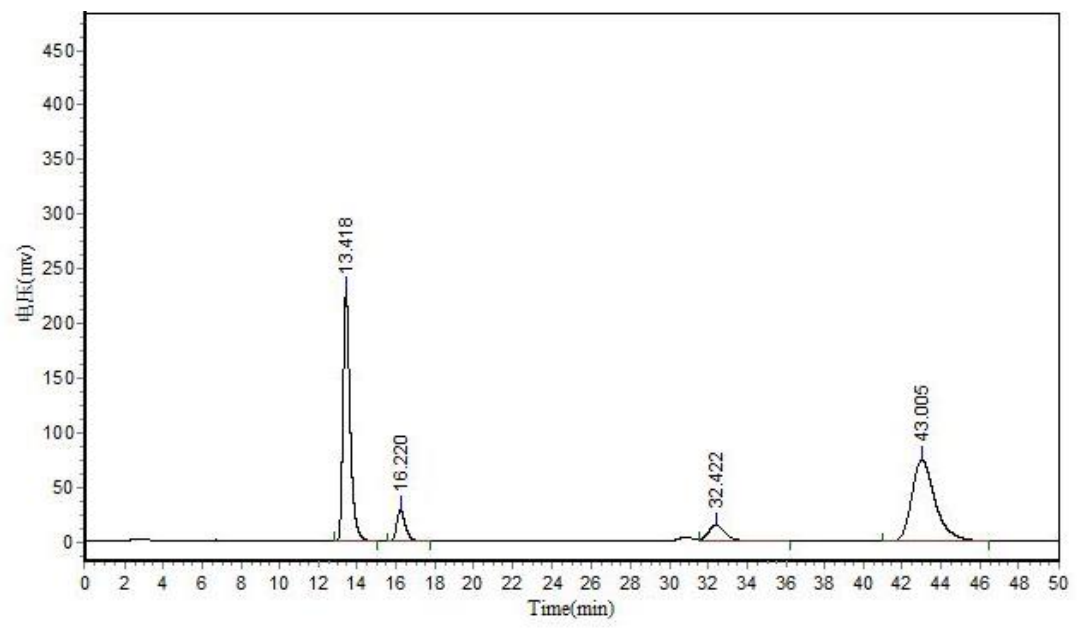

\section{Results}

\begin{tabular}{|c|c|c|c|c|c|}
\hline \\
\hline Peak No. & Peak ID & Ret Time & Height & Area & Conc. \\
\hline 1 & & 13.418 & 228945.734 & 5806399.500 & 41.6869 \\
\hline 2 & & 16.220 & 29782.564 & 902722.375 & 6.4811 \\
\hline 3 & & 32.422 & 15236.500 & 953972.188 & 6.8490 \\
\hline 4 & & 43.005 & 75007.992 & 6265502.000 & 44.9830 \\
\hline Total & & & 348972.791 & 13928596.063 & 100.0000 \\
\hline
\end{tabular}

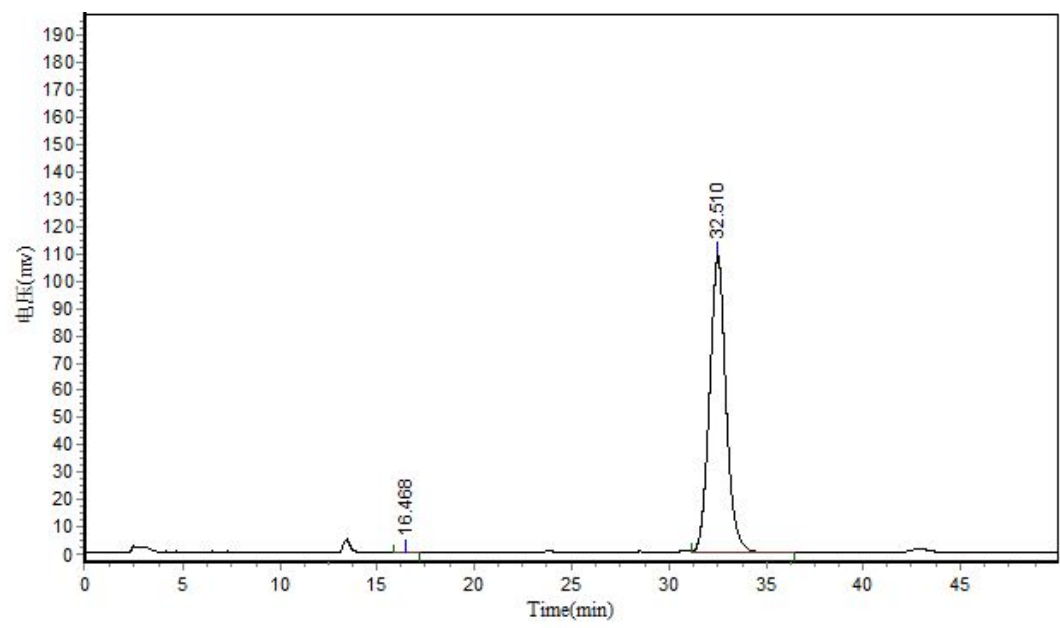

Results

\begin{tabular}{|c|c|c|c|c|c|}
\hline \multicolumn{6}{|c|}{ Kesuits } \\
\hline Peak No. & Peak ID & Ret Time & Height & Area & Conc. \\
\hline 1 & & 16.468 & 90.728 & 3174.998 & 0.0490 \\
\hline 2 & & 32.510 & 108413.227 & 6475140.000 & 99.9510 \\
\hline Total & & & 108503.955 & 6478314.998 & 100.0000 \\
\hline
\end{tabular}




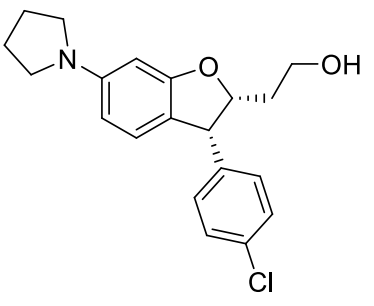

Colorless oil, $30.7 \mathrm{mg}, 90 \%$ yield, $97: 3 \mathrm{dr}, 99 \%$ ee; $[\alpha]_{\mathrm{D}}^{20}=-15.4(c$ 1.0, $\left.\mathrm{CHCl}_{3}\right) ;{ }^{1} \mathrm{H}$ NMR $\left(400 \mathrm{MHz}, \mathrm{CDCl}_{3}\right) \delta 7.22(\mathrm{~d}, J=8.4 \mathrm{~Hz}, 2 \mathrm{H})$, $6.92(\mathrm{~d}, J=8.4 \mathrm{~Hz}, 2 \mathrm{H}), 6.87(\mathrm{~d}, J=7.6 \mathrm{~Hz}, 1 \mathrm{H}), 6.13-6.11(\mathrm{~m}, 2 \mathrm{H})$, 5.06-5.00 (m, 1H), $4.45(\mathrm{~d}, J=8.0 \mathrm{~Hz}, 1 \mathrm{H}), 3.80-3.77(\mathrm{~m}, 2 \mathrm{H}), 3.28(\mathrm{t}$, $J=6.4 \mathrm{~Hz}, 4 \mathrm{H}), 2.05-1.96(\mathrm{~m}, 5 \mathrm{H}), 1.51-1.40(\mathrm{~m}, 2 \mathrm{H}) ;{ }^{13} \mathrm{C} \mathrm{NMR}(100$ $\left.\mathrm{MHz}, \mathrm{CDCl}_{3}\right) \delta 161.1,149.5,139.6,132.9,130.6,128.8,126.0,117.3$, 105.3, 93.6, 85.9, 61.1, 50.9, 48.2, 34.9, 25.8; HRMS (ESI) for $\mathrm{C}_{20} \mathrm{H}_{23}{ }^{35} \mathrm{ClNO}_{2}[\mathrm{M}+\mathrm{H}]^{+}$: calcd 344.1412, found 344.1408; $\mathrm{C}_{20} \mathrm{H}_{23}{ }^{37} \mathrm{ClNO}_{2}[\mathrm{M}+\mathrm{H}]^{+}$: calcd 346.1382, found: 346.1397 .

HPLC: Chiralpak AD-H column $(250 \mathrm{~mm})$; detected at $220 \mathrm{~nm}$; hexane $/ i$-propanol $=90 / 10$; flow $=1.1 \mathrm{~mL} / \mathrm{min}$; Retention time: $11.1 \mathrm{~min}, 13.9 \mathrm{~min}$ (major).

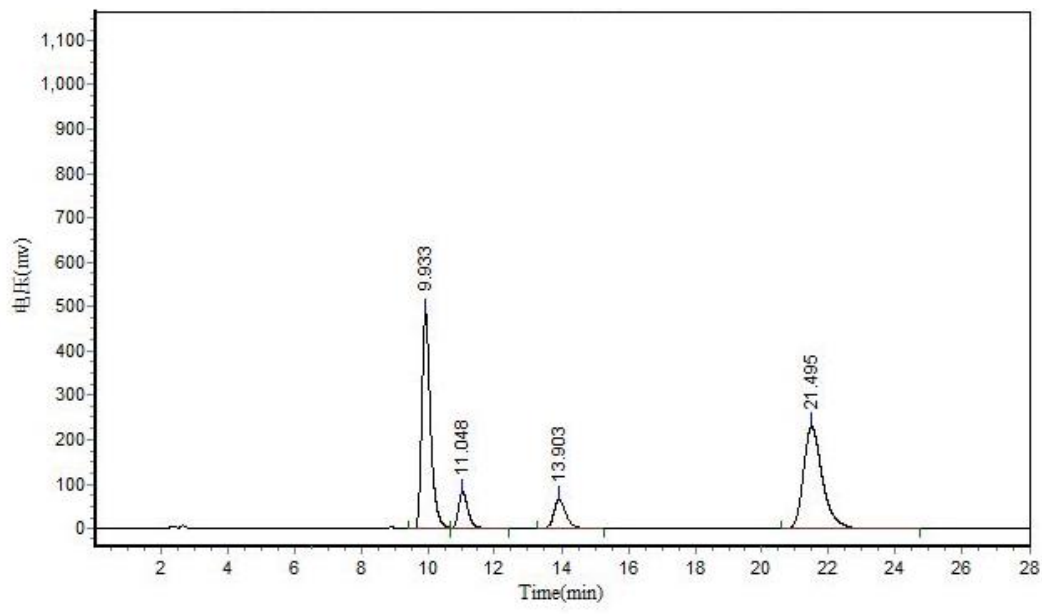

Results

\begin{tabular}{|c|c|c|c|c|c|}
\hline \multicolumn{6}{|c|}{ 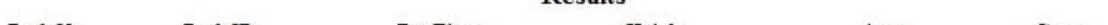 } \\
\hline Peak No. & Peak ID & Ret Time & Height & Area & Conc. \\
\hline 1 & & 9.933 & 484673.750 & 8868383.000 & 41.2614 \\
\hline 2 & & 11.048 & 84050.891 & 1705837.000 & 7.9366 \\
\hline 3 & & 13.903 & 67000.547 & 1742103.250 & 8.1054 \\
\hline 4 & & 21.495 & 230225.141 & 9176871.000 & 42.6966 \\
\hline Total & & & 865950.328 & 21493194.250 & 100.0000 \\
\hline
\end{tabular}

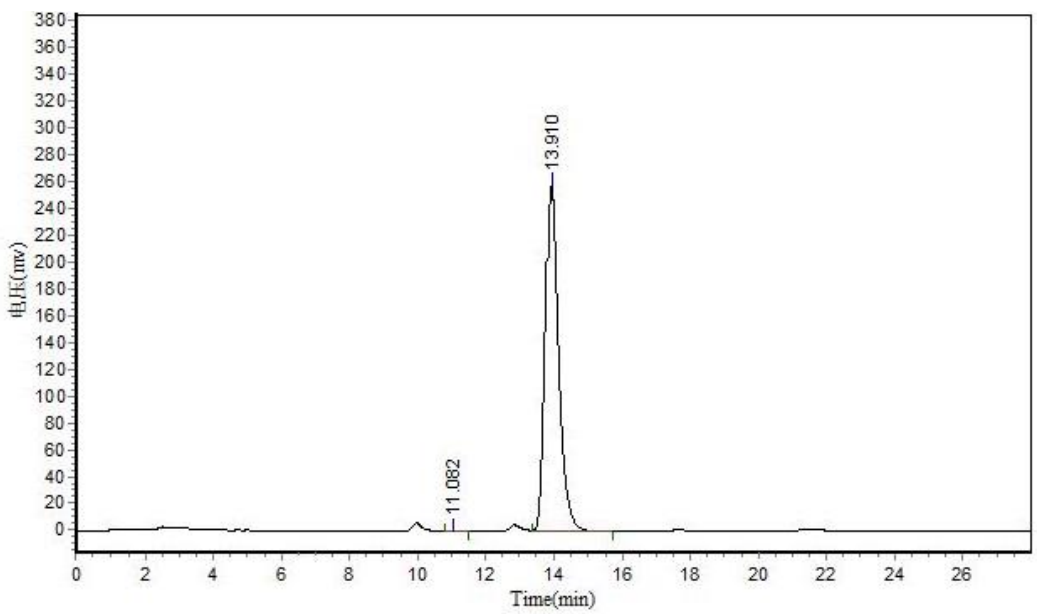

Results

\begin{tabular}{|c|c|c|c|c|c|}
\hline Peak No. & Peak ID & Ret Time & Height & Area & Conc. \\
\hline 1 & & 11.082 & 74.425 & 1381.400 & 0.0208 \\
\hline 2 & & 13.910 & 257469.328 & 6628526.000 & 99.9792 \\
\hline Total & & & 257543.753 & 6629907.400 & 100.0000 \\
\hline
\end{tabular}




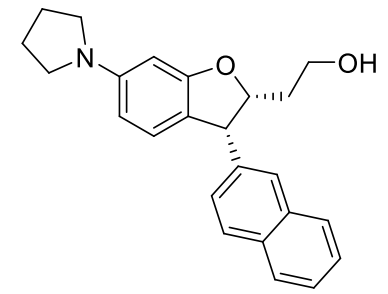

Colorless oil, $33.0 \mathrm{mg}, 92 \%$ yield, $98: 2 \mathrm{dr}$, 99\% ee; $[\alpha]_{\mathrm{D}}^{20}=-62.5(c$ $\left.1.0, \mathrm{CHCl}_{3}\right) ;{ }^{1} \mathrm{H}$ NMR $\left(400 \mathrm{MHz}, \mathrm{CDCl}_{3}\right) \delta$ 7.81-7-72 (m, 3H), $7.52(\mathrm{~s}$, $1 \mathrm{H}), 7.48-7.42(\mathrm{~m}, 2 \mathrm{H}), 7.11(\mathrm{~d}, J=8.4 \mathrm{~Hz}, 1 \mathrm{H}), 6.93(\mathrm{~d}, J=8.0 \mathrm{~Hz}$, $1 \mathrm{H}), 6.21(\mathrm{~s}, 1 \mathrm{H}), 6.15(\mathrm{~d}, J=8.0 \mathrm{~Hz}, 1 \mathrm{H}), 5.16-5.11(\mathrm{~m}, 1 \mathrm{H}), 4.67(\mathrm{~d}$, $J=8.8 \mathrm{~Hz}, 1 \mathrm{H}), 3.81-3.72(\mathrm{~m}, 2 \mathrm{H}), 3.31(\mathrm{t}, J=6.0 \mathrm{~Hz}, 4 \mathrm{H}), 2.04-1.96$ (m, 5H), 1.63-1.54 (m, 1H), 1.50-1.43 (m, 1H); ${ }^{13} \mathrm{C}$ NMR (100 MHz, $\left.\mathrm{CDCl}_{3}\right) \delta 161.2,149.4,138.6,133.6,132.8,128.2,128.1,127.9,127.7,127.6,126.4,126.2,125.9$, $117.8,105.3,93.6,86.3,61.2,51.7,48.2,35.0,25.8$; HRMS (ESI) for $\mathrm{C}_{24} \mathrm{H}_{26} \mathrm{NO}_{2}[\mathrm{M}+\mathrm{H}]^{+}$: calcd 360.1958 , found 360.1958 .

HPLC: Chiralpak AD-H column $(250 \mathrm{~mm})$; detected at $220 \mathrm{~nm}$; hexane $/ \mathrm{i}$-propanol $=90 / 10$; flow $=1.1 \mathrm{~mL} / \mathrm{min}$; Retention time: $14.0 \mathrm{~min}, 16.7 \mathrm{~min}$ (major).

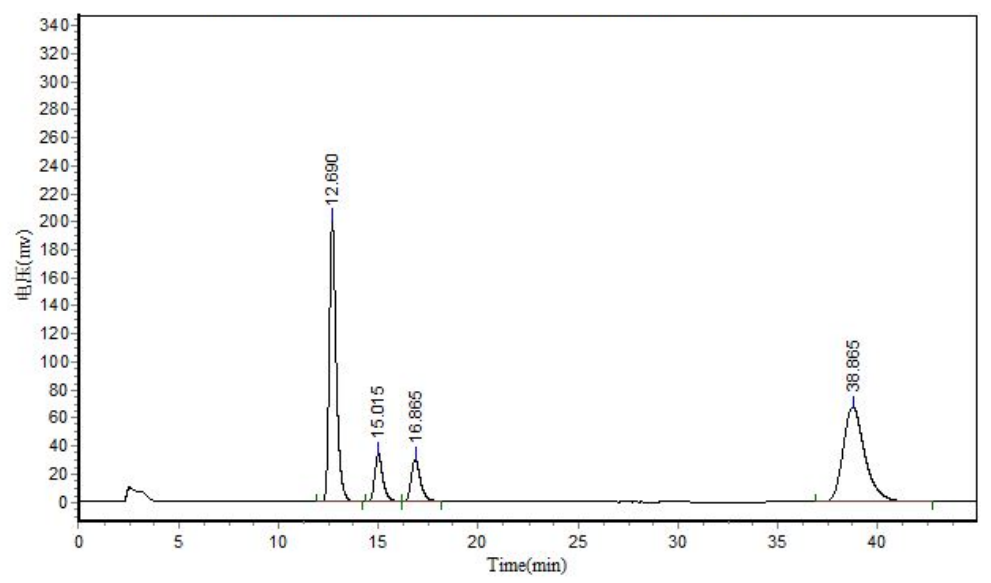

\begin{tabular}{|c|c|c|c|c|c|}
\hline \multicolumn{6}{|c|}{ Results } \\
\hline Peak No. & Peak ID & Ret Time & Height & Area & Conc. \\
\hline 1 & & 12.690 & 200296.625 & 4885418.000 & 40.1760 \\
\hline 2 & & 15.015 & 34417.031 & 1001617.375 & 8.2370 \\
\hline 3 & & 16.865 & 30922.205 & 1045197.625 & 8.5953 \\
\hline 4 & & 38.865 & 68060.688 & 5227818.000 & 42.9917 \\
\hline Total & & & 333696.549 & 12160051.000 & 100.0000 \\
\hline
\end{tabular}

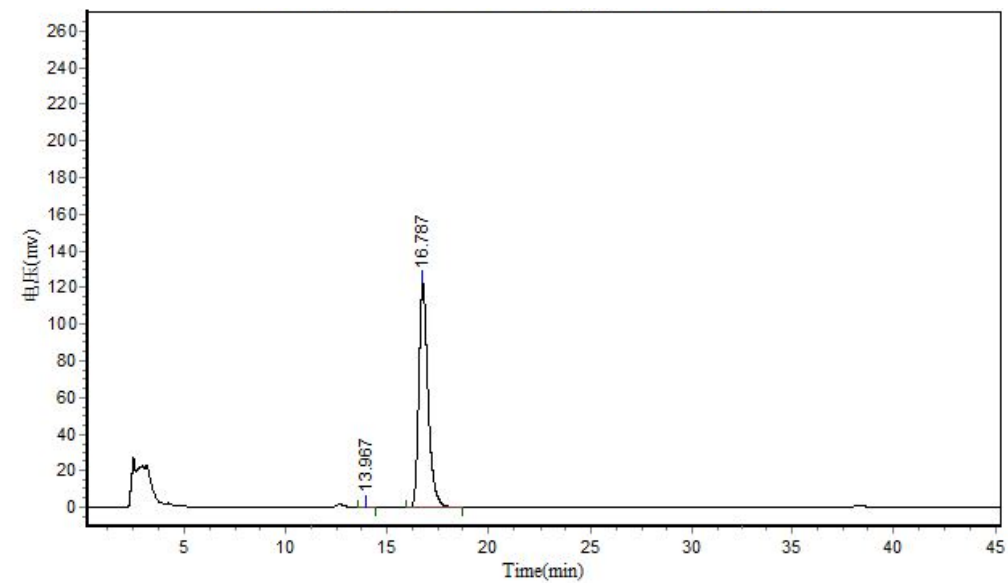

\begin{tabular}{|c|c|c|c|c|c|}
\hline \multicolumn{6}{|c|}{ Results } \\
\hline Peak No. & Peak ID & Ret Time & Height & Area & Conc. \\
\hline 1 & & 13.967 & 67.164 & 1447.700 & 0.0372 \\
\hline 2 & & 16.787 & 121943.609 & 3886851.500 & 99.9628 \\
\hline Total & & & 122010.774 & 3888299.200 & 100.0000 \\
\hline \multicolumn{6}{|c|}{ S40 } \\
\hline
\end{tabular}




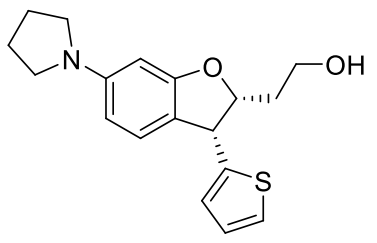

Colorless oil, $31.3 \mathrm{mg}, 99 \%$ yield, $98: 2 \mathrm{dr}$, 99\% ee; $[\alpha]_{\mathrm{D}}{ }^{20}=39.1(c$ 1.22, $\left.\mathrm{CHCl}_{3}\right) ;{ }^{1} \mathrm{H}$ NMR $\left(400 \mathrm{MHz}, \mathrm{CDCl}_{3}\right) \delta 7.16(\mathrm{~d}, J=5.2 \mathrm{~Hz}, 1 \mathrm{H})$, $7.01(\mathrm{~d}, J=8.0 \mathrm{~Hz}, 1 \mathrm{H}), 6.94(\mathrm{dd}, J=4.8,3.6 \mathrm{~Hz}, 1 \mathrm{H}), 6.74(\mathrm{~d}, J=$ $3.2 \mathrm{~Hz}, 1 \mathrm{H}), 6.15-6.12(\mathrm{~m}, 2 \mathrm{H}), 5.02-4.97(\mathrm{~m}, 1 \mathrm{H}), 4.79(\mathrm{~d}, J=8.0 \mathrm{~Hz}$, $1 \mathrm{H}), 3.85-3.80(\mathrm{~m}, 2 \mathrm{H}), 3.28(\mathrm{t}, J=6.4 \mathrm{~Hz}, 4 \mathrm{H}), 2.05-1.97(\mathrm{~m}, 5 \mathrm{H})$, 1.69-1.60 (m, 2H); $\left.{ }^{13} \mathrm{C} \mathrm{NMR} \mathrm{(100} \mathrm{MHz,} \mathrm{CDCl}_{3}\right) \delta 160.7,149.6,144.8,127.1,126.2,126.0,124.7$, 117.3, 105.2, 93.6, 85.9, 61.1, 48.1, 46.4, 34.3, 25.8; HRMS (ESI) for $\mathrm{C}_{18} \mathrm{H}_{22} \mathrm{NO}_{2} \mathrm{~S}[\mathrm{M}+\mathrm{H}]^{+}$: calcd 316.1366 , found 316.1369 .

HPLC: Chiralpak OD-H column $(250 \mathrm{~mm})$; detected at $220 \mathrm{~nm}$; hexane $/ i$-propanol $=85 / 15$; flow $=1.1 \mathrm{~mL} / \mathrm{min}$; Retention time: $19.1 \mathrm{~min}$ (major), $30.8 \mathrm{~min}$.

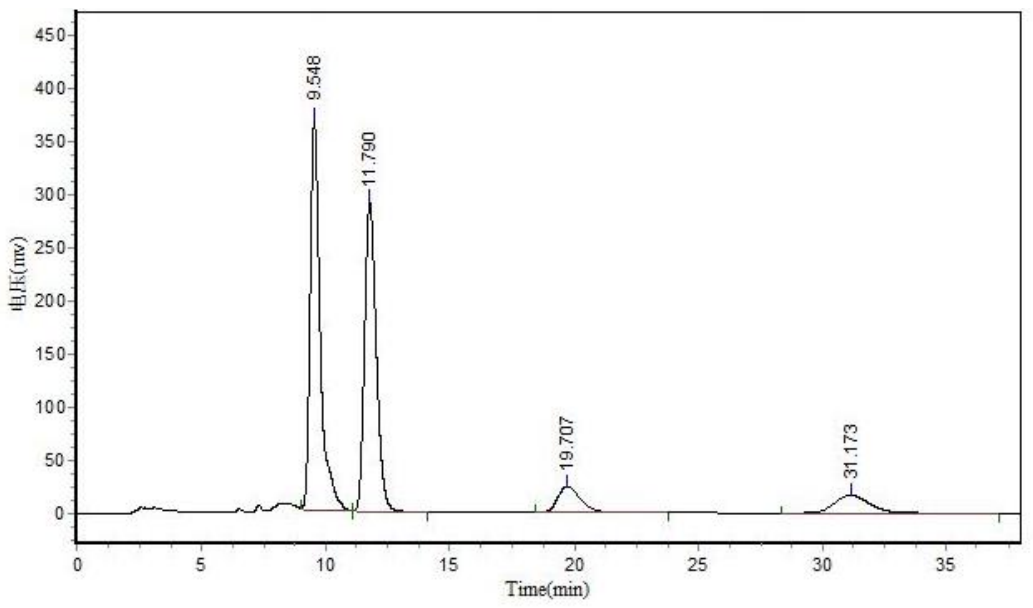

Results

\begin{tabular}{|c|c|c|c|c|c|}
\hline \\
\hline Peak No. & Peak ID & Ret Time & Height & Area & Conc. \\
\hline 1 & & 9.548 & 365622.813 & 10341497.000 & 44.5861 \\
\hline 2 & & 11.790 & 290853.750 & 9558625.000 & 41.2108 \\
\hline 3 & & 19.707 & 24547.652 & 1599167.500 & 6.8946 \\
\hline 4 & & 31.173 & 17109.891 & 1695174.000 & 7.3085 \\
\hline Total & & & 698134.105 & 23194463.500 & 100.000 \\
\hline
\end{tabular}

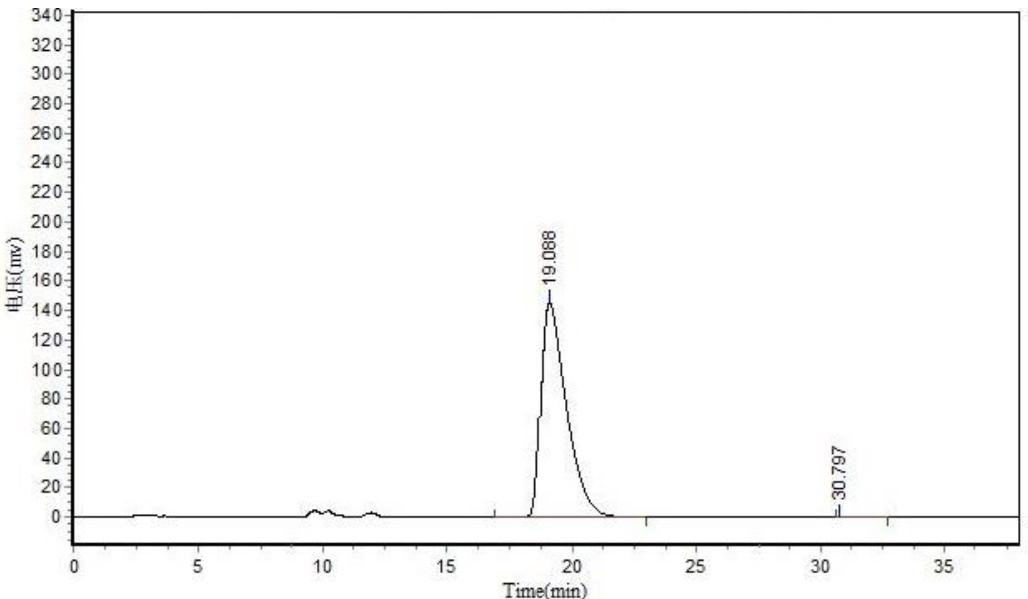

\begin{tabular}{|c|c|c|c|c|c|}
\hline \multicolumn{6}{|c|}{ Results } \\
\hline Peak No. & Peak ID & Ret Time & Height & Area & Conc. \\
\hline 1 & & 19.088 & 145911.781 & 10264913.000 & 99.9887 \\
\hline 2 & & 30.797 & 25.831 & 1163.048 & 0.0113 \\
\hline Total & & & 145937.612 & 10266076.048 & 100.000 \\
\hline
\end{tabular}


2-((2R,3S)-6-(dimethylamino)-3-phenyl-2,3-dihydrobenzofuran-2-yl)ethanol (4aa)<smiles>CN(C)c1ccc2c(c1)OC(CCO)C2c1ccccc1</smiles>

White solid, $24.2 \mathrm{mg}, 86 \%$ yield, $98: 2 \mathrm{dr}, 99 \%$ ee; m.p. $77.6-78.3^{\circ} \mathrm{C}$, $[\alpha]_{\mathrm{D}}^{20}=11.0\left(\right.$ c $\left.0.90, \mathrm{CHCl}_{3}\right) ;{ }^{1} \mathrm{H}$ NMR $\left(400 \mathrm{MHz}, \mathrm{CDCl}_{3}\right) \delta$ 7.34-7.21 $(\mathrm{m}, 5 \mathrm{H}), 6.81(\mathrm{~d}, J=8.0 \mathrm{~Hz}, 1 \mathrm{H}), 6.29-6.26(\mathrm{~m}, 2 \mathrm{H}), 4.72-4.67(\mathrm{~m}, 1 \mathrm{H})$, $4.24(\mathrm{~d}, J=8.4 \mathrm{~Hz}, 1 \mathrm{H}), 3.88(\mathrm{~d}, J=5.2 \mathrm{~Hz}, 2 \mathrm{H}), 2.94(\mathrm{~s}, 6 \mathrm{H}), 2.14-1.99$ $(\mathrm{m}, 3 \mathrm{H}) ;{ }^{13} \mathrm{C}$ NMR $\left(100 \mathrm{MHz}, \mathrm{CDCl}_{3}\right) \delta 161.1,152.2,143.0,129.1$, 128.5, 127.4, 125.6, 118.5, 105.9, 94.7, 91.5, 60.9, 54.8, 41.2, 37.6; HRMS (ESI) for $\mathrm{C}_{18} \mathrm{H}_{22} \mathrm{NO}_{2}$ $[\mathrm{M}+\mathrm{H}]^{+}$: calcd 284.1645, found 284.1648.

HPLC: Chiralpak OD-H column $(250 \mathrm{~mm})$; detected at $254 \mathrm{~nm}$; hexane $/ i$-propanol $=90 / 10$; flow $=0.7 \mathrm{~mL} / \mathrm{min}$; Retention time: $15.4 \mathrm{~min}$ (major), $18.1 \mathrm{~min}$.

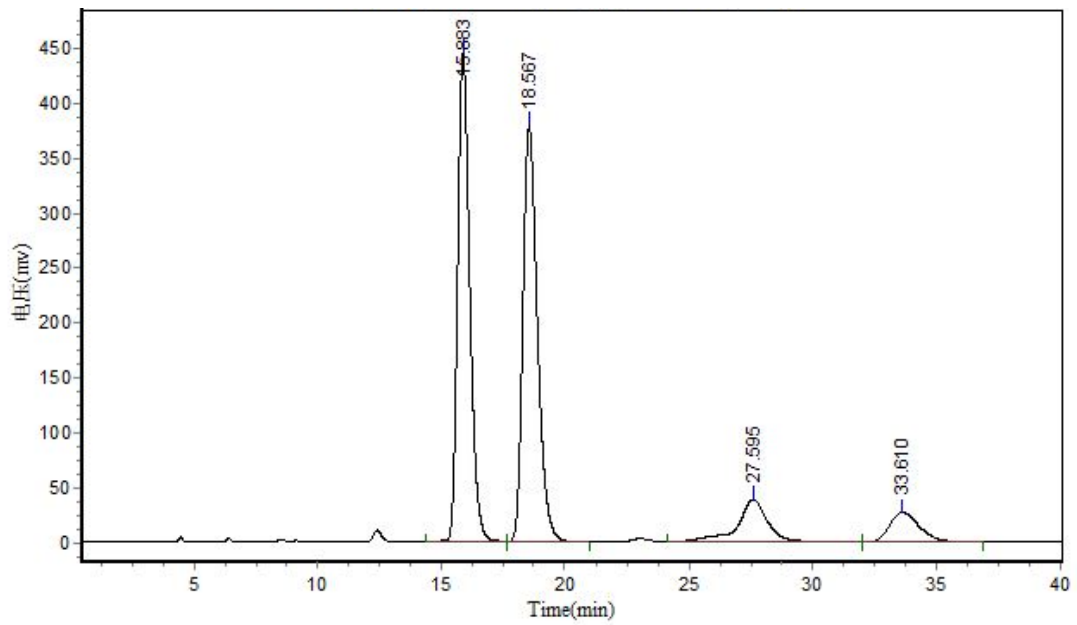

\begin{tabular}{|c|c|c|c|c|c|}
\hline \multicolumn{6}{|c|}{ Results } \\
\hline Peak No. & Peak ID & Ret Time & Height & Area & Conc. \\
\hline 1 & & 15.883 & 444359.969 & 15278567.000 & 41.9656 \\
\hline 2 & & 18.567 & 377903.063 & 15450704.000 & 42.4384 \\
\hline 3 & & 27.595 & 38617.996 & 3373483.000 & 9.2659 \\
\hline 4 & & 33.610 & 27481.867 & 2304626.500 & 6.3301 \\
\hline Total & & & 888362.895 & 36407380.500 & 100.0000 \\
\hline
\end{tabular}

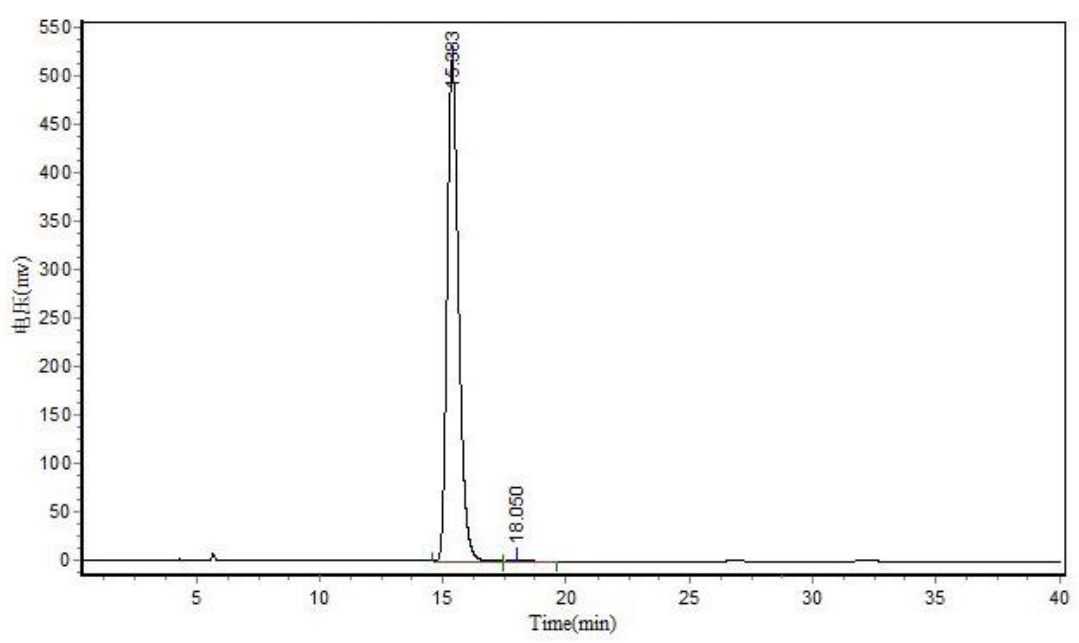

Results

\begin{tabular}{|c|c|c|c|c|c|}
\hline \multicolumn{6}{|c|}{ Nesuits } \\
\hline Peak No. & Peak ID & Ret Time & Height & Area & Conc. \\
\hline 1 & & 15.383 & 517071.125 & 16890062.000 & 99.5162 \\
\hline 2 & & 18.050 & 1749.637 & 82107.094 & 0.4838 \\
\hline Total & & & 518820.762 & 16972169.094 & 100.0000 \\
\hline
\end{tabular}


2-((2R,3S)-6-(diethylamino)-3-phenyl-2,3-dihydrobenzofuran-2-yl)ethanol (4ab)<smiles>CCN(CC)c1ccc2c(c1)OC(CCO)C2c1ccccc1</smiles>

Colorless oil, $27.0 \mathrm{mg}, 87 \%$ yield, $99: 1 \mathrm{dr}$, 99\% ee; $[\alpha]_{\mathrm{D}}^{20}=12.9(c$ $\left.1.02, \mathrm{CHCl}_{3}\right) ;{ }^{1} \mathrm{H}$ NMR $\left(400 \mathrm{MHz}, \mathrm{CDCl}_{3}\right) \delta 7.34-7.23(\mathrm{~m}, 5 \mathrm{H}), 6.77$ $(\mathrm{d}, J=8.0 \mathrm{~Hz}, 1 \mathrm{H}), 6.23-6.20(\mathrm{~m}, 2 \mathrm{H}), 4.70-4.65(\mathrm{~m}, 1 \mathrm{H}), 4.23(\mathrm{~d}, J=$ $8.0 \mathrm{~Hz}, 1 \mathrm{H}), 3.88(\mathrm{~d}, J=5.6 \mathrm{~Hz}, 2 \mathrm{H}), 3.33(\mathrm{q}, J=7.2 \mathrm{~Hz}, 4 \mathrm{H})$, 2.18-2.04 (m, 3H), $1.17(\mathrm{t}, J=6.8 \mathrm{~Hz}, 6 \mathrm{H}) ;{ }^{13} \mathrm{C}$ NMR $(100 \mathrm{MHz}$, $\left.\mathrm{CDCl}_{3}\right) \delta 161.3,149.4,143.1,129.0,128.5,127.3,125.7,117.2,105.0,93.6,91.4,60.8,54.8,45.0$, 37.5, 12.9; HRMS (ESI) for $\mathrm{C}_{20} \mathrm{H}_{26} \mathrm{NO}_{2}[\mathrm{M}+\mathrm{H}]^{+}$: calcd 312.1958, found 312,1957.

HPLC: Chiralpak OD-H column $(250 \mathrm{~mm})$; detected at $254 \mathrm{~nm}$; hexane $/ i$-propanol $=95 / 5$; flow $=$ $1.2 \mathrm{~mL} / \mathrm{min}$; Retention time: $15.4 \mathrm{~min}$ (major), $20.9 \mathrm{~min}$.

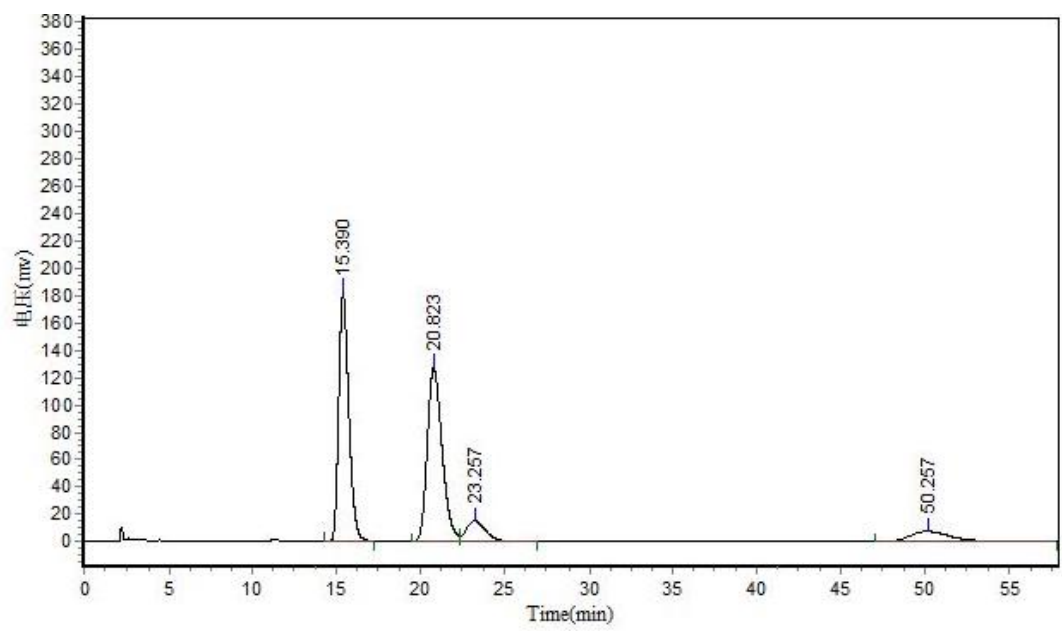

\begin{tabular}{|c|c|c|c|c|c|}
\hline \multicolumn{6}{|c|}{ Results } \\
\hline Peak No. & Peak ID & Ret Time & Height & Area & Conc. \\
\hline 1 & & 15.390 & 181420.719 & 7608994.500 & 42.2606 \\
\hline 2 & & 20.823 & 126841.430 & 7862476.500 & 43.6684 \\
\hline 3 & & 23.257 & 15726.574 & 1247456.000 & 6.9284 \\
\hline 4 & & 50.257 & 7505.571 & 1286017.625 & 7.1426 \\
\hline Total & & & 331494.294 & 18004944.625 & 100.0000 \\
\hline
\end{tabular}

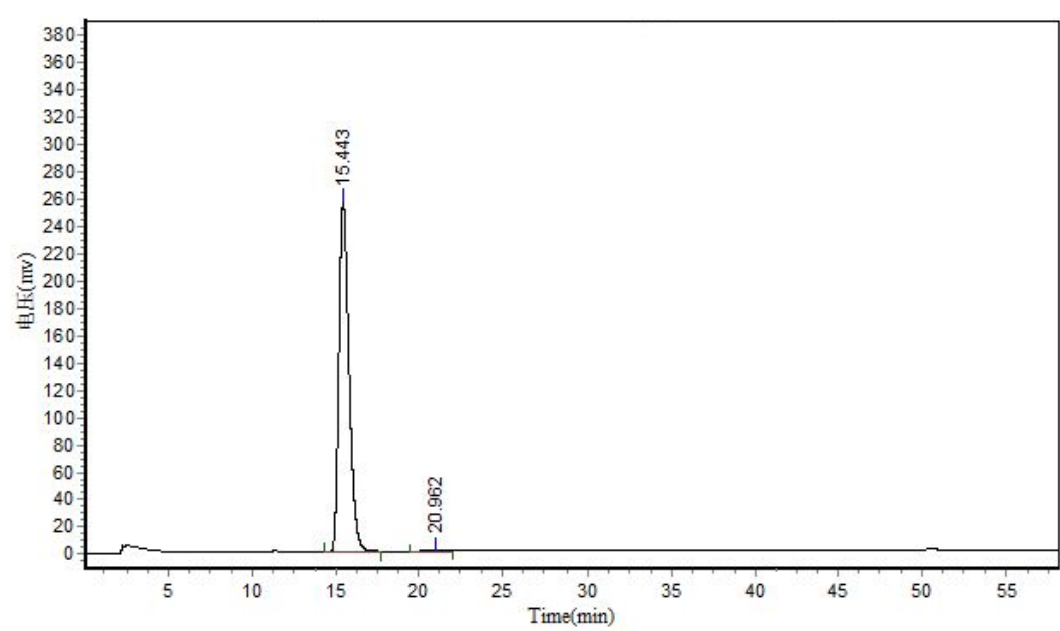

Results

\begin{tabular}{|c|c|c|c|c|c|}
\hline Peak No. & Peak ID & Ret Time & Height & Area & Conc. \\
\hline 1 & & 15.443 & 255958.297 & 10795499.000 & 99.2831 \\
\hline 2 & & 20.962 & 742.192 & 77946.992 & 0.7169 \\
\hline Total & & & 256700.489 & 10873445.992 & 100.0000 \\
\hline
\end{tabular}


2-((2R,3S)-6-(dibenzylamino)-3-phenyl-2,3-dihydrobenzofuran-2-yl)ethanol (4ac)<smiles>OCCC1Oc2cc(N(Cc3ccccc3)Cc3ccccc3)ccc2C1c1ccccc1</smiles>

White solid, $38.7 \mathrm{mg}, 89 \%$ yield, $99: 1 \mathrm{dr}$, $98 \%$ ee; m.p. $120.1-121.8^{\circ} \mathrm{C}$, $[\alpha]_{\mathrm{D}}{ }^{20}=13.1\left(c 1.0, \mathrm{CHCl}_{3}\right) ;{ }^{1} \mathrm{H} \mathrm{NMR}\left(400 \mathrm{MHz}, \mathrm{CDCl}_{3}\right) \delta$ 7.37-7.24 $(\mathrm{m}, 15 \mathrm{H}), 6.74(\mathrm{~d}, J=8.4 \mathrm{~Hz}, 1 \mathrm{H}), 6.30-6.27(\mathrm{~m}, 2 \mathrm{H}), 4.70-4.61(\mathrm{~m}$, $5 \mathrm{H}), 4.23(\mathrm{~d}, J=8.4 \mathrm{~Hz}, 1 \mathrm{H}), 3.84(\mathrm{~d}, J=6.0 \mathrm{~Hz}, 2 \mathrm{H}), 2.13-2.00(\mathrm{~m}$, $3 \mathrm{H}) ;{ }^{13} \mathrm{C}$ NMR $\left(100 \mathrm{MHz}, \mathrm{CDCl}_{3}\right) \delta 161.2,150.9,142.7,138.8,129.0$, 128.9, 128.6, 127.4, 127.2, 126.9, 125.6, 118.7, 105.7, 94.5, 91.5, 60.8, 54.9, 54.8, 37.4; HRMS (ESI) for $\mathrm{C}_{30} \mathrm{H}_{30} \mathrm{NO}_{2}[\mathrm{M}+\mathrm{H}]^{+}$: calcd 436.2271, found 436.2269.

HPLC: Chiralpak OD-H column $(250 \mathrm{~mm})$; detected at $254 \mathrm{~nm}$; hexane $/ i$-propanol $=90 / 10$; flow $=0.7 \mathrm{~mL} / \mathrm{min}$; Retention time: $24.8 \mathrm{~min}$ (major), $36.2 \mathrm{~min}$.

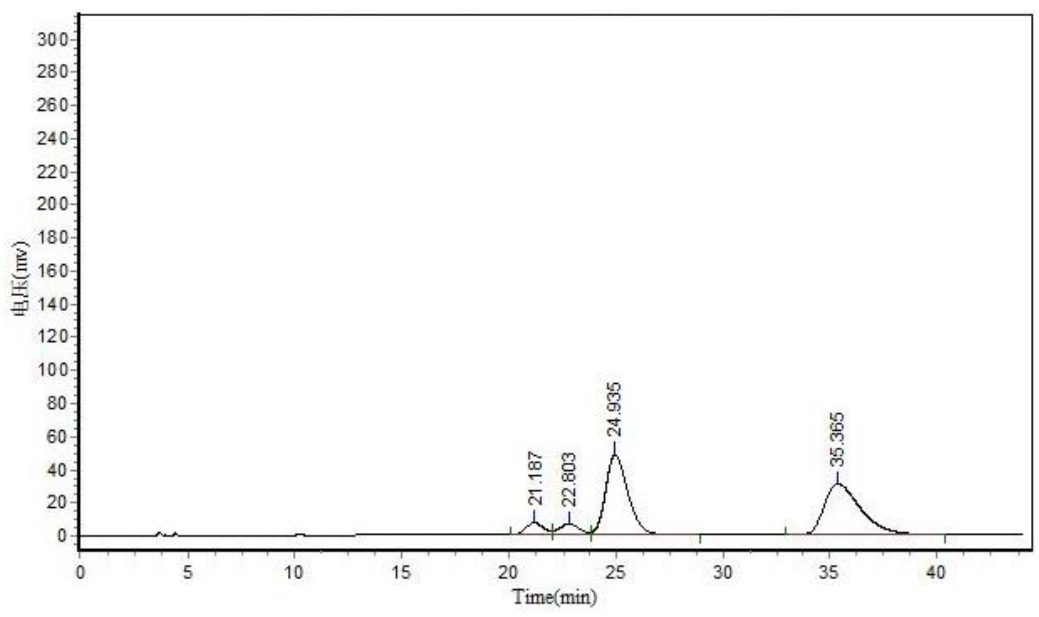

\begin{tabular}{|c|c|c|c|c|c|}
\hline \multicolumn{6}{|c|}{ Results } \\
\hline Peak No. & Peak ID & Ret Time & Height & Area & Conc. \\
\hline 1 & & 21.187 & 7208.800 & 425523.031 & 5.3006 \\
\hline 2 & & 22.803 & 6517.118 & 431953.344 & 5.3807 \\
\hline 3 & & 24.935 & 48412.168 & 3533943.500 & 44.0210 \\
\hline 4 & & 35.365 & 30719.078 & 3636436.250 & 45.2977 \\
\hline Total & & & 92857.164 & 8027856.125 & 100.000 \\
\hline
\end{tabular}

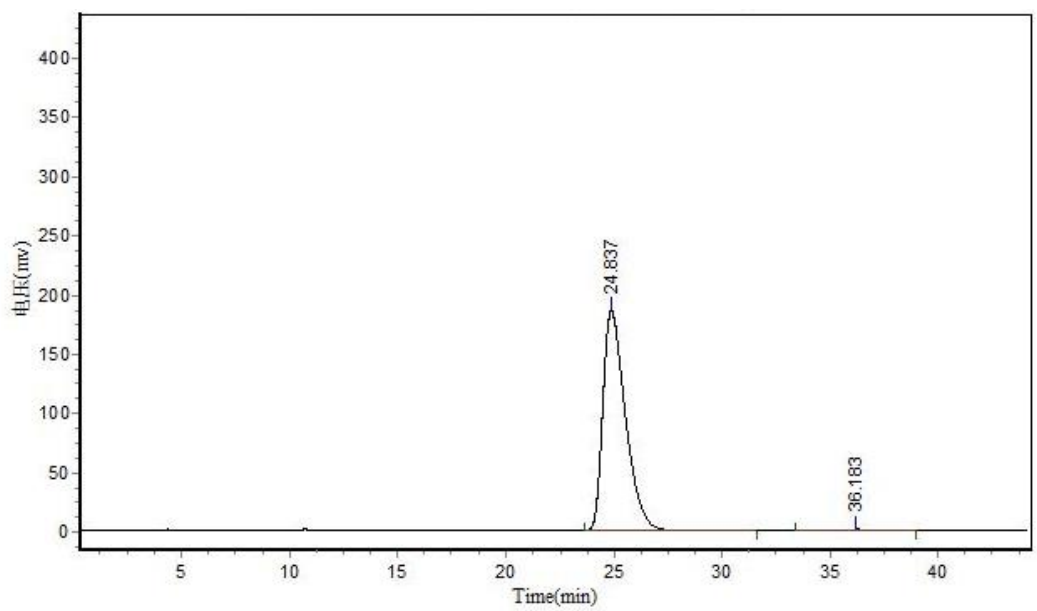

\begin{tabular}{|c|c|c|c|c|c|}
\hline \multicolumn{6}{|c|}{ Results } \\
\hline Peak No. & Peak ID & Ret Time & Height & Area & Conc. \\
\hline$\overline{1}$ & & 24.837 & 185545.813 & 13636739.000 & 99.0306 \\
\hline 2 & & 36.183 & 1065.963 & 133488.875 & 0.9694 \\
\hline Total & & & 186611.776 & 13770227.875 & 100.000 \\
\hline
\end{tabular}




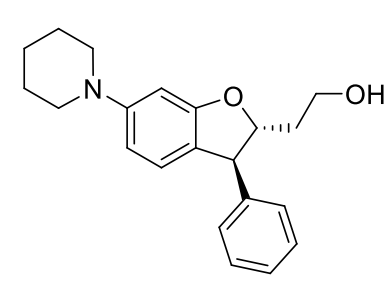

White solid, $29.4 \mathrm{mg}, 91 \%$ yield, $99: 1 \mathrm{dr}, 99 \%$ ee; m.p. $74.5-75.9^{\circ} \mathrm{C}$, $[\alpha]_{\mathrm{D}}{ }^{20}=18.1\left(c 1.0, \mathrm{CHCl}_{3}\right) ;{ }^{1} \mathrm{H}$ NMR $\left(400 \mathrm{MHz}, \mathrm{CDCl}_{3}\right) \delta 7.33-7.20$ $(\mathrm{m}, 5 \mathrm{H}), 6.80(\mathrm{~d}, J=8.4 \mathrm{~Hz}, 1 \mathrm{H}), 6.47-6.45(\mathrm{~m}, 2 \mathrm{H}), 4.72-4.66(\mathrm{~m}$, $1 \mathrm{H}), 4.23(\mathrm{~d}, J=8.4 \mathrm{~Hz}, 1 \mathrm{H}), 3.87(\mathrm{t}, J=5.2 \mathrm{~Hz}, 2 \mathrm{H}), 3.14(\mathrm{t}, J=5.2$ $\mathrm{Hz}, 4 \mathrm{H}), 2.15-2.00(\mathrm{~m}, 3 \mathrm{H}), 1.73-1.67(\mathrm{~m}, 4 \mathrm{H}), 1.60-1.54(\mathrm{~m}, 2 \mathrm{H}) ;{ }^{13} \mathrm{C}$ NMR $\left(100 \mathrm{MHz}, \mathrm{CDCl}_{3}\right) \delta 160.9,153.8,142.8,129.1,128.5,127.4$, $125.5,109.8,98.4,91.5,60.9,54.9,51.2,37.6,26.2,24.7$; HRMS (ESI) for $\mathrm{C}_{21} \mathrm{H}_{26} \mathrm{NO}_{2}[\mathrm{M}+\mathrm{H}]^{+}$: calcd 324.1958, found 324.1962.

HPLC: Chiralpak AD-H column $(250 \mathrm{~mm})$; detected at $254 \mathrm{~nm}$; hexane $/ i$-propanol $=90 / 10$; flow $=1.0 \mathrm{~mL} / \mathrm{min}$; Retention time: $10.7 \mathrm{~min}, 19.5 \mathrm{~min}$ (major).
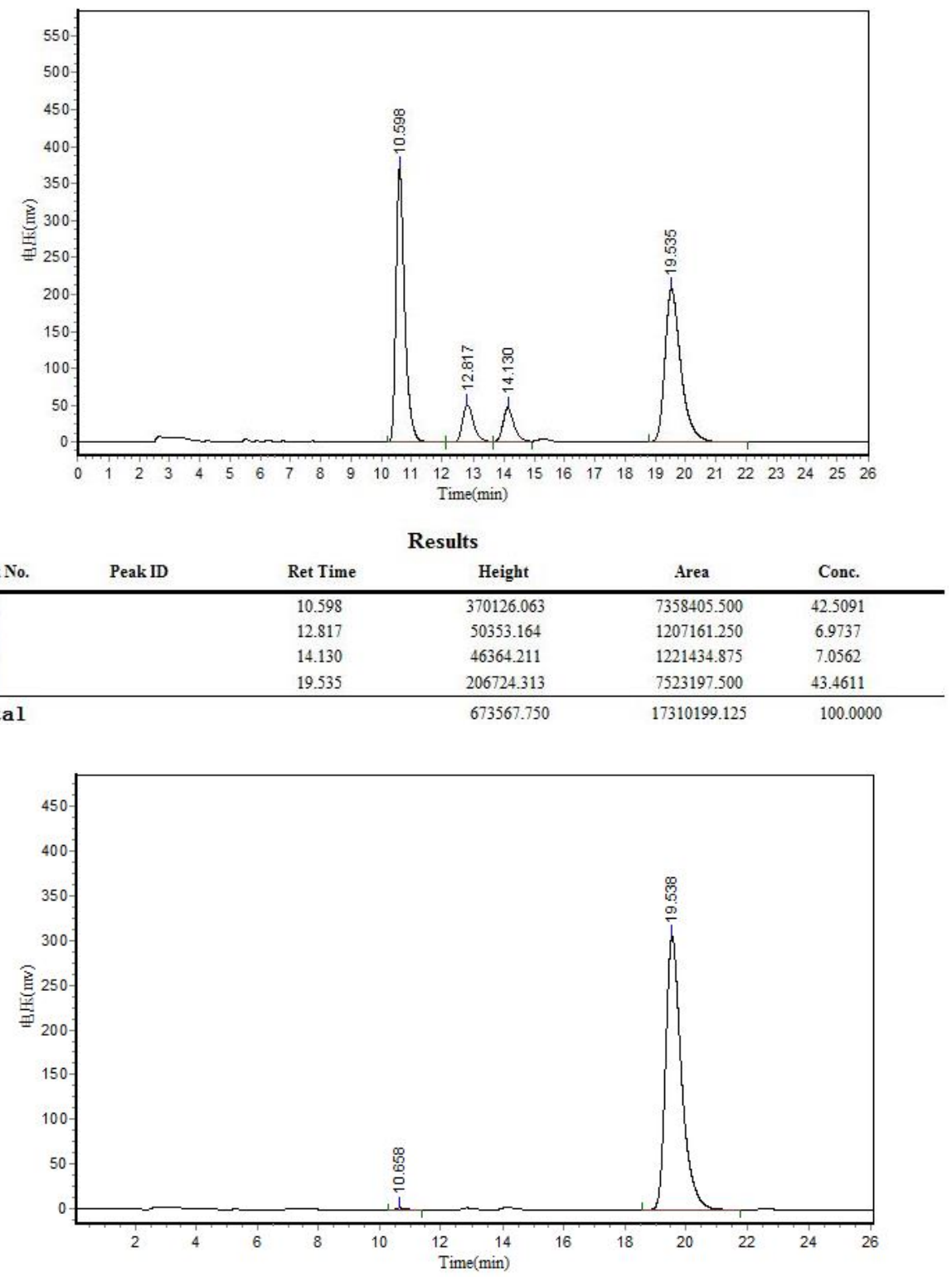

\begin{tabular}{|c|c|c|c|c|c|}
\hline \multicolumn{6}{|c|}{ Results } \\
\hline Peak No. & Peak ID & Ret Time & Height & Area & Conc. \\
\hline 1 & & 10.658 & 2021.095 & 39614.902 & 0.3592 \\
\hline 2 & & 19.538 & 305801.156 & 10988970.000 & 99.6408 \\
\hline Total & & & 307822.251 & 11028584.902 & 100.0000 \\
\hline
\end{tabular}




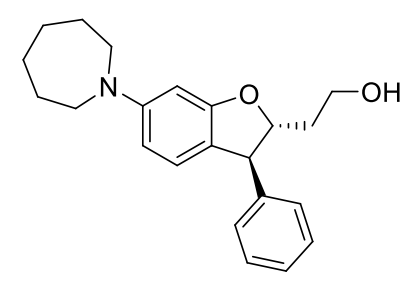

Pale yellow solid, $30.0 \mathrm{mg}$, $89 \%$ yield, 99:1 dr, 99\% ee; m.p. 80.1-81.8 ${ }^{\circ} \mathrm{C},[\alpha]_{\mathrm{D}}{ }^{20}=15.7$ (c 1.0, $\left.\mathrm{CHCl}_{3}\right) ;{ }^{1} \mathrm{H}$ NMR $(400 \mathrm{MHz}$, $\left.\mathrm{CDCl}_{3}\right) \delta$ 7.34-7.23 (m, 5H), $6.76(\mathrm{~d}, J=8.4 \mathrm{~Hz}, 1 \mathrm{H}), 6.23-6.20(\mathrm{~m}$, $2 \mathrm{H}), 4.70-4.64(\mathrm{~m}, 1 \mathrm{H}), 4.23(\mathrm{~d}, J=8.0 \mathrm{~Hz}, 1 \mathrm{H}), 3.87(\mathrm{t}, J=5.2 \mathrm{~Hz}$, 2H), $3.42(\mathrm{t}, J=5.2 \mathrm{~Hz}, 4 \mathrm{H}), 2.18-2.02(\mathrm{~m}, 3 \mathrm{H}), 1.83-1.79(\mathrm{~m}, 4 \mathrm{H})$, 1.57-1.53 (m, 4H); ${ }^{13} \mathrm{C}$ NMR $\left(100 \mathrm{MHz}, \mathrm{CDCl}_{3}\right) \delta 161.3,150.6$, 143.1, 129.0, 128.5, 127.3, 125.7, 117.0, 104.5, 93.1, 91.5, 60.9, 54.9, 49.8, 37.5, 28.1, 27.5; HRMS (ESI) for $\mathrm{C}_{22} \mathrm{H}_{28} \mathrm{NO}_{2}[\mathrm{M}+\mathrm{H}]^{+}$: calcd 338.2115, found 338.2116.

HPLC: Chiralpak OD-H column $(250 \mathrm{~mm})$; detected at $254 \mathrm{~nm}$; hexane $/ i$-propanol $=90 / 10$; flow $=0.7 \mathrm{~mL} / \mathrm{min}$; Retention time: $16.0 \mathrm{~min}$ (major), $17.6 \mathrm{~min}$.
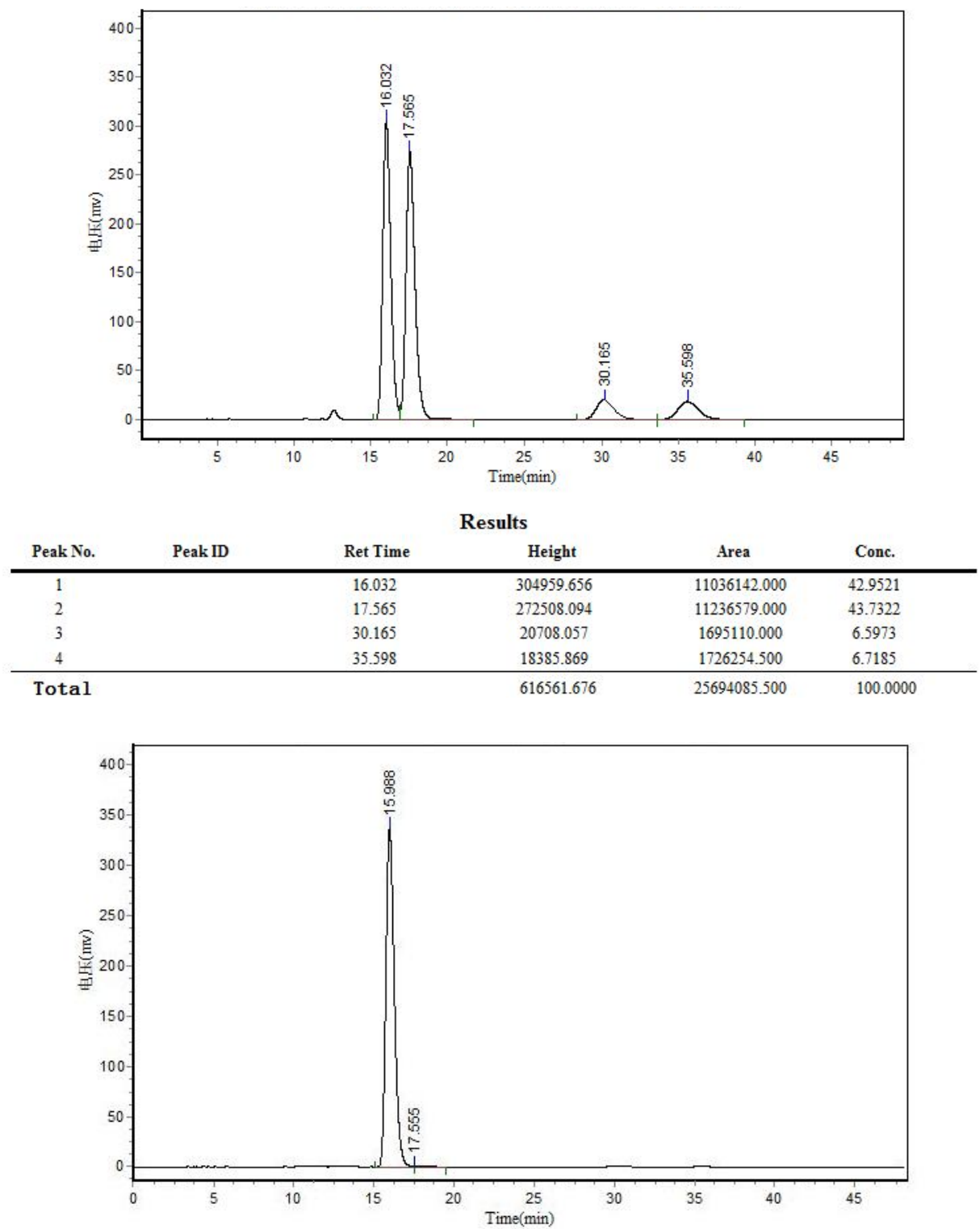

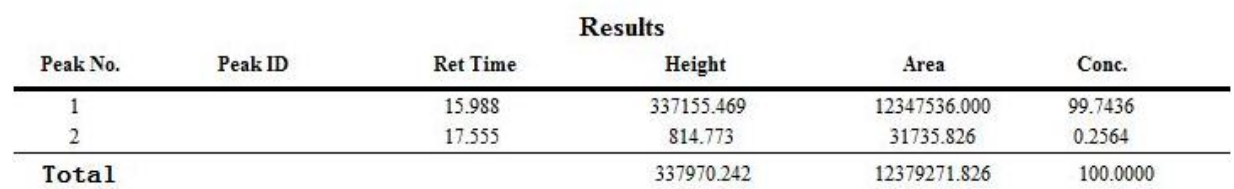




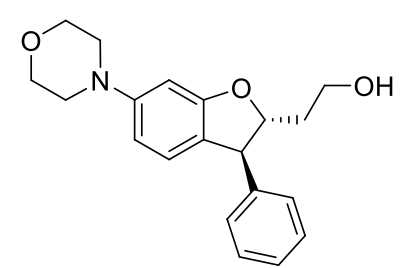

White solid, $26.4 \mathrm{mg}, 81 \%$ yield, $98: 2 \mathrm{dr}$, $99 \%$ ee; m.p. $74.3-76.5^{\circ} \mathrm{C}$, $[\alpha]_{\mathrm{D}}^{20}=19.4\left(c\right.$ 1.07, $\left.\mathrm{CHCl}_{3}\right) ;{ }^{1} \mathrm{H}$ NMR $\left(400 \mathrm{MHz}, \mathrm{CDCl}_{3}\right) \delta$ 7.34-7.20 (m, 5H), $6.84(\mathrm{~d}, J=8.0 \mathrm{~Hz}, 1 \mathrm{H}), 6.45-6.39(\mathrm{~m}, 2 \mathrm{H})$, 4.74-4.69 (m, 1H), $4.24(\mathrm{~d}, J=8.4 \mathrm{~Hz}, 1 \mathrm{H}), 3.88-3.84(\mathrm{~m}, 6 \mathrm{H}), 3.14$ (t, $J=4.4 \mathrm{~Hz}, 4 \mathrm{H}), 2.13-2.02(\mathrm{~m}, 3 \mathrm{H}) ;{ }^{13} \mathrm{C}$ NMR $\left(100 \mathrm{MHz}, \mathrm{CDCl}_{3}\right) \delta$ $160.9,152.9,142.6,129.1,128.5,127.5,125.7,122.1,108.9,97.9$, 91.5, 67.2, 60.7, 54.8, 49.9, 37.6; HRMS (ESI) for $\mathrm{C}_{20} \mathrm{H}_{24} \mathrm{NO}_{3}[\mathrm{M}+\mathrm{H}]^{+}$: calcd 326.1751, found 326.1752 .

HPLC: Chiralpak AD-H column $(250 \mathrm{~mm})$; detected at $254 \mathrm{~nm}$; hexane $/ i$-propanol $=90 / 10$; flow $=0.7 \mathrm{~mL} / \mathrm{min}$; Retention time: $21.0 \mathrm{~min}, 41.0 \mathrm{~min}$ (major).

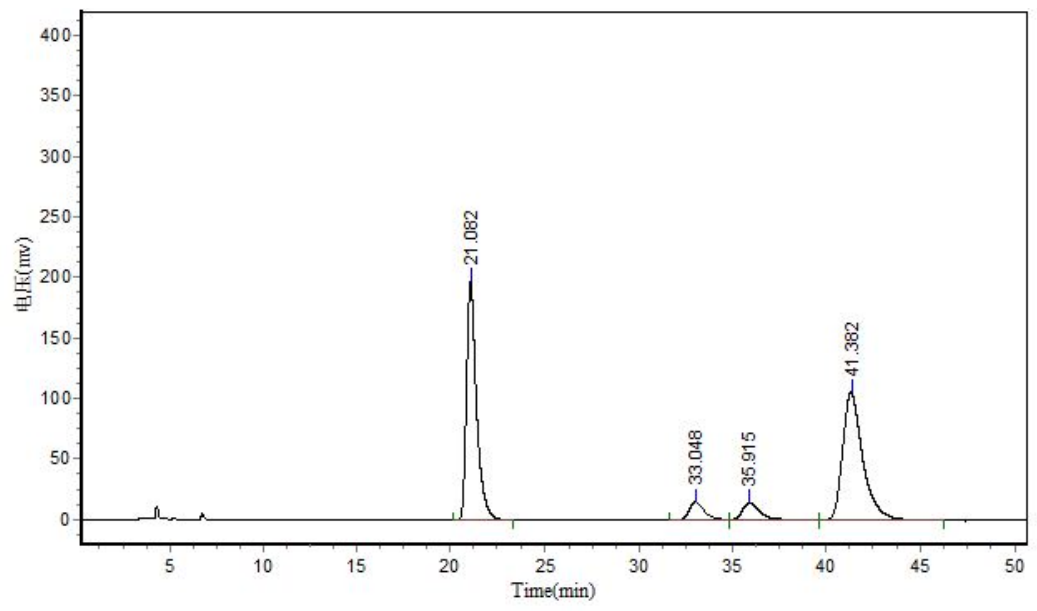

\begin{tabular}{|c|c|c|c|c|c|}
\hline \multicolumn{6}{|c|}{ Results } \\
\hline Peak No. & Peak ID & Ret Time & Height & Area & Conc. \\
\hline 1 & & 21.082 & 196047.578 & 7297531.500 & 43.1160 \\
\hline 2 & & 33.048 & 15108.074 & 866667.250 & 5.1205 \\
\hline 3 & & 35.915 & 13961.127 & 900253.375 & 5.3190 \\
\hline 4 & & 41.382 & 104887.859 & 7860892.000 & 46.4445 \\
\hline Total & & & 330004.639 & 16925344.125 & 100.000 \\
\hline
\end{tabular}

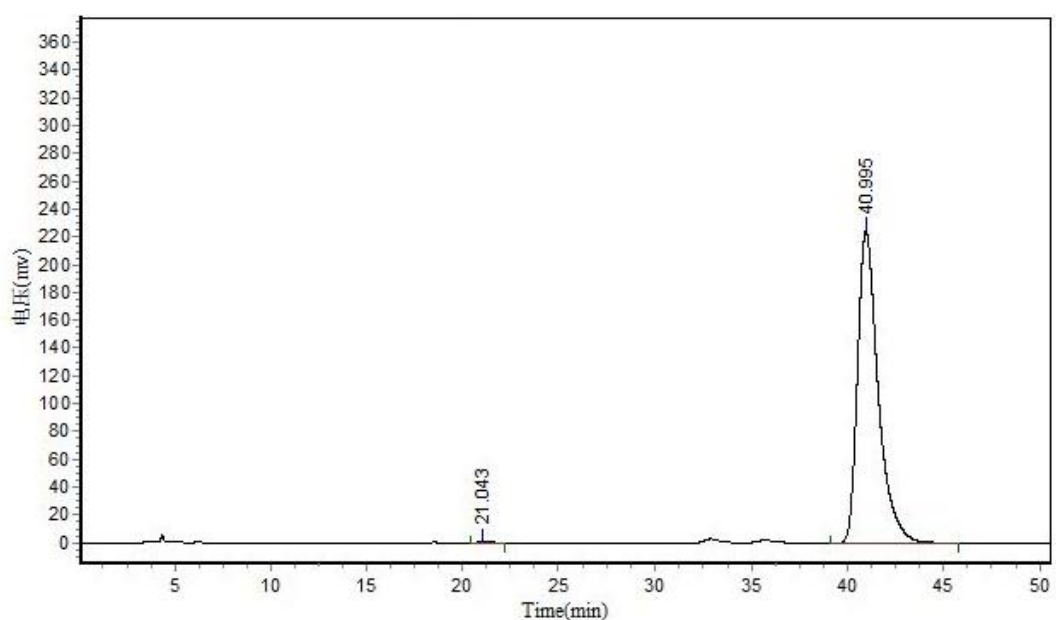

\begin{tabular}{|c|c|c|c|c|c|}
\hline \multicolumn{6}{|c|}{ Results } \\
\hline Peak No. & Peak ID & Ret Time & Height & Area & Conc. \\
\hline 1 & & 21.043 & 1226.893 & 43236.699 & 0.2563 \\
\hline 2 & & 40.995 & 224057.781 & 16826290.000 & 99.7437 \\
\hline Total & & & 225284.674 & 16869526.699 & 100.0000 \\
\hline
\end{tabular}


tert-butyl 4-((2R,3S)-2-(2-hydroxyethyl)-3-phenyl-2,3-dihydrobenzofuran-6-yl)piperazine

-1-carboxylate (4ag)

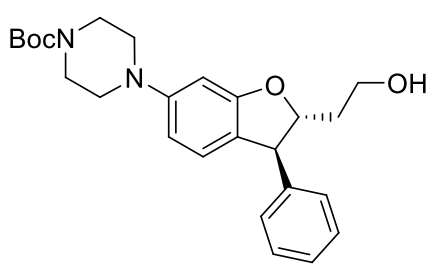

Pale yellow oil, $39.0 \mathrm{mg}, 92 \%$ yield, $99: 1 \mathrm{dr}, 99 \%$ ee; $[\alpha]_{\mathrm{D}}{ }^{20}=17.3$ (c 1.0, $\left.\mathrm{CHCl}_{3}\right) ;{ }^{1} \mathrm{H} \mathrm{NMR}\left(400 \mathrm{MHz}, \mathrm{CDCl}_{3}\right) \delta$ 7.34-7.19 (m, 5H), $6.82(\mathrm{~d}, J=8.0 \mathrm{~Hz}, 1 \mathrm{H}), 6.45-6.42(\mathrm{~m}, 2 \mathrm{H}), 4.74-4.68(\mathrm{~m}, 1 \mathrm{H})$, $4.23(\mathrm{~d}, J=8.0 \mathrm{~Hz}, 1 \mathrm{H}), 3.86(\mathrm{t}, J=6.0 \mathrm{~Hz}, 2 \mathrm{H}), 3.56(\mathrm{t}, J=4.8$ $\mathrm{Hz}, 4 \mathrm{H}), 3.11$ (t, $J=4.4 \mathrm{~Hz}, 4 \mathrm{H}), 2.13-2.04(\mathrm{~m}, 3 \mathrm{H}), 1.48$ (s, 9H); ${ }^{13} \mathrm{C}$ NMR $\left(100 \mathrm{MHz}, \mathrm{CDCl}_{3}\right) \delta 160.9,155.1,152.9,142.6,129.1$, $128.5,127.5,125.7,122.3,109.8,98.7,91.5,80.3,60.7,54.8,49.9,37.6,28.8$; HRMS (ESI) for $\mathrm{C}_{25} \mathrm{H}_{33} \mathrm{~N}_{2} \mathrm{O}_{4}[\mathrm{M}+\mathrm{H}]^{+}$: calcd 425.2435, found 425.2437.

HPLC: Chiralpak AD-H column $(250 \mathrm{~mm})$; detected at $254 \mathrm{~nm}$; hexane $/ i$-propanol $=90 / 10$; flow $=0.7 \mathrm{~mL} / \mathrm{min}$; Retention time: $23.4 \mathrm{~min}, 32.6 \mathrm{~min}$ (major).

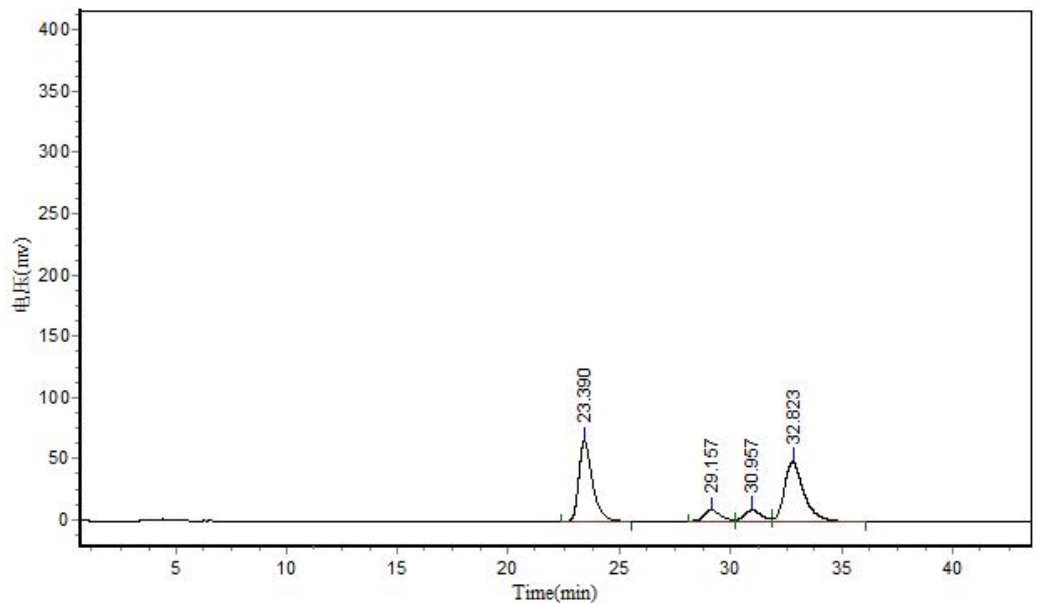

\begin{tabular}{|c|c|c|c|c|c|}
\hline \multicolumn{6}{|c|}{ Results } \\
\hline Peak No. & Peak ID & Ret Time & Height & Area & Conc. \\
\hline 1 & & 23.390 & 66389.492 & 2865168.750 & 41.7891 \\
\hline 2 & & 29.157 & 9602.333 & 510464.094 & 7.4452 \\
\hline 3 & & 30.957 & 9042.333 & 493848.688 & 7.2029 \\
\hline 4 & & 32.823 & 48562.668 & 2986778.500 & 43.5628 \\
\hline Total & & & 133596.826 & 6856260.031 & 100.0000 \\
\hline
\end{tabular}

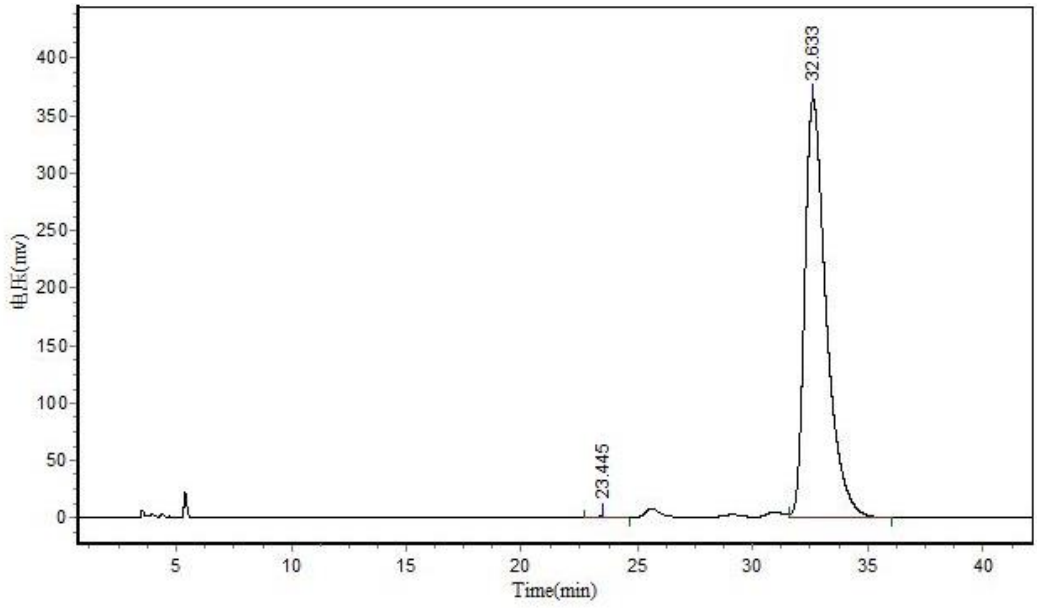

\begin{tabular}{|c|c|c|c|c|c|}
\hline \multicolumn{6}{|c|}{ Results } \\
\hline Peak No. & Peak ID & Ret Time & Height & Area & Conc. \\
\hline 1 & & 23.445 & 997.447 & 40628.871 & 0.1716 \\
\hline 2 & & 32.633 & 364653.563 & 23637370.000 & 99.8284 \\
\hline Total & & & 365651.009 & 23677998.871 & 100.0000 \\
\hline
\end{tabular}




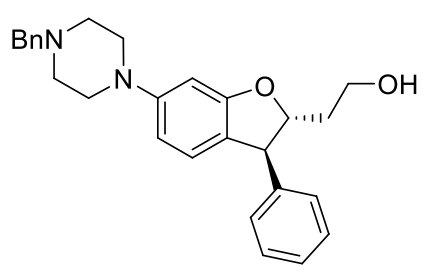

Colorless oil, $38.1 \mathrm{mg}, 92 \%$ yield, $97: 3 \mathrm{dr}, 99 \%$ ee; $[\alpha]_{\mathrm{D}}^{20}=20.0(c$ 1.0, $\left.\mathrm{CHCl}_{3}\right) ;{ }^{1} \mathrm{H}$ NMR $\left(400 \mathrm{MHz}, \mathrm{CDCl}_{3}\right) \delta 7.36-7.20(\mathrm{~m}, 10 \mathrm{H})$, $6.82(\mathrm{~d}, J=8.0 \mathrm{~Hz}, 1 \mathrm{H}), 6.45-6.43(\mathrm{~m}, 2 \mathrm{H}), 4.73-4.68(\mathrm{~m}, 1 \mathrm{H})$, $4.23(\mathrm{~d}, J=8.0 \mathrm{~Hz}, 1 \mathrm{H}), 3.86$ (t, $J=6.0 \mathrm{~Hz}, 2 \mathrm{H}), 3.57$ (s, 2H), 3.19 (t, $J=4.8 \mathrm{~Hz}, 4 \mathrm{H}), 2.61(\mathrm{t}, J=4.4 \mathrm{~Hz}, 4 \mathrm{H}), 2.20-2.01(\mathrm{~m}, 3 \mathrm{H}) ;{ }^{13} \mathrm{C}$ NMR $\left(100 \mathrm{MHz}, \mathrm{CDCl}_{3}\right) \delta 160.9,152.9,142.7,138.1,129.6$, 129.1, 128.6, 128.5, 127.5, 127.4, 125.6, 121.6, 109.3, 98.1, 91.4, 63.4, 60.7, 54.8, 53.4, 49.6, 37.6; HRMS (ESI) for $\mathrm{C}_{27} \mathrm{H}_{31} \mathrm{~N}_{2} \mathrm{O}_{2}[\mathrm{M}+\mathrm{H}]^{+}$: calcd 415.238, found 415.2376.

HPLC: Chiralpak AD-H column $(250 \mathrm{~mm})$; detected at $254 \mathrm{~nm}$; hexane $/ i$-propanol $=90 / 10$; flow $=1.0 \mathrm{~mL} / \mathrm{min}$; Retention time: $14.1 \mathrm{~min}, 31.0 \mathrm{~min}$ (major).
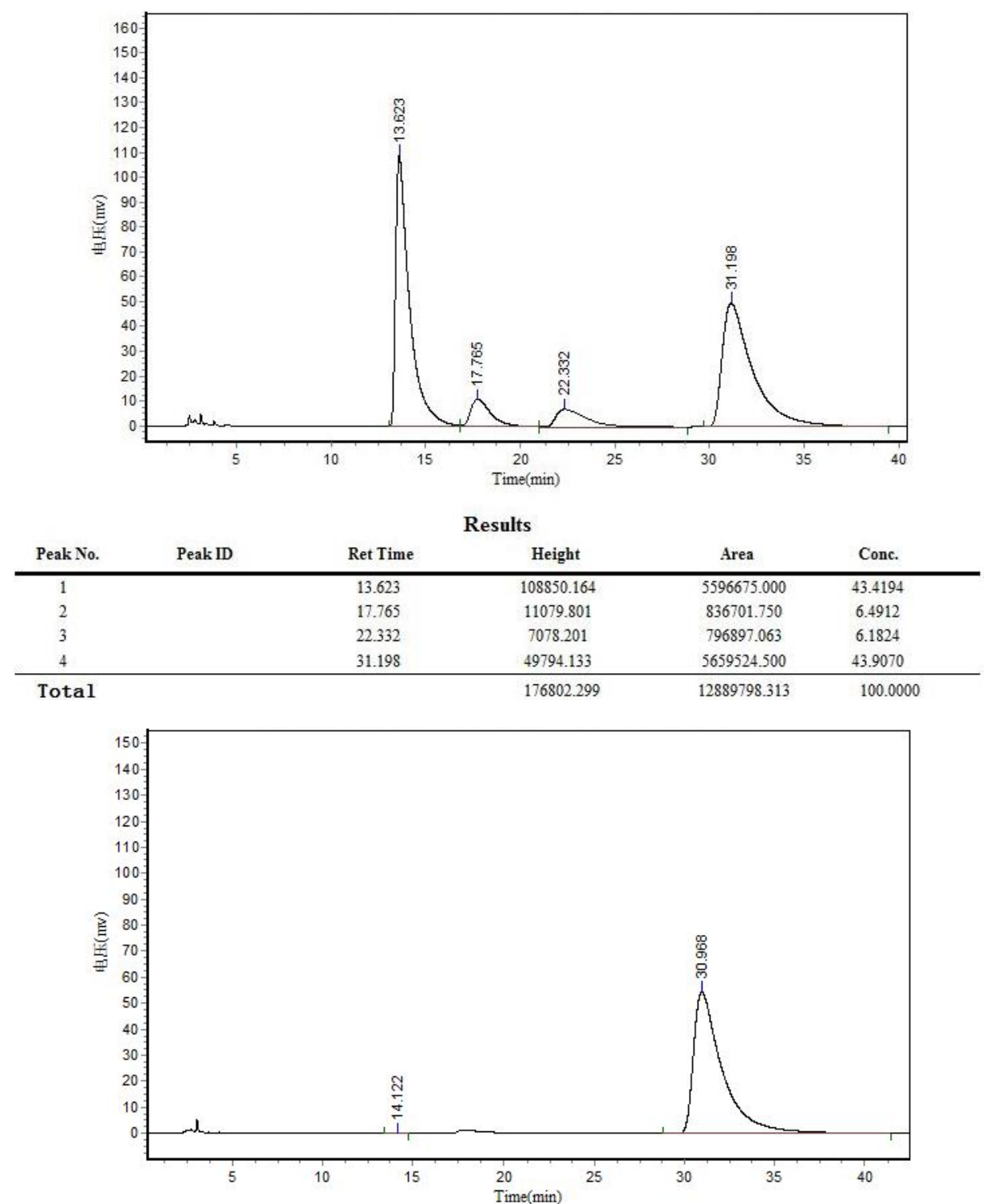

\begin{tabular}{|c|c|c|c|c|c|}
\hline \multicolumn{6}{|c|}{ Results } \\
\hline Peak No. & Peak ID & Ret Time & Height & Area & Conc. \\
\hline 1 & & 14.122 & 177.842 & 7682.400 & 0.1254 \\
\hline 2 & & 30.968 & 54587.484 & 6118932.000 & 99.8746 \\
\hline Total & & & 54765.326 & 6126614.400 & 100.000 \\
\hline
\end{tabular}




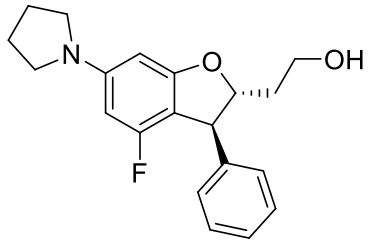

Pale yellow oil, $28.8 \mathrm{mg}, 88 \%$ yield, $97: 3 \mathrm{dr}, 99 \%$ ee; $[\alpha]_{\mathrm{D}}^{20}=51.9(c$ $\left.1.0, \mathrm{CHCl}_{3}\right) ;{ }^{1} \mathrm{H} \mathrm{NMR}\left(400 \mathrm{MHz}, \mathrm{CDCl}_{3}\right) \delta 7.33-7.20(\mathrm{~m}, 5 \mathrm{H}), 5.90(\mathrm{~s}$, $1 \mathrm{H}), 5.79\left(\mathrm{~d}, J_{\mathrm{HF}}=11.6 \mathrm{~Hz}, 1 \mathrm{H}\right), 4.75-4.70(\mathrm{~m}, 1 \mathrm{H}), 4.32(\mathrm{~d}, J=6.0 \mathrm{~Hz}$ $1 \mathrm{H}), 3.87(\mathrm{t}, J=6.0 \mathrm{~Hz}, 2 \mathrm{H}), 3.24(\mathrm{t}, J=6.0 \mathrm{~Hz}, 4 \mathrm{H}), 2.14-2.00(\mathrm{~m}$, $6 \mathrm{H}), 1.87$ (brs, $1 \mathrm{H}) ;{ }^{13} \mathrm{C}$ NMR $\left(100 \mathrm{MHz}, \mathrm{CDCl}_{3}\right) \delta 162.5$ (d, $J=10.1$ $\mathrm{Hz}), 160.6(\mathrm{~d}, J=242.8 \mathrm{~Hz}), 150.6(\mathrm{~d}, J=12.1 \mathrm{~Hz}), 142.6,129.0,127.7,127.3,103.2(\mathrm{~d}, J=20.7$ $\mathrm{Hz}), 92.1$ (d, $J=24.7 \mathrm{~Hz}), 91.6,89.6(\mathrm{~d}, J=2.4 \mathrm{~Hz}), 60.5,52.4,48.2,37.9,25.8$; HRMS (ESI) for $\mathrm{C}_{20} \mathrm{H}_{23} \mathrm{FNO}_{2}[\mathrm{M}+\mathrm{H}]^{+}$: calcd 328.1707, found 328.1704.

HPLC: Chiralpak IG+AD-H column $(250 \mathrm{~mm})$; detected at $254 \mathrm{~nm}$; hexane $/ i$-propanol $=95 / 5$; flow $=1.0 \mathrm{~mL} / \mathrm{min}$; Retention time: $19.8 \mathrm{~min}, 31.8 \mathrm{~min}$ (major).

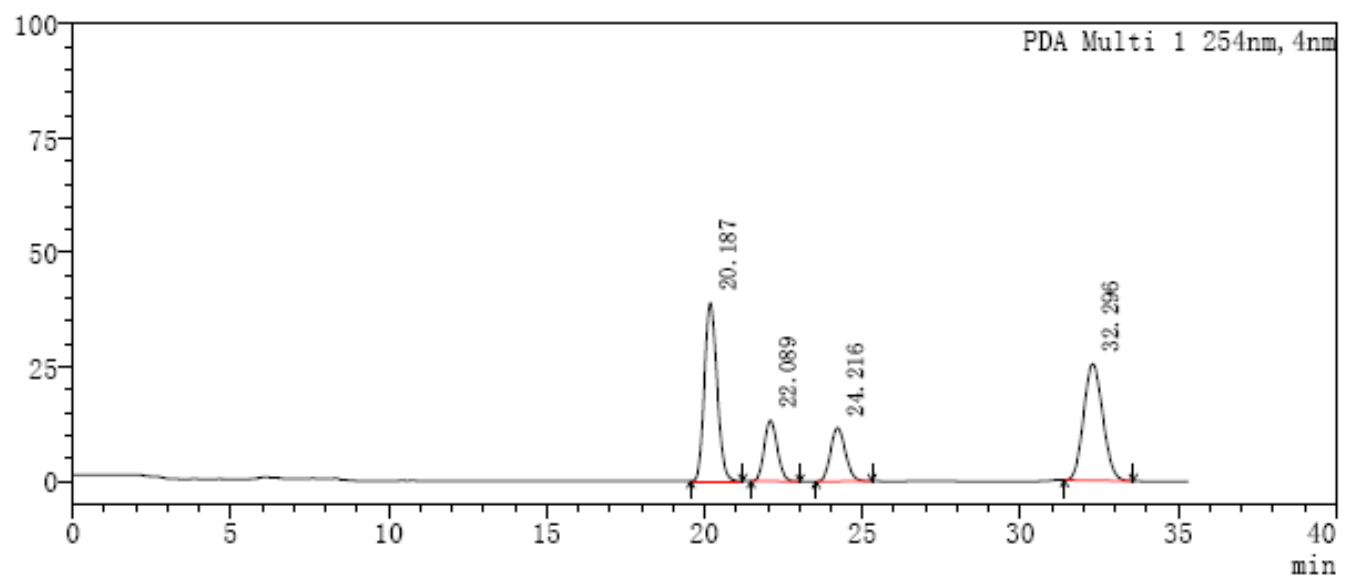

PDA Ch1 254nm
\begin{tabular}{|c|c|c|c|c|}
\hline Number & Retention & Area & Height & Area\% \\
\hline 1 & 20.187 & 1086348 & 38992 & 36.987 \\
\hline 2 & 22.089 & 383206 & 13318 & 13.047 \\
\hline 3 & 24.216 & 384531 & 11705 & 13.092 \\
\hline 4 & 32.296 & 1083056 & 25508 & 36.875 \\
\hline
\end{tabular}

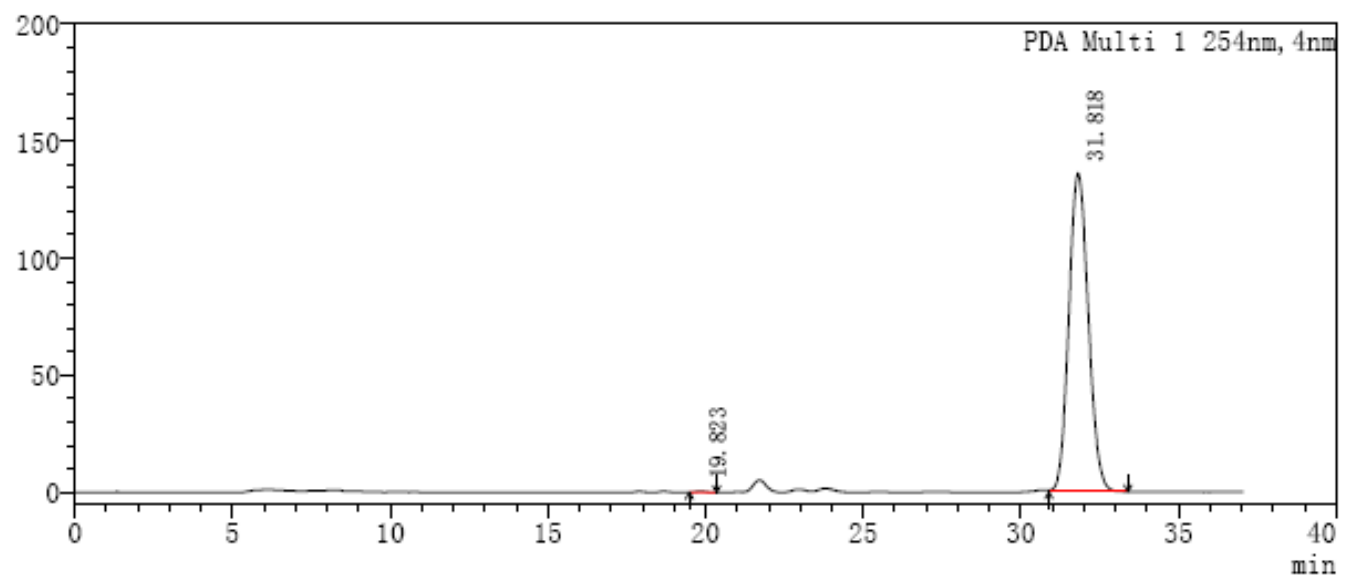

PDA Ch1 $254 \mathrm{~nm}$
\begin{tabular}{|c|c|c|c|c|}
\hline Number & Retention & Area & Height & Area\% \\
\hline 1 & 19.823 & 8652 & 353 & 0.147 \\
\hline 2 & 31.818 & 5863849 & 136355 & 99.853 \\
\hline
\end{tabular}


2-((2R,3S)-5-fluoro-3-phenyl-6-(pyrrolidin-1-yl)-2,3-dihydrobenzofuran-2-yl)ethanol (4aj)

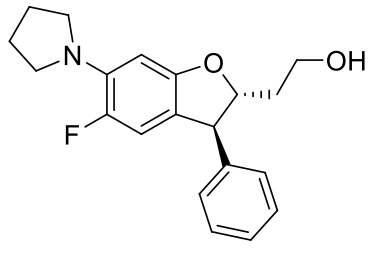

Pale yellow oil, $29.6 \mathrm{mg}, 91 \%$ yield, $98: 2 \mathrm{dr}, 99 \%$ ee; $[\alpha]_{\mathrm{D}^{20}}=37.2(c$ 0.87, $\left.\mathrm{CHCl}_{3}\right) ;{ }^{1} \mathrm{H}$ NMR $\left(400 \mathrm{MHz}, \mathrm{CDCl}_{3}\right) \delta$ 7.34-7.19 (m, 5H), 6.58 $\left(\mathrm{d}, J^{\mathrm{HF}}=12.8 \mathrm{~Hz}, 1 \mathrm{H}\right), 6.22\left(\mathrm{~d}, J^{\mathrm{HF}}=7.2 \mathrm{~Hz}, 1 \mathrm{H}\right), 4.71-4.66(\mathrm{~m}, 1 \mathrm{H})$, $4.22(\mathrm{~d}, J=8.4 \mathrm{~Hz}, 1 \mathrm{H}), 3.86(\mathrm{t}, J=5.6 \mathrm{~Hz}, 2 \mathrm{H}), 3.36-3.34(\mathrm{~m}, 4 \mathrm{H})$, 2.15-1.92 (m, 7H); ${ }^{13} \mathrm{C}$ NMR $\left(100 \mathrm{MHz}, \mathrm{CDCl}_{3}\right) \delta 156.2,147.9(\mathrm{~d}, J=$ $231.9 \mathrm{~Hz}), 142.5,138.2$ (d, $J=11.7 \mathrm{~Hz}), 129.2,128.4,127.5,117.7$ (d, $J=7.7 \mathrm{~Hz}), 112.7$ (d, $J=$ $24.2 \mathrm{~Hz}$ ), 96.8 (d, $J=4.9 \mathrm{~Hz}$ ), 91.7, 60.8, 55.2, 50.3 (d, $J=5.3 \mathrm{~Hz}$ ), 37.5, 25.6; HRMS (ESI) for $\mathrm{C}_{20} \mathrm{H}_{23} \mathrm{FNO}_{2}[\mathrm{M}+\mathrm{H}]^{+}$: calcd 328.1707, found 328.1708.

HPLC: Chiralpak IG-3 column $(250 \mathrm{~mm})$; detected at $254 \mathrm{~nm}$; hexane $/ i$-propanol $=80 / 20$; flow $=$ $0.5 \mathrm{~mL} / \mathrm{min}$; Retention time: $17.6 \mathrm{~min}, 24.2 \mathrm{~min}$ (major).

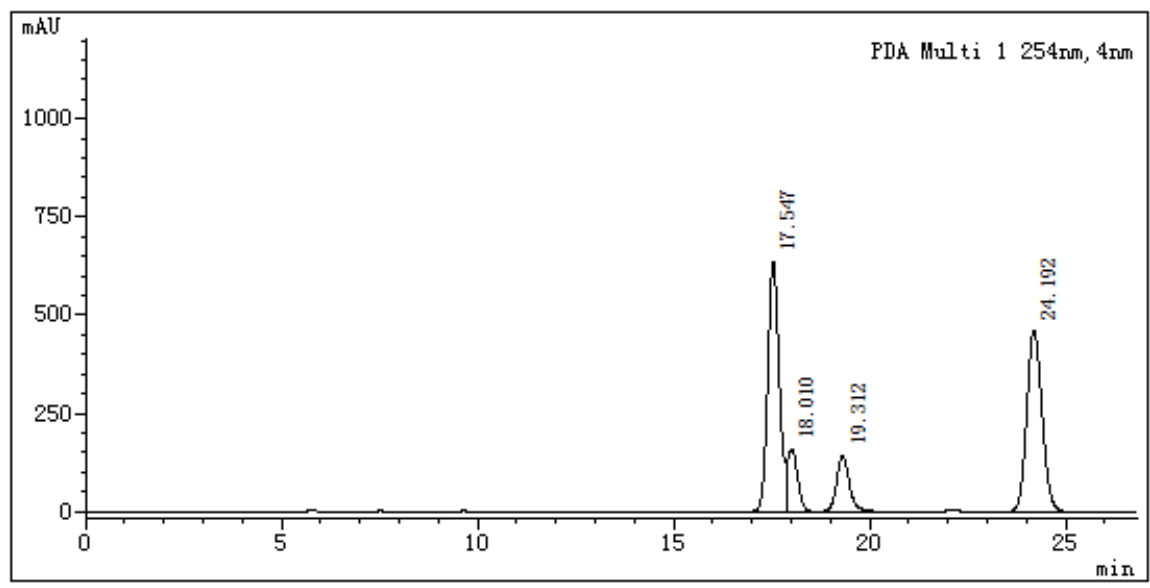

\begin{tabular}{|c|c|c|c|c|}
\hline PDA Ch1 254nm & \multicolumn{3}{l|}{} \\
\hline Humber & Retention & Area & Height & Area\& \\
\hline 1 & 17.547 & 12900816 & 636909 & 40.941 \\
\hline 2 & 18.010 & 2874233 & 158122 & 9.121 \\
\hline 3 & 19.312 & 3228412 & 141088 & 10.245 \\
\hline 4 & 24.192 & 12507169 & 461187 & 39.692 \\
\hline
\end{tabular}

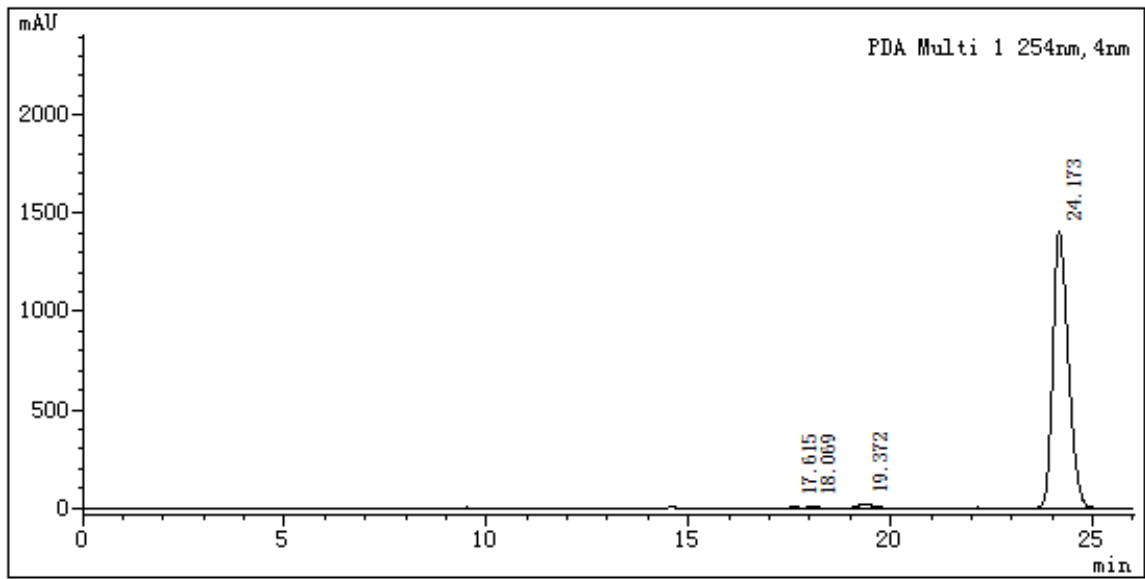

\begin{tabular}{|c|c|c|c|c|}
\hline PDA Ch1 254nm & \multicolumn{3}{|c|}{} \\
\hline Humber & Retention & krea & Height & krea\% \\
\hline 1 & 17.615 & 54081 & 2916 & 0.143 \\
\hline 2 & 18.069 & 96112 & 4969 & 0.253 \\
\hline 3 & 19.372 & 532549 & 23083 & 1.404 \\
\hline 4 & 24.173 & 37237569 & 1406585 & 98.200 \\
\hline
\end{tabular}




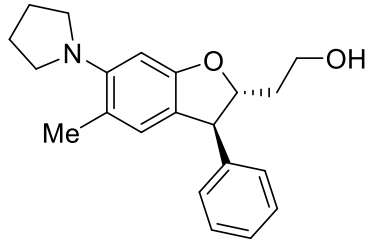

Pale yellow oil, $27.2 \mathrm{mg}, 84 \%$ yield, $98: 2 \mathrm{dr}, 98 \%$ ee; $[\alpha]_{\mathrm{D}}{ }^{20}=17.3(c$ $\left.1.1, \mathrm{CHCl}_{3}\right) ;{ }^{1} \mathrm{H}$ NMR $\left(400 \mathrm{MHz}, \mathrm{CDCl}_{3}\right) \delta 7.35-7.22(\mathrm{~m}, 5 \mathrm{H}), 6.68(\mathrm{~s}$, $1 \mathrm{H}), 6.47(\mathrm{~s}, 1 \mathrm{H}), 4.71-4.66(\mathrm{~m}, 1 \mathrm{H}), 4.23(\mathrm{~d}, J=8.0 \mathrm{~Hz}, 1 \mathrm{H}), 3.86(\mathrm{t}$, $J=6.0 \mathrm{~Hz}, 2 \mathrm{H}), 3.16-3.10(\mathrm{~m}, 4 \mathrm{H}), 2.19(\mathrm{~s}, 3 \mathrm{H}), 2.16-2.02(\mathrm{~m}, 3 \mathrm{H})$, 1.96-1.91 (m, 4H); ${ }^{13} \mathrm{C}$ NMR $\left(100 \mathrm{MHz}, \mathrm{CDCl}_{3}\right) \delta 158.6,142.8,129.1$, 128.6, 127.9, 127.4, 121.7, 98.1, 91.4, 60.9, 55.2, 51.5, 37.5, 25.2, 20.5; HRMS (ESI) for $\mathrm{C}_{21} \mathrm{H}_{26} \mathrm{NO}_{2}[\mathrm{M}+\mathrm{H}]^{+}$: calcd 324.1958, found 324.1957.

HPLC: Chiralpak OD-H column $(250 \mathrm{~mm})$; detected at $254 \mathrm{~nm}$; hexane $/ i$-propanol $=90 / 10$; flow $=0.7 \mathrm{~mL} / \mathrm{min}$; Retention time: $9.9 \mathrm{~min}$ (major), $14.5 \mathrm{~min}$.

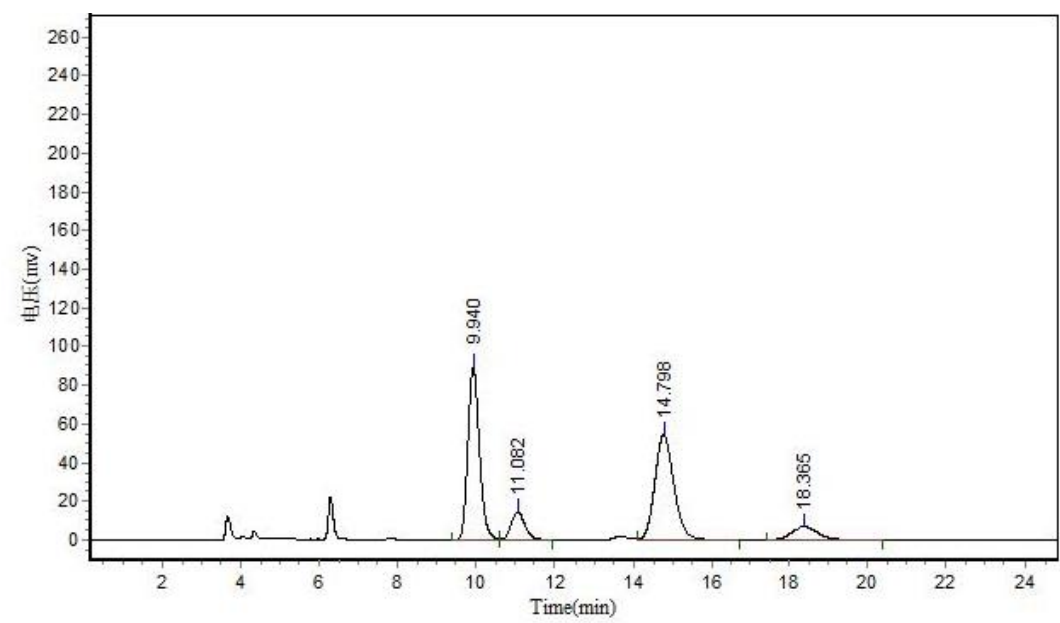

\begin{tabular}{|c|c|c|c|c|c|}
\hline \multicolumn{6}{|c|}{ Results } \\
\hline Peak No. & Peak ID & Ret Time & Height & Area & Conc. \\
\hline$\overline{1}$ & & 9.940 & 89352.023 & 1839366.250 & 42.2830 \\
\hline 2 & & 11.082 & 14534.247 & 358042.469 & 8.2306 \\
\hline 3 & & 14.798 & 54232.996 & 1843779.000 & 42.3844 \\
\hline 4 & & 18.365 & 6808.364 & 308944.000 & 7.1019 \\
\hline Total & & & 164927.630 & 4350131.719 & 100.000 \\
\hline
\end{tabular}
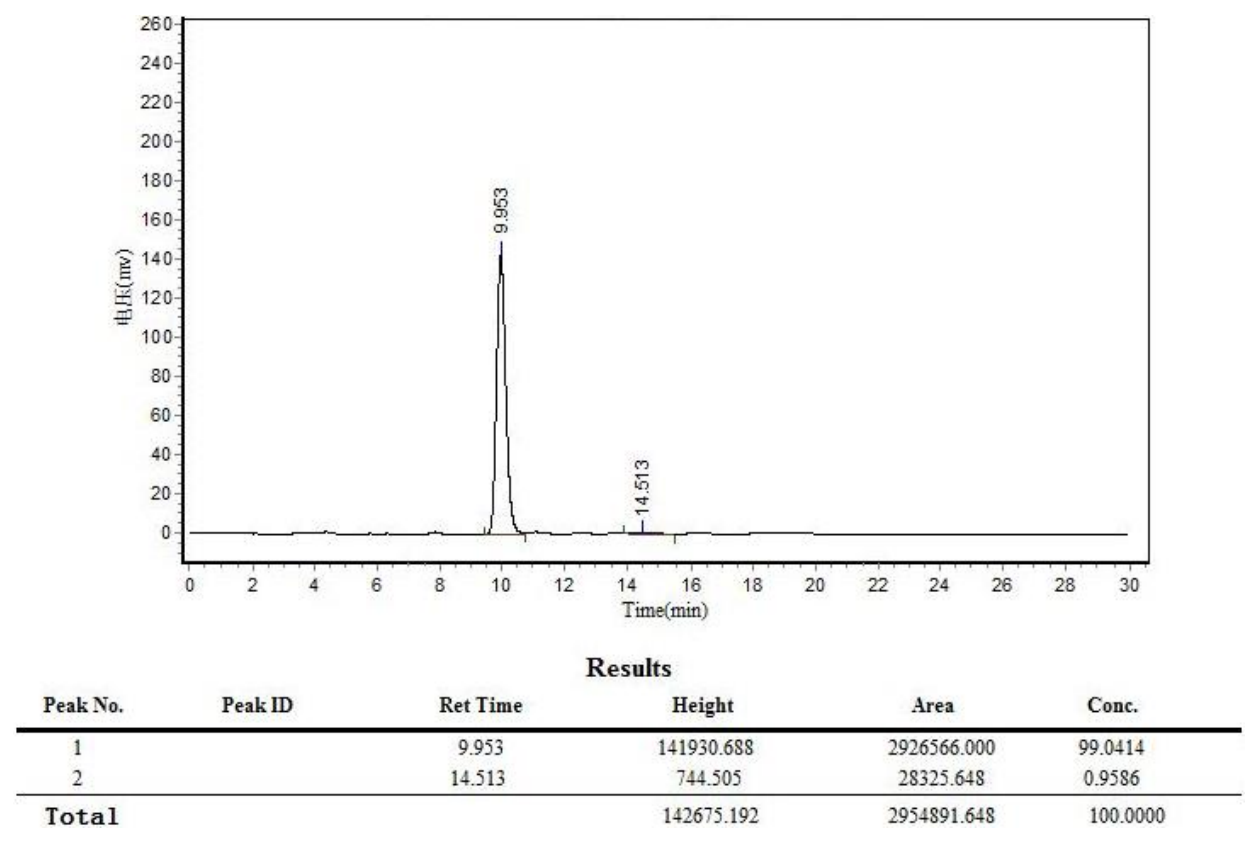
<smiles>O=[W]c1c(N2CCCC2)ccc2c1OC(CCO)C2c1ccccc1</smiles>

Pale yellow oil, $29.4 \mathrm{mg}, 91 \%$ yield, $99: 1 \mathrm{dr}, 99 \%$ ee; $[\alpha]_{\mathrm{D}}^{20}=-17.7(c$ $\left.1.0, \mathrm{CHCl}_{3}\right) ;{ }^{1} \mathrm{H}$ NMR $\left(400 \mathrm{MHz}, \mathrm{CDCl}_{3}\right) \delta 7.34-7.22(\mathrm{~m}, 5 \mathrm{H}), 6.68(\mathrm{~d}$ $J=8.0 \mathrm{~Hz}, 1 \mathrm{H}), 6.44(\mathrm{~d}, J=8.0 \mathrm{~Hz}, 1 \mathrm{H}), 4.72-4.67(\mathrm{~m}, 1 \mathrm{H}), 4.27$ (d, $J$ $=8.4 \mathrm{~Hz}, 1 \mathrm{H}), 3.89(\mathrm{t}, J=6.0 \mathrm{~Hz}, 2 \mathrm{H}), 3.21-3.13(\mathrm{~m}, 4 \mathrm{H}), 2.22(\mathrm{~s}, 3 \mathrm{H})$, 2.18-2.03 (m, 3H), 1.94-1.91 (m, 4H); $\left.{ }^{13} \mathrm{C} \mathrm{NMR} \mathrm{(100} \mathrm{MHz,} \mathrm{CDCl}_{3}\right) \delta$ 159.6, 150.6, 142.8, 129.1, 128.5, 127.4, 122.1, 122.0, 111.0, 109.0, 91.1, 61.1, 55.5, 51.7, 37.5, 25.2, 12.9; HRMS (ESI) for $\mathrm{C}_{21} \mathrm{H}_{26} \mathrm{NO}_{2}[\mathrm{M}+\mathrm{H}]^{+}$: calcd 324.1958, found 324.1953.

HPLC: Chiralpak AD-H column $(250 \mathrm{~mm})$; detected at $254 \mathrm{~nm}$; hexane $/ i$-propanol $=97 / 3$; flow $=$ $0.5 \mathrm{~mL} / \mathrm{min}$; Retention time: $26.7 \mathrm{~min}, 40.5 \mathrm{~min}$ (major).

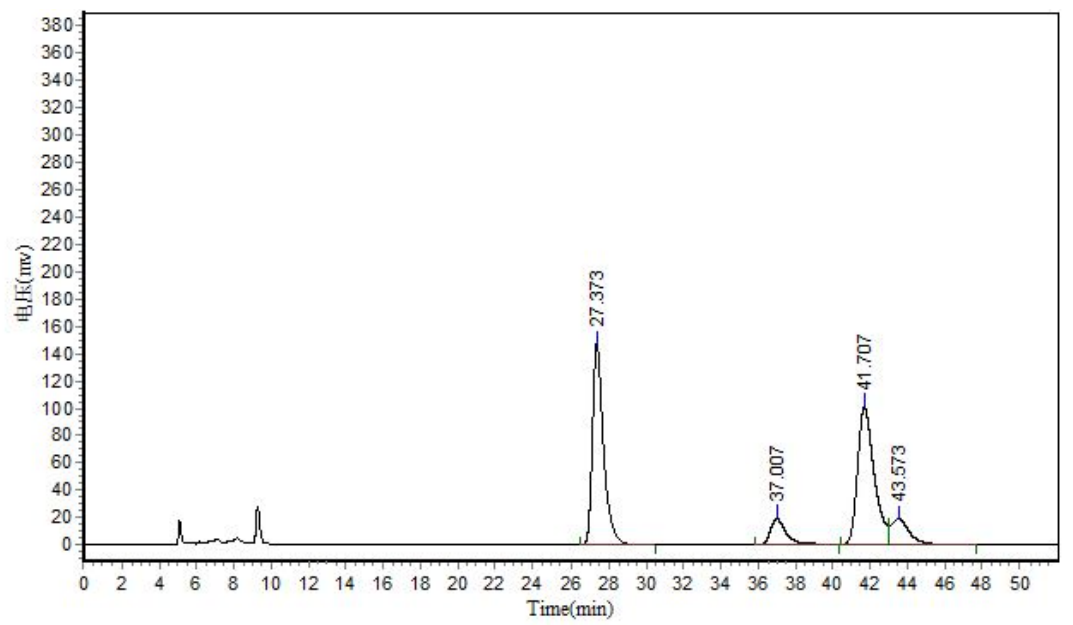

Results

\begin{tabular}{|c|c|c|c|c|c|}
\hline \\
\hline Peak No. & Peak ID & Ret Time & Height & Area & Conc. \\
\hline 1 & & 27.373 & 147419.938 & 6127288.000 & 41.0409 \\
\hline 2 & & 37.007 & 19046.268 & 1179259.375 & 7.8987 \\
\hline 3 & & 41.707 & 100150.469 & 6330588.000 & 42.4026 \\
\hline 4 & & 43.573 & 19072.000 & 1292577.750 & 8.6578 \\
\hline Total & & & 285688.674 & 14929713.125 & 100.0000 \\
\hline
\end{tabular}

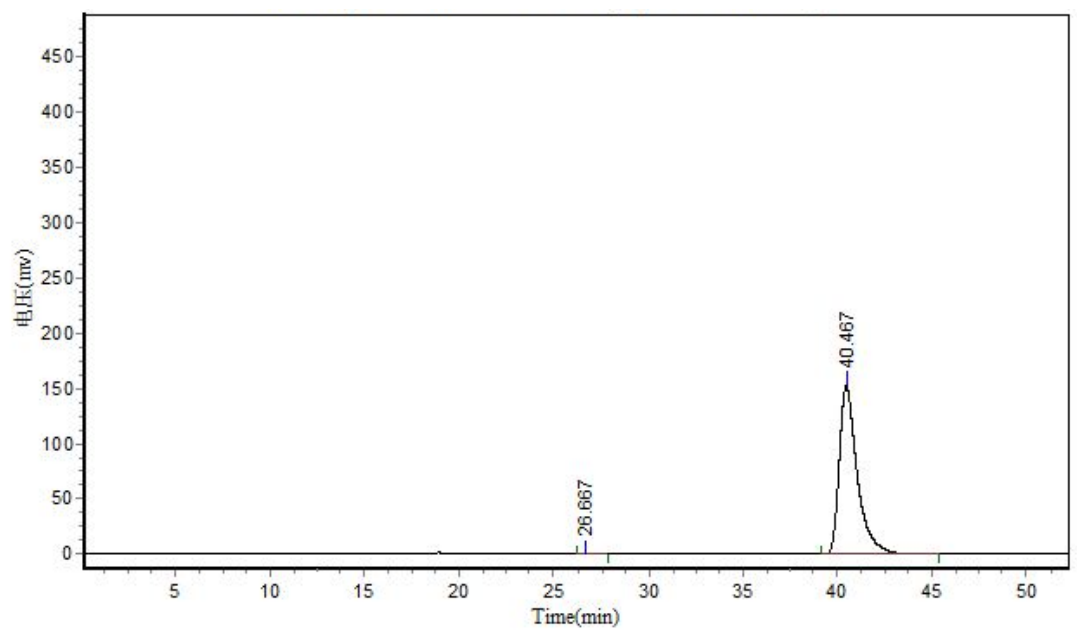

Results

\begin{tabular}{|c|c|c|c|c|c|}
\hline \multicolumn{6}{|c|}{. } \\
\hline Peak No. & Peak ID & Ret Time & Height & Area & Conc. \\
\hline 1 & & 26.667 & 411.139 & 16479.150 & 0.1626 \\
\hline 2 & & 40.467 & 151687.438 & 10119498.000 & 99.8374 \\
\hline Total & & & 152098.577 & 10135977.150 & 100.0000 \\
\hline \multicolumn{6}{|c|}{ S53 } \\
\hline
\end{tabular}




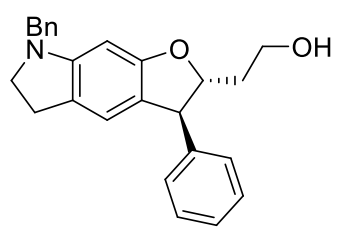

Pale yellow solid, $34.2 \mathrm{mg}$, 92\% yield, 99:1 dr, 99\% ee; m.p. 123.3-125.6 ${ }^{\circ} \mathrm{C},[\alpha]_{\mathrm{D}}{ }^{20}=24.1\left(c 1.0, \mathrm{CHCl}_{3}\right) ;{ }^{1} \mathrm{H} \mathrm{NMR}\left(400 \mathrm{MHz}, \mathrm{CDCl}_{3}\right)$ $\delta$ 7.39-7.23 (m, 10H), $6.64(\mathrm{~s}, 1 \mathrm{H}), 6.08(\mathrm{~s}, 1 \mathrm{H}), 4.72-4.67(\mathrm{~m}, 1 \mathrm{H})$, 4.23-4.19 (m, 3H), 3.85 (d, $J=5.6 \mathrm{~Hz}, 2 \mathrm{H}), 3.36-3.30$ (m, 2H), 2.84 (t, $J$ $=8.0 \mathrm{~Hz}, 2 \mathrm{H}), 2.12-2.01(\mathrm{~m}, 3 \mathrm{H}) ;{ }^{13} \mathrm{C} \mathrm{NMR}\left(100 \mathrm{MHz}, \mathrm{CDCl}_{3}\right) \delta 159.7$, 153.8, 143.2, 138.6, 129.1, 128.9, 128.5, 128.2, 127.5, 127.4, 122.9, 121.1, 120.7, 91.6, 90.6, 60.9, 55.1, 54.8, 54.1, 37.5, 28.3; HRMS (ESI) for $\mathrm{C}_{25} \mathrm{H}_{26} \mathrm{NO}_{2}[\mathrm{M}+\mathrm{H}]^{+}$: calcd 372.1958, found 372.1954 .

HPLC: Chiralpak AD-H column $(250 \mathrm{~mm})$; detected at $254 \mathrm{~nm}$; hexane $/ i$-propanol $=90 / 10$; flow $=0.7 \mathrm{~mL} / \mathrm{min}$; Retention time: $11.5 \mathrm{~min}, 13.6 \mathrm{~min}$ (major).
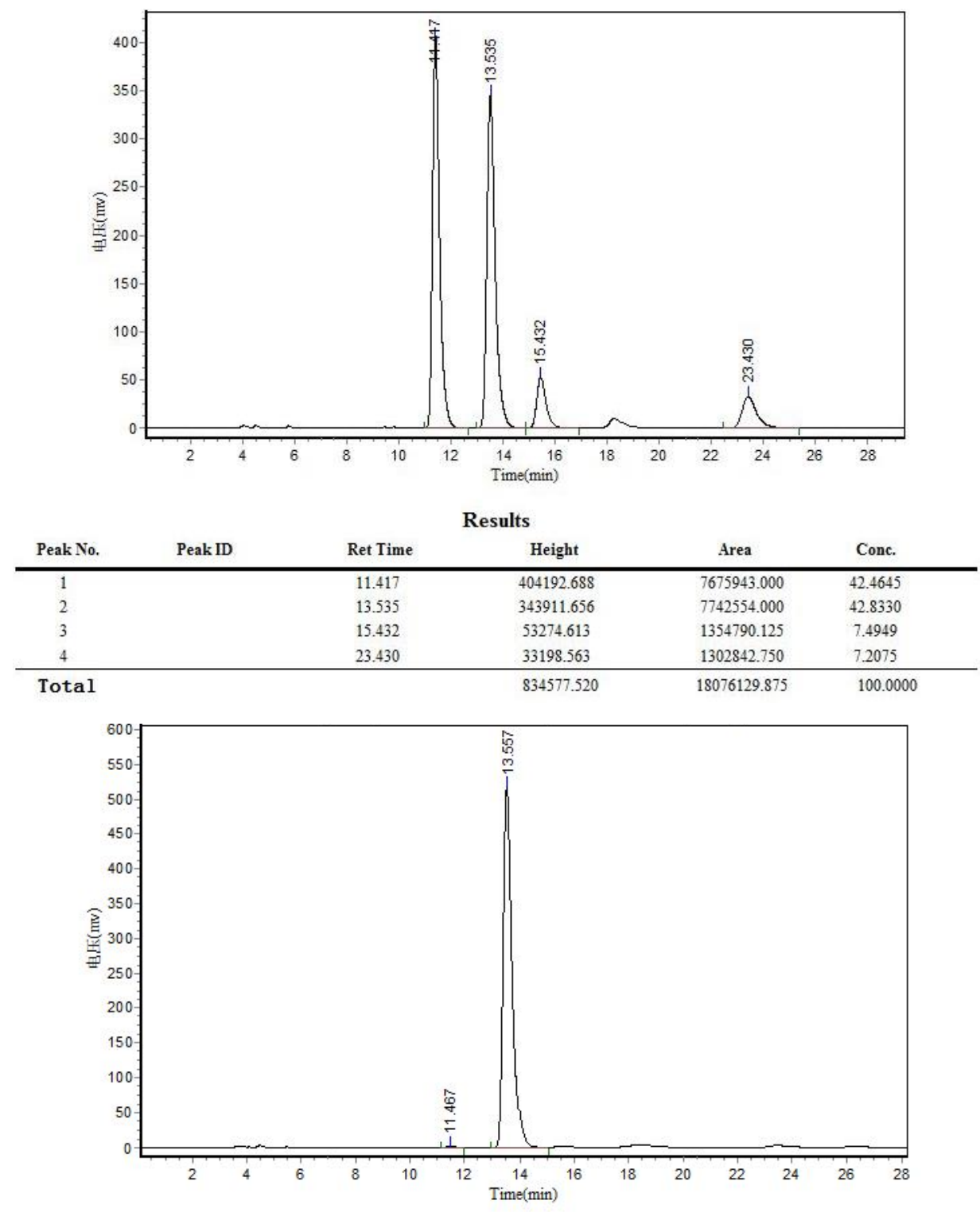

Results

\begin{tabular}{|c|c|c|c|c|c|}
\hline \\
\hline Peak No. & Peak ID & Ret Time & Height & Area & Conc. \\
\hline 1 & & 11.467 & 1085.240 & 18685.549 & 0.1595 \\
\hline 2 & & 13.557 & 517059.938 & 11695757.000 & 99.8405 \\
\hline Total & & & 518145.177 & 11714442.549 & 100.0000 \\
\hline
\end{tabular}


2-((2R,3S)-6-benzyl-3-phenyl-3,6,7,8-tetrahydro-2H-furo[2,3-e]indol-2-yl)ethanol (4an)

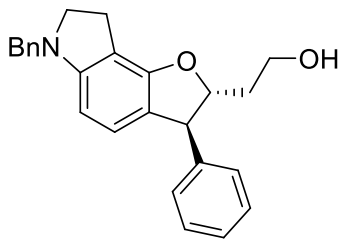

Colorless oil, $31.8 \mathrm{mg}, 86 \%$ yield, $98: 2 \mathrm{dr}, 99 \%$ ee; $[\alpha]_{\mathrm{D}}{ }^{20}=18.1(c 0.68$, $\left.\mathrm{CHCl}_{3}\right) ;{ }^{1} \mathrm{H} \mathrm{NMR}\left(400 \mathrm{MHz}, \mathrm{CDCl}_{3}\right) \delta$ 7.37-7.23 (m, 10H), 6.64 (d, $J=$ $7.6 \mathrm{~Hz}, 1 \mathrm{H}), 6.08(\mathrm{~d}, J=8.0 \mathrm{~Hz}, 1 \mathrm{H}), 4.74-4.69(\mathrm{~m}, 1 \mathrm{H}), 4.24(\mathrm{~s}, 2 \mathrm{H})$, $4.22(\mathrm{~d}, J=8.4 \mathrm{~Hz}, 1 \mathrm{H}), 3.88(\mathrm{q}, J=5.2 \mathrm{~Hz}, 2 \mathrm{H}), 3.40-3.34(\mathrm{~m}, 2 \mathrm{H})$, $2.96(\mathrm{t}, J=6.0 \mathrm{~Hz}, 2 \mathrm{H}), 2.15-2.02(\mathrm{~m}, 3 \mathrm{H}) ;{ }^{13} \mathrm{C} \mathrm{NMR}\left(100 \mathrm{MHz}, \mathrm{CDCl}_{3}\right)$ $\delta 156.0,155.0,142.9,138.8,129.1,128.8,128.6,128.2,127.4,127.3,124.2,121.3,110.1,100.5$, 91.9, 60.9, 54.9, 54.4, 54.2, 37.5, 25.3; HRMS (ESI) for $\mathrm{C}_{25} \mathrm{H}_{26} \mathrm{NO}_{2}[\mathrm{M}+\mathrm{H}]^{+}$: calcd 372.1958, found 372.1955 .

HPLC: Chiralpak AD-H column $(250 \mathrm{~mm})$; detected at $254 \mathrm{~nm}$; hexane $/$ - propanol $=90 / 10$; flow $=0.7 \mathrm{~mL} / \mathrm{min}$; Retention time: $15.0 \mathrm{~min}, 20.6 \mathrm{~min}$ (major).

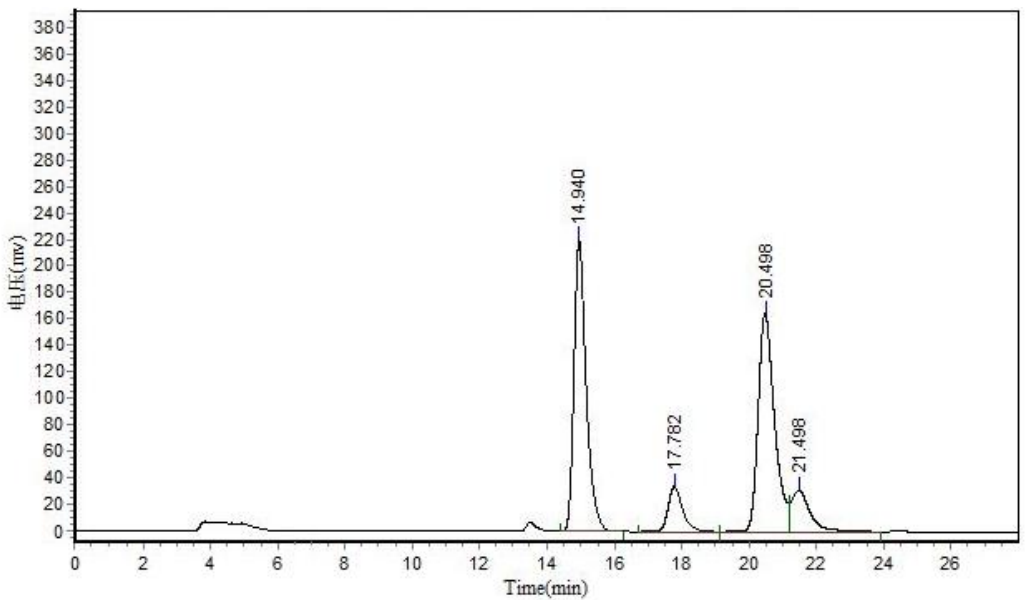

Results

\begin{tabular}{|c|c|c|c|c|c|}
\hline Peak No. & Peak ID & Ret Time & Height & Area & Conc. \\
\hline 1 & & 14.940 & 219500.828 & 5587405.500 & 41.4482 \\
\hline 2 & & 17.782 & 34325.629 & 1047302.688 & 7.7690 \\
\hline 3 & & 20.498 & 164809.859 & 5611852.000 & 41.6296 \\
\hline 4 & & 21.498 & 31702.977 & 1233889.500 & 9.1532 \\
\hline Total & & & 450339.293 & 13480449.688 & 100.0000 \\
\hline
\end{tabular}

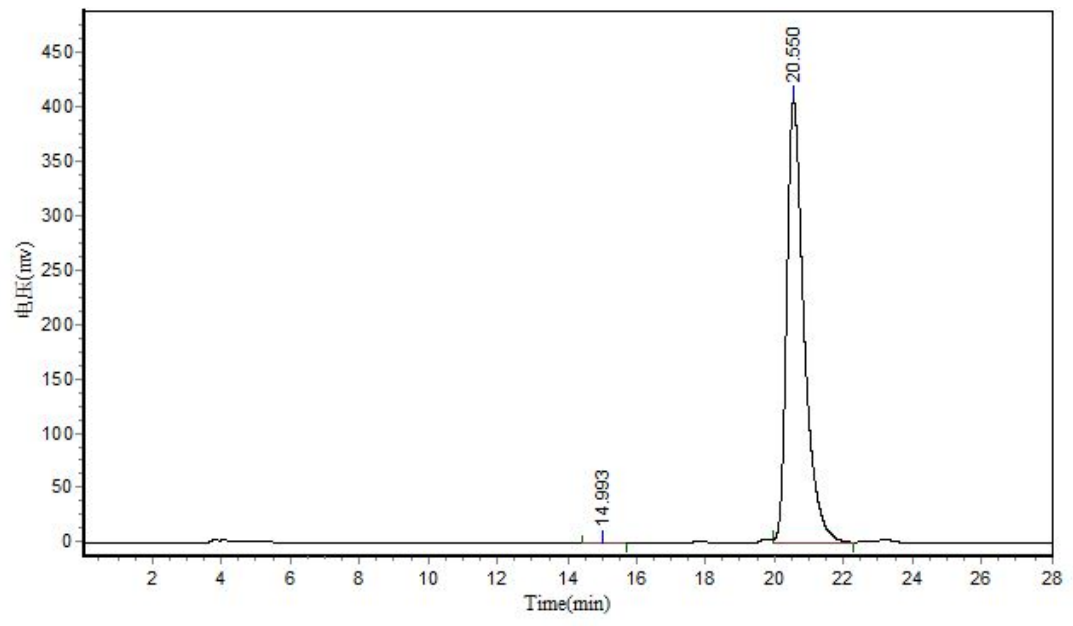

Results

\begin{tabular}{|c|c|c|c|c|c|}
\hline \multicolumn{6}{|c|}{ Results } \\
\hline Peak No. & Peak ID & Ret Time & Height & Area & Conc. \\
\hline 1 & & 14.993 & 590.278 & 15284.805 & 0.1068 \\
\hline 2 & & 20.550 & 408112.469 & 14297692.000 & 99.8932 \\
\hline Total & & & 408702.747 & 14312976.805 & 100.0000 \\
\hline
\end{tabular}




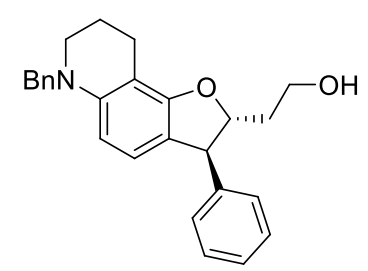

Pale yellow solid, $33.9 \mathrm{mg}, 88 \%$ yield, 97:3 dr, 99\% ee; m.p. 86.2-88.5 ${ }^{\circ} \mathrm{C},[\alpha]_{\mathrm{D}}^{20}=-17.4\left(c 1.0, \mathrm{CHCl}_{3}\right){ }^{1} \mathrm{H}$ NMR $\left(400 \mathrm{MHz}, \mathrm{CDCl}_{3}\right) \delta$ 7.33-7.22 (m, 10H), $6.55(\mathrm{~d}, J=8.0 \mathrm{~Hz}, 1 \mathrm{H}), 6.09(\mathrm{~d}, J=8.0 \mathrm{~Hz}, 1 \mathrm{H})$, 4.71-4.66 (m, 1H), 4.51-4.40 (m, 2H), $4.22(\mathrm{~d}, J=8.4 \mathrm{~Hz}, 1 \mathrm{H}), 3.89$ (t, $J=5.6 \mathrm{~Hz}, 2 \mathrm{H}), 3.35(\mathrm{t}, J=5.2 \mathrm{~Hz}, 2 \mathrm{H}), 2.81-2.68(\mathrm{~m}, 2 \mathrm{H}), 2.18-1.99$ $(\mathrm{m}, 5 \mathrm{H}) ;{ }^{13} \mathrm{C} \mathrm{NMR}\left(100 \mathrm{MHz}, \mathrm{CDCl}_{3}\right) \delta 158.0,146.8,143.0,139.5$, $129.0,128.9,128.5,127.3,127.1,126.9,123.0,117.6,104.8,104.5,91.8,61.3,56.3,55.3,50.1$, 37.5, 22.1, 21.6; HRMS (ESI) for $\mathrm{C}_{26} \mathrm{H}_{28} \mathrm{NO}_{2}[\mathrm{M}+\mathrm{H}]^{+}$: calcd 386.2115, found 386.2119.

HPLC: Chiralpak AD-H column $(250 \mathrm{~mm})$; detected at $254 \mathrm{~nm}$; hexane $/ i$-propanol $=95 / 5$; flow $=$ $0.7 \mathrm{~mL} / \mathrm{min}$; Retention time: $20.2 \mathrm{~min}, 23.6 \mathrm{~min}$ (major).

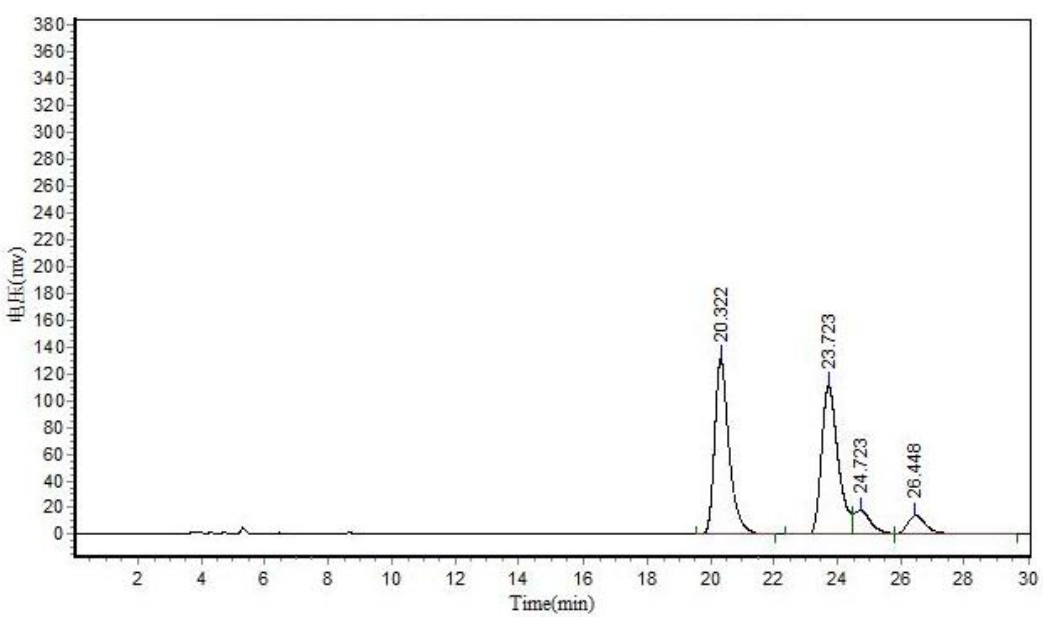

\begin{tabular}{|c|c|c|c|c|c|}
\hline \multicolumn{6}{|c|}{ Results } \\
\hline Peak No. & Peak ID & Ret Time & Height & Area & Conc. \\
\hline 1 & & 20.322 & 130674.508 & 4136656.750 & 43.4659 \\
\hline 2 & & 23.723 & 111283.328 & 4068593.500 & 42.7507 \\
\hline 3 & & 24.723 & 18215.051 & 681352.875 & 7.1593 \\
\hline 4 & & 26.448 & 14430.193 & 630422.250 & 6.6242 \\
\hline Total & & & 274603.080 & 9517025.375 & 100.000 \\
\hline
\end{tabular}

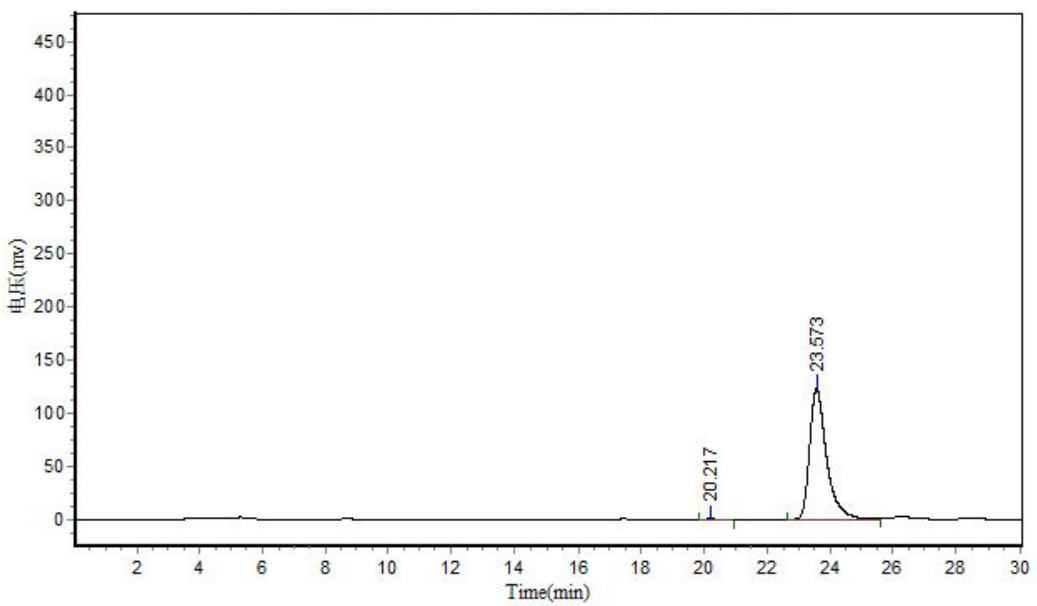

\begin{tabular}{|c|c|c|c|c|c|}
\hline \multicolumn{6}{|c|}{ Results } \\
\hline Peak No. & Peak ID & Ret Time & Height & Area & Conc. \\
\hline 1 & & 20.217 & 306.721 & 8768.950 & 0.1899 \\
\hline 2 & & 23.573 & 122681.258 & 4608195.000 & 99.8101 \\
\hline Total & & & 122987.979 & 4616963.950 & 100.000 \\
\hline
\end{tabular}


<smiles>OCC[C@H]1Oc2cc3c(cc2[C@@H]1c1ccccc1)CCC[NH2+]3</smiles>

Pale yellow solid, $35.0 \mathrm{mg}$, 91\% yield, 99:1 dr, 99\% ee; m.p. 100.6-102. ${ }^{\circ} \mathrm{C},[\alpha]_{\mathrm{D}}^{20}=41.5\left(c 1.0, \mathrm{CHCl}_{3}\right) ;{ }^{1} \mathrm{H} \mathrm{NMR}\left(400 \mathrm{MHz}, \mathrm{CDCl}_{3}\right)$ $\delta$ 7.36-7.24 (m, 10H), $6.56(\mathrm{~s}, 1 \mathrm{H}), 6.08(\mathrm{~s}, 1 \mathrm{H}), 4.64-4.59(\mathrm{~m}, 1 \mathrm{H})$, $4.52-4.43(\mathrm{~m}, 2 \mathrm{H}), 4.20(\mathrm{~d}, J=8.8 \mathrm{~Hz}, 1 \mathrm{H}), 3.82(\mathrm{t}, J=6.0 \mathrm{~Hz}, 2 \mathrm{H})$, $3.36(\mathrm{t}, J=5.2 \mathrm{~Hz}, 2 \mathrm{H}), 2.69(\mathrm{t}, J=6.0 \mathrm{~Hz}, 2 \mathrm{H}), 2.09-1.96(\mathrm{~m}, 5 \mathrm{H}) ;{ }^{13} \mathrm{C}$ NMR $\left(100 \mathrm{MHz}, \mathrm{CDCl}_{3}\right) \delta 159.4,146.6,143.0,139.1,129.0,128.9,128.6,127.3,127.2,126.9$, 125.4, 117.4, 115.4, 93.1, 91.2, 60.9, 55.9, 55.1, 50.3, 37.4, 28.3, 23.0; HRMS (ESI) for $\mathrm{C}_{26} \mathrm{H}_{28} \mathrm{NO}_{2}[\mathrm{M}+\mathrm{H}]^{+}$: calcd 386.2115, found 386.2114.

HPLC: Chiralpak AD-H column $(250 \mathrm{~mm})$; detected at $254 \mathrm{~nm}$; hexane $/ i$-propanol $=90 / 10$; flow $=0.7 \mathrm{~mL} / \mathrm{min}$; Retention time: $9.6 \mathrm{~min}, 10.3 \mathrm{~min}$ (major).
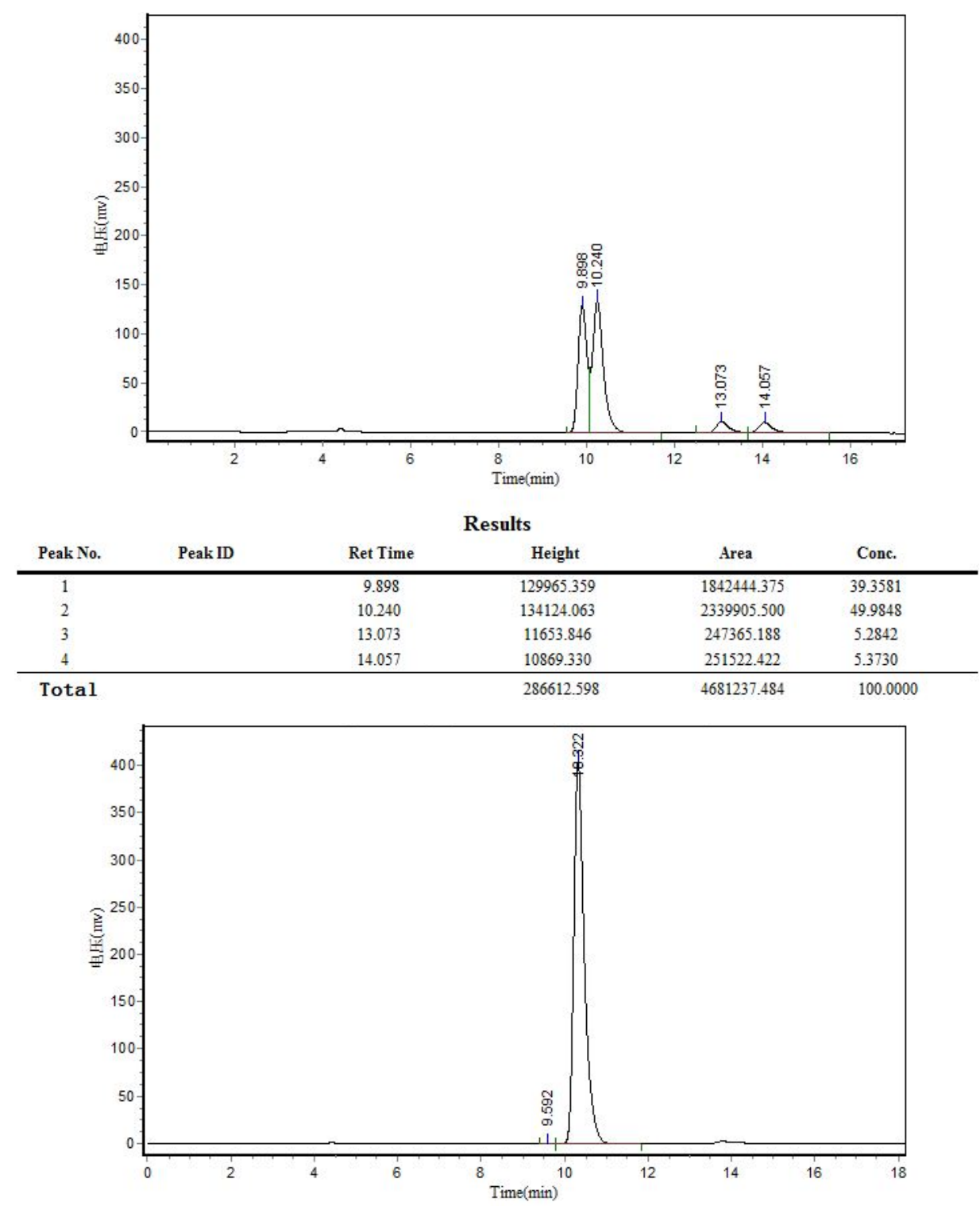

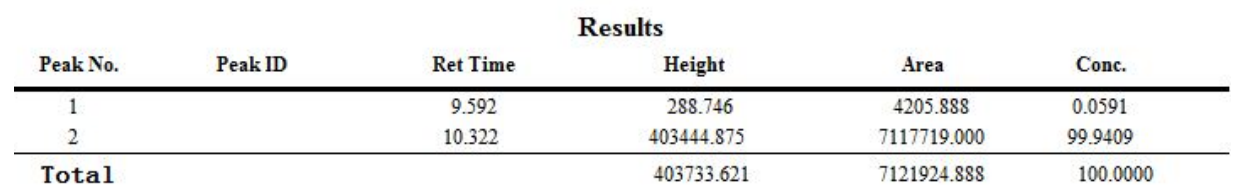




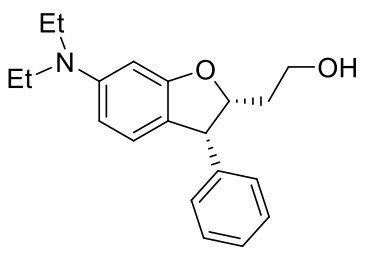

Pale yellow oil, $29.9 \mathrm{mg}, 96 \%$ yield, $96: 4 \mathrm{dr}, 99 \%$ ee; $[\alpha]_{\mathrm{D}}^{20}=-2.7(c$ 1.0, $\left.\mathrm{CHCl}_{3}\right) ;{ }^{1} \mathrm{H}$ NMR $\left(400 \mathrm{MHz}, \mathrm{CDCl}_{3}\right) \delta$ 7.29-7.20 (m, 3H), $7.02(\mathrm{~d}$, $J=7.2 \mathrm{~Hz}, 2 \mathrm{H}), 6.89(\mathrm{~d}, J=8.0 \mathrm{~Hz}, 1 \mathrm{H}), 6.26-6.22(\mathrm{~m}, 2 \mathrm{H}), 5.08-5.03$ (m, 1H), 4.49 (d, $J=8.4 \mathrm{~Hz}, 1 \mathrm{H}), 3.81-3.75(\mathrm{~m}, 2 \mathrm{H}), 3.35(\mathrm{q}, J=6.8$ $\mathrm{Hz}, 4 \mathrm{H}), 2.00$ (brs, $1 \mathrm{H}), 1.57-1.48(\mathrm{~m}, 1 \mathrm{H}), 1.46-1.39(\mathrm{~m}, 1 \mathrm{H}), 1.18(\mathrm{t}$, $J=6.8 \mathrm{~Hz}, 6 \mathrm{H}) ;{ }^{13} \mathrm{C} \mathrm{NMR}\left(100 \mathrm{MHz}, \mathrm{CDCl}_{3}\right) \delta 161.4,149.4,140.9,129.4,128.6,127.2,126.2$, 117.6, 105.3, 93.7, 86.3, 61.2, 51.5, 45.0, 34.9, 12.9; HRMS (ESI) for $\mathrm{C}_{20} \mathrm{H}_{26} \mathrm{NO}_{2}[\mathrm{M}+\mathrm{H}]^{+}$: calcd 312.1958, found 312.1960.

HPLC: Chiralpak OD-H column $(250 \mathrm{~mm})$; detected at $254 \mathrm{~nm}$; hexane $/ i$-propanol $=95 / 5$; flow $=$ $1.2 \mathrm{~mL} / \mathrm{min}$; Retention time: $22.1 \mathrm{~min}$ (major), $46.6 \mathrm{~min}$.
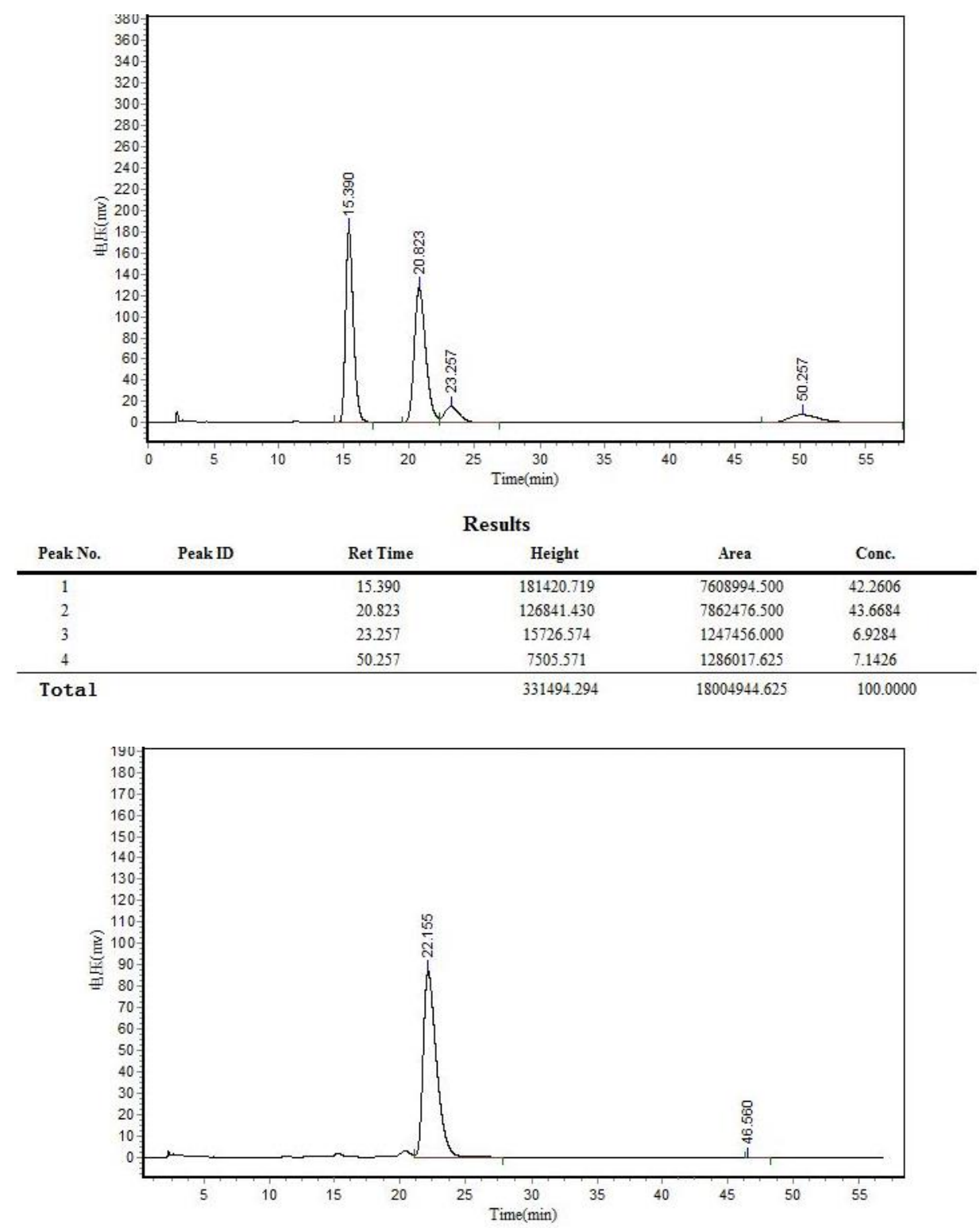

\begin{tabular}{|c|c|c|c|c|c|}
\hline \multicolumn{6}{|c|}{ Results } \\
\hline Peak No. & Peak ID & Ret Time & Height & Area & Conc. \\
\hline 1 & & 22.155 & 86807.469 & 6138650.500 & 99.9492 \\
\hline 2 & & 46.560 & 41.543 & 3118.421 & 0.0508 \\
\hline Total & & & 86849.012 & 6141768.921 & 100.0000 \\
\hline
\end{tabular}




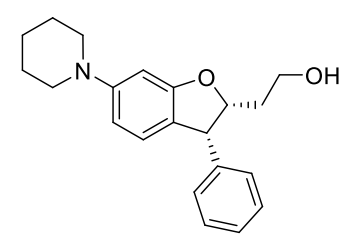

Pale yellow oil, $30.0 \mathrm{mg}, 93 \%$ yield, $98: 2 \mathrm{dr}, 99 \%$ ee; $[\alpha]_{\mathrm{D}}^{20}=5.9(c 1.0$, $\left.\mathrm{CHCl}_{3}\right) ;{ }^{1} \mathrm{H}$ NMR $\left(400 \mathrm{MHz}, \mathrm{CDCl}_{3}\right) \delta$ 7.28-7.20 (m, 3H), $6.99(\mathrm{~d}, J=$ $7.2 \mathrm{~Hz}, 2 \mathrm{H}), 6.92(\mathrm{~d}, J=8.4 \mathrm{~Hz}, 1 \mathrm{H}), 6.50-6.48(\mathrm{~m}, 2 \mathrm{H}), 5.09-5.03(\mathrm{~m}$, $1 \mathrm{H}), 4.50(\mathrm{~d}, J=8.4 \mathrm{~Hz}, 1 \mathrm{H}), 3.79-3.75(\mathrm{~m}, 2 \mathrm{H}), 3.16(\mathrm{t}, J=5.6 \mathrm{~Hz}, 4 \mathrm{H})$, 2.00 (brs, $1 \mathrm{H}), 1.71-1.68(\mathrm{~m}, 4 \mathrm{H}), 1.61-1.55(\mathrm{~m}, 2 \mathrm{H}), 1.54-1.40(\mathrm{~m}, 2 \mathrm{H})$; ${ }^{13} \mathrm{C}$ NMR $\left(100 \mathrm{MHz}, \mathrm{CDCl}_{3}\right) \delta 161.0,153.8,140.5,129.4,128.6,127.3,125.9,121.4,110.0,98.4$, 86.3, 61.1, 51.5, 51.2, 34.8, 26.2, 24.6; HRMS (ESI) for $\mathrm{C}_{21} \mathrm{H}_{26} \mathrm{NO}_{2}[\mathrm{M}+\mathrm{H}]^{+}$: calcd 324.1958, found 324.1962 .

HPLC: Chiralpak AD-H column $(250 \mathrm{~mm})$; detected at $254 \mathrm{~nm}$; hexane $/ i$-propanol $=90 / 10$; flow $=1.0 \mathrm{~mL} / \mathrm{min}$; Retention time: $12.0 \mathrm{~min}, 14.1 \mathrm{~min}$ (major).

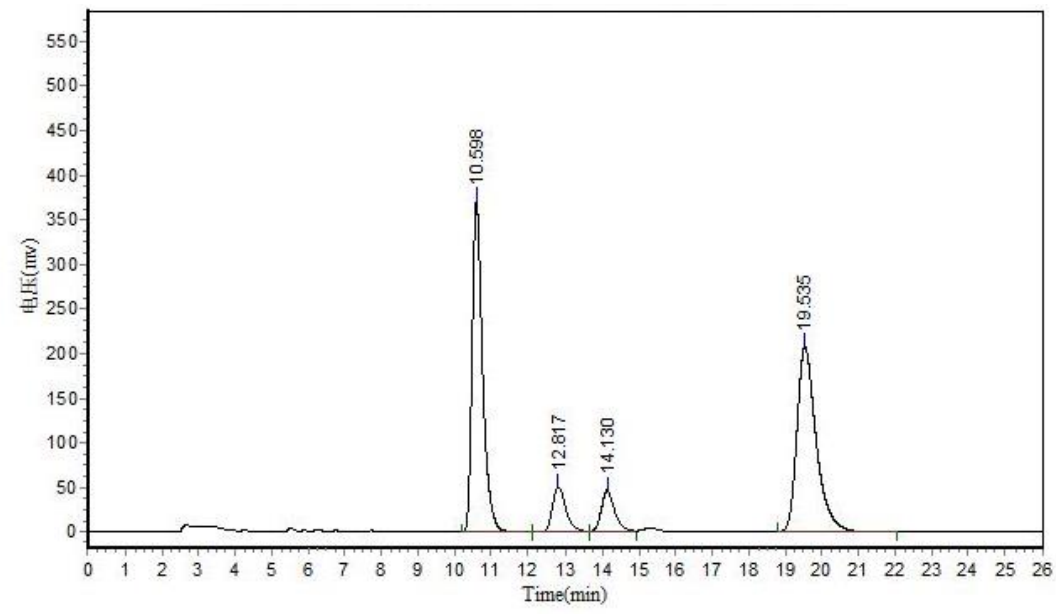

\begin{tabular}{|c|c|c|c|c|c|}
\hline \multicolumn{6}{|c|}{ Results } \\
\hline Peak No. & Peak ID & Ret Time & Height & Area & Conc. \\
\hline 1 & & 10.598 & 370126.063 & 7358405.500 & 42.5091 \\
\hline 2 & & 12.817 & 50353.164 & 1207161.250 & 6.9737 \\
\hline 3 & & 14.130 & 46364.211 & 1221434.875 & 7.0562 \\
\hline 4 & & 19.535 & 206724.313 & 7523197.500 & 43.4611 \\
\hline Total & & & 673567.750 & 17310199.125 & 100.000 \\
\hline
\end{tabular}

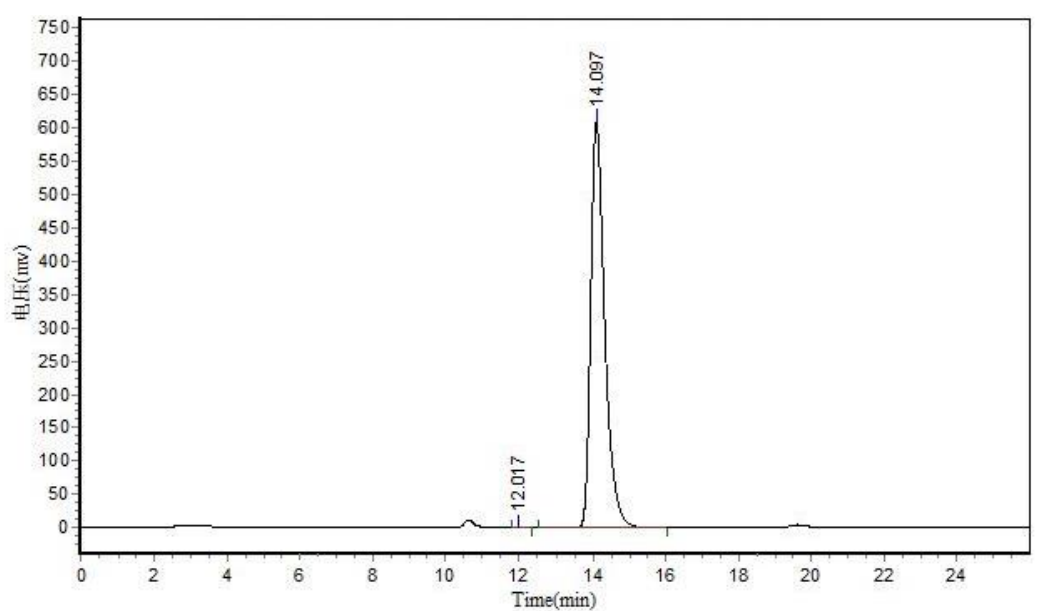

\begin{tabular}{|c|c|c|c|c|c|}
\hline \multicolumn{6}{|c|}{ Results } \\
\hline Peak No. & Peak ID & Ret Time & Height & Area & Conc. \\
\hline$\overline{1}$ & & 12.017 & 36.667 & 588.500 & 0.0036 \\
\hline 2 & & 14.097 & 607500.250 & 16359195.000 & 99.9964 \\
\hline Total & & & 607536.917 & 16359783.500 & 100.0000 \\
\hline \multicolumn{6}{|c|}{ S59 } \\
\hline
\end{tabular}




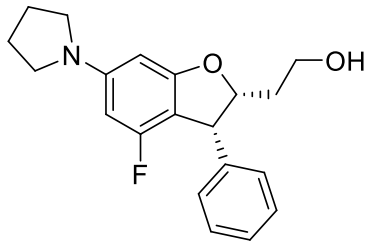

Pale yellow oil, $31.0 \mathrm{mg}, 95 \%$ yield, $96: 4 \mathrm{dr}, 99 \%$ ee; $[\alpha]_{\mathrm{D}}^{20}=1.7(c$ 1.0, $\left.\mathrm{CHCl}_{3}\right) ;{ }^{1} \mathrm{H}$ NMR $\left(400 \mathrm{MHz}, \mathrm{CDCl}_{3}\right) \delta$ 7.27-7.19 (m, 3H), $6.99(\mathrm{~d}$, $J=7.6 \mathrm{~Hz}, 2 \mathrm{H}), 5.93(\mathrm{~s}, 1 \mathrm{H}), 5.81(\mathrm{~d}, J=11.6 \mathrm{~Hz}, 1 \mathrm{H}), 5.07-5.02(\mathrm{~m}$, $1 \mathrm{H}), 4.55(\mathrm{~d}, J=8.0 \mathrm{~Hz}, 1 \mathrm{H}), 3.79-3.73(\mathrm{~m}, 2 \mathrm{H}), 3.25(\mathrm{t}, J=6.0 \mathrm{~Hz}$, $4 \mathrm{H}), 2.04-1.98(\mathrm{~m}, 4 \mathrm{H}), 1.84(\mathrm{~s}, 1 \mathrm{H}), 1.52-1.47(\mathrm{~m}, 2 \mathrm{H}) ;{ }^{13} \mathrm{C} \mathrm{NMR}$ $\left(100 \mathrm{MHz}, \mathrm{CDCl}_{3}\right) \delta 162.7(\mathrm{~d}, J=11.9 \mathrm{~Hz}), 160.3(\mathrm{~d}, J=243.0 \mathrm{~Hz}), 150.5(\mathrm{~d}, J=11.9 \mathrm{~Hz}), 139.5$, 128.8, 128.6, 127.4, 104.5 (d, $J=21.4 \mathrm{~Hz}), 92.2$ (d, $J=24.8 \mathrm{~Hz}), 89.5$ (d, $J=2.5 \mathrm{~Hz}), 86.7,61.0$, 49.1, 48.3, 34.7, 25.8; HRMS (ESI) for $\mathrm{C}_{20} \mathrm{H}_{23} \mathrm{FNO}_{2}[\mathrm{M}+\mathrm{H}]^{+}$: calcd 328.1707, found 328.1708.

HPLC: Chiralpak IG+AD-H column $(250 \mathrm{~mm})$; detected at $220 \mathrm{~nm}$; hexane/i-propanol $=90 / 10$; flow $=1.0 \mathrm{~mL} / \mathrm{min}$; Retention time: $22.0 \mathrm{~min}$ (major), $24.3 \mathrm{~min}$.

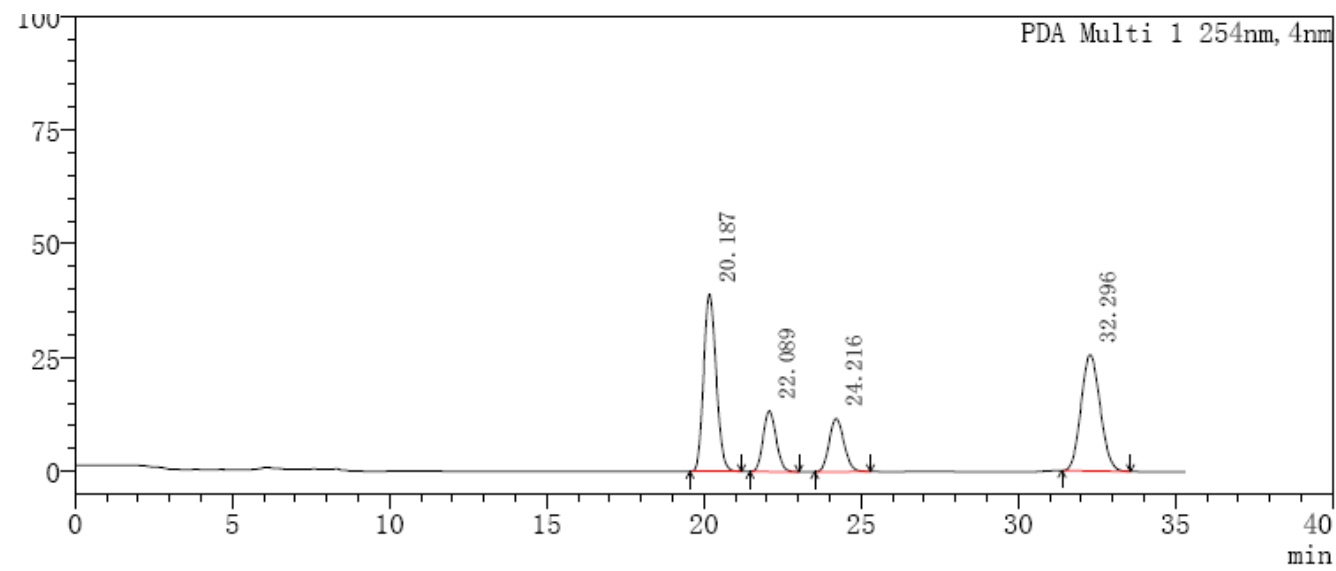

PDA Ch1 $254 \mathrm{~nm}$
\begin{tabular}{|c|c|c|c|c|}
\hline Number & Retention & Area & Height & Area $\%$ \\
\hline 1 & 20.187 & 1086348 & 38992 & 36.987 \\
\hline 2 & 22.089 & 383206 & 13318 & 13.047 \\
\hline 3 & 24.216 & 384531 & 11705 & 13.092 \\
\hline 4 & 32.296 & 1083056 & 25508 & 36.875 \\
\hline
\end{tabular}

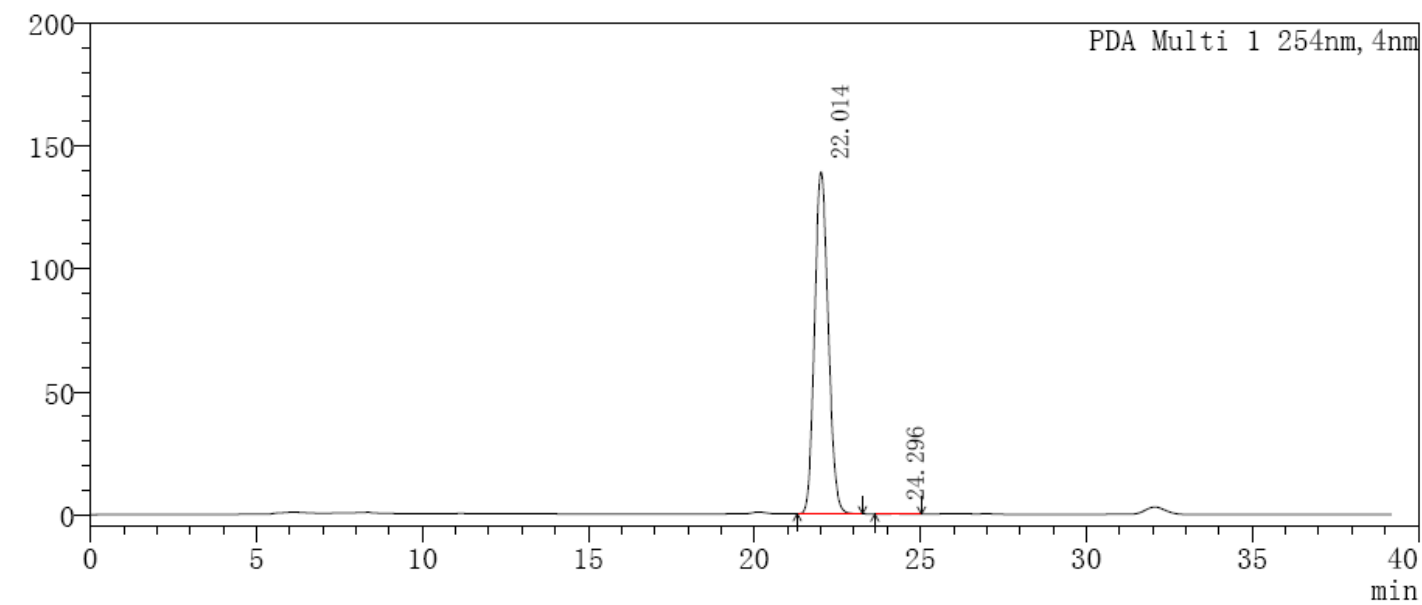

PDA Ch1 254nm
\begin{tabular}{|c|c|c|c|c|}
\hline Number & Retention & Area & Height & Area $\%$ \\
\hline 1 & 22.014 & 3988245 & 139120 & 99.940 \\
\hline 2 & 24.296 & 2404 & 68 & 0.060 \\
\hline
\end{tabular}




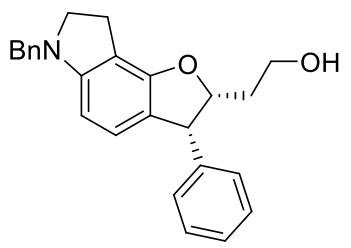

Pale yellow oil, $34.8 \mathrm{mg}$, 94\% yield, $99: 1 \mathrm{dr}, 99 \%$ ee; $[\alpha]_{\mathrm{D}}^{20}=9.7(c 1.0$, $\left.\mathrm{CHCl}_{3}\right) ;{ }^{1} \mathrm{H}$ NMR $\left(400 \mathrm{MHz}, \mathrm{CDCl}_{3}\right) \delta$ 7.39-7.21 (m, 8H), 7.03 (d, $J=$ $7.6 \mathrm{~Hz}, 2 \mathrm{H}), 6.78(\mathrm{~d}, J=8.0 \mathrm{~Hz}, 1 \mathrm{H}), 6.13(\mathrm{~d}, J=7.6 \mathrm{~Hz}, 1 \mathrm{H}), 5.10-5.05$ $(\mathrm{m}, 1 \mathrm{H}), 4.51(\mathrm{~d}, J=8.4 \mathrm{~Hz}, 1 \mathrm{H}), 4.30-4.22(\mathrm{~m}, 2 \mathrm{H}), 3.81-3.75(\mathrm{~m}, 2 \mathrm{H})$, 3.42-3.33 (m, 2H), $2.98(\mathrm{t}, J=8.0 \mathrm{~Hz}, 2 \mathrm{H}), 1.99(\mathrm{~s}, 1 \mathrm{H}), 1.61-1.52(\mathrm{~m}$, 1H), 1.47-1.40 (m, 1H); ${ }^{13} \mathrm{C}$ NMR $\left(100 \mathrm{MHz}, \mathrm{CDCl}_{3}\right) \delta 156.1,154.9,140.8,138.8,129.4,128.8$, 128.6, 128.3, 127.4, 127.2, 124.6, 121.7, 110.2, 100.7, 86.8, 61.2, 54.5, 54.2, 51.5, 34.8, 25.4; HRMS (ESI) for $\mathrm{C}_{25} \mathrm{H}_{26} \mathrm{NO}_{2}[\mathrm{M}+\mathrm{H}]^{+}$: calcd 372.1958, found 372.1962.

HPLC: Chiralpak AD-H column $(250 \mathrm{~mm})$; detected at $220 \mathrm{~nm}$; hexane $/ i$-propanol $=90 / 10$; flow $=1.1 \mathrm{~mL} / \mathrm{min}$; Retention time: $17.7 \mathrm{~min}$ (major), $22.3 \mathrm{~min}$.

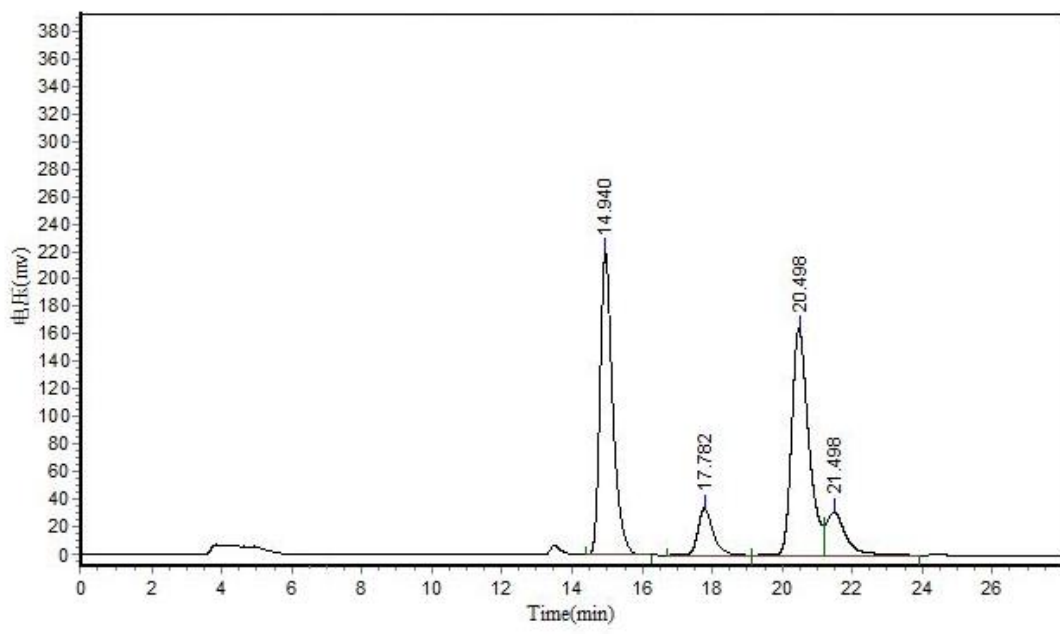

Results

\begin{tabular}{|c|c|c|c|c|c|}
\hline \multicolumn{6}{|c|}{ 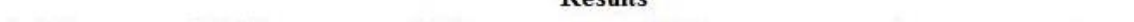 } \\
\hline Peak No. & Peak ID & Ret Time & Height & Area & Conc. \\
\hline 1 & & 14.940 & 219500.828 & 5587405.500 & 41.4482 \\
\hline 2 & & 17.782 & 34325.629 & 1047302.688 & 7.7690 \\
\hline 3 & & 20.498 & 164809.859 & 5611852.000 & 41.6296 \\
\hline 4 & & 21.498 & 31702.977 & 1233889.500 & 9.1532 \\
\hline Total & & & 450339.293 & 13480449.688 & 100.000 \\
\hline
\end{tabular}

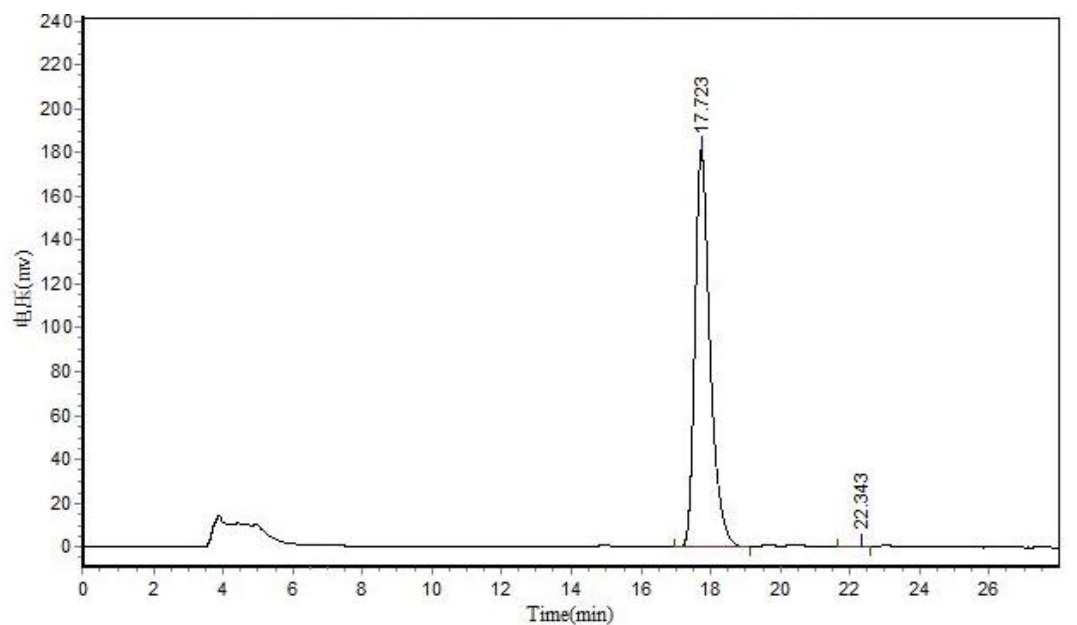

Results

\begin{tabular}{|c|c|c|c|c|c|}
\hline \multicolumn{6}{|c|}{ Kesuits } \\
\hline Peak No. & Peak ID & Ret Time & Height & Area & Conc. \\
\hline 1 & & 17.723 & 181155.563 & 5512059.500 & 99.7492 \\
\hline 2 & & 22.343 & 409.508 & 13860.580 & 0.2508 \\
\hline Total & & & 181565.071 & 5525920.080 & 100.0000 \\
\hline
\end{tabular}




\section{Optimization of Reaction Conditions for Stereodivergent Synthesis of 3,4-Disubstituted Isochromans 6}

\subsection{One-pot reaction attempt}

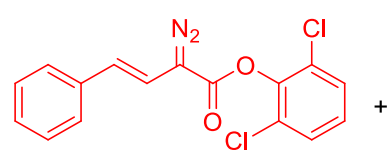

$1 a$

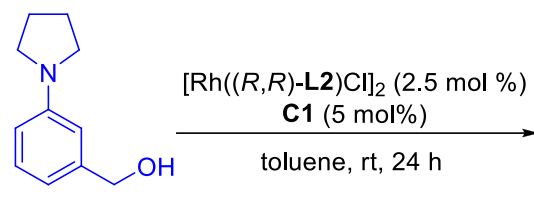

$2 r$

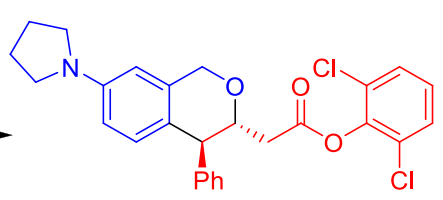

6

trace

Encouraged by the success of stereodivergent synthesis of 2,3-disubstituted dihydrobenzofurans, the same one-pot cascade strategy was applied to the divergent synthesis of 3,4-disubstituted isochromans. Unfortunately, the reaction of (E)-2,6-dichlorophenyl 2-diazo-4-phenylbut-3-enoate 1a with (3-(pyrrolidin-1-yl)phenyl)methanol $\mathbf{2 r}$ under the standard conditions gave to only trace amount of the expected product 6, partly due to the lower reactivity of (3-(pyrrolidin-1-yl)phenyl)methanol compared with 3-(pyrrolidin-1-yl)phenol in the initial intermolecular $\mathrm{C}-\mathrm{H}$ functionalization and poor reactivity of alcoholic hydroxyl compared with phenolic hydroxyl in the subsequent intramolecular oxa-Michael addition. To our disappointment, no syntheticly useful results were obtained by one-pot fashion when varying the reaction parameters. 


\subsection{Optimization of reaction conditions for two-step sequence}

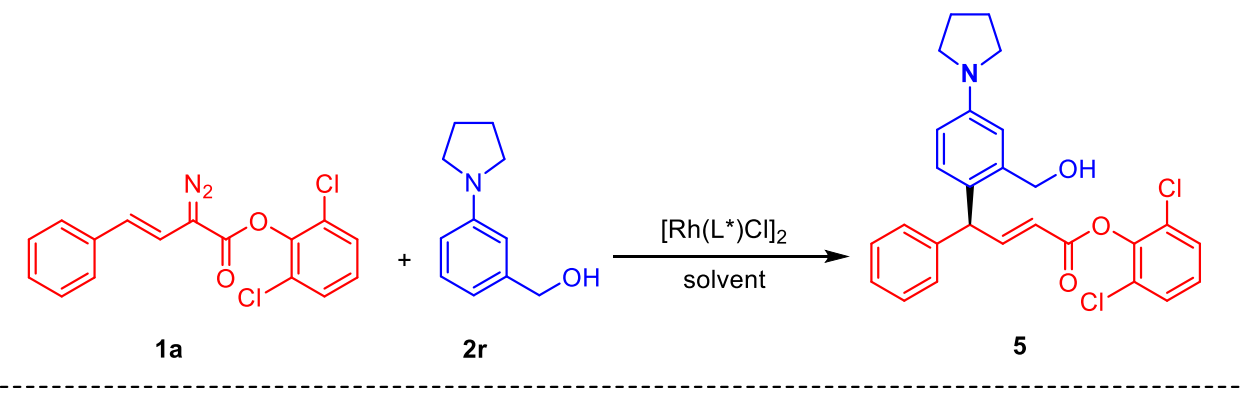

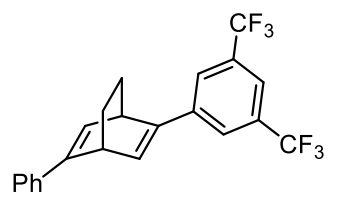

L1

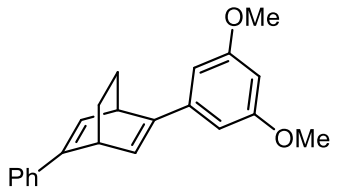

L4

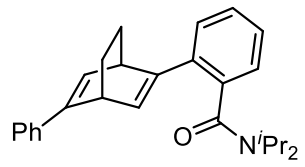

L2

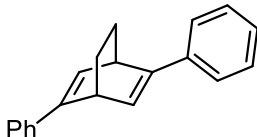

L5

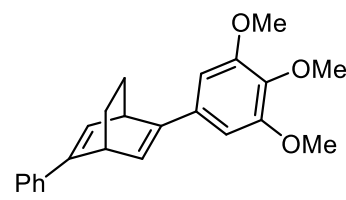

L3

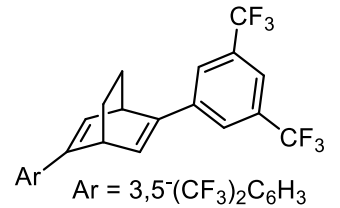

L6

\begin{tabular}{ccccc}
\hline entry $^{a}$ & Ligand & solvent & ${\text { yield }(\%)^{b}}^{b}$ & ee (\%) \\
\hline 1 & L1 & DCM & 69 & 84 \\
2 & L2 & DCM & 21 & 80 \\
3 & L3 & DCM & 48 & 74 \\
4 & L4 & DCM & 31 & 66 \\
5 & L5 & DCM & 44 & 82 \\
6 & L6 & DCM & 75 & 70 \\
7 & L1 & CHCl & 68 & 86 \\
8 & L1 & toluene & 17 & 83 \\
9 & L1 & THF & trace & - \\
\hline
\end{tabular}

${ }^{a}$ Reactions were performed with $\mathbf{1 a}(0.2 \mathrm{mmol})$ and $\mathbf{2 r}(0.4 \mathrm{mmol})$ in the presence of $1.5 \mathrm{~mol} \%$ of $\left[\mathrm{Rh}\left(\mathrm{L}^{*}\right) \mathrm{Cl}\right]_{2}$ in solvent $(4.0 \mathrm{~mL})$ at room temperature for 6 h. ${ }^{b}$ Isolated yield. ${ }^{c}$ ee was determined by chiral HPLC.

The failure in one-pot attempt led us to perform this reaction in a two-step fashion. At the outset of this study, the C-H functionalization of (E)-2,6-dichlorophenyl 2-diazo-4-phenylbut-3-enoate 1a and (3-(pyrrolidin-1-yl)phenyl)methanol 2r was investigated, the product 5 was obtained in 69\% yield with $84 \%$ ee when $\mathbf{L 1}$ was used as ligand and DCM as solvent (entry 1). In order to improve the enantioselective of $\mathbf{5}$, a series of ligand with different steric and electronic properties were evaluated. However, no higher enantioselective was obtained (entries 2-6). To our delight, the replacement of DCM with $\mathrm{CHCl}_{3}$ led to a slightly increased enantioselectivity, furnishing the product 5 in 68\% yield and $86 \%$ ee. Changing to other solvents did not give better results (entries 
7-9). Then the following oxa-Michael reaction was investigated by using the obtained product 5 .

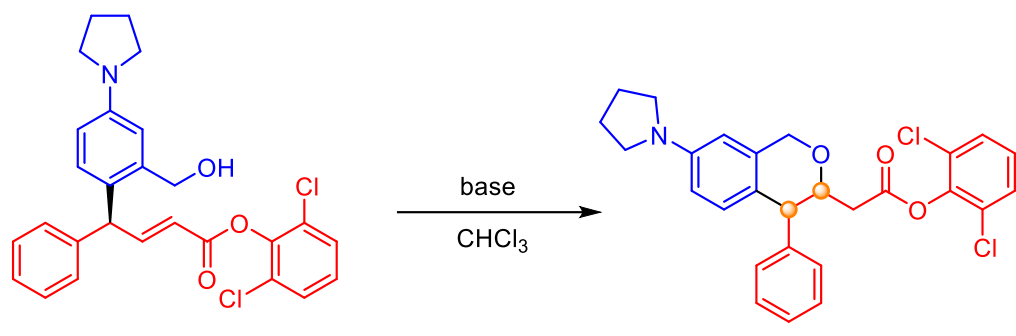

$586 \%$ ee

\begin{tabular}{cccccc}
\hline entry $^{\mathrm{a}}$ & ee of substrate & base & ${\text { yield }(\%)^{b}}$ & $\mathrm{dr}_{(\operatorname{anti} / \mathrm{syn})^{c}}$ & ${\text { ee }(\%)^{c}}^{c}$ \\
\hline 1 & $86(S)$ & C1 & 79 & $92: 8$ & 95 \\
2 & $86(S)$ & C2 & 92 & $16: 84$ & 99 \\
3 & $86(S)$ & $\mathbf{C 3}$ & 64 & $17: 83$ & 99 \\
4 & $86(S)$ & $\mathbf{C 4}$ & 90 & $91: 9$ & 95 \\
5 & $86(S)$ & C5 & 99 & $94: 6$ & 95 \\
6 & $86(S)$ & C6 & 99 & $14: 86$ & 99 \\
7 & $86(S)$ & C7 & 99 & $13: 87$ & 99 \\
8 & $86(S)$ & C8 & 99 & $92: 8$ & 95 \\
9 & $86(R)$ & C5 & 95 & $30: 70$ & 99 \\
10 & $86(R)$ & C7 & 99 & $93: 7$ & 98 \\
\hline
\end{tabular}<smiles>C=CC1CC2CCN1C2[C@H](NC(=S)NCCCCCCC)c1ccnc2ccc(OC)cc12</smiles>

C1

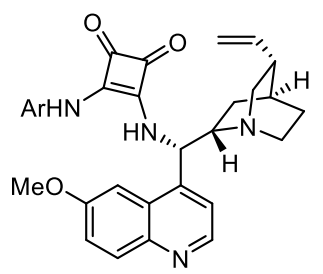

C5

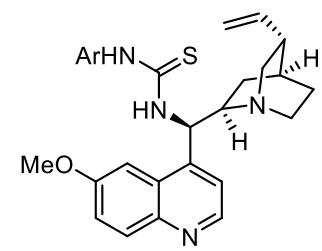

C2

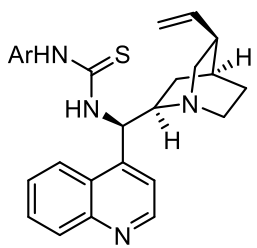

C3

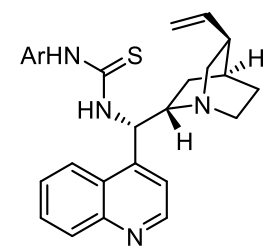

C4

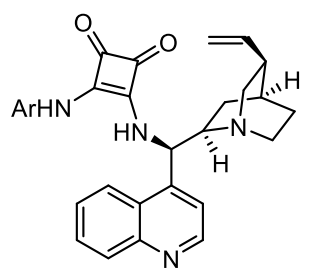

C7

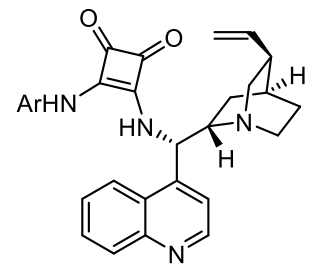

C8

C1-C8, $\mathrm{Ar}=3,5-\left(\mathrm{CF}_{3}\right)_{2} \mathrm{C}_{6} \mathrm{H}_{3}$

${ }^{a}$ Reactions were performed with $\mathrm{C}-\mathrm{H}$ functionalization product $(0.1 \mathrm{mmol})$ in the presence of $5.0 \mathrm{~mol} \%$ base in $\mathrm{CHCl}_{3}(4.0 \mathrm{~mL})$ for $24 \mathrm{~h} .{ }^{b}$ Isolated yield of two diastereomers of the product. ${ }^{c} \mathrm{dr}$ and ee were determined by chiral HPLC.

With the $\mathrm{C}-\mathrm{H}$ functionalization product $5(86 \%$ ee) in hand, the oxa-Michael reaction was investigated. Based on the excellent catalytic performance of cinchona alkaloid-based bifunctional organocatalysts in the stereodivergent synthesis of 2,3-disubstituted dihydrobenzofuran, organocatalysts C1-C8 were examined. Generally, squaramide-based catalyst gives better results 
than thiourea-based catalyst. After careful investigation, we found that the quinine-derived catalysts and cinchonidine-derived catalysts furnished the anti-6 as major product while quinidine-derived catalysts and cinchonine-derived catalysts gave the syn-6 as major product (entries 1, 4, 5, 8 vs entries 2, 3, 6, 7). Finally, we found that quinine-derived squaramide C5 gave the anti-product $(3 R, 4 S)-6$ with the best results (99\% yield, $94: 6 \mathrm{dr}, 95 \%$ ee) (entry 5). Moreover, the syn-product $(3 S, 4 S)-6$ could be obtained in $99 \%$ yield with $87: 13 \mathrm{dr}$ and $99 \%$ ee by replaceing C5 to cinchonine-derived squaramide C7 (entry 7). Accordingly, two other diastereoisomers $(3 R, 4 R)-6$ (entry 9) and $(3 S, 4 R)-6$ (entry 10$)$ could also be obtained when $(R)-5$ was used as substrate. 
2,6-dichlorophenyl 2-((3R,4S)-4-phenyl-7-(pyrrolidin-1-yl)isochroman-3-yl)acetate $((3 R, 4 S)-6 \mathrm{a})$<smiles>O=C(COc1c(Cl)cccc1Cl)CC1OCc2cc(N3CCCC3)ccc2C1c1ccccc1</smiles>

White solid, $48.0 \mathrm{mg}$, 99\% yield, 94:6 dr, 95\% ee; m.p. 147.9-149.6 ${ }^{\circ} \mathrm{C},[\alpha]_{\mathrm{D}}{ }^{20}=-35.1\left(\right.$ c $\left.0.72, \mathrm{CHCl}_{3}\right) ;{ }^{1} \mathrm{H}$ NMR $\left(400 \mathrm{MHz}, \mathrm{CDCl}_{3}\right) \delta$ 7.36-7.28 (m, 5H), 7.23-7.21 (m, 2H), $7.12(\mathrm{t}, J=8.0 \mathrm{~Hz}, 1 \mathrm{H}), 6.60$ $(\mathrm{d}, J=8.4 \mathrm{~Hz}, 1 \mathrm{H}), 6.35(\mathrm{~d}, J=8.0 \mathrm{~Hz}, 1 \mathrm{H}), 6.22(\mathrm{~s}, 1 \mathrm{H}), 5.05(\mathrm{~d}, J$ $=15.2 \mathrm{~Hz}, 1 \mathrm{H}), 4.93(\mathrm{~d}, J=15.2 \mathrm{~Hz}, 1 \mathrm{H}), 4.36(\mathrm{td}, J=10.0,3.6 \mathrm{~Hz}$, $1 \mathrm{H}), 3.99(\mathrm{~d}, J=10.0 \mathrm{~Hz}, 1 \mathrm{H}), 3.25(\mathrm{t}, J=6.0 \mathrm{~Hz}, 4 \mathrm{H}), 2.88-2.77(\mathrm{~m}, 2 \mathrm{H}), 1.98(\mathrm{t}, J=6.0 \mathrm{~Hz}, 4 \mathrm{H})$; ${ }^{13} \mathrm{C}$ NMR $\left(100 \mathrm{MHz}, \mathrm{CDCl}_{3}\right) \delta 168.2,146.7,144.3,142.4,135.3,130.4,129.9,129.3,129.1$, 128.9, 127.4, 127.3, 123.9, 111.1, 106.1, 78.5, 69.2, 49.6, 47.9, 38.9, 25.8; HRMS (ESI) for $\mathrm{C}_{27} \mathrm{H}_{26} \mathrm{Cl}_{2} \mathrm{NO}_{3}[\mathrm{M}+\mathrm{H}]^{+}$:calcd 482.1284 , found 482.1288 .

HPLC: Chiralpak OD-H column $(250 \mathrm{~mm})$; detected at $254 \mathrm{~nm}$; hexane $/ i$-propanol $=90 / 10$; flow $=0.7 \mathrm{~mL} / \mathrm{min}$; Retention time: $13.1 \mathrm{~min}$ (major), $25.2 \mathrm{~min}$.

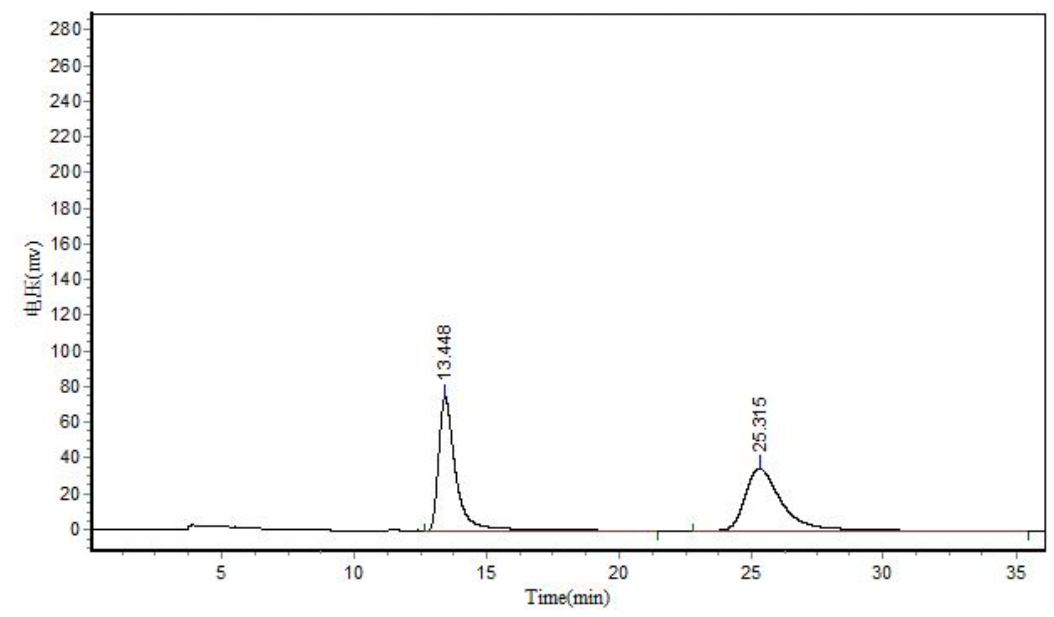

\begin{tabular}{|c|c|c|c|c|c|}
\hline \multicolumn{6}{|c|}{ Results } \\
\hline Peak No. & Peak ID & Ret Time & Height & Area & Conc. \\
\hline 1 & & 13.448 & 75032.367 & 3619928.750 & 49.7977 \\
\hline 2 & & 25.315 & 35280.898 & 3649339.750 & 50.2023 \\
\hline Total & & & 110313.266 & 7269268.500 & 100.000 \\
\hline
\end{tabular}

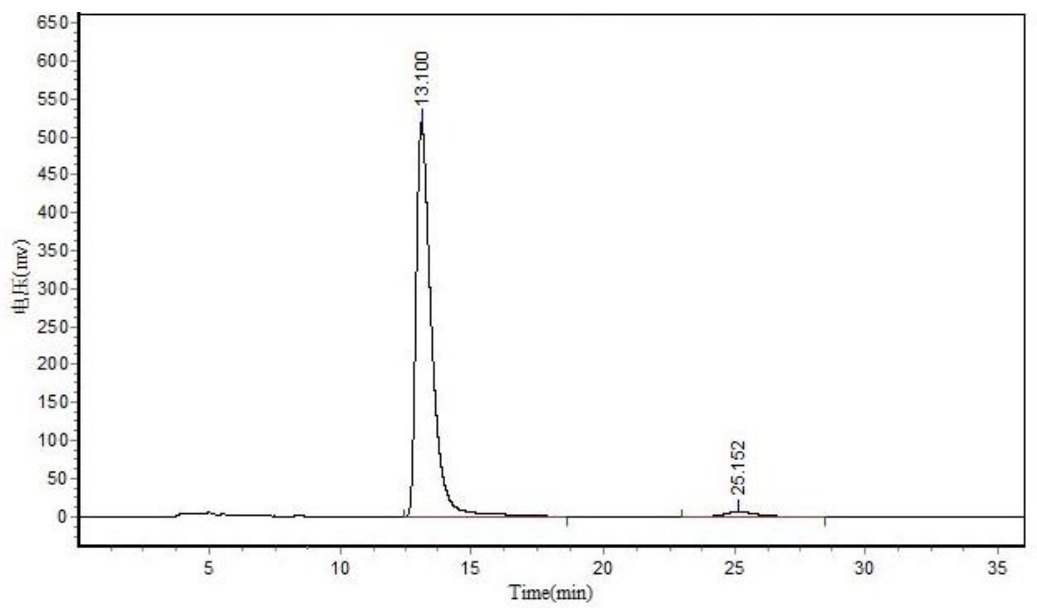

\begin{tabular}{|c|c|c|c|c|c|}
\hline \multicolumn{6}{|c|}{ Results } \\
\hline Peak No. & Peak ID & Ret Time & Height & Area & Conc. \\
\hline 1 & & 13.100 & 518325.375 & 21371046.000 & 97.4481 \\
\hline 2 & & 25.152 & 6385.932 & 559650.313 & 2.5519 \\
\hline Total & & & 524711.307 & 21930696.313 & $100.000 \mathrm{C}$ \\
\hline
\end{tabular}


2,6-dichlorophenyl 2-((3S,4S)-4-phenyl-7-(pyrrolidin-1-yl)isochroman-3-yl)acetate-

\section{$((3 S, 4 S)-6 a)$}

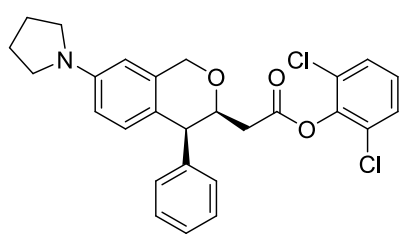

White solid, $48.2 \mathrm{mg}$, 99\% yield, $87: 13 \mathrm{dr}$, 99\% ee; m.p. 78.4-80.5 ${ }^{\circ} \mathrm{C},[\alpha]_{\mathrm{D}}{ }^{20}=160.0\left(\right.$ c $\left.1.0, \mathrm{CHCl}_{3}\right) ;{ }^{1} \mathrm{H}$ NMR $\left(400 \mathrm{MHz}, \mathrm{CDCl}_{3}\right) \delta$ $7.37(\mathrm{~d}, J=8.4 \mathrm{~Hz}, 2 \mathrm{H}), 7.31-7.21(\mathrm{~m}, 5 \mathrm{H}), 7.15(\mathrm{t}, J=8.0 \mathrm{~Hz}, 1 \mathrm{H})$, $6.90(\mathrm{~d}, J=8.4 \mathrm{~Hz}, 1 \mathrm{H}), 6.44(\mathrm{dd}, J=8.4,1.6 \mathrm{~Hz}, 1 \mathrm{H}), 6.27(\mathrm{~s}, 1 \mathrm{H})$, $5.05(\mathrm{~d}, J=15.6 \mathrm{~Hz}, 1 \mathrm{H}), 5.02(\mathrm{~d}, J=15.6 \mathrm{~Hz}, 1 \mathrm{H}), 4.63-4.59(\mathrm{~m}$, $1 \mathrm{H}), 4.00(\mathrm{~d}, J=2.8 \mathrm{~Hz}, 1 \mathrm{H}), 3.30-3.26(\mathrm{~m}, 4 \mathrm{H}), 2.70-2.65(\mathrm{~m}, 1 \mathrm{H}), 2.57-2.51(\mathrm{~m}, 1 \mathrm{H}), 2.01(\mathrm{t}, J$ $=6.0 \mathrm{~Hz}, 4 \mathrm{H}) ;{ }^{13} \mathrm{C} \mathrm{NMR}\left(100 \mathrm{MHz}, \mathrm{CDCl}_{3}\right) \delta 168.2,146.9,144.2,142.3,134.7,131.2,130.1$, $129.3,128.9,128.5,127.5,126.9,123.8,111.6,106.0,74.9,69.5,47.9,46.8,38.9,25.8$; $\mathrm{C}_{27} \mathrm{H}_{26} \mathrm{Cl}_{2} \mathrm{NO}_{3}[\mathrm{M}+\mathrm{H}]^{+}$:calcd 482.1284 , found 482.1288 .

HPLC: Chiralpak AD-H column $(250 \mathrm{~mm})$; detected at $254 \mathrm{~nm}$; hexane/ $i$-propanol $=90 / 10$; flow $=0.7 \mathrm{~mL} / \mathrm{min}$; Retention time: $6.3 \mathrm{~min}, 12.2 \mathrm{~min}$ (major).

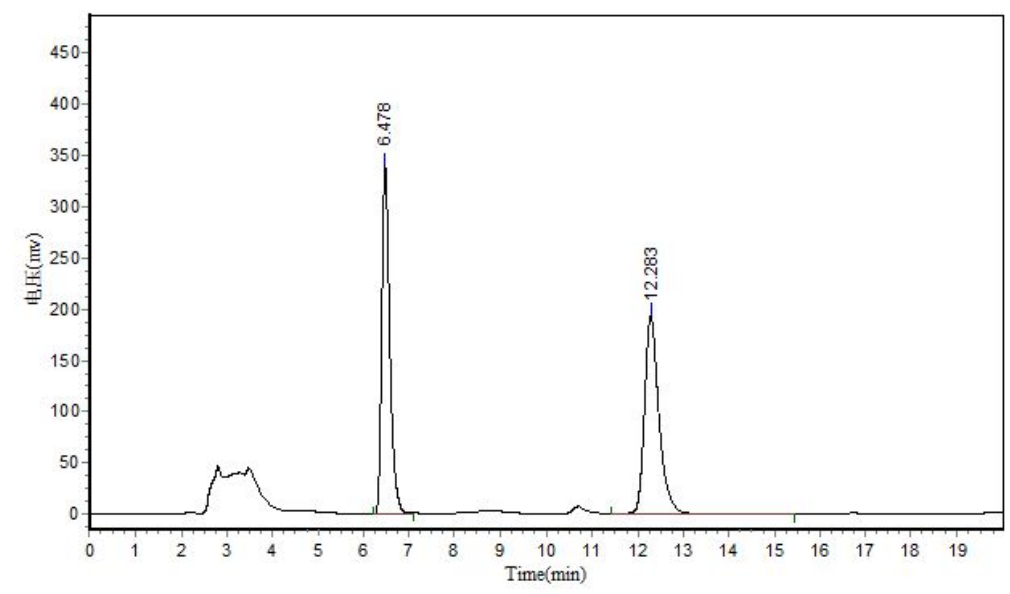

\begin{tabular}{|c|c|c|c|c|c|}
\hline \multicolumn{6}{|c|}{ Results } \\
\hline Peak No. & Peak ID & Ret Time & Height & Area & Conc. \\
\hline 1 & & 6.478 & 337540.563 & 4128246.250 & 48.9074 \\
\hline 2 & & 12.283 & 192430.906 & 4312704.000 & 51.0926 \\
\hline Total & & & 529971.469 & 8440950.250 & 100.000 \\
\hline
\end{tabular}

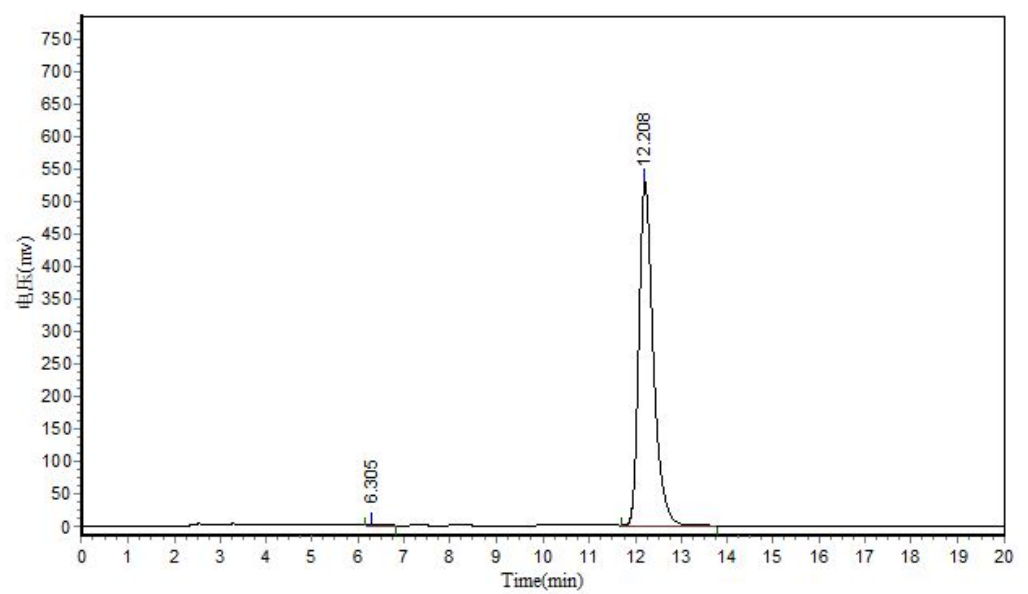

\begin{tabular}{|c|c|c|c|c|c|}
\hline \multicolumn{6}{|c|}{ Results } \\
\hline Peak No. & Peak ID & Ret Time & Height & Area & Conc. \\
\hline 1 & & 6.305 & 464.223 & 9169.900 & 0.0794 \\
\hline 2 & & 12.208 & 529208.875 & 11536324.000 & 99.9206 \\
\hline Total & & & 529673.098 & 11545493.900 & $100.000 \mathrm{c}$ \\
\hline
\end{tabular}


2,6-dichlorophenyl 2-((3S,4R)-4-phenyl-7-(pyrrolidin-1-yl)isochroman-3-yl)acetate-

\section{$((3 S, 4 R)-6 a)$}

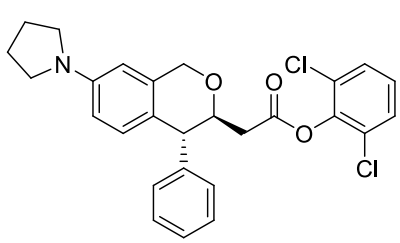

White solid, $48.0 \mathrm{mg}$, 99\% yield, 93:7 dr, 98\% ee; m.p. 151.4-153.2 ${ }^{\circ} \mathrm{C},[\alpha]_{\mathrm{D}}{ }^{20}=33.8\left(c 1.0, \mathrm{CHCl}_{3}\right) ;{ }^{1} \mathrm{H}$ NMR $\left(400 \mathrm{MHz}, \mathrm{CDCl}_{3}\right) \delta$ 7.36-7.28 (m, 5H), 7.23-7.21 (m, 2H), $7.12(\mathrm{t}, J=8.0 \mathrm{~Hz}, 1 \mathrm{H}), 6.60$ $(\mathrm{d}, J=8.4 \mathrm{~Hz}, 1 \mathrm{H}), 6.35(\mathrm{dd}, J=8.0,2.0 \mathrm{~Hz}, 1 \mathrm{H}), 6.21(\mathrm{~d}, J=2.0$ $\mathrm{Hz}, 1 \mathrm{H}), 5.04(\mathrm{~d}, J=15.2 \mathrm{~Hz}, 1 \mathrm{H}), 4.92(\mathrm{~d}, J=15.2 \mathrm{~Hz}, 1 \mathrm{H}), 4.36$ (td, $J=10.0,3.6 \mathrm{~Hz}, 1 \mathrm{H}), 3.98$ (d, $J=10.0 \mathrm{~Hz}, 1 \mathrm{H}), 3.25(\mathrm{t}, J=6.0 \mathrm{~Hz}, 4 \mathrm{H}), 2.88-2.76(\mathrm{~m}, 2 \mathrm{H})$, 1.99-1.96 (m, 4H); ${ }^{13} \mathrm{C}$ NMR $\left(100 \mathrm{MHz}, \mathrm{CDCl}_{3}\right) \delta 168.2,146.7,144.3,142.4,135.3,130.4,129.9$, 129.3, 129.1, 128.9, 127.4, 127.3, 123.9, 111.1, 106.1, 78.5, 69.2, 49.6, 47.9, 38.9, 25.8; HRMS (ESI) for $\mathrm{C}_{27} \mathrm{H}_{26} \mathrm{Cl}_{2} \mathrm{NO}_{3}[\mathrm{M}+\mathrm{H}]^{+}$:calcd 482.1284 , found 482.1288 .

HPLC: Chiralpak OD-H column $(250 \mathrm{~mm})$; detected at $254 \mathrm{~nm}$; hexane $/ i$-propanol $=90 / 10$; flow $=0.7 \mathrm{~mL} / \mathrm{min}$; Retention time: $13.6 \mathrm{~min}, 25.1 \mathrm{~min}$ (major).

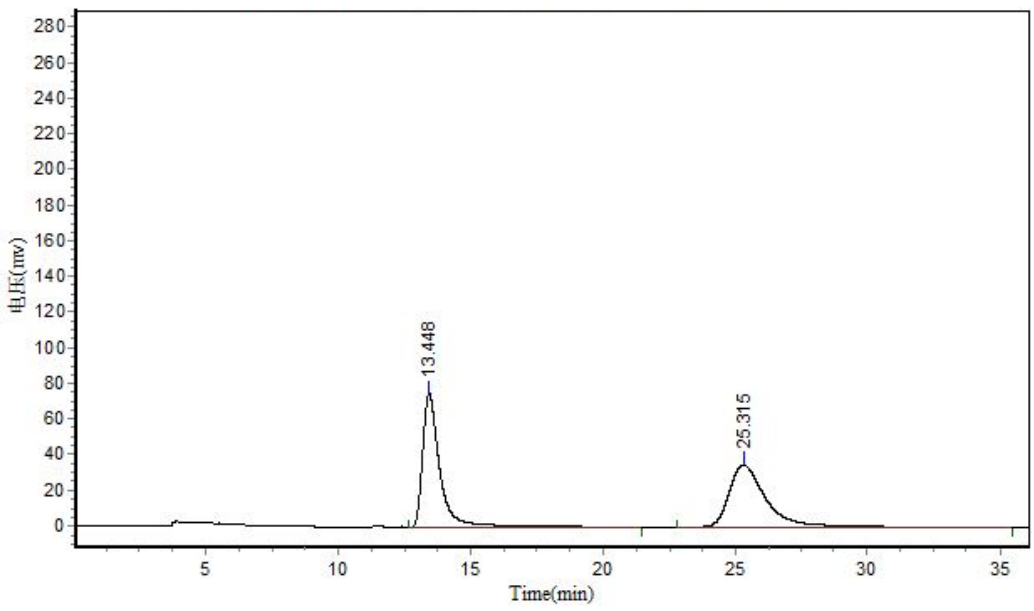

\begin{tabular}{|c|c|c|c|c|c|}
\hline \multicolumn{6}{|c|}{ Results } \\
\hline Peak No. & Peak ID & Ret Time & Height & Area & Conc. \\
\hline 1 & & 13.448 & 75032.367 & 3619928.750 & 49.7977 \\
\hline 2 & & 25.315 & 35280.898 & 3649339.750 & 50.2023 \\
\hline Total & & & 110313.266 & 7269268.500 & 100.000 \\
\hline
\end{tabular}

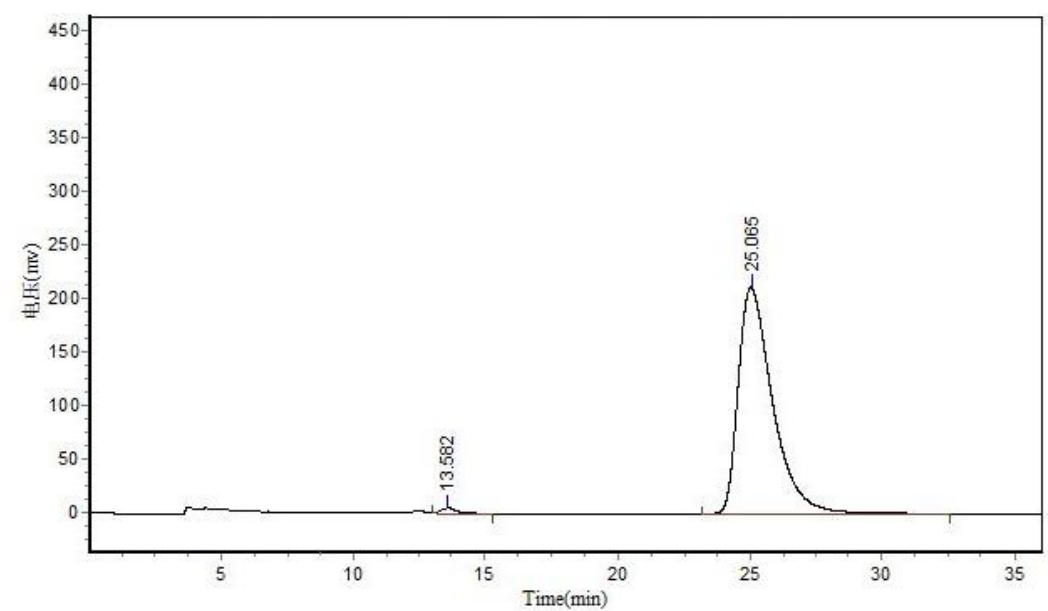

\begin{tabular}{|c|c|c|c|c|c|}
\hline \multicolumn{6}{|c|}{ Results } \\
\hline Peak No. & Peak ID & Ret Time & Height & Area & Conc. \\
\hline 1 & & 13.582 & 4994.310 & 204017.281 & 1.0507 \\
\hline 2 & & 25.065 & 211947.375 & 19214140.000 & 98.9493 \\
\hline Total & & & 216941.685 & 19418157.281 & 100.0000 \\
\hline
\end{tabular}


2,6-dichlorophenyl2-((3R,4R)-4-phenyl-7-(pyrrolidin-1-yl)isochroman-3-yl)acetate$((3 R, 4 R)-6 a)$

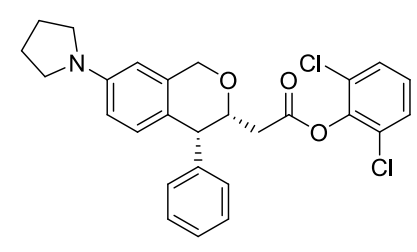

White solid, $45.6 \mathrm{mg}, 95 \%$ yield, 70:30 dr, 99\% ee; m.p. 76.2-78.9 ${ }^{\circ} \mathrm{C},[\alpha]_{\mathrm{D}}{ }^{20}=-163.5\left(\right.$ c $\left.1.0, \mathrm{CHCl}_{3}\right) ;{ }^{1} \mathrm{H}$ NMR $\left(400 \mathrm{MHz}, \mathrm{CDCl}_{3}\right) \delta$ $7.36(\mathrm{~d}, J=8.4 \mathrm{~Hz}, 2 \mathrm{H}), 7.30-7.20(\mathrm{~m}, 5 \mathrm{H}), 7.13(\mathrm{t}, J=8.0 \mathrm{~Hz}, 1 \mathrm{H})$, $6.89(\mathrm{~d}, J=8.4 \mathrm{~Hz}, 1 \mathrm{H}), 6.43(\mathrm{dd}, J=8.4,2.0 \mathrm{~Hz}, 1 \mathrm{H}), 6.26(\mathrm{~s}, 1 \mathrm{H})$, $5.05(\mathrm{~d}, J=15.6 \mathrm{~Hz}, 1 \mathrm{H}), 5.02(\mathrm{~d}, J=15.6 \mathrm{~Hz}, 1 \mathrm{H}), 4.62-4.57(\mathrm{~m}$, $1 \mathrm{H}), 3.99(\mathrm{~d}, J=3.2 \mathrm{~Hz}, 1 \mathrm{H}), 3.29-3.25(\mathrm{~m}, 4 \mathrm{H}), 2.69-2.63(\mathrm{~m}, 1 \mathrm{H}), 2.56-2.50(\mathrm{~m}, 1 \mathrm{H}), 2.01-1.98$ (m, 4H); ${ }^{13} \mathrm{C}$ NMR $\left(100 \mathrm{MHz}, \mathrm{CDCl}_{3}\right) \delta 168.2,146.9,144.2,142.3,134.7,131.2,130.1,129.3$, $128.9,128.5,127.5,126.9,123.8,111.6,106.0,74.9,69.5,47.9,46.8,38.9,25.8 ; \mathrm{C}_{27} \mathrm{H}_{26} \mathrm{Cl}_{2} \mathrm{NO}_{3}$ $[\mathrm{M}+\mathrm{H}]^{+}$:calcd 482.1284, found 482.1288 .

HPLC: Chiralpak AD-H column $(250 \mathrm{~mm})$; detected at $254 \mathrm{~nm}$; hexane $/ i$-propanol $=90 / 10$; flow $=0.7 \mathrm{~mL} / \mathrm{min}$; Retention time: $6.4 \mathrm{~min}$ (major), $12.2 \mathrm{~min}$.

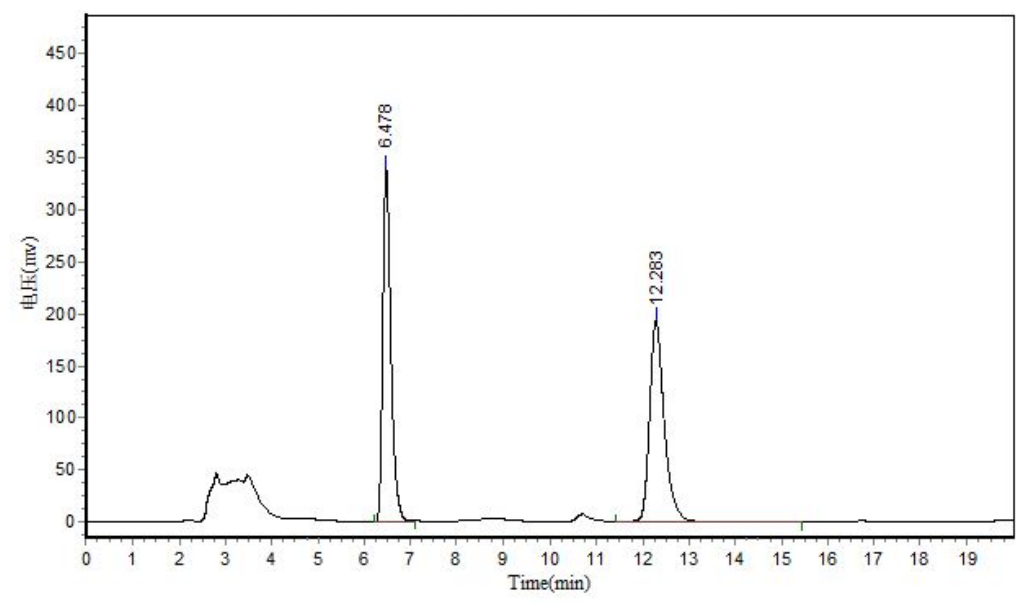

\begin{tabular}{|c|c|c|c|c|c|}
\hline \multicolumn{6}{|c|}{ Results } \\
\hline Peak No. & Peak ID & Ret Time & Height & Area & Conc. \\
\hline 1 & & 6.478 & 337540.563 & 4128246.250 & 48.9074 \\
\hline 2 & & 12.283 & 192430.906 & 4312704.000 & 51.0926 \\
\hline Total & & & 529971.469 & 8440950.250 & 100.000 \\
\hline
\end{tabular}
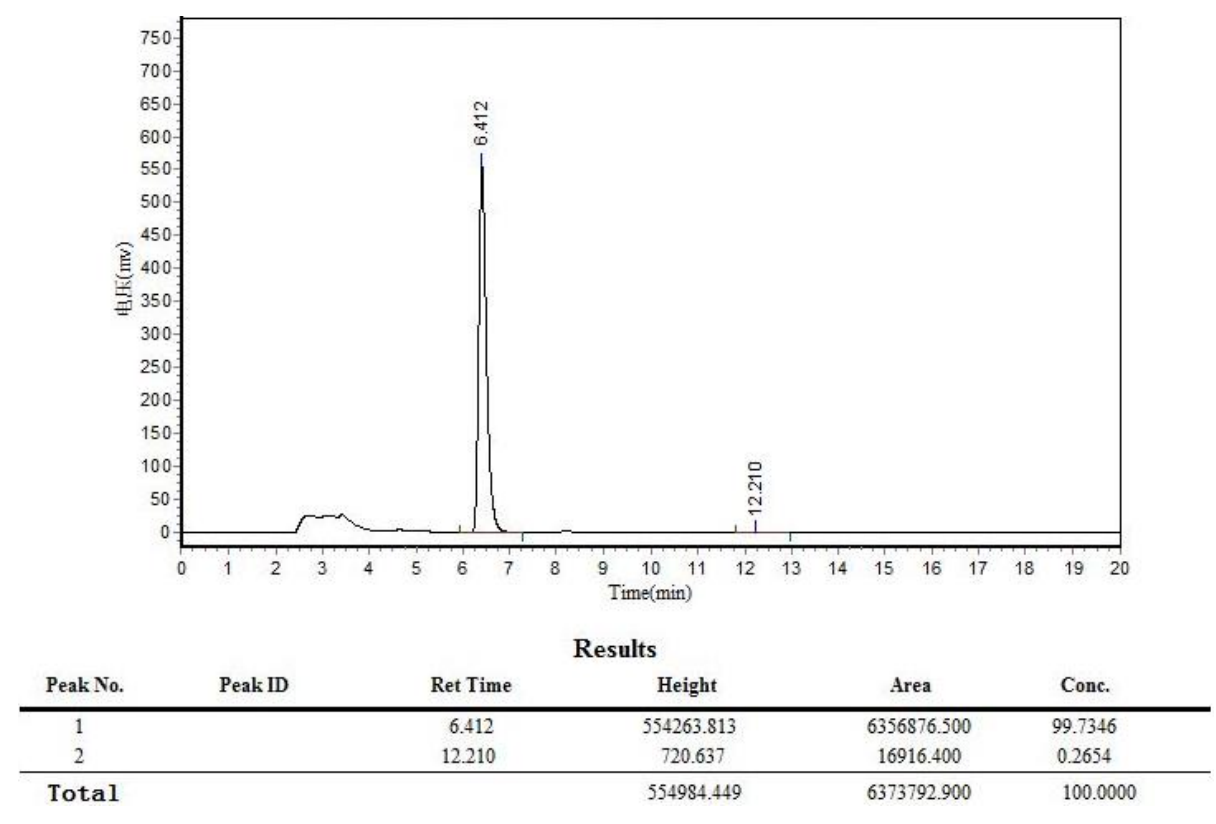


\section{The Gram-Scale Synthesis of 3ac and Its Derivatization}

\subsection{The gram-scale synthesis of 3ac}<smiles>N#CC(=CC=Cc1ccccc1)C(=O)Oc1c(Cl)cccc1Cl</smiles>

$1 \mathrm{a}$

$2 \mathrm{mmol}$<smiles>Oc1cccc([18OH])c1</smiles>

2c

$3 \mathrm{mmol}$

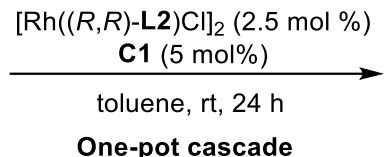

One-pot cascade

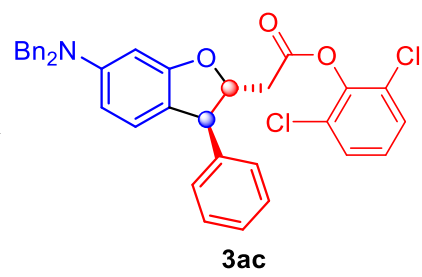

$898 \mathrm{mg}, 76 \%$ yield, $99 \%$ ee

Under Ar atmosphere, one tube with $[\mathrm{Rh}(\mathbf{L} 2) \mathrm{Cl}]_{2}(52 \mathrm{mg}, 0.05 \mathrm{mmol}, 2.5 \mathrm{~mol} \%)$ in $20 \mathrm{~mL}$ of toluene was stirred at room temperature. A mixture of (E)-2,6-dichlorophenyl 2-diazo-4-phenylbut-3-enoate (666 mg, $2 \mathrm{mmol}$ ), 3-(dibenzylamino)phenol (867 mg, $3 \mathrm{mmol}$ ) and cinchona alkaloid catalyst $\mathbf{C 1}(60 \mathrm{mg}, 0.01 \mathrm{mmol}, 5 \mathrm{~mol} \%)$ in $60 \mathrm{~mL}$ of toluene was added in one portion and then the resulting mixture was stirred at $\mathrm{rt}$ for $24 \mathrm{~h}$. The solvent was removed under reduced pressure and the residue was purified by silica gel column chromatography using petroleum ether/ethyl acetate to afford the corresponding product 3ac (898 mg, 76\% yield).

${ }^{1} \mathrm{H}$ NMR $\left(400 \mathrm{MHz}, \mathrm{CDCl}_{3}\right) \delta 7.36-7.24(\mathrm{~m}, 17 \mathrm{H}), 7.13(\mathrm{t}, J=8.0 \mathrm{~Hz}, 1 \mathrm{H}), 6.79(\mathrm{~d}, J=8.0 \mathrm{~Hz}$, $1 \mathrm{H}), 6.35(\mathrm{~d}, J=2.4 \mathrm{~Hz}, 1 \mathrm{H}), 6.29(\mathrm{dd}, J=8.0,2.0 \mathrm{~Hz}, 1 \mathrm{H}), 5.09$ (q, $J=6.0 \mathrm{~Hz}, 1 \mathrm{H}), 4.64-4.60$ $(\mathrm{m}, 4 \mathrm{H}), 4.36(\mathrm{~d}, J=6.4 \mathrm{~Hz}, 1 \mathrm{H}), 3.24-3.11(\mathrm{~m}, 2 \mathrm{H}) ;{ }^{13} \mathrm{C} \mathrm{NMR}\left(100 \mathrm{MHz}, \mathrm{CDCl}_{3}\right) \delta 167.2,161.0$, 151.1, 144.1, 142.9, 138.8, 129.2, 129.1, 129.0, 128.9, 128.3, 127.6, 127.4, 127.2, 127.0, 125.8, $118.1,106.0,94.9,87.4,54.8,54.0,39.4$.

HPLC: Chiralpak AD-H column $(250 \mathrm{~mm})$; detected at $254 \mathrm{~nm}$; hexane $/ i$-propanol $=90 / 10$; flow $=1.2 \mathrm{~mL} / \mathrm{min}$; Retention time: $11.4 \mathrm{~min}$ (major), $18.2 \mathrm{~min}$.

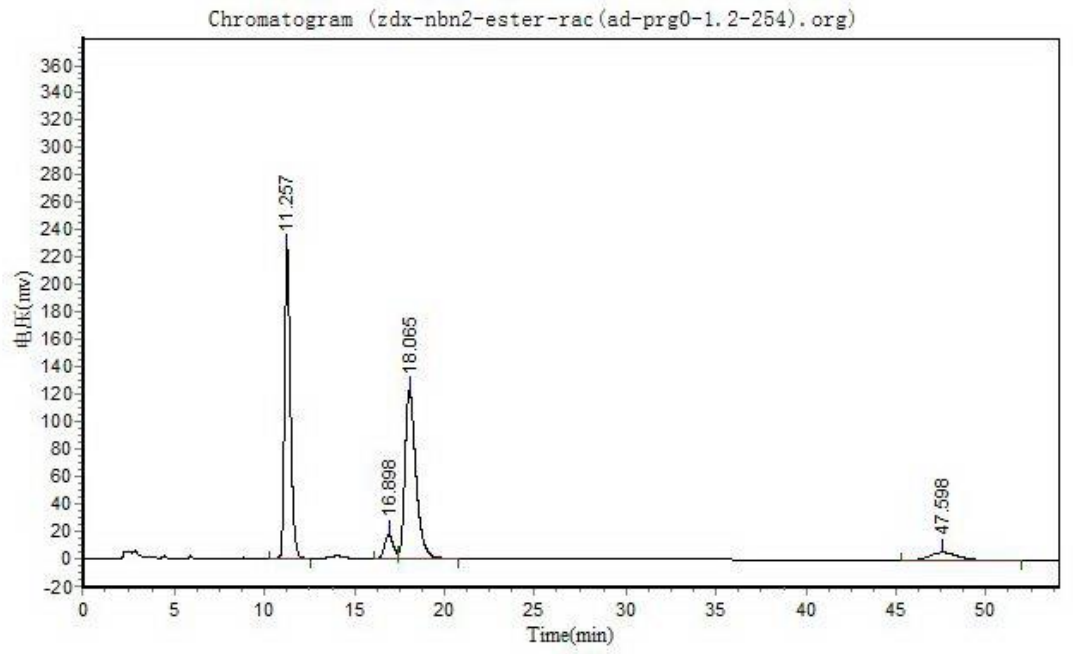

\begin{tabular}{|c|c|c|c|c|c|}
\hline \multicolumn{6}{|c|}{ Results } \\
\hline Peak No. & Peak ID & Ret Time & Height & Area & Conc. \\
\hline 1 & & 11.257 & 226164.859 & 5144868.500 & 44.5193 \\
\hline 2 & & 16.898 & 17734.096 & 588270.063 & 5.0904 \\
\hline 3 & & 18.065 & 122827.633 & 5171198.000 & 44.7471 \\
\hline 4 & & 47.598 & 5806.254 & 652150.625 & 5.6432 \\
\hline Total & & & 372532.842 & 11556487.188 & 100.0000 \\
\hline
\end{tabular}




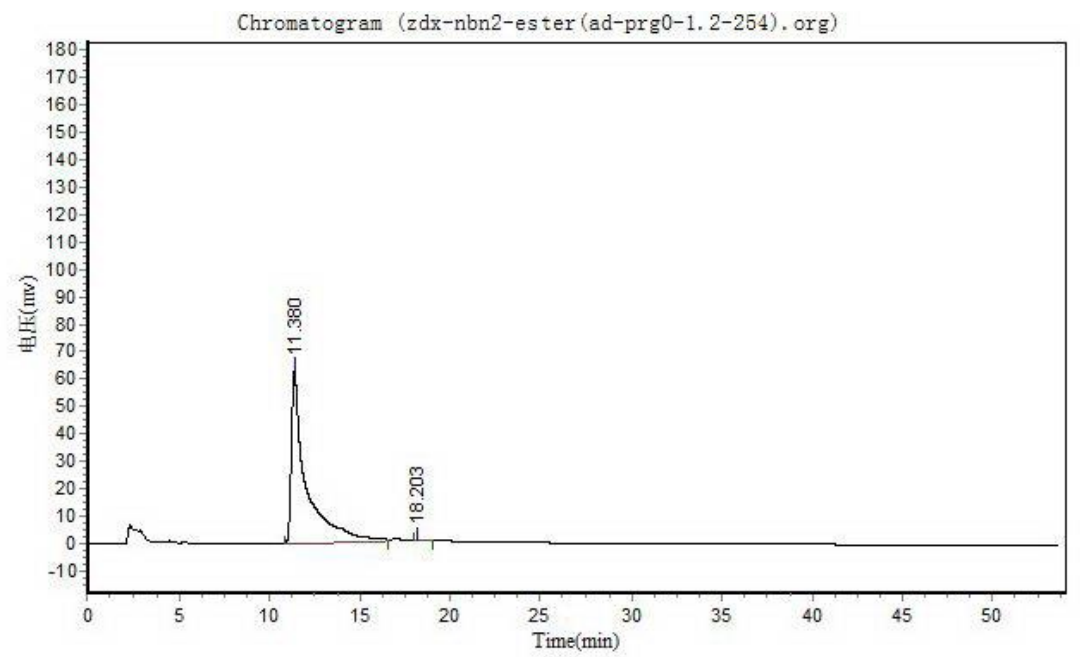

\begin{tabular}{|c|c|c|c|c|c|}
\hline \multicolumn{6}{|c|}{ Results } \\
\hline Peak No. & Peak ID & Ret Time & Height & Area & Conc. \\
\hline 1 & & 11.380 & 62461.547 & 3476205.750 & 99.8654 \\
\hline 2 & & 18.203 & 142.510 & 4686.530 & 0.1346 \\
\hline Total & & & 62604.056 & 3480892.280 & 100.0000 \\
\hline
\end{tabular}

\subsection{Reduction of 3 ac}

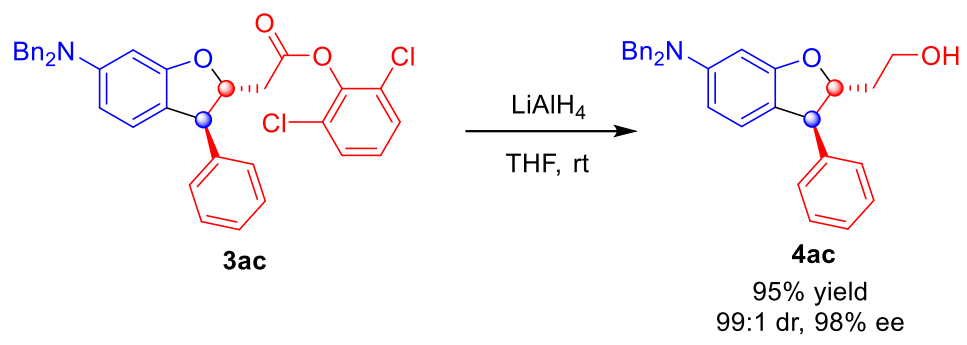

To a solution of $3 \mathbf{a c}(593 \mathrm{mg}, 1 \mathrm{mmol})$ in $20 \mathrm{~mL}$ of anhydrous THF was added $\mathrm{LiAlH}_{4}(152 \mathrm{mg}$, $4 \mathrm{mmol}$ ), After stirring the mixture at room temperature for $10 \mathrm{~min}$, the reaction was quenched with ice water and saturated aqueous sodium potassium tartrate was added, and the solution was stirred at room temperature for another $30 \mathrm{~min}$. then the aqueous layer was extracted with EA for three times, the combined organic layers were dried over $\mathrm{Na}_{2} \mathrm{SO}_{4}$ and concentrated under reduced pressure. The crude product was purified by silica gel column chromatography using petroleum ether/ethyl acetate (4:1) to afford the corresponding alcohol 4 ac (413 mg).

\section{2-((2R,3S)-6-(dibenzylamino)-3-phenyl-2,3-dihydrobenzofuran-2-yl)ethanol (4ac)}

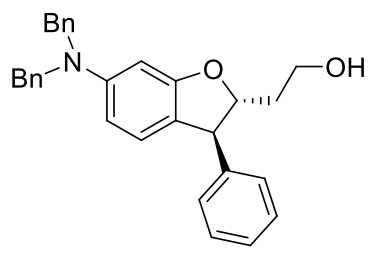

95\% yield, 99:1 dr, 98\% ee; $[\alpha]_{\mathrm{D}}{ }^{20}=13.1\left(c 1.0, \mathrm{CHCl}_{3}\right) ;{ }^{1} \mathrm{H}$ NMR $\left(400 \mathrm{MHz}, \mathrm{CDCl}_{3}\right) \delta$ 7.37-7.24 (m, $\left.15 \mathrm{H}\right), 6.74(\mathrm{~d}, J=8.4 \mathrm{~Hz}, 1 \mathrm{H})$, 6.30-6.27 (m, 2H), 4.70-4.61 (m, 5H), $4.23(\mathrm{~d}, J=8.4 \mathrm{~Hz}, 1 \mathrm{H}), 3.84(\mathrm{~d}$, $J=6.0 \mathrm{~Hz}, 2 \mathrm{H}), 2.13-2.00(\mathrm{~m}, 3 \mathrm{H}) ;{ }^{13} \mathrm{C} \mathrm{NMR}\left(100 \mathrm{MHz}, \mathrm{CDCl}_{3}\right) \delta$ $161.2,150.9,142.7,138.8,129.0,128.9,128.6,127.4,127.2,126.9$, 125.6, 118.7, 105.7, 94.5, 91.5, 60.8, 54.9, 54.8, 37.4; HRMS (ESI) for $\mathrm{C}_{30} \mathrm{H}_{30} \mathrm{NO}_{2}[\mathrm{M}+\mathrm{H}]^{+}$: calcd 436.2271, found 436.2269. 
HPLC: Chiralpak OD-H column $(250 \mathrm{~mm})$; detected at $254 \mathrm{~nm}$; hexane $/ i$-propanol $=90 / 10$; flow $=0.7 \mathrm{~mL} / \mathrm{min}$; Retention time: $24.8 \mathrm{~min}$ (major), $36.2 \mathrm{~min}$.

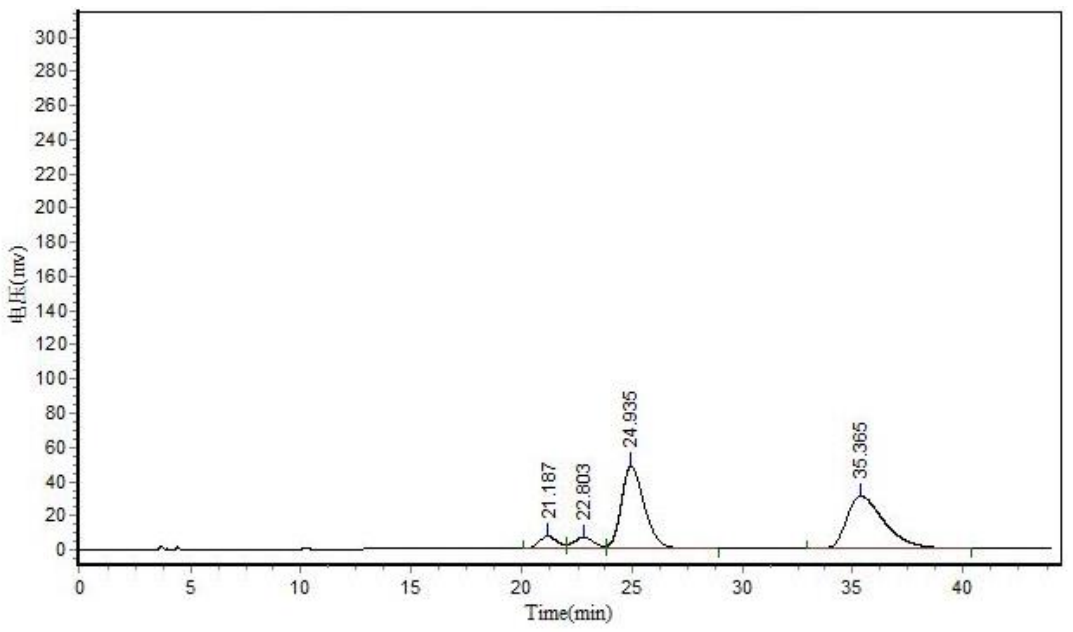

Results

\begin{tabular}{|c|c|c|c|c|c|}
\hline \multicolumn{6}{|c|}{ ( } \\
\hline Peak No. & Peak ID & Ret Time & Height & Area & Conc. \\
\hline 1 & & 21.187 & 7208.800 & 425523.031 & 5.3006 \\
\hline 2 & & 22.803 & 6517.118 & 431953.344 & 5.3807 \\
\hline 3 & & 24.935 & 48412.168 & 3533943.500 & 44.0210 \\
\hline 4 & & 35.365 & 30719.078 & 3636436.250 & 45.2977 \\
\hline Total & & & 92857.164 & 8027856.125 & 100.000 \\
\hline
\end{tabular}

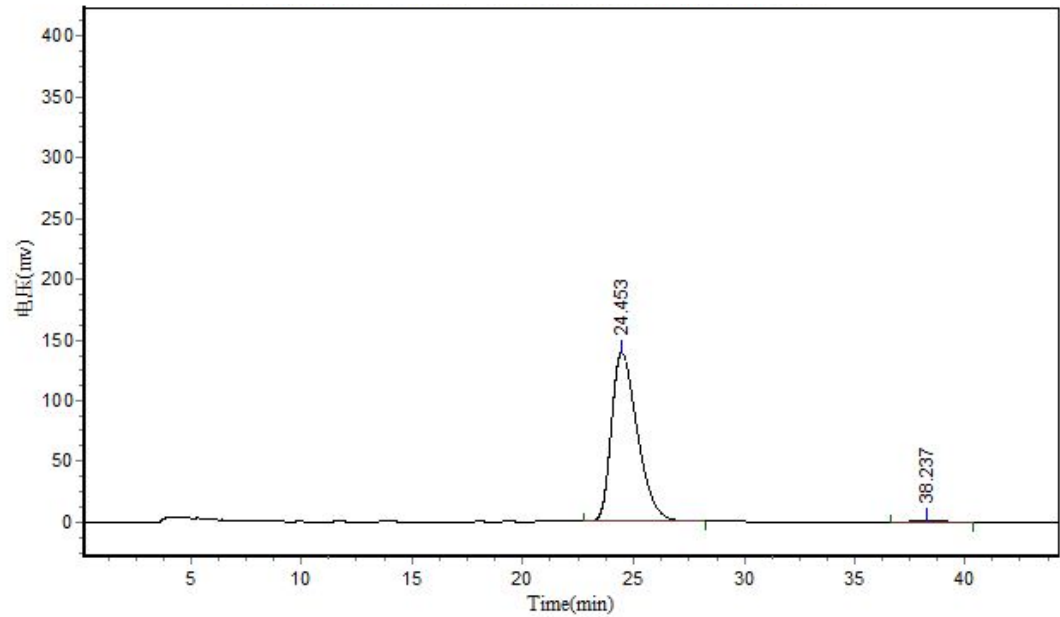

Results

\begin{tabular}{|c|c|c|c|c|c|}
\hline Peak No. & Peak ID & Ret Time & Height & Area & Conc. \\
\hline 1 & & 24.453 & 139434.922 & 11476899.000 & 98.9743 \\
\hline 2 & & 38.237 & 960.814 & 118933.602 & 1.0257 \\
\hline Total & & & 140395.736 & 11595832.602 & 100.000 \\
\hline
\end{tabular}




\section{3 $N$-Debenzylation of 4ac}

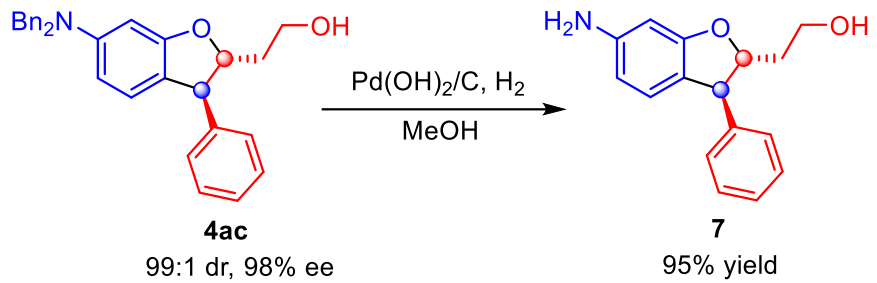

The alcohol product $4 \mathrm{ac}(174 \mathrm{mg}, 0.4 \mathrm{mmol})$ was dissolved in $\mathrm{MeOH}(20.0 \mathrm{~mL})$ and hydrogenated in a flow of hydrogen in the presence of $\mathrm{Pd}(\mathrm{OH})_{2} / \mathrm{C}(10 \mathrm{mg})$ at room temperature for $10 \mathrm{~h}$. After 4 ac was complete disappeared determined by TLC, the reaction mixture was filtered, the solid was washed with $\mathrm{MeOH}$ and the combined filtrate was concentrated in vacuo. The crude product was purified by silica gel column chromatography using petroleum ether/ethyl acetate to afford the corresponding solid product 7 ( $97 \mathrm{mg}, 95 \%$ yield).

\section{2-((2R,3S)-6-amino-3-phenyl-2,3-dihydrobenzofuran-2-yl)ethanol (7)}

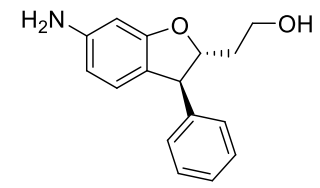

White solid, m.p.106.8-108.5 ${ }^{\circ} \mathrm{C}, 95 \%$ yield; $[\alpha]_{\mathrm{D}}{ }^{20}=9.9\left(c 1.0, \mathrm{CHCl}_{3}\right) ;{ }^{1} \mathrm{H}$ NMR $\left(400 \mathrm{MHz}, \mathrm{CDCl}_{3}\right) \delta 7.34-7.20(\mathrm{~m}, 5 \mathrm{H}), 6.71(\mathrm{~d}, J=7.6 \mathrm{~Hz}, 1 \mathrm{H})$, 6.22-6.19 (m, 2H), 4.71-4.66 (m, 1H), $4.20(\mathrm{~d}, J=8.0 \mathrm{~Hz}, 1 \mathrm{H}), 3.86(\mathrm{t}, J=$ $6.0 \mathrm{~Hz}, 2 \mathrm{H}), 2.16-2.00(\mathrm{~m}, 2 \mathrm{H}) ;{ }^{13} \mathrm{C}$ NMR $\left(100 \mathrm{MHz}, \mathrm{CDCl}_{3}\right) \delta 160.9$, $147.7,142.8,129.1,128.5,127.4,126.0,120.7,108.3,97.2$, 91.6, 60.8, 54.8, 37.5; HRMS (ESI) for $\mathrm{C}_{16} \mathrm{H}_{18} \mathrm{NO}_{2}[\mathrm{M}+\mathrm{H}]^{+}$:calcd 256.1332, found 256.1337.

\subsection{Determine the enantiomeric excess of 7}

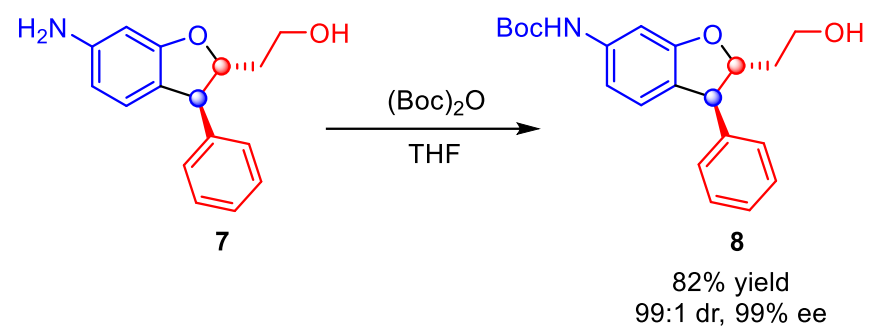

To a solution of 7 (52 mg, $0.2 \mathrm{mmol})$ in $20 \mathrm{~mL}$ of anhydrous THF was added (Boc) $)_{2} \mathrm{O}(52 \mathrm{mg}$, $0.24 \mathrm{mmol}$ ), then the mixture was stirred at room temperature until the substrate 7 was complete disappeared. Then the solvent was removed under reduced pressure and the residue was diluted with water, extracted with ethyl acetate for 3 times, the combined organic phase washed with brine, dried over $\mathrm{Na}_{2} \mathrm{SO}_{4}$, the solvent was removed under reduced pressure and the residue was purified by flash column chromatography afforded the corresponding oil product 8 (58 $\mathrm{mg}, 82 \%$ yield, 99:1 dr, 99\% ee). 
tert-butyl (2R,3S)-2-(2-hydroxyethyl)-3-phenyl-2,3-dihydrobenzofuran-6-ylcarbamate (8)

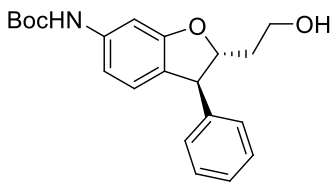

Colorless oil, $82 \%$ yield, $99: 1 \mathrm{dr}, 99 \%$ ee; $[\alpha]_{\mathrm{D}}^{20}=29.0\left(c 0.96, \mathrm{CHCl}_{3}\right)$; ${ }^{1} \mathrm{H}$ NMR $\left(400 \mathrm{MHz}, \mathrm{CDCl}_{3}\right) \delta$ 7.33-7.23 (m, 3H), $7.19(\mathrm{~d}, J=7.2 \mathrm{~Hz}$, 2H), $7.01(\mathrm{~s}, 1 \mathrm{H}), 6.83(\mathrm{~d}, J=8.0 \mathrm{~Hz}, 1 \mathrm{H}), 6.76(\mathrm{~d}, J=8.0 \mathrm{~Hz}, 1 \mathrm{H}), 6.58$ (s, 1H), $4.72(\mathrm{td}, J=8.0,4.0 \mathrm{~Hz}, 1 \mathrm{H}), 4.24(\mathrm{~d}, J=8.0 \mathrm{~Hz}, 1 \mathrm{H}), 3.86(\mathrm{t}, J$ $=6.0 \mathrm{~Hz}, 2 \mathrm{H}), 2.10-2.01(\mathrm{~m}, 2 \mathrm{H}), 1.51(\mathrm{~s}, 9 \mathrm{H}) ;{ }^{13} \mathrm{C} \mathrm{NMR}\left(100 \mathrm{MHz}, \mathrm{CDCl}_{3}\right) \delta 160.4,153.0$, 142.4, 139.3, 129.1, 128.5, 127.5, 125.5, 125.3, 111.6, 101.0, 91.5, 80.9, 60.6, 54.8, 37.6, 28.6; HRMS (ESI) for $\mathrm{C}_{21} \mathrm{H}_{24} \mathrm{NO}_{4}[\mathrm{M}-\mathrm{H}]$ : :calcd 354.1711, found 354.1706 .

HPLC: Chiralpak AD-H column $(250 \mathrm{~mm})$; detected at $254 \mathrm{~nm}$; hexane $/ i$-propanol $=95 / 5$; flow $=$ $0.7 \mathrm{~mL} / \mathrm{min}$; Retention time: $12.3 \mathrm{~min}, 26.1 \mathrm{~min}$ (major).

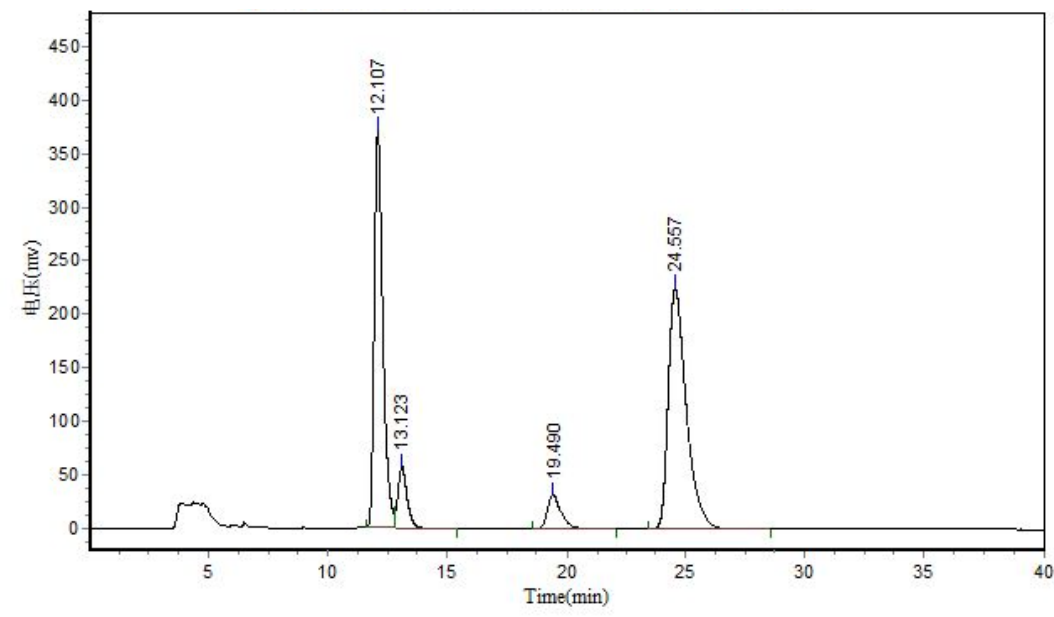

\begin{tabular}{|c|c|c|c|c|c|}
\hline \multicolumn{6}{|c|}{ Results } \\
\hline Peak No. & Peak ID & Ret Time & Height & Area & Conc. \\
\hline$\overline{1}$ & & 12.107 & 371140.875 & 9414720.000 & 38.8019 \\
\hline 2 & & 13.123 & 58609.363 & 1609086.250 & 6.6317 \\
\hline 3 & & 19.490 & 31951.250 & 1287209.625 & 5.3051 \\
\hline 4 & & 24.557 & 224467.109 & 11952514.000 & 49.2612 \\
\hline Total & & & 686168.598 & 24263529.875 & 100.000 \\
\hline
\end{tabular}

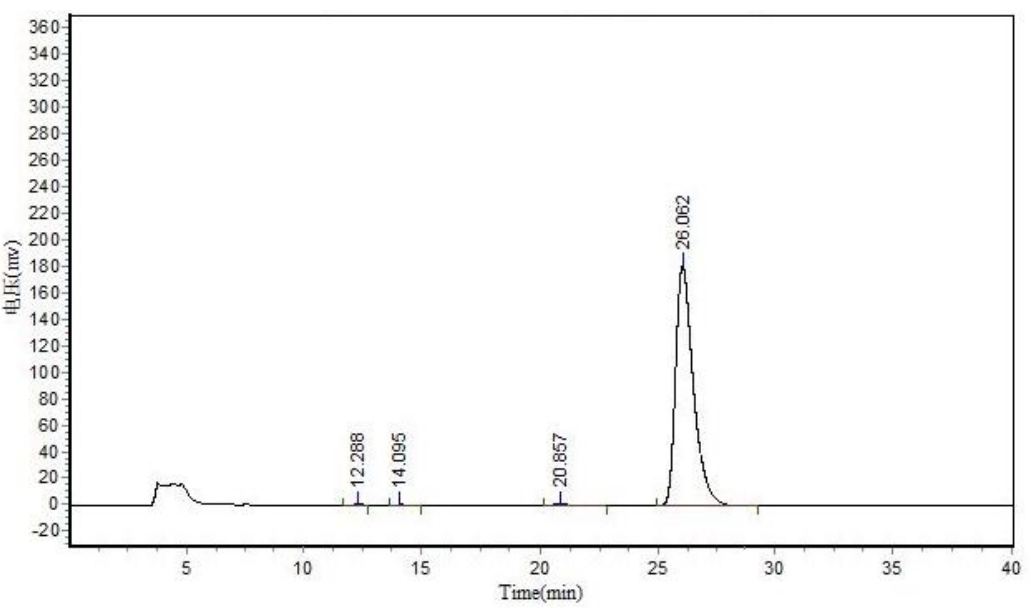

\begin{tabular}{|c|c|c|c|c|c|}
\hline \multicolumn{6}{|c|}{ Results } \\
\hline Peak No. & Peak ID & Ret Time & Height & Area & Conc. \\
\hline 1 & & 12.288 & 1184.442 & 28727.951 & 0.2985 \\
\hline 2 & & 14.095 & 749.497 & 19653.402 & 0.2042 \\
\hline 3 & & 20.857 & 1433.847 & 59334.855 & 0.6165 \\
\hline 4 & & 26.062 & 181008.969 & 9517238.000 & 98.8809 \\
\hline Total & & & 184376.754 & 9624954.209 & 100.0000 \\
\hline
\end{tabular}




\section{Copies of ${ }^{1} \mathrm{H}$ NMR and ${ }^{13} \mathrm{C}$ NMR Spectra of Products}

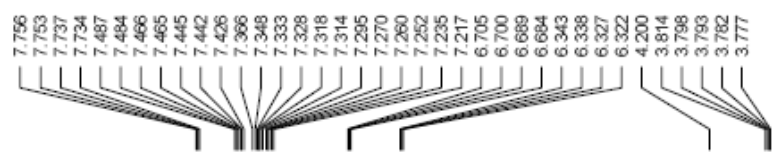

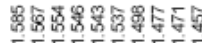
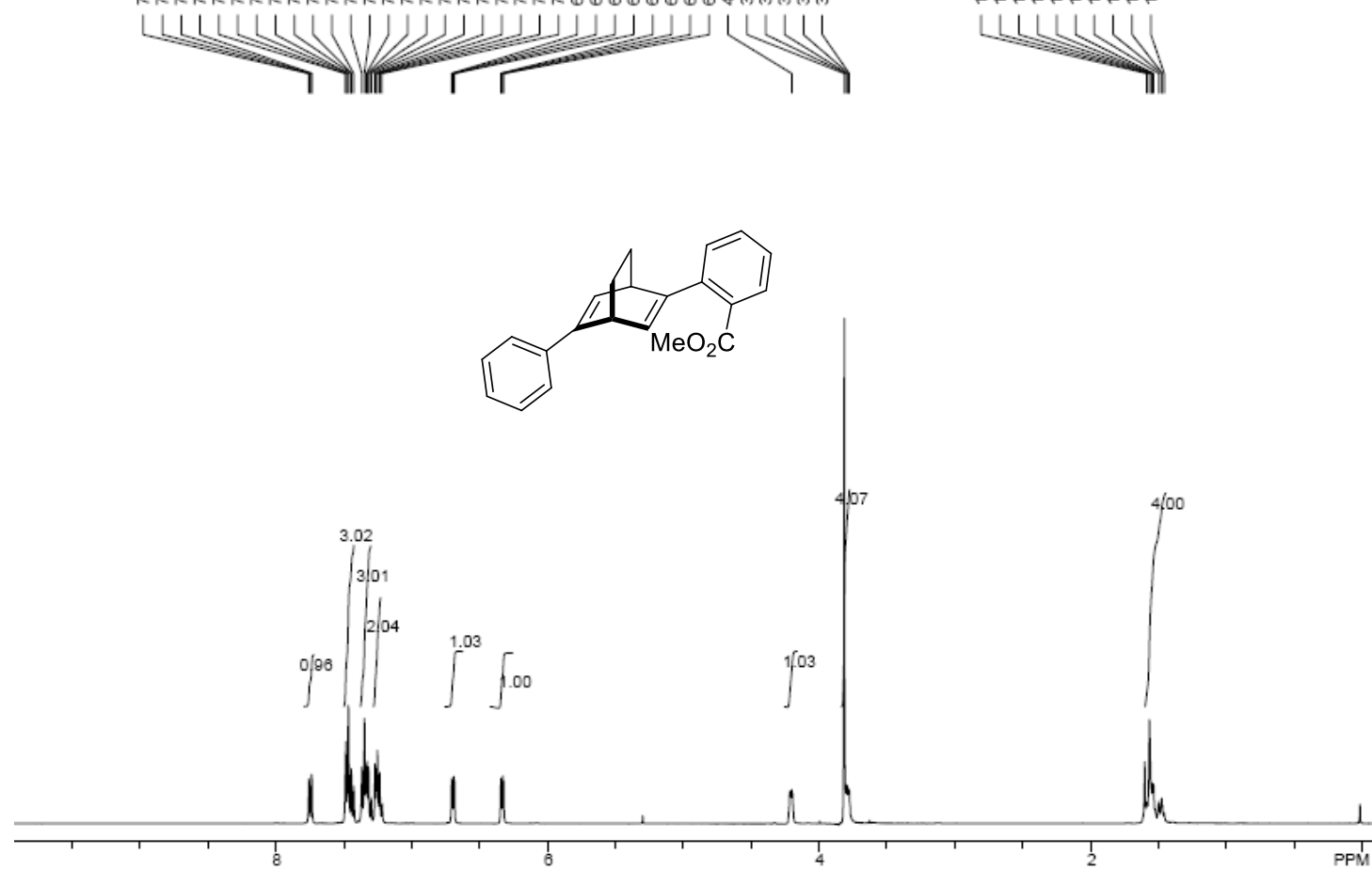

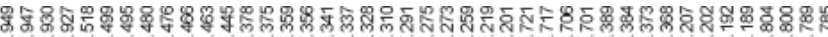
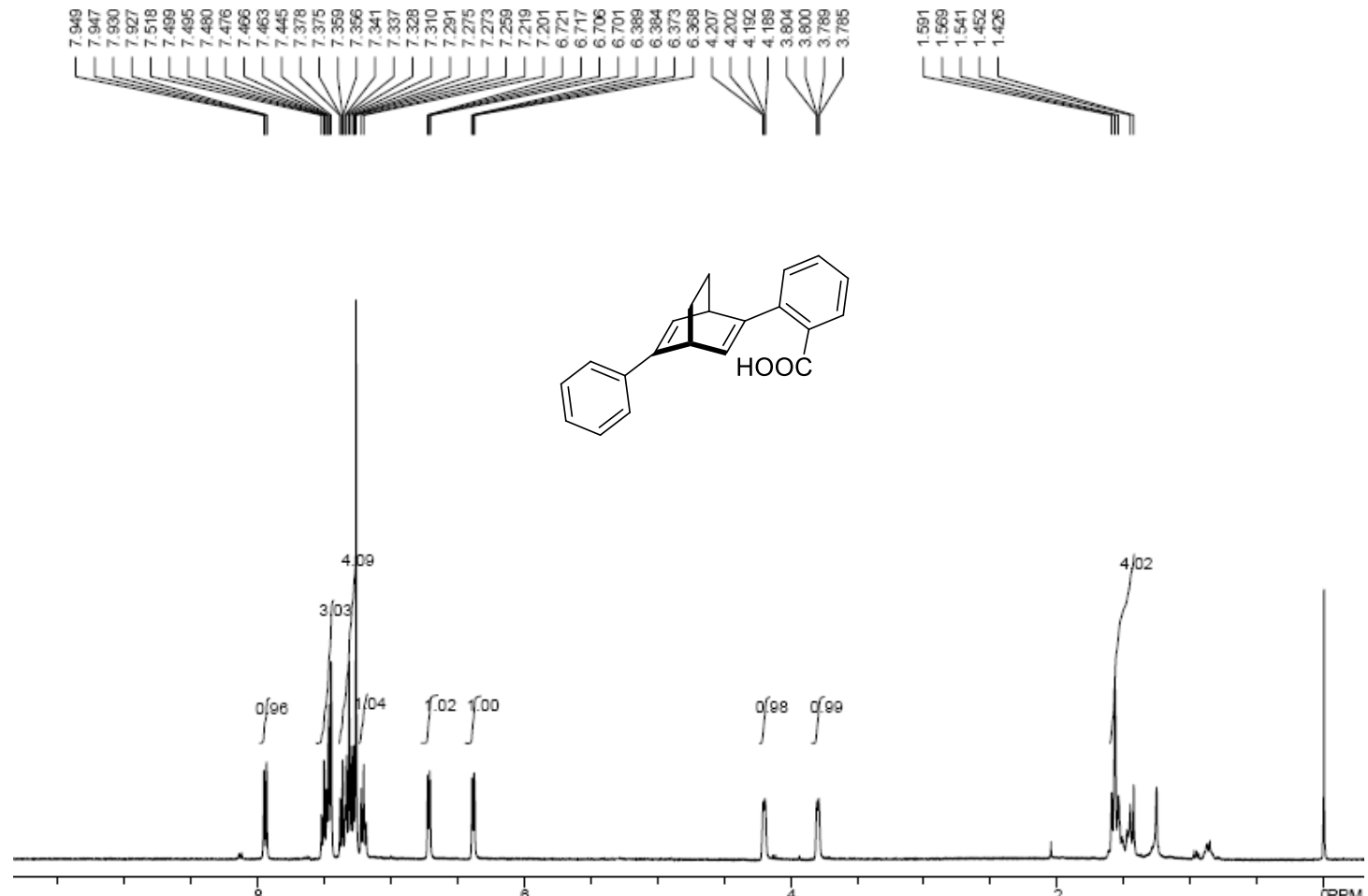


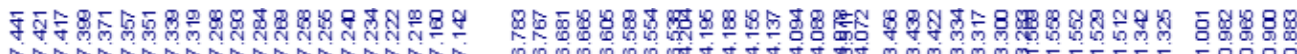

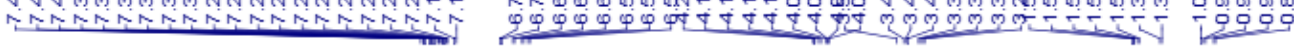
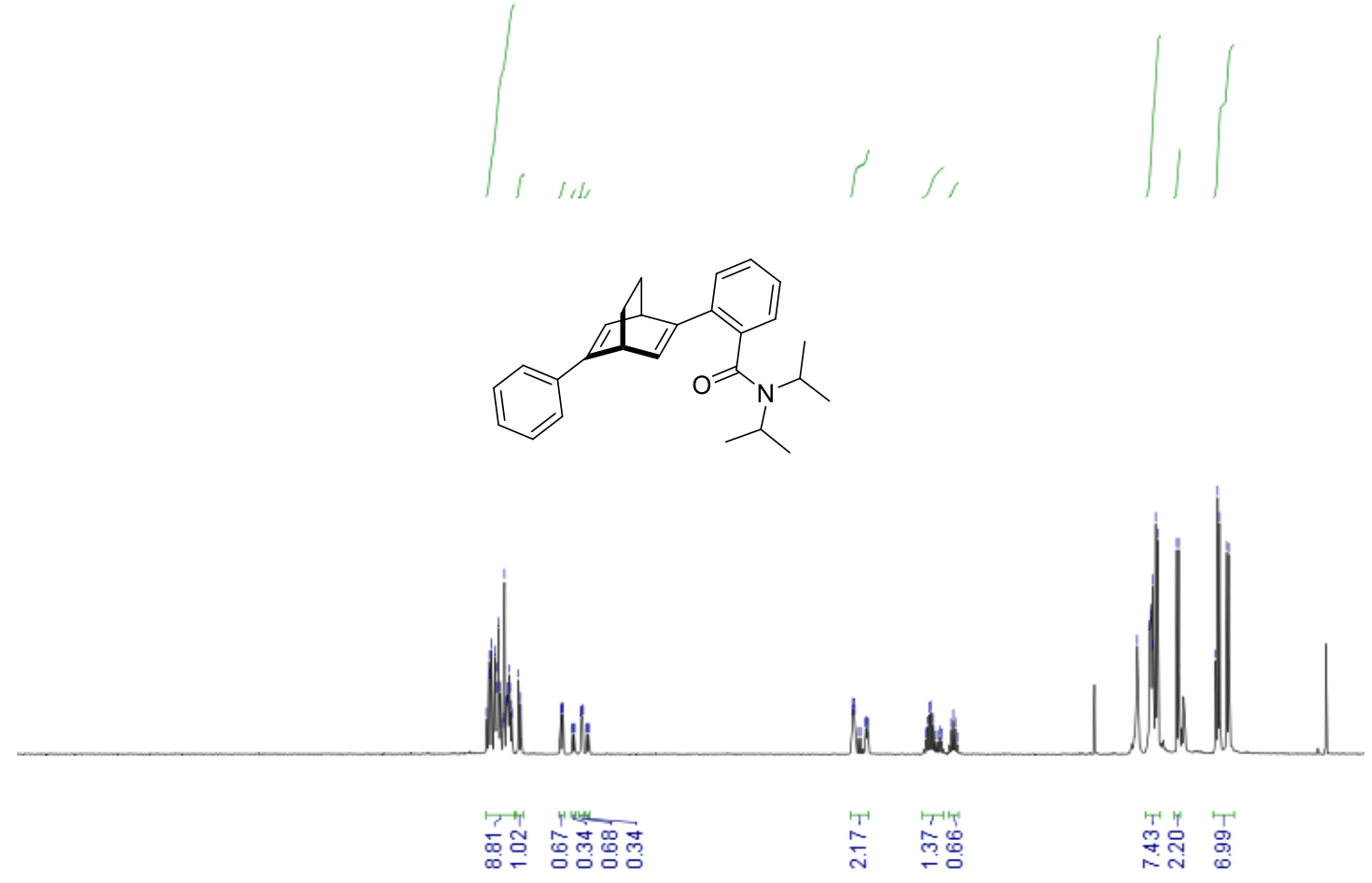

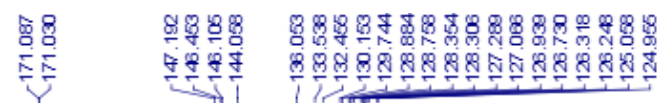

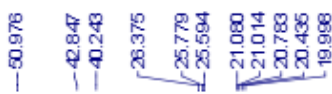

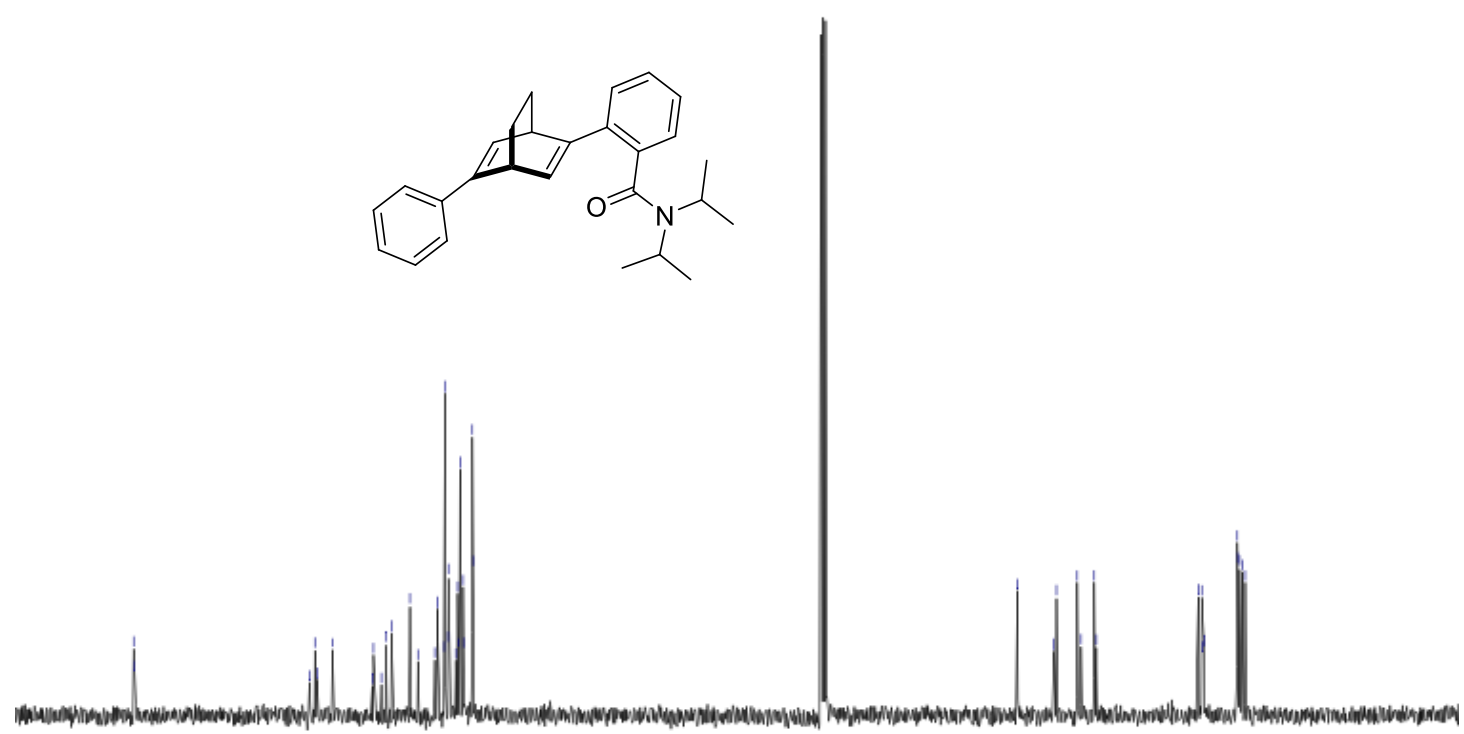



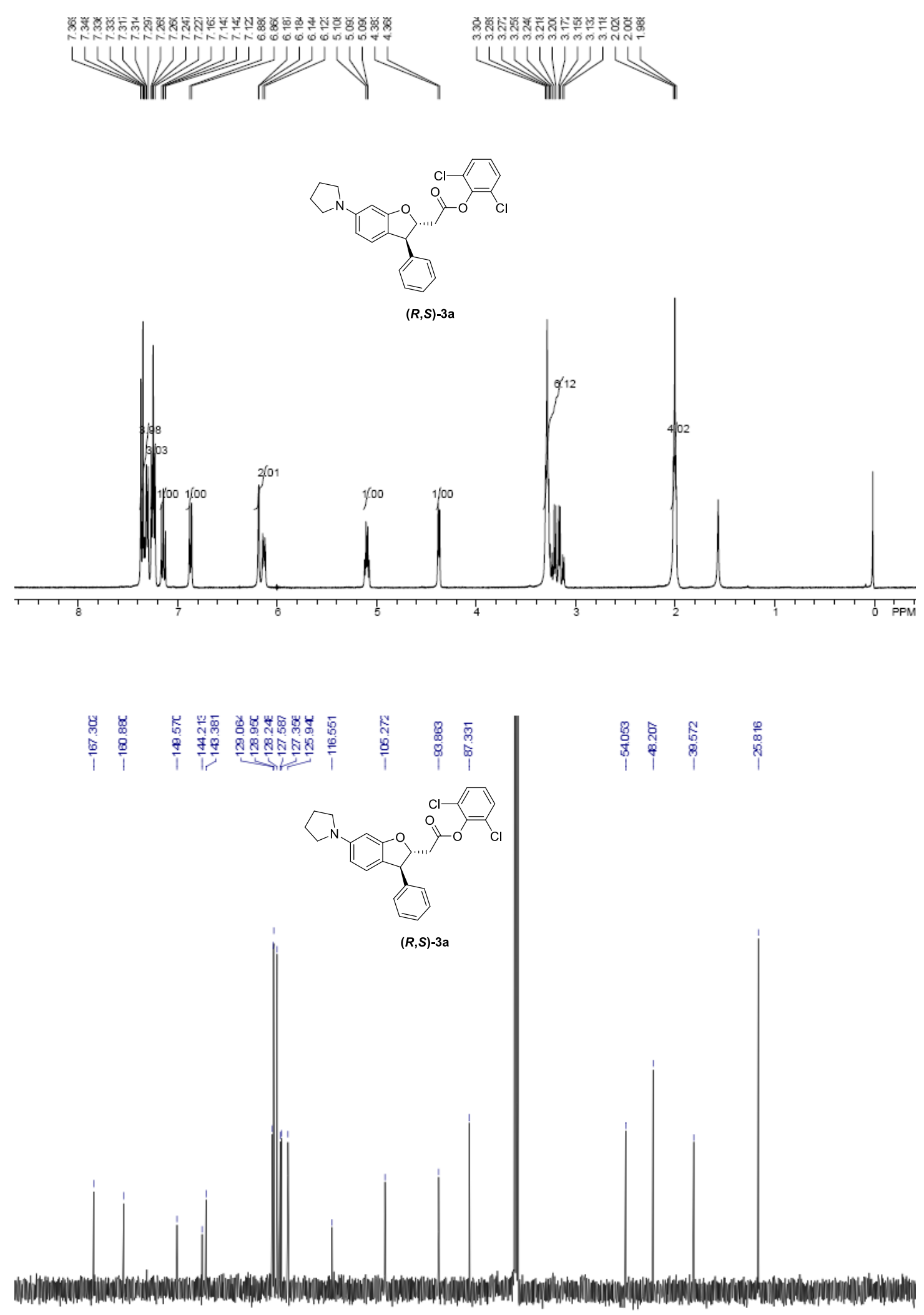

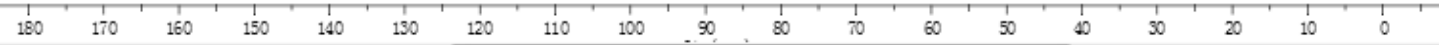




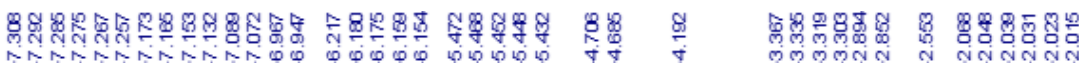

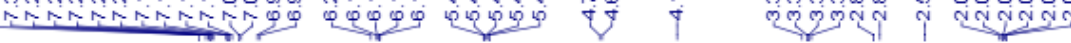
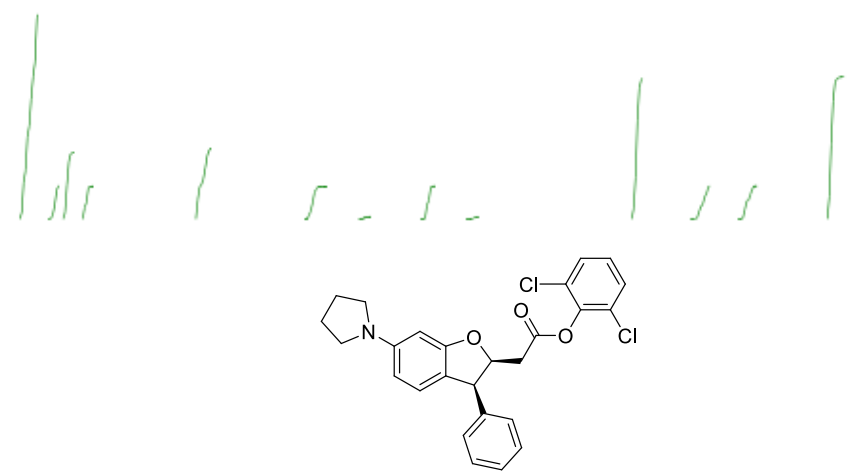

$(s, s)-3 a$

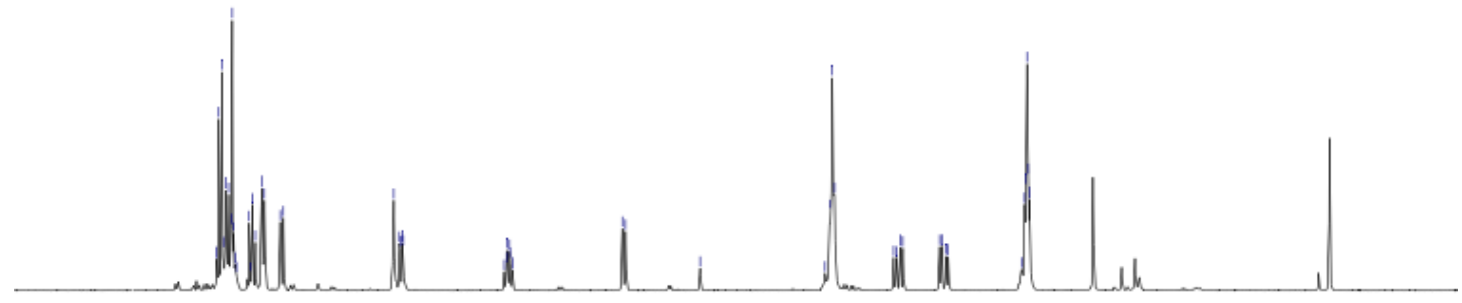

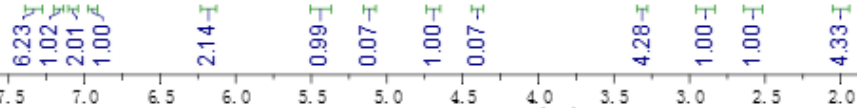

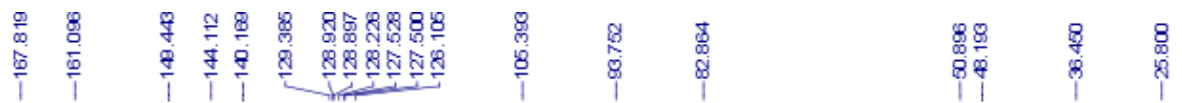<smiles>O=C(CC1Oc2cc(N3CCCC3)ccc2C1c1ccccc1)Oc1c(Cl)cccc1Cl</smiles>

$(s, s)-3 a$

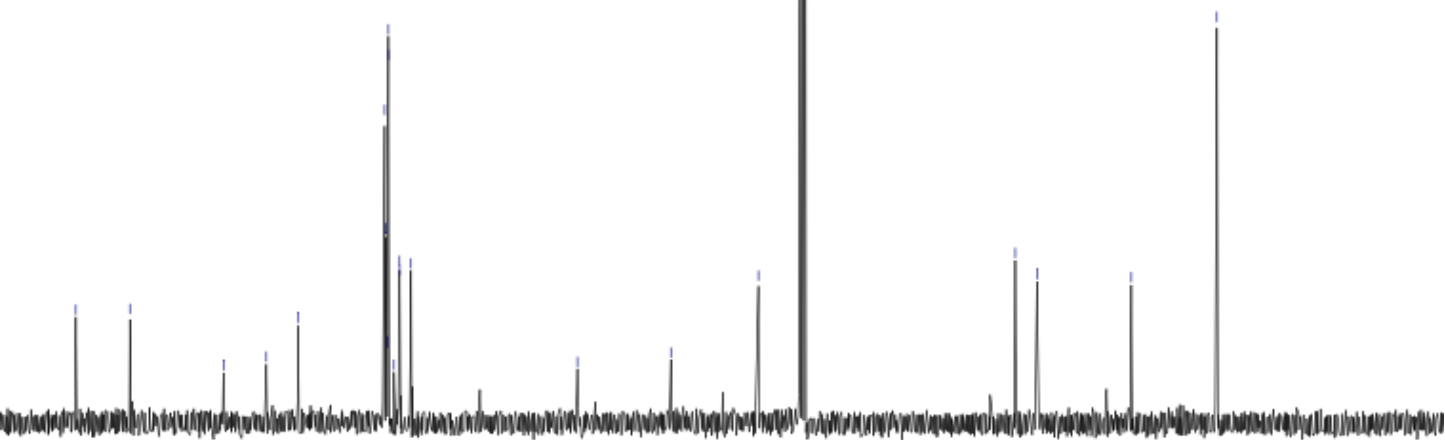



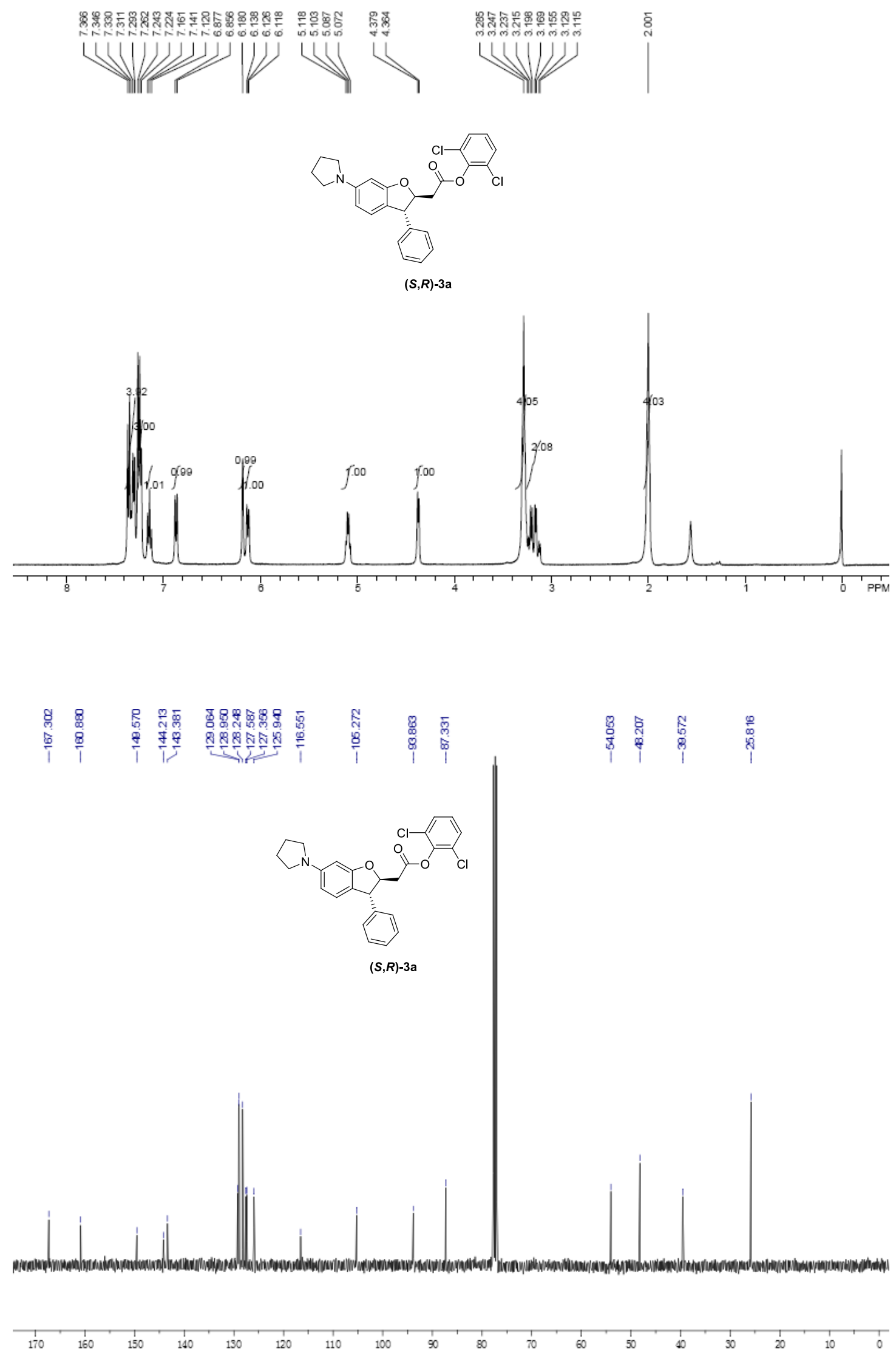


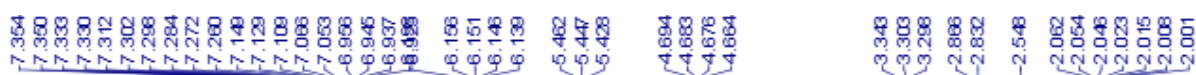
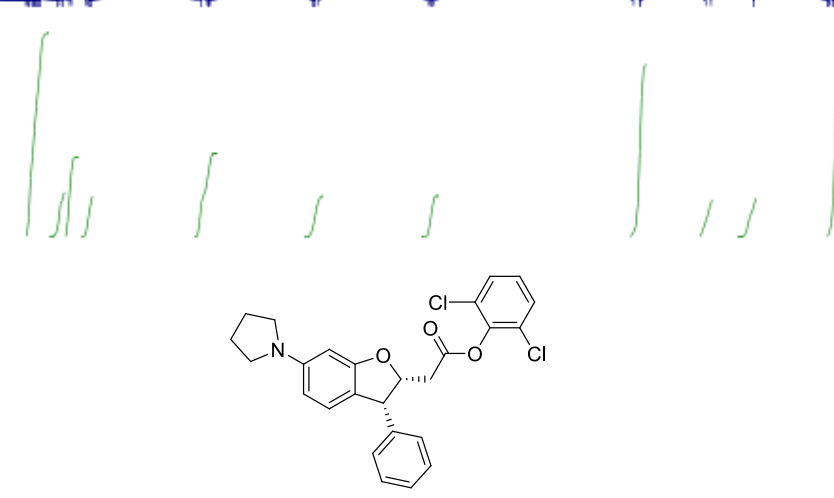

$(R, R)-3 a$

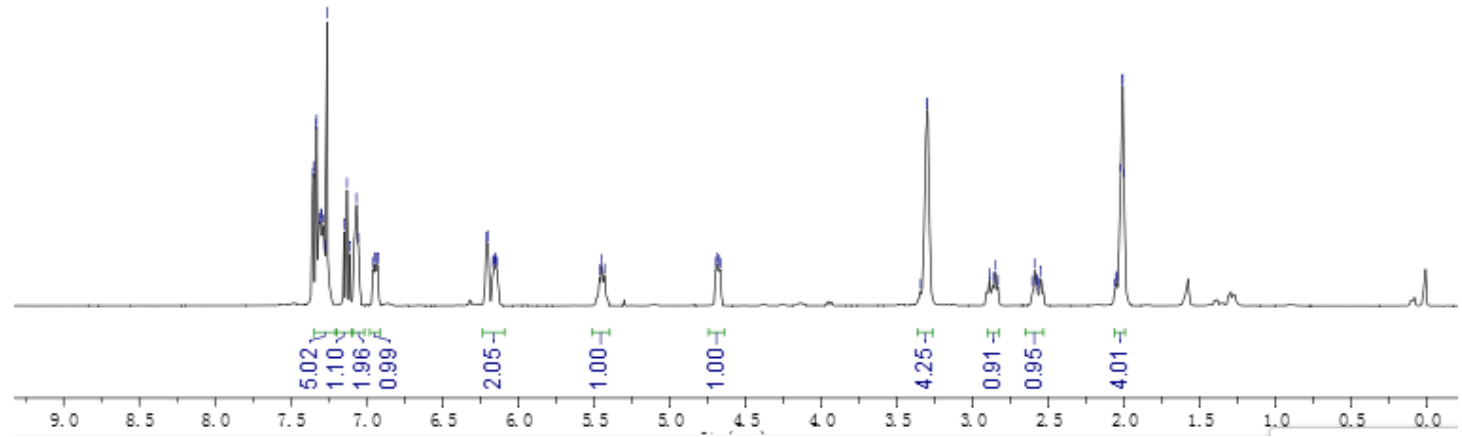

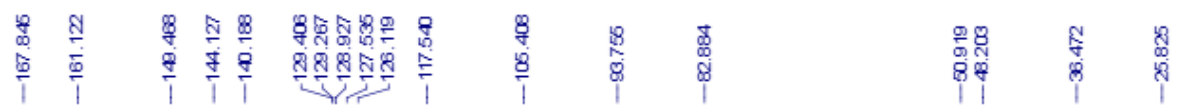<smiles>O=C(C[C@H]1Cc2ccc(N3CCCC3)cc2O1)Oc1c(Cl)cccc1Cl</smiles>

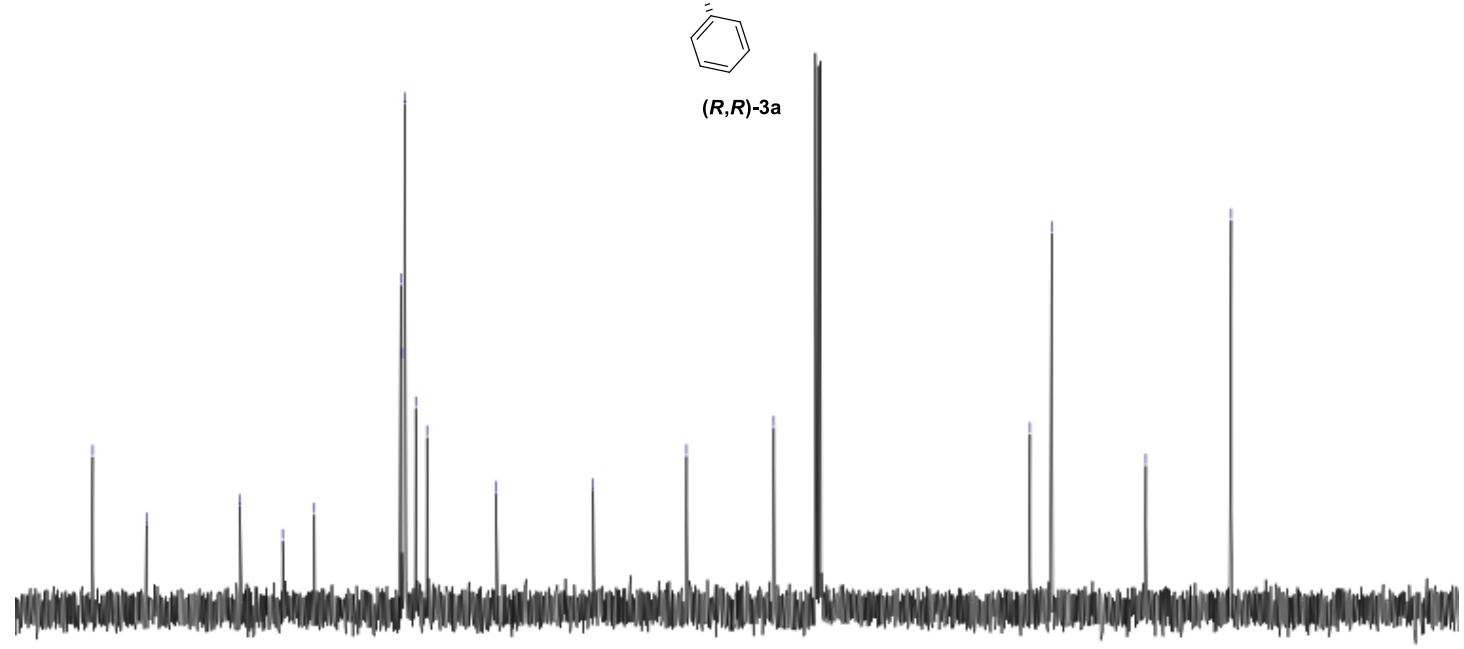

170

$160 \quad 150 \quad 140$

$130 \quad 120$

$110 \quad 100$

80

$10 \quad 30, \frac{1}{20}, 10, \frac{1}{10}$ 

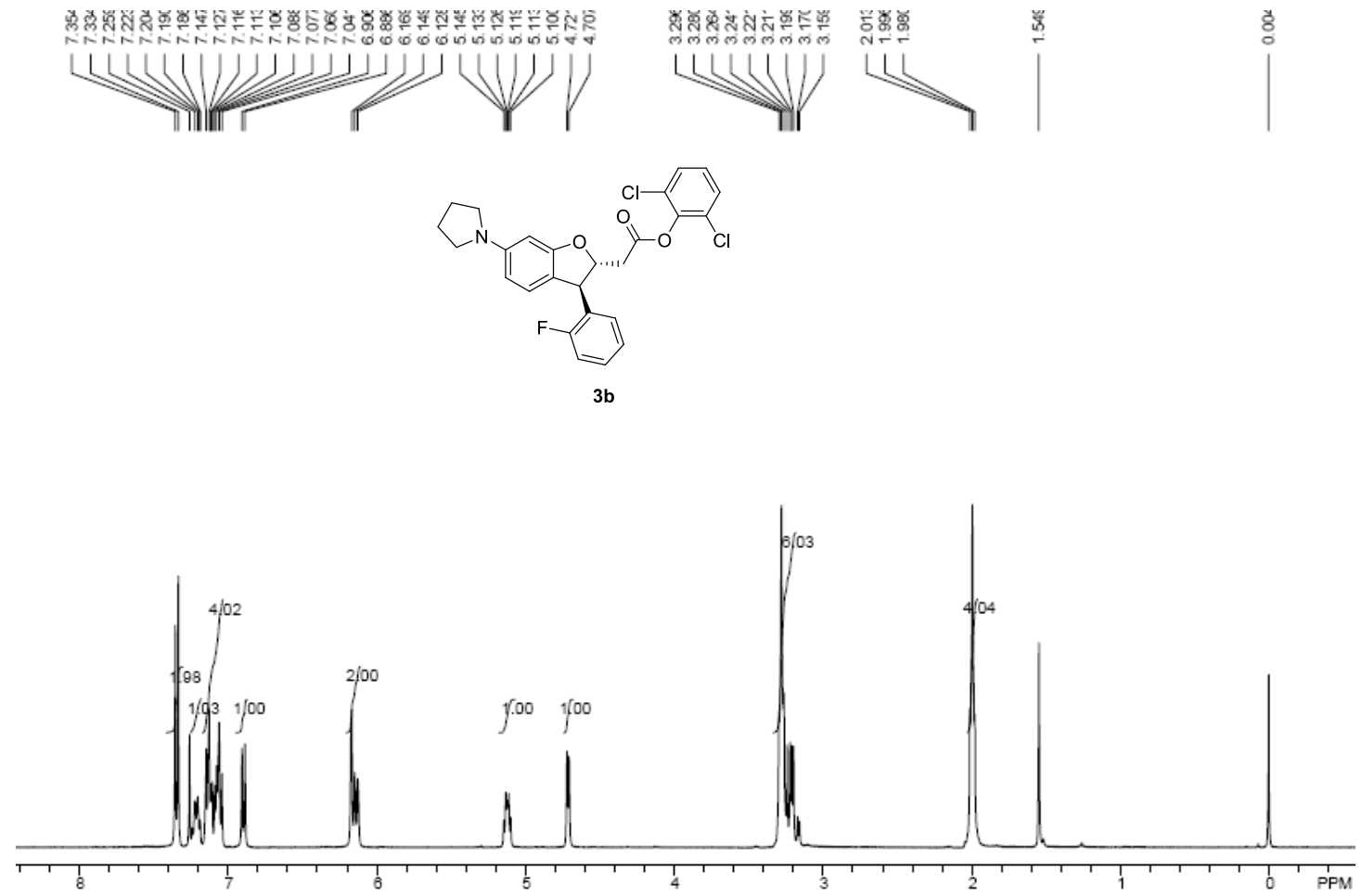

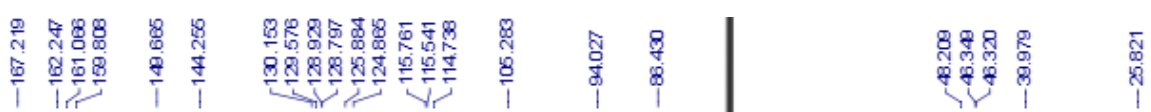

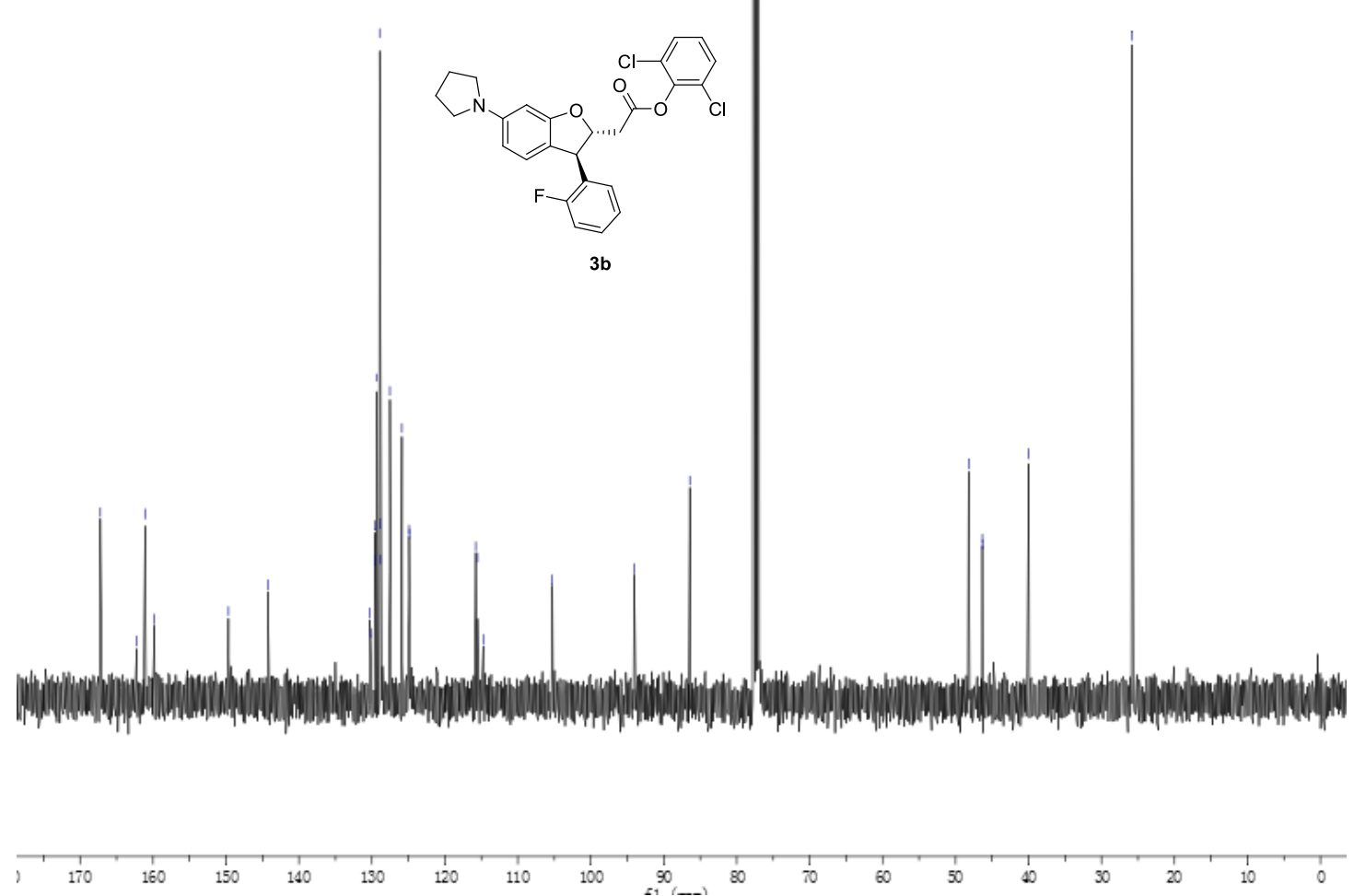




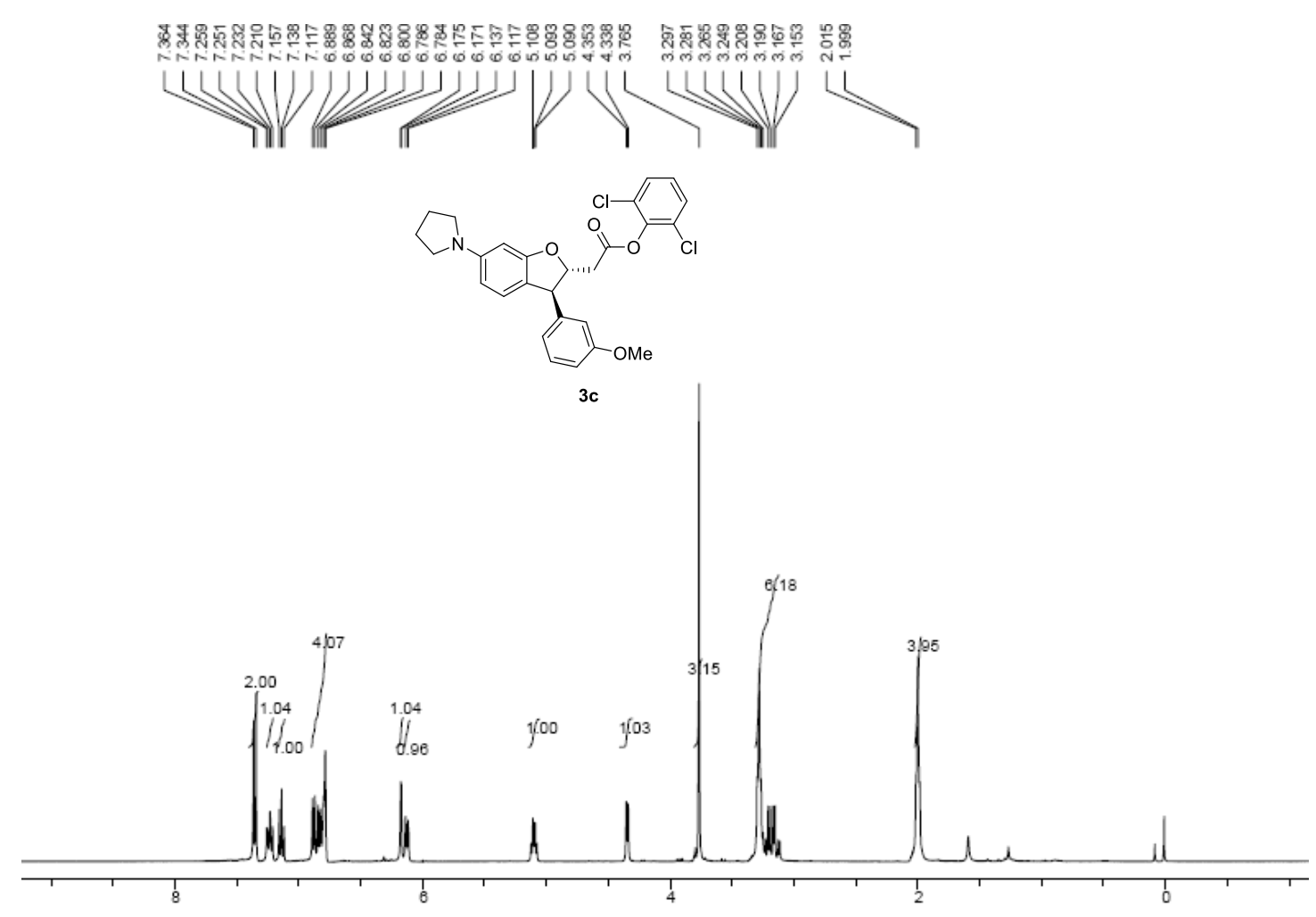

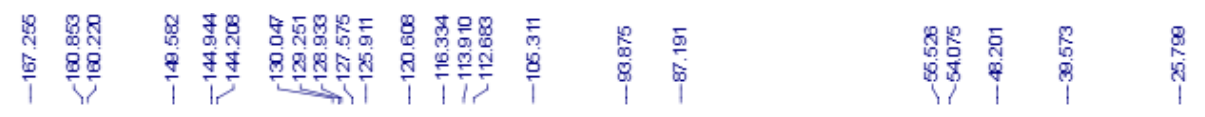
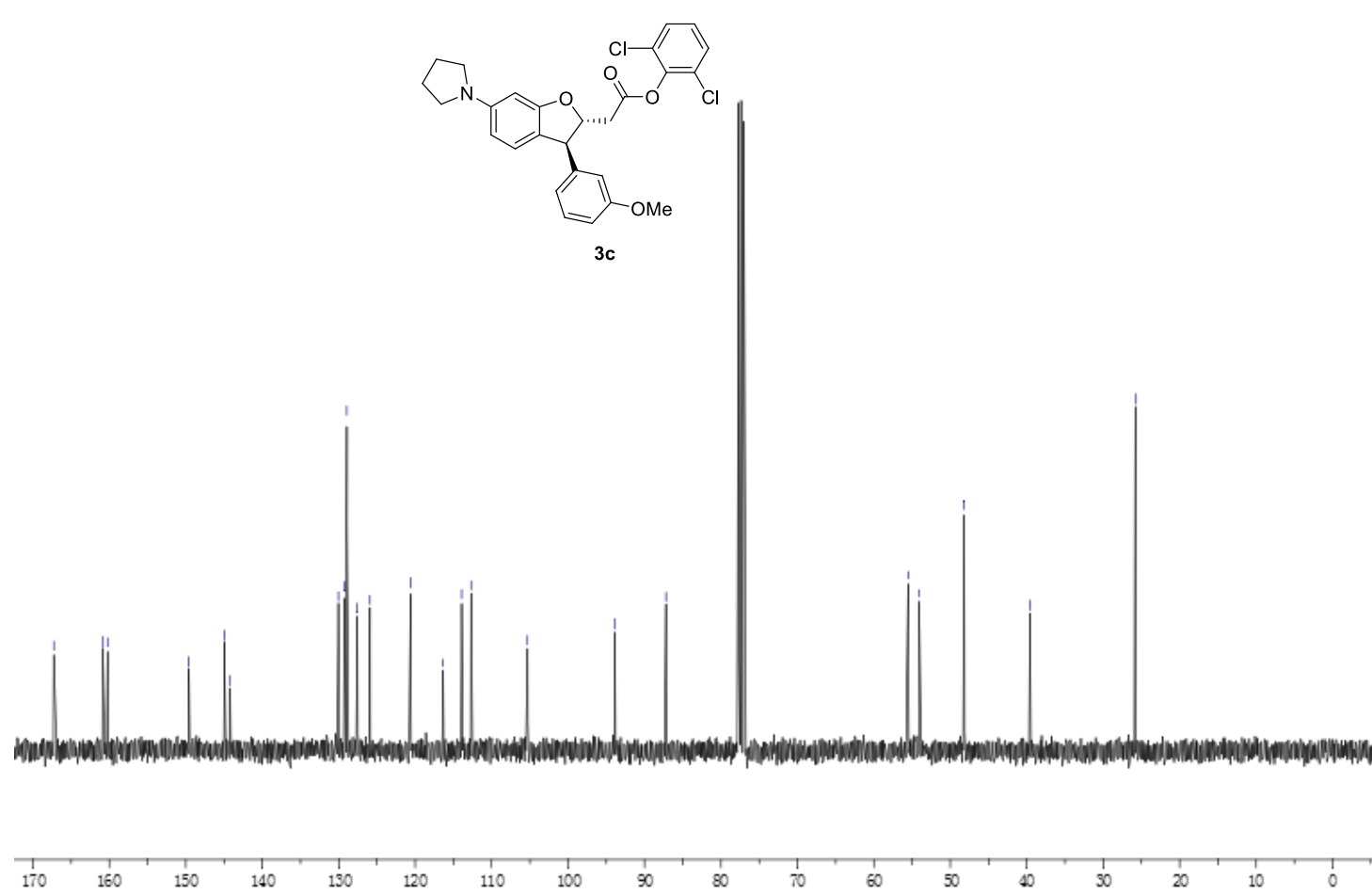

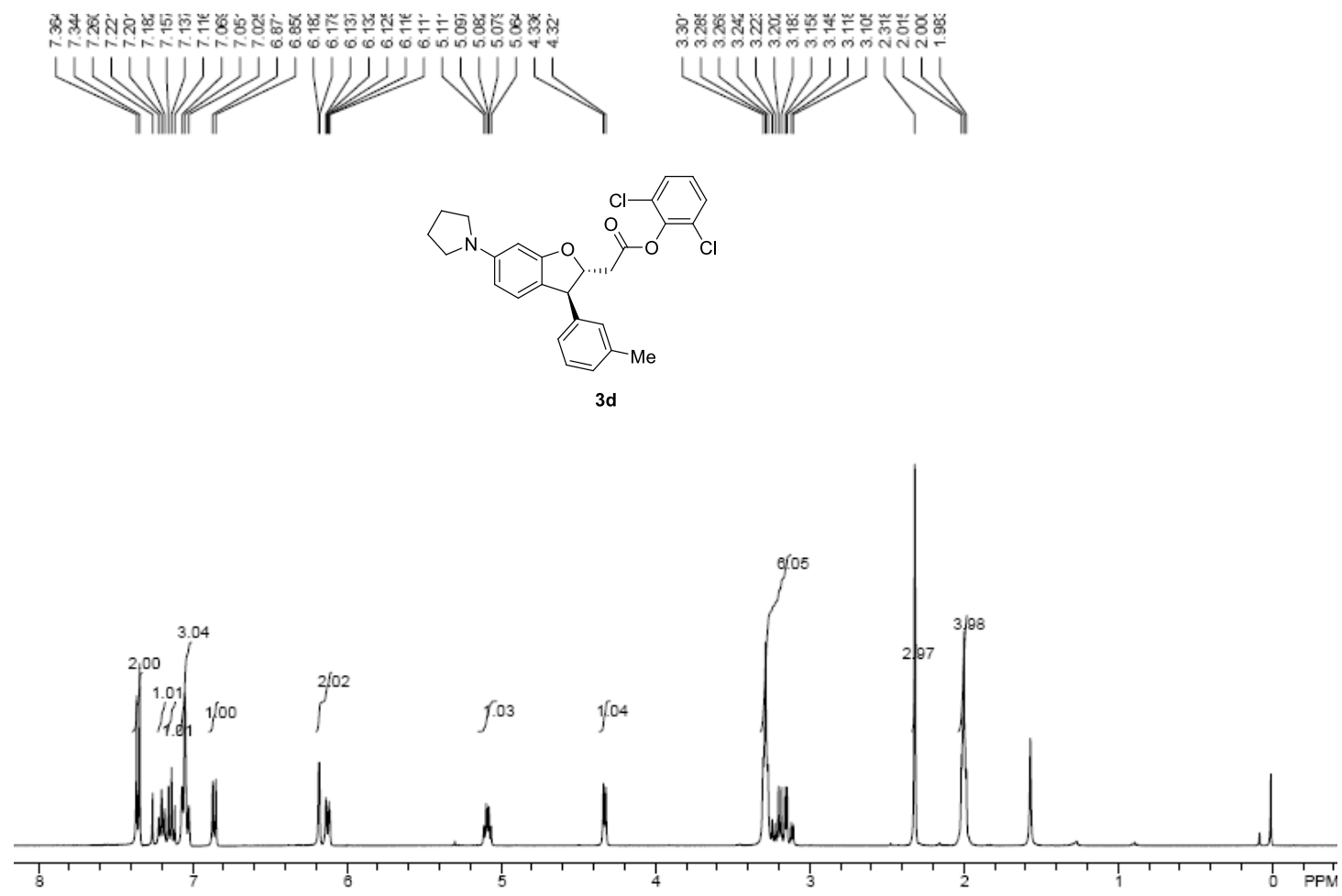

$H H$ H
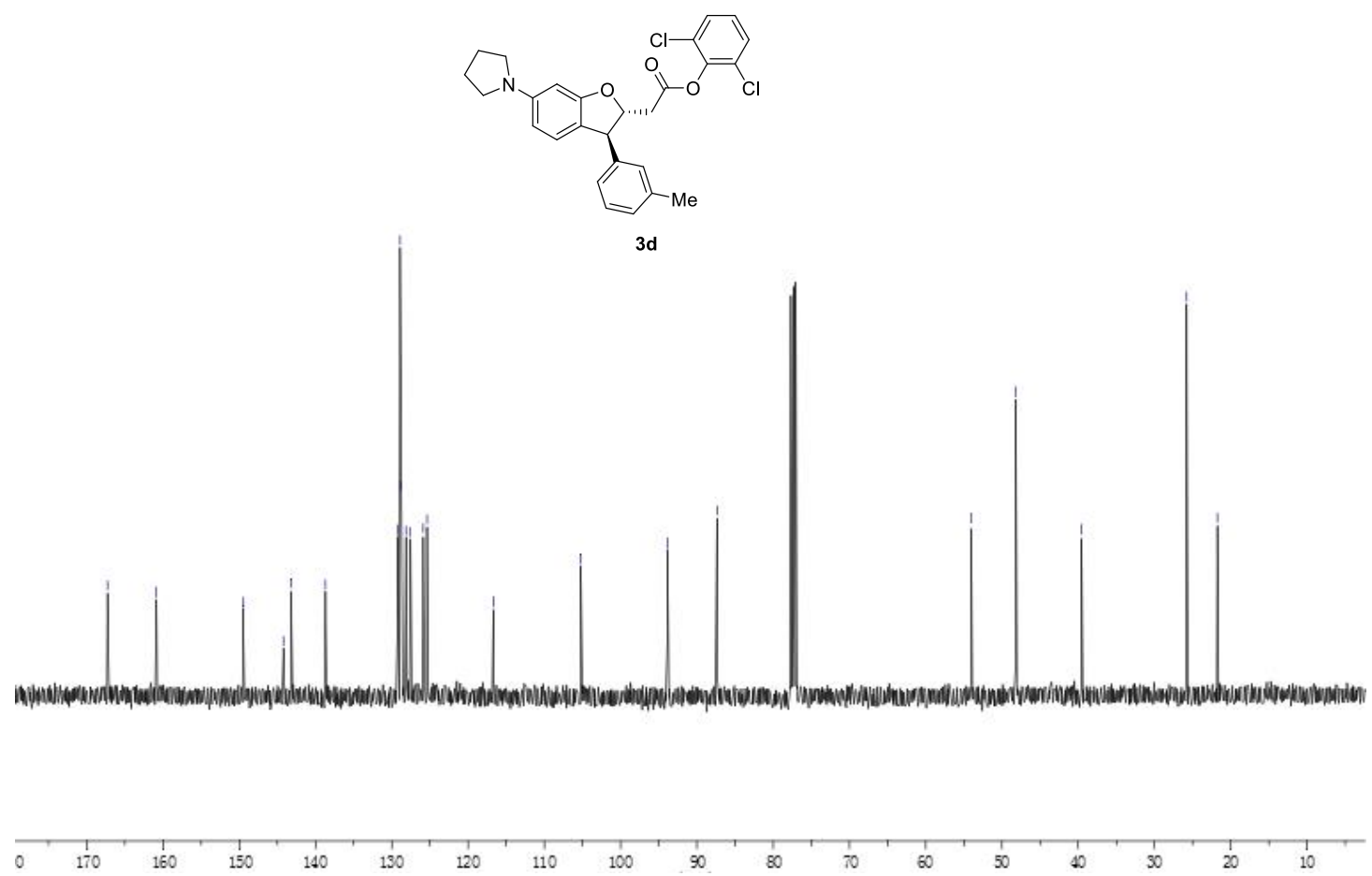


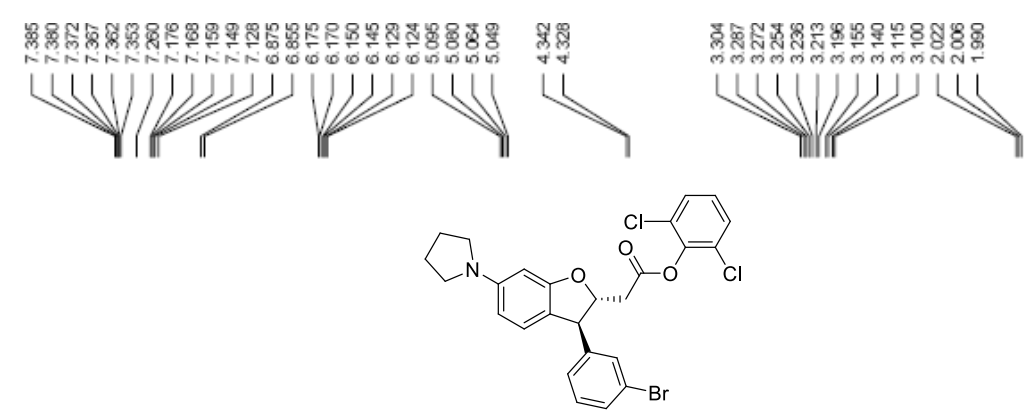

3e

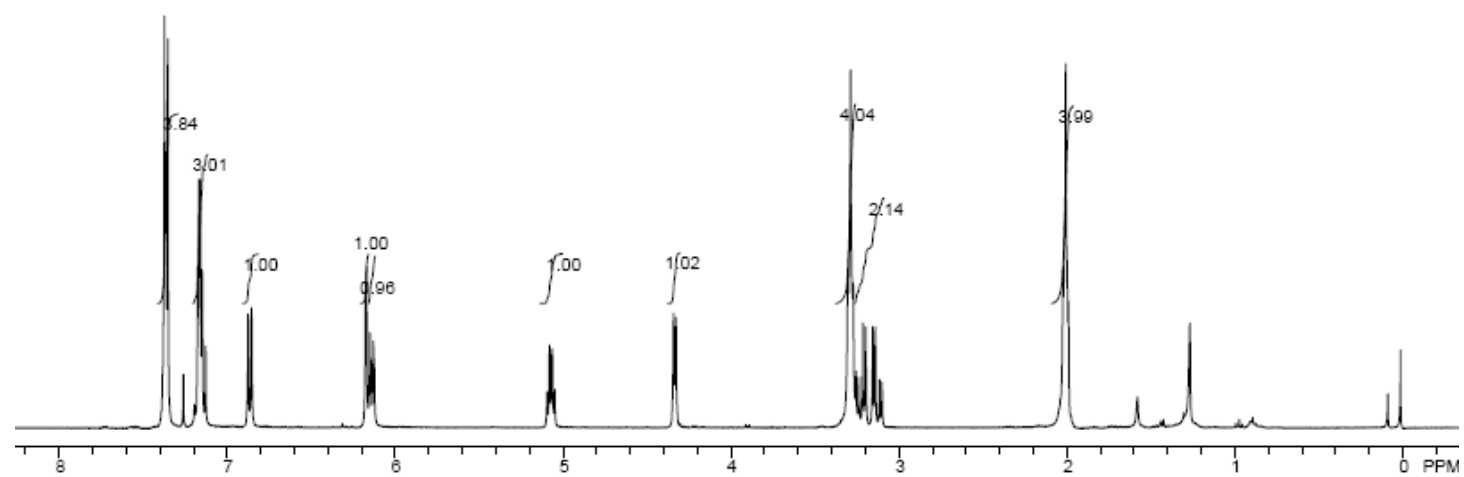

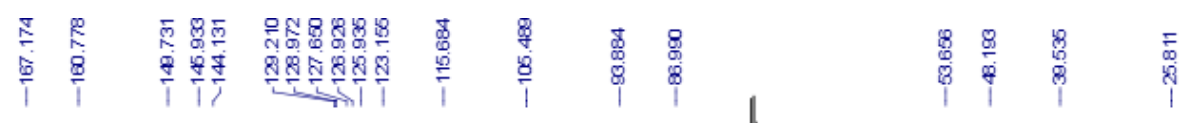

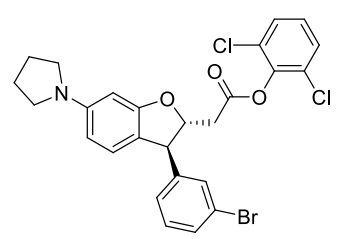

$3 e$

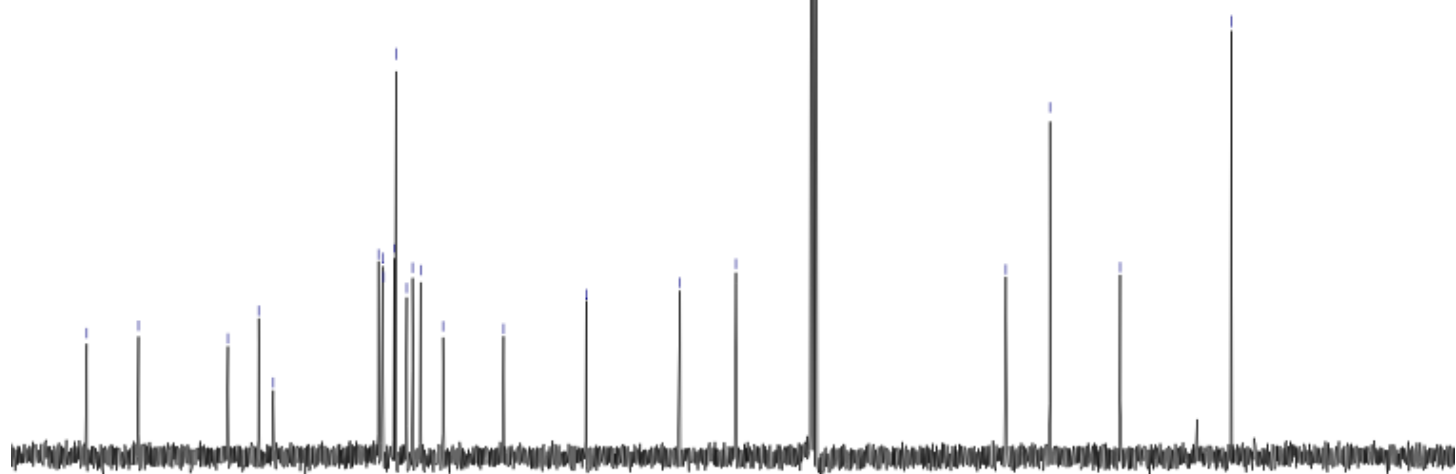

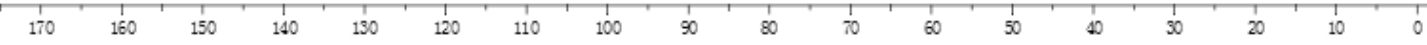




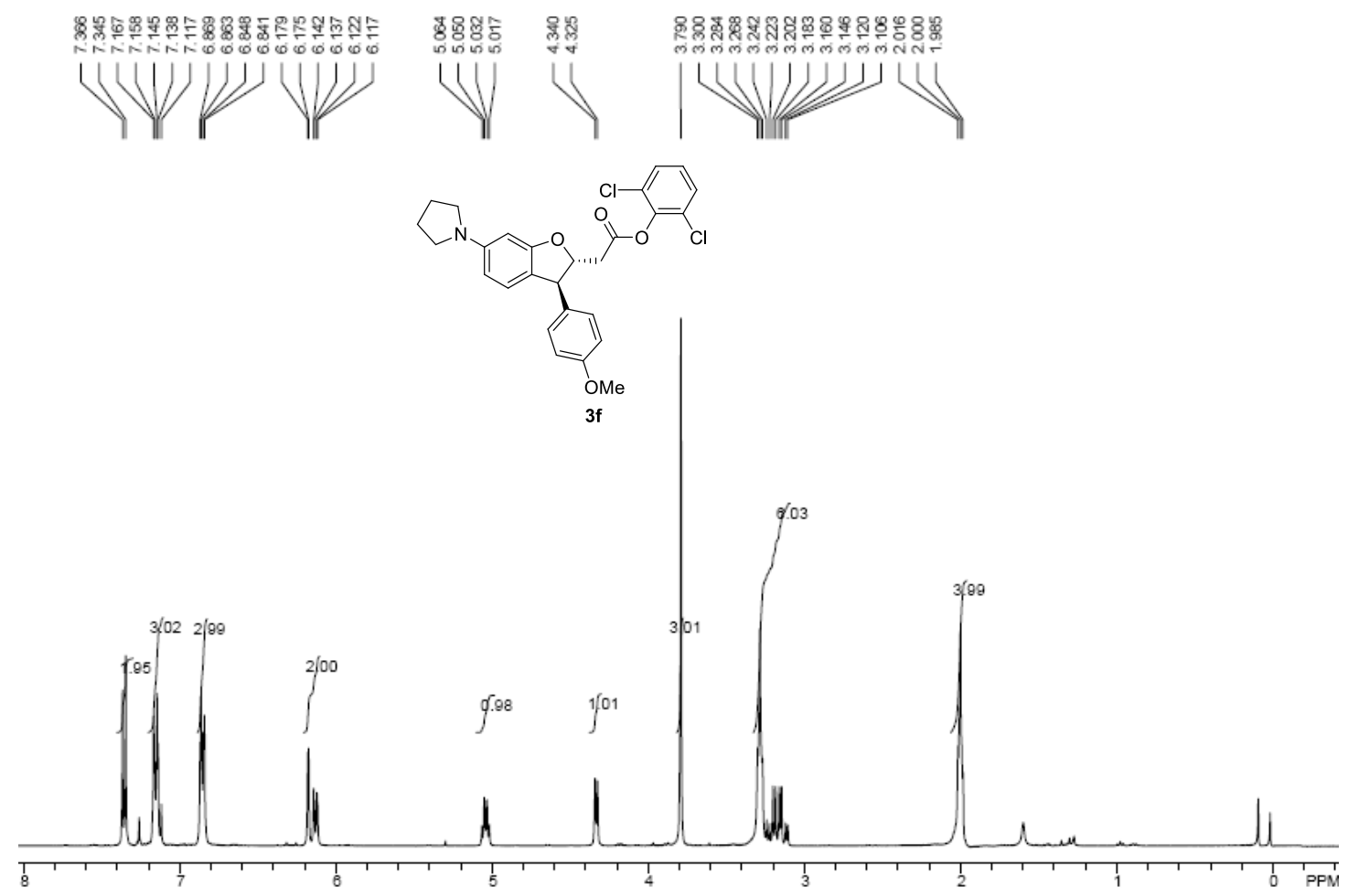

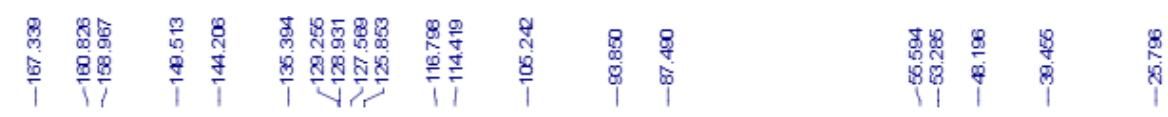
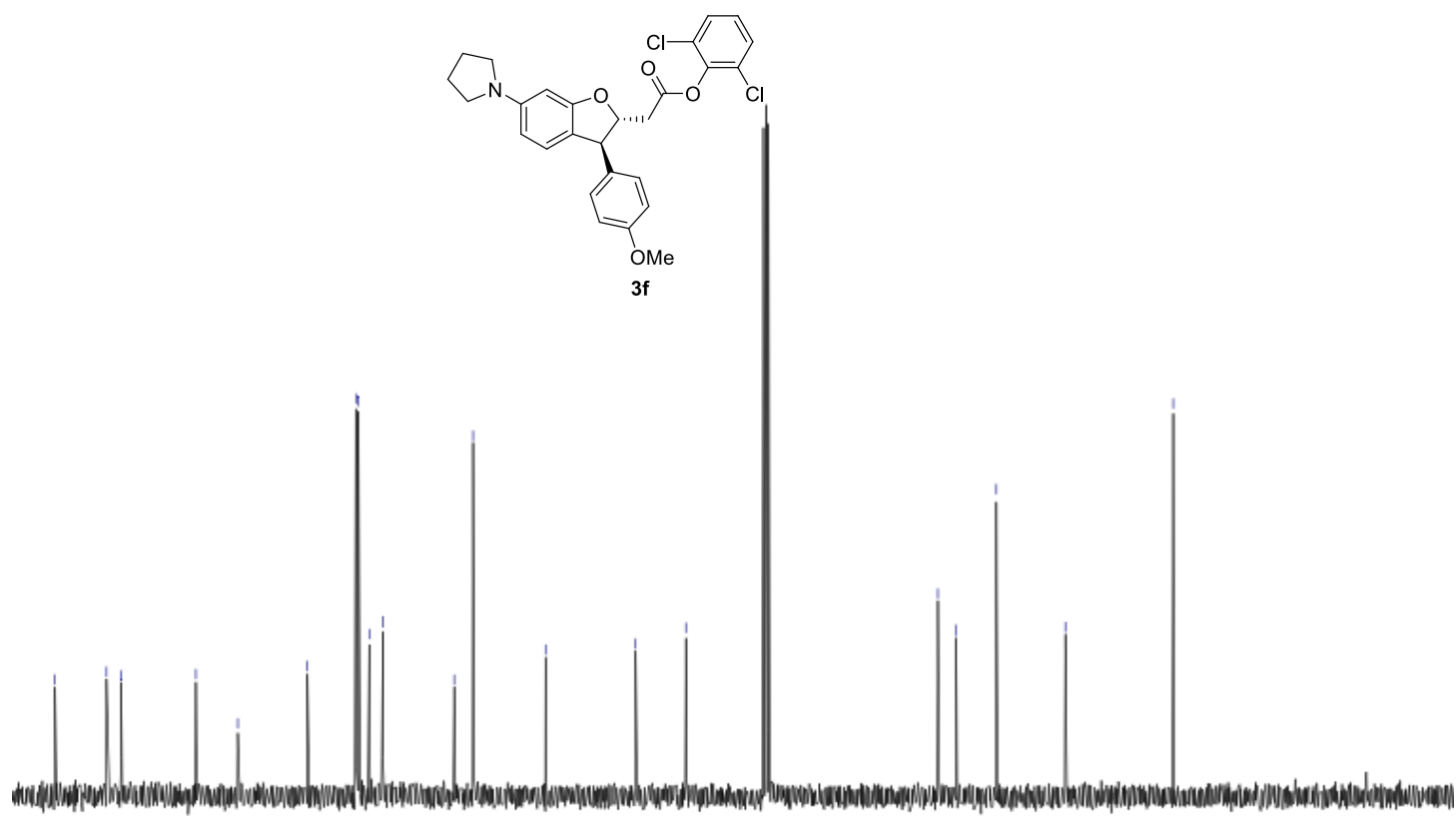

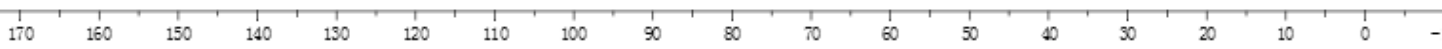




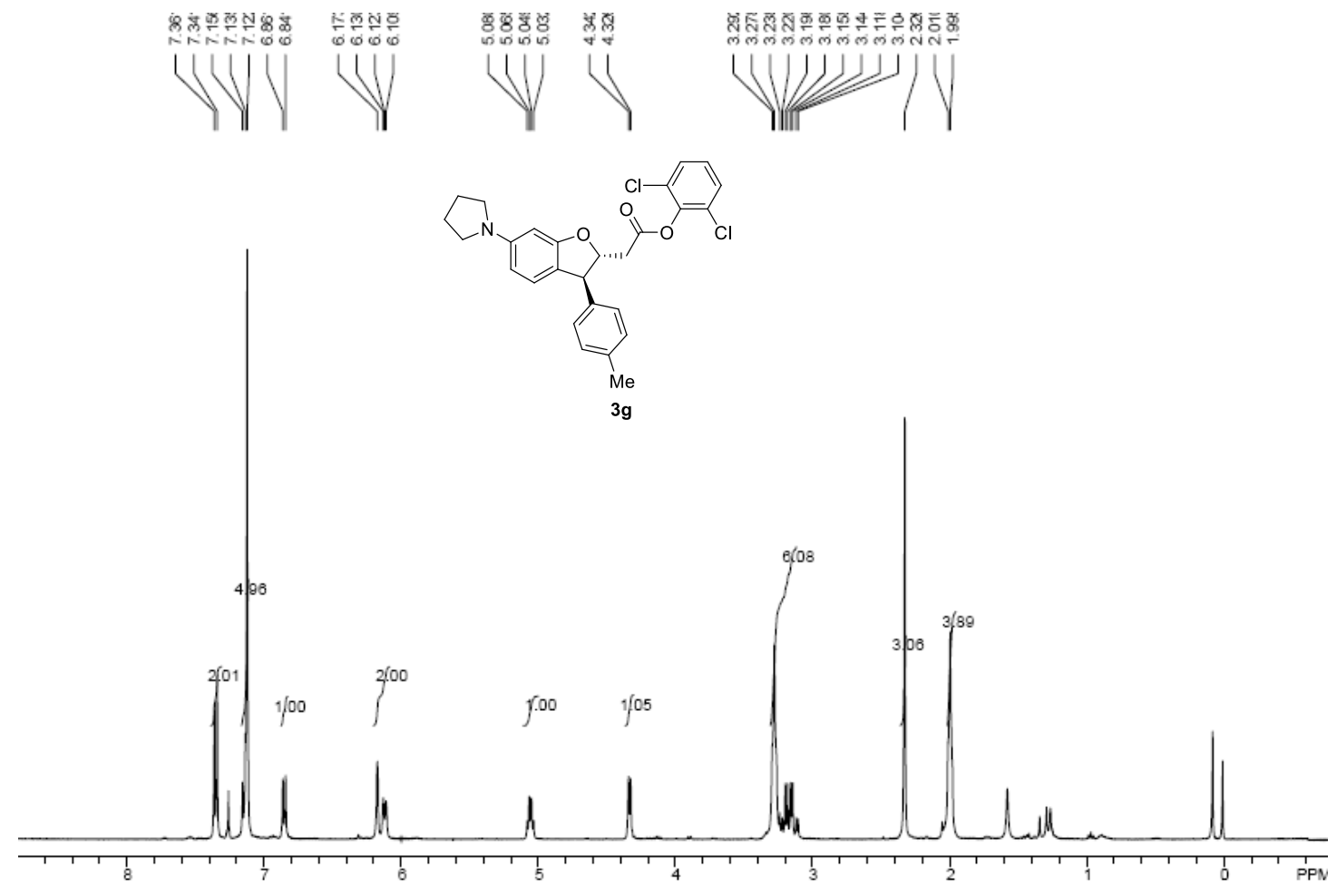

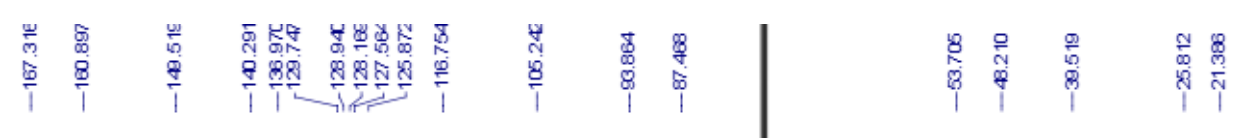<smiles>C[14c]1ccc(C(CC2Oc3cccc(Cl)c3O2)c2ccc(N3CCCC3)cc2)cc1</smiles>
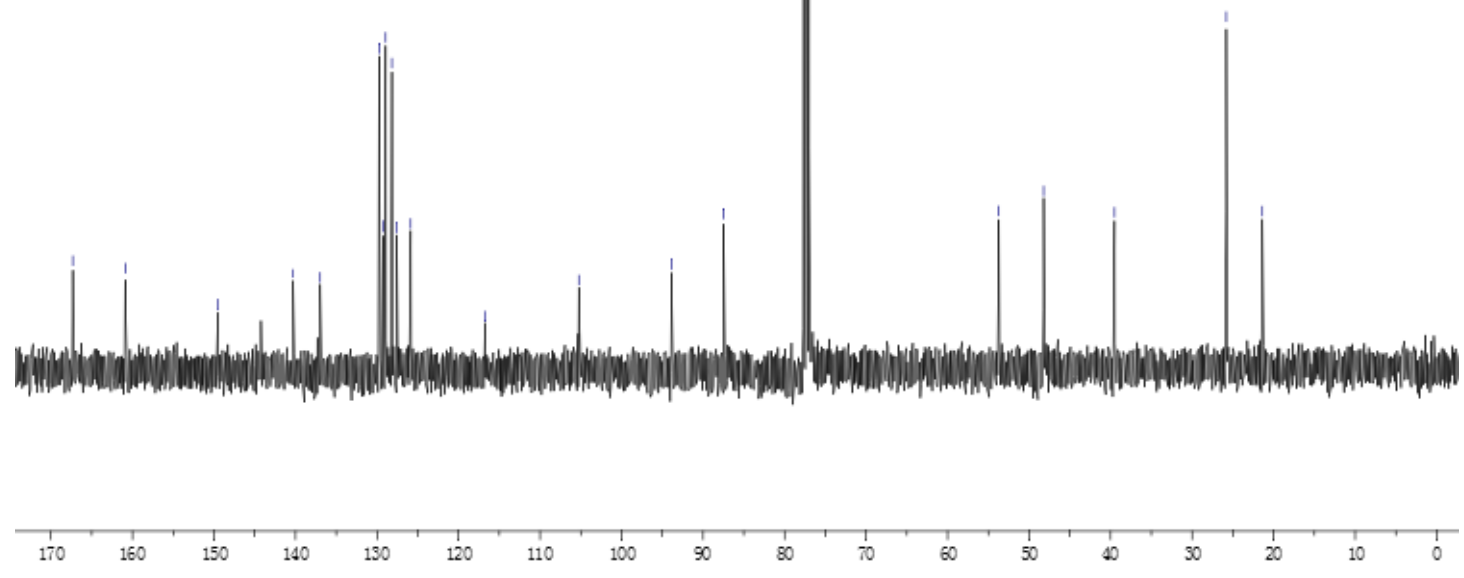


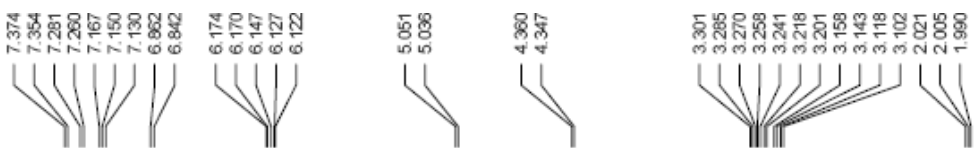<smiles>O=C(COc1cc(N2CCCC2)ccc1Cl)Oc1ccccc1Cl</smiles>

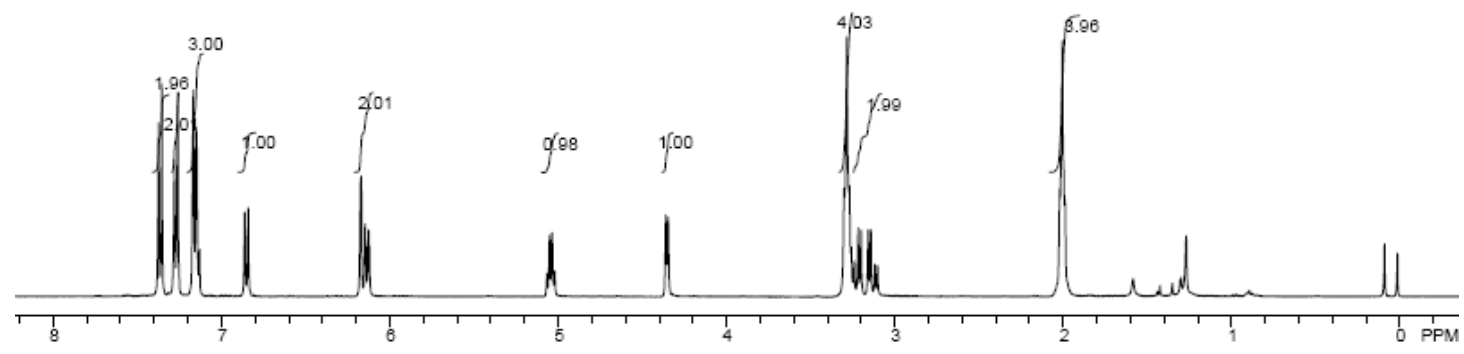

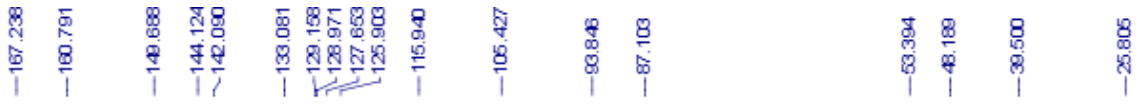
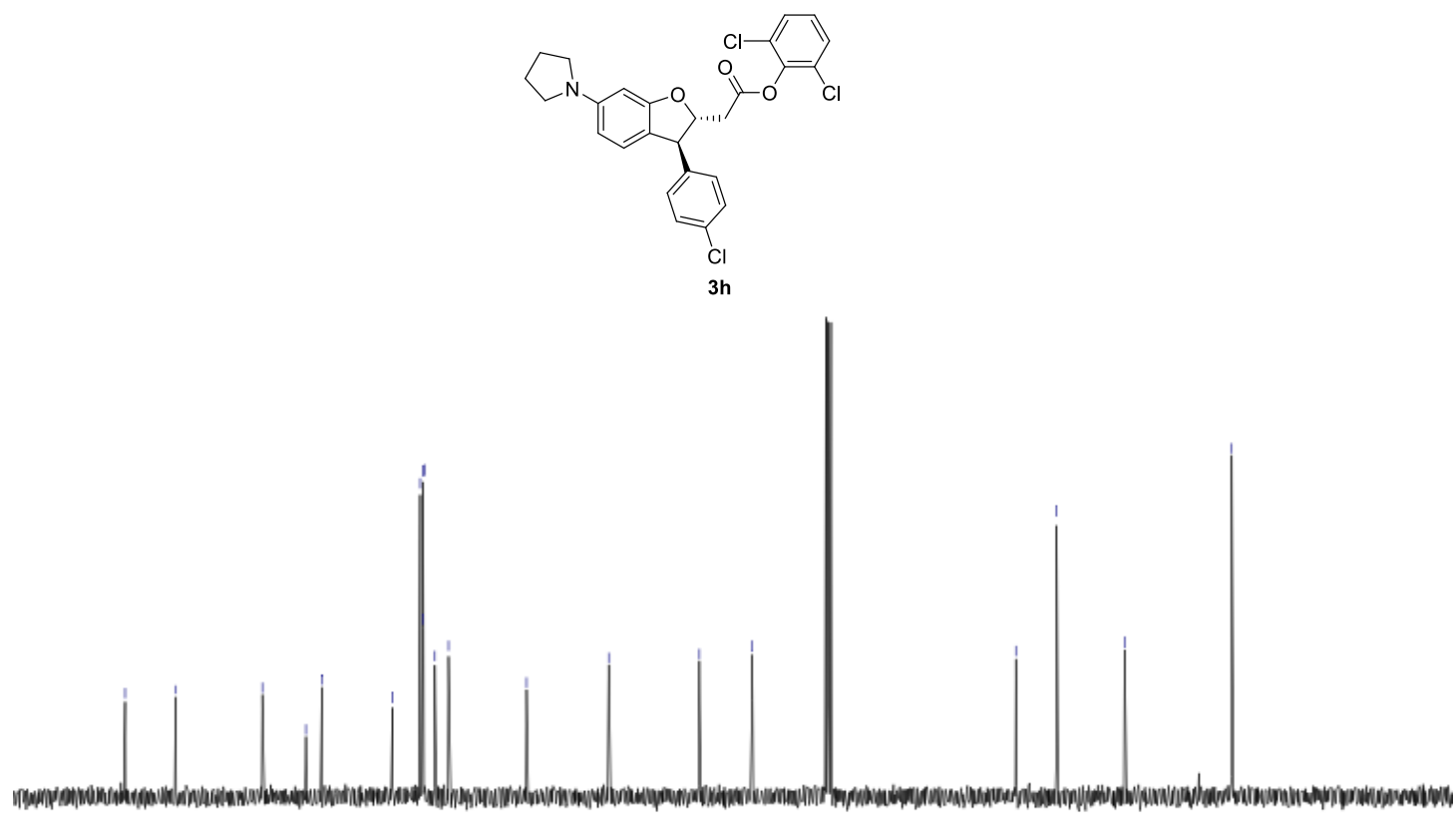

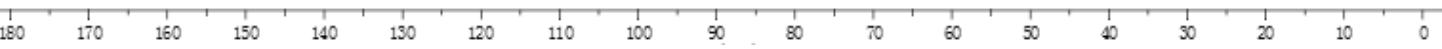




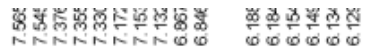

ulvV)

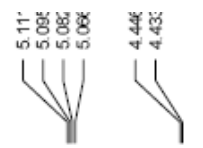

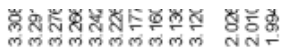

(U)<smiles>O=C(C[C@H]1Oc2cc(N3CCCC3)ccc2[C@H]1c1ccc(C(F)(F)F)cc1)Oc1c(Cl)cccc1Cl</smiles>

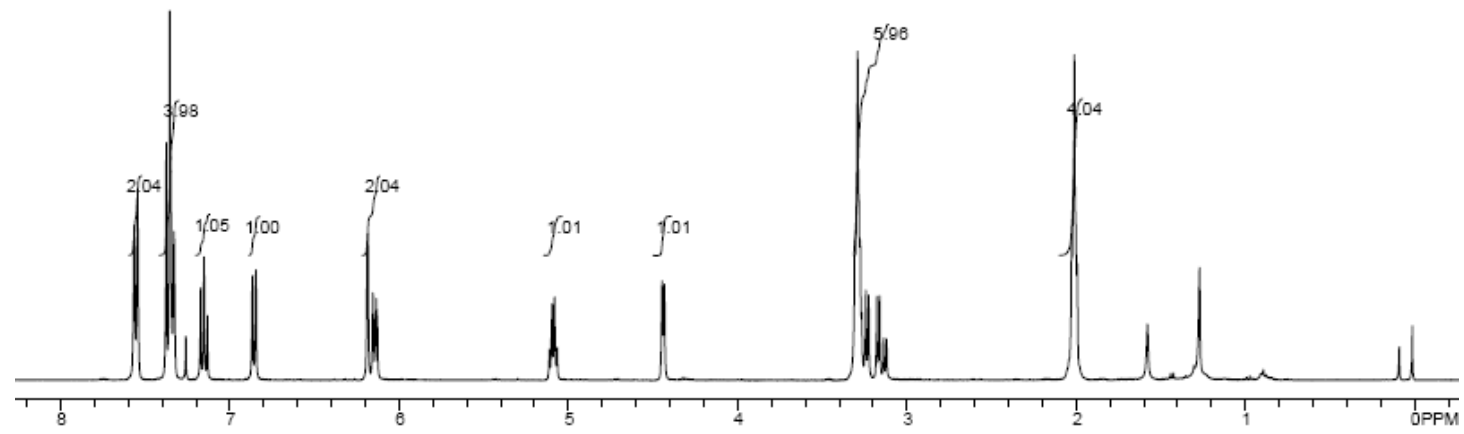

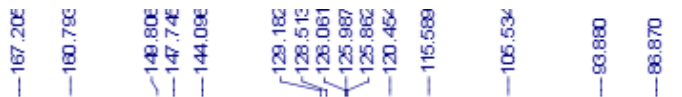

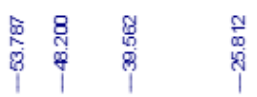<smiles>O=C(C[C@H]1Oc2cc(N3CCCC3)ccc2[C@H]1c1ccc(C(F)(F)F)cc1)Oc1c(Cl)cccc1Cl</smiles>

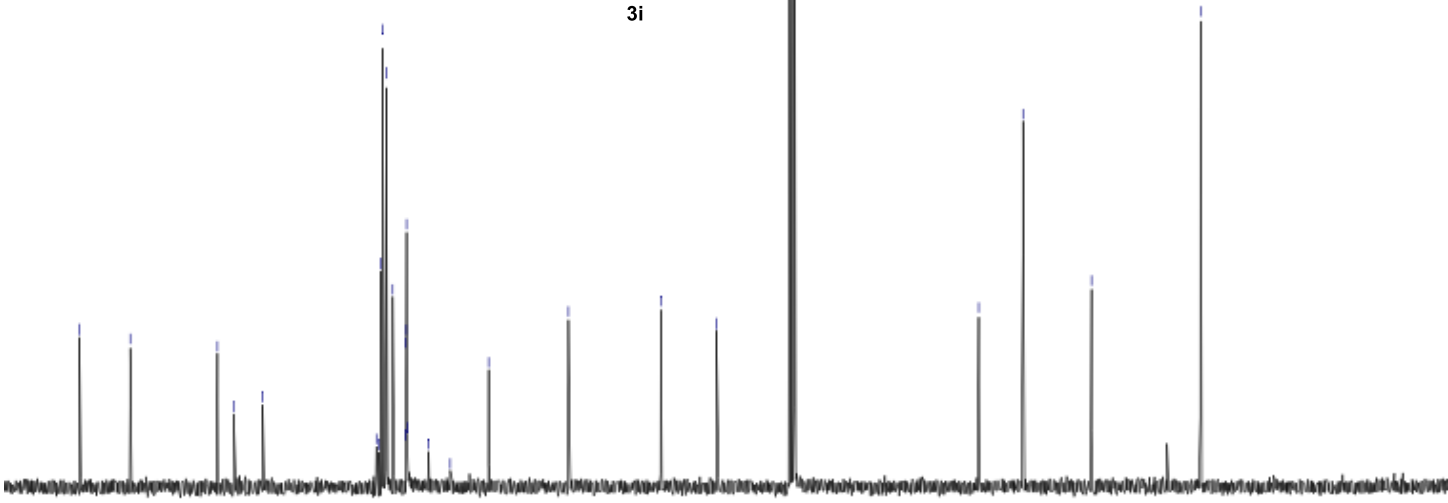

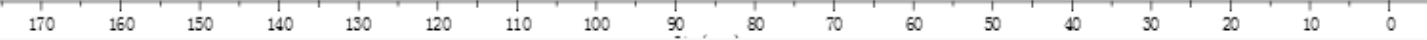



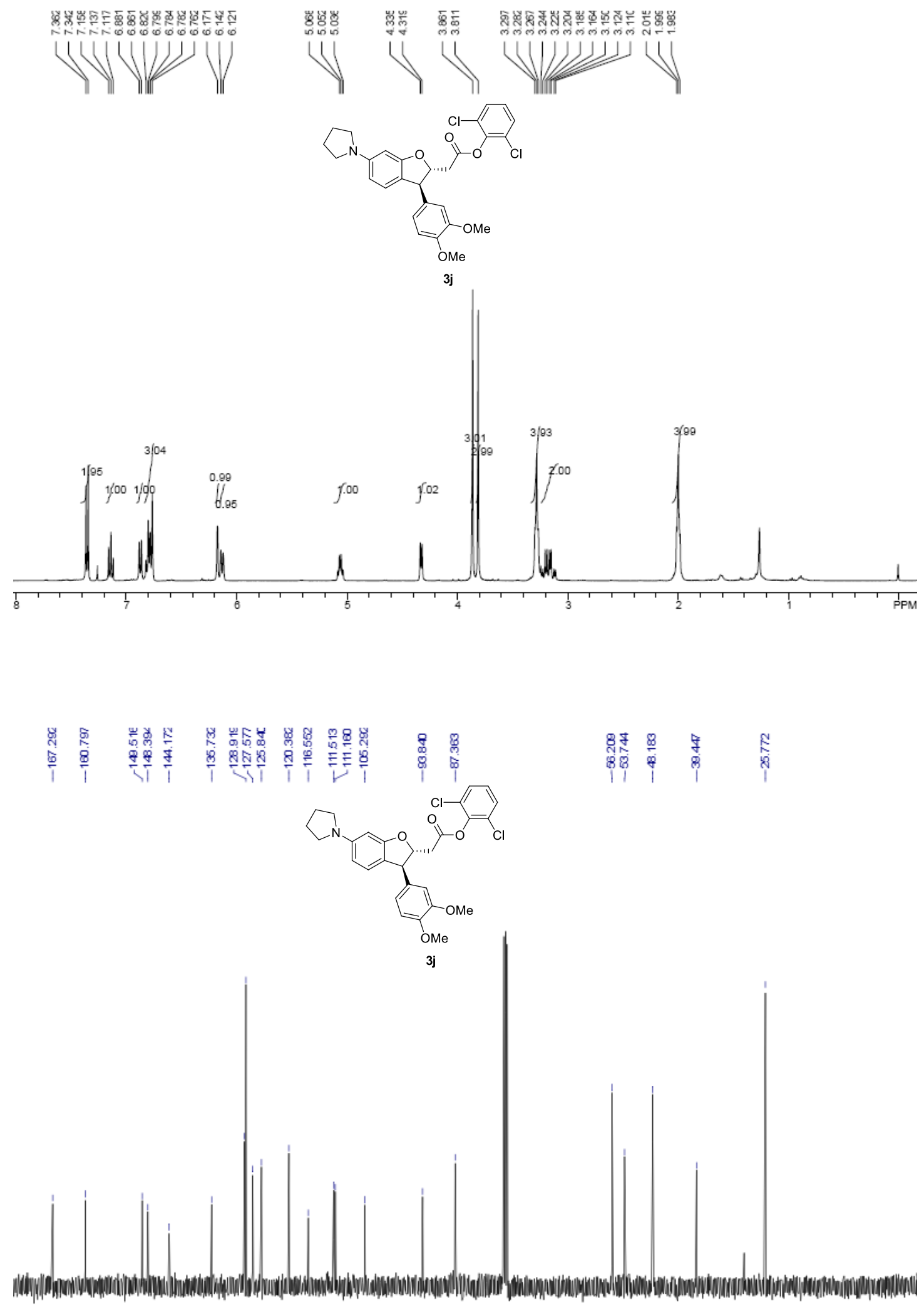

\begin{tabular}{llllllllllllllllllll}
\hline 170 & 160 & 150 & 140 & 130 & 120 & 110 & 100 & 90 & 80 & 70 & 60 & 50 & 10 & 30 & 10 & 10 & 1
\end{tabular} 


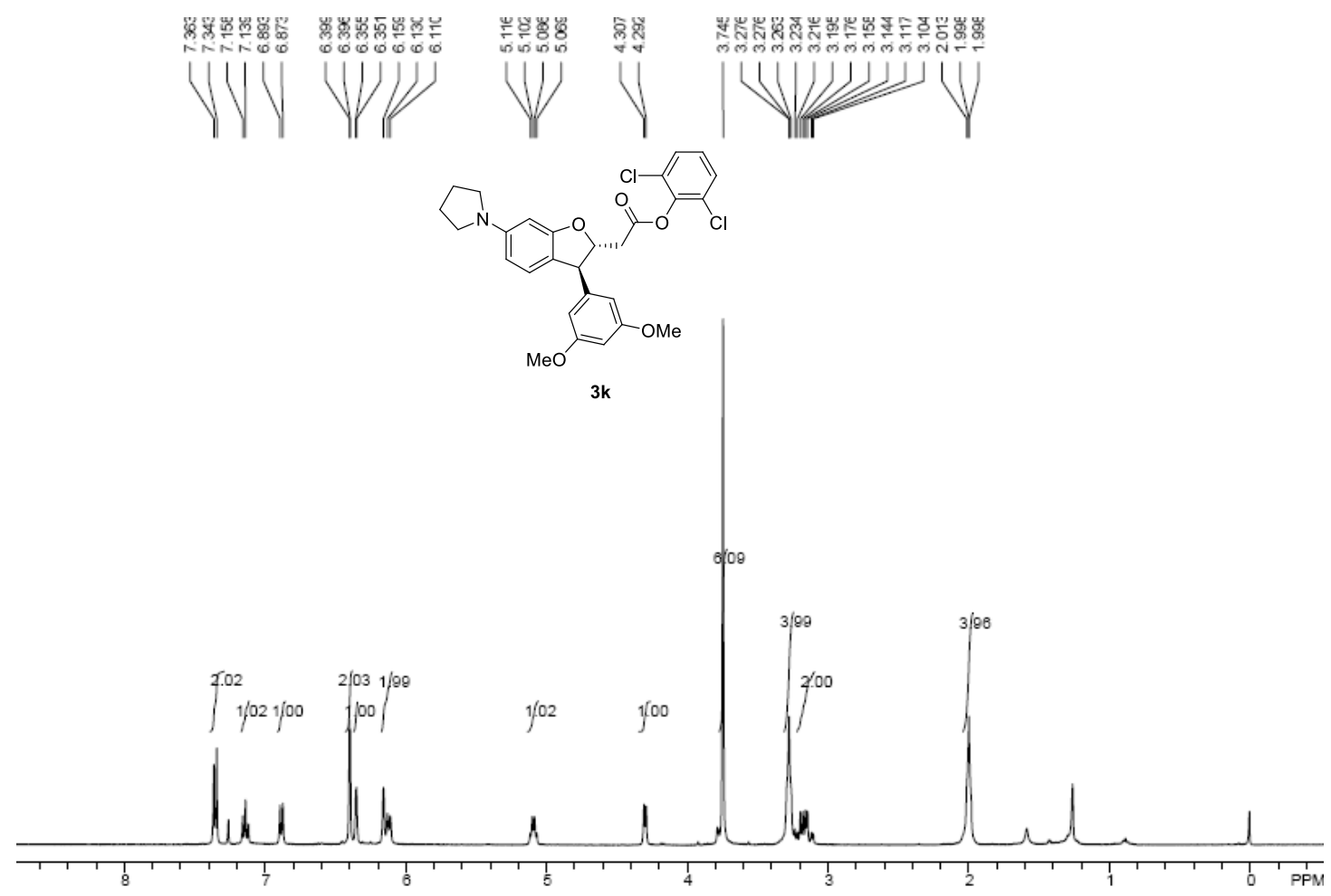

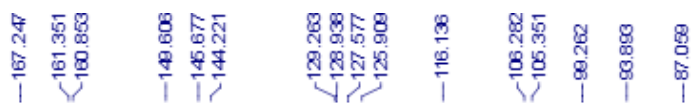

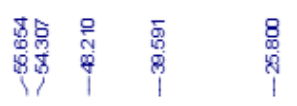

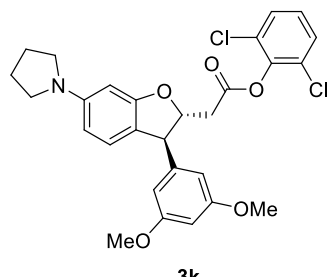

$3 \mathbf{k}$

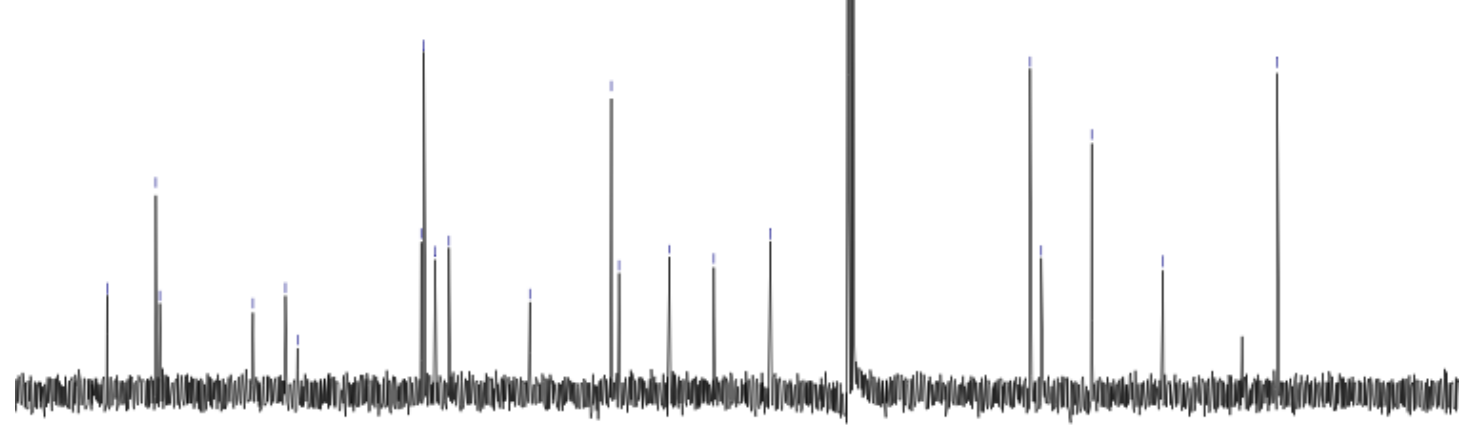

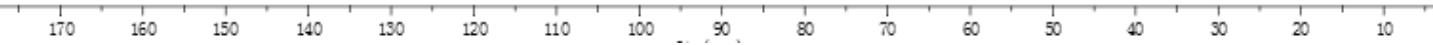




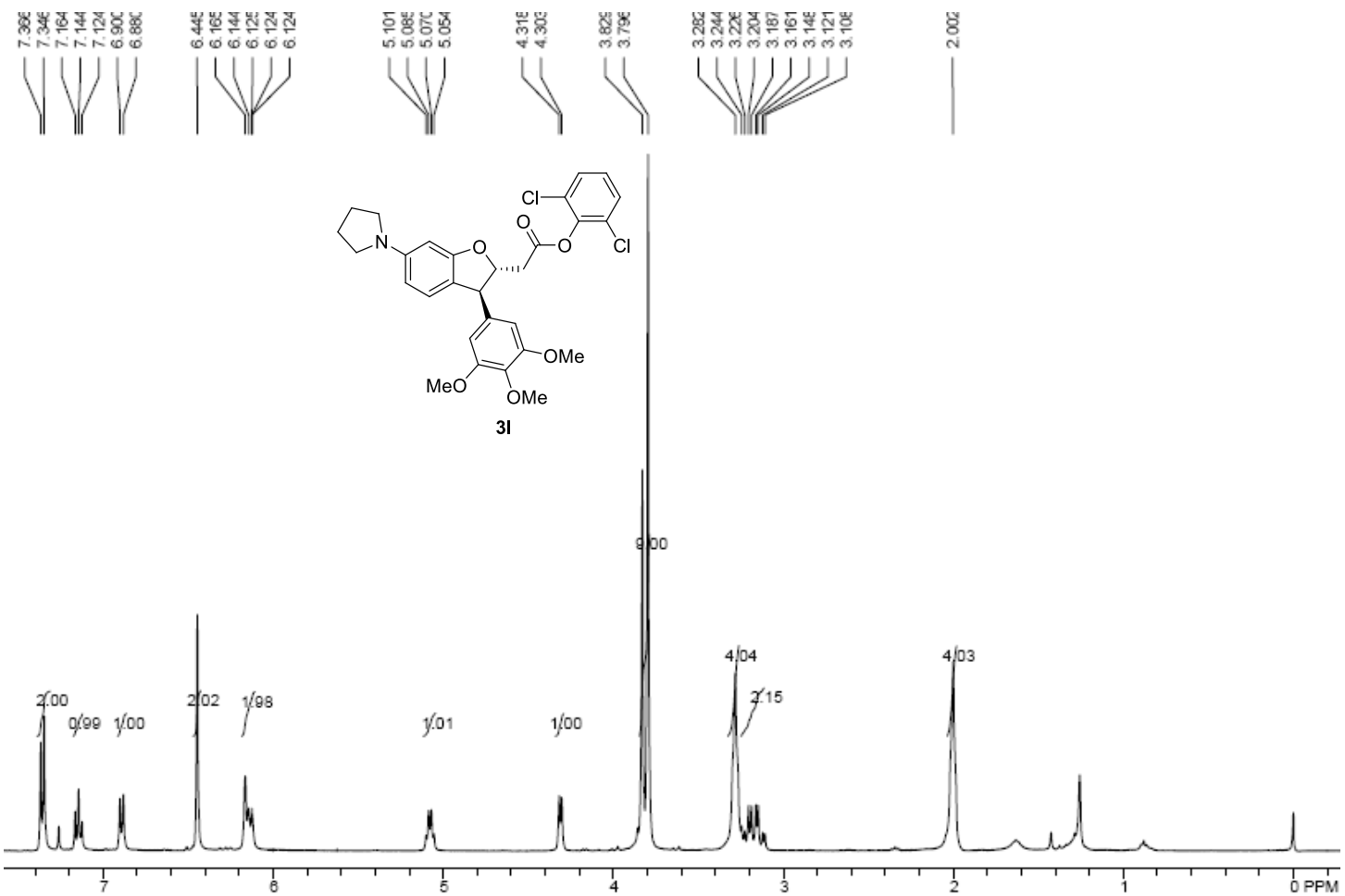

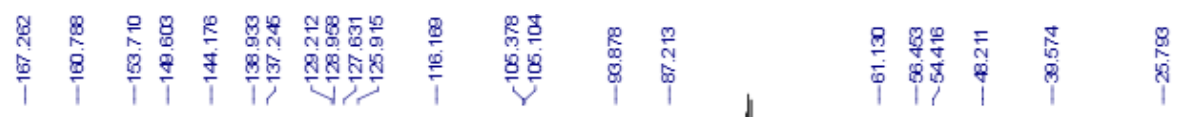<smiles>COc1cc(C2Oc3cc(N4CCCC4)ccc3C2CC(=O)Oc2c(Cl)cccc2Cl)cc(OC)c1OC</smiles>
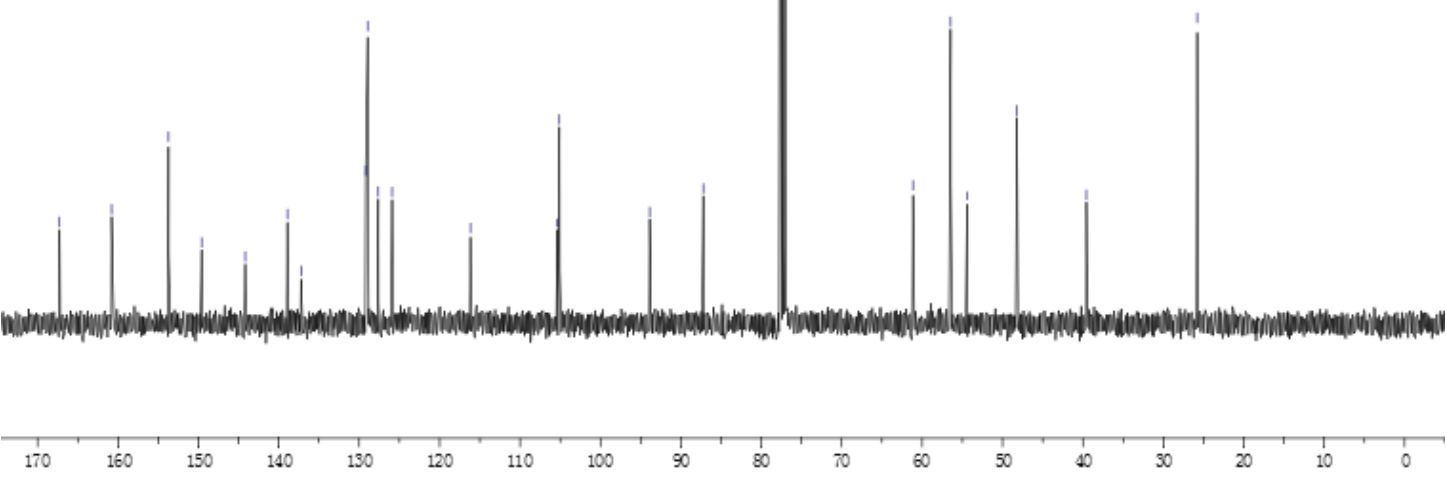

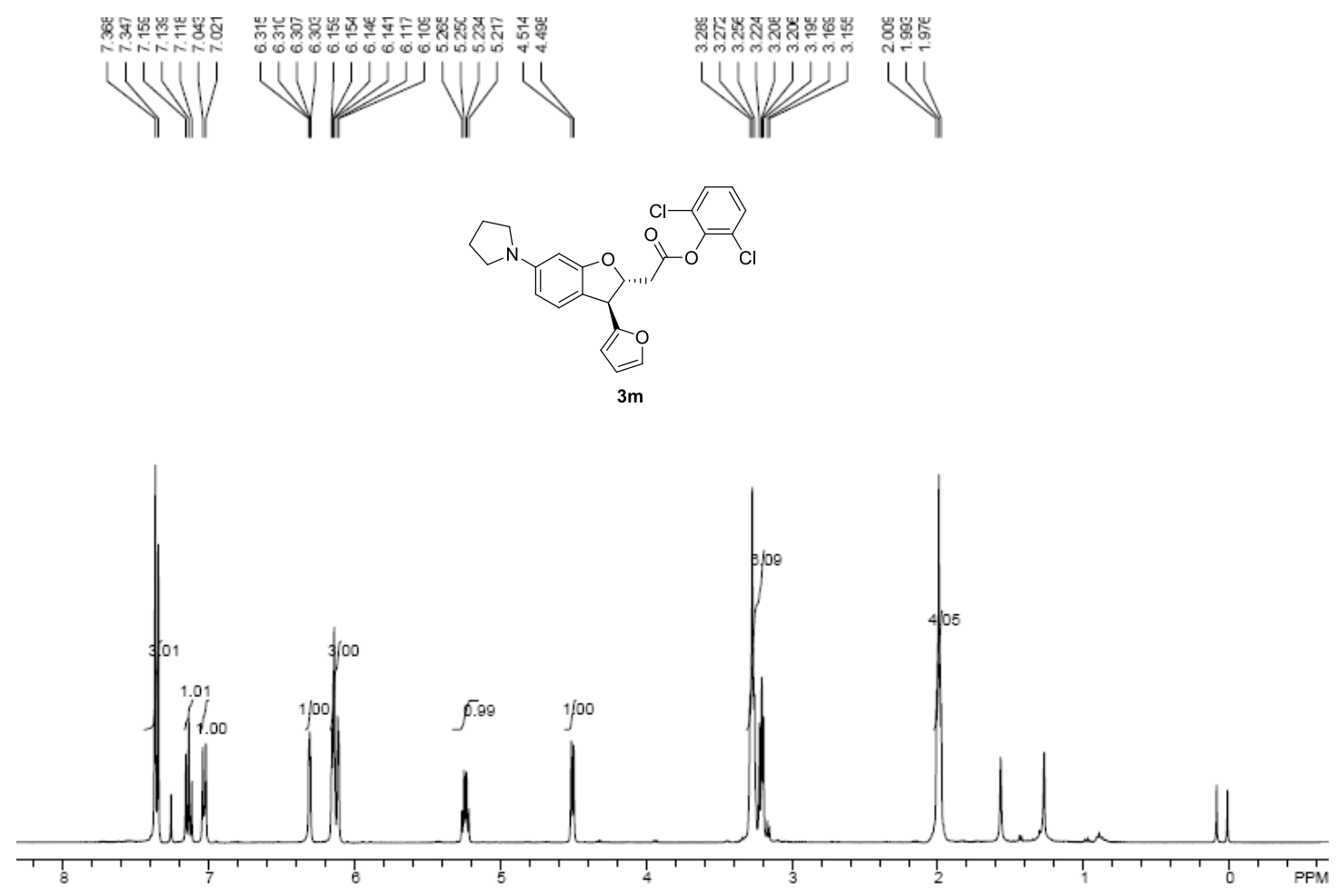

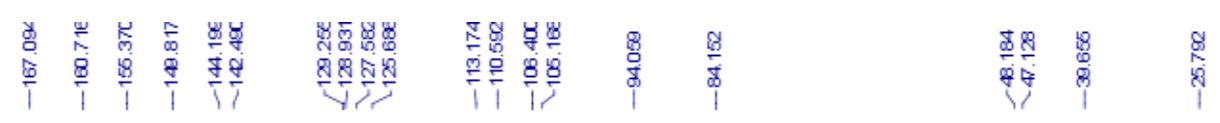<smiles></smiles>
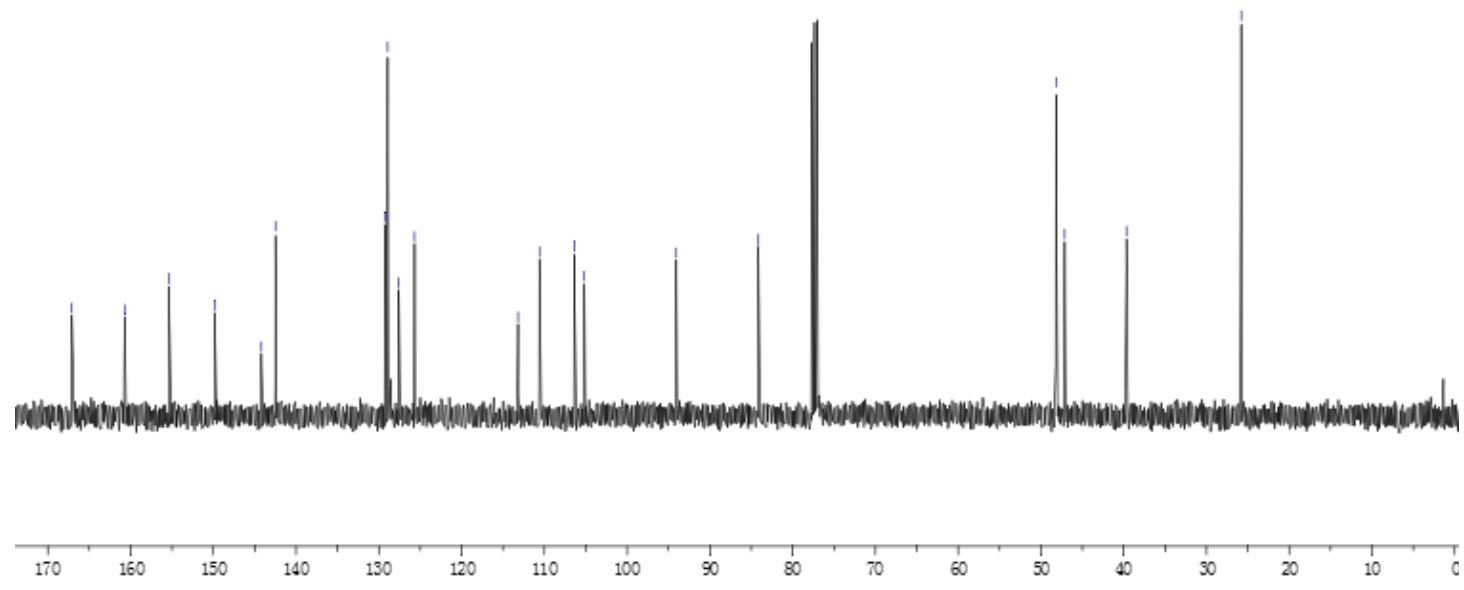


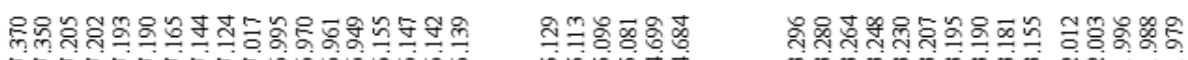

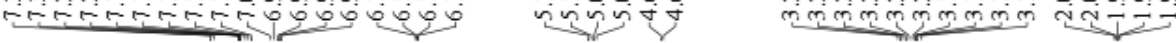

$\int \| d x$
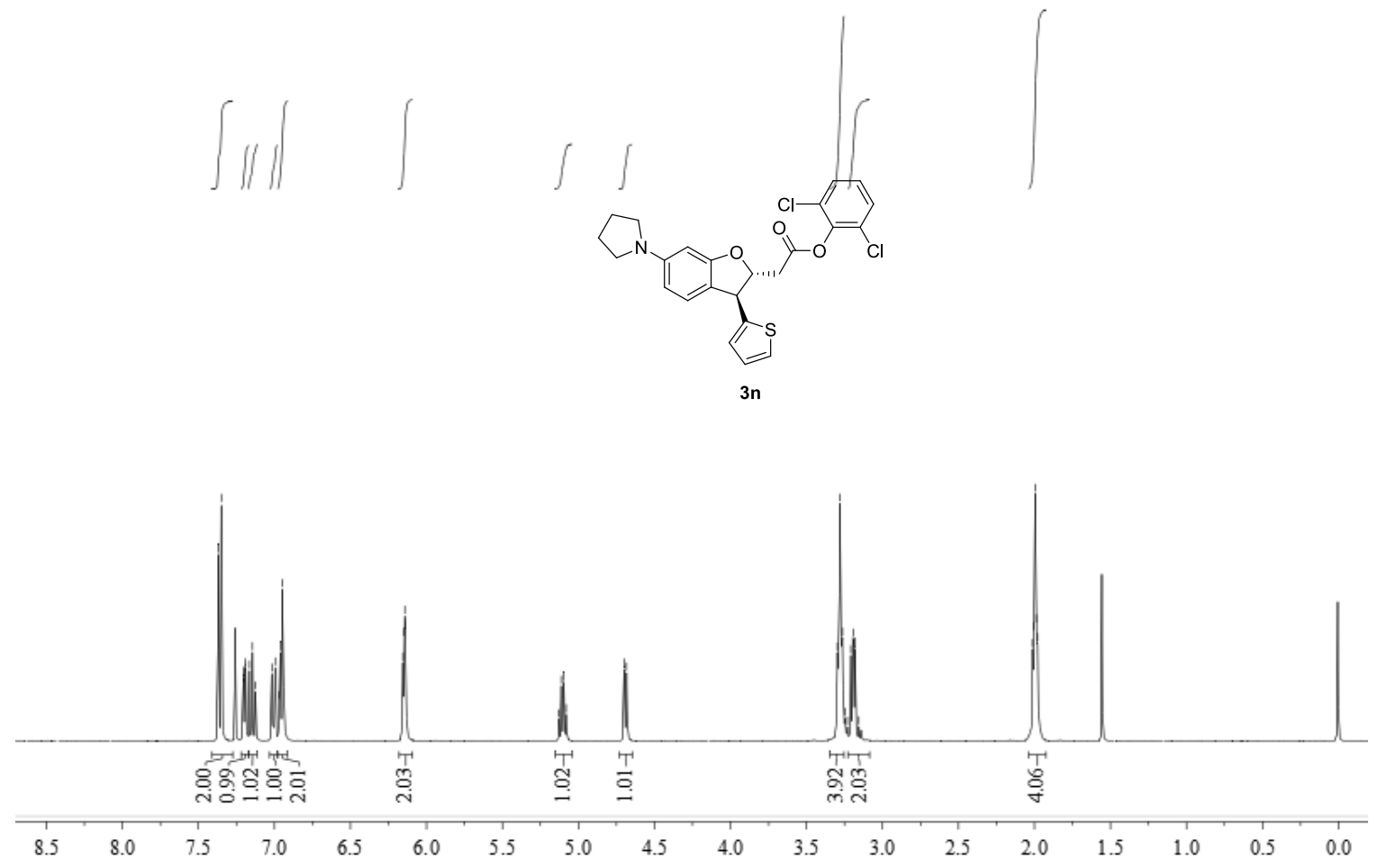

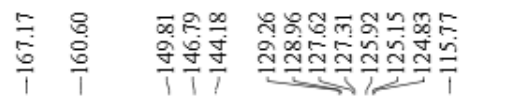

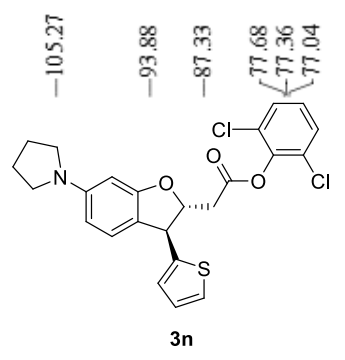

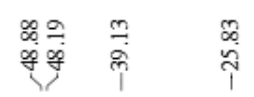

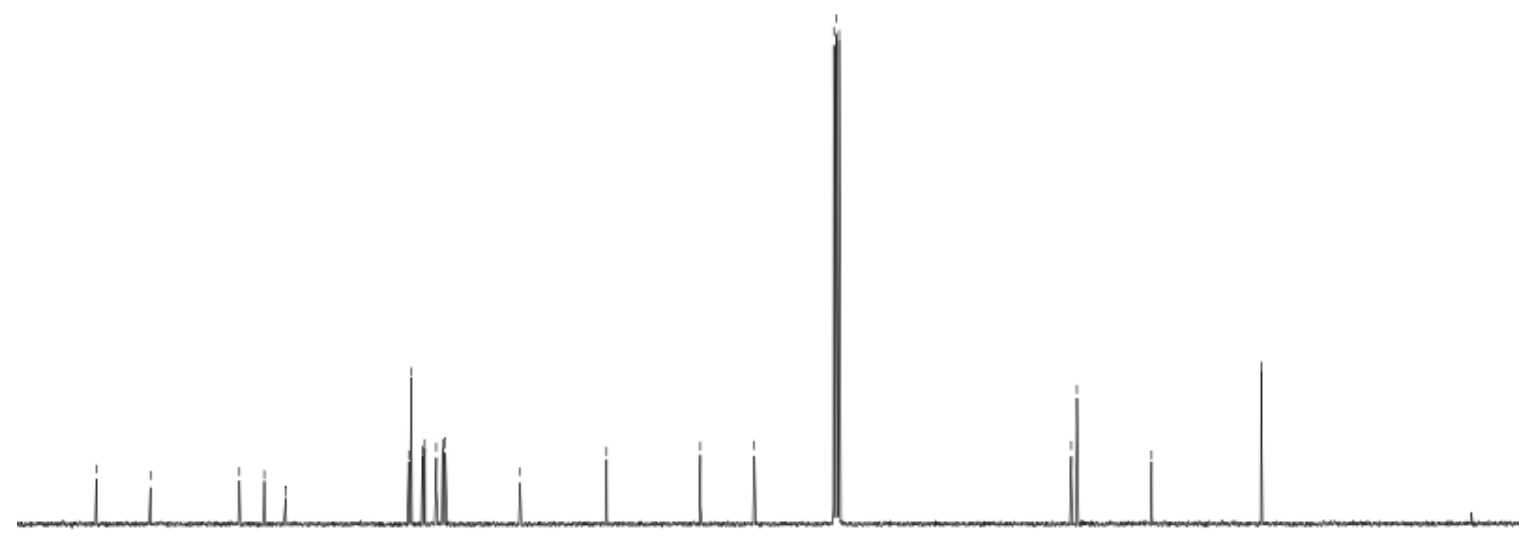

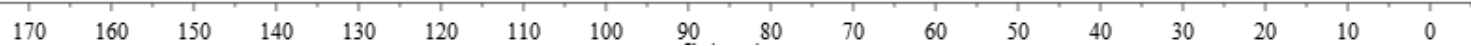




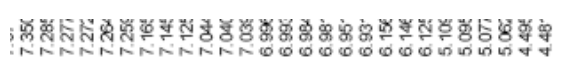

HUI<smiles>Clc1cccc2ccccc12</smiles>

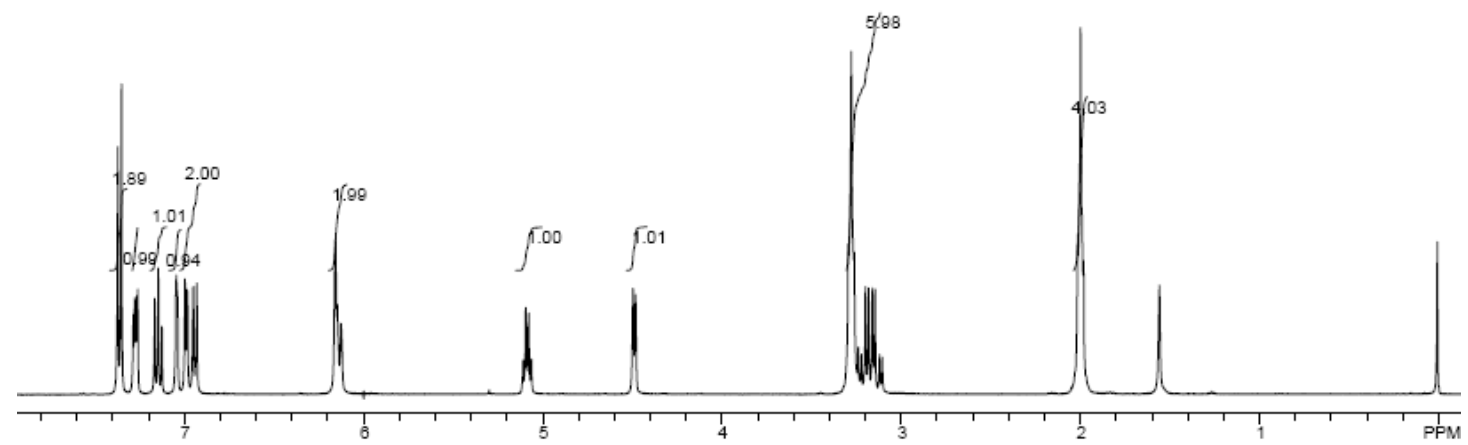

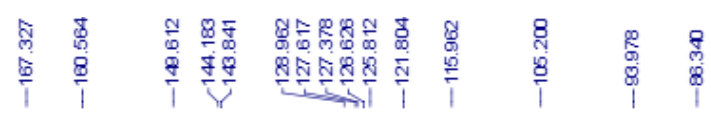

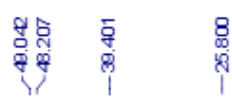
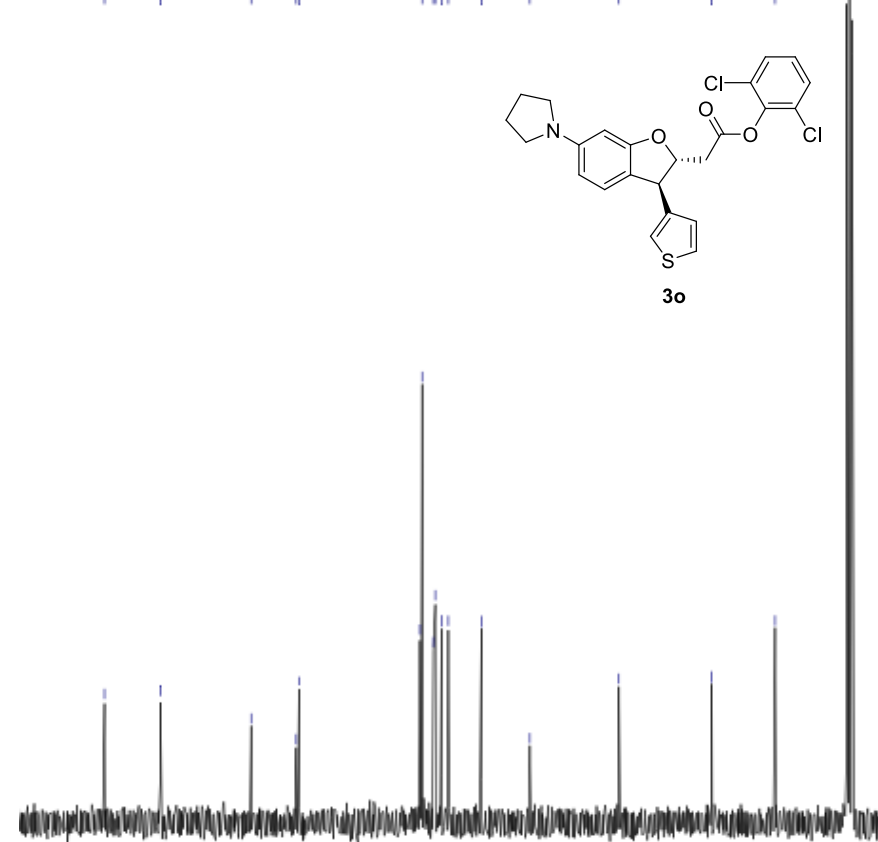

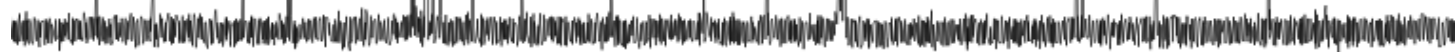

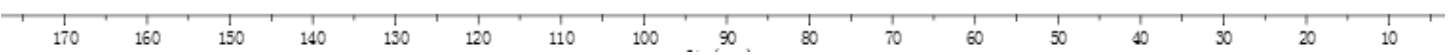



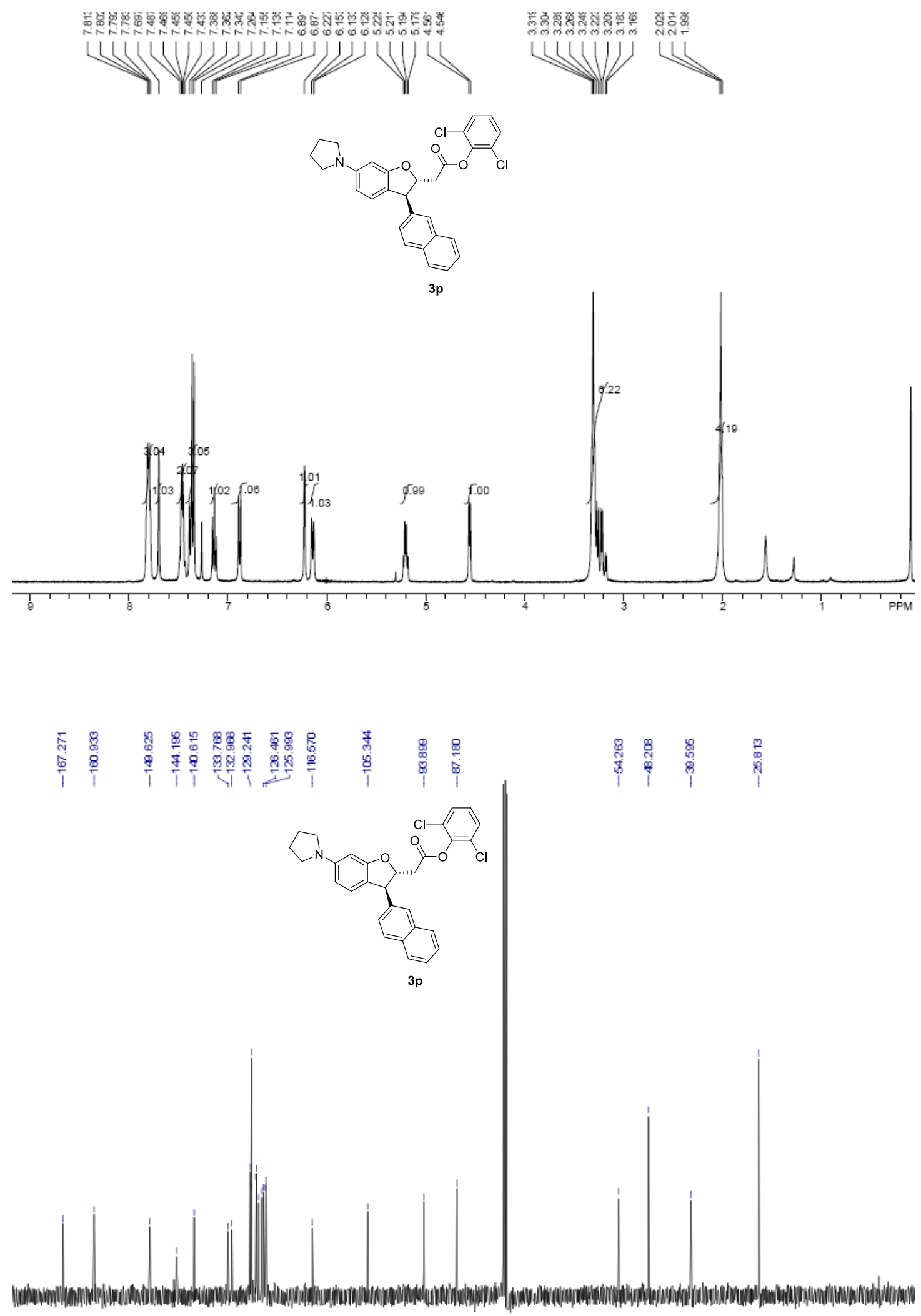

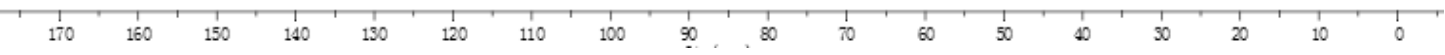



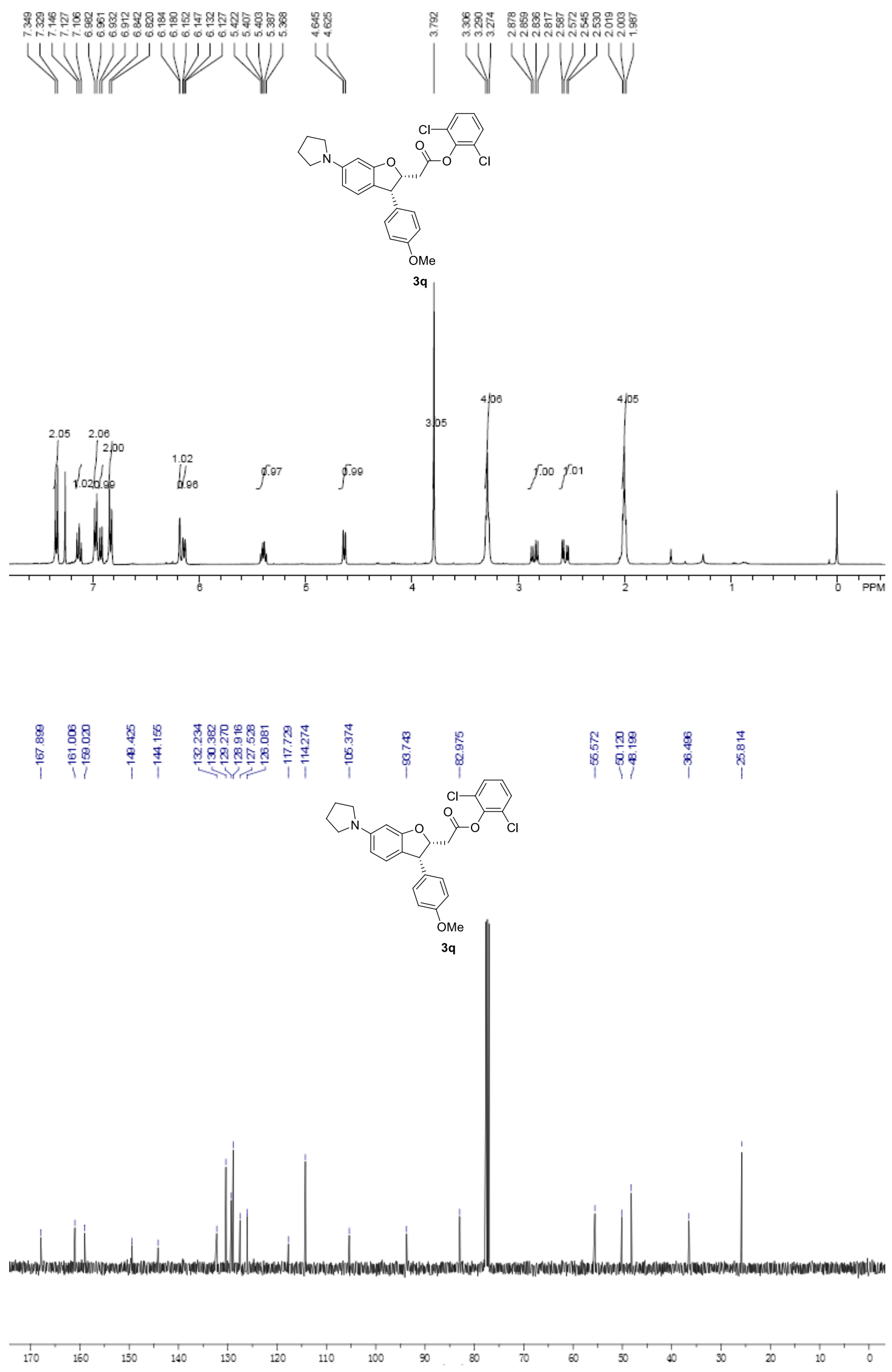


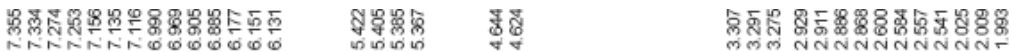

UUV
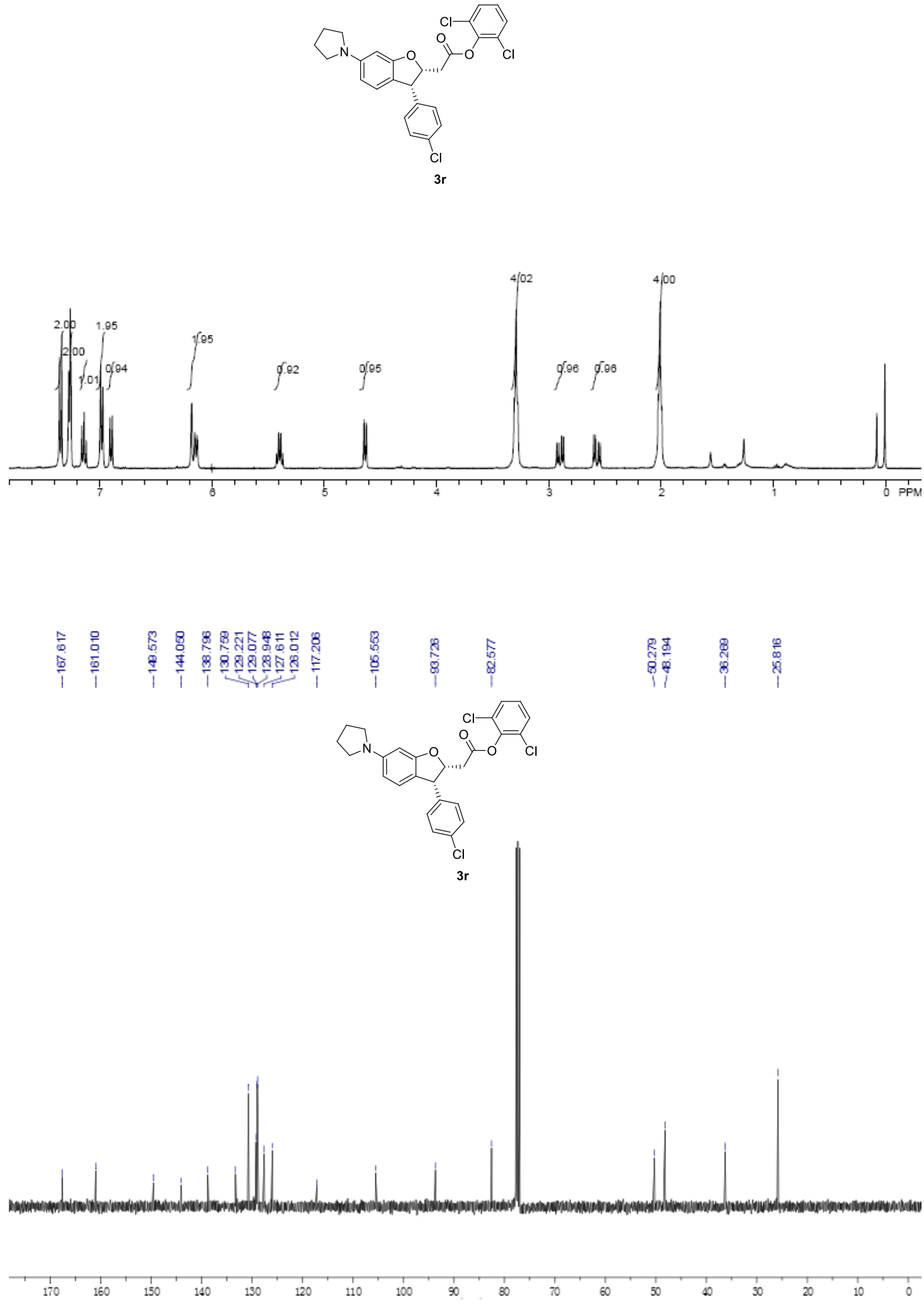

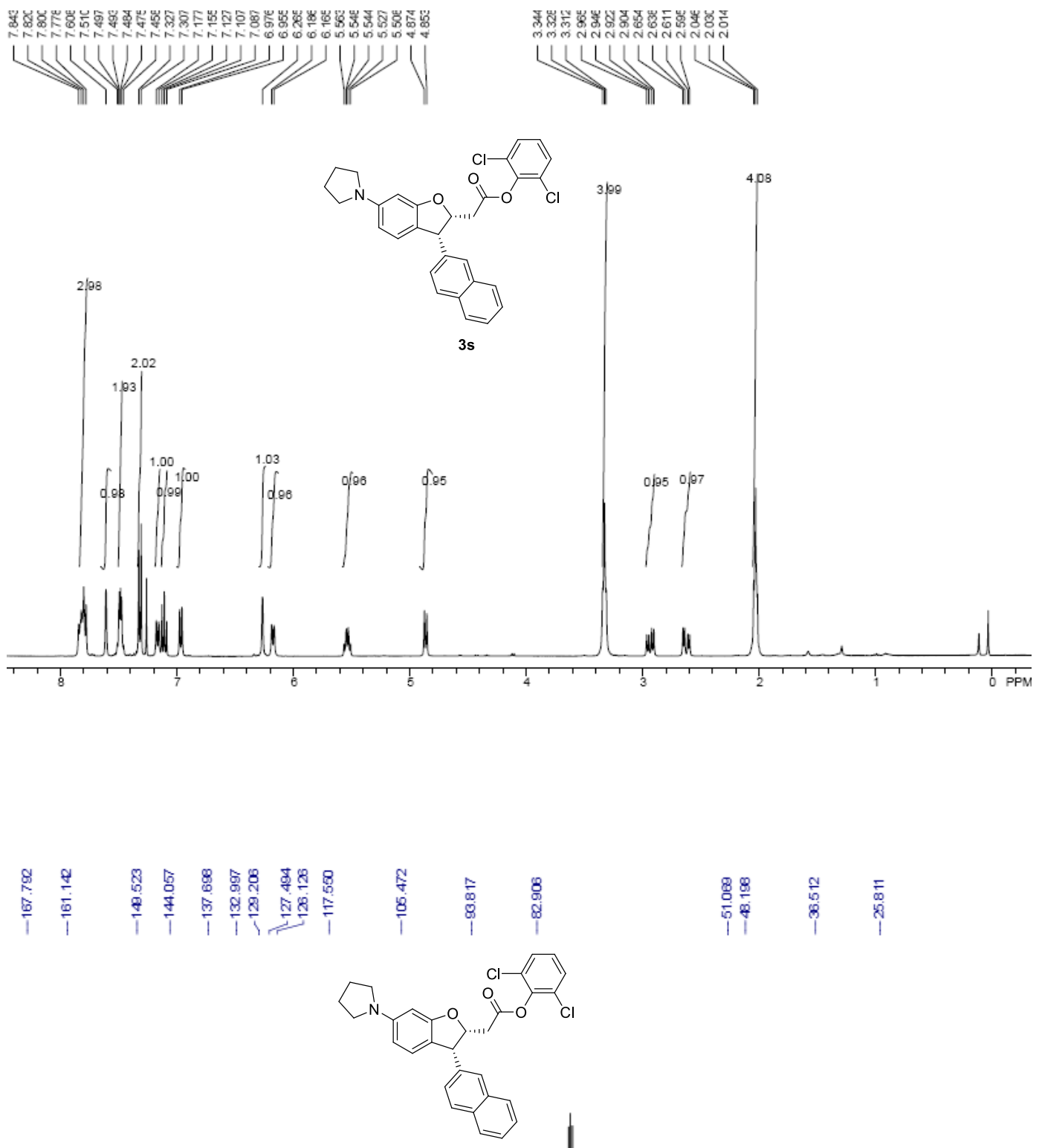

$3 s$
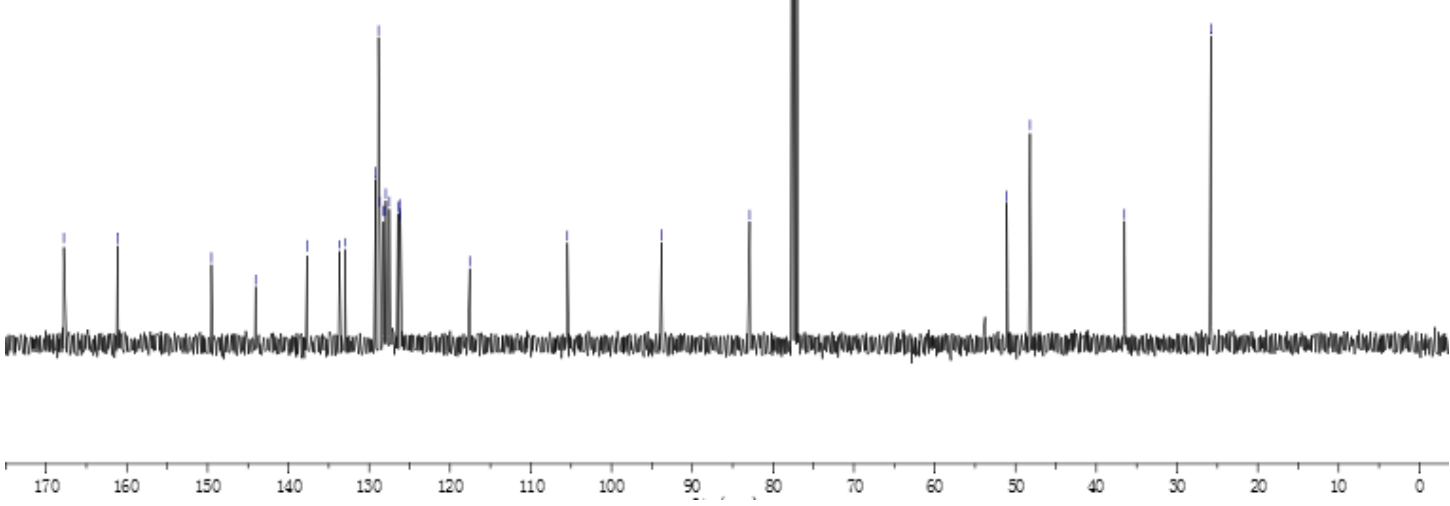

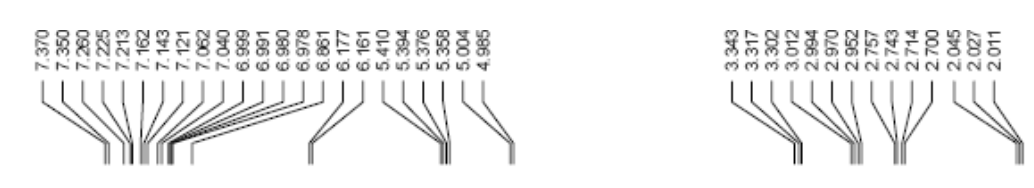

$$
\text { angar }
$$
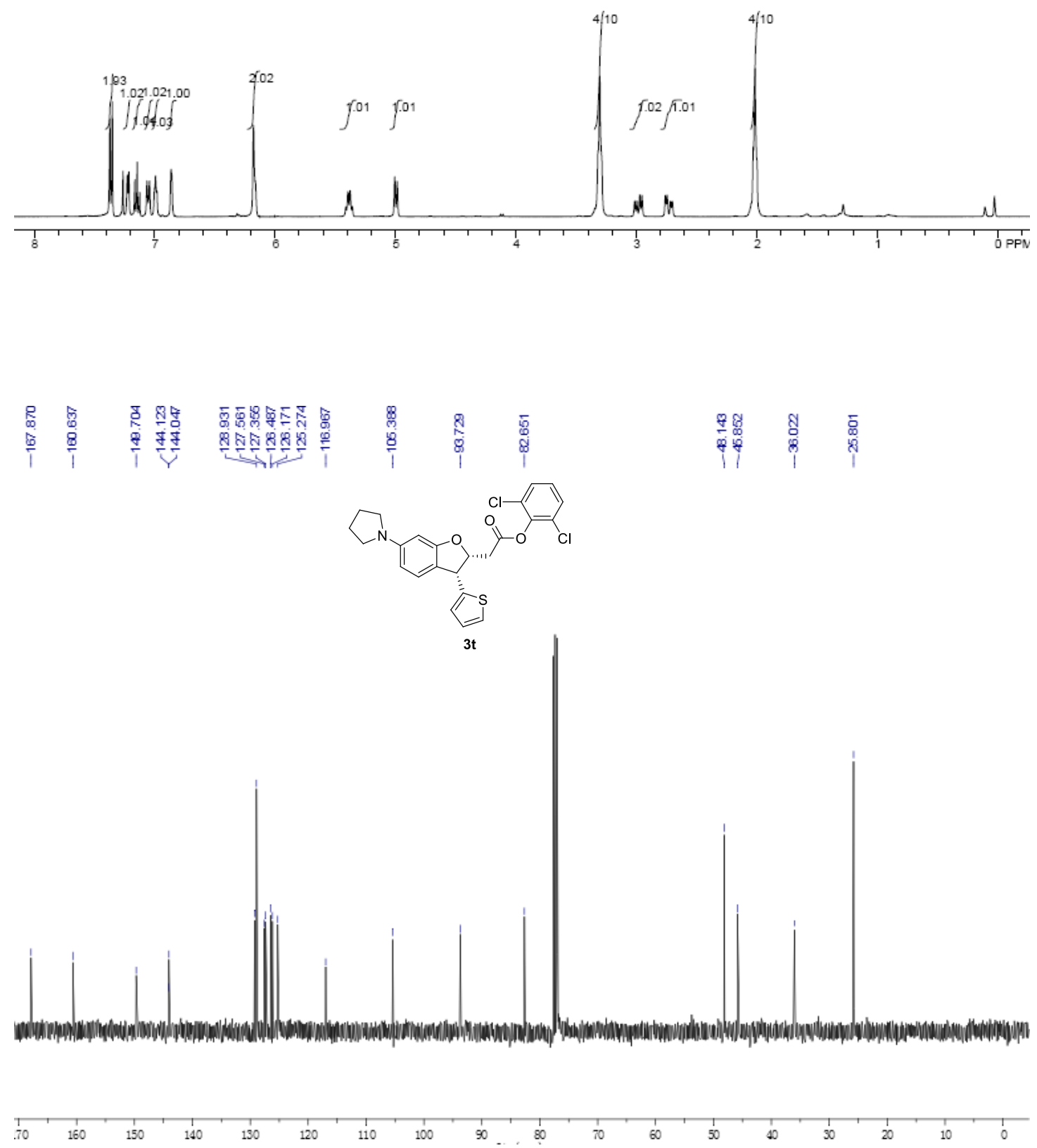

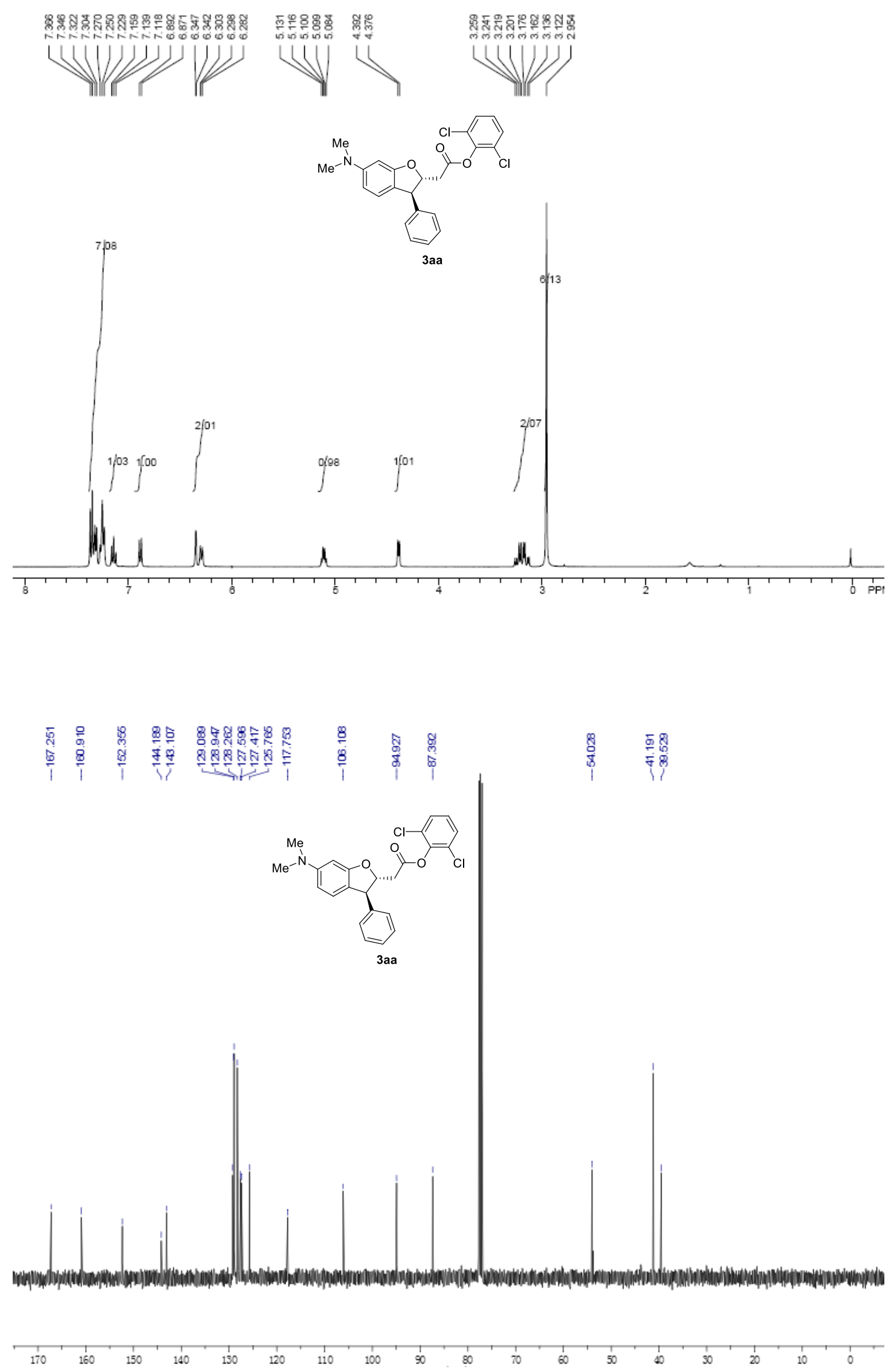

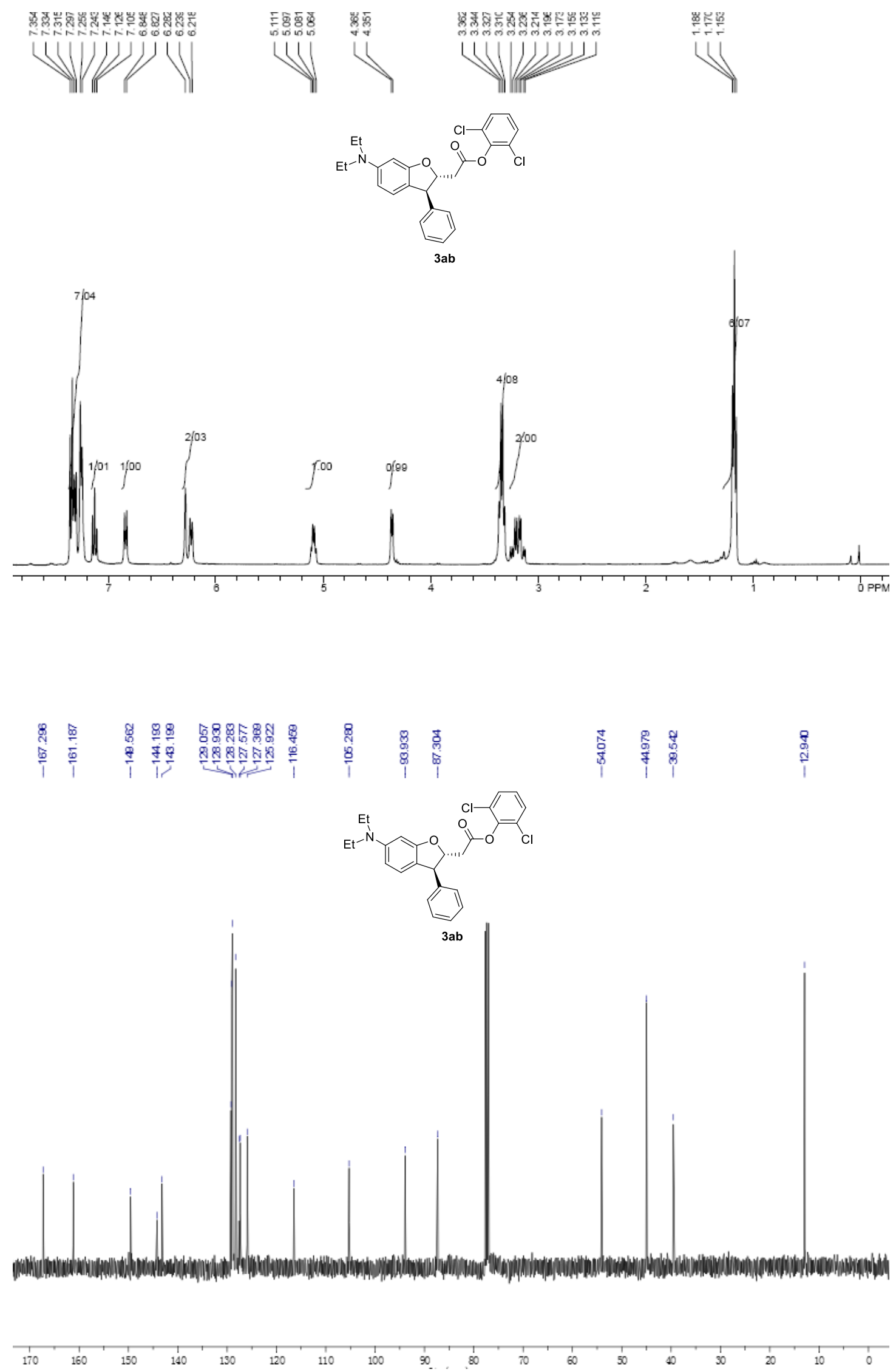

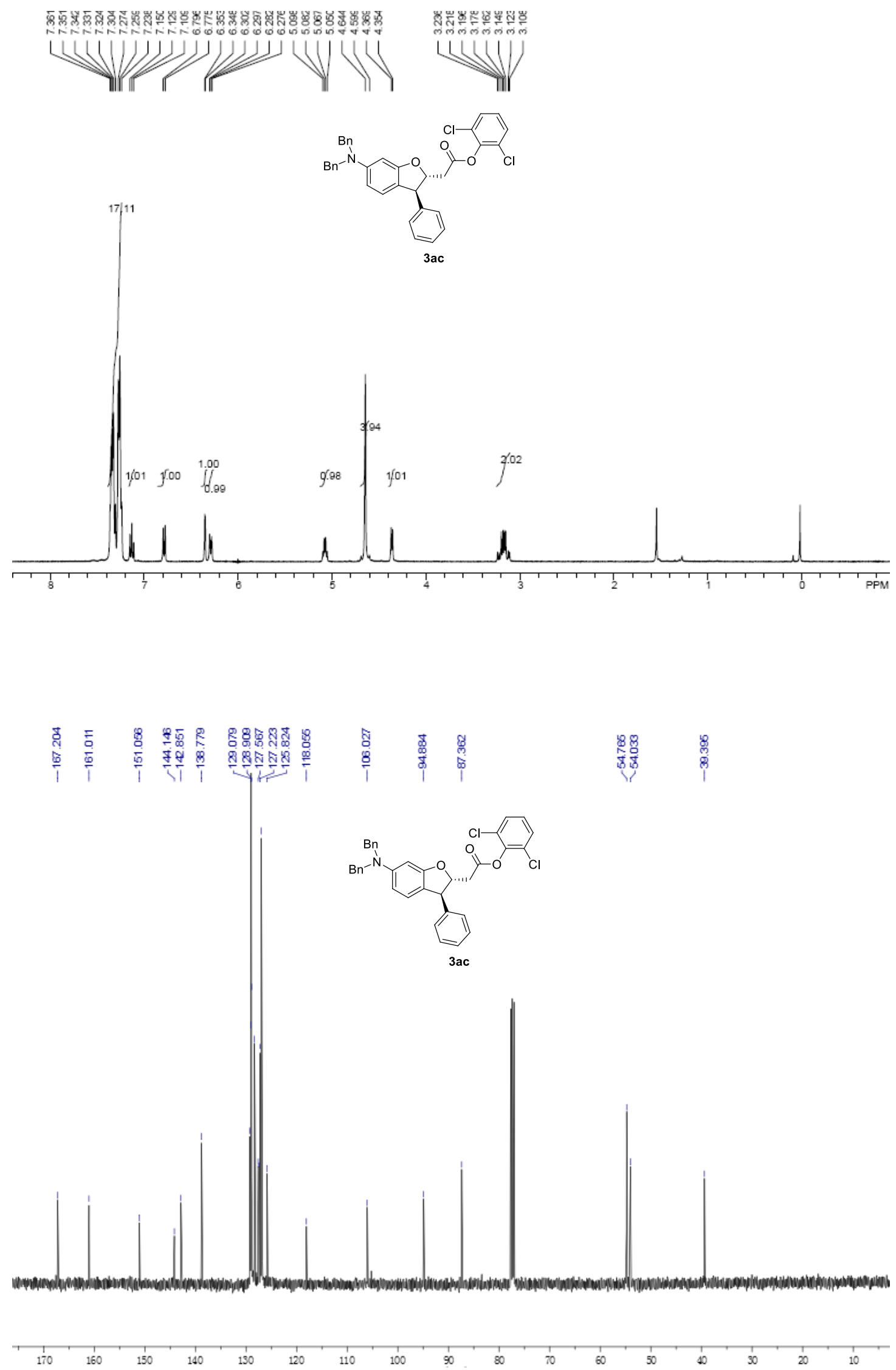

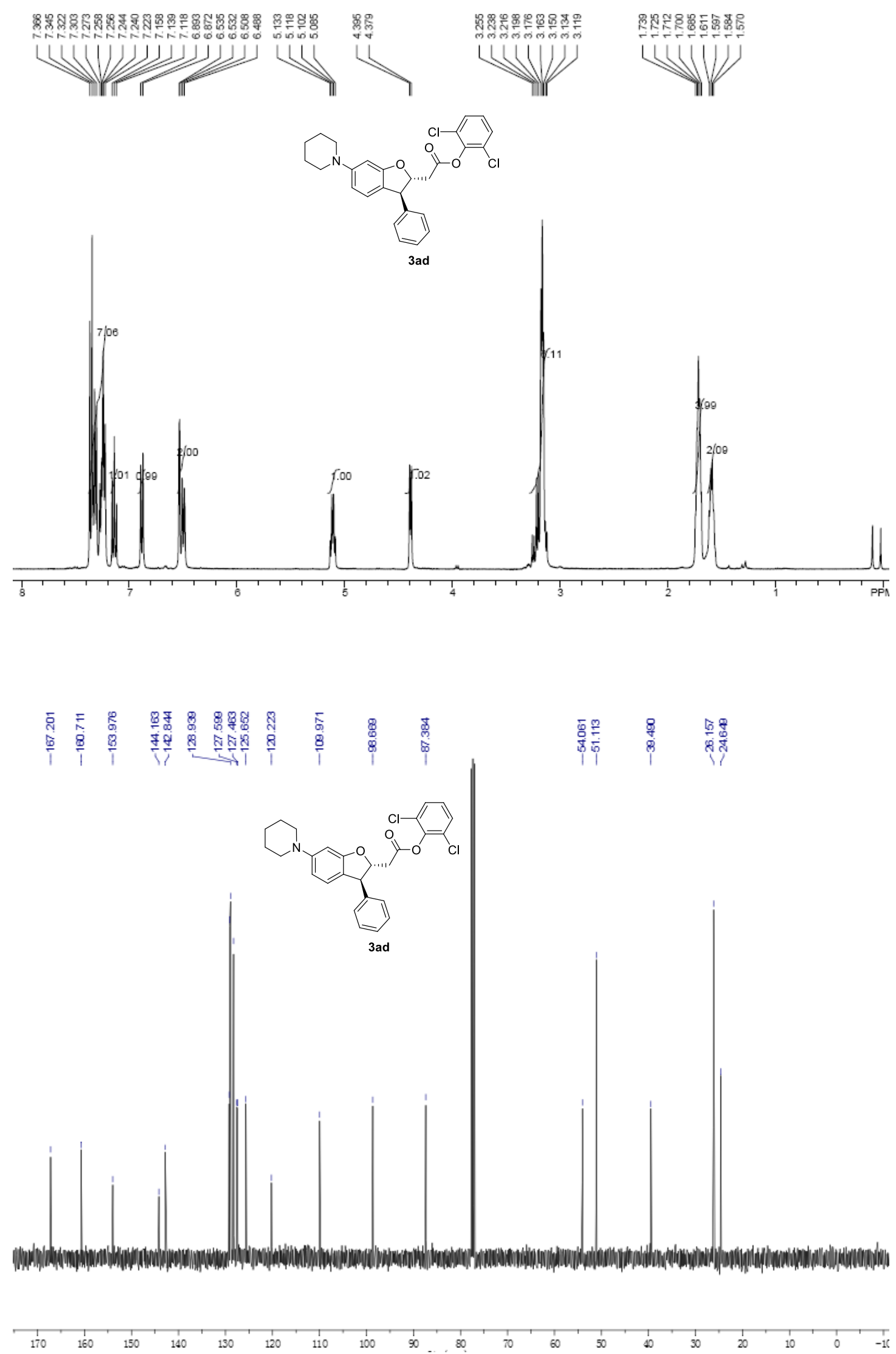

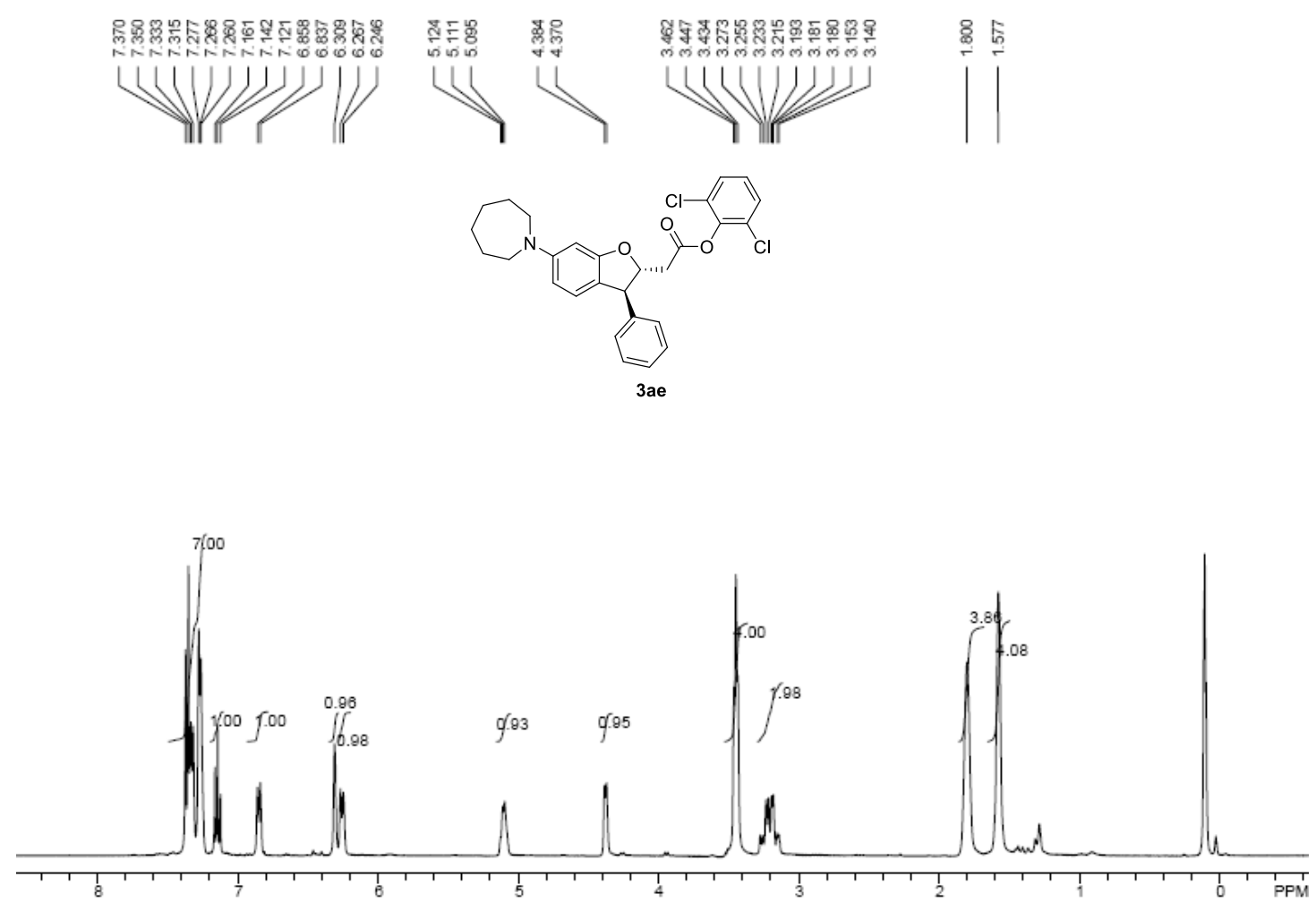

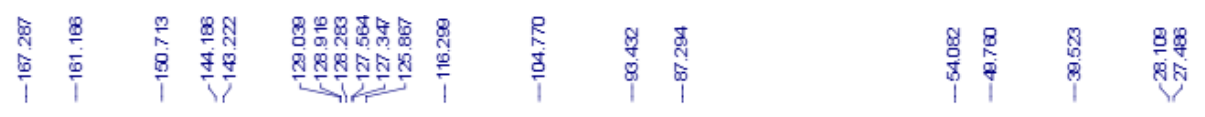

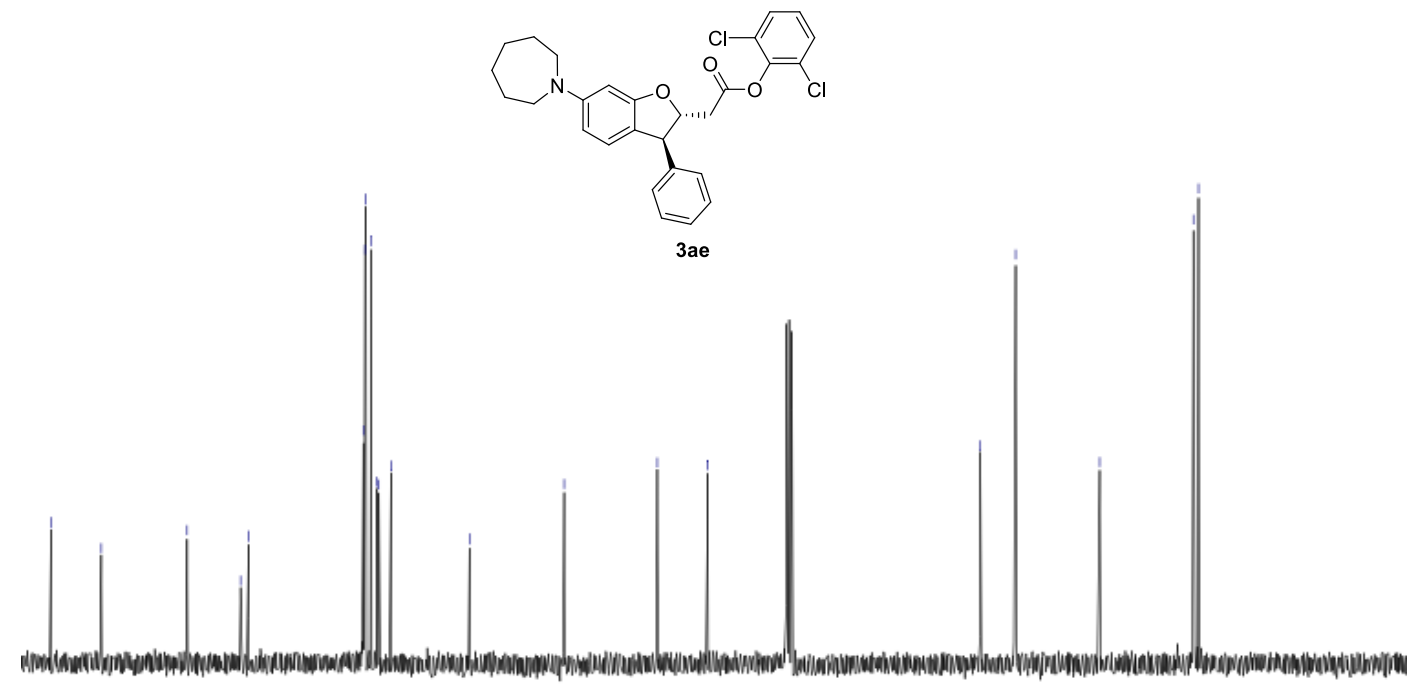

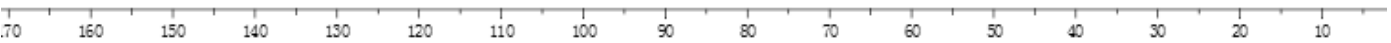




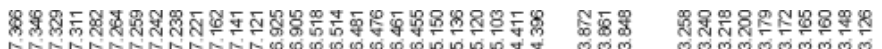

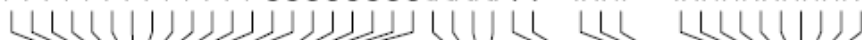

N<smiles>Clc1cccc2ccccc12</smiles>

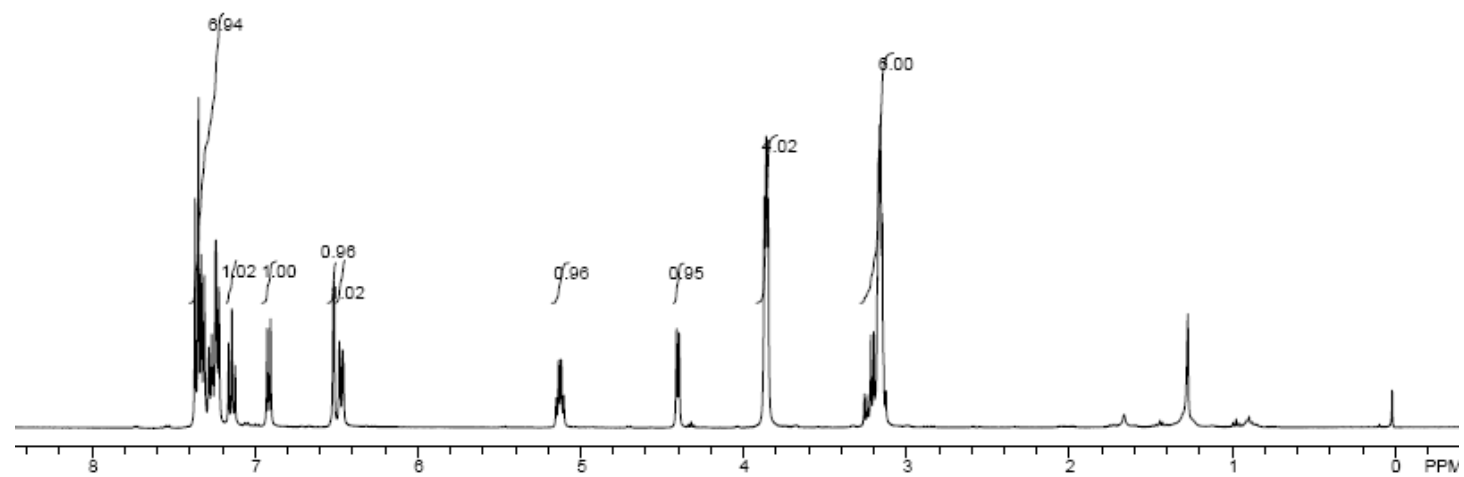

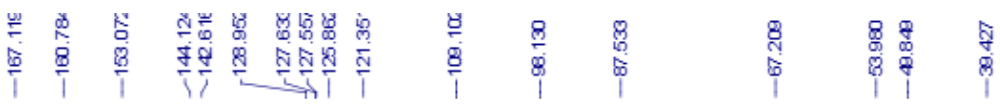
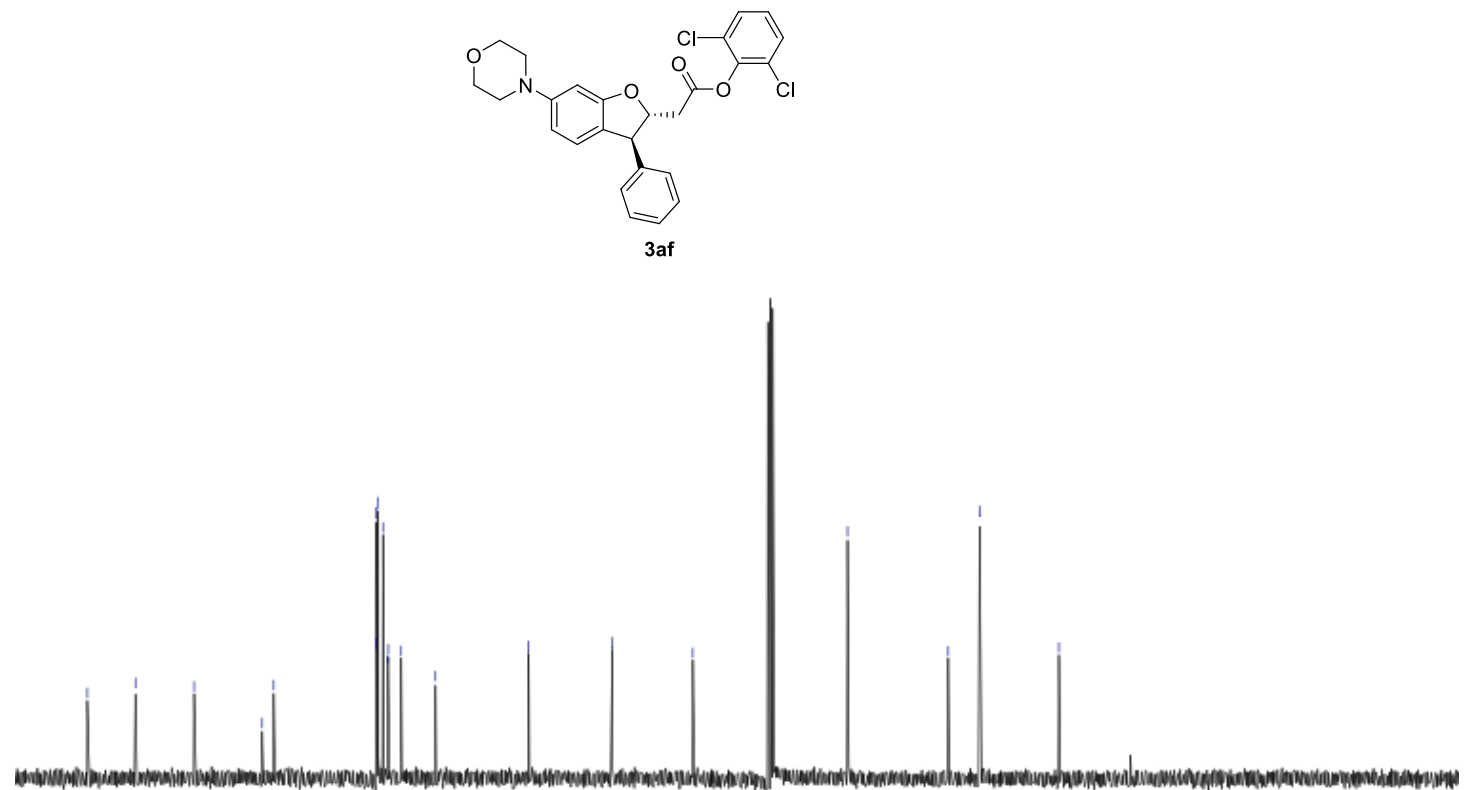

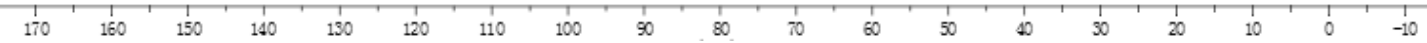



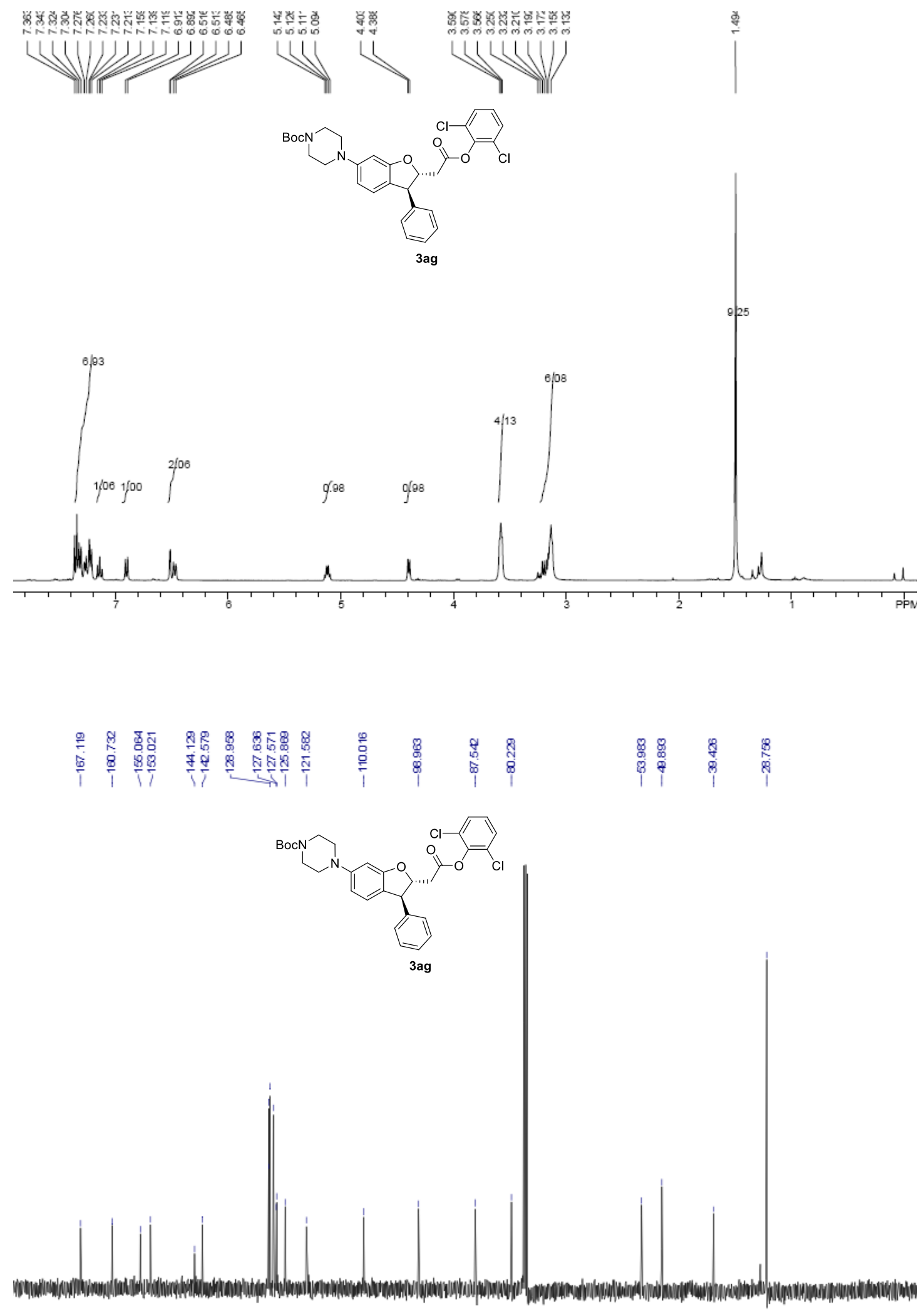

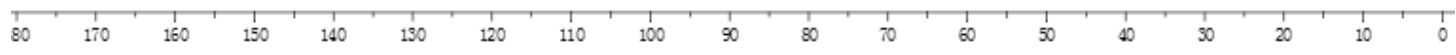




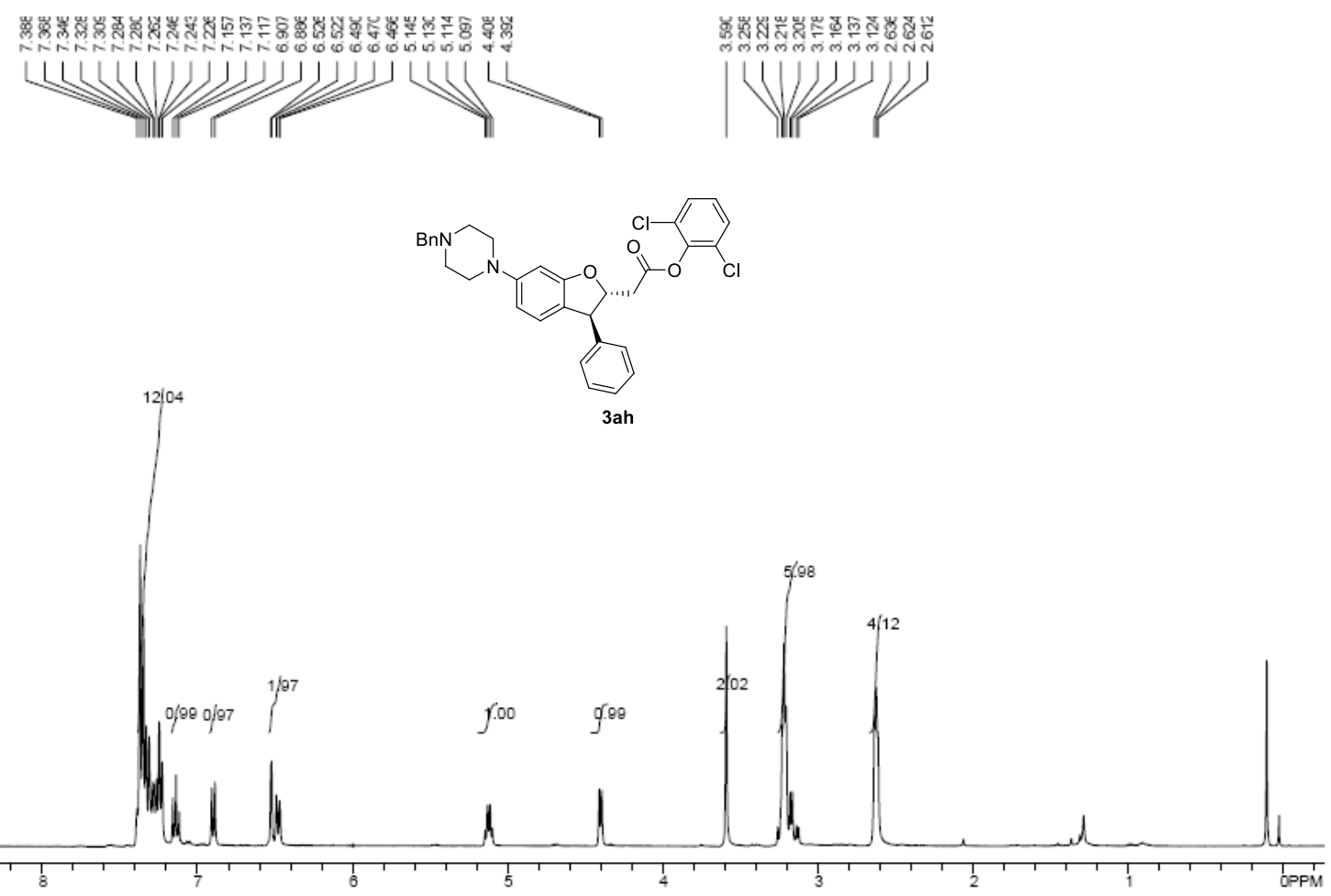

$\begin{array}{lllllllllllll}1 & 1 & 1 & 1 & 1 & 1 & 1\end{array}$
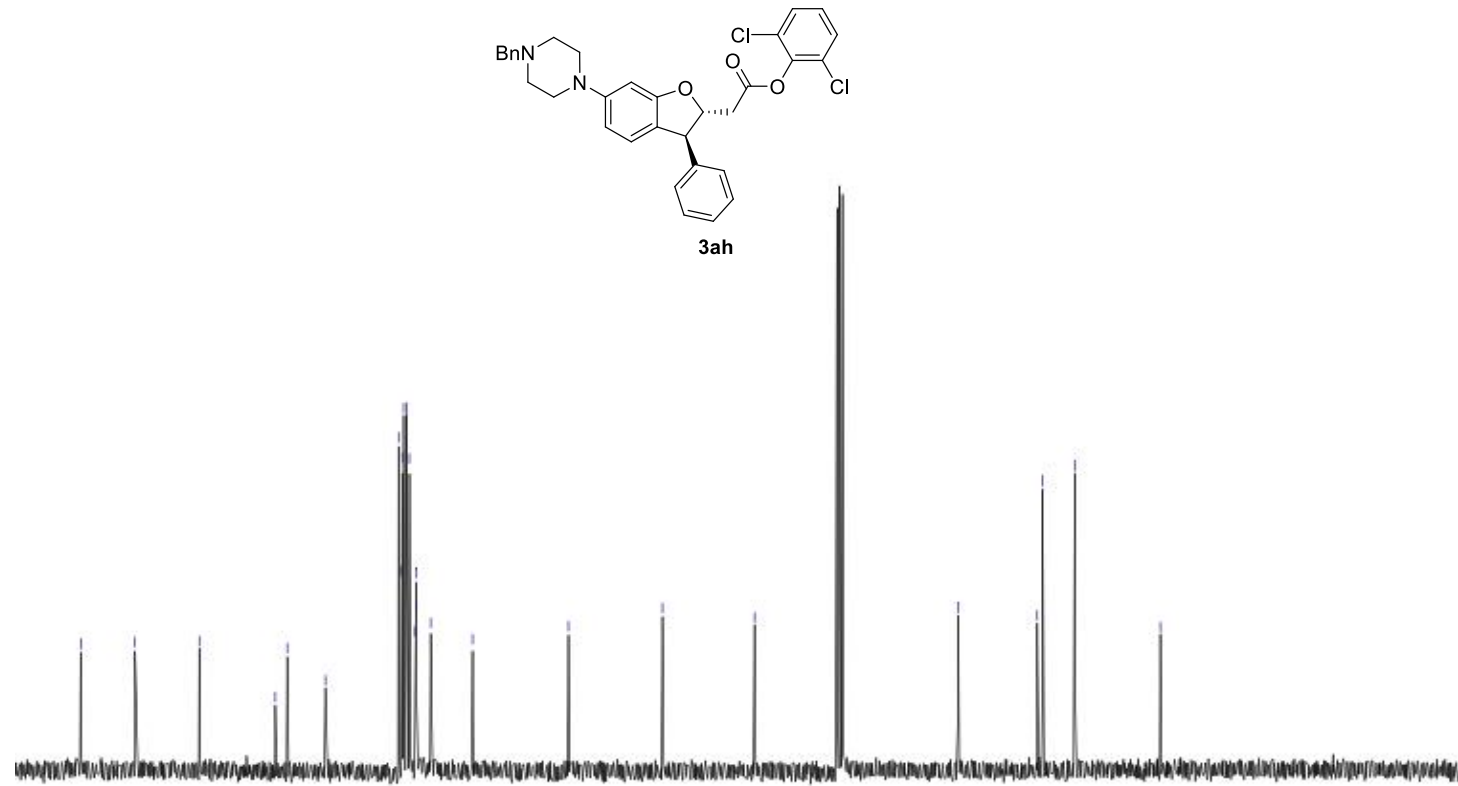

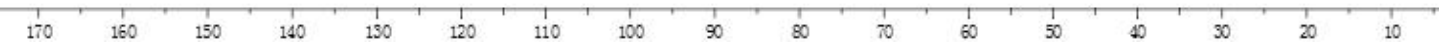




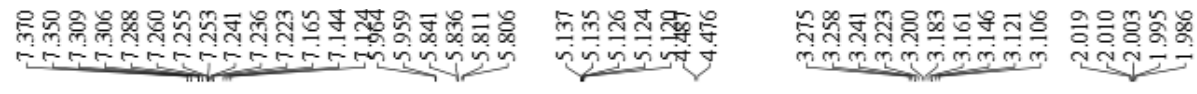

$\| 1 /$<smiles>C=CC(C)C=C</smiles>
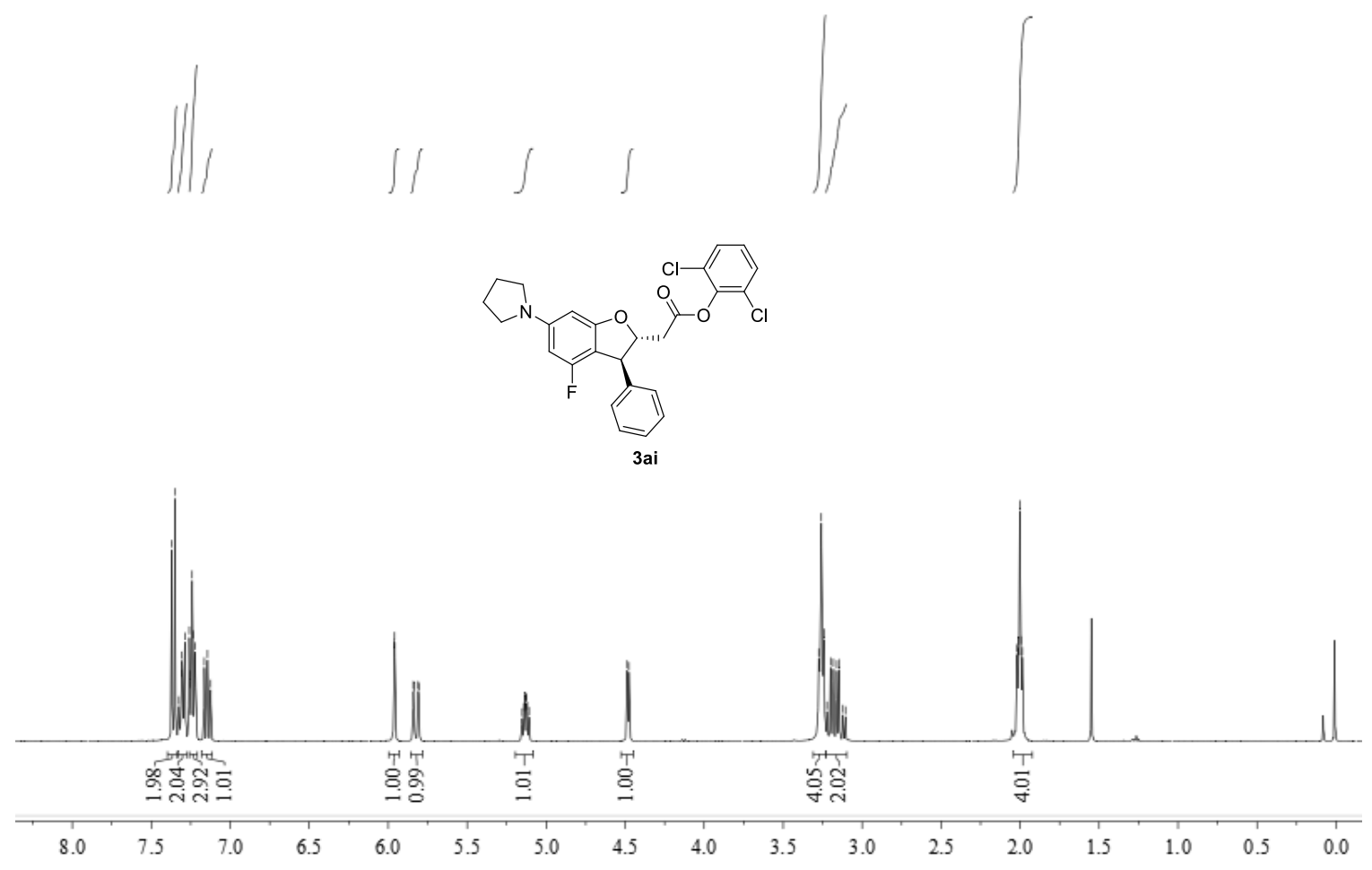

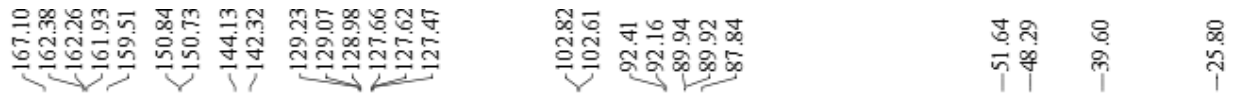

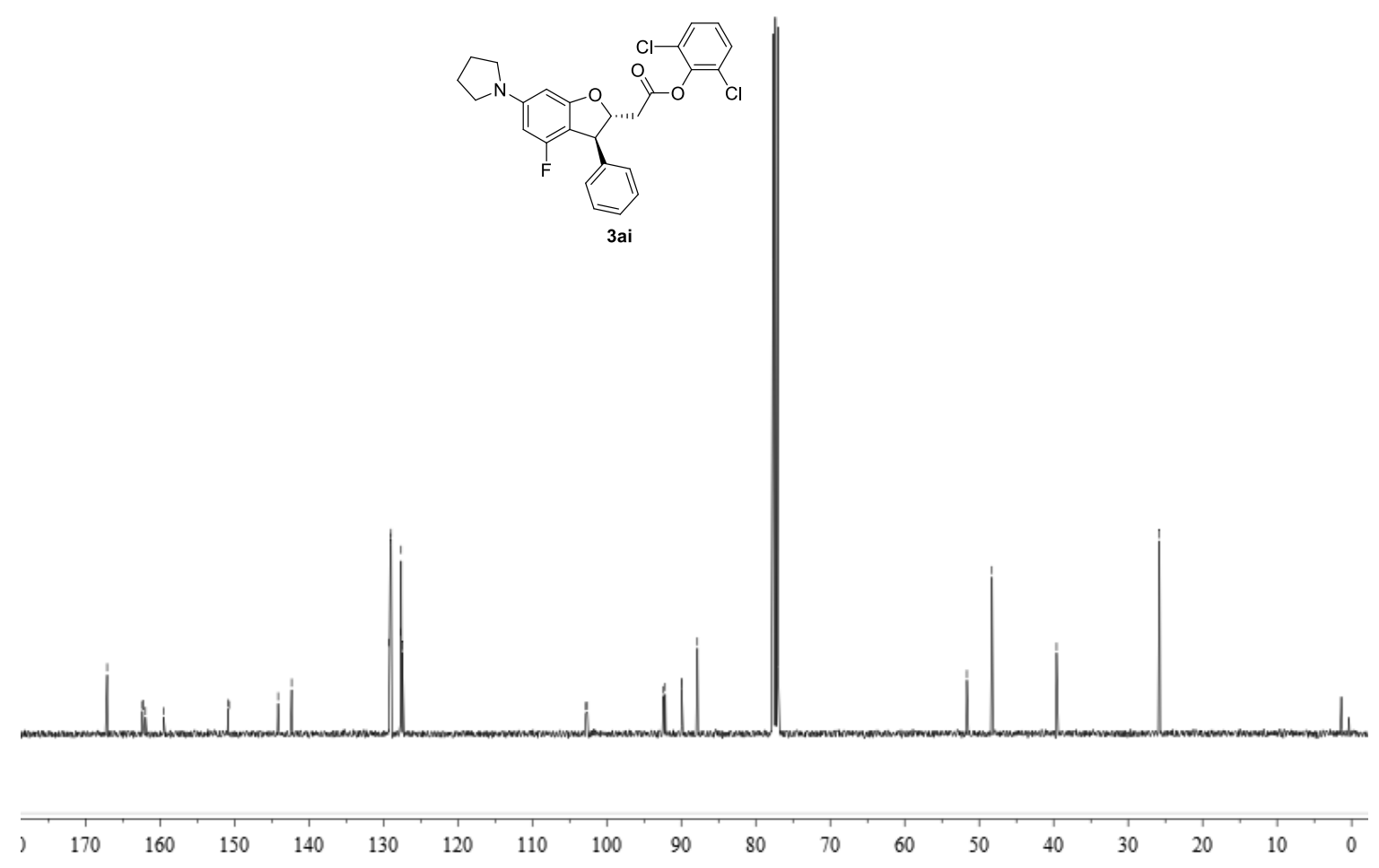



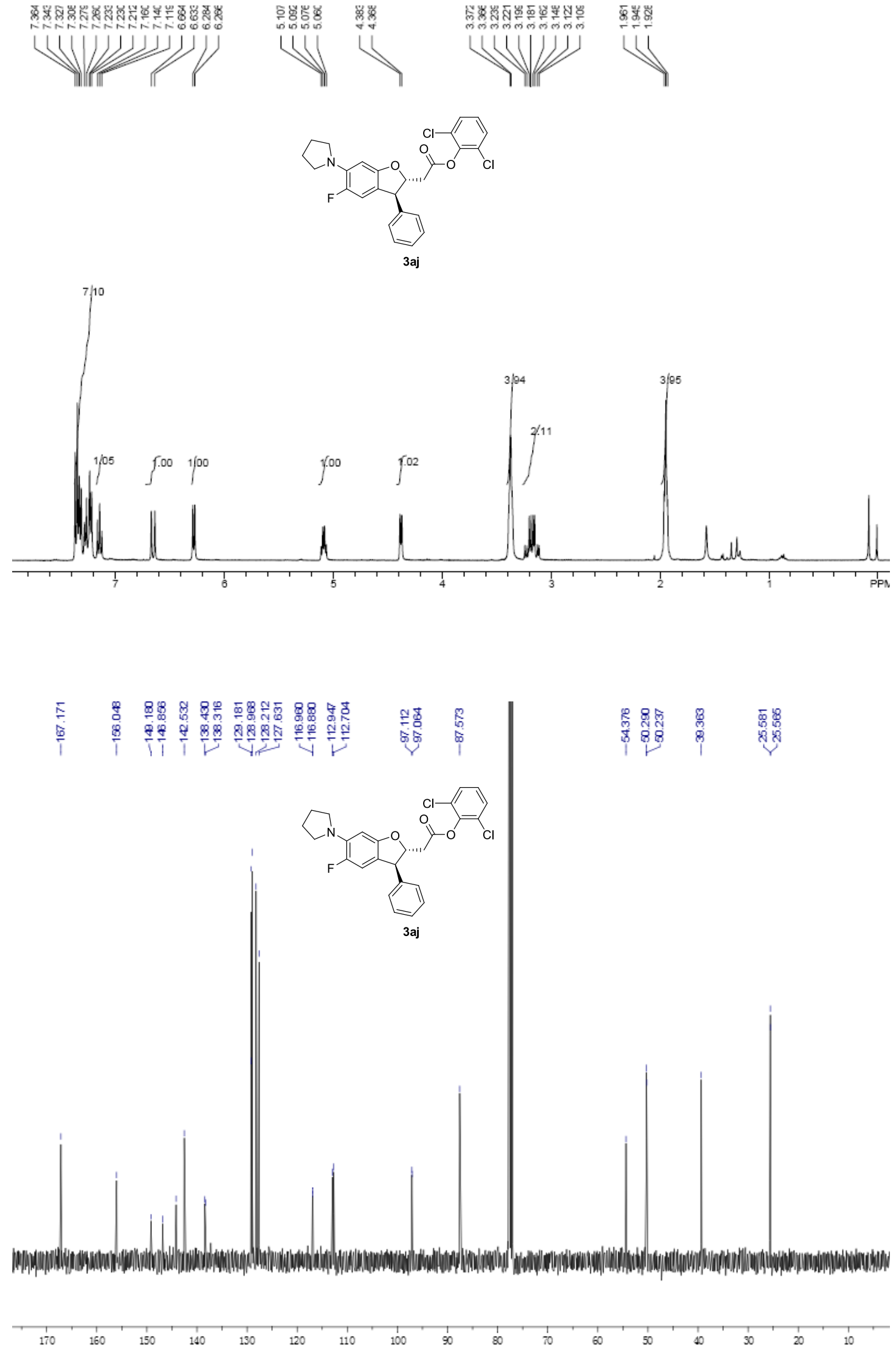

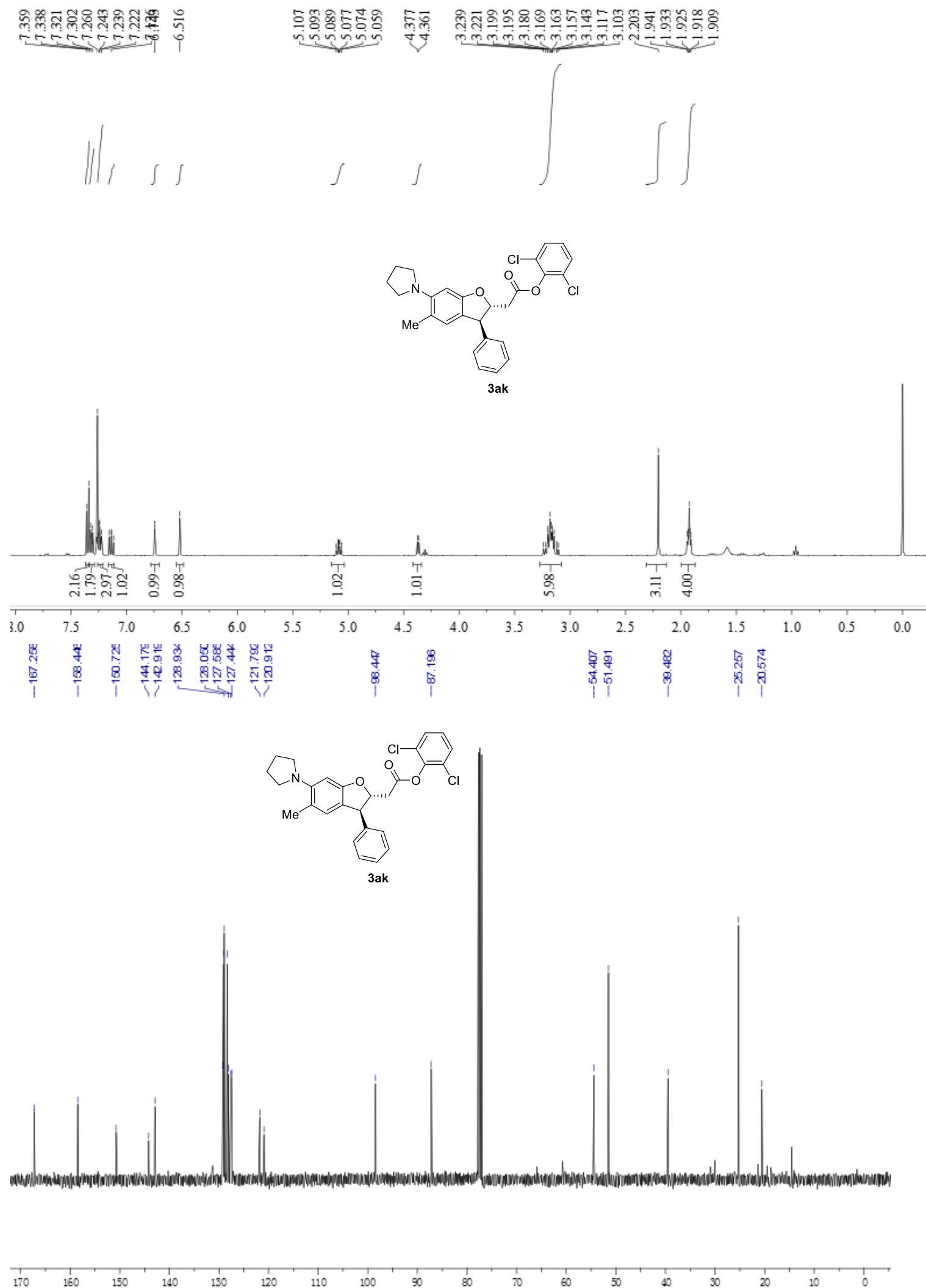

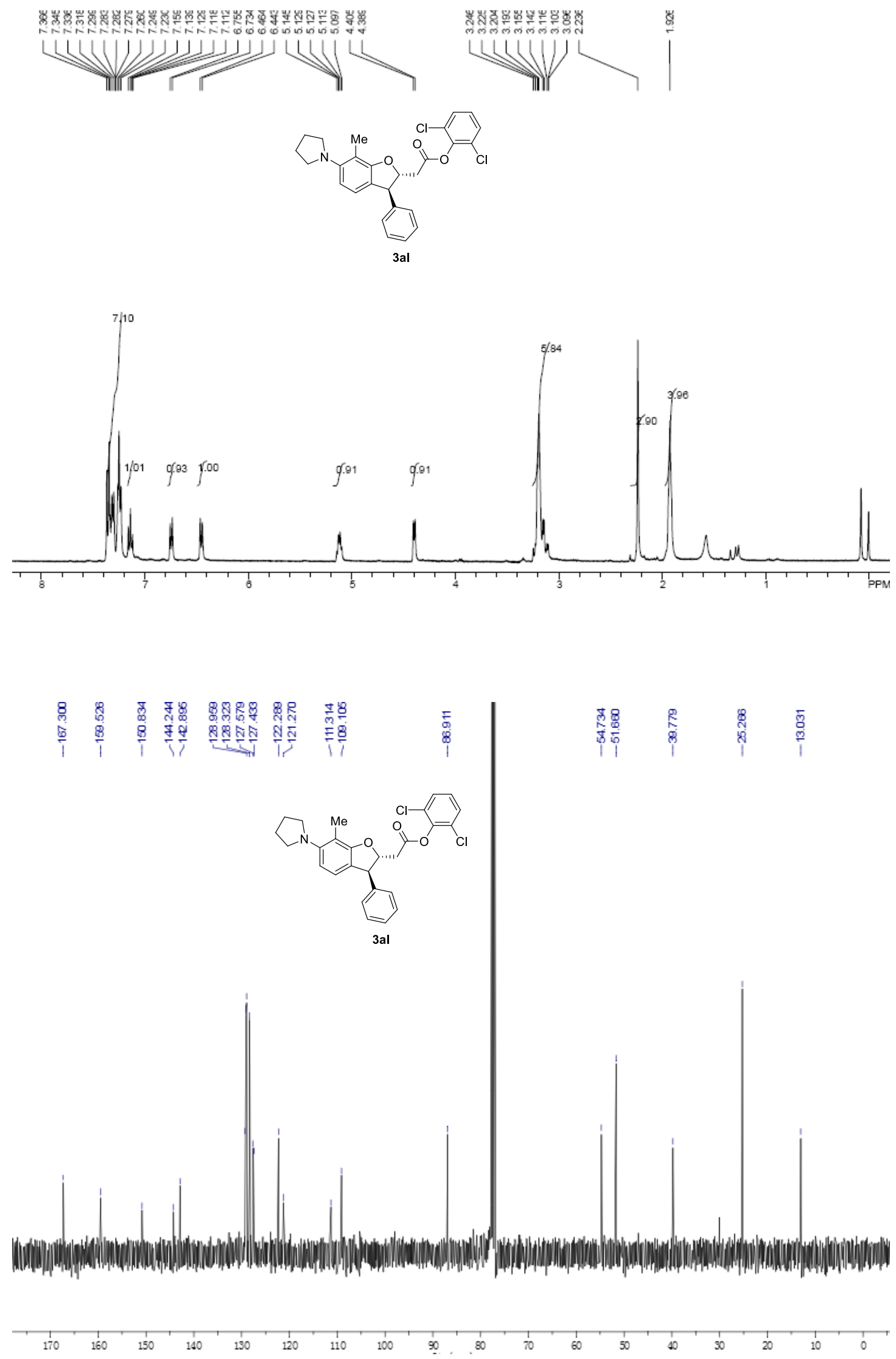

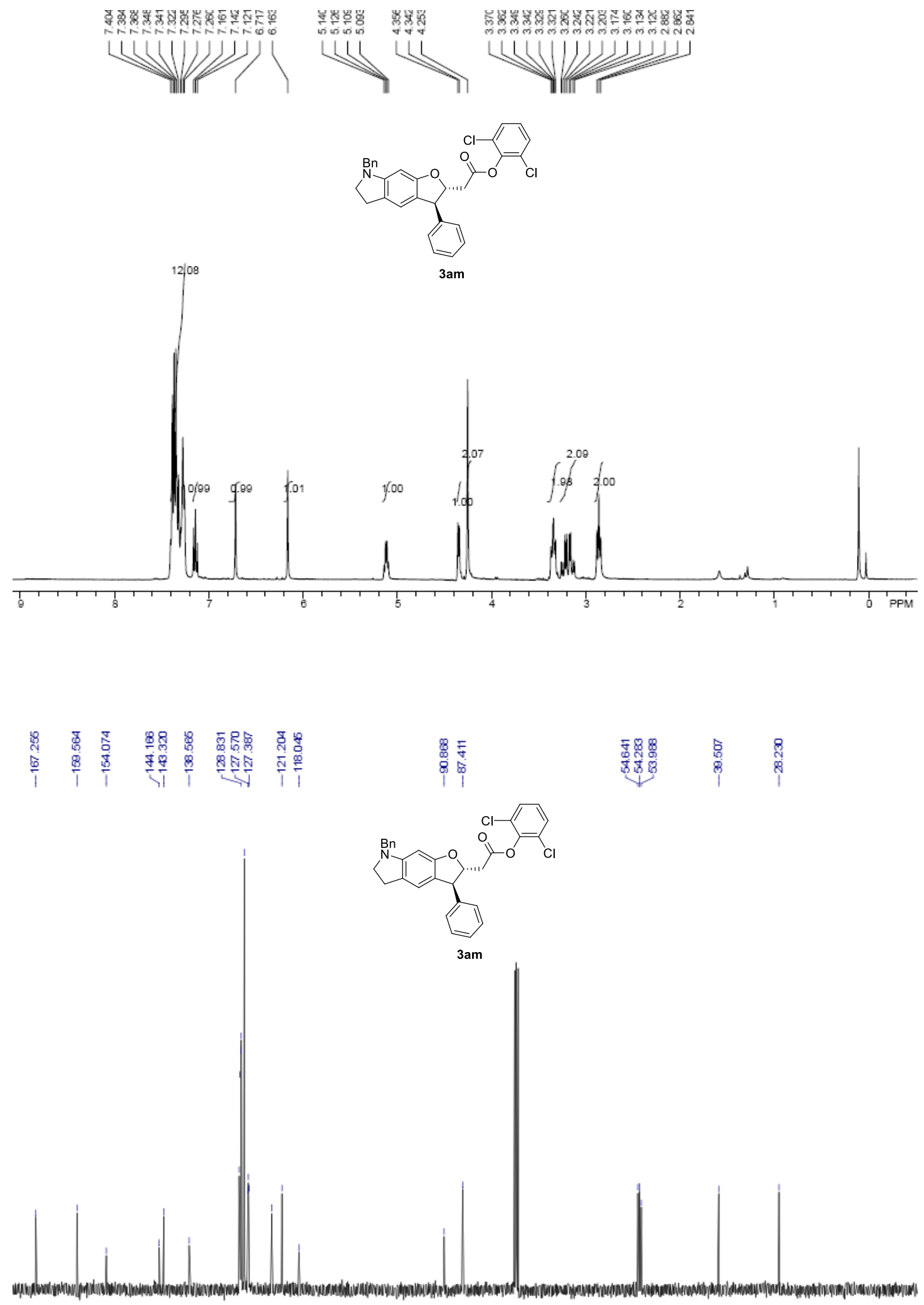

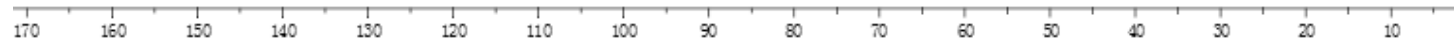




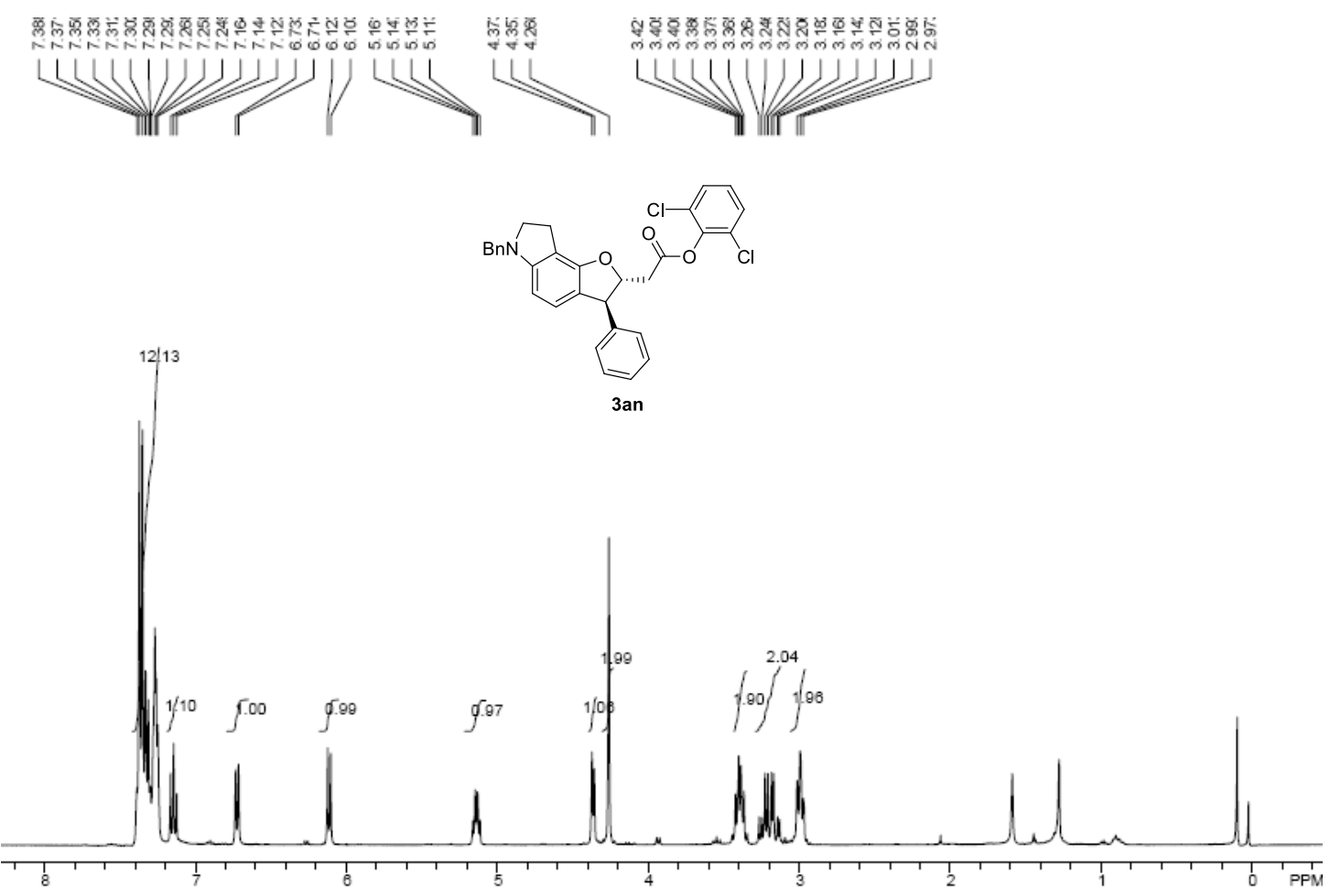

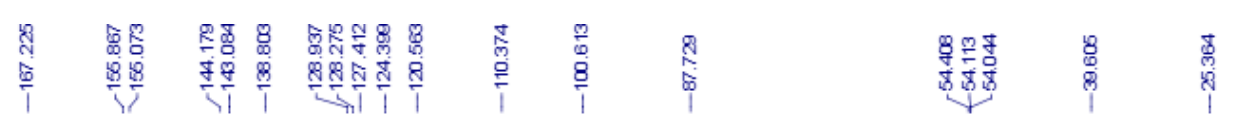

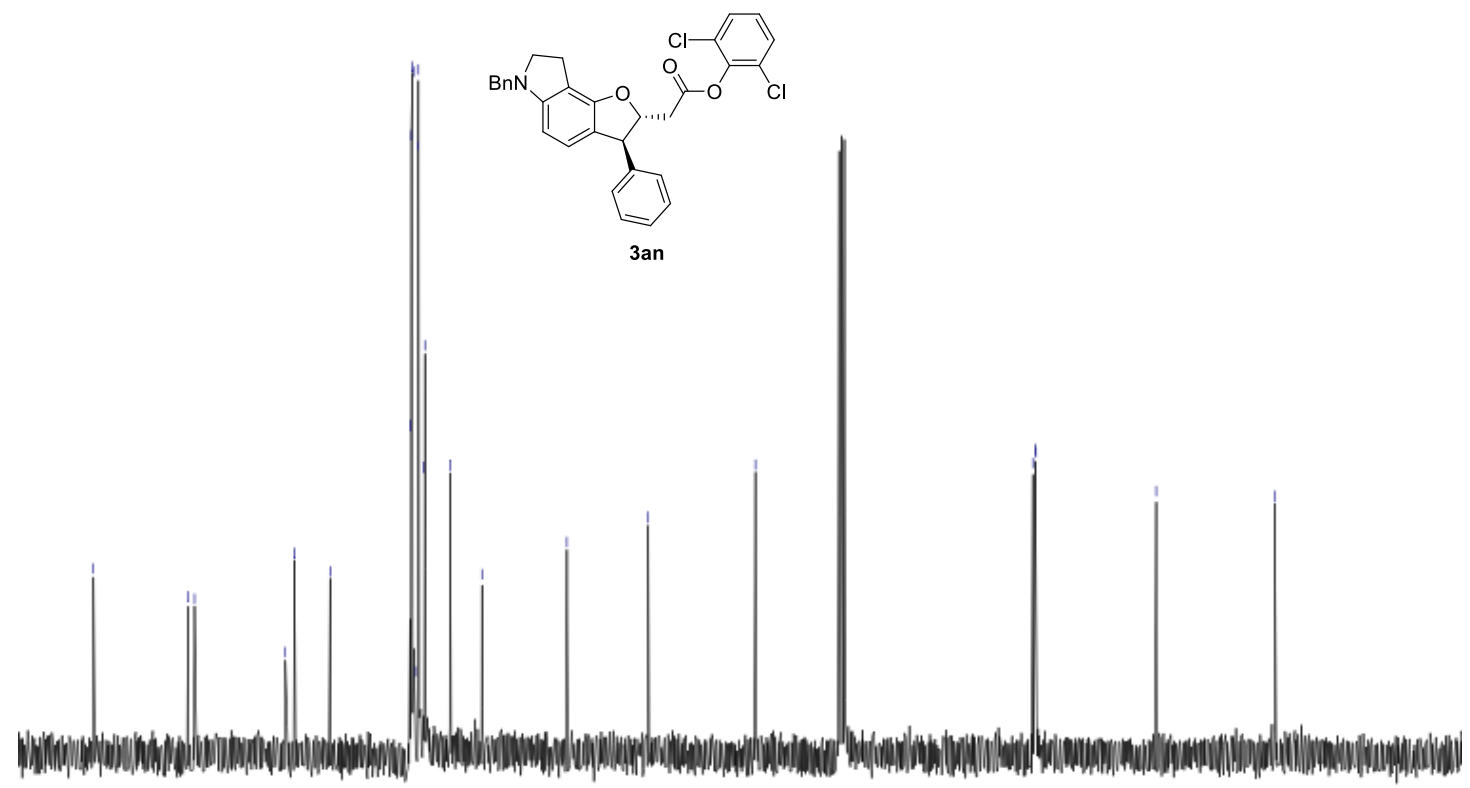

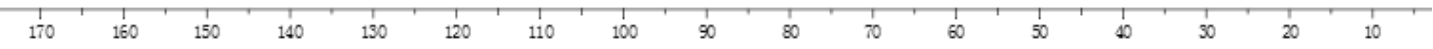




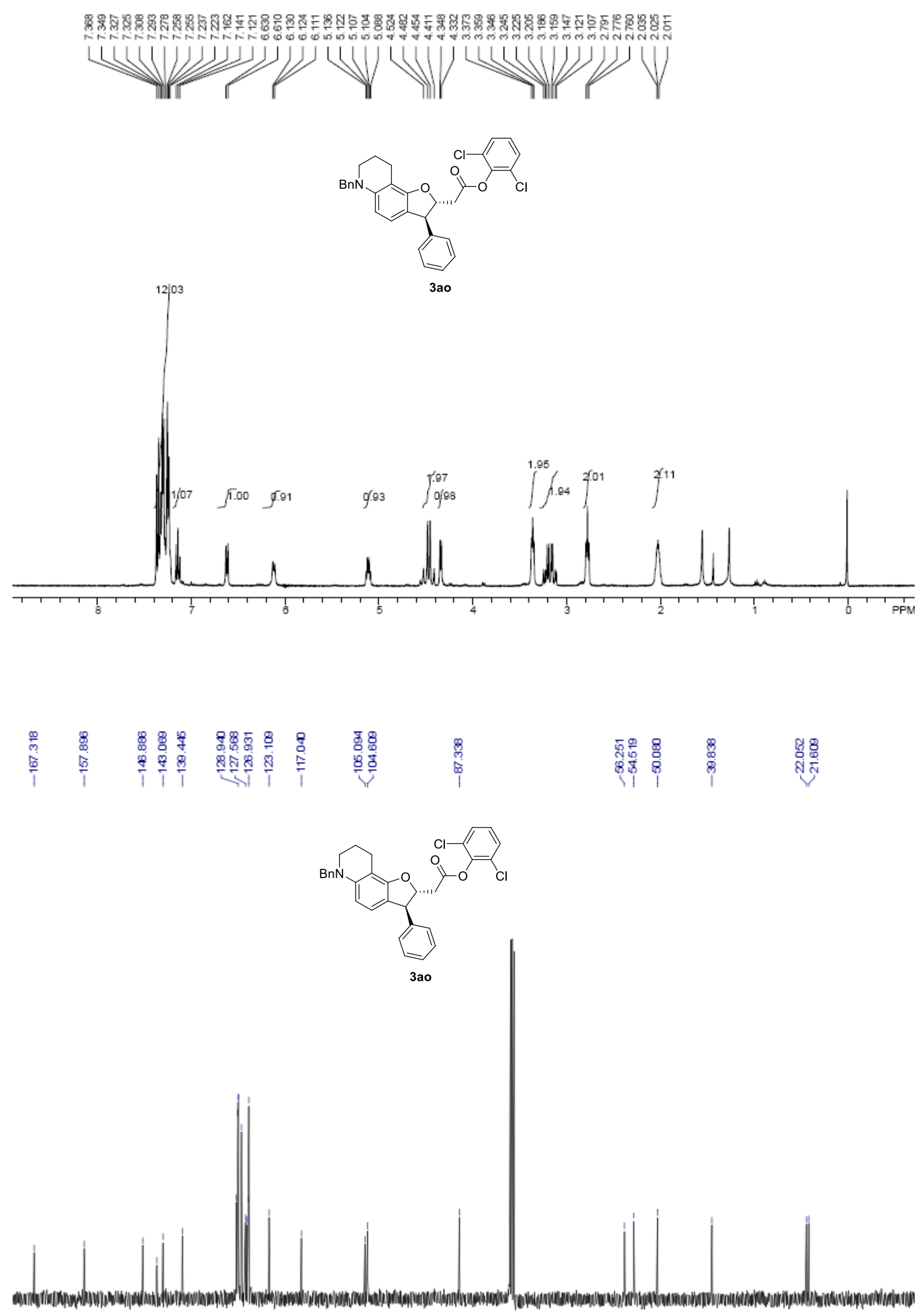

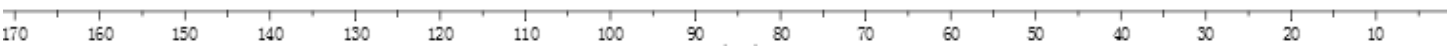



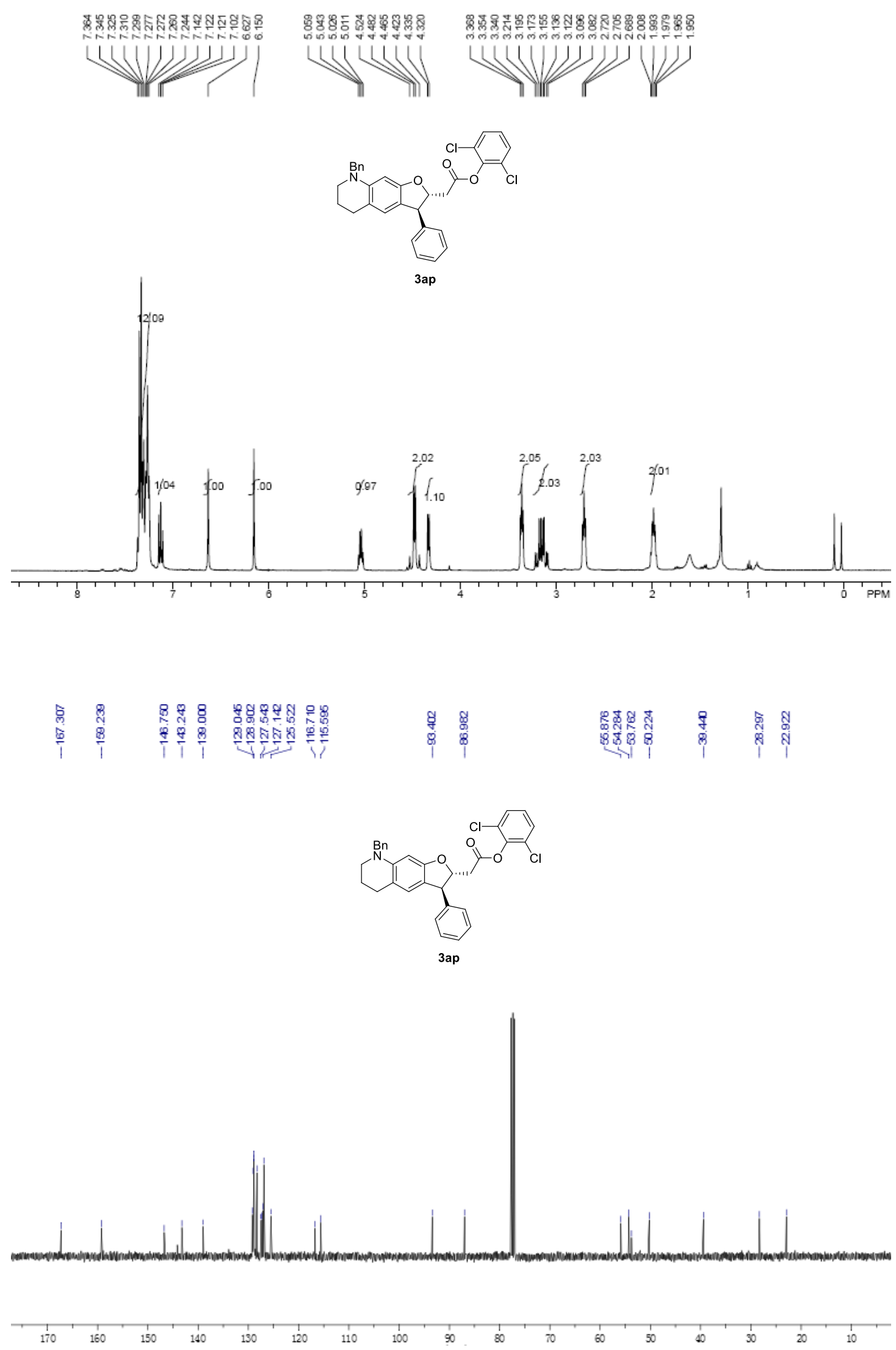

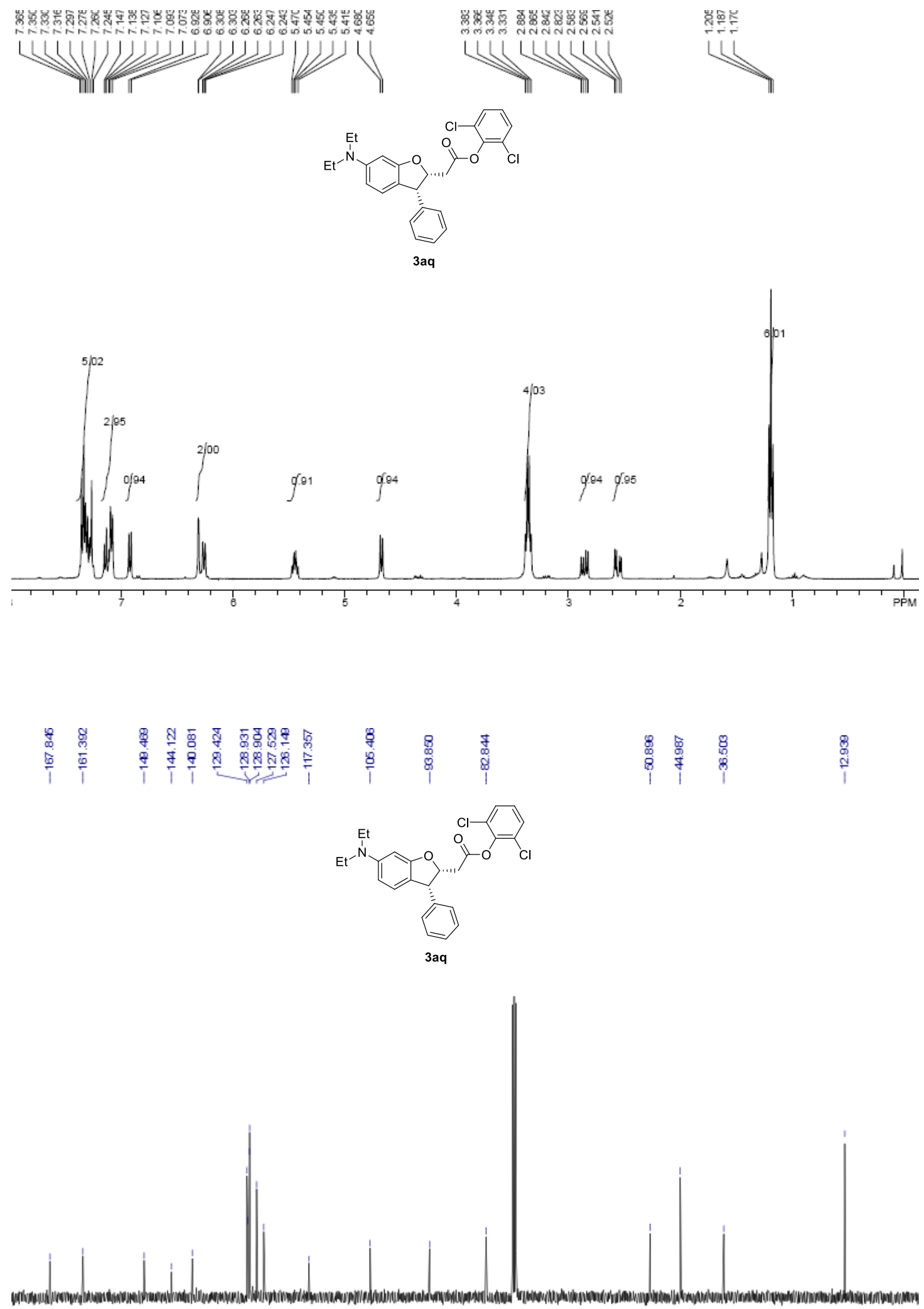

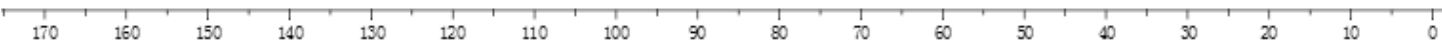



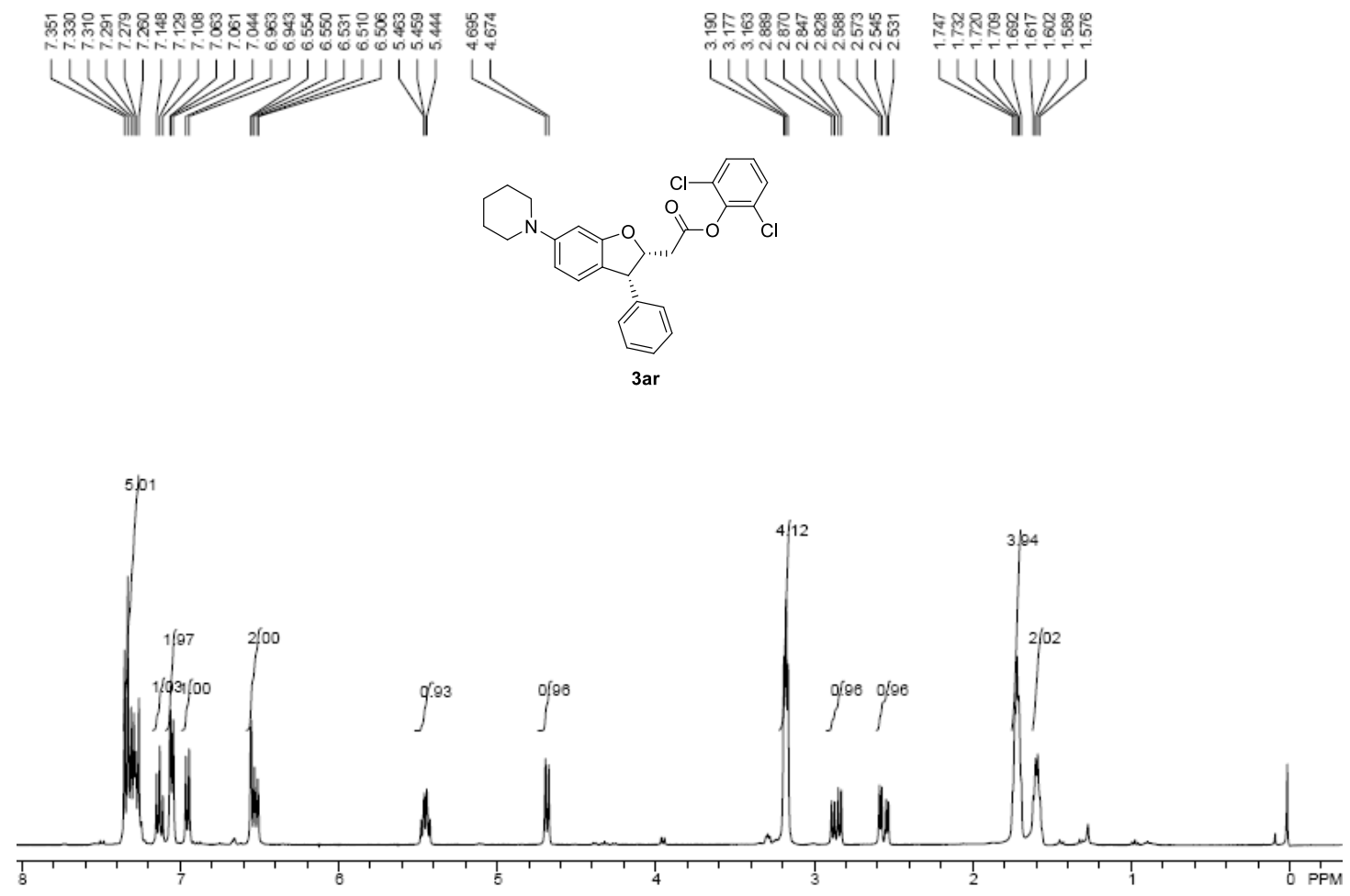

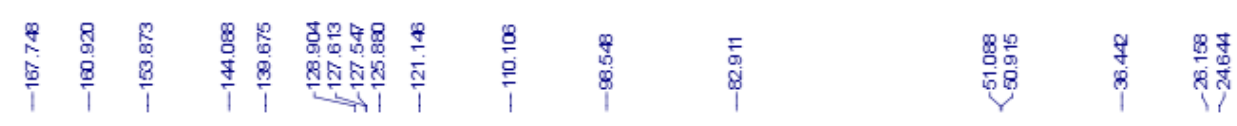
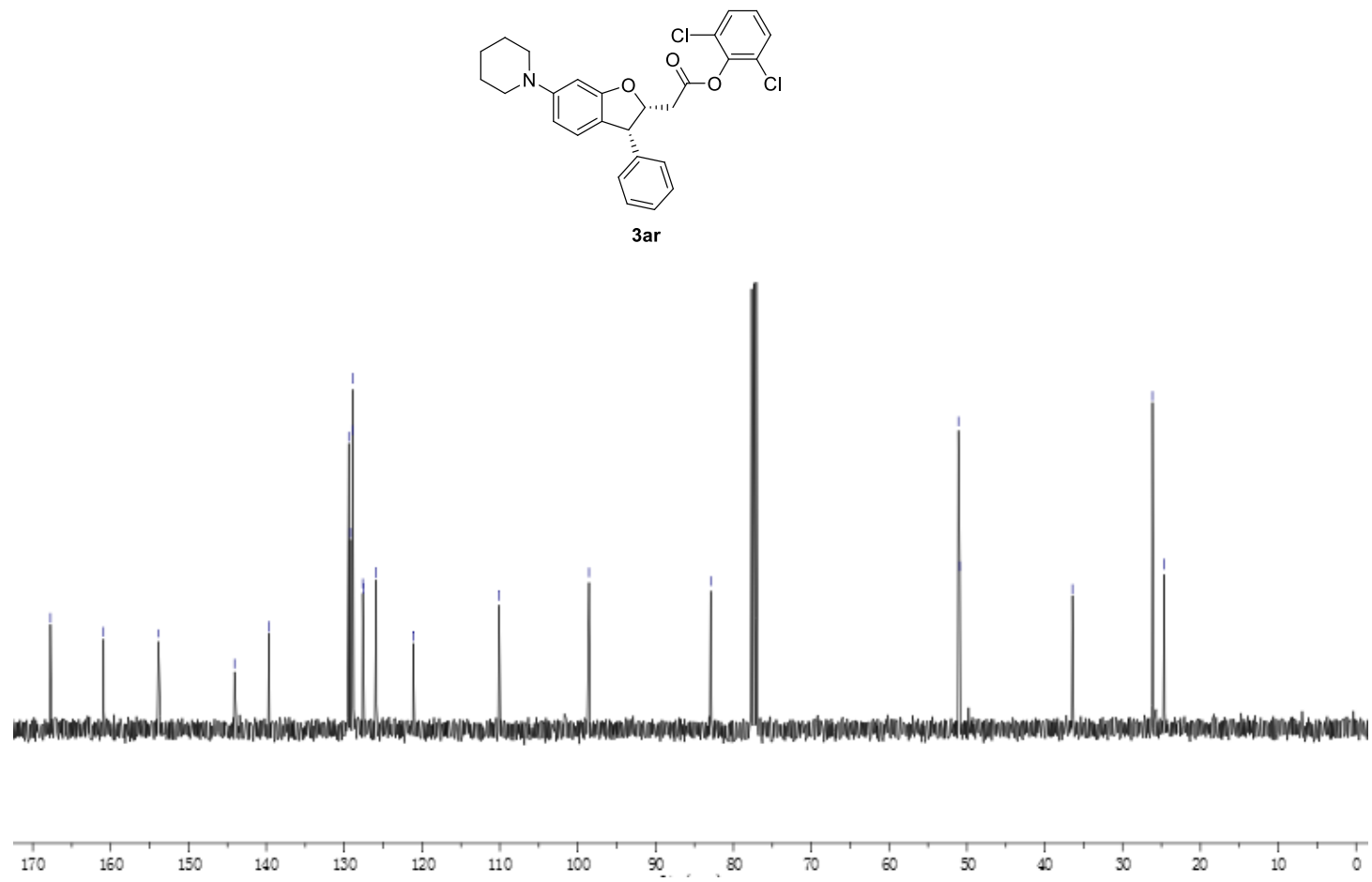

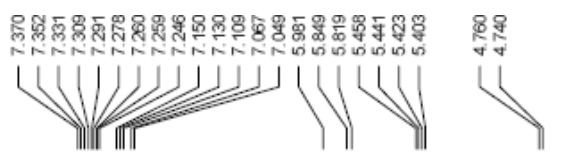

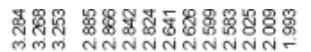
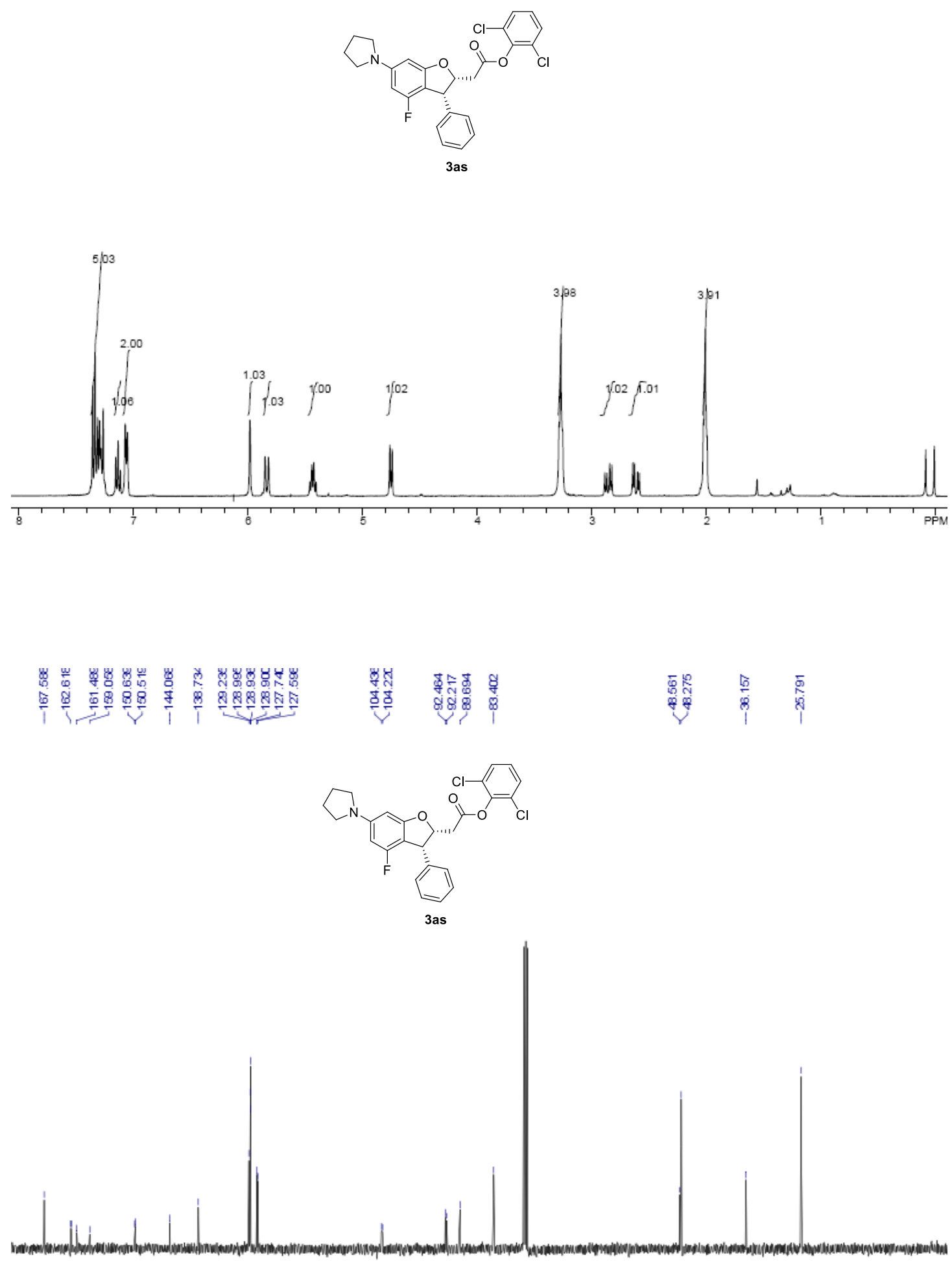

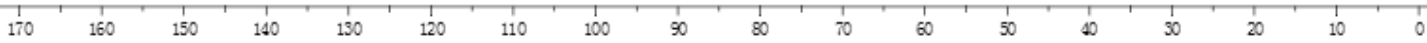



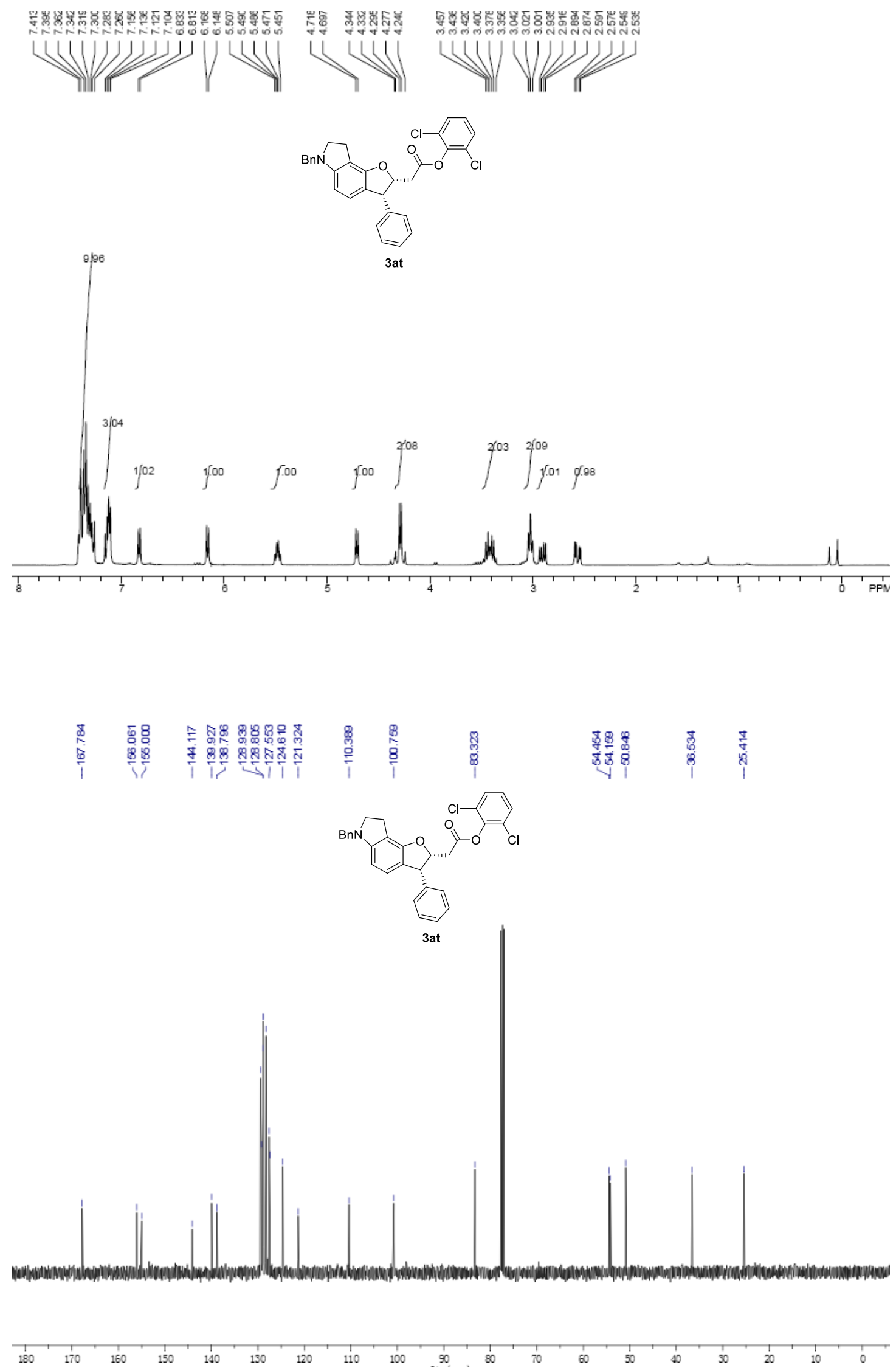


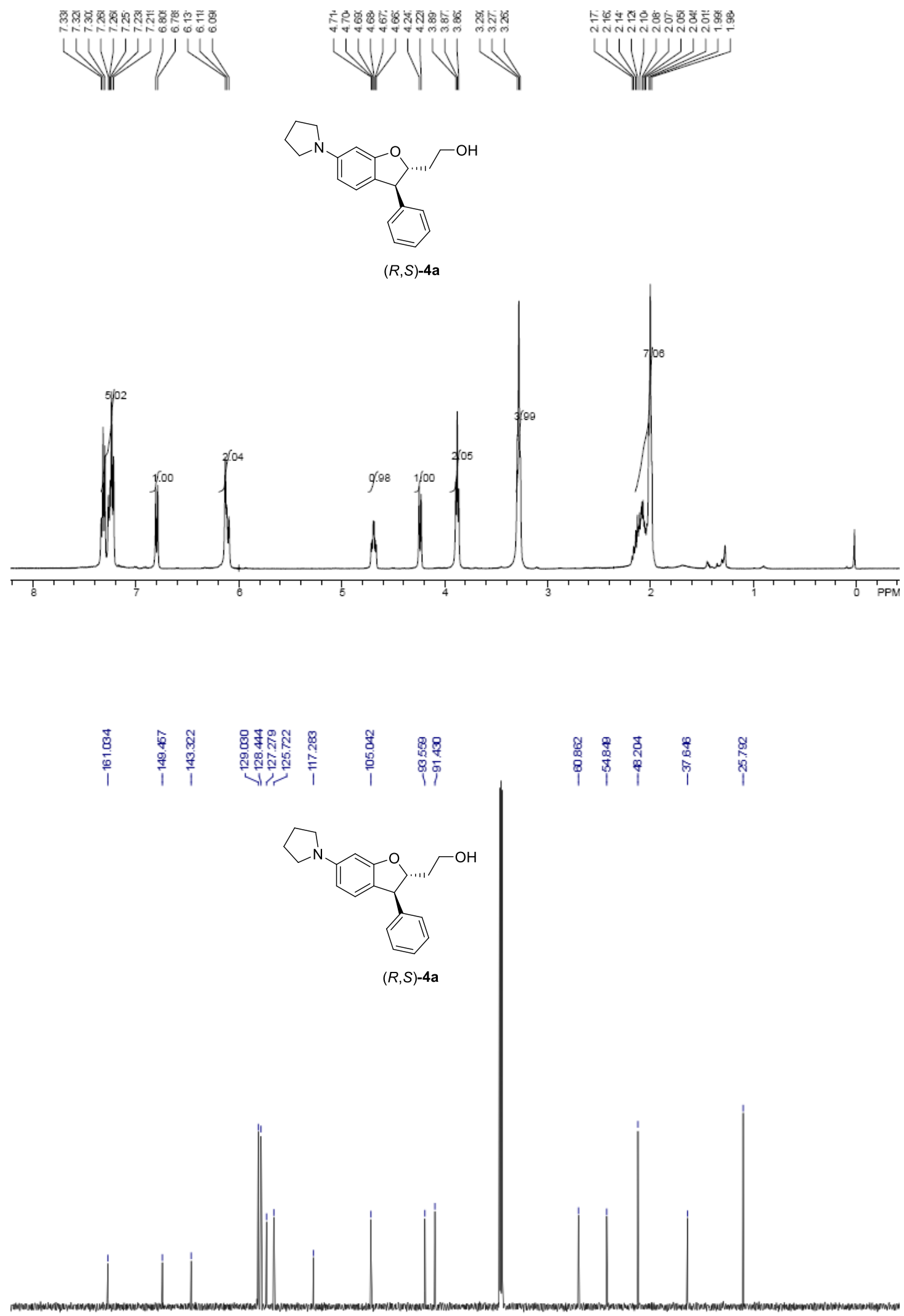

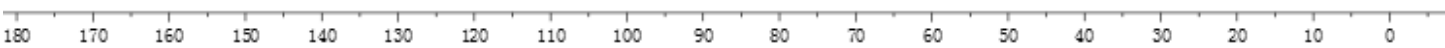




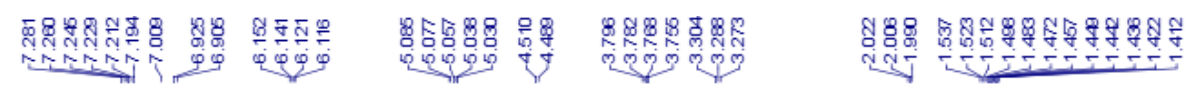
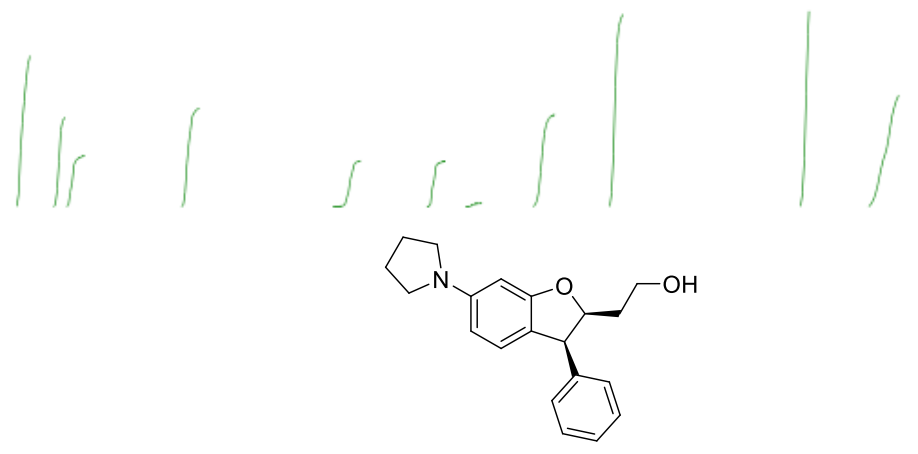

$(S, S)-4 a$

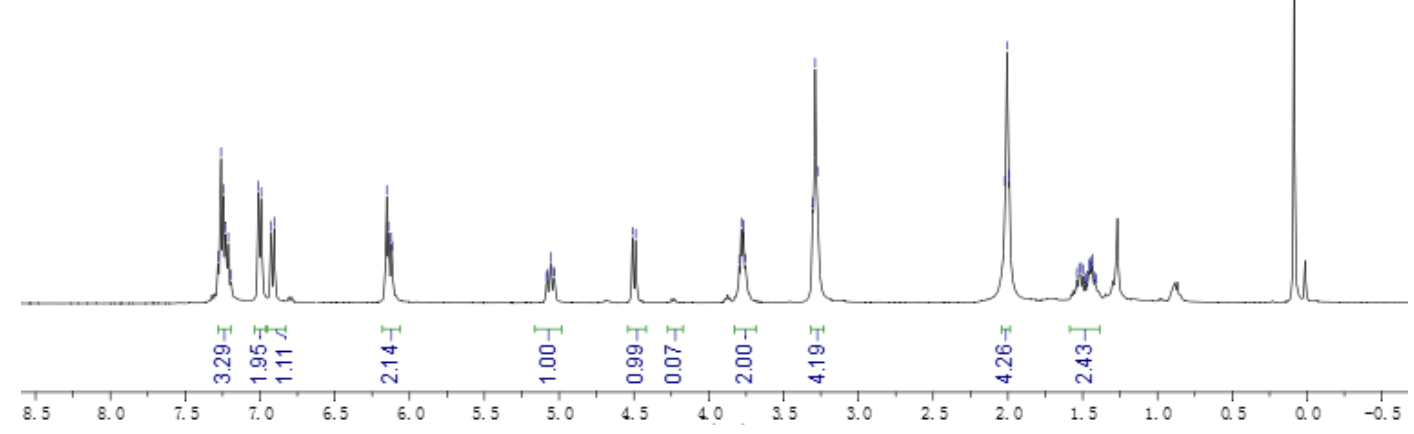

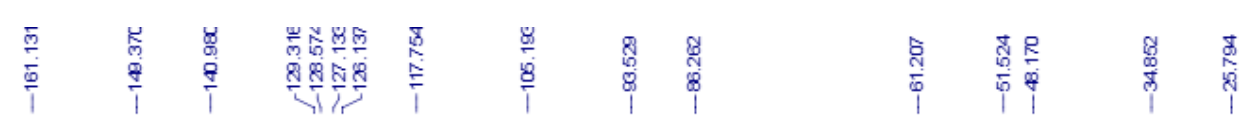

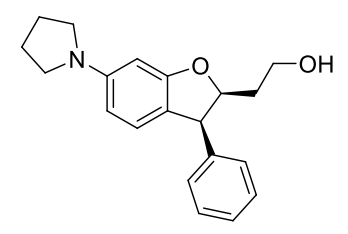

$(S, S)-\mathbf{4 a}$

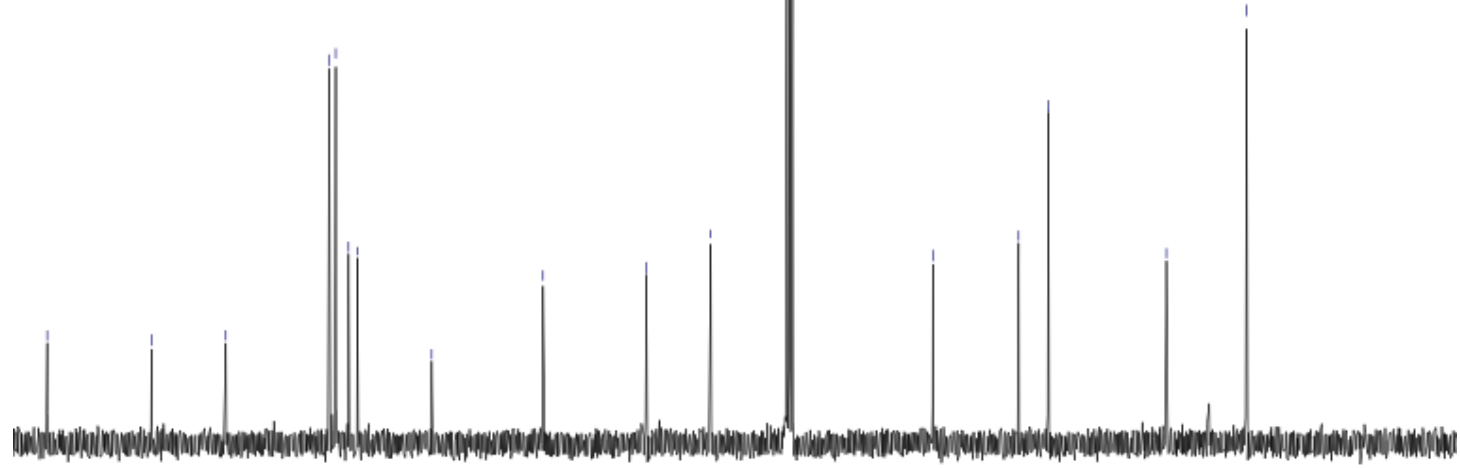

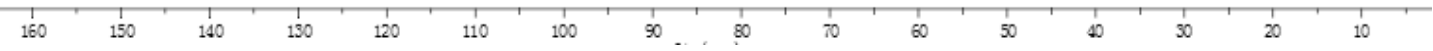




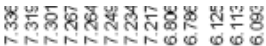

LU)

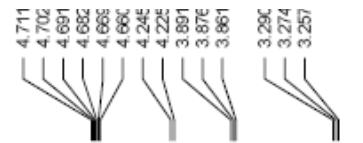

$(S, R)-4 a$

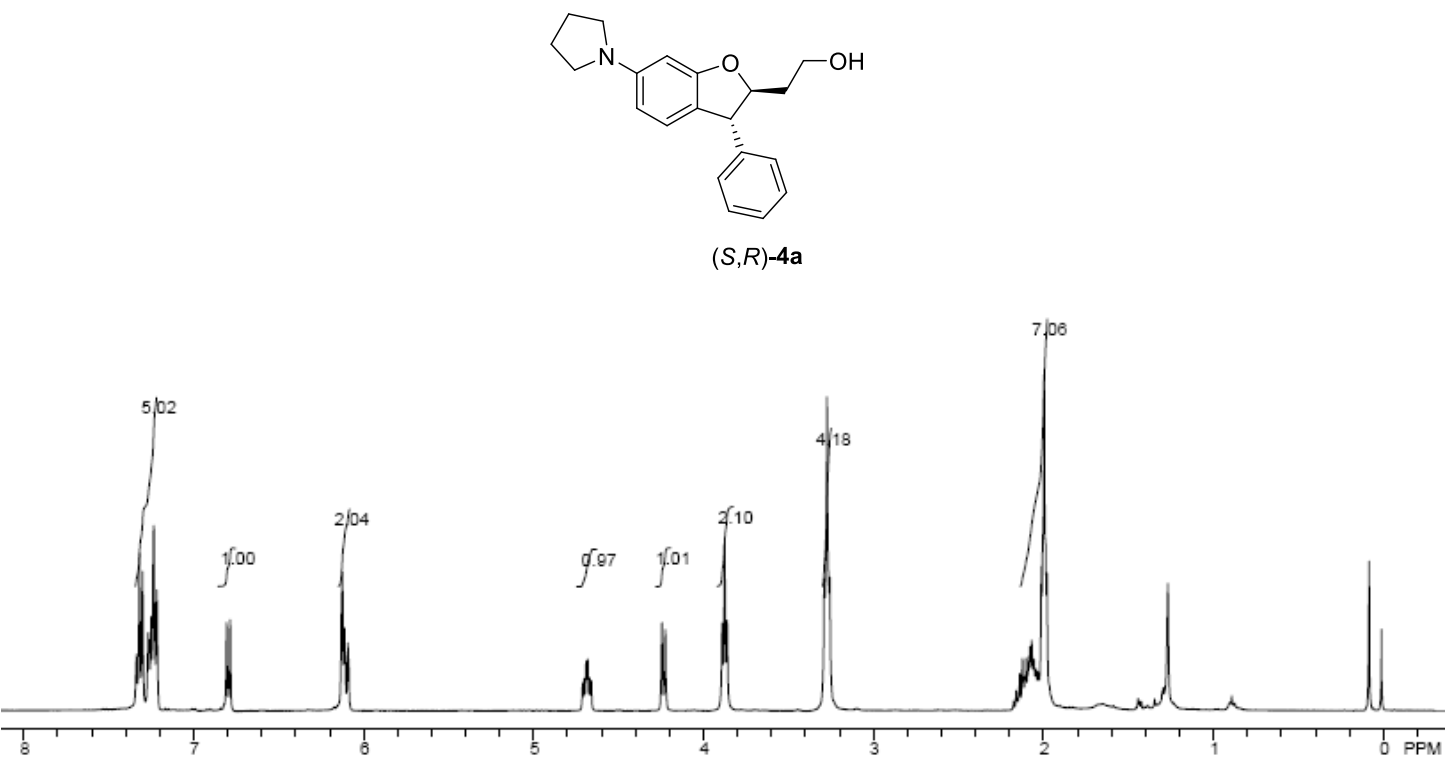

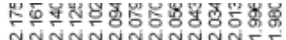

WUUHUDH

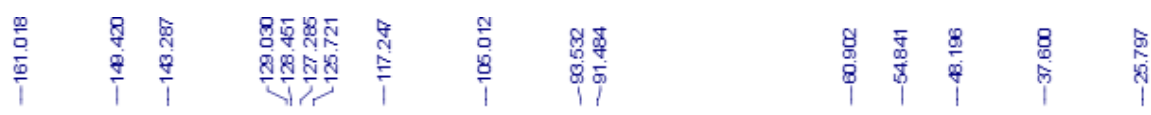

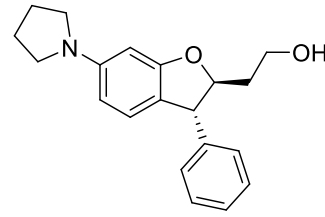

$(S, R)-\mathbf{4 a}$

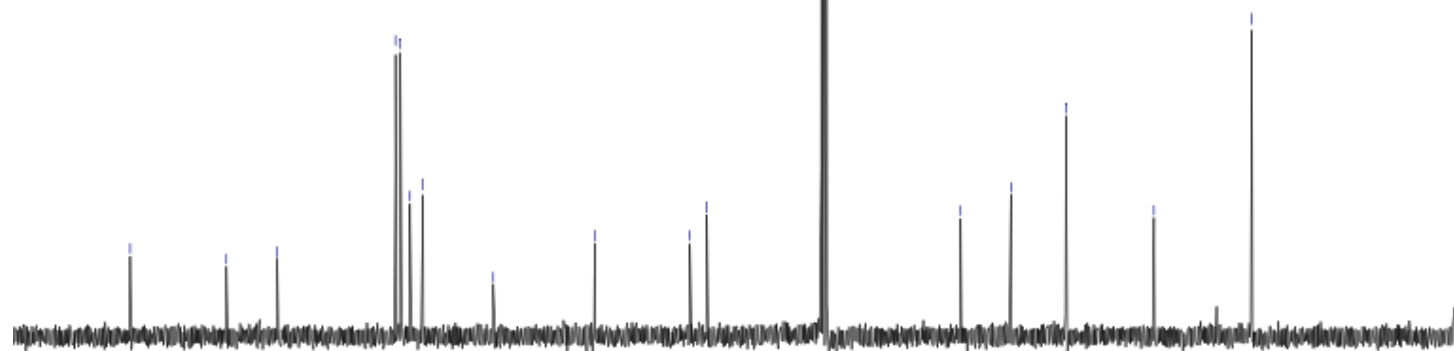

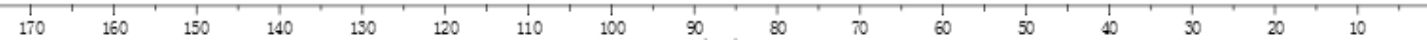




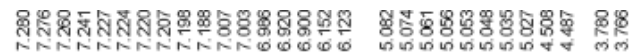
(U)

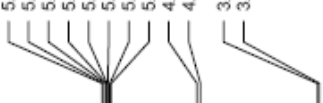<smiles>OCCC1Oc2cc(N3CCCC3)ccc2C1c1ccccc1</smiles>

$(R, R)-\mathbf{4 a}$

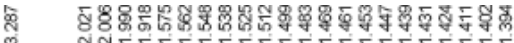

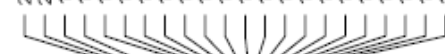

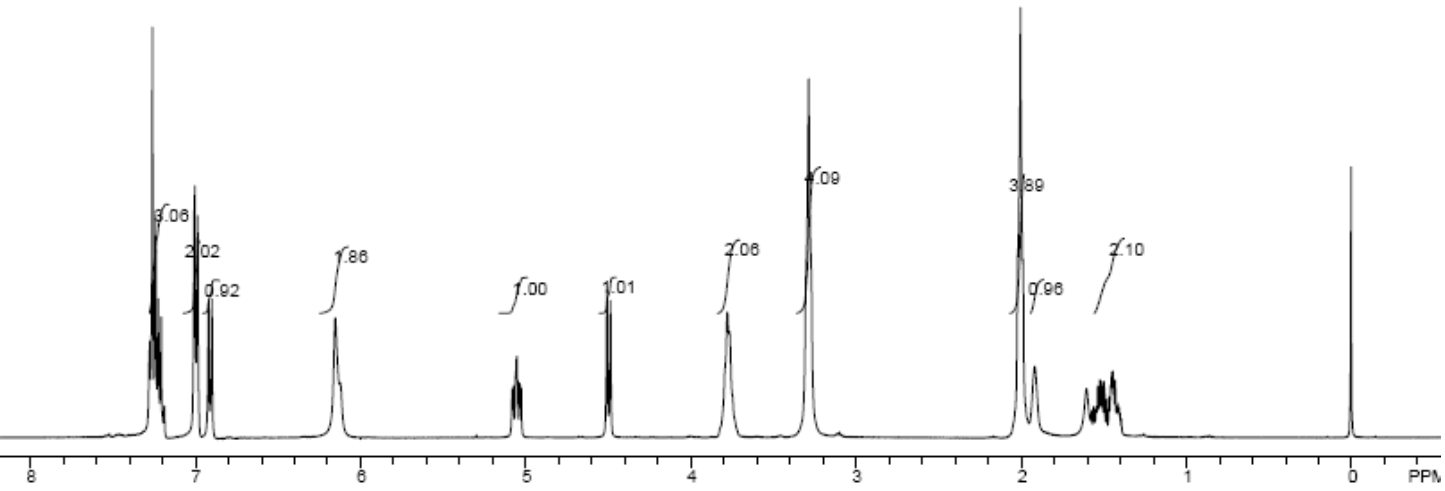

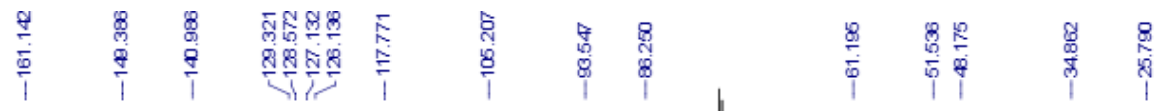<smiles>CCC1Oc2cc(N3CCCC3)ccc2C1c1ccccc1</smiles>

$(R, R)-4 \mathrm{a}$

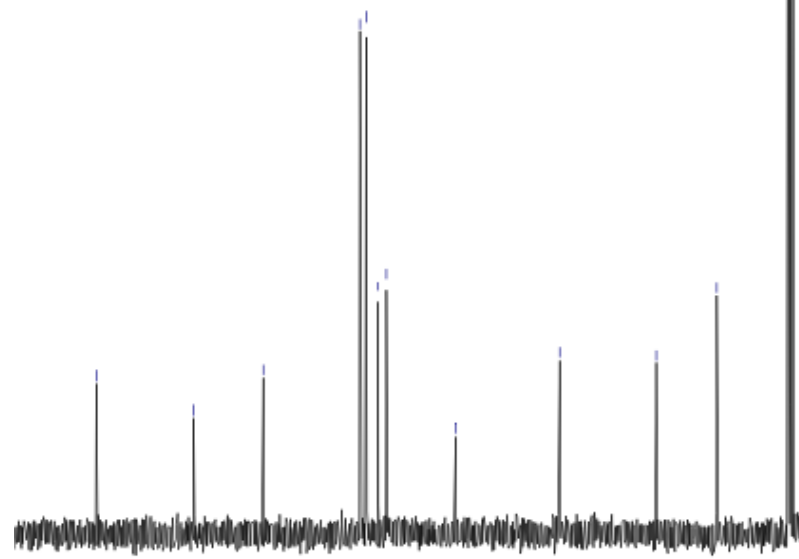

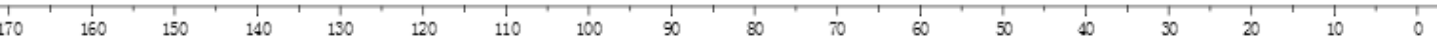



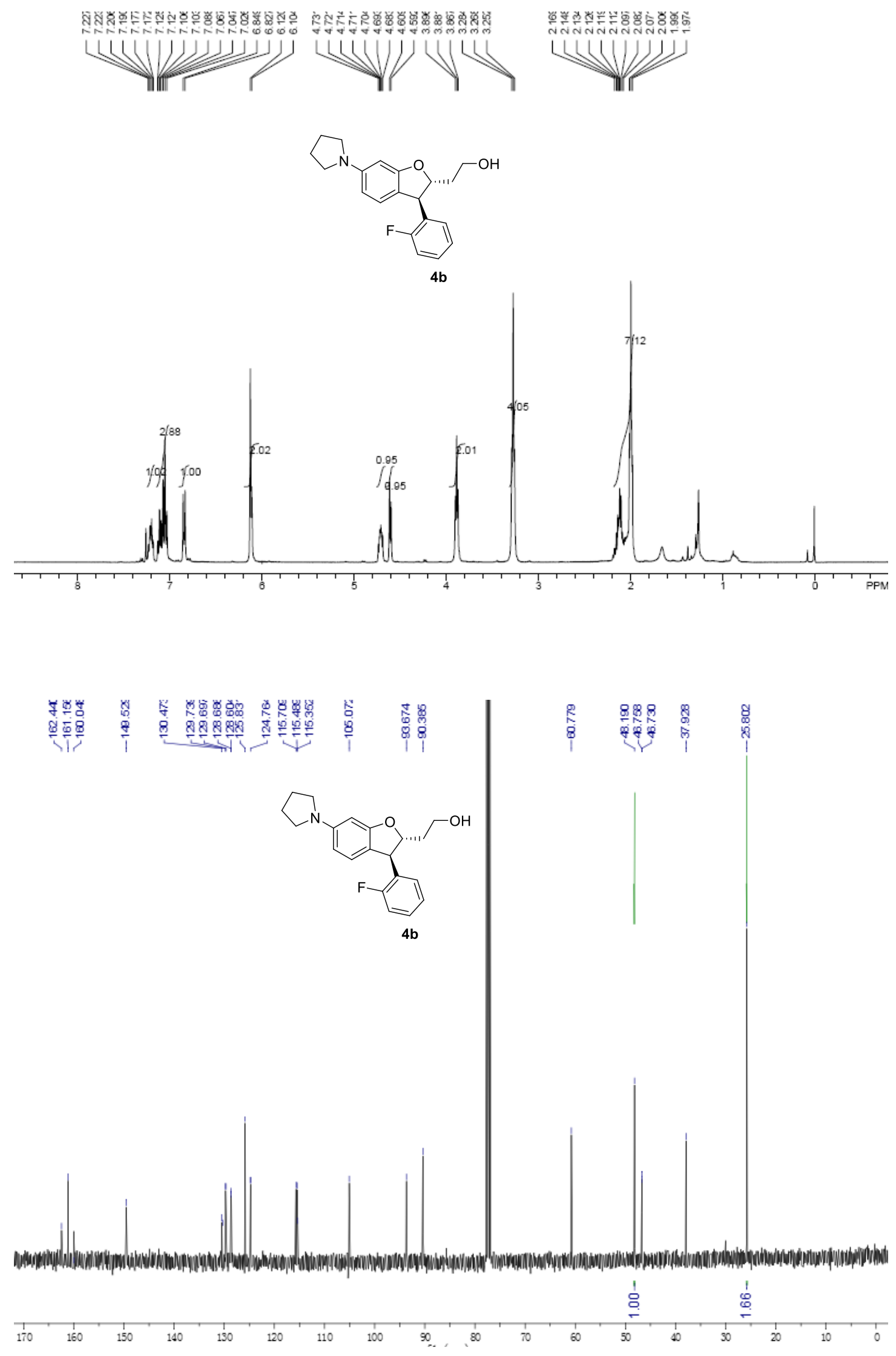

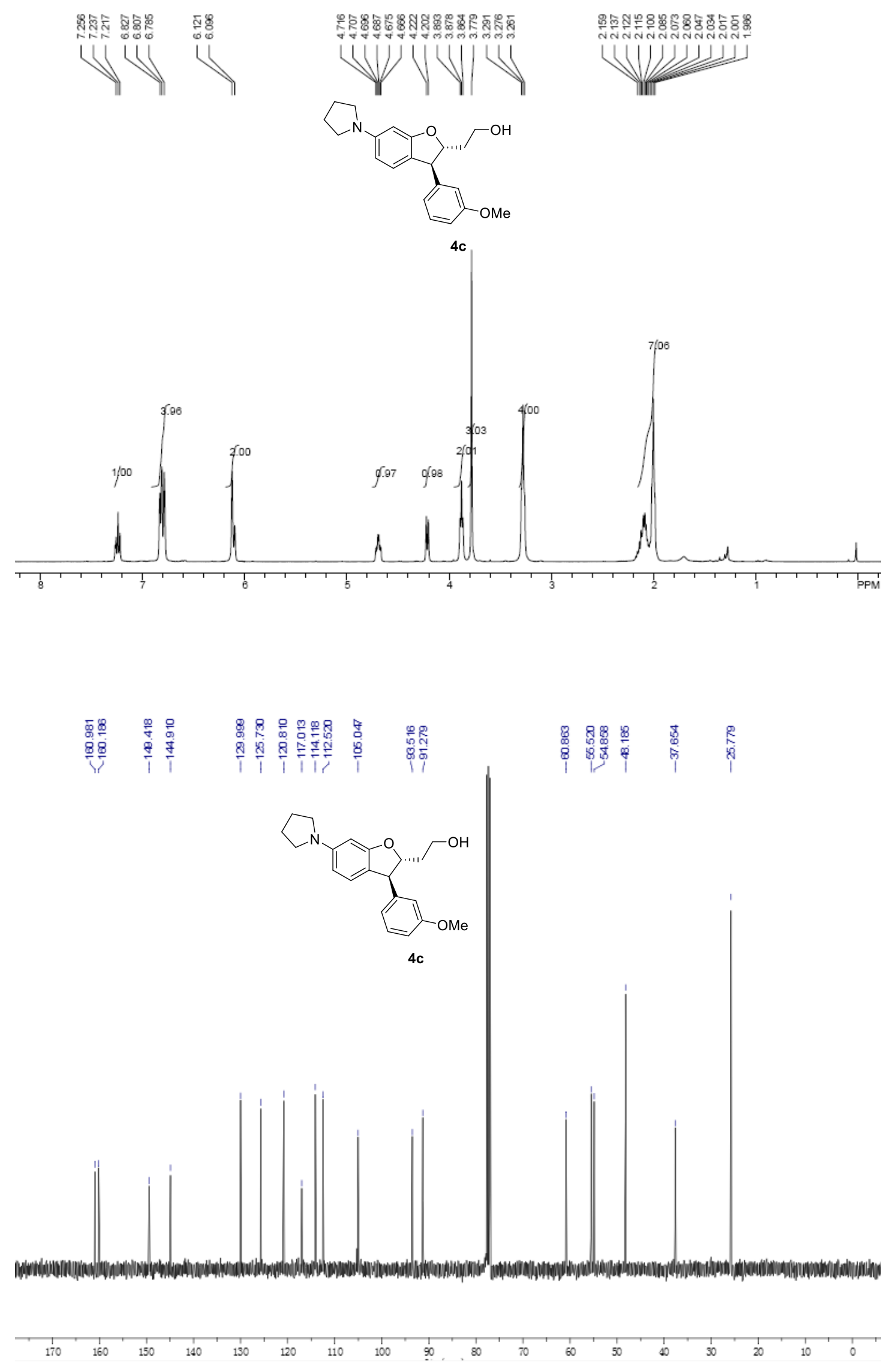

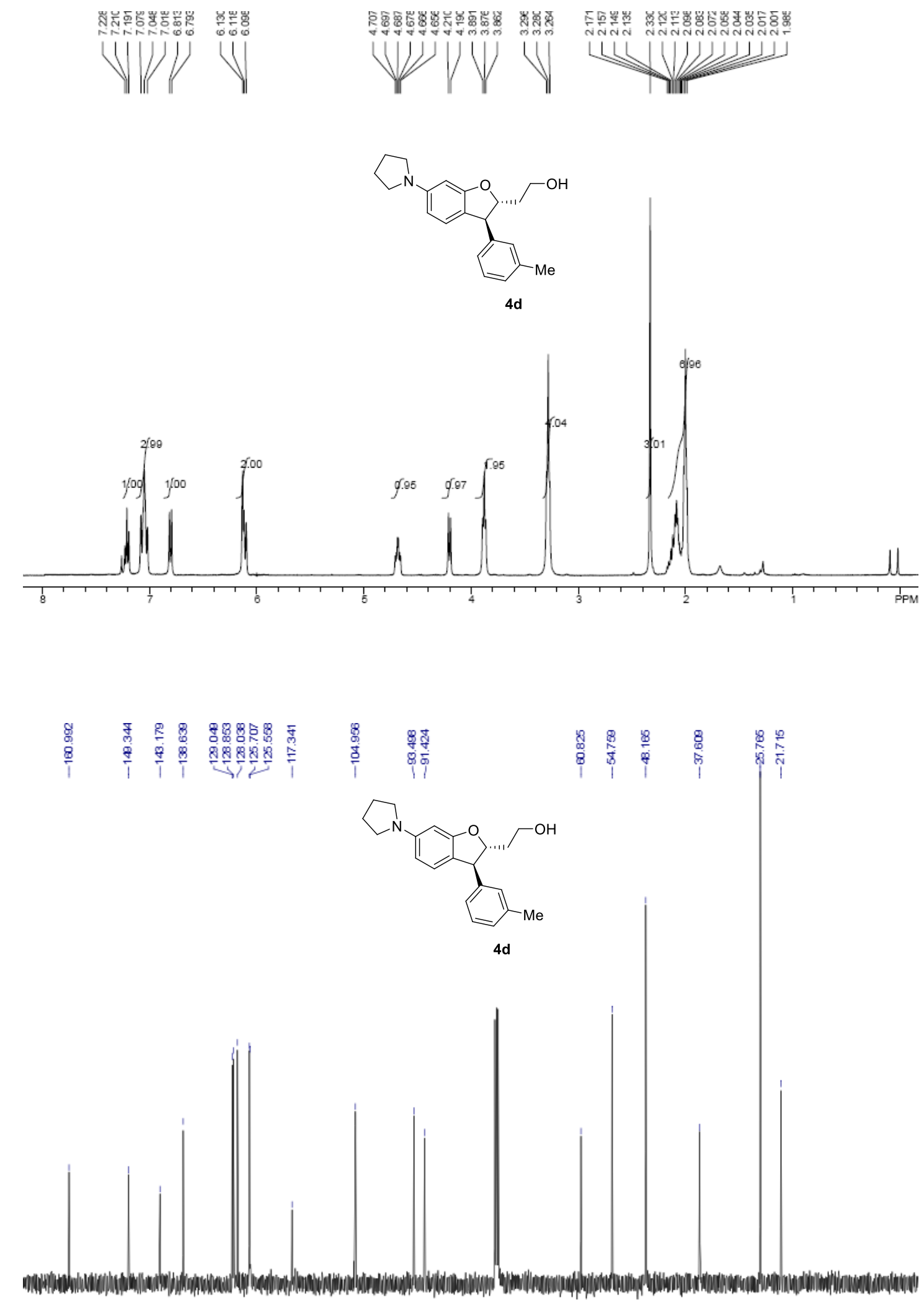

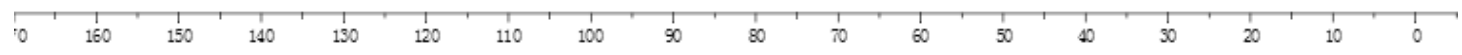




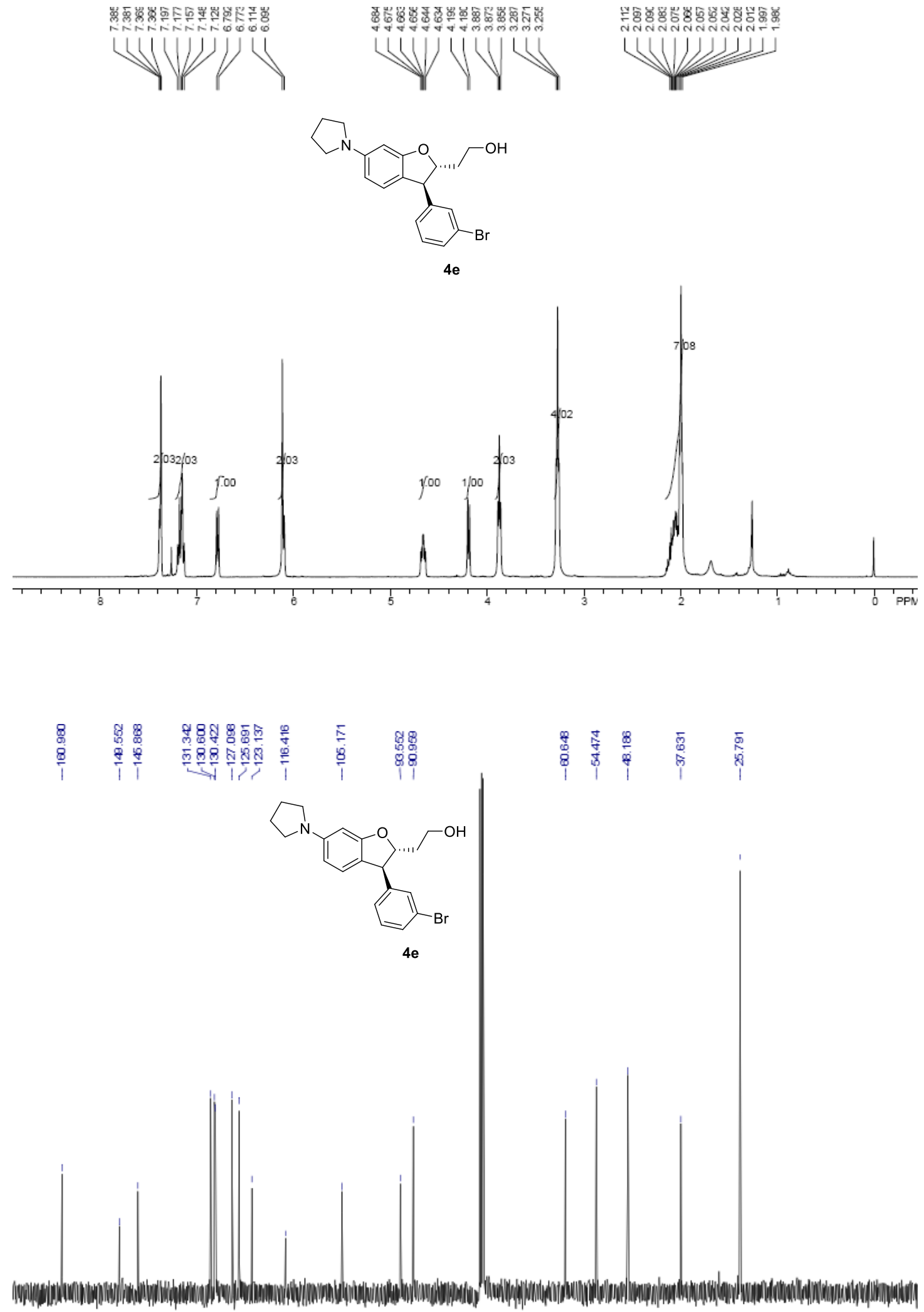

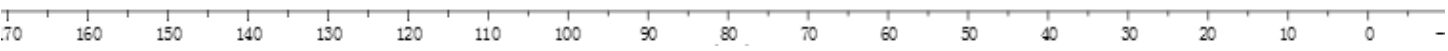



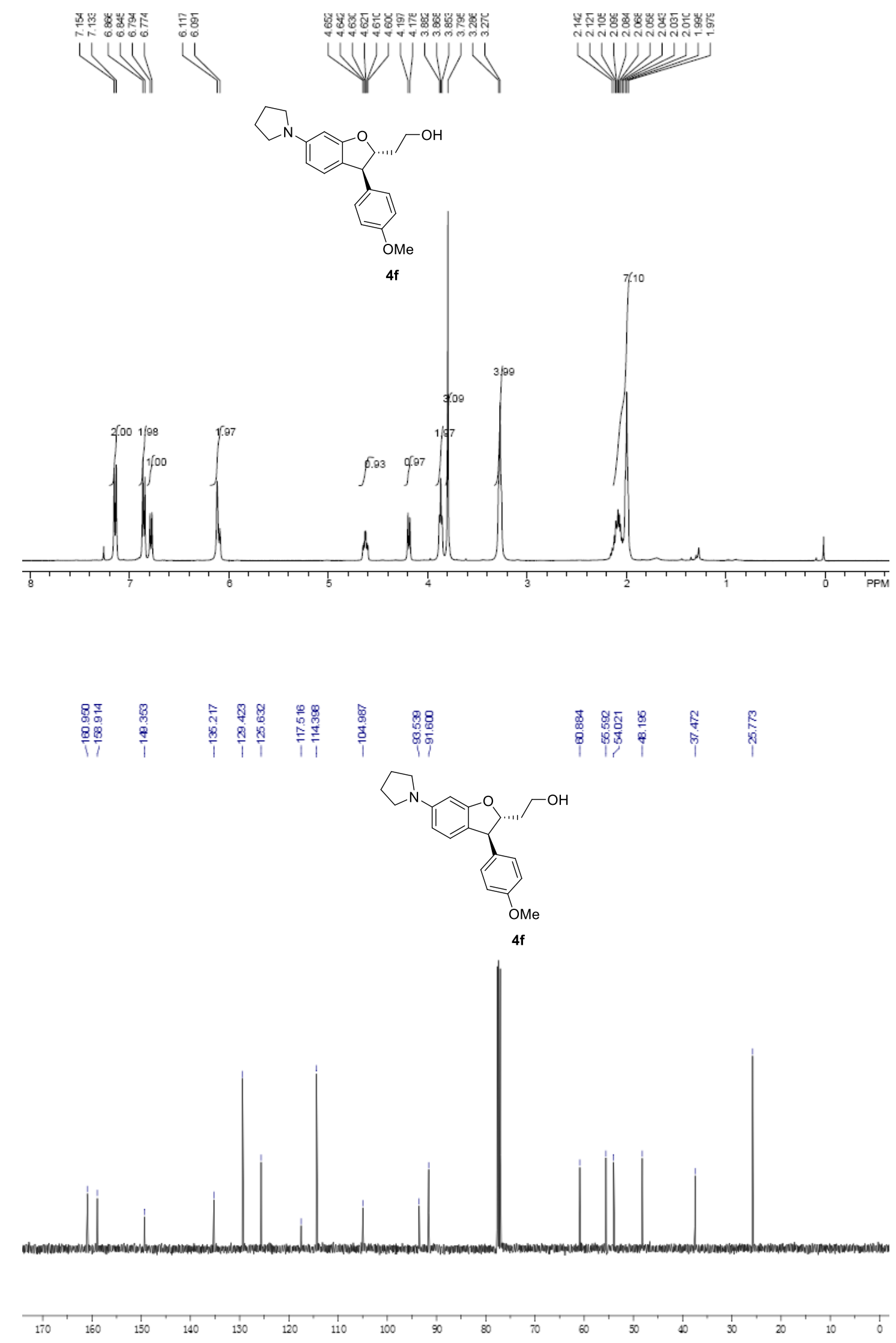

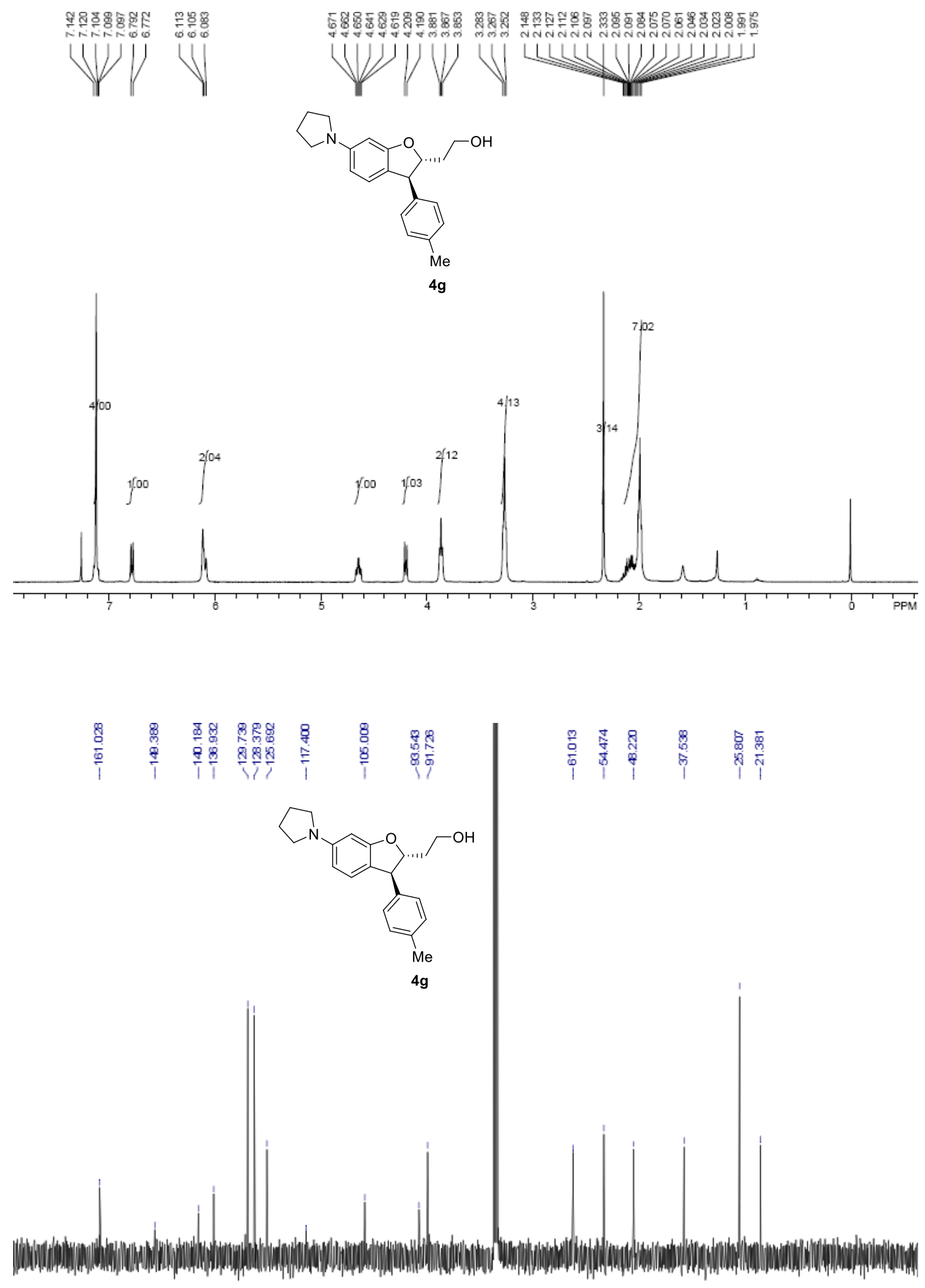

\begin{tabular}{llllllllllllllllllllllll}
\hline & 170 & 160 & 150 & 140 & 130 & 120 & 110 & 100 & 90 & 80 & 70 & 60 & 50 & 40 & 30 & 20 & 10 & 0 & -10
\end{tabular}



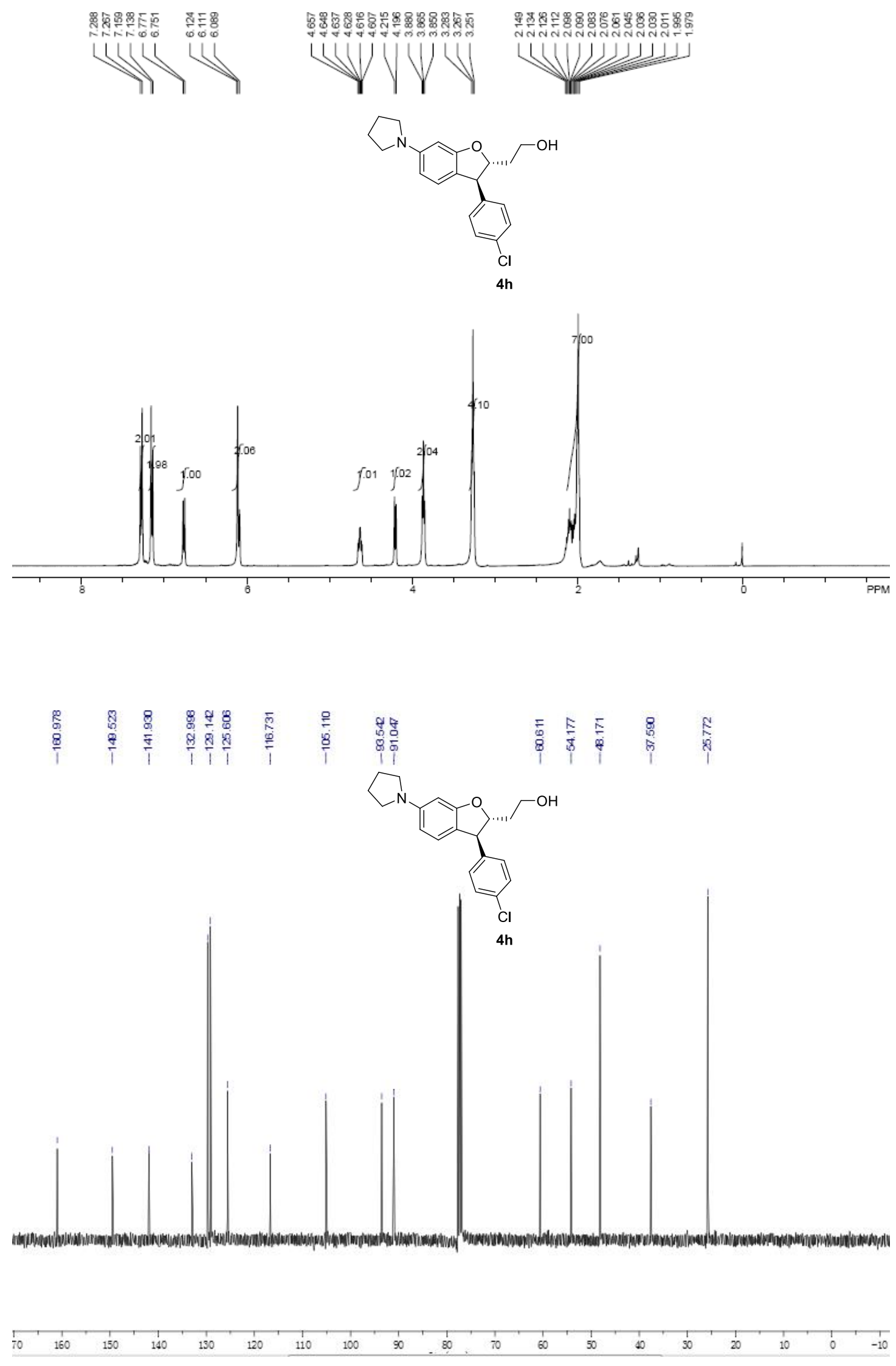

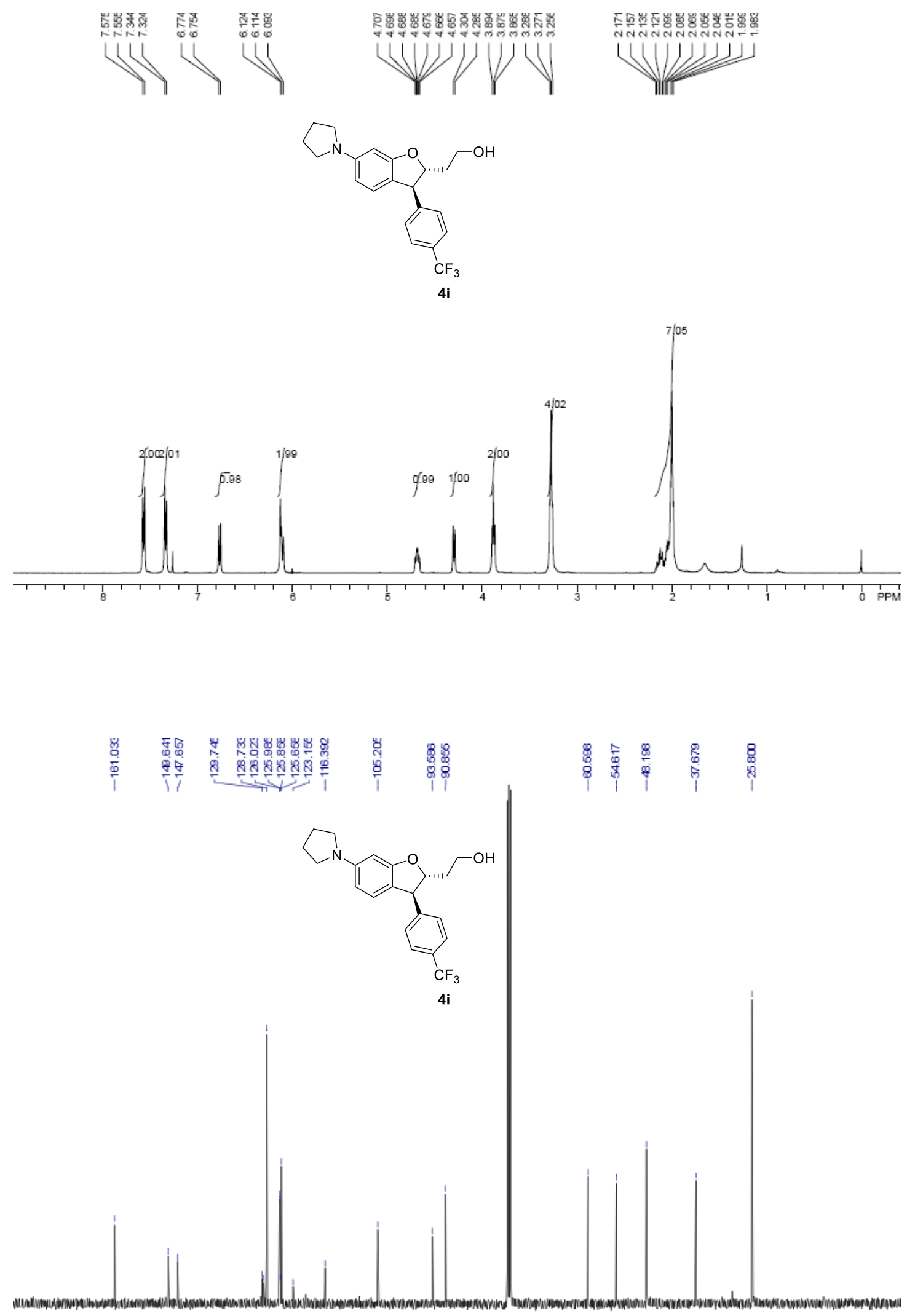


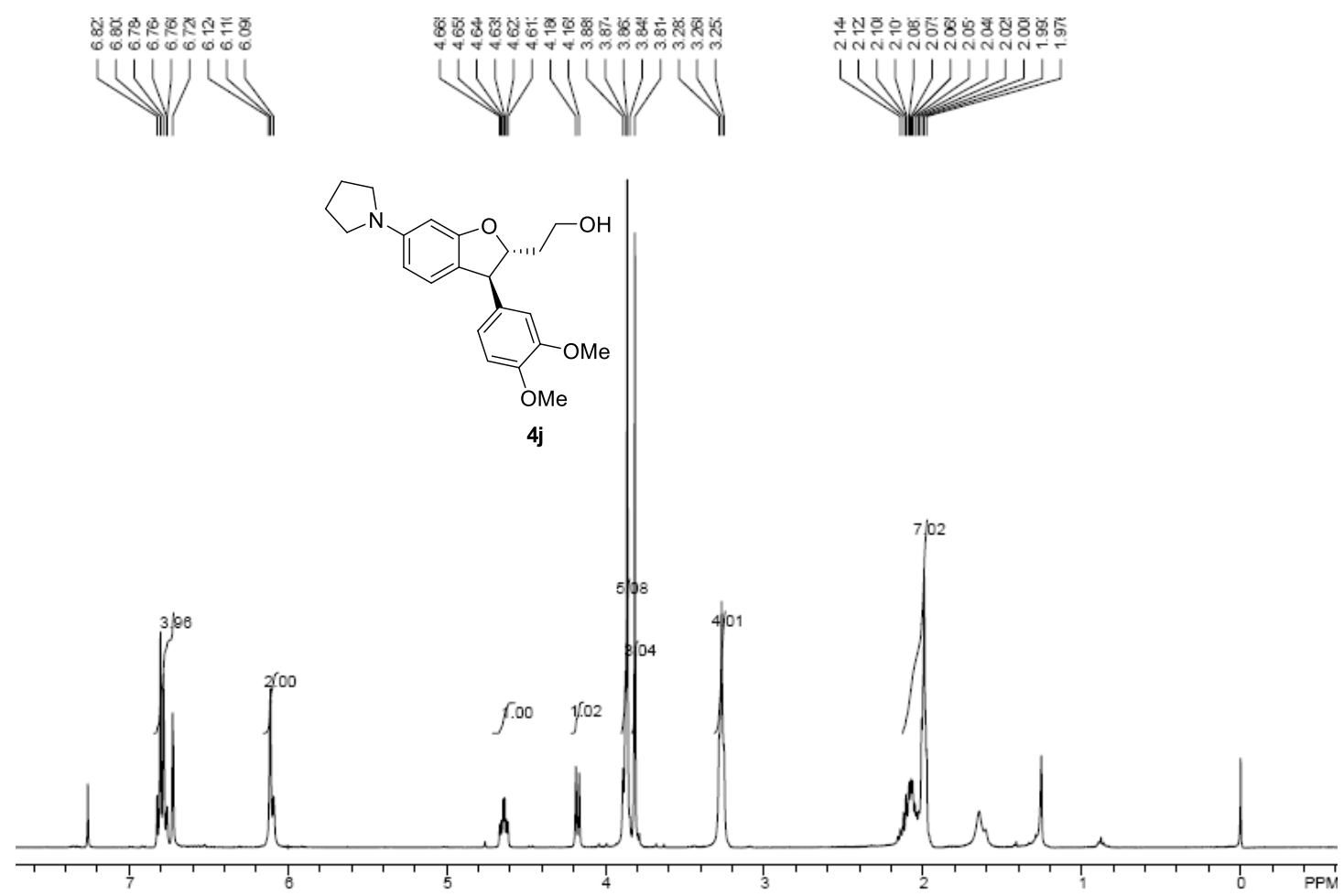

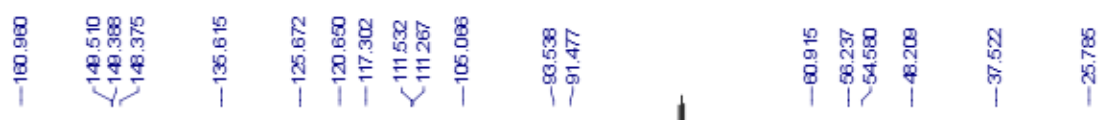
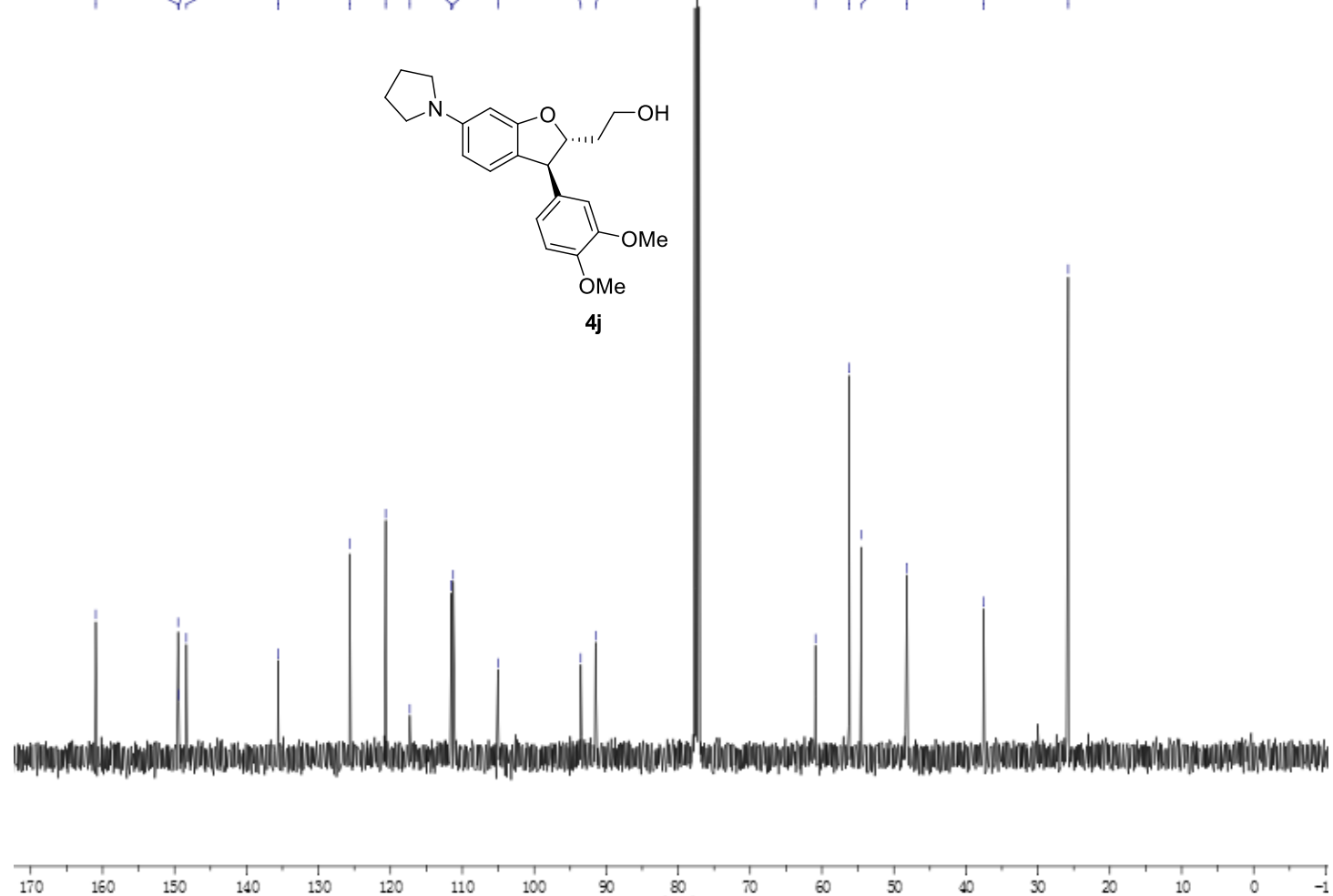


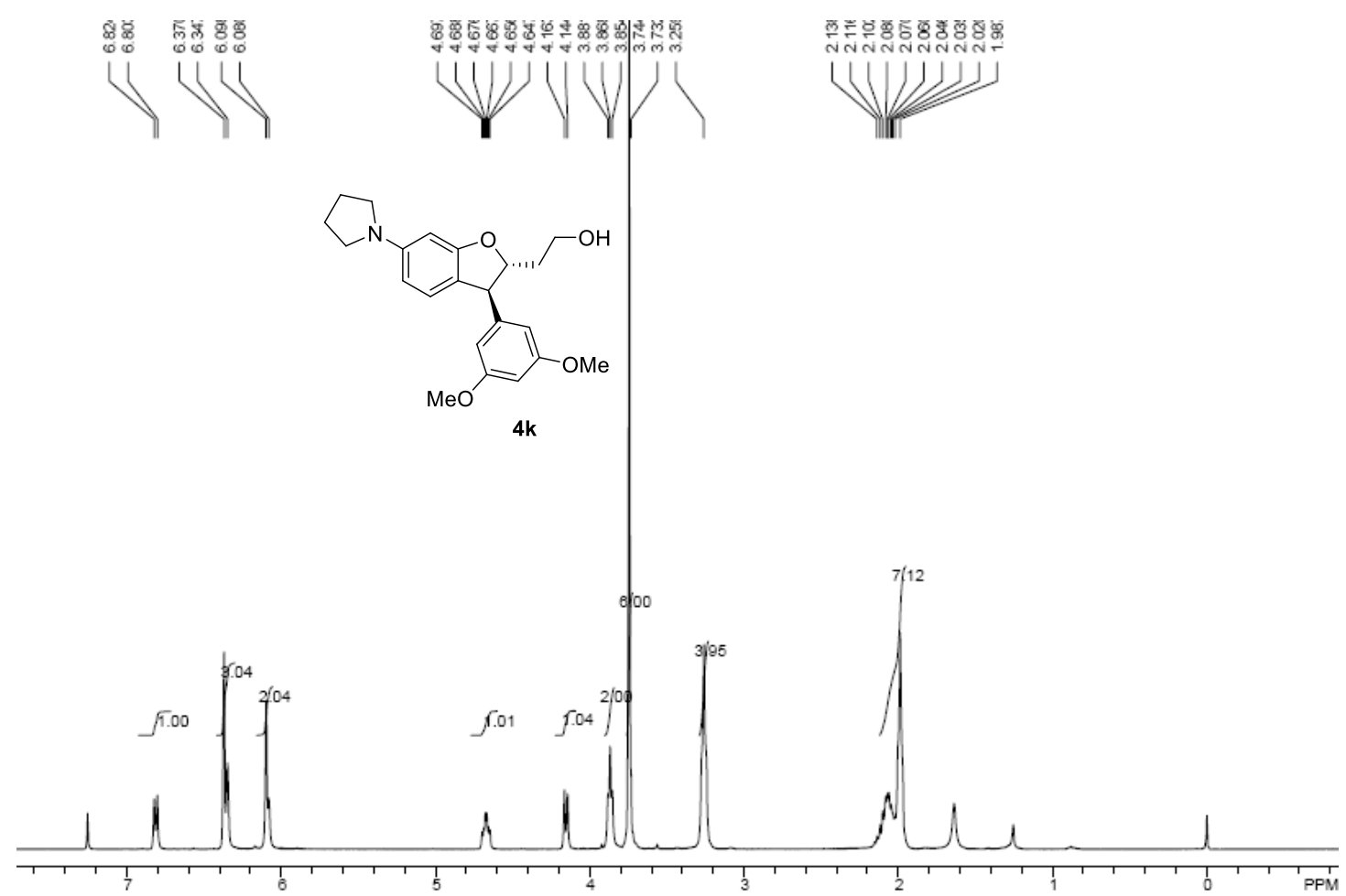

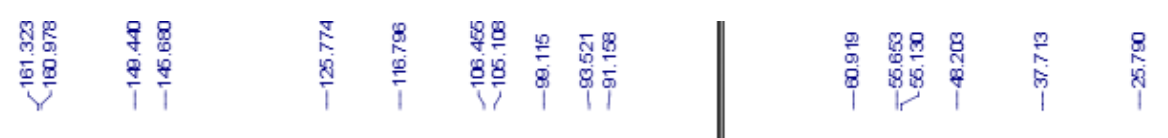

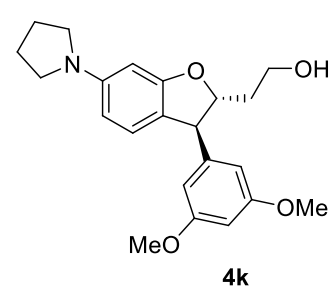

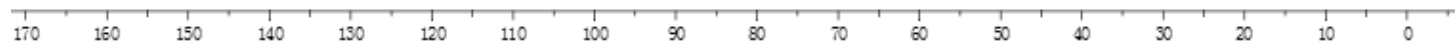



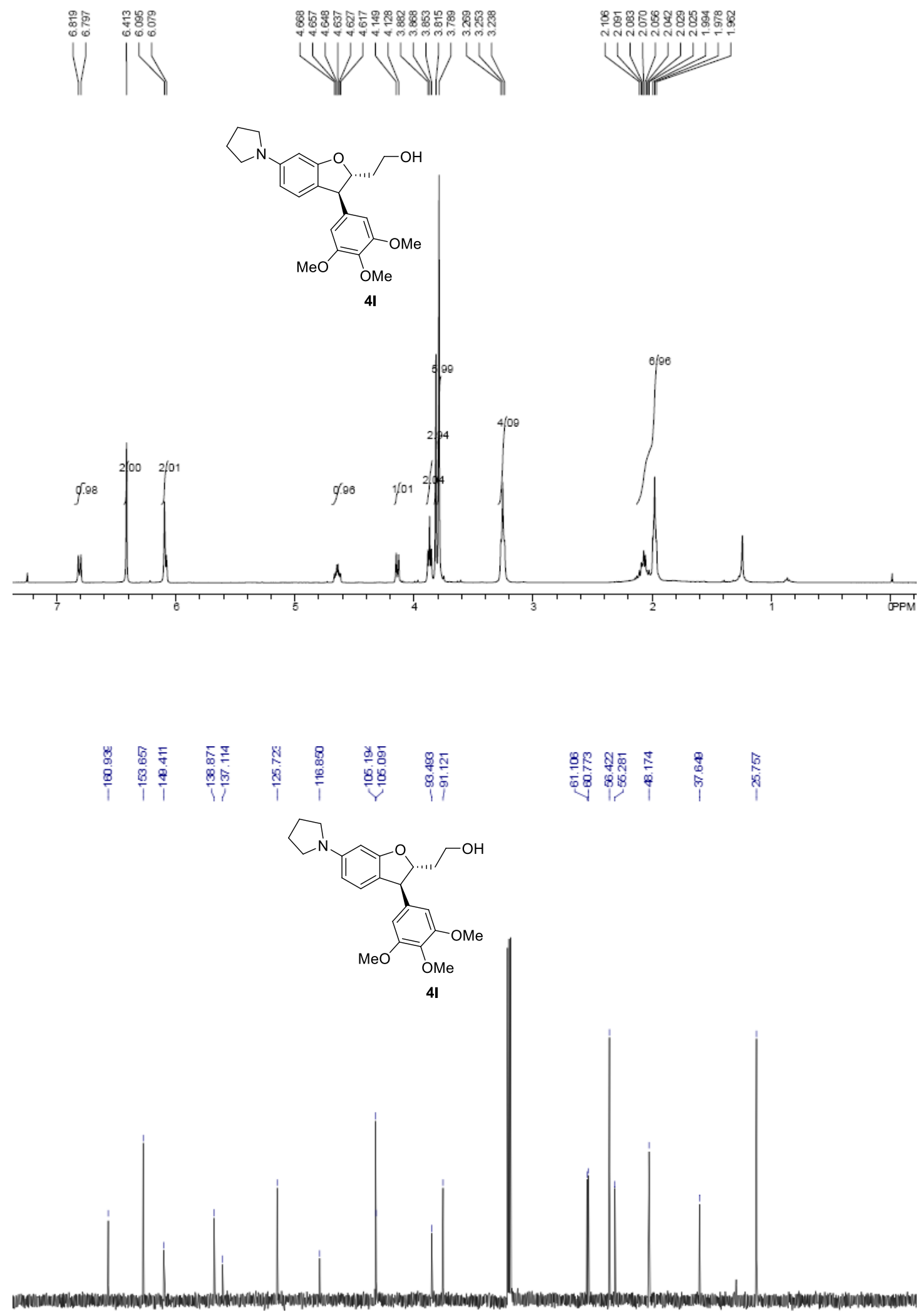

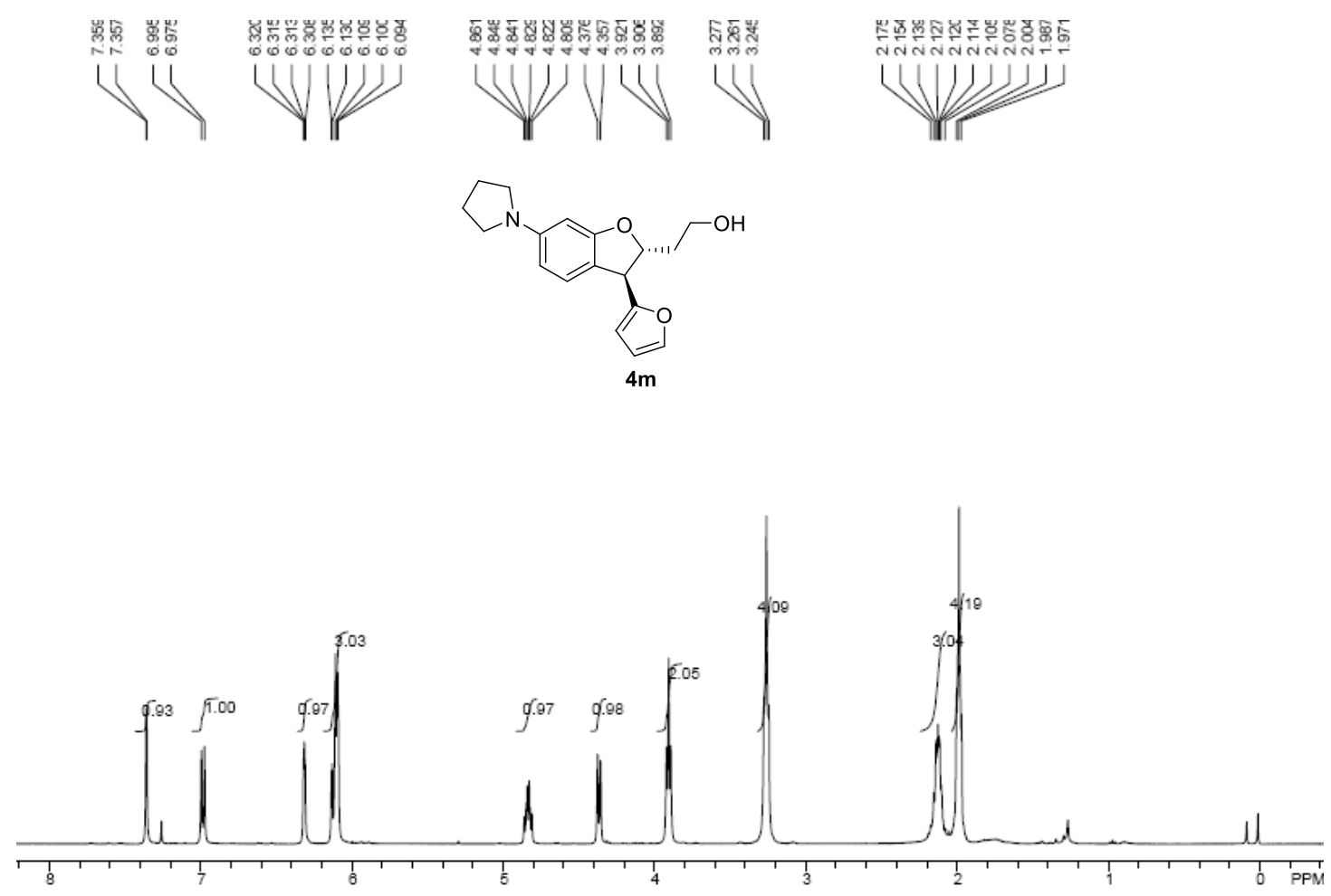

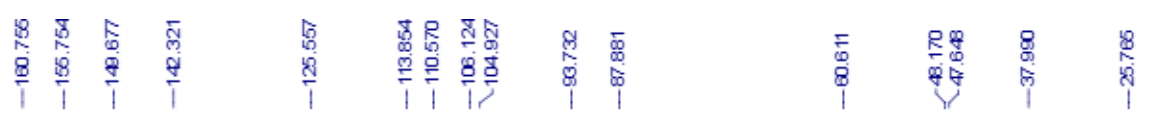

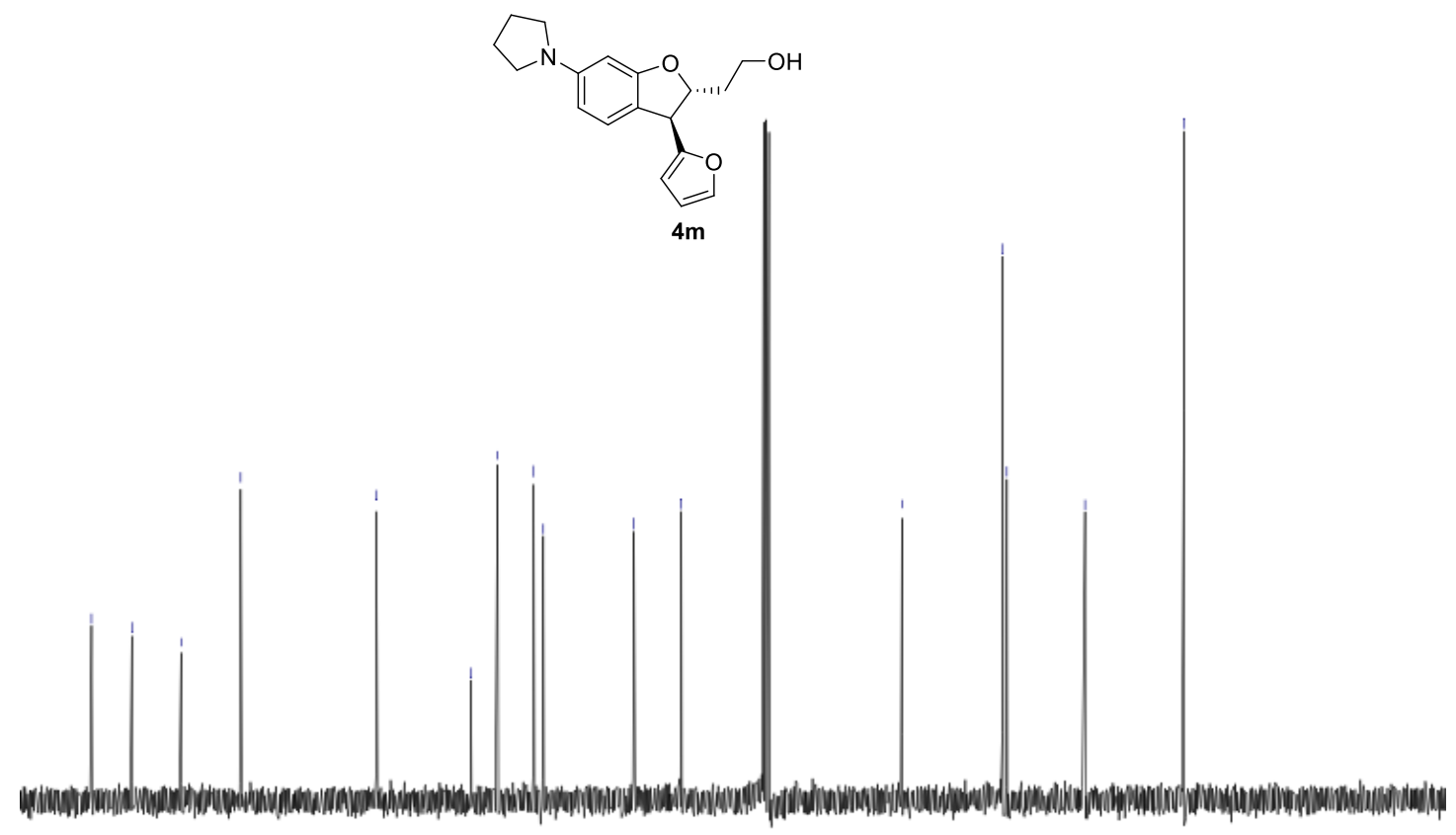

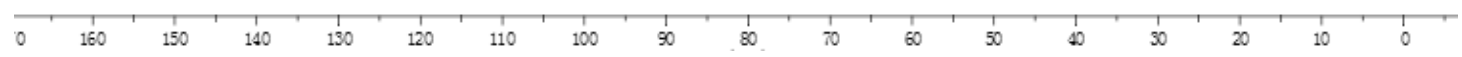




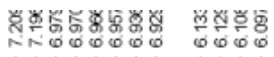

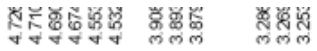

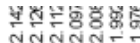

WW W

WV V V

W.

(n)

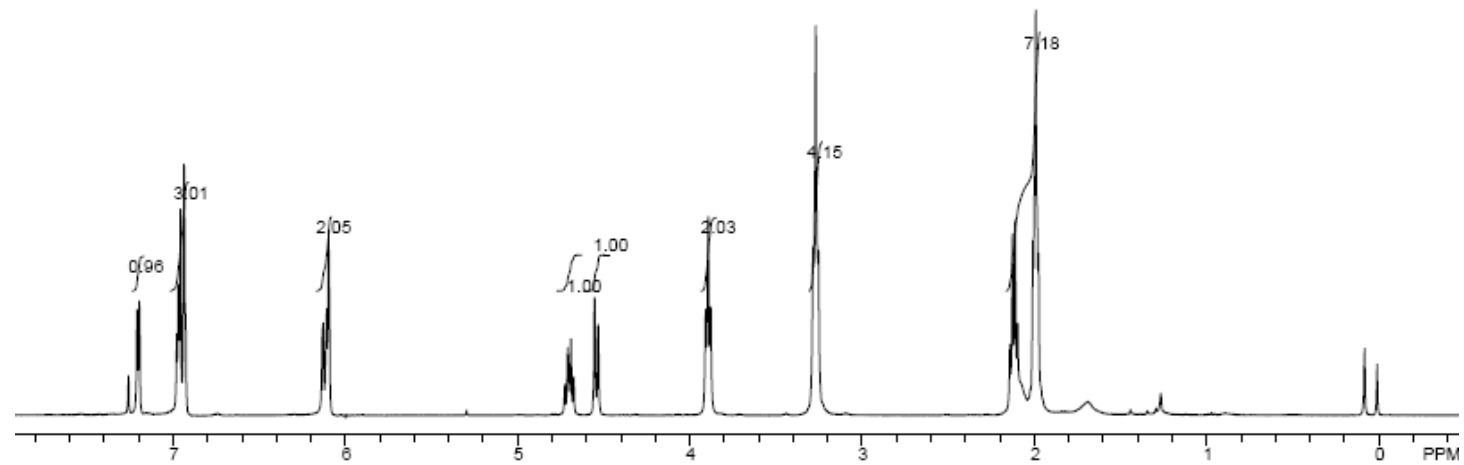

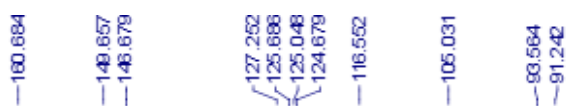

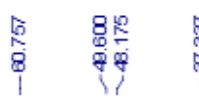
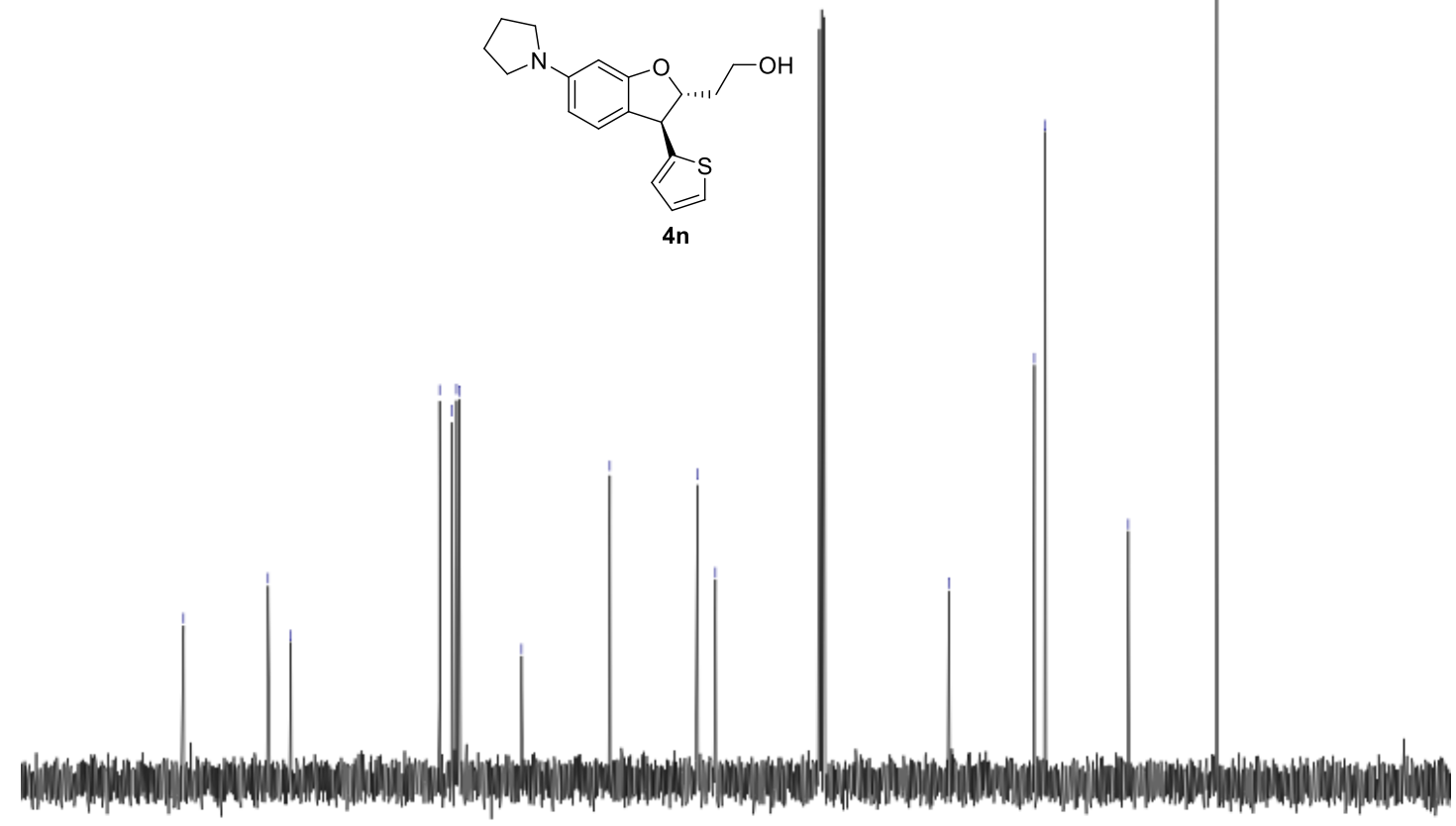

8
0
0

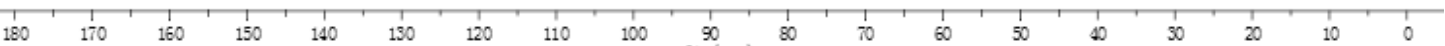



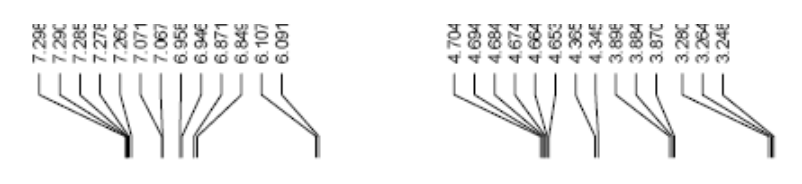

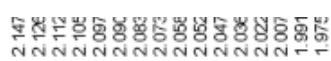

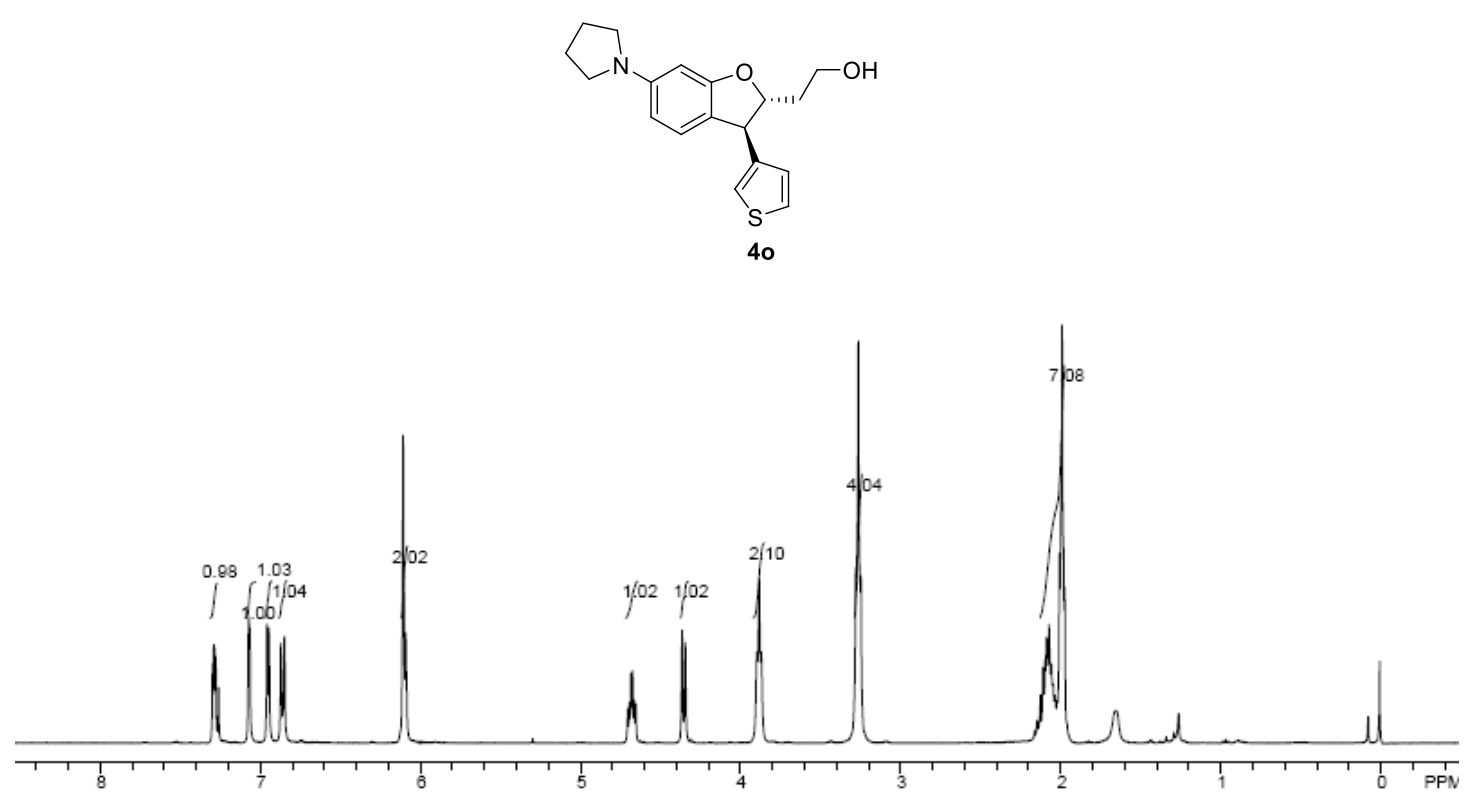

WW

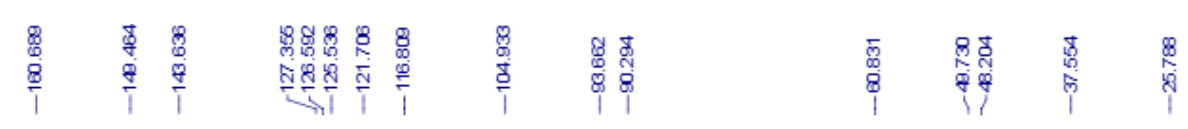
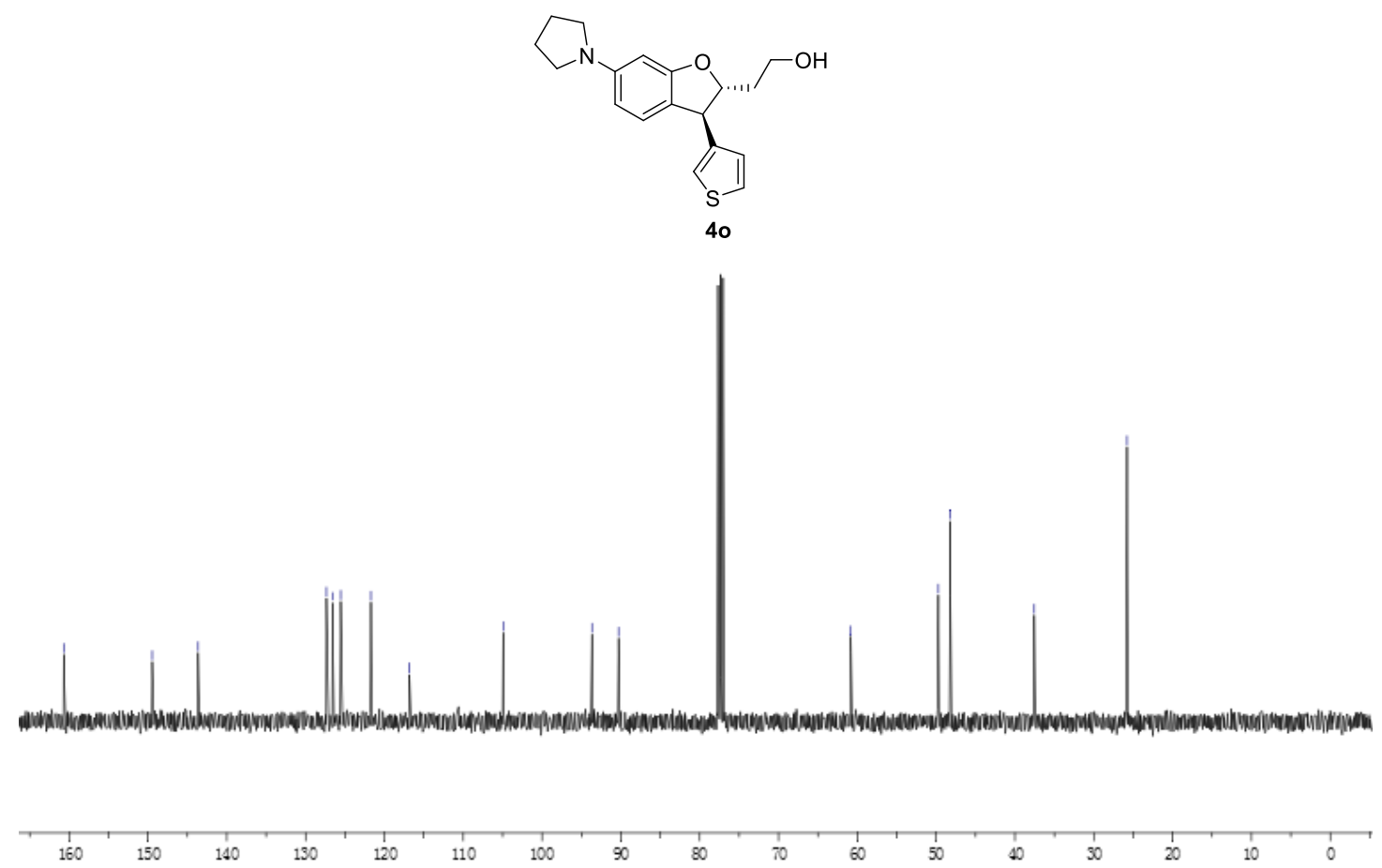

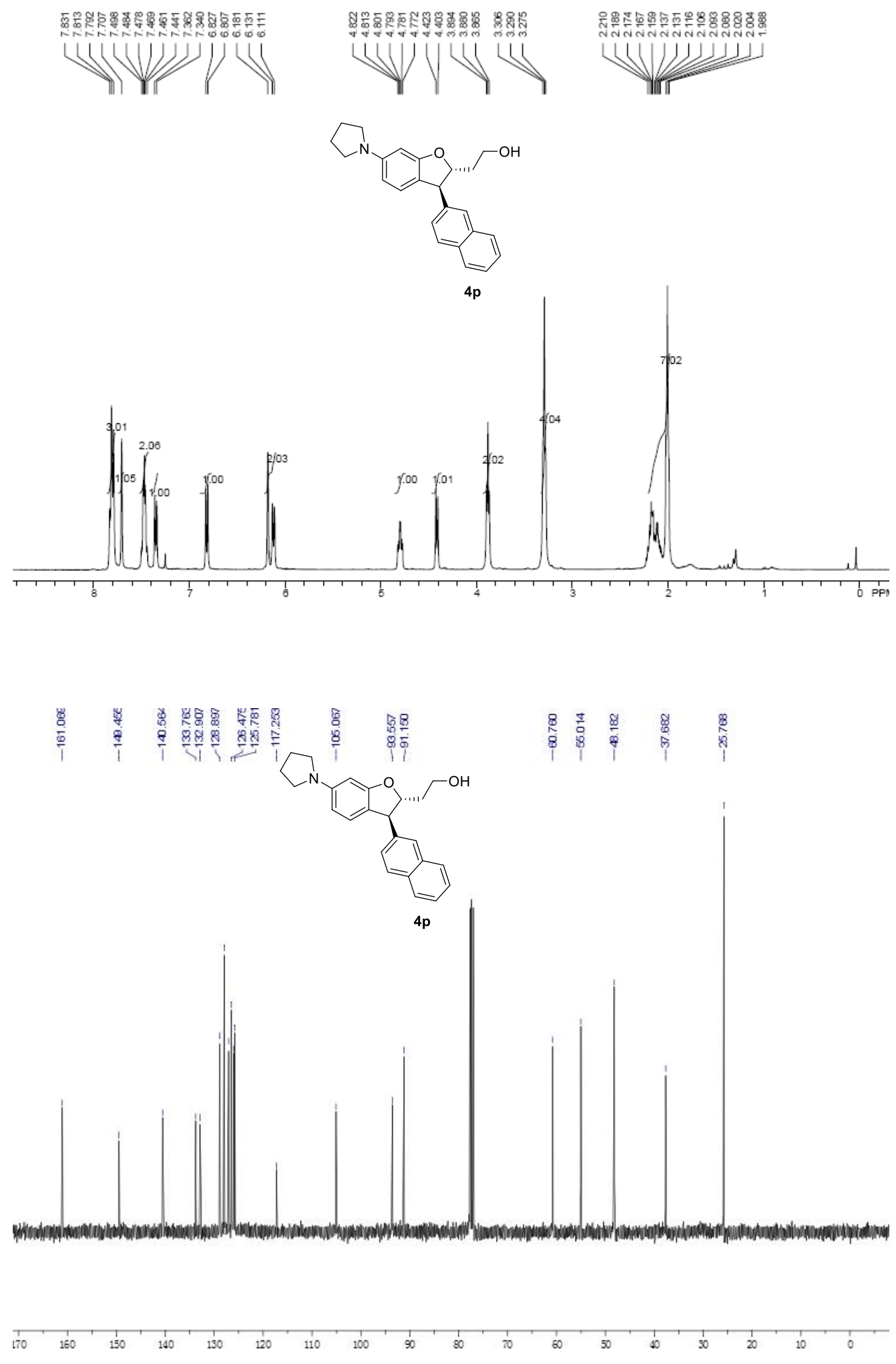

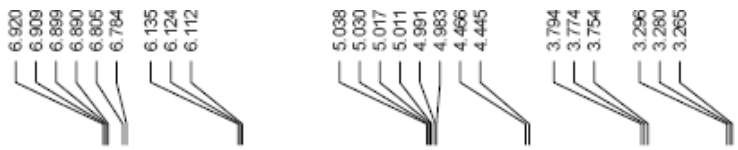

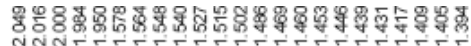

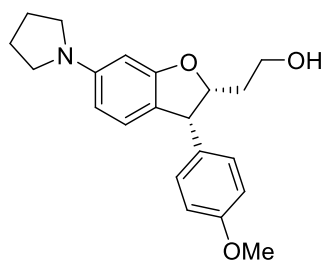

WWHWWJWdoded

$+1$

$4 \mathrm{q}$

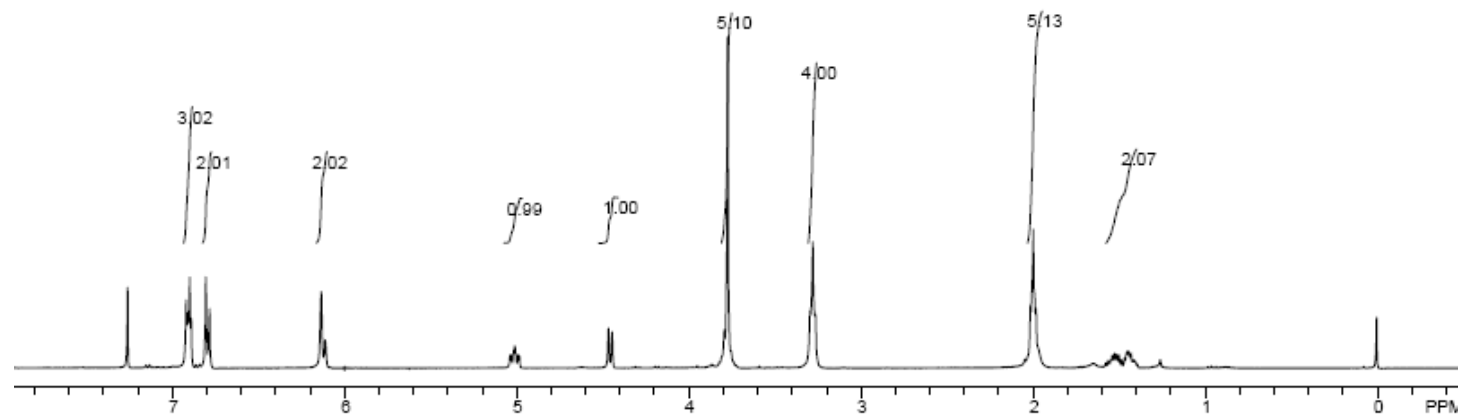

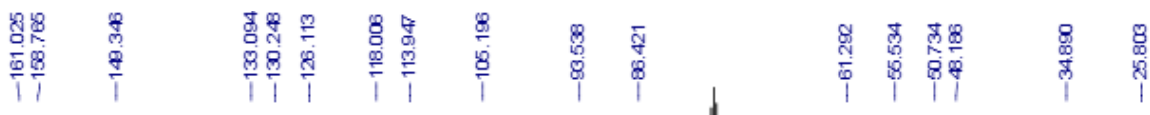<smiles>OCCC1Cc2ccc(N3CCCC3)cc2O1</smiles><smiles>COc1ccc(C)cc1</smiles>

$4 q$
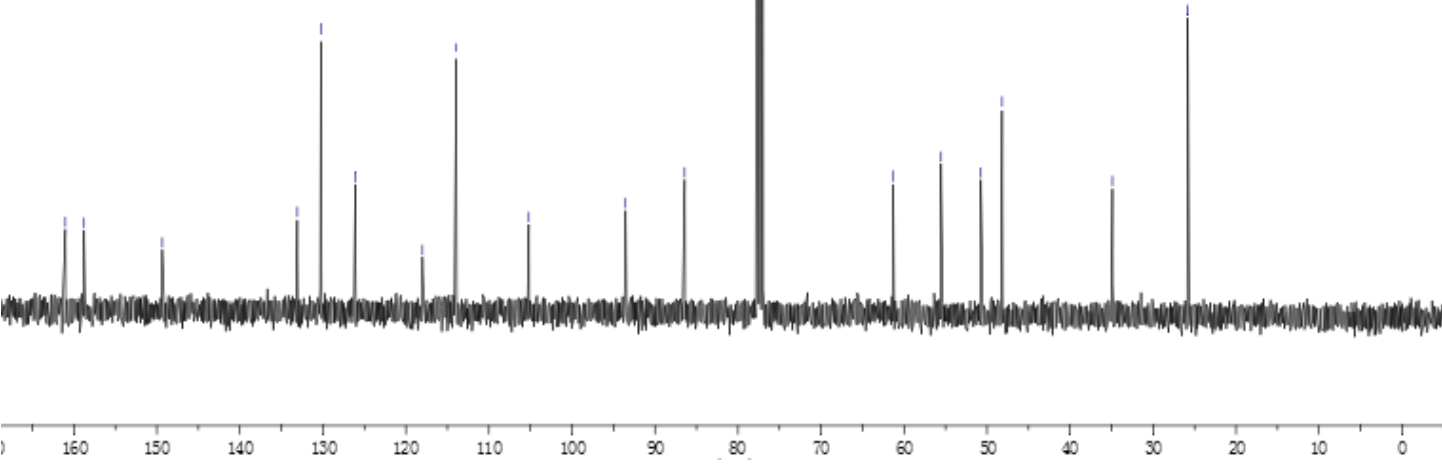

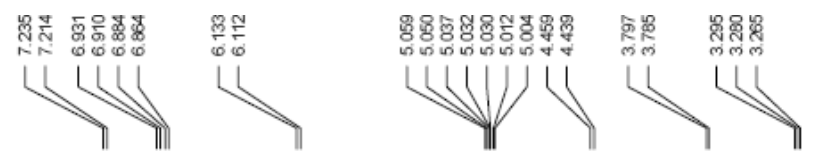

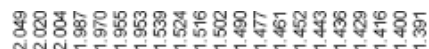

O

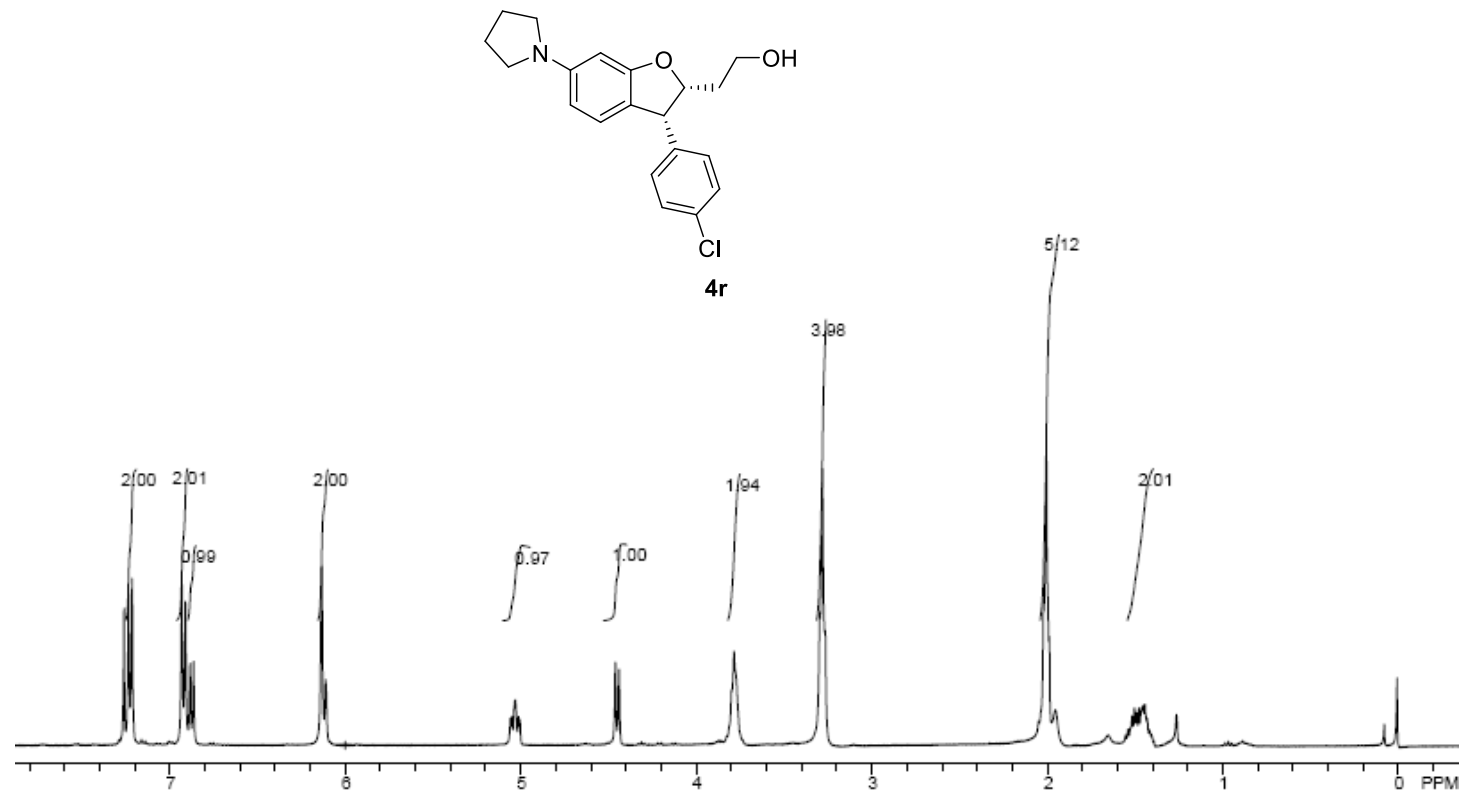

㢈

WWWWHJJIJU

W Wir

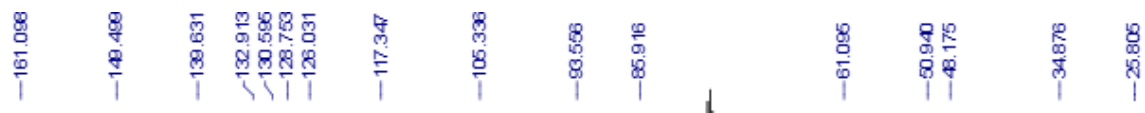

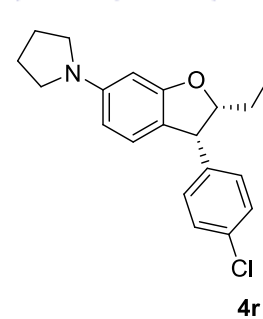

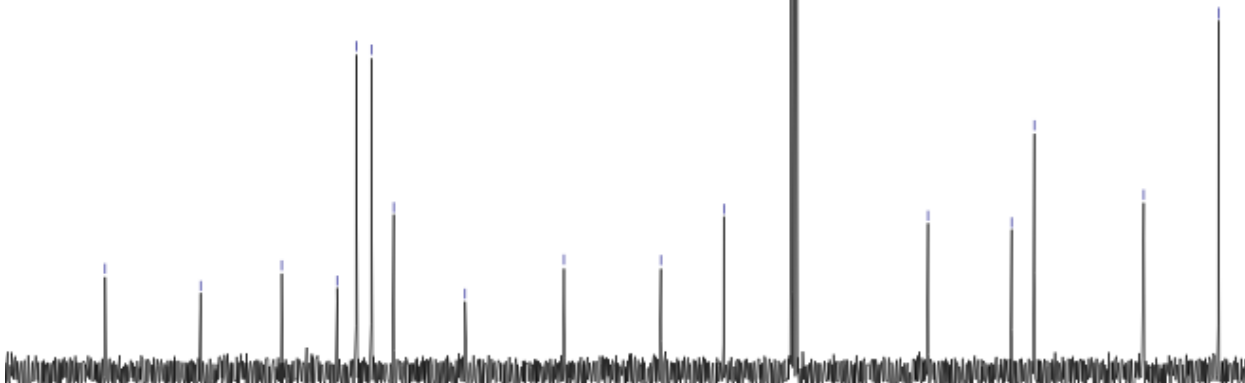

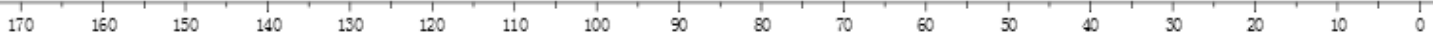




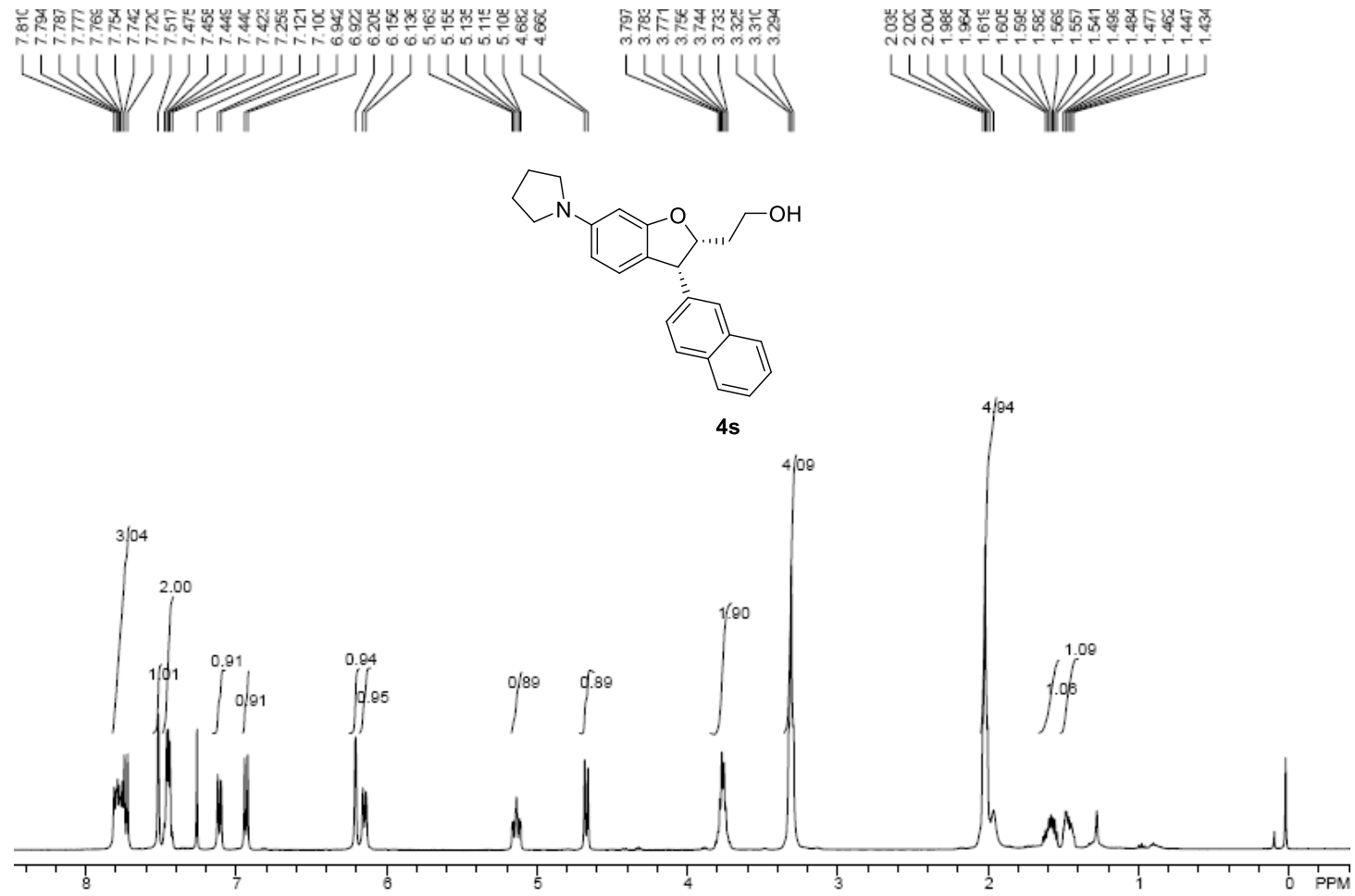

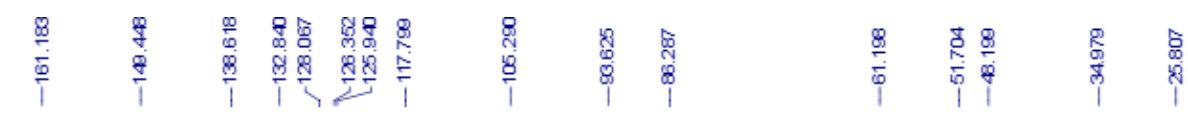

$$
\begin{aligned}
& \text { (N) }
\end{aligned}
$$

4s
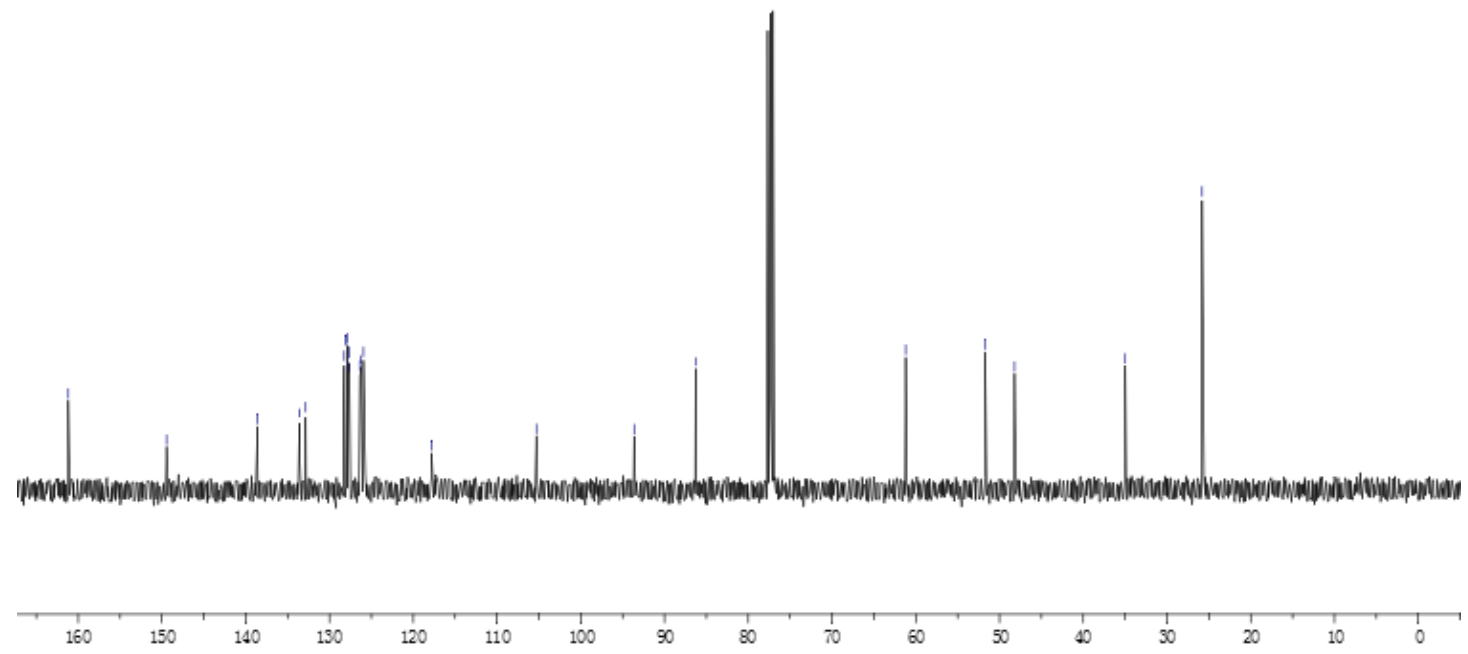


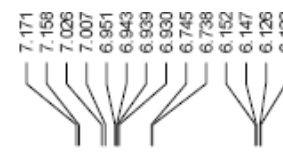

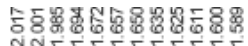

YW

UUUU)<smiles>OCCC1Oc2cc(N3CCCC3)ccc2C1c1cccs1</smiles>

4t

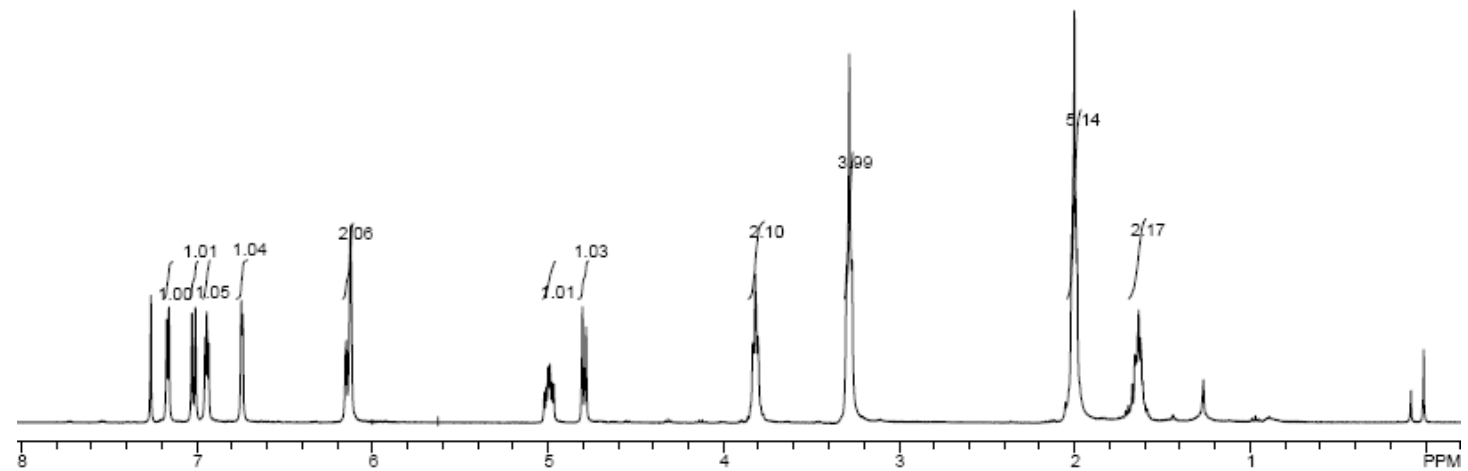

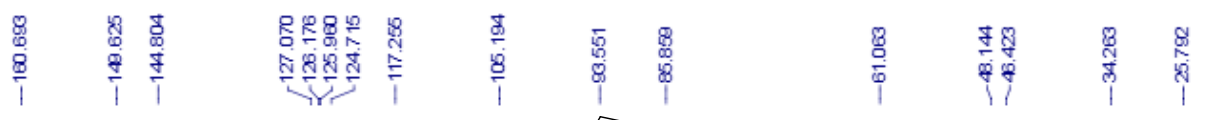<smiles>OCCC1Oc2cc(N3CCCC3)ccc2C1c1cccs1</smiles>

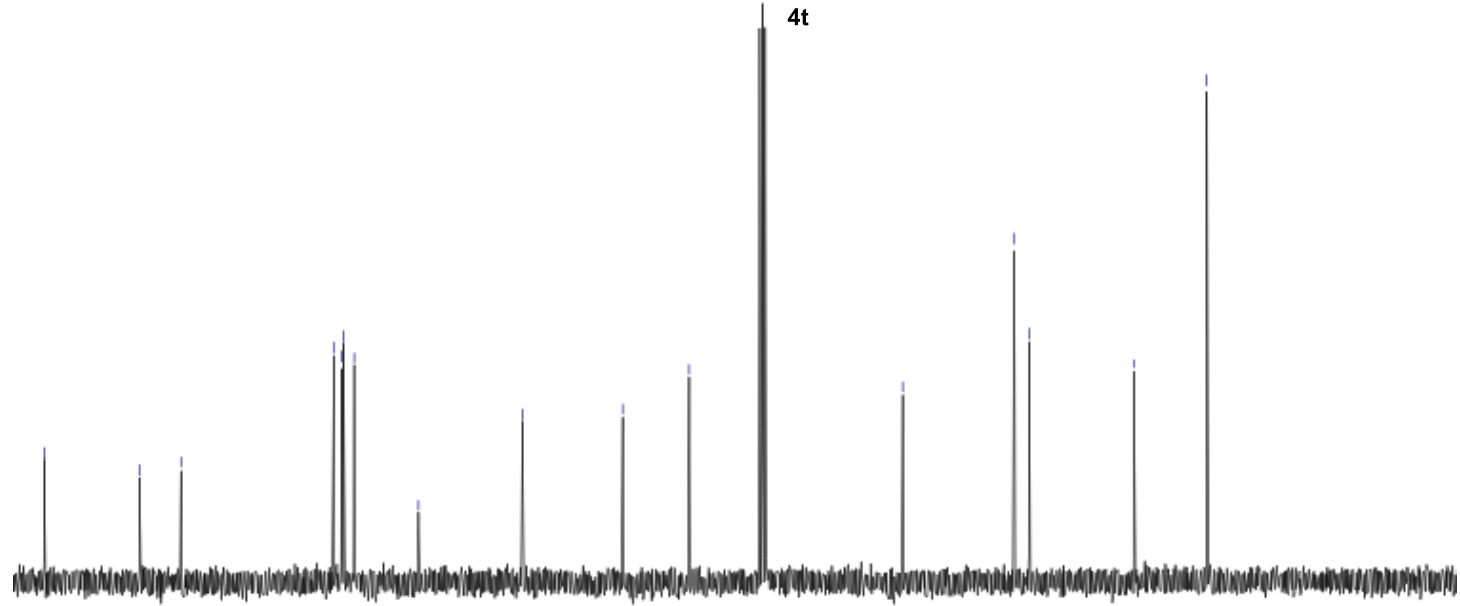

160 $130,120,110,100$

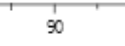

80.70 

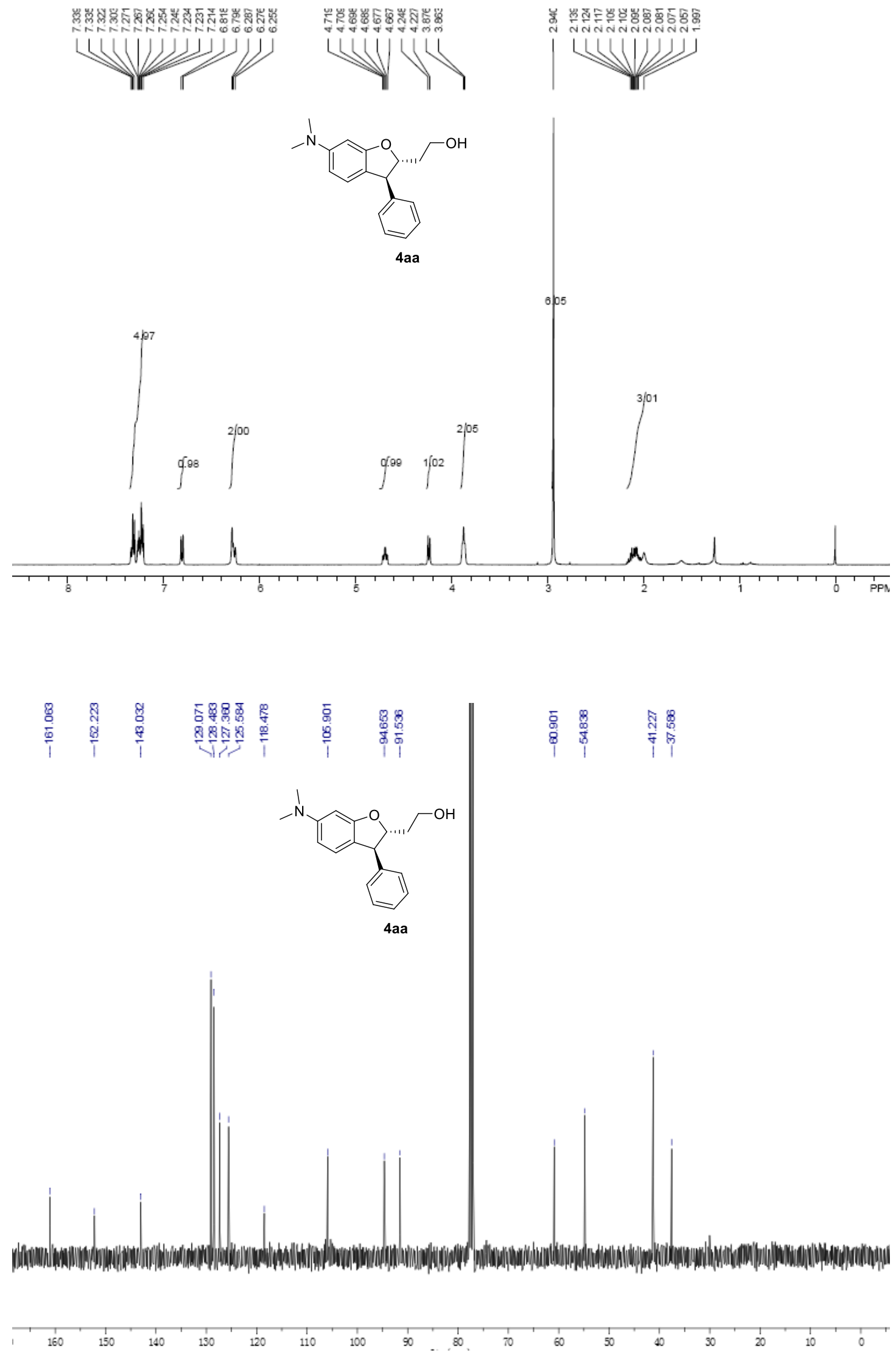

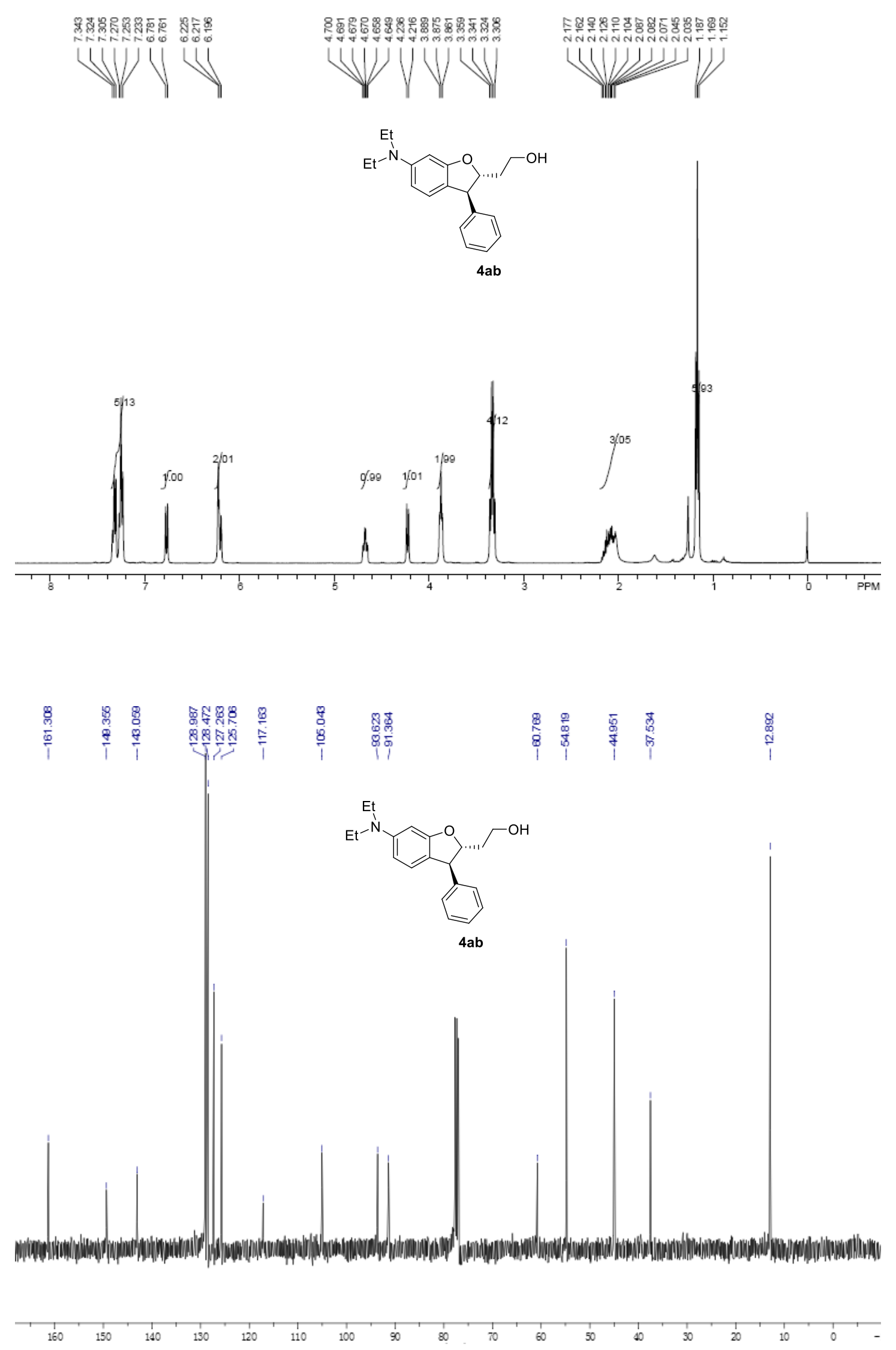

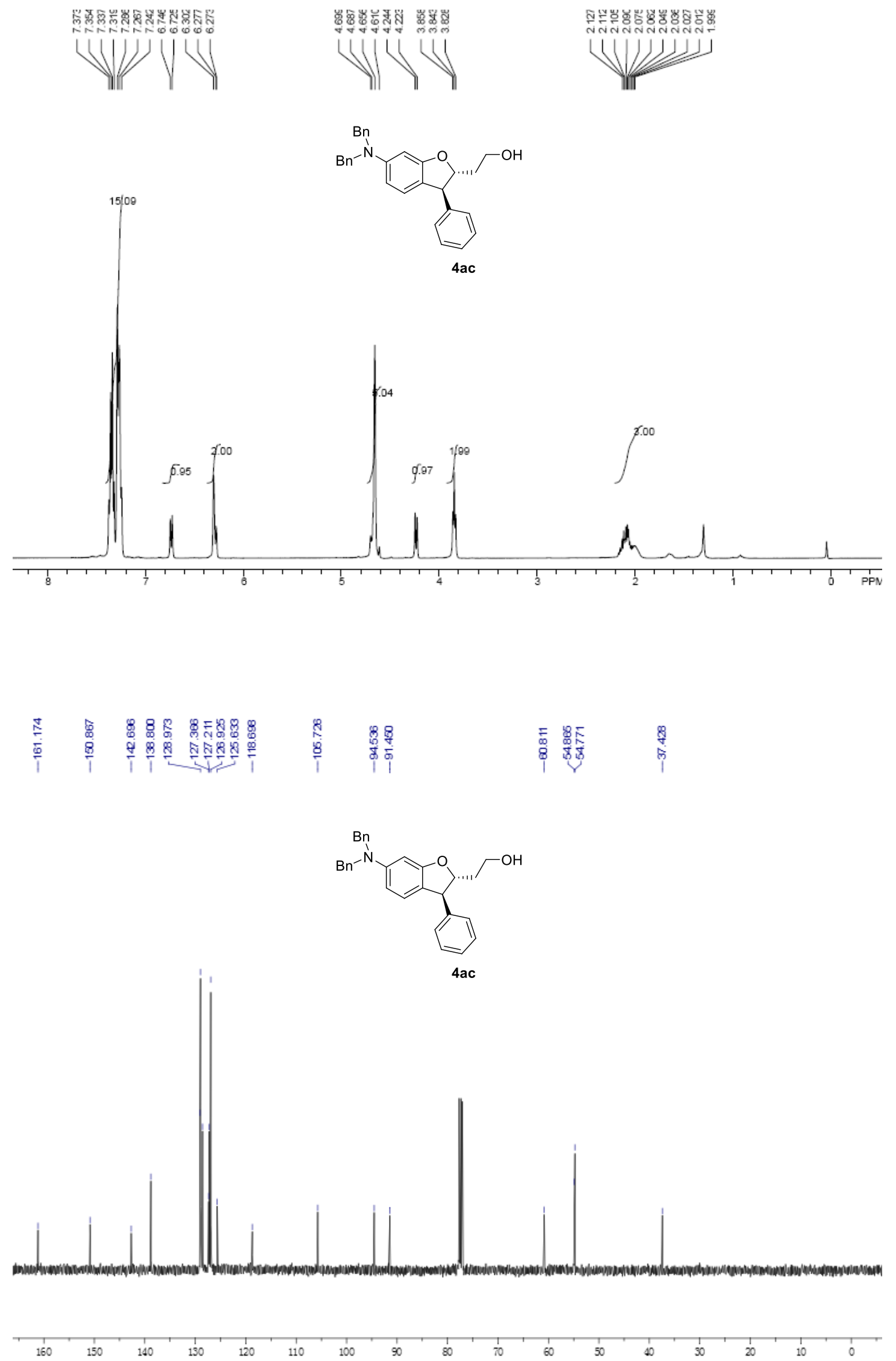

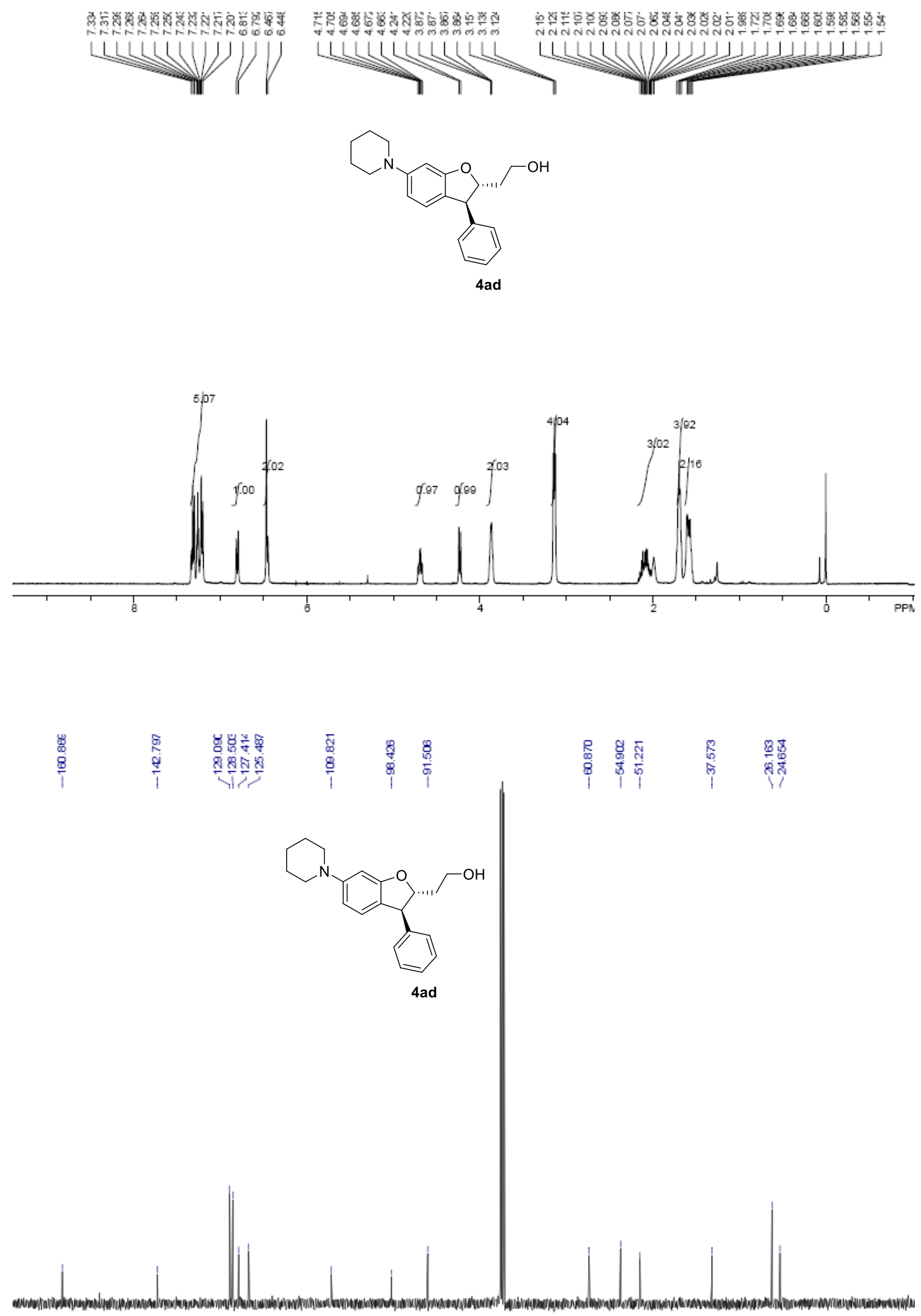


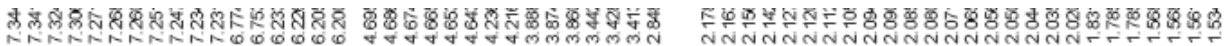

WW W W<smiles>OCCC1Oc2cc(N3CCCCC3)ccc2C1c1ccccc1</smiles>

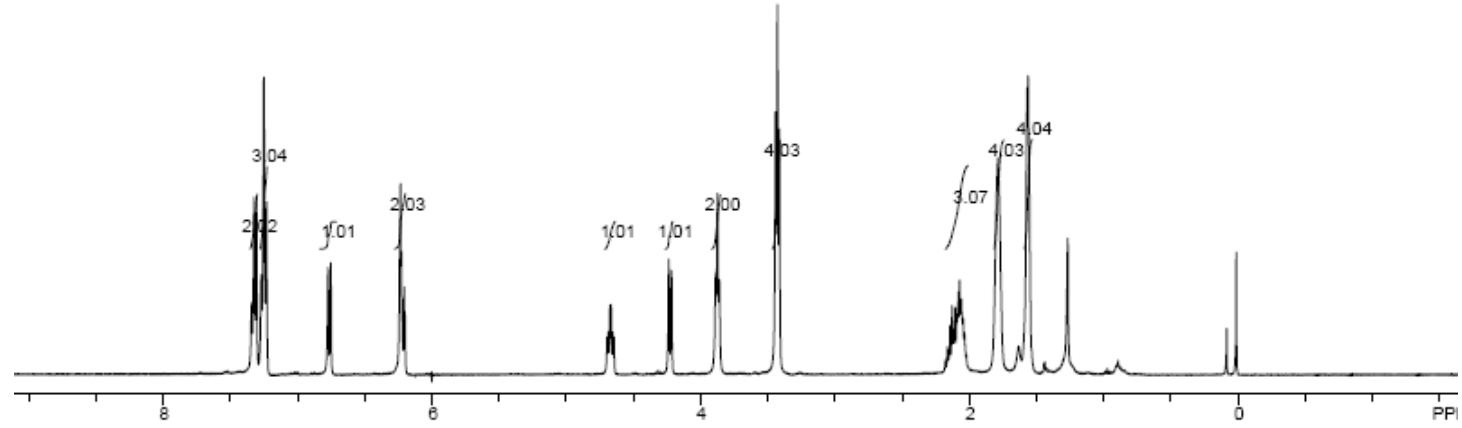

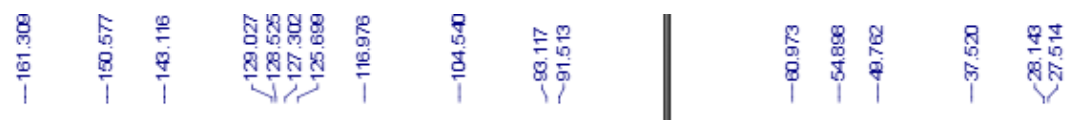
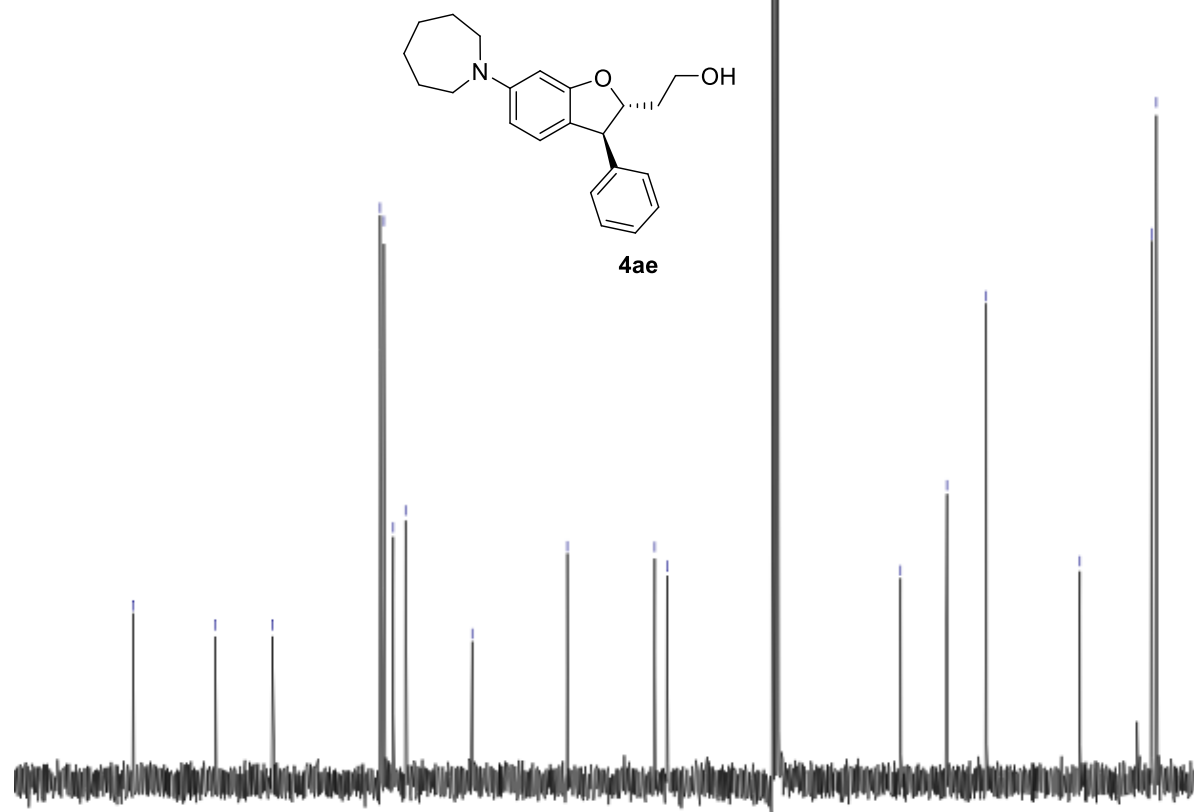

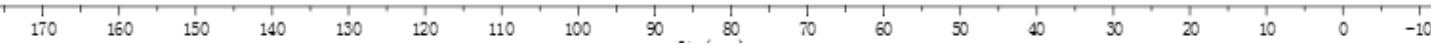




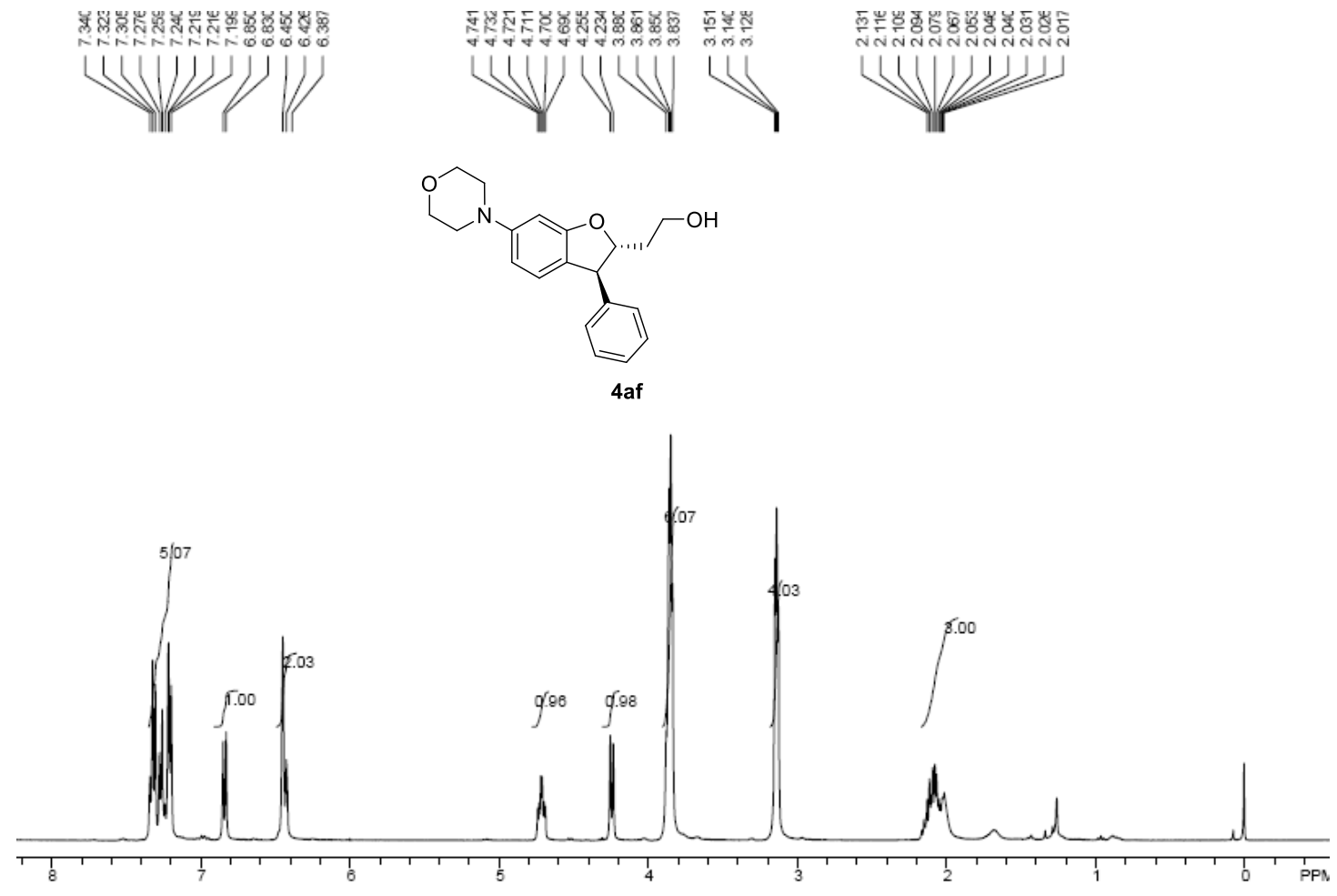

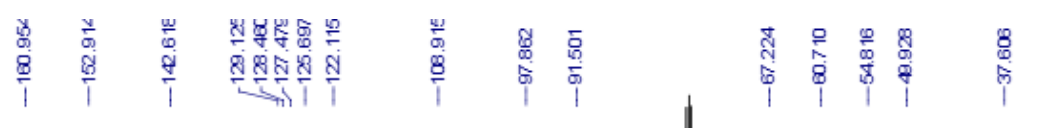<smiles>OCC[C@H]1Oc2cc(N3CCOCC3)ccc2[C@H]1c1ccccc1</smiles>

4af

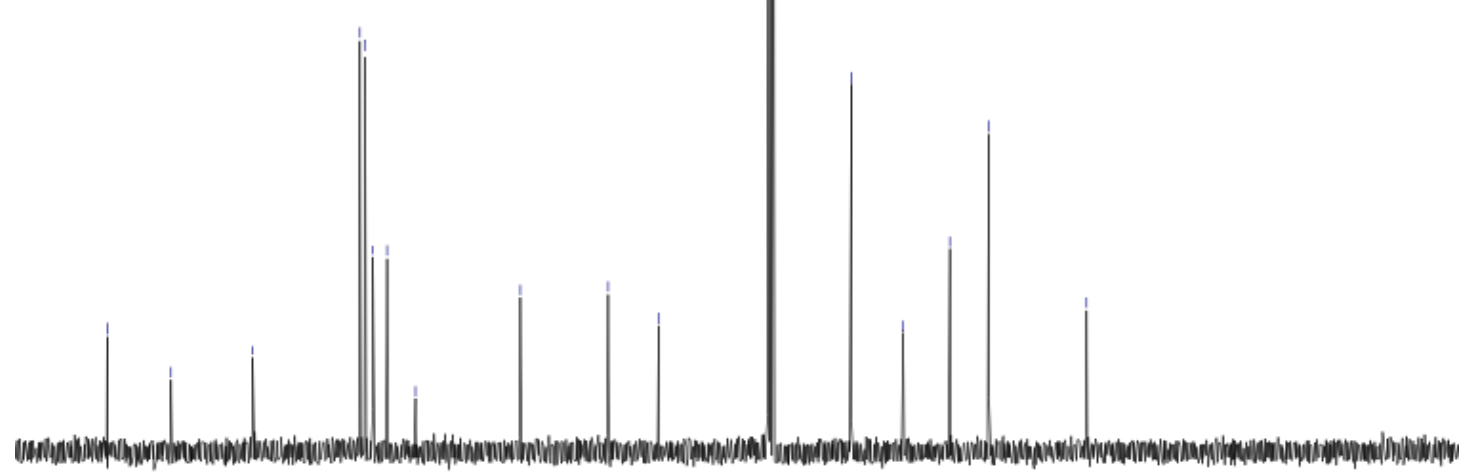

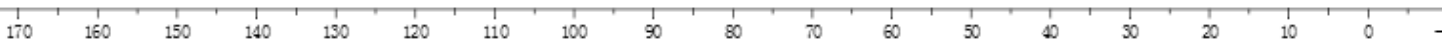




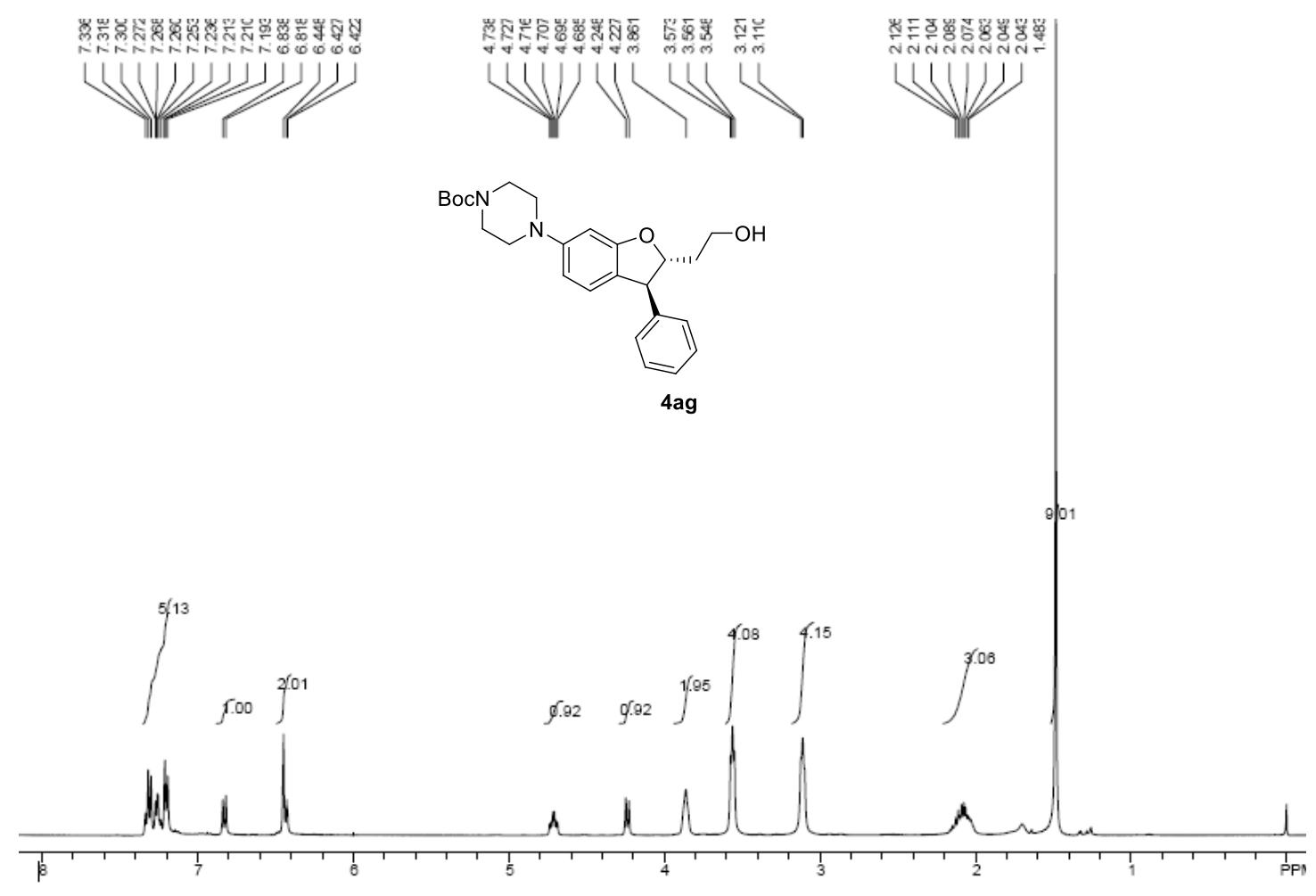

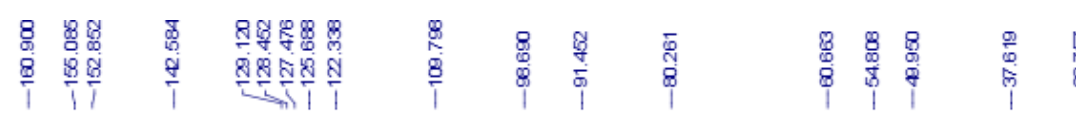

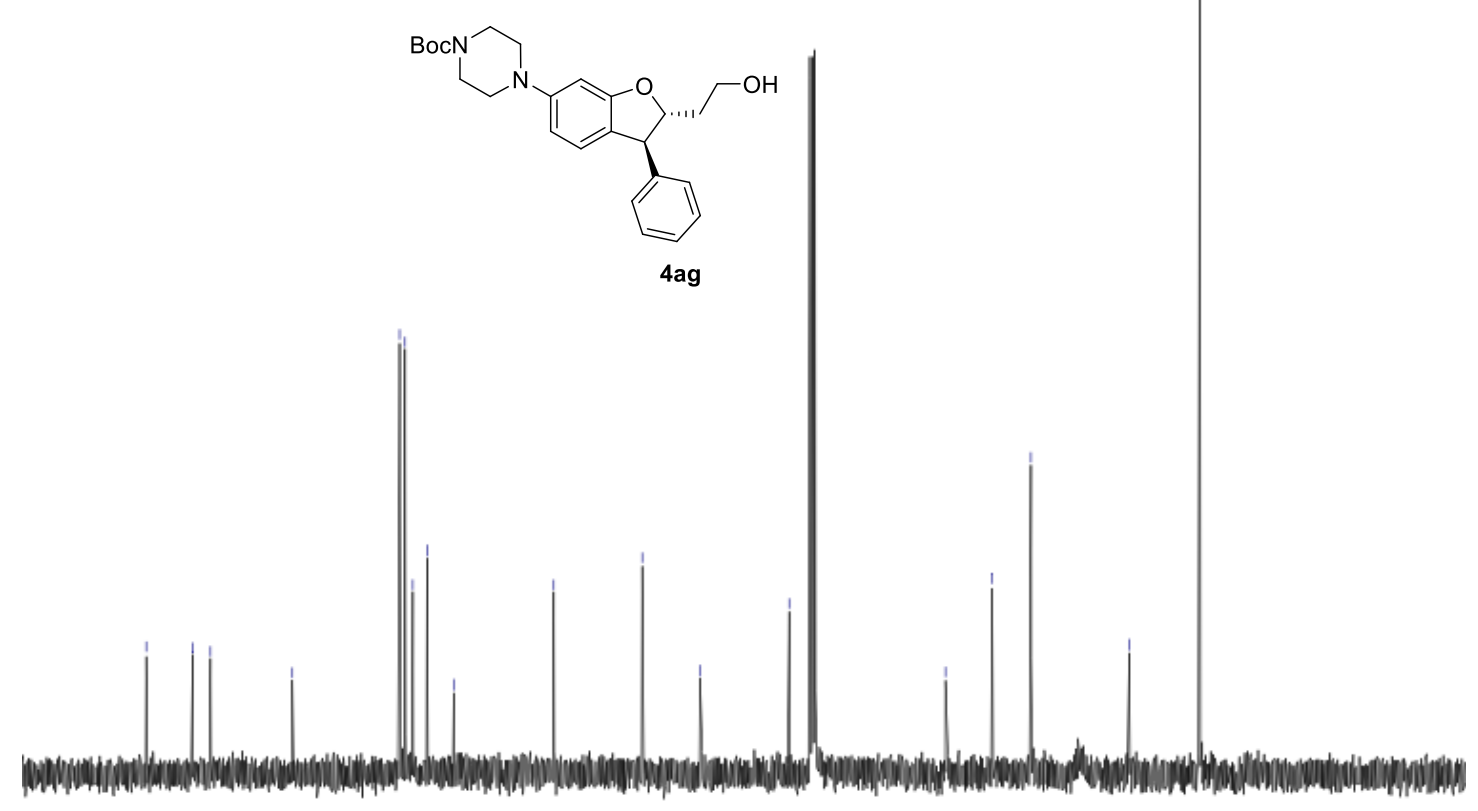

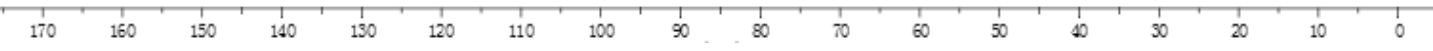



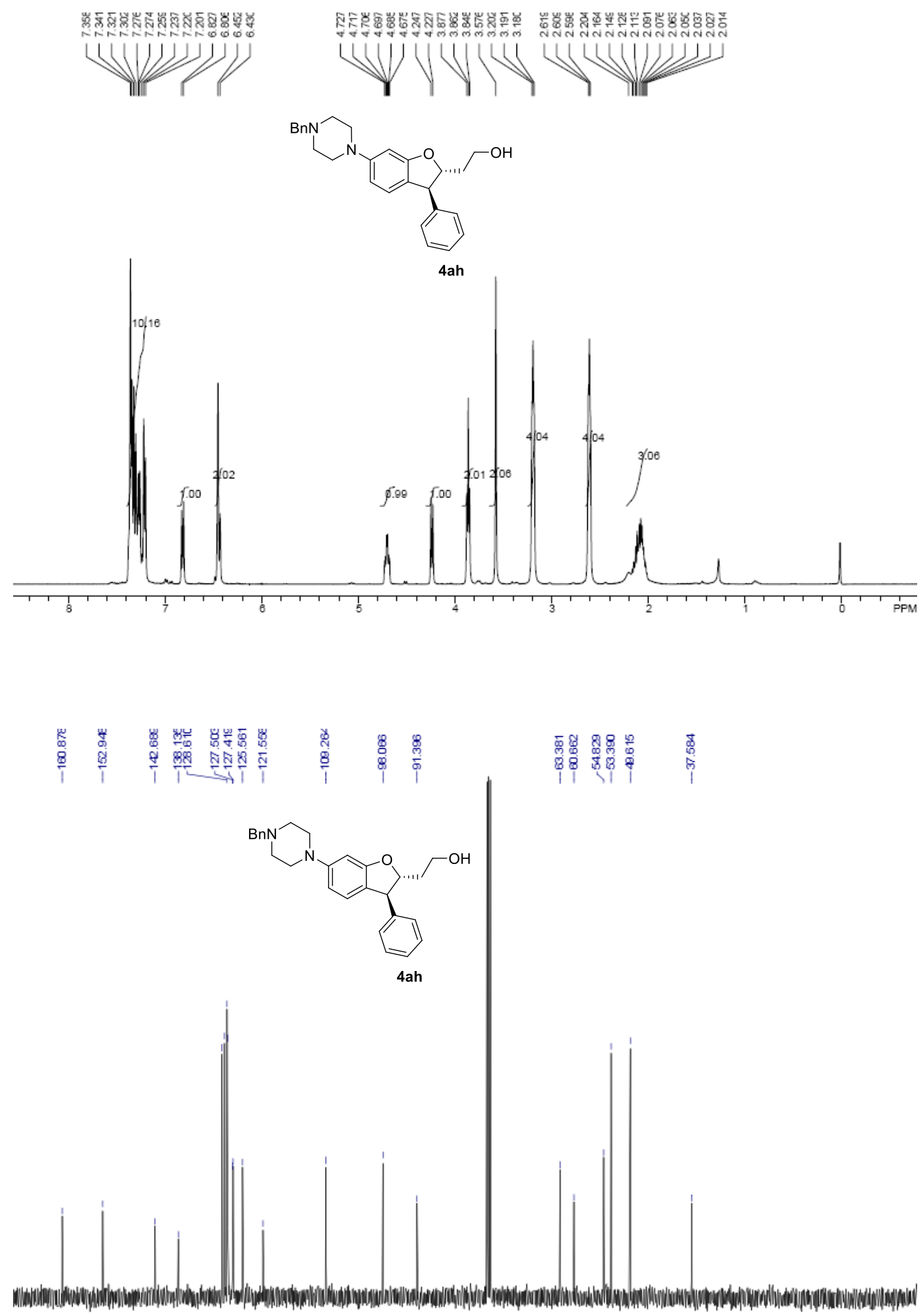

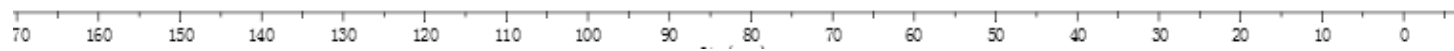




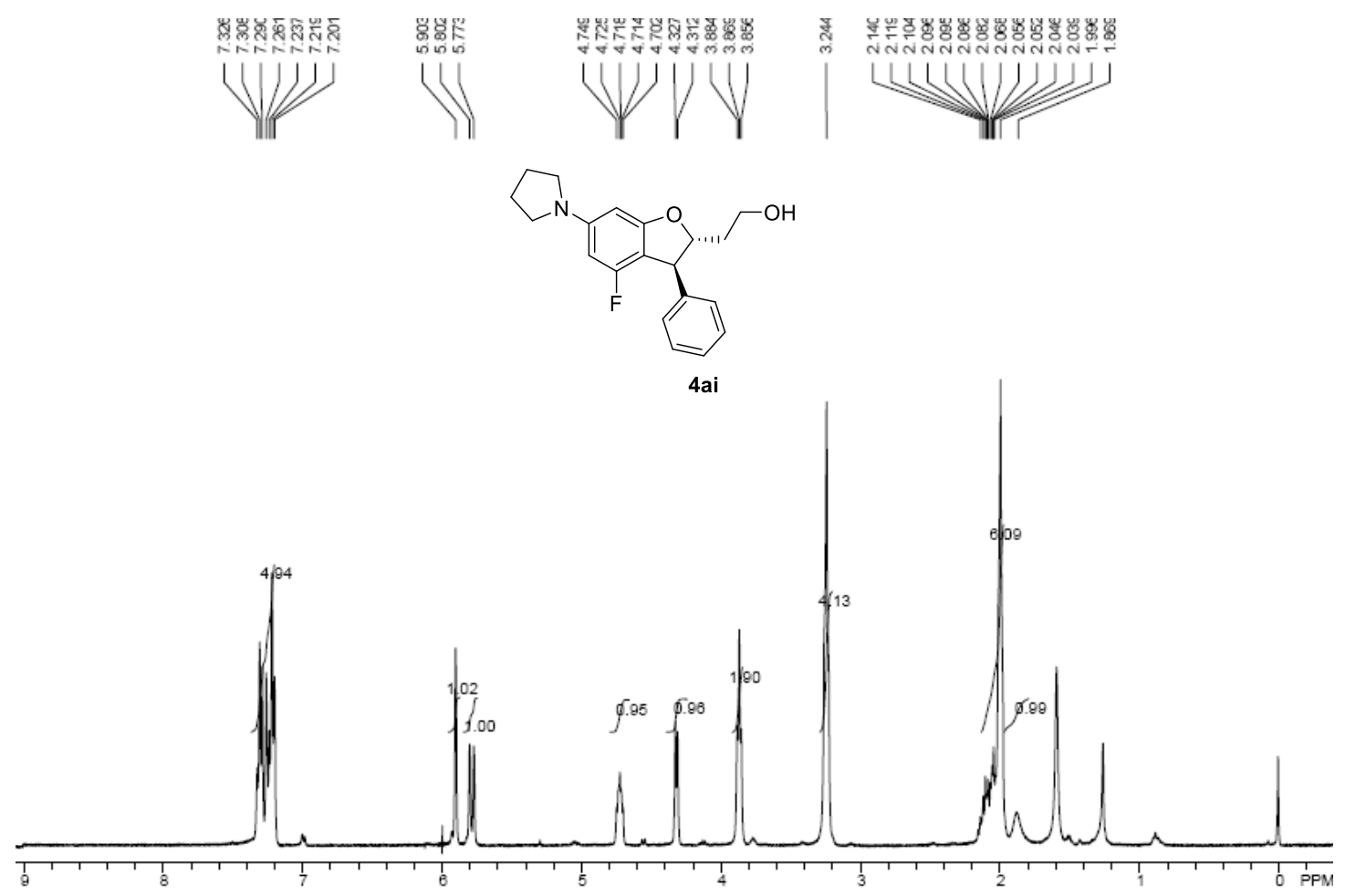

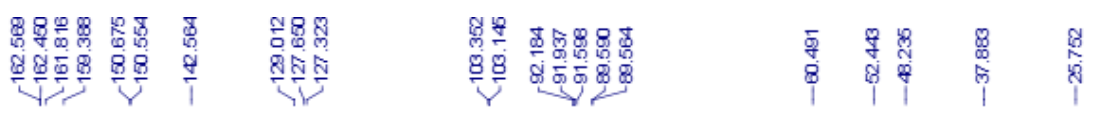

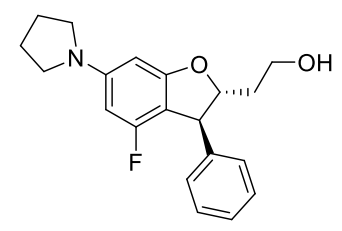

4ai

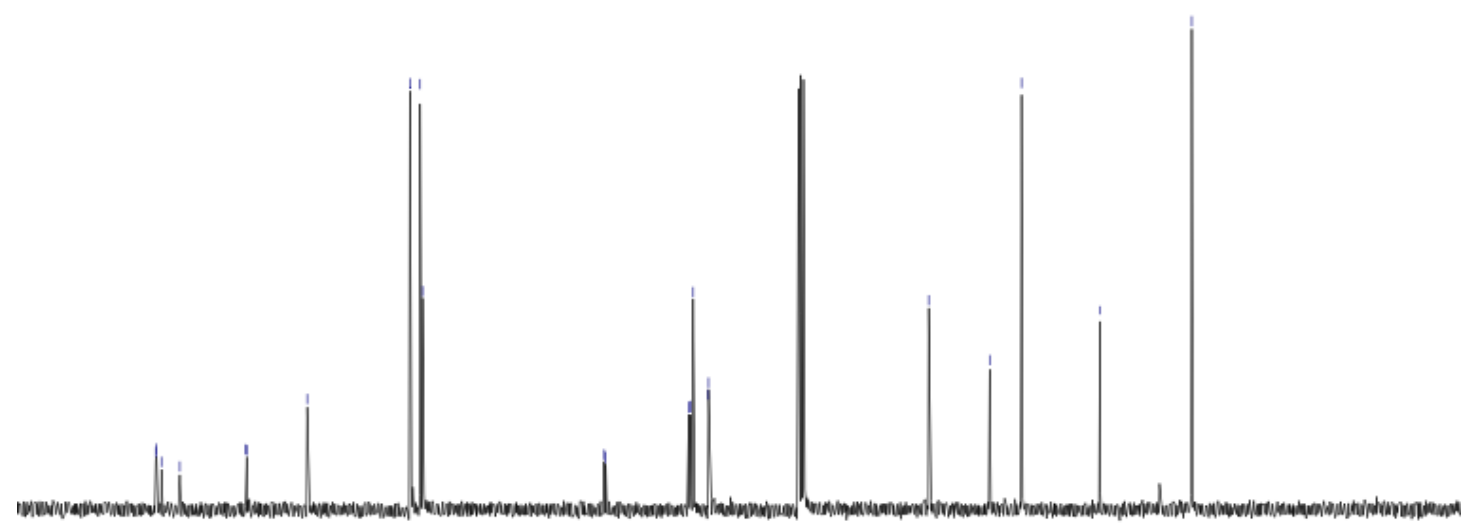

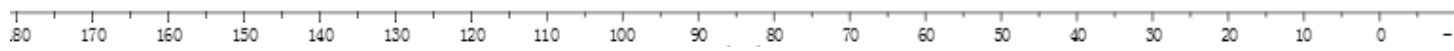




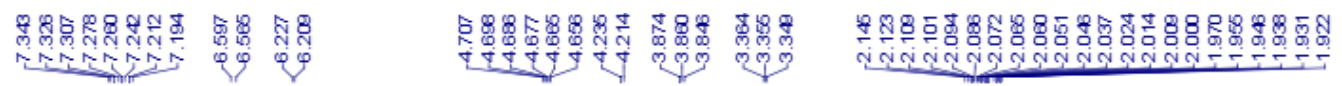

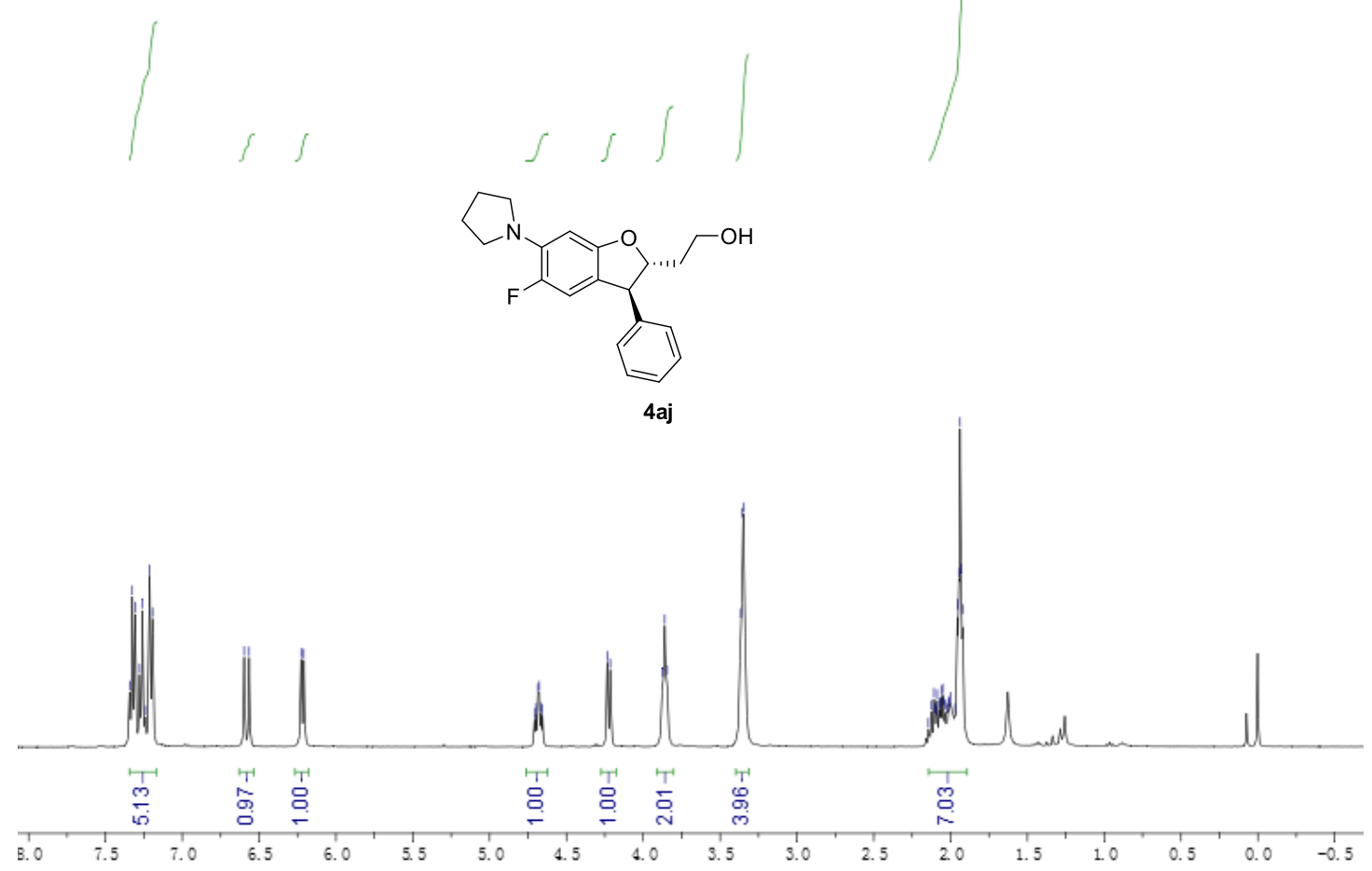

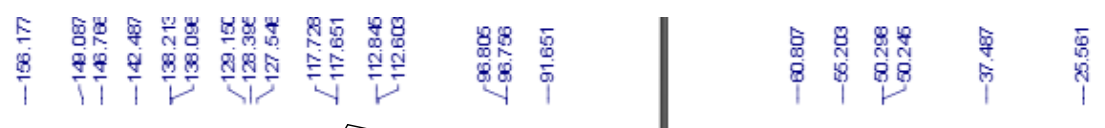

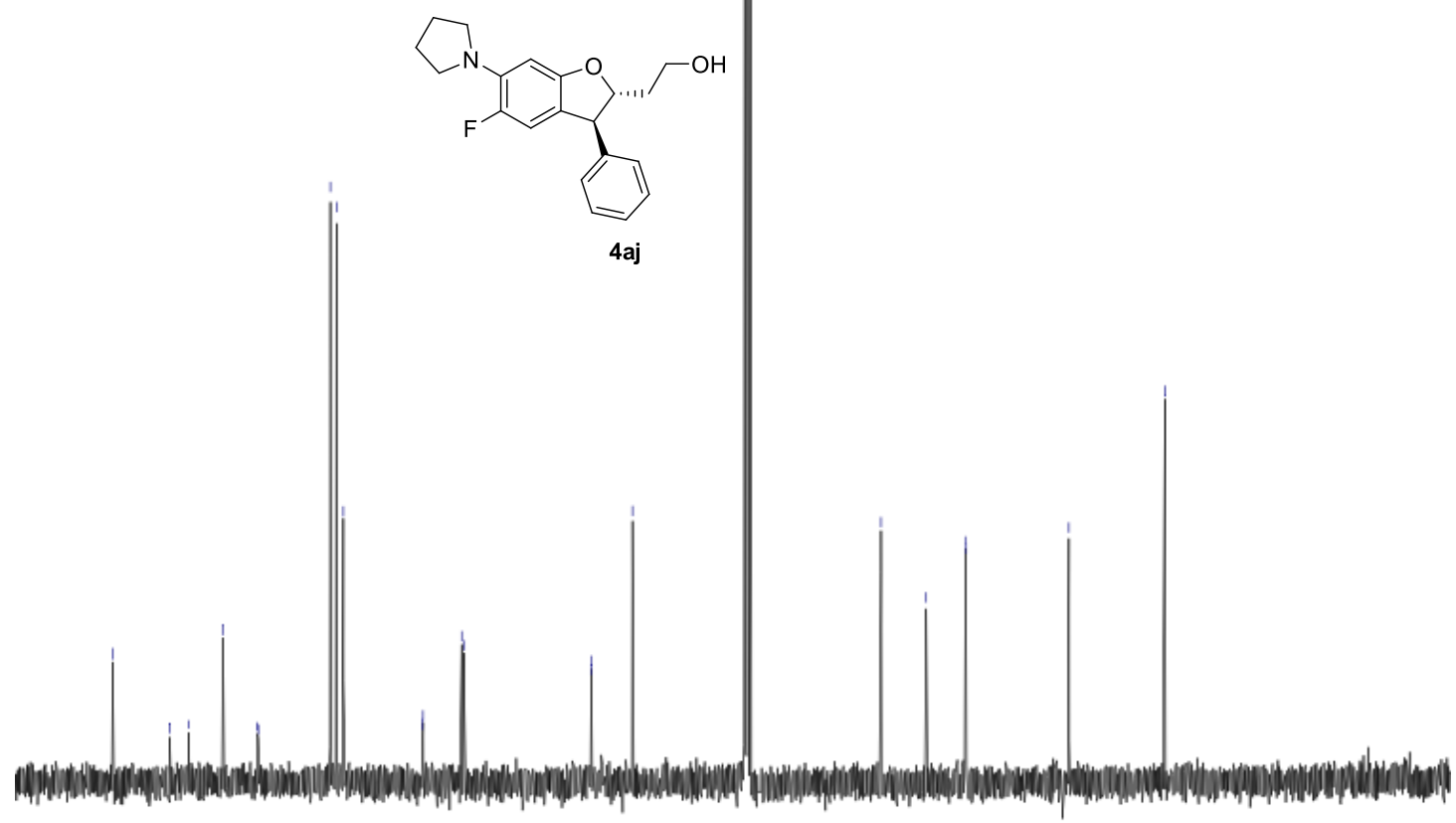

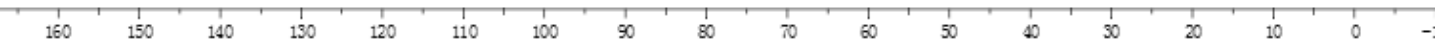



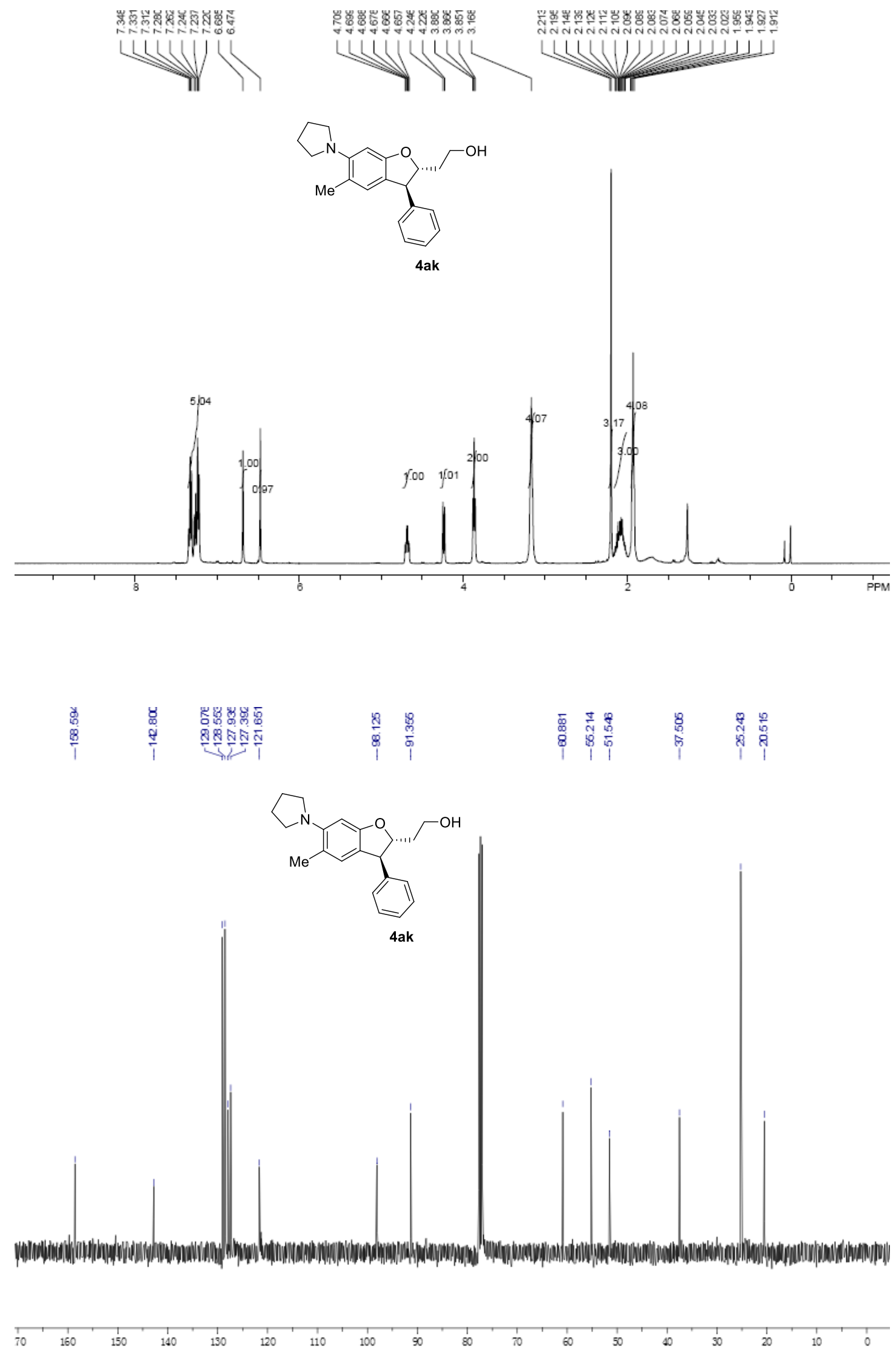


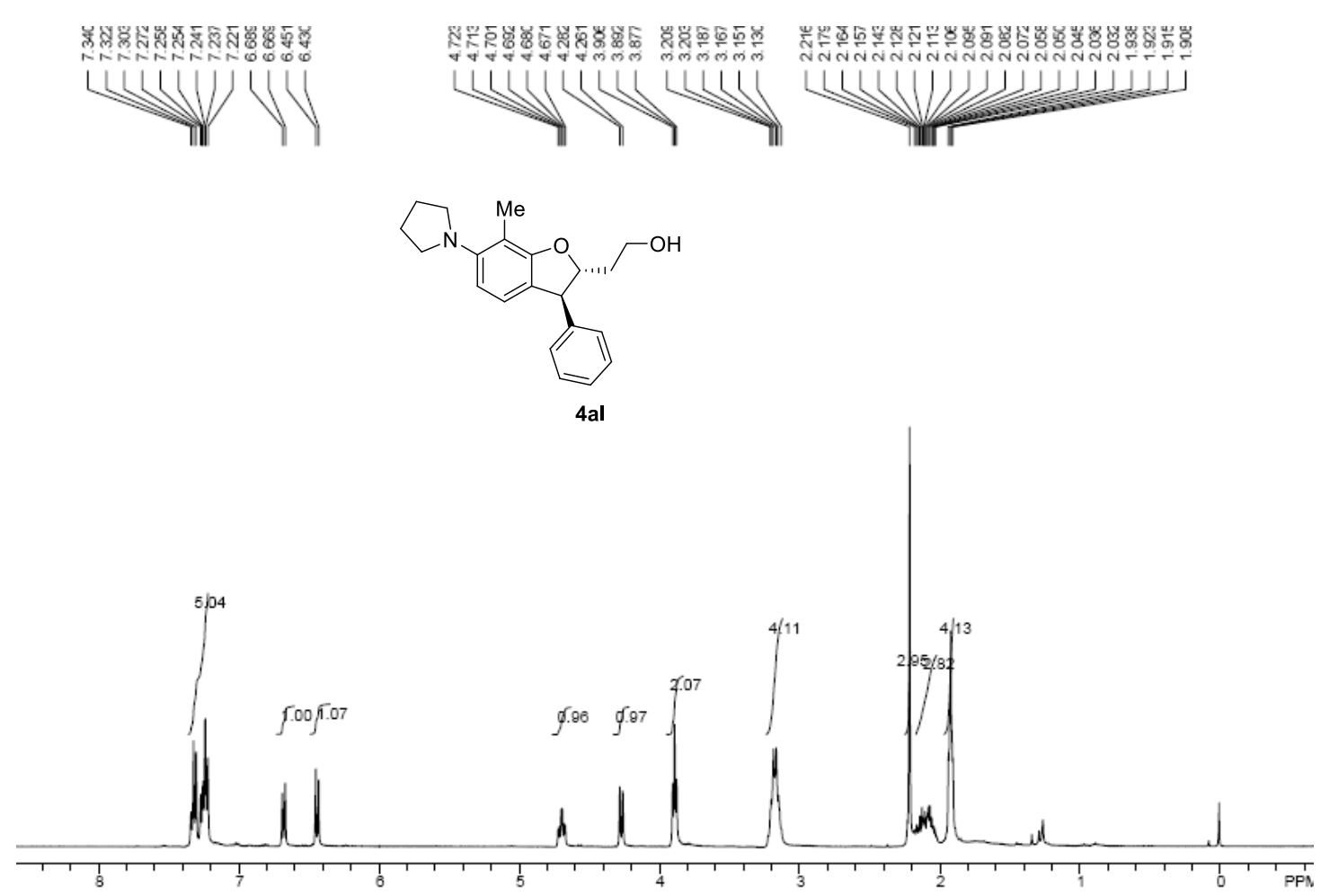

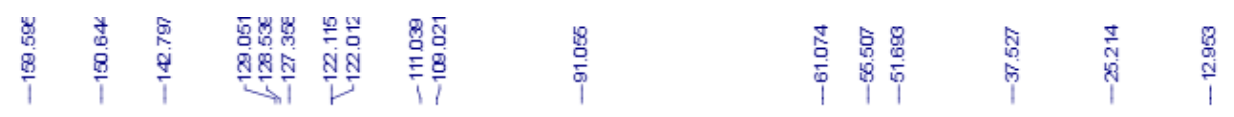
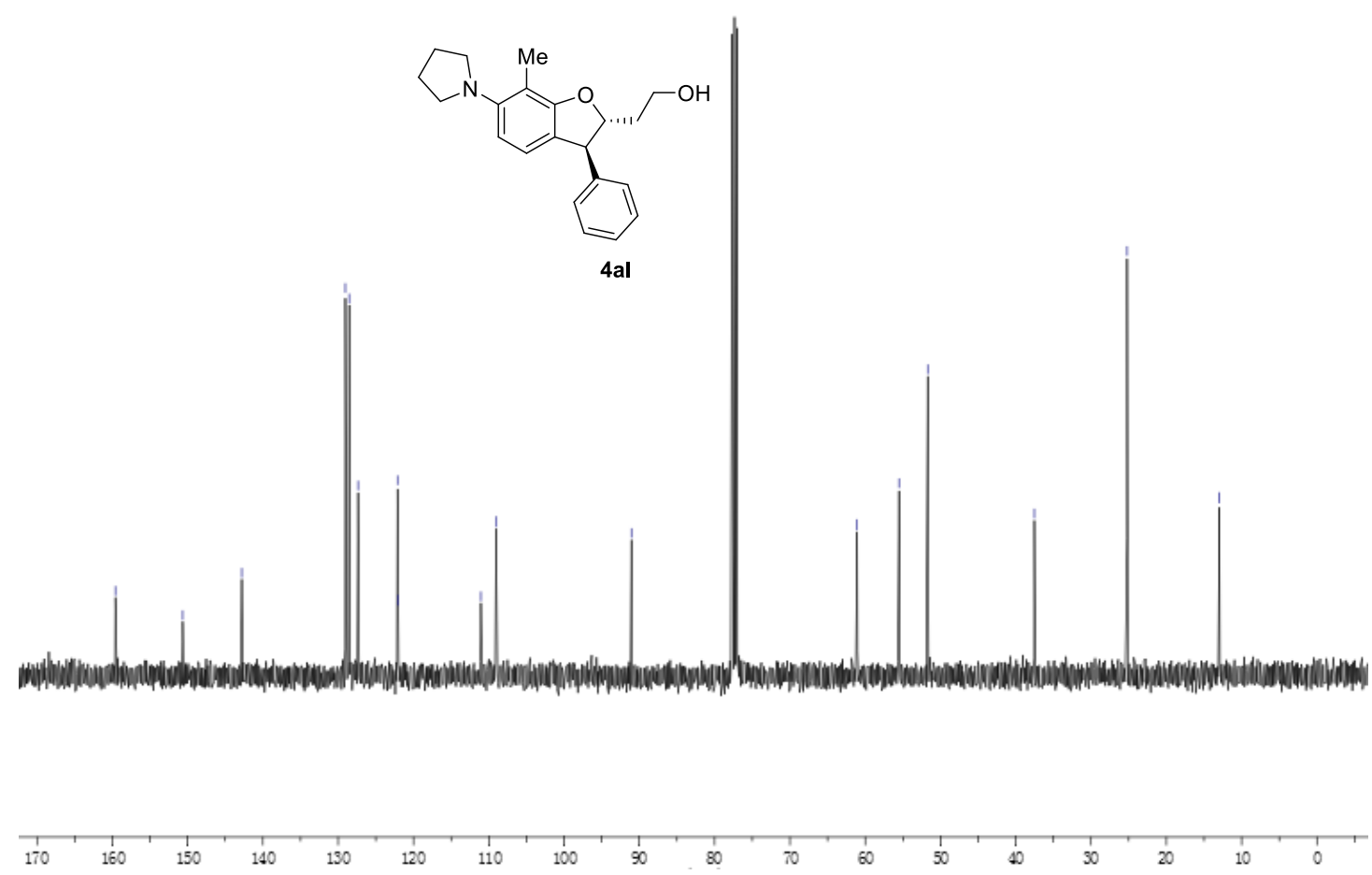


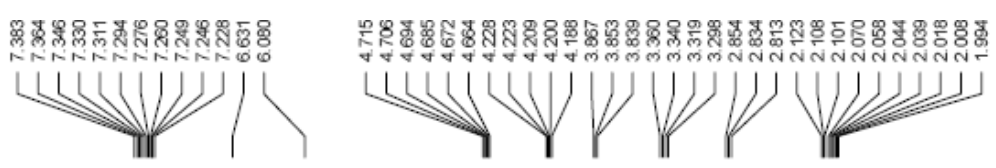
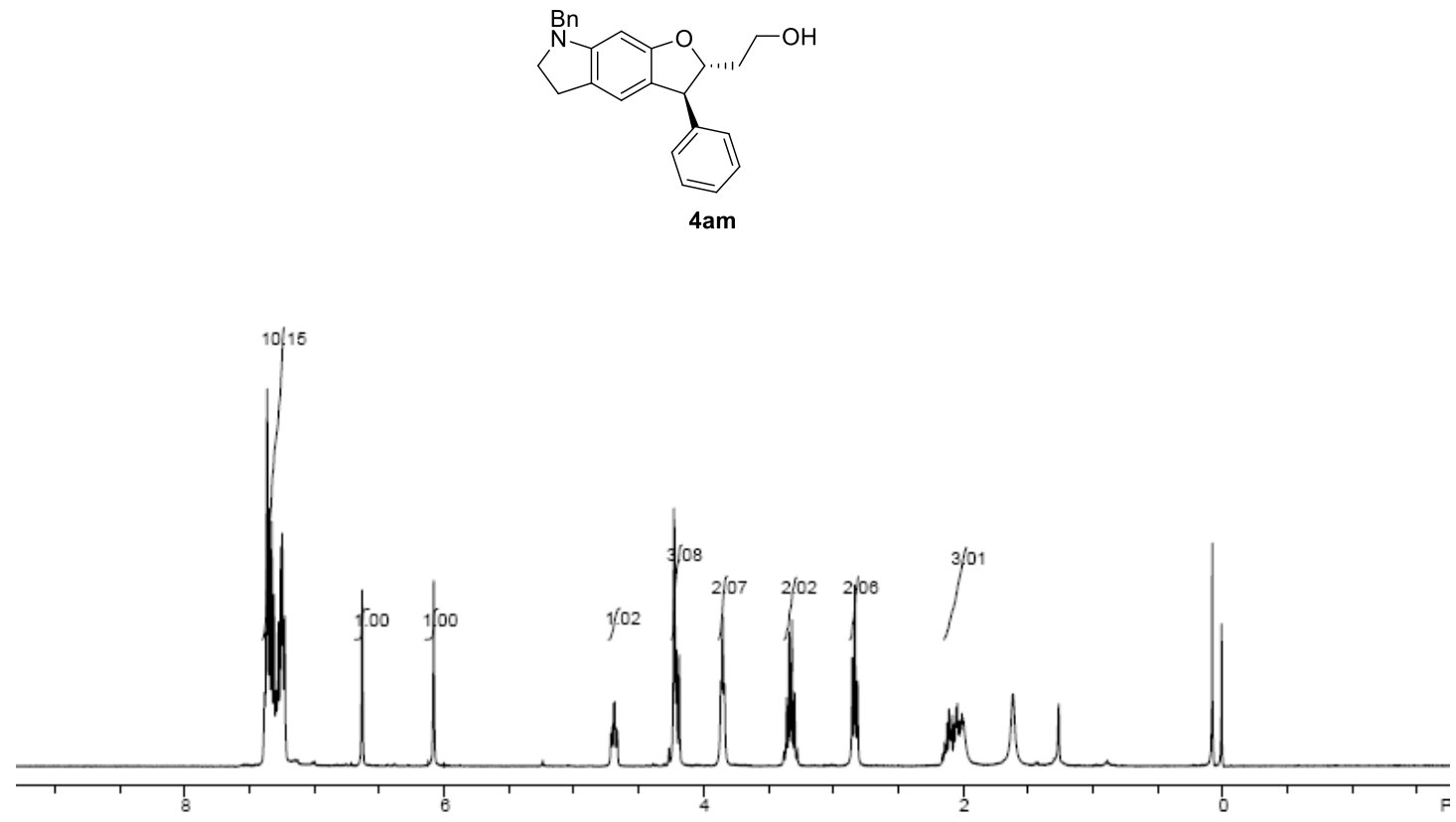

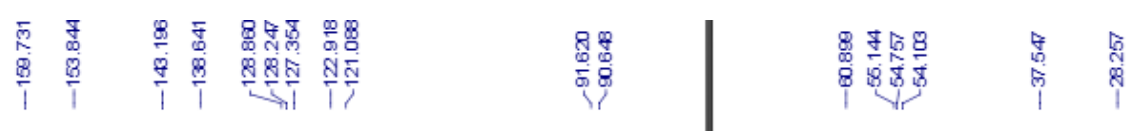
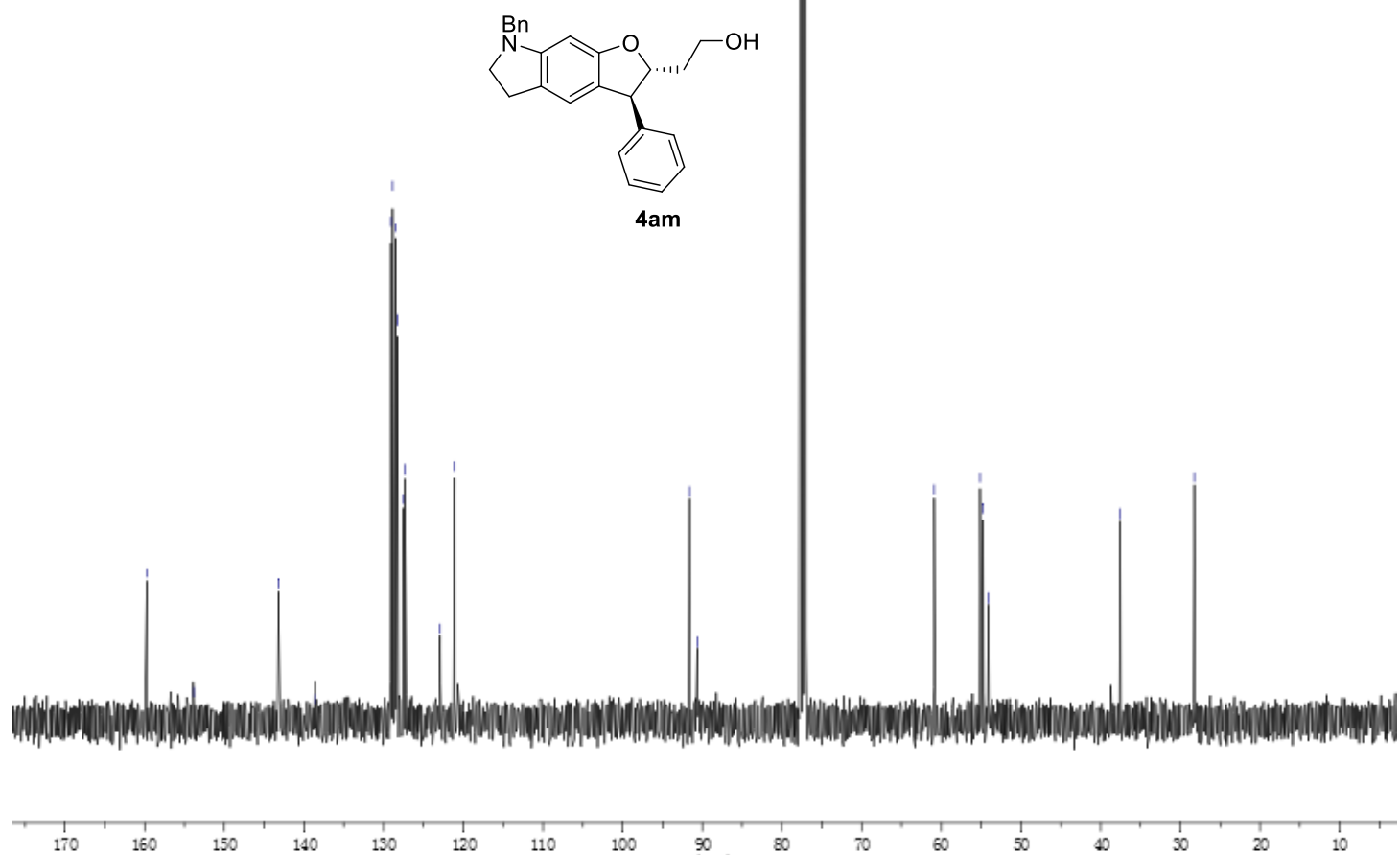


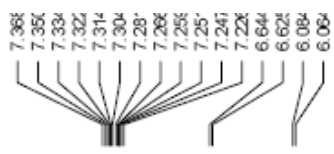

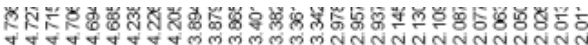

LUV V V V V V V<smiles>OCCC1Oc2c(ccc3c2CC[C@@H]3Br)C1c1ccccc1</smiles>

4an

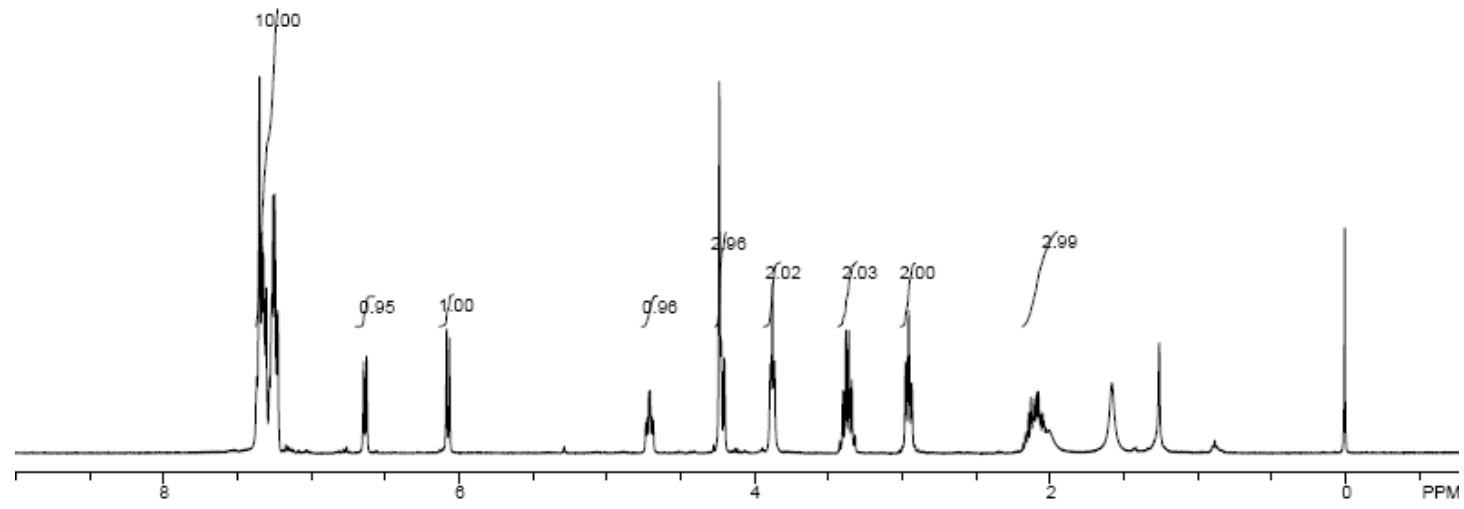

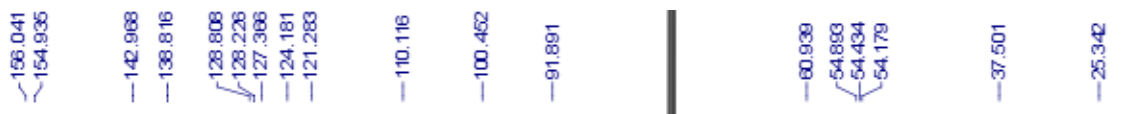

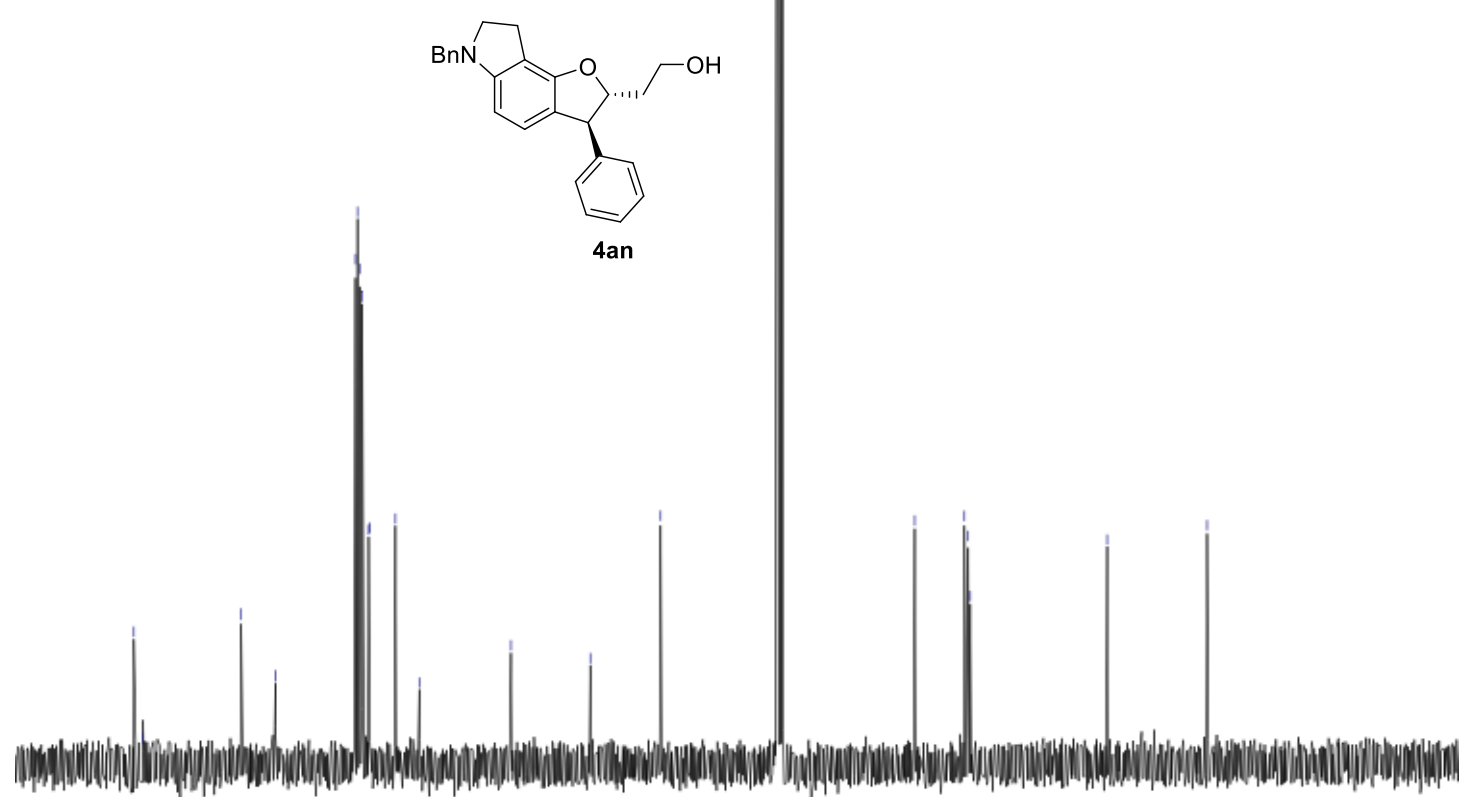

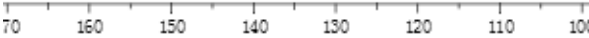



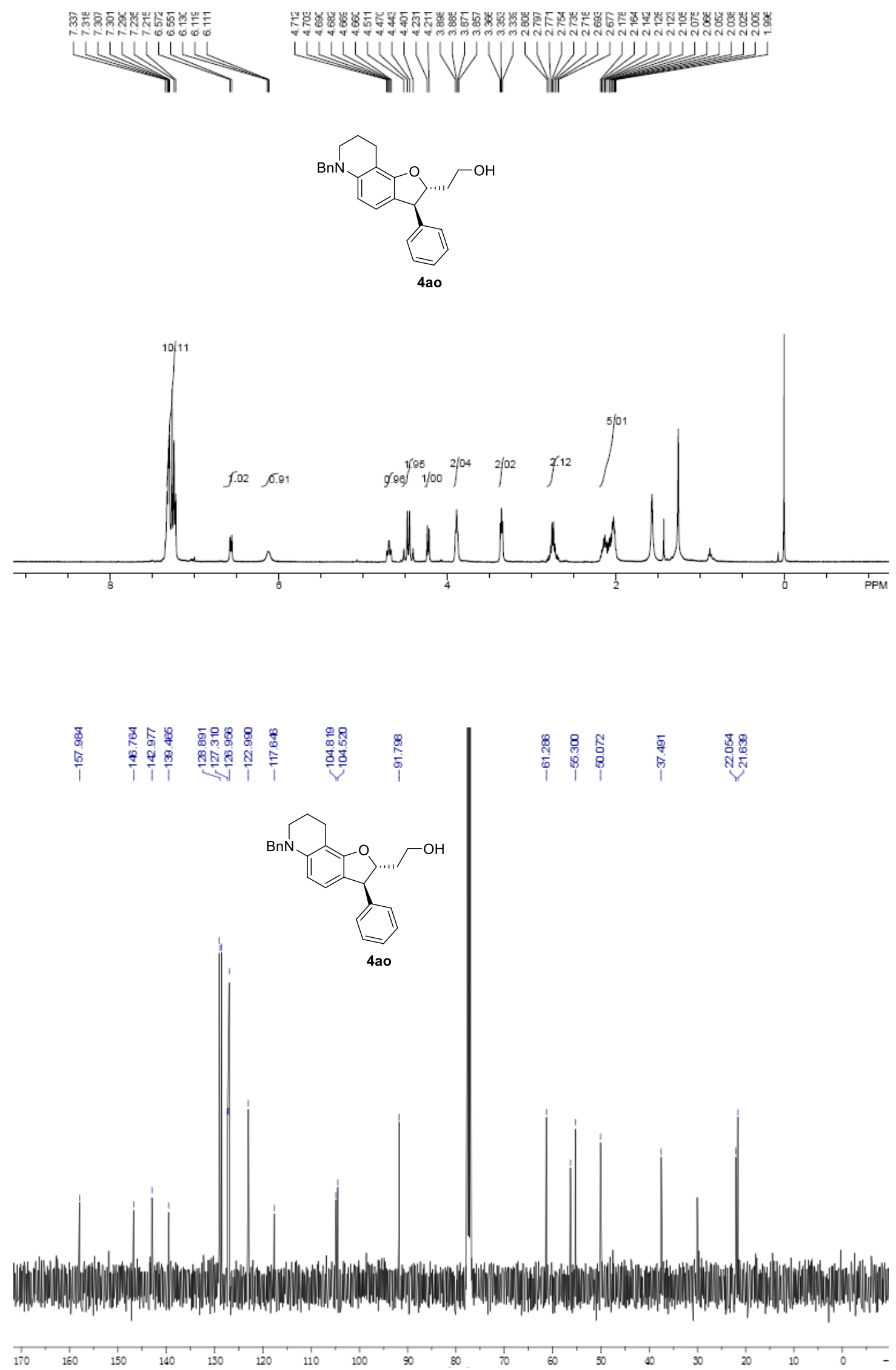


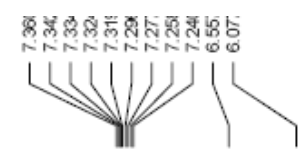

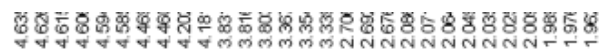

WP W W WW

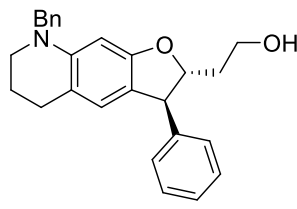

4ap

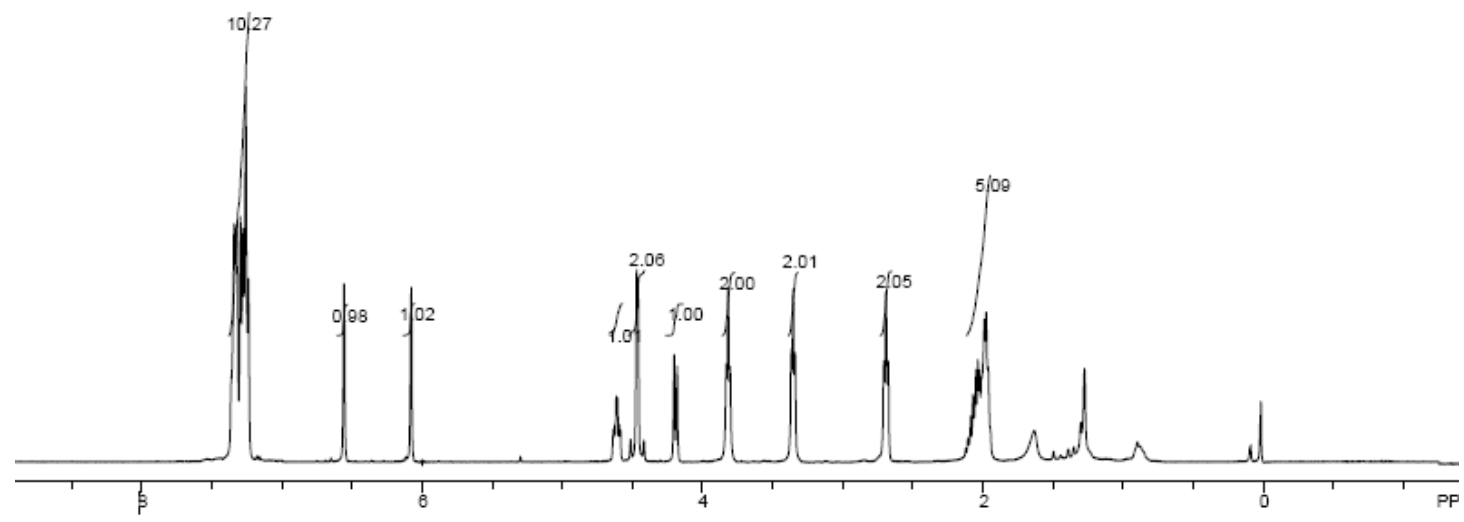

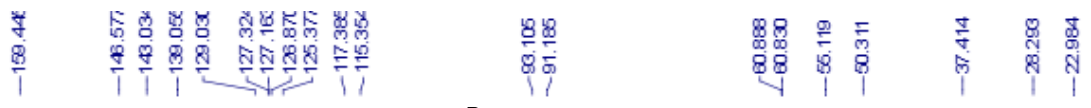<smiles>OCC[C@H]1Oc2cc3c(cc2[C@@H]1c1ccccc1)CCCN3Cc1ccccc1</smiles>
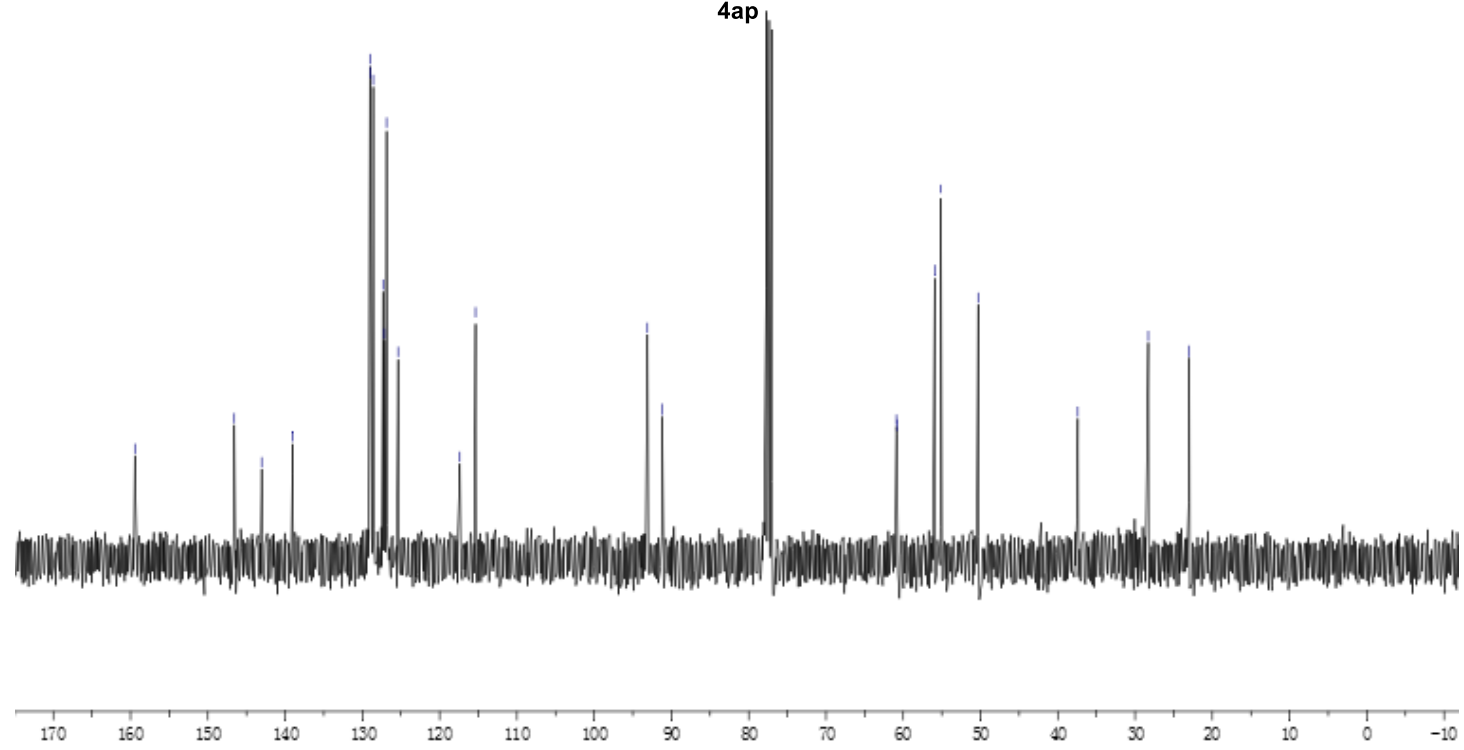

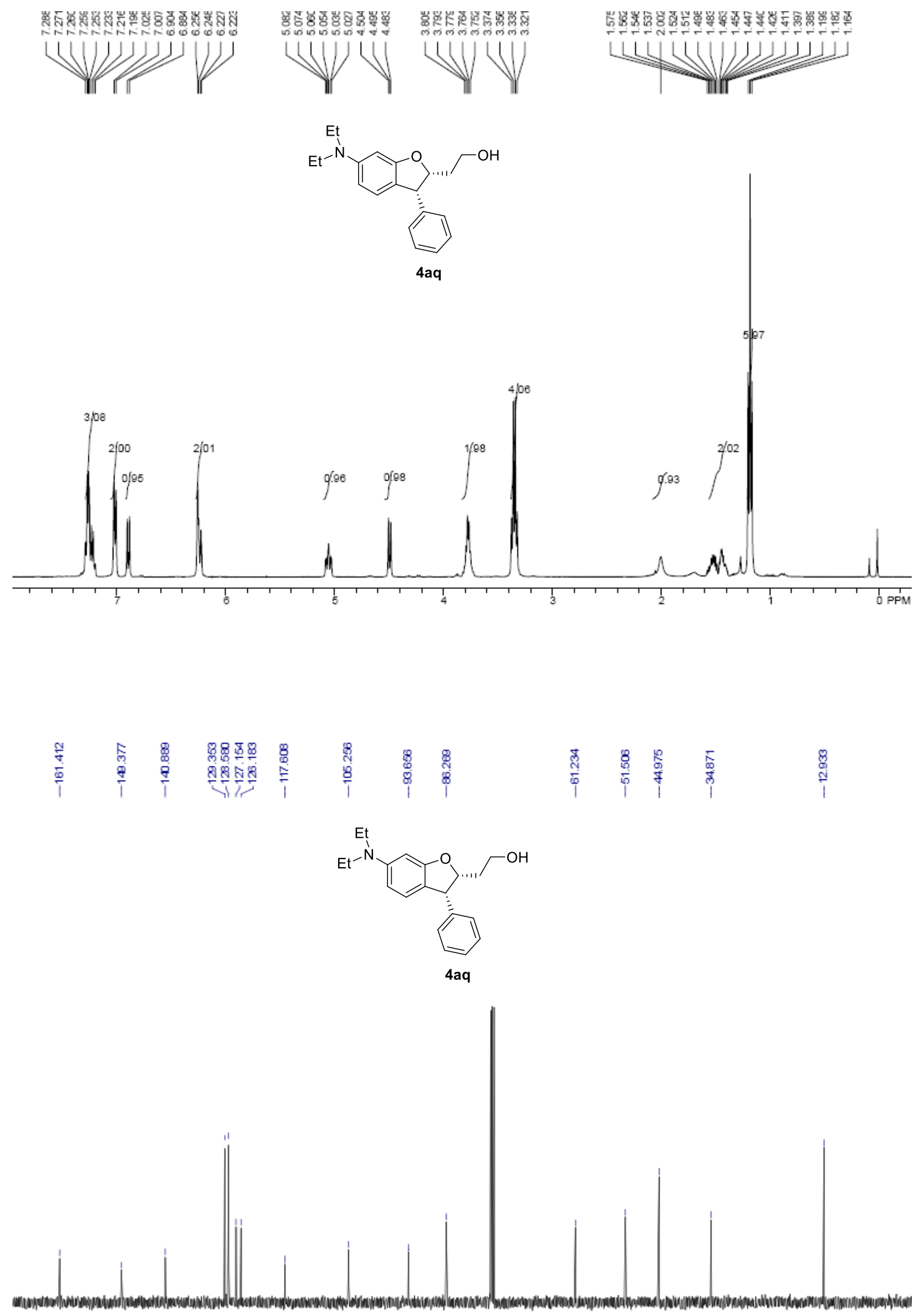

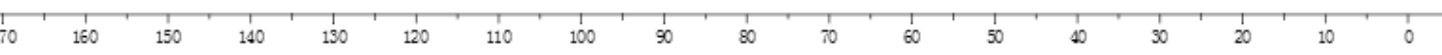




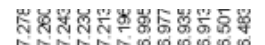

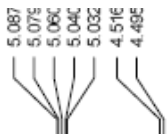

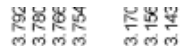

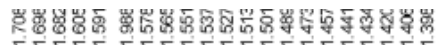

WW

$\bigcup^{\infty ल m}$

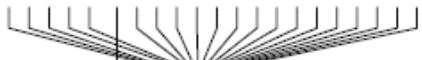<smiles>OCCC1Oc2cc(N3CCCCC3)ccc2C1c1ccccc1</smiles>

4ar

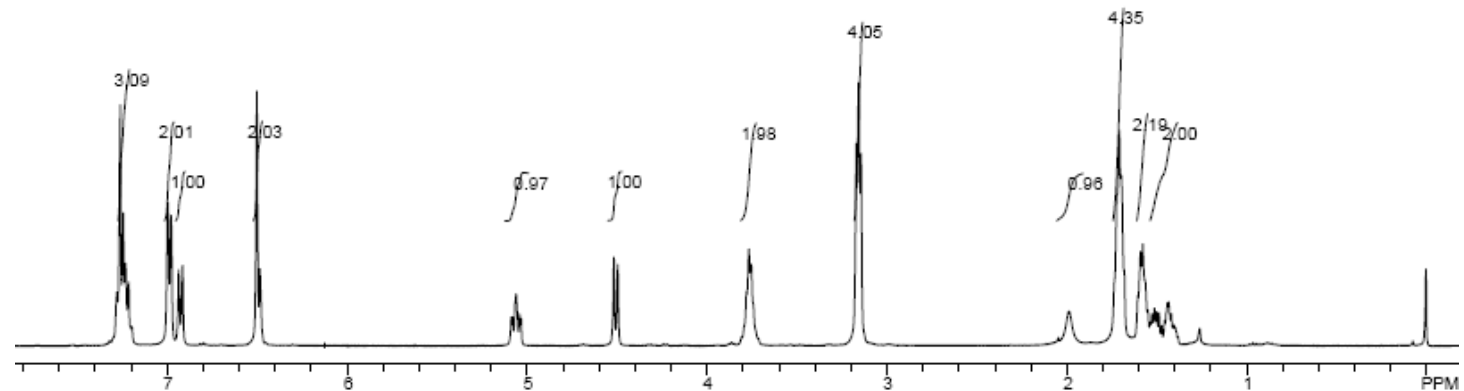

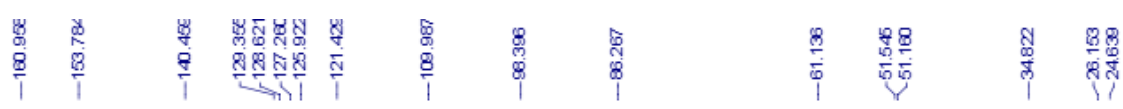

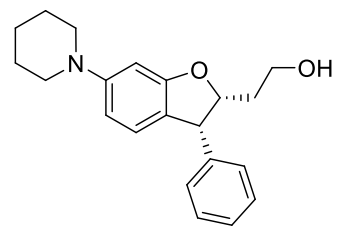

4ar
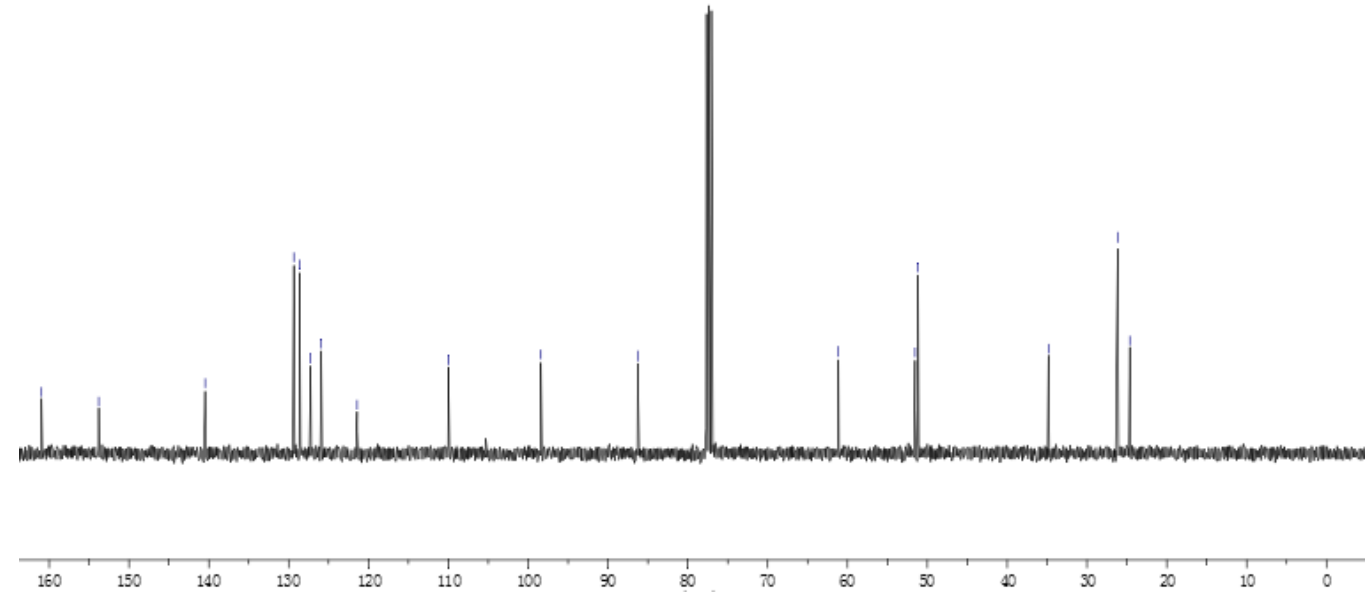

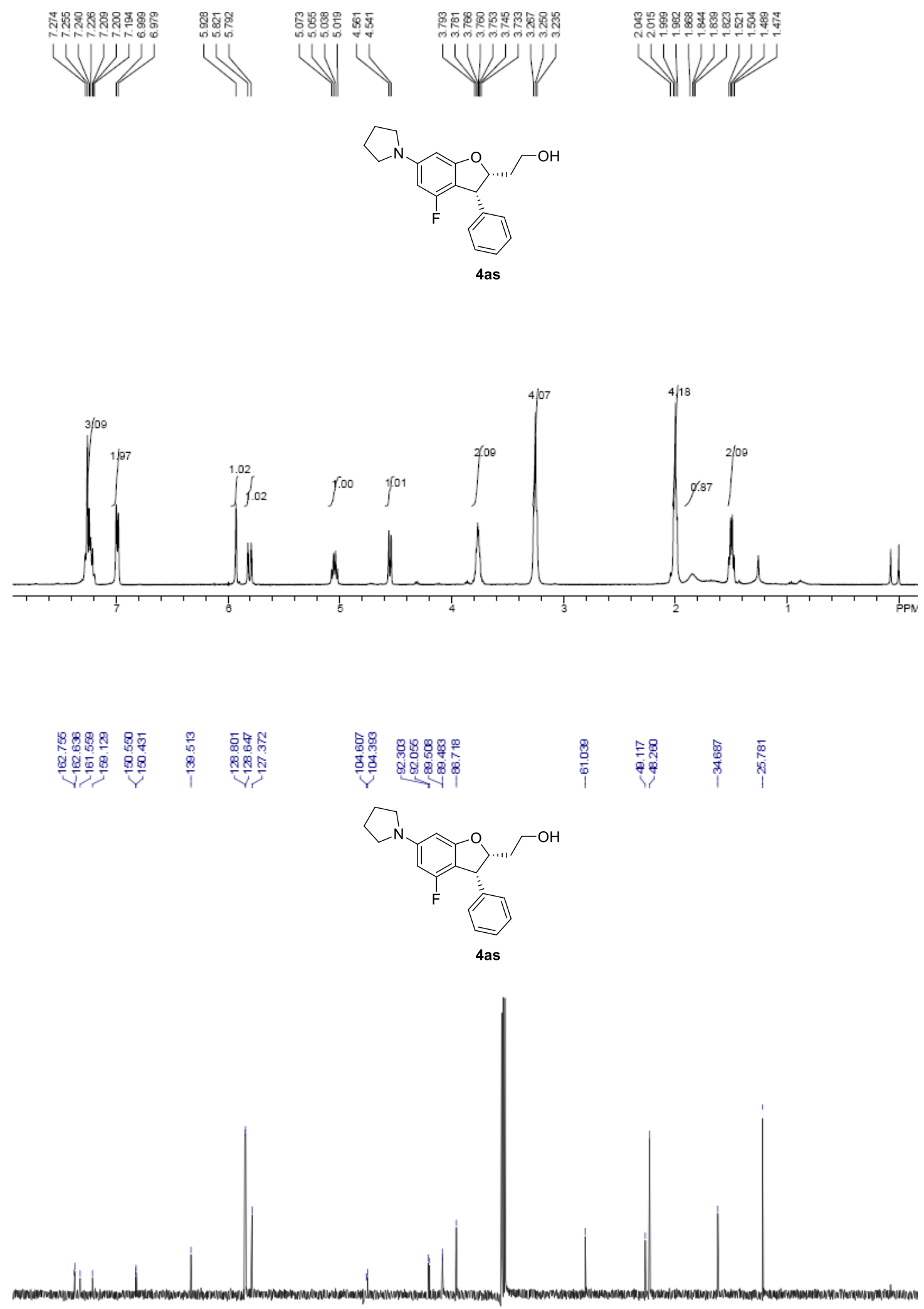


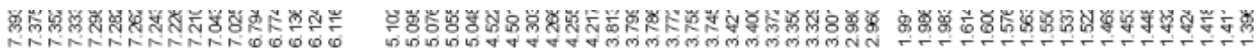

UW
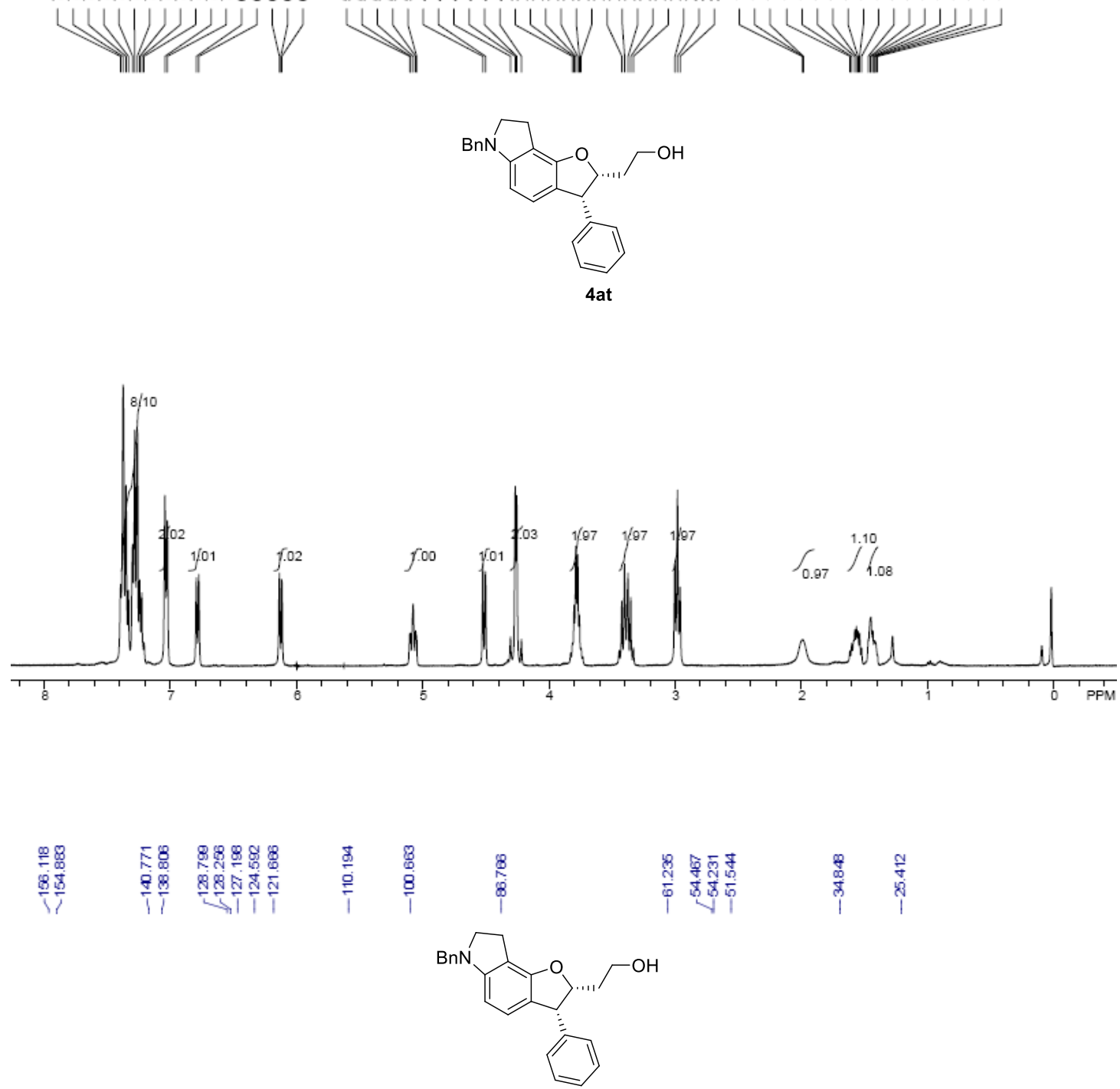

4at

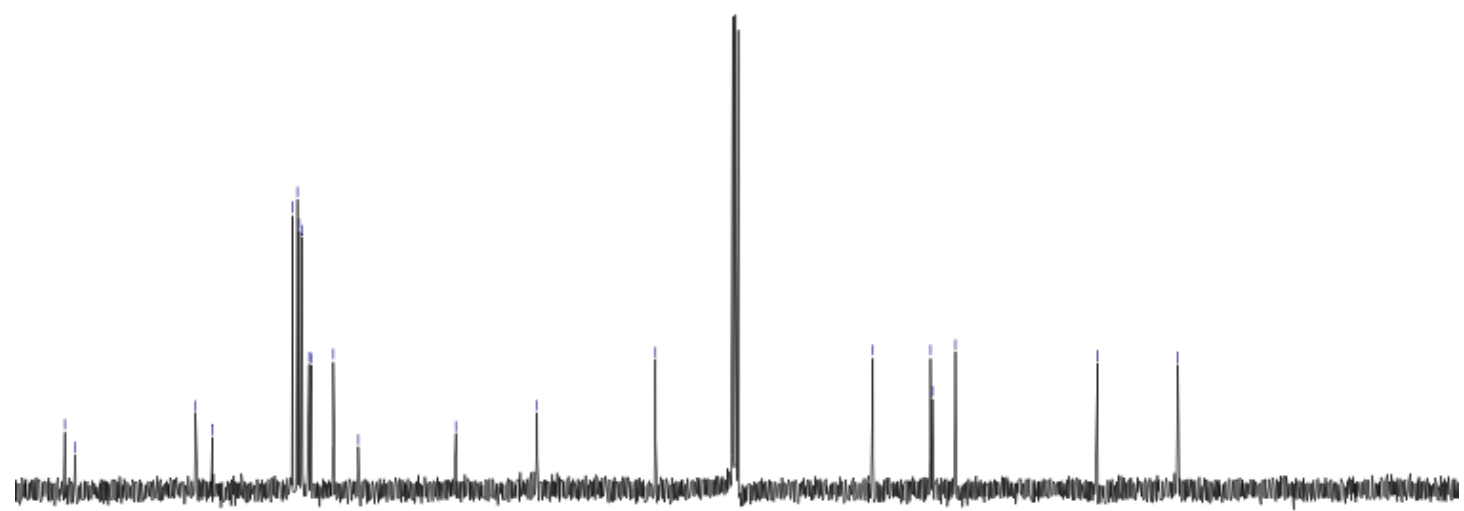

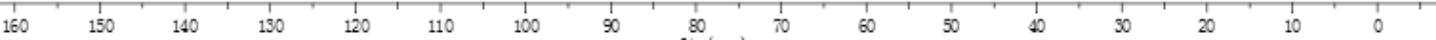




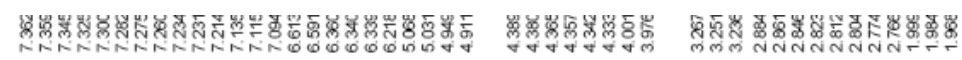

WW W W W W<smiles>O=C(Cc1ccccc1C1OCc2cc(N3CCCC3)ccc21)CC1COc2c(Cl)cccc21</smiles>

$(3 R, 4 S)-6 \mathbf{a}$

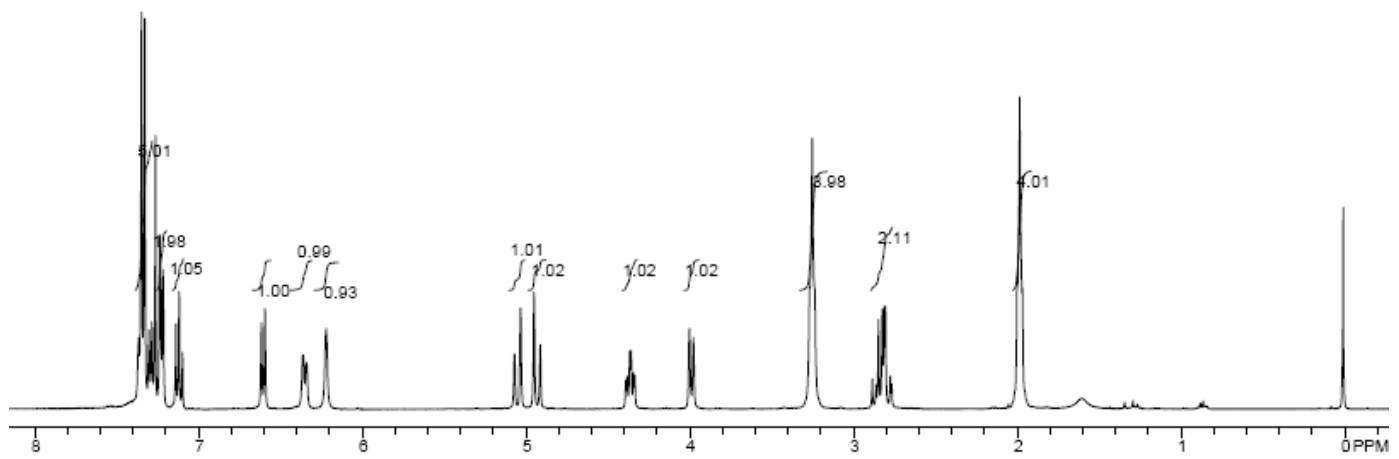

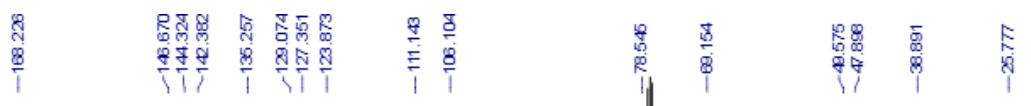

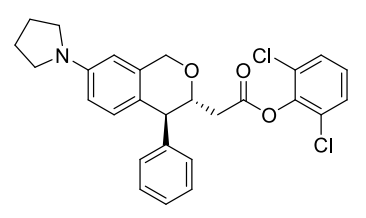

$(3 R, 4 S)-6 \mathbf{a}$

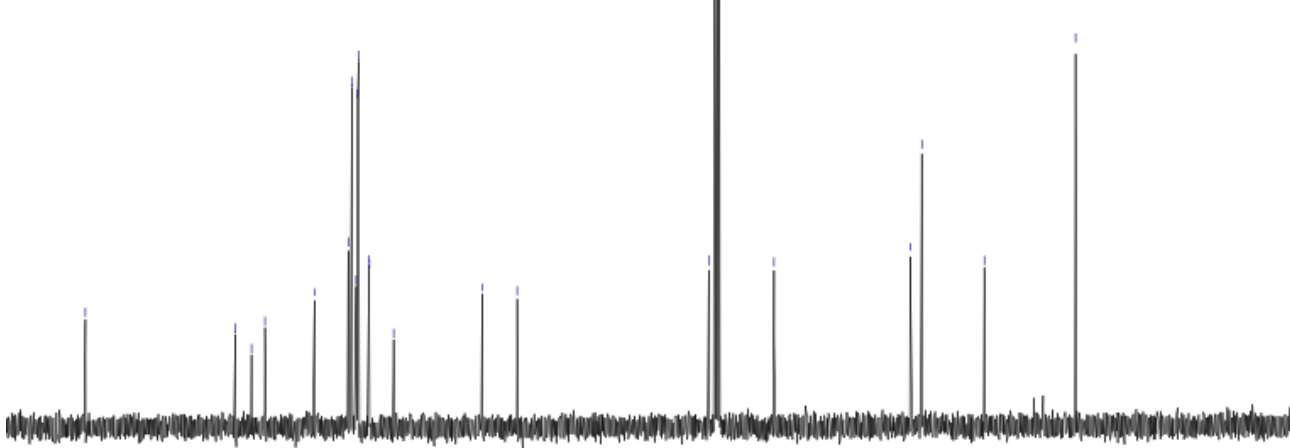

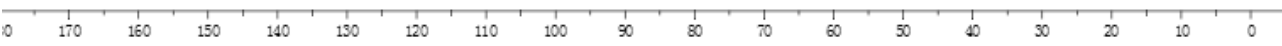




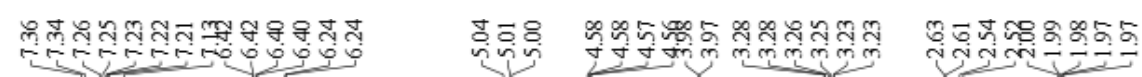
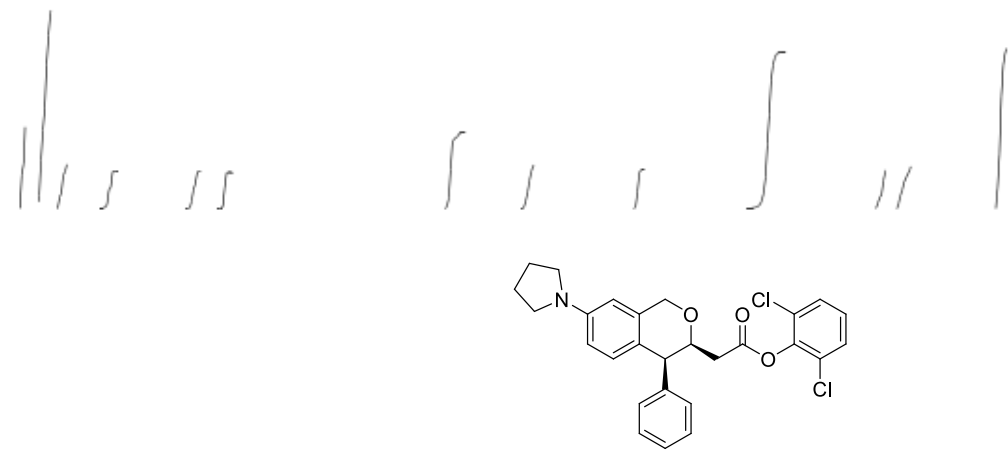

$(3 S, 4 S)-6 \mathbf{a}$
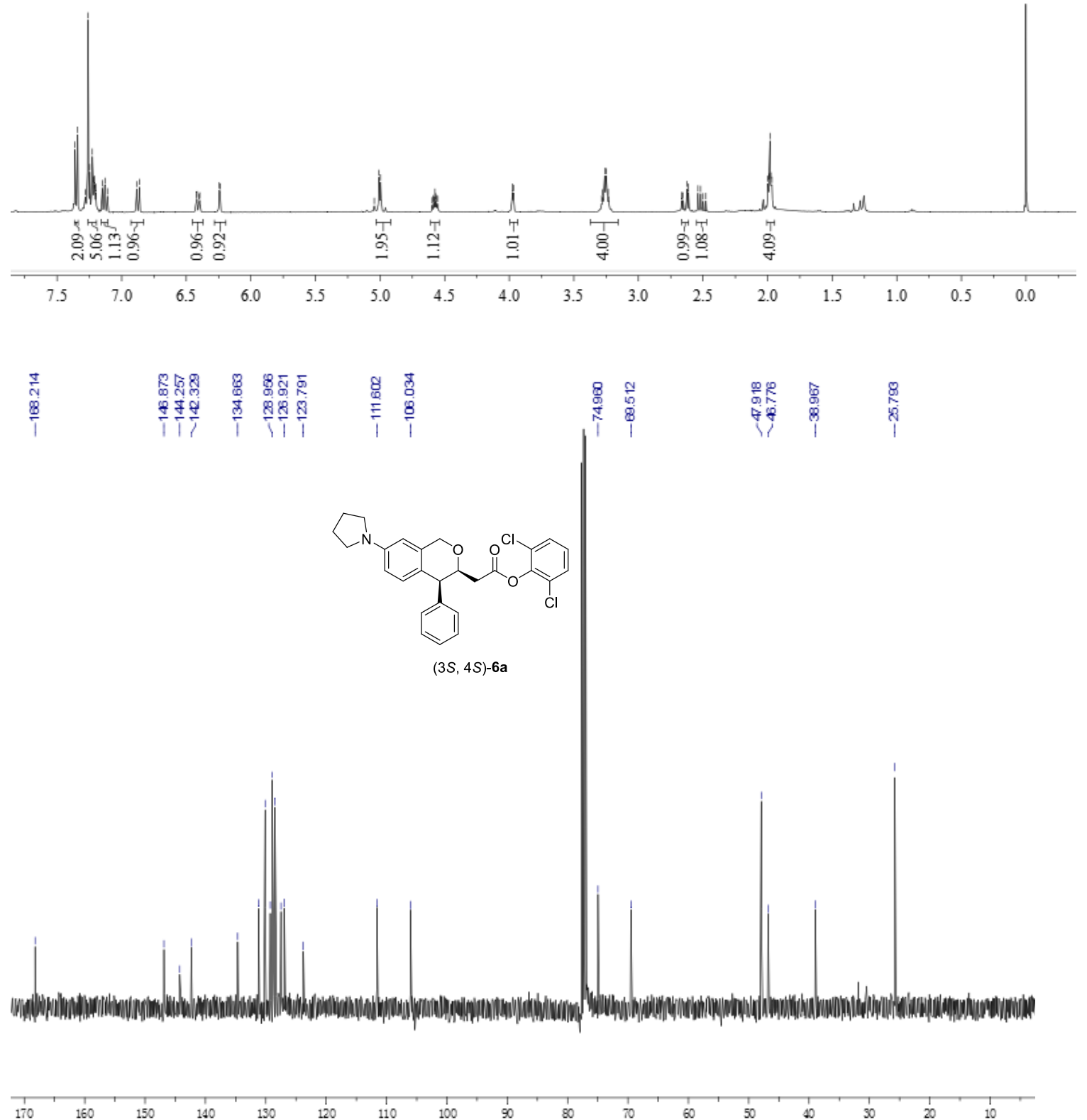


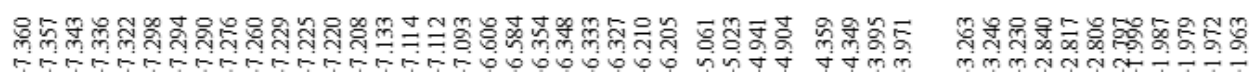

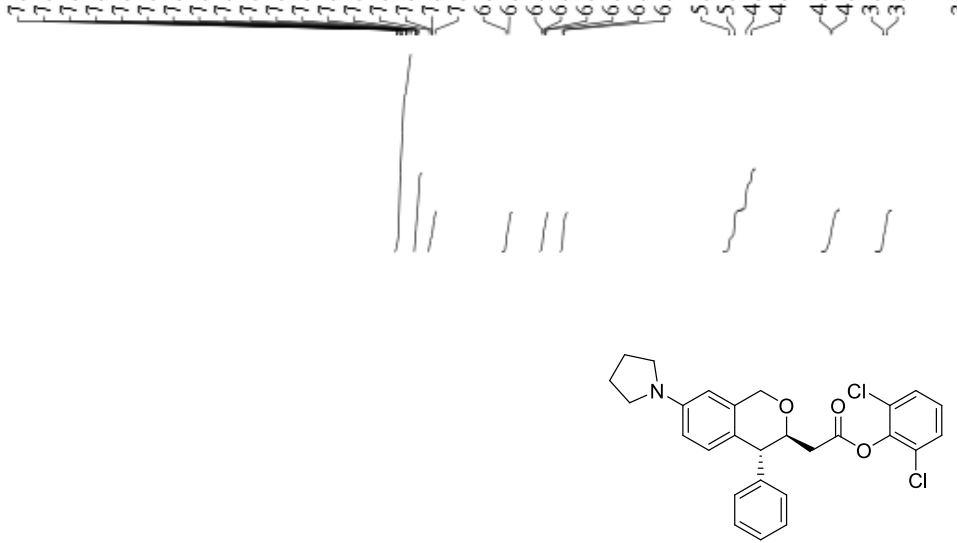

$(3 S, 4 R)-6 \mathbf{a}$

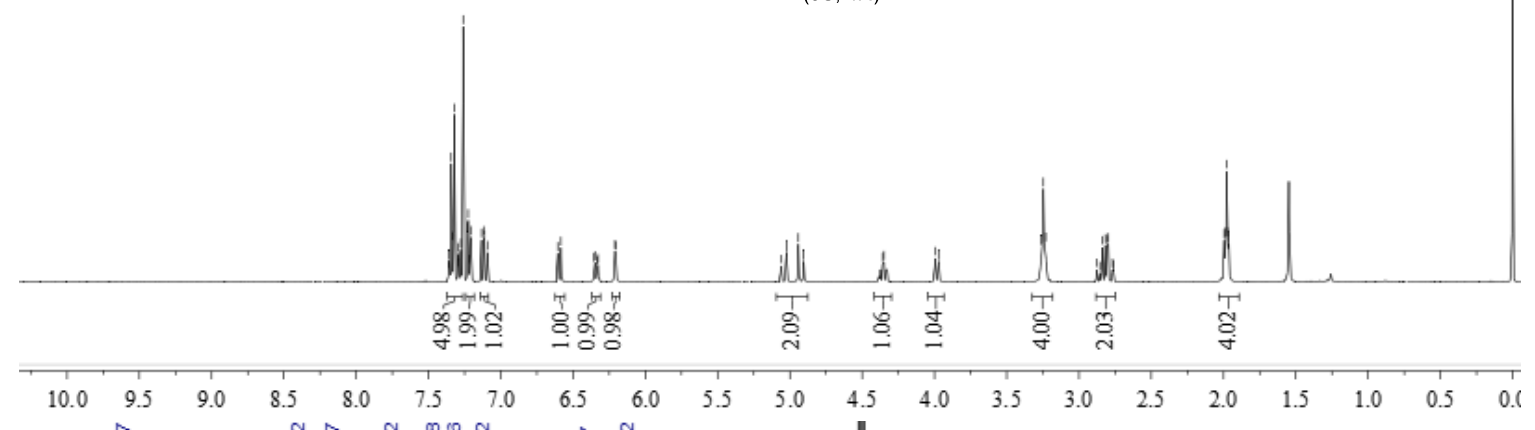

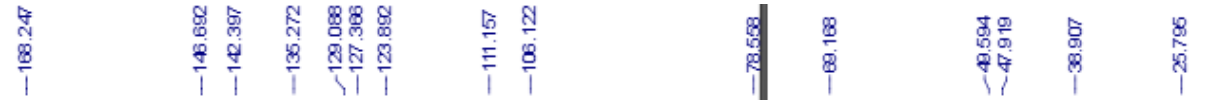

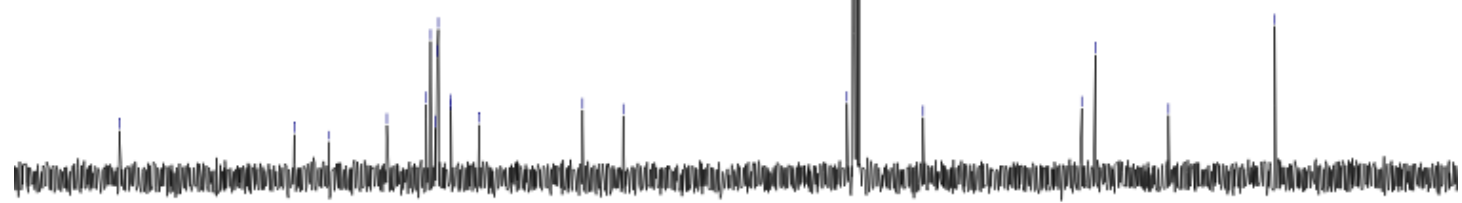

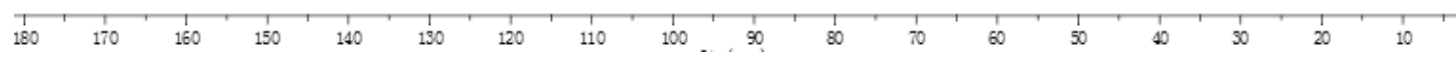




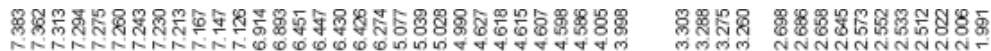

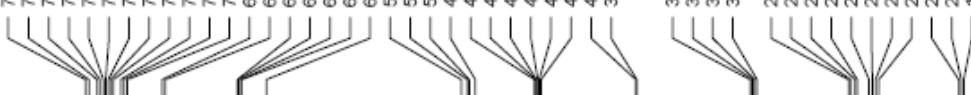<smiles>O=C(C[C@H]1OCc2cc(N3CCCC3)ccc2[C@H]1c1ccccc1)Oc1c(Cl)cccc1Cl</smiles>

$(3 R, 4 R)-6 \mathbf{a}$

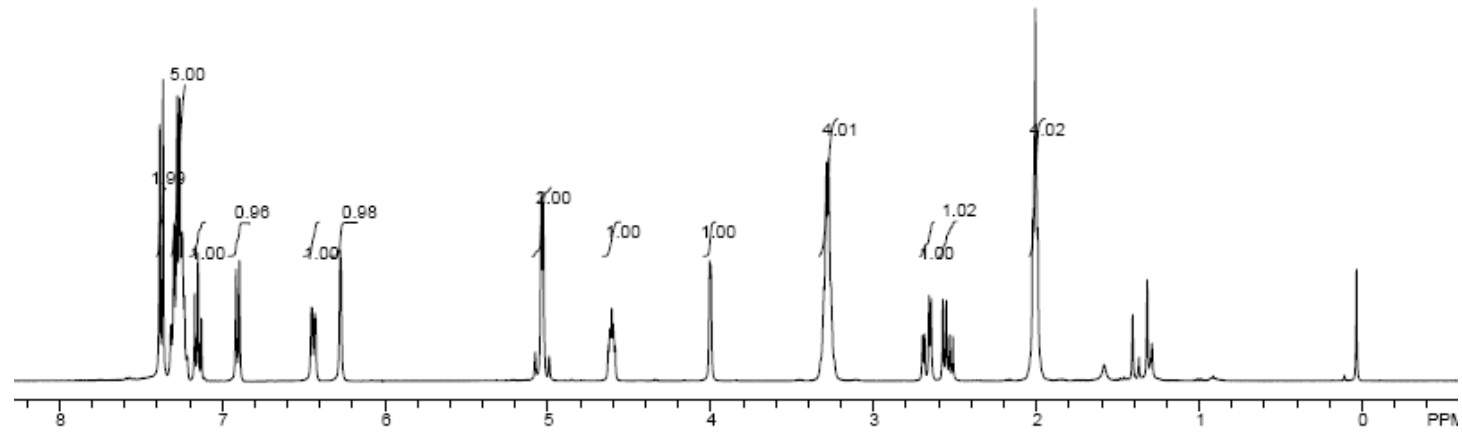

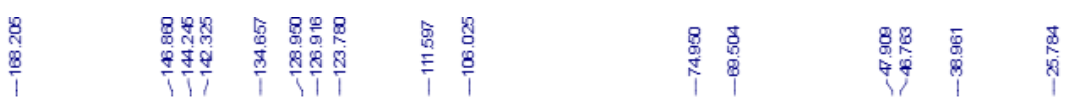

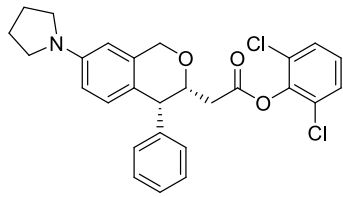

$(3 R, 4 R)-6 \mathrm{a}$

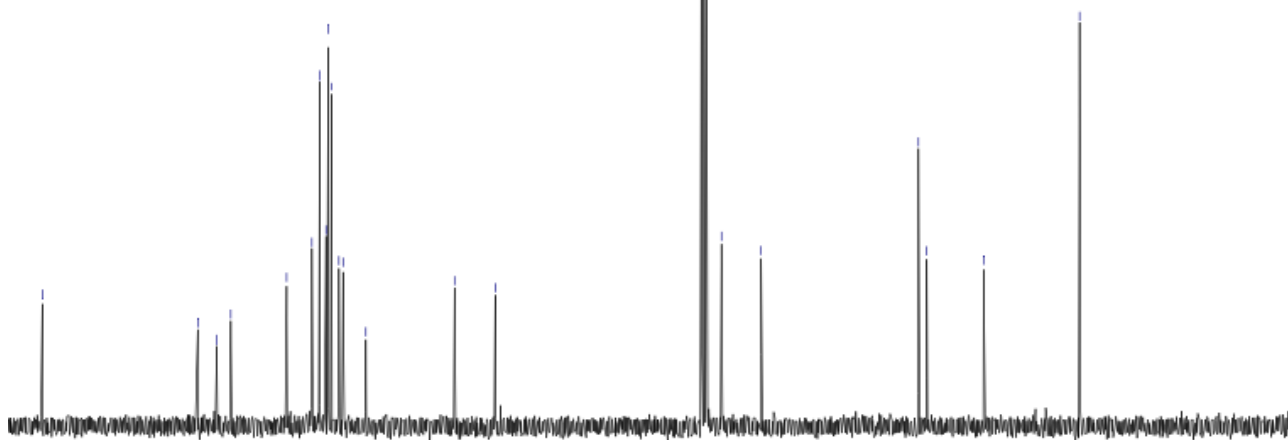

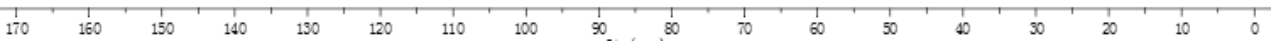




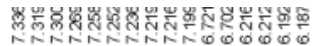

WW W

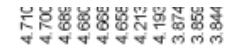

WW

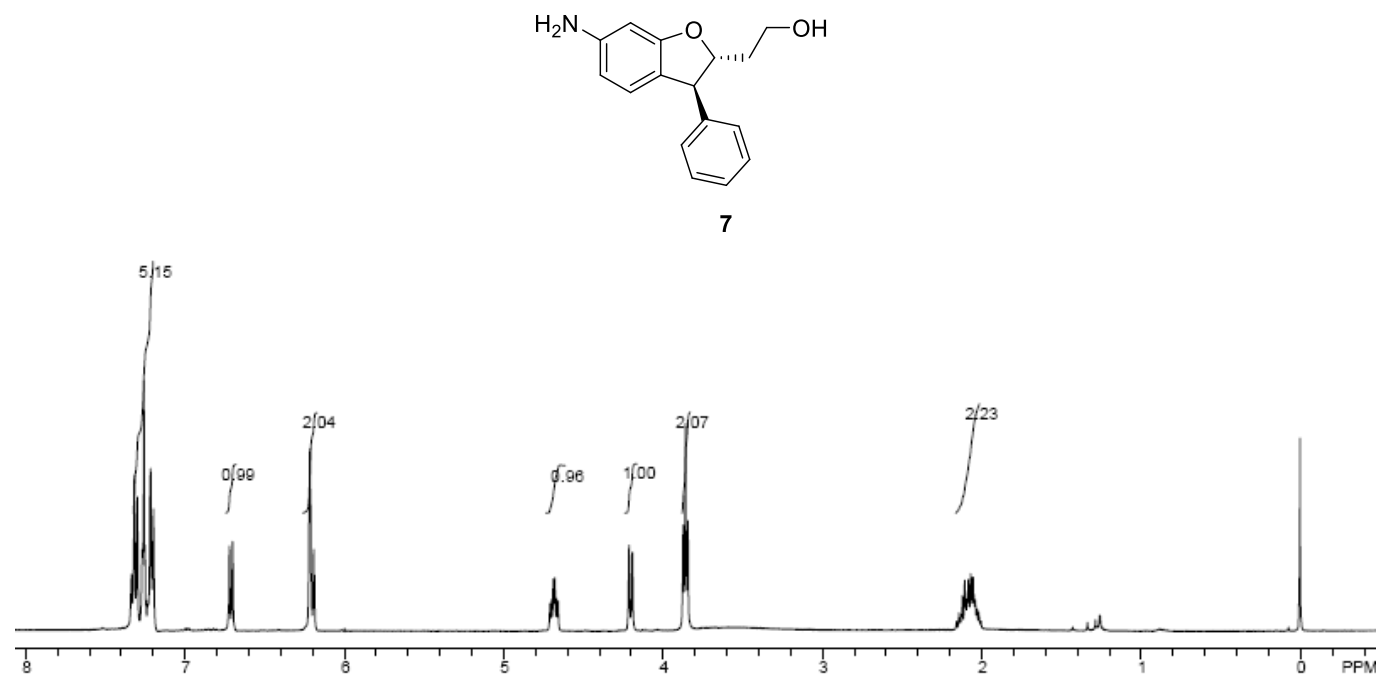

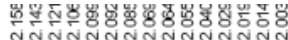

WWWHIJ]

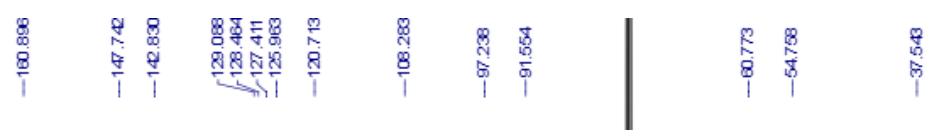<smiles></smiles>

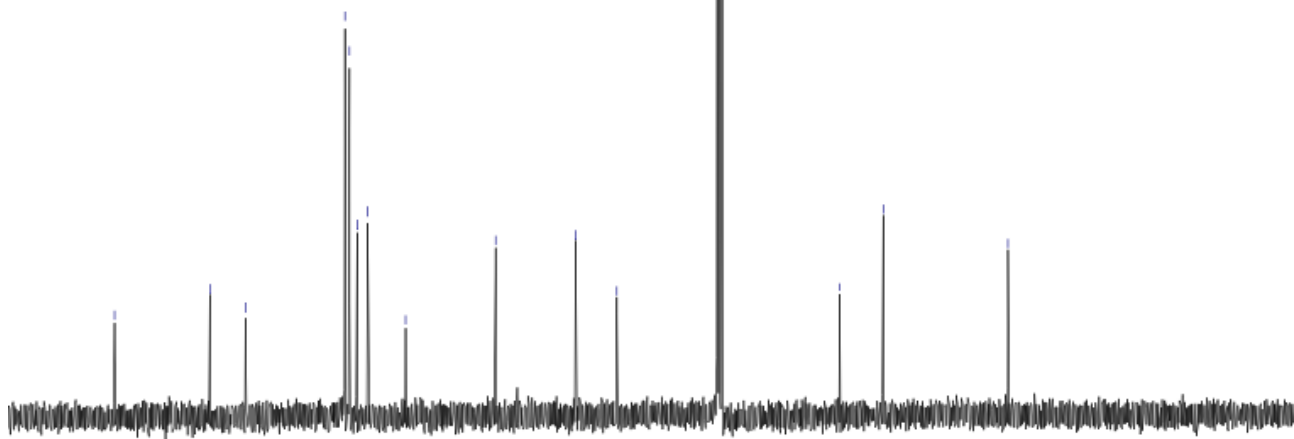

$\begin{array}{llllllllllllllllllll}170 & 160 & 150 & 190 & 130 & 120 & 110 & 100 & 90 & 80 & 10 & 60 & 1 & 10 & 30 & 20 & 10 & 1 & 1 & 1\end{array}$ 

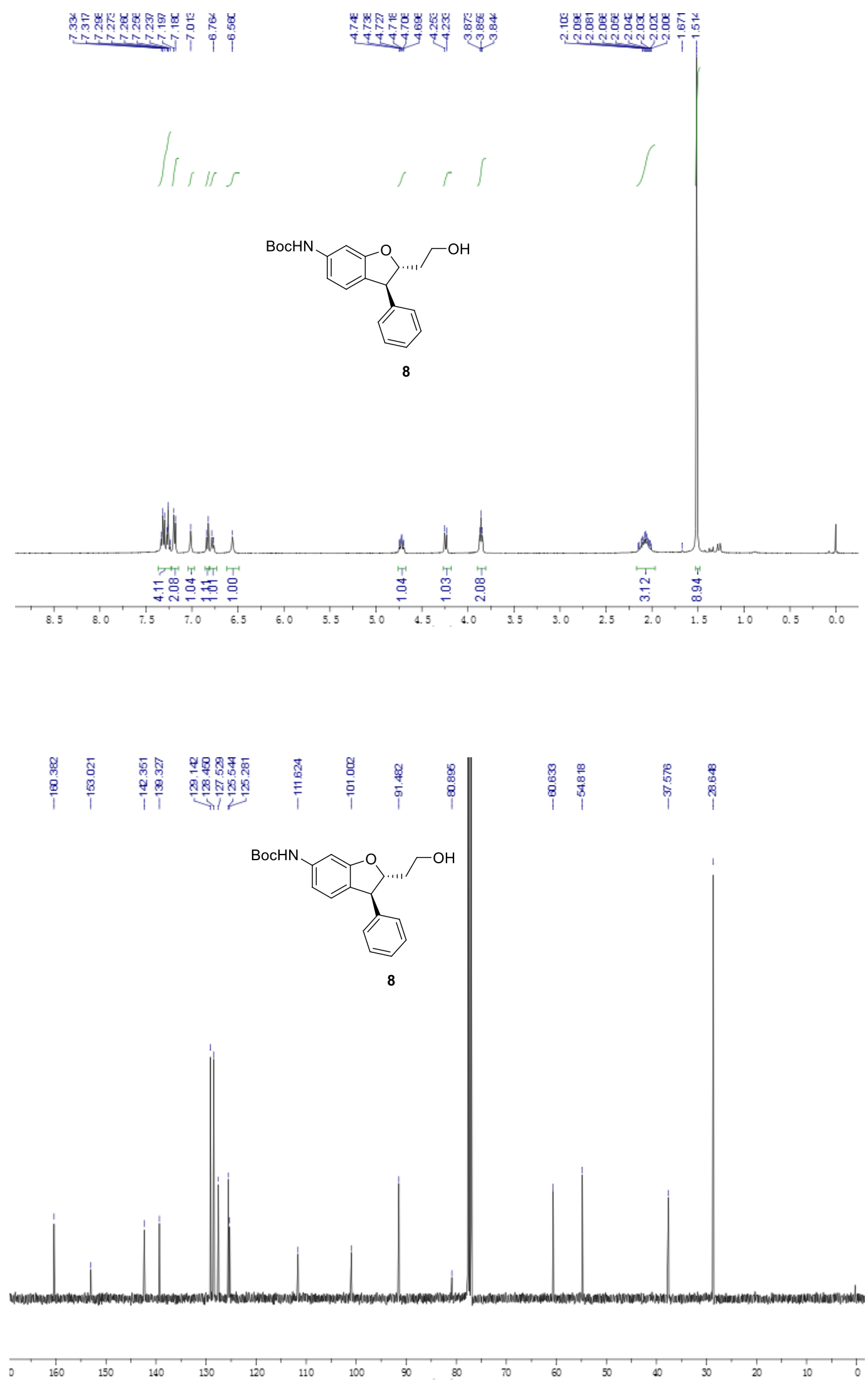